\title{
Olfaction in vector-host interactions
}

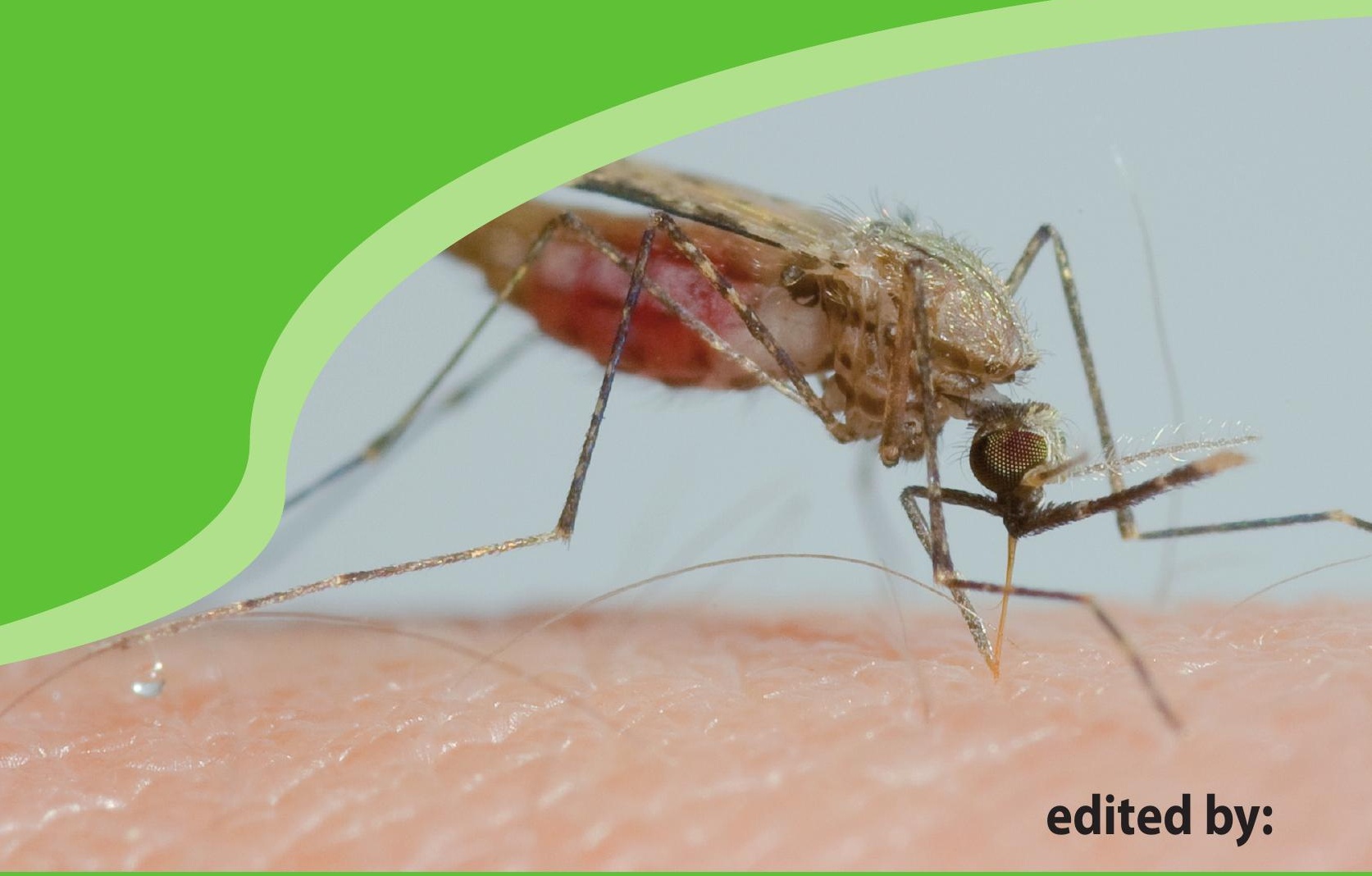

Willem Takken and Bart G.J. Knols

Ecology and control of vector-borne diseases

Volume 2 
Olfaction in vector-host interactions 



\section{Olfaction \\ in vector-host interactions}

Ecology and control of vector-borne diseases

Volume 2

edited by:

Willem Takken

and

Bart G.J. Knols

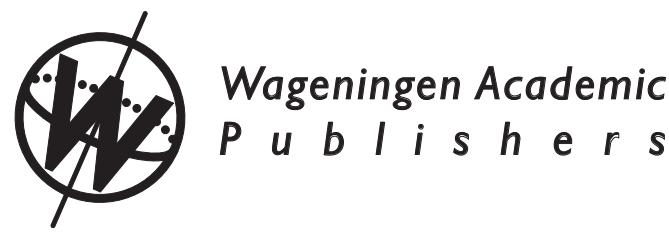


ISBN: 978-90-8686-091-3

e-ISBN: 978-90-8686-698-4

DOI: 10.3920/978-90-8686-698-4

ISSN: 1875-0699

Cover photo: Hans Smid www.bugsinthepicture.com

First published, 2010

(c) Wageningen Academic Publishers

The Netherlands, 2010
This work is subject to copyright. All rights are reserved, whether the whole or part of the material is concerned. Nothing from this publication may be translated, reproduced, stored in a computerised system or published in any form or in any manner, including electronic, mechanical, reprographic or photographic, without prior written permission from the publisher:

Wageningen Academic Publishers, P.O. Box 220, 6700 AE Wageningen, the Netherlands, Copyright@WageningenAcademic.com, www.WageningenAcademic.com

The individual contributions in this publication and any liabilities arising from them remain the responsibility of the authors.

The publisher is not responsible for possible damages, which could be a result of content derived from this publication. 


\section{Ecology and control of vector-borne diseases}

In the past century, many advances were made in the control of vector-borne diseases. Malaria disappeared from the northern hemisphere, diseases such as typhus, Bartonella and yellow fever were seriously reduced in prevalence and in many countries effective methods of disease control contributed to a greatly reduced incidence of such diseases. Most of these advances were beneficial to the industrialised world, whereas underdeveloped countries continued to suffer much as before. Indeed, several diseases such as malaria, Rift Valley fever and African sleeping sickness are still highly prevalent in specific countries. 'New' vector-borne diseases such as dengue, chikungunya fever and West Nile fever, have emerged and are invading previously disease-free regions. The discovery of new drugs and vaccines has made great advances and allows for the effective treatment and control of many diseases. In contrast, vector control has lagged behind in development, even though it is realised that effective vector control would allow for an immediate interruption of the transmission of disease, and aid in disease control and eradication. In the last 20 years new initiatives on vector control have been undertaken, leading to a rapid development of effective and lasting methods of vector control. The development of molecular genetics has provided new insight in vector biology and behaviour, which is being used for developing new strategies of vector control. Advances in geographic information systems allow for precision targeting of interventions. The collective information on new developments in Vector Ecology and Control of Vector-borne Diseases is scattered over numerous periodicals and electronic databases. This book series intends to bring together this information in sequential volumes arranged around selected themes that are currently of interest. Forthcoming themes will include 'Surveillance methods of vectors of infectious diseases,' 'Recent advances in biological control of mosquitoes,' 'Transgenic tools for vector management' and 'Integrated vector management', but also fundamental biological topics such as 'Mating behaviour of disease vectors', 'Oviposition behaviour of disease vectors' and 'Parasite-vector interactions'. Other topics will be added as perceived relevant.

Willem Takken and Bart Knols are well-known experts in the field of Medical and Veterinary Entomology. Both have extensive experience from field work in the tropics and ecological studies in the laboratory and field. They also have a wide experience in publishing and other methods of research dissemination. Willem Takken is Professor in Medical and Veterinary Entomology at Wageningen University. Bart Knols is visiting scientist at the Division of Infectious Diseases, Tropical Medicine \& AIDS of the Academic Medical Centre in Amsterdam, the Netherlands, as well as director of K\&S Consulting, a company engaged in the global dissemination of medical information, organisation building, and consultancies in infectious disease control. 



\section{Table of contents}

$\begin{array}{ll}\text { Preface } & 11\end{array}$

1. Strategic use of chemical ecology for vector-borne disease control 13

Willem Takken and Bart G.J. Knols

2. Molecular regulation of olfaction in mosquitoes

Jonathan D. Bohbot, Tan Lu and Laurence J. Zwiebel

3. Olfactory physiology of blood-feeding vector mosquitoes

Yu Tong Qiu and Joop J.A. van Loon

4. Odour coding and neural connections

63

Rickard Ignell, Meryem S. Sengul, Sharon R. Hill and Bill S. Hansson

5. The detection of carbon dioxide and its role in the orientation to hosts by haematophagous insects

Alan J. Grant and Robert J. O'Connell

6. Host finding by female mosquitoes: mechanisms of orientation to host odours and other cues

Ring T. Cardé and Gabriella Gibson

7. Host-seeking behaviour of mosquitoes: responses to olfactory stimuli in the laboratory Renate C. Smallegange and Willem Takken

8. Host-seeking behaviour of Afrotropical anophelines: field and semi-field studies Wolfgang R. Mukabana, Evelyn A. Olanga and Bart G.J. Knols

Daniel P. Bray, Richard D. Ward and James G.C. Hamilton

10. Understanding and exploiting olfaction for the surveillance and control of Culicoides biting midges

James G. Logan, James I. Cook, A. Jennifer Mordue (Luntz) and Dan L. Kline

11. Black fly interactions with their hosts

James F. Sutcliffe

12. Olfaction in Glossina - host interactions: a tale of two tsetse

Steve J. Torr and Philippe Solano

13. Behavioural modalities of 'non-vector' biting Diptera: from olfaction to feeding

Steve Schofield and Steve J. Torr

14. The role of olfaction in host seeking of triatomine bugs

Pablo G. Guerenstein and Claudio R. Lazzari 
15. Chemical ecology of tick-host interactions

Sandra A. Allan

16. Parasite-mediated enhancement of transmission by haematophagous insects

Hilary Hurd

17. Practical application of olfactory cues for monitoring and control of Aedes aegypti in Brazil: a case study

Alvaro E. Eiras, Martin Geier, Andreas Rose and Owen Jones

18. Exploitation of olfactory-mediated behaviour

Willem Takken and Bart G.J. Knols

About the editors

Contributors

411

Reviewers

Keyword index 




\section{Preface}

The series Ecology and Control of Vector-borne Diseases was launched in 2007 with Volume I providing an overview on the emergence of pests and vector-borne diseases in Europe. This first volume has been well received, and was the starting point of an overview of the state of the art of various topics that are relevant for this field of science. In the past decade the world has witnessed an increasing number of outbreaks of vector-borne diseases as well as invasions of vectors and/ or pathogens. Whereas in the previous century many of these diseases were eliminated from the temperate climate zones, some of them are found to expand their distribution, with invasions of Europe, North America and Australia. Many of these events are associated with environmental change and find their explanation by the often complex ecological processes that drive the transmission of the pathogens. The prevention and control of these diseases requires in-depth knowledge of the arthropods that transmit the pathogens. This book series aims for highlighting specific aspects of the ecology of these vectors that can be exploited for the prevention and control of these diseases.

The current volume addresses the topic of how blood-feeding arthropods interact with their vertebrate hosts. As the transmission of infectious vector-borne pathogens is much dependent on the contact between vector and host, the efficacy of host location is of profound importance. Interruption of vector-host contact is considered one of the most effective means of vector-borne disease control, as is currently witnessed by the successful use of insecticide-treated bed nets for malaria prevention in sub-Saharan Africa.

As with Volume I, this book is a collaboration of a large number of scientists, each experts in their specific field, who have written comprehensive overviews of specific aspects of olfaction-mediated arthropod behaviour. Each chapter was reviewed by independent reviewers, and subsequently adjusted as needed. We are grateful to the anonymous reviewers, as we realise that this takes time and effort. We thank Mike Jacobs and Marijn van der Gaag of Wageningen Academic Publishers for their patience and useful advice. We thank Hans Smid for once more having contributed to the cover design. Special thanks to Françoise Kaminker, who has language-edited several chapters, and provided useful advice.

Wageningen, 8 March 2010

Willem Takken and Bart Knols 



\title{
1. Strategic use of chemical ecology for vector-borne disease control
}

\author{
Willem Takken and Bart G.J. Knols
}

\begin{abstract}
In the epidemiology and control of vector-borne diseases an accurate estimate of parasite and pathogen transmission is required. This can be achieved by monitoring the vector population using traps provided with attractive cues. Volatile chemicals are the principle cues with which the disease vectors locate their vertebrate hosts. Natural and synthetic blends of such chemicals can be used to manipulate the vectors, so that transmission risk can be determined and, in some cases, the vector population can be killed. Novel scientific developments, including molecular genetics, analytic chemistry and odour-release technologies provide opportunities for the effective use of chemical ecology in vector-borne disease control.
\end{abstract}

Keywords: olfaction, insects, ticks, behaviour, disease transmission, nuisance, attractant, repellent

\section{Introduction}

In the last decade nuisance insects and disease vectors have received increasing attention because of their continuing and devastating effects on human and animal health. Examples are recent outbreaks of chikungunya fever in the Indian Ocean, SE India and Italy (Erin Staples et al. 2009), the large number of recorded dengue outbreaks in South America (Barclay 2008) and the arrival of bluetongue virus in NW Europe (Carpenter et al. 2009). In addition, the rapid growth of the world population coupled with increasing poverty in resource-poor countries is the cause of much suffering from neglected vector-borne diseases such as malaria, leishmaniasis and trypanosomiasis (Mathers et al. 2007). Lastly, the current debate on climate change and its potential effects on human health is causing global concern about emerging diseases such as West Nile virus, Lyme disease and other tick-borne diseases (Epstein 2001, Gould and Higgs 2009, Jones et al. 2008). Whereas for several of these diseases effective intervention strategies are available, and hence their control can be implemented, for many of them the current control strategy is inadequate. With some exceptions, effective vaccines are lacking, parasites have developed resistance against drugs, and vector control efforts are hampered by insecticide resistance, technologically inadequate tools and lack of resources.

It is widely agreed that the first step in monitoring vector-borne disease is accurate risk assessment (Hay et al. 2004, Mathers et al. 2007). This information is needed to study the epidemiology of the disease, and to predict its course in time and space. The basic reproductive number $\left(R_{0}\right)$ (Hartemink et al. 2008, Smith et al. 2007) is used to express disease risk, and as the biting rate of disease vectors is one of the critical parameters in $\mathrm{R}_{0}$, tools to accurately measure this are essential. Examples in which these data are being widely used are malaria and dengue control programmes: in endemic situations vector densities provide knowledge on spatial and temporal distribution of biting intensities, while in epidemic situations, vector populations are measured so that the course of the outbreak can be monitored. Also, the outcome of vector-targeted interventions, aimed at the interruption of parasite/pathogen transmission, can be assessed when accurate data on vector densities are available. Vectors are also collected to determine their infections with disease agents: the intensity of disease transmission the product of vector density and parasite/ pathogen infection rate. In spite of the importance of accurate sampling of disease vectors, the methodologies used for insect collection are in many cases based on perception rather than on 
accurate knowledge of vector behaviour. This is because information on vector-host interactions (at which level the parasites/pathogens are being transferred) is in many cases poor or lacking. It is therefore of relevance that this knowledge be expanded in order to obtain better, more precise, data on vector biting density and intensity of parasite transmission.

Since the discovery of the role of arthropods in disease transmission, entomologists have worked on the development of tools for vector surveillance, and a wide range of traps is available with which the vector population can be monitored (Qiu et al. 2007, Service 1993). Traps are designed to attract the target insect(s) using sensory cues such as sound, colour and odour. As chemical signals are the principal cues with which arthropods identify their blood hosts, many traps employ odorants to lure the vectors to the trap. The odorants used vary from natural host odours, synthetic blends of compounds mimicking host odours, or non-host related chemical compounds that have been found to be attractive to the vectors. Harris (1938) and Van Thiel (1935) were among the first to report on the use of host odours to trap tsetse flies and mosquitoes, respectively. It was also established that carbon dioxide $\left(\mathrm{CO}_{2}\right)$ acts as a universal attractant for many bloodfeeding insects, and many traps are baited with this compound. For example, the Centre for Disease Control miniature light trap (CDC light trap) (Sudia and Chamberlain 1962) was reported to produce significantly-enhanced catches of mosquitoes when baited with dry ice (Newhouse et al. 1966). The $\mathrm{CO}_{2}$-baited CDC trap was the gold standard for establishing mosquito densities for many years. In the tropics, $\mathrm{CO}_{2}$ was replaced by natural human emanations by operating the $\mathrm{CDC}$ trap next to a bed net under which a human spent the night (Garrett-Jones and Magayuka 1975). Although these odour-baited traps collected disease vectors, the trap collections represented only crude data on vector densities, and more accurate measurements were needed.

In the 1970s, Vale (1974), dissatisfied with the variable results of odour-baited traps, began to experiment with natural host odours to investigate the behaviour of tsetse flies in Zimbabwe. By using an elegant design of electrified grids and artificial host models, Vale demonstrated that natural host odours were the principle cues that led tsetse flies to the vicinity of their blood hosts (Vale 1993). The studies on tsetse olfactory behaviour are still considered to be classic methods for investigating insect behaviour with respect to their vertebrate hosts. Gillies and Wilkes (1968) demonstrated that mosquitoes could also be manipulated with host odours, and it was soon found that synthetic blends of odorants could be used to manipulate mosquito populations (Takken and Kline 1989). These behavioural studies were gradually augmented with physiological studies to reveal the sensory processes that made the insects respond to the odorants (Cork 1996, Davis and Sokolove 1976). The advent of molecular genetics has allowed for detailed insight in the genetics and molecular regulation of insect olfaction, which provides fundamental knowledge on the regulation of insect behaviour (Jacquin Joly and Merlin 2004, Lessing and Carlson 1999, Touhara and Vosshall 2009). This, in turn is being used for the identification of chemical compounds that affect vector-host interactions, so that the target vectors can be more effectively lured to sampling devices and/or removed from the environment as a tool for disease control.

In future, odorants will increasingly be used for the monitoring of vector-borne disease transmission risk as well as for vector control. It is therefore appropriate that the current volume of the Ecology and Control of Vector-borne Diseases has brought together reviews of the stateof-the-art of this topic, from the molecular regulation of olfactory senses (Chapter 2) to field applications of odorants for disease control (Chapter 17). As many disease vectors belong to the insect order of Diptera, this group of insects is discussed in detail, with mosquitoes being the example for detailed explanation of olfactory regulation. Chapters on blood-feeding bugs, vectors of Chagas disease, and blood-feeding ticks, vectors of a variety of parasites and pathogens, are 
included as well. Finally, as it is increasingly becoming apparent that the olfactory behaviour of insects and ticks is often mediated by parasites, a chapter on the latest knowledge of this field of science is also included. Odorants are not only used to attract insects and ticks, but they can also be used to protect blood hosts from being attacked (Katz et al. 2008). In many cases odorants act as deterrents or repellents, and where applicable, this aspect of olfaction is discussed as well.

The ecology of vector-borne diseases is much determined by the association between vectors, parasites and hosts, which is in turn strongly mediated by chemical cues. The chapters in this volume demonstrate that detailed studies on insect olfaction contribute to our understanding of the individual components regulating this process, and contribute to finding effective solutions for disease control.

\section{References}

Barclay E (2008) Is climate change affecting dengue in the Americas? The Lancet 371: 973-974.

Carpenter S, Wilson A and Mellor PS (2009) Culicoides and the emergence of bluetongue virus in northern Europe. Trends Microb 17: 172-178.

Cork A (1996) Olfactory basis of host location by mosquitoes and other haematophagous Diptera. In: Bock GR and Cardew G (eds) Olfaction in mosquito host interactions. CIBA Foundation Symposium 200. Wiley, Chichester, UK, pp 71-88.

Davis EE and Sokolove PG (1976) Lactic acid-sensitive receptors on the antennae of the mosquito, Aedes aegypti. J Comp Physiol 105: 43-54.

Epstein PR (2001) Climate change and emerging infectious diseases: review. Microbes and Infection 3: 747-754.

Erin Staples J, Breiman RF and Powers AM (2009) Chikungunya fever: an epidemiological review of a re-emerging infectious disease. Clinical Inf Dis 49: 942-948.

Garrett-Jones C and Magayuka SA (1975) Studies on the natural incidence of Plasmodium and Wuchereria infections on Anopheles in rural East Africa: I-assessment of densities by trapping hungry female Anopheles gambiae Giles, Species A. WHO/VBC 75.145 Geneva Switzerland.

Gillies MT and Wilkes TJ (1968) A comparison of the range of attraction of animal baits and of carbon dioxide for some West African mosquitoes. Bull Entomol Res 59: 441-456.

Gould EA and Higgs S (2009) Impact of climate change and other factors on emerging arbovirus diseases. Trans Royal Soc Trop Med and Hygiene 103: 109-121.

Harris RHTP (1938) The control and possible extermination of the tsetse by trapping. Acta Conventus Tertii de Tropicis Atque Malariae Morbis 1:663-677.

Hartemink NA, Randolph SE, Davis SA and Heesterbeek JA (2008) The basic reproduction number for complex disease systems: defining R(0) for tick-borne infections. American Naturalist 171: 743-754.

Hay SI, Guerra CA, Tatem AJ, Noor AM and Snow RW (2004) The global distribution and population at risk of malaria: past, present, and future. Lancet Infectious Diseases 4: 327-336.

Jacquin Joly E and Merlin C (2004) Insect olfactory receptors: contributions of molecular biology to chemical ecology. J Chem Ecology 30: 2359-2397.

Jones KE, Patel NG, Levy MA, Storeygard A, Balk D, Gittleman JL and Daszak P (2008) Global trends in emerging infectious diseases. Nature 451: 990-993.

Katz TM, Miller JH and Hebert AA (2008) Insect repellents: historical perspectives and new developments. J Am Acad Derm 58: 865-871.

Lessing D and Carlson JR (1999) Chemosensory behaviour: the path from stimulus to response. Curr Opinion Neurobiol 9: 766-771.

Mathers CD, Ezzati M and Lopez AD (2007) Measuring the burden of neglected tropical diseases: the global burden of disease framework. PLoS Negl Trop Dis 1: e114. 
Newhouse VF, Chamberlain RW, Johnston JG and Sudia WD (1966) Use of dry ice to increase mosquito catches of the CDC miniature light trap. Mosquito News 26: 30-35.

Qiu YT, Spitzen J, Smallegange RC and Knols BGJ (2007) Monitoring systems for adult insect pests and disease vectors. In: Takken W and Knols BGJ (eds) Ecology and control of vector-borne diseases, Vol. 1: emerging pests and vectorborne diseases in Europe. Wageningen Academic Publishers, Wageningen, the Netherlands, pp 329-353.

Service MW (1993) Mosquito ecology - field sampling methods. second edn. Elsevier Applied Science, London, UK.

Smith DL, McKenzie FE, Snow RW and Hay SI (2007) Revisiting the basic reproductive number for malaria and its implications for malaria control. Plos Biology 5: e42.

Sudia WD and Chamberlain RW (1962) Battery-operated light trap, an improved model. Mosquito News 22: 126-129.

Takken W and Kline DL (1989) Carbon dioxide and 1-octen-3-ol as mosquito attractants. J Am Mosquito Control Ass 5:311-316.

Touhara K and Vosshall LB (2009) Sensing odorants and pheromones with chemosensory receptors. Annual Rev Physiol 71: 307-332.

Vale GA (1974) New field methods for studying the responses of tsetse flies (Diptera, Glossinidae) to hosts. Bull Ent Res 64: 199-208.

Vale GA (1993) Development of baits for Tsetse flies (Diptera: Glossinidae) in Zimbabwe. J Med Ent 30: 831-842.

Van Thiel PH (1935) Onderzoekingen omtrent den gedrag van Anopheles ten opzichte van mensch en dier, mede in verband met rassenstudie bij Anopheles maculipennis (Studies on the behaviour of Anopheles around humans and animals, associated with a species-specific study of Anopheles maculipennis). Gen Tijdschr Ned Indië 75:2101-2181. 


\title{
2. Molecular regulation of olfaction in mosquitoes
}

\author{
Jonathan D. Bohbot, Tan Lu and Laurence J. Zwiebel
}

\begin{abstract}
Recent advances in our understanding of the molecular mechanisms of mosquito olfaction have opened opportunities to approach behavioural disruption strategies of disease vectors from a new vantage point. The genome sequence projects of Anopheles gambiae, Aedes aegypti and Drosophila melanogaster have allowed the identification of complete gene families that are involved in odorant sensing and signal regulation. Odorant receptor proteins, in particular, have been shown to be directly involved in olfactory transduction. The function of these receptors in various head appendages of the larval and adult mosquitoes has also raised new fundamental questions pertaining to the nature and mechanisms of olfaction in these insects.
\end{abstract}

Keywords: gene, g-protein coupled receptor (gpcr), labellum, maxillary palp, odorant-binding protein (obp), odorant receptor (or), signal transduction

\section{Introduction}

Eighty years have elapsed from the initial published (Rudolfs 1922) evidence of olfactory-driven behaviour of mosquitoes to the most recent discovery and characterisation of several olfactory gene families in Anopheles gambiae Giles (Fox et al. 2001, Hill et al. 2002), Aedes aegypti (L.) (Bohbot et al. 2007, Nene et al. 2007) and Culex pipiens quinquefasciatus Say (unpublished at the time of writing). Emphasis on these three mosquito species derives from their roles as vectors of the pathogens responsible for several critical human infectious diseases including malaria, West Nile disease, dengue fever, yellow fever, Rift Valley fever, chikungunya disease, leishmaniasis, filiariasis, various arboviral encephalitis and others. In these cases the mosquito's vectorial capacity is not only regulated by internal factors (physiological state, see Chapter 2 in this volume), but is also mediated by sensory inputs derived from heat, visual and most importantly, olfactory stimuli (Figure 1).

Adult anophelines are attracted to odorant stimuli that range from single compounds to complex odour blends (reviewed in Knols 1996). For example, Ae. aegypti is attracted by a mixture of $\mathrm{CO}_{2}$, ammonia, L-lactic acid and fatty acids (Bosch et al. 2000, Geier et al. 1999, Kellogg 1970, Steib et al. 2001) while An. gambiae is attracted by $\mathrm{CO}_{2}$, lactic acid, acetone and 1-octen-3-ol (Dekker et al. 2002, Healy and Copland 1995, 2000, Smallegange et al. 2005, Takken et al. 1997). In nature, mosquitoes rely principally on olfaction to locate and choose oviposition (Takken and Knols 1999), nectar-feeding sites (Foster 1995) and mates (Cabrera and Jaffe 2007, Nijhout and Craig 1971) to name but a few critical life-cycle behaviours.

The availability of the complete Ae. aegypti (Nene et al. 2007) and An. gambiae (Holt et al. 2002) genomes has facilitated the identification and annotation of a handful of gene families, which are potentially involved in the regulation of olfactory behaviours. This effort was further enhanced by the pioneering studies undertaken to similarly analyse the Drosophila melanogaster Meigen genome (Adams et al. 2000). Indeed, comparative genomics using these three mosquito species has provided insights into the evolution of many gene families and the possible conserved function shared by homologous genes. Functional characterisation of olfactory mechanisms in mosquitoes is a more recent development and combines information from electrophysiological 


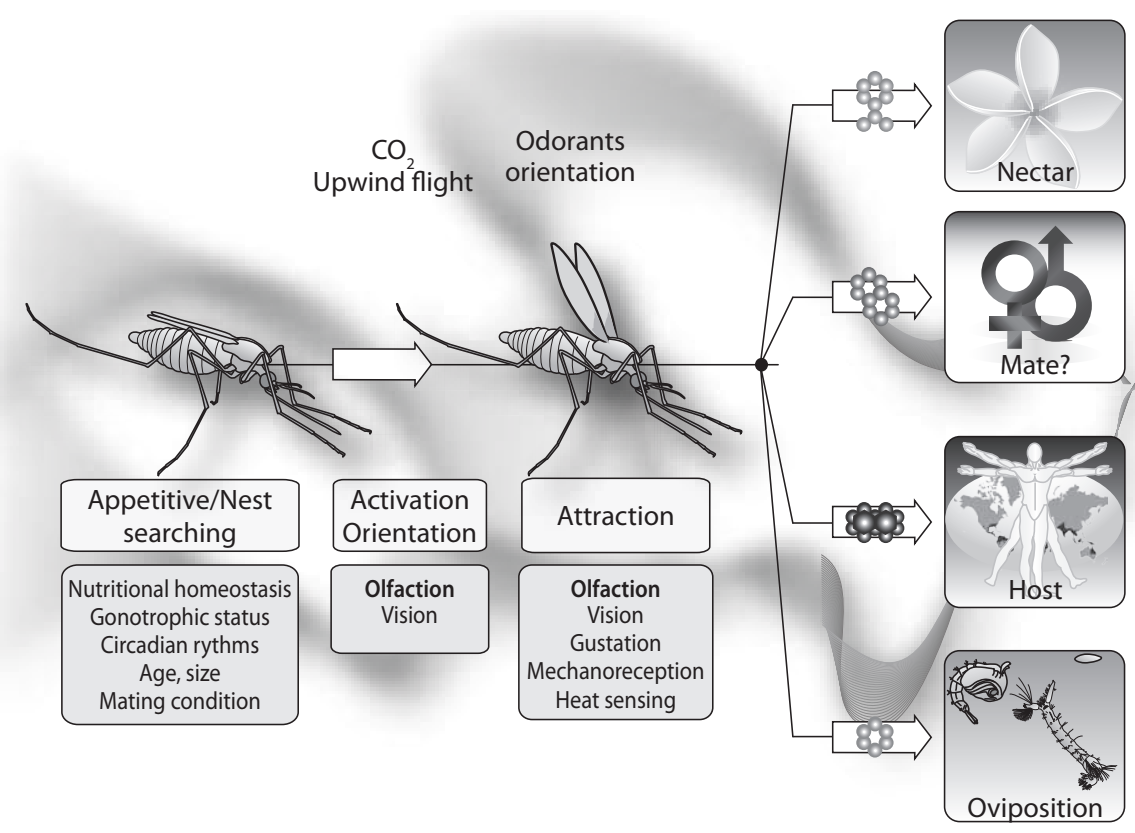

Figure 1. The physiological and sensory basis for mosquito behaviour. Foraging, lekking, preying and nesting behaviours are modulated by internal and external signals. The reproductive, sexual, nutritional and developmental states and temporal factors including the circadian clock all influence the mosquito's responses to external stimuli, which rely on a combination of sensory modalities.

studies as well as advances in the use of heterologous expression systems including cell-based assays and the use of Drosophila as a transgenic platform for the study of mosquito genes.

The sensory modality of olfaction is a form of chemoreception specifically dealing with volatile stimuli and essentially represents the interface between the organism and its immediate environment. It requires the activity of highly complex peripheral signal transduction cascades to transform air-borne chemical signals into precise patterns of neuronal activity. At the cellular level in insects this process is focused on a collection of olfactory receptor neurons (ORNs) that are encased in small, perforated cuticular structures called sensilla and are distributed on the surface of various head appendages (Figure 2). The olfactory signal transduction events take place on the ORN dendrites and their underlying mechanism remains largely uncharacterised and controversial despite intensive research efforts.

This chapter will review the current knowledge of the molecular components implicated in insect and especially mosquito olfactory transduction. It will attempt to provide the most recent theory of olfactory transduction based on current experimental data focusing on two mosquito species, An. gambiae and Ae. aegypti, and from Drosophila. While Drosophila remains the most robust genetic model for the study of insect olfaction, significant progress has been made toward the identification and functional characterisation of mosquito olfactory genes. In the following paragraphs, we will look at the contribution of genome sequencing projects to the identification and phylogenetic analyses of olfactory proteins. Rather than merely cataloguing the olfactory 


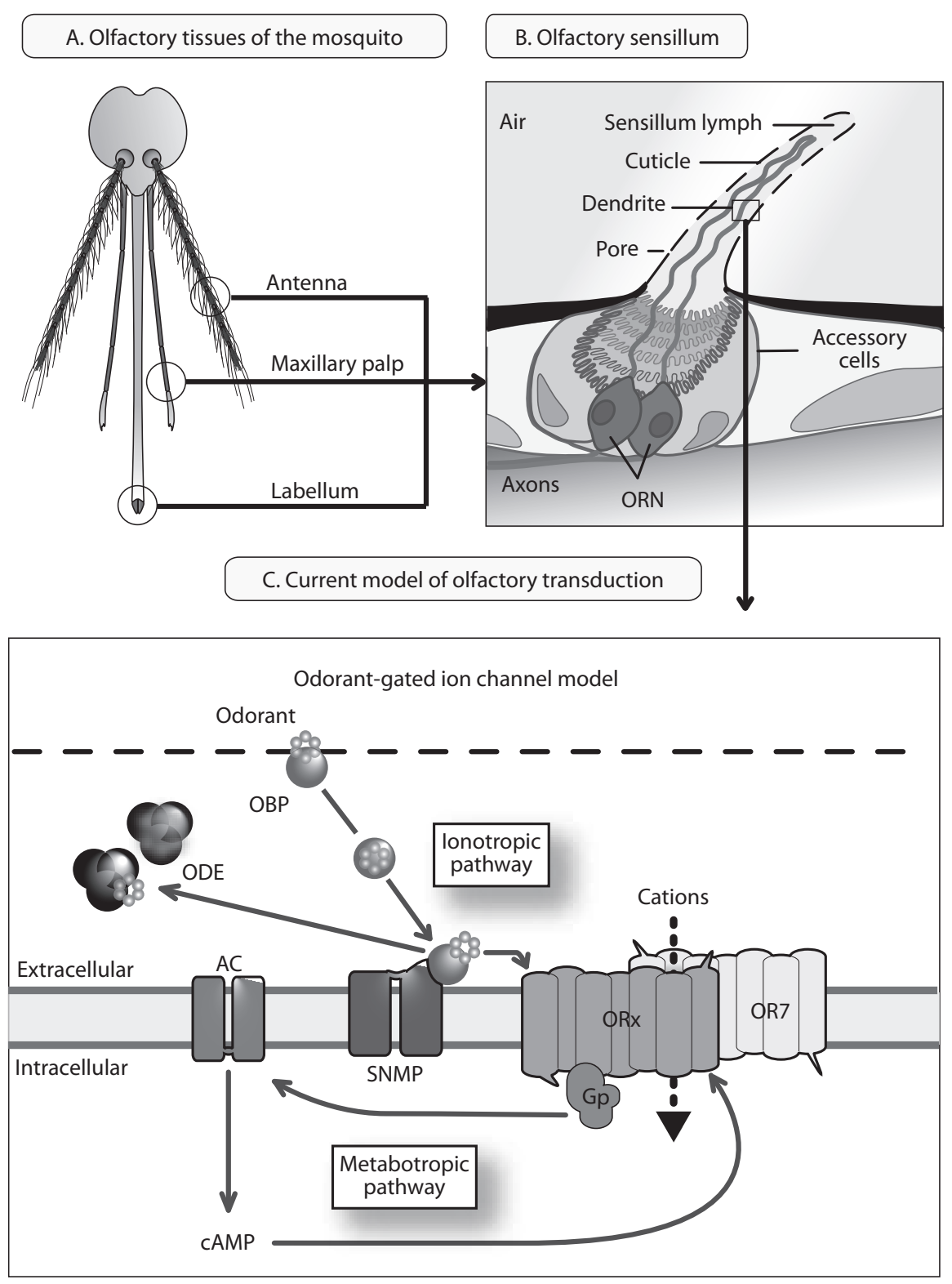

Figure 2. Olfactory transduction theory in mosquitoes. (A) The antenna, the maxillary palp and the labellum of mosquitoes are the three sites of olfactory sensing. (B) Sensory organs called sensilla cover these tissues and house Odorant Receptor Neurons (ORNs), which are dedicated to convert odorant signals into electrical outputs. Several molecular players have been involved in this transduction pathway. (C) Odorant molecules access the sensillum lymph through pores in the cuticles and are shuttled to a membrane protein complex constituted of a Sensory Neuron Membrane Protein (SNMP) and an Odorant Receptor (OR) heteromer (ORx/OR7). In this model, $O R$ activation triggers two responses. The fast response involves a direct effect on the receptors, which act as an odorant-gated ion channel. A slower response is mediated via a $G$ protein (Gp) dependent secondary messenger pathway including Adenyl Cyclase (AC) and cyclic AMP (CAMP) (Sato et al. 2008, Wicher et al. 2008). 
gene families and their hypothetical operative machinery, we will attempt to provide a biological context for these olfactory components as derived from functional data as well as studies that have elucidated the distribution of olfactory genes in various mosquito olfactory tissues and developmental stages.

\section{Mosquito olfactory transduction: a road map under construction}

Olfaction is a key sensory modality that modulates host-seeking and other behaviours in mosquitoes (Figure 1). Over two decades of research on invertebrate olfaction have identified several molecular components that may safely be stated to be involved in the biochemical processes responsible for the detection and transduction of odorant stimuli. These include arrestins (Arrs), G-proteins, odorant-binding proteins (OBPs), odorant degrading enzymes (ODEs), odorant receptors (ORs) and sensory neuron membrane proteins (SNMPs).

While the structures of insect ORs have not been precisely determined, there is little doubt that they function as a heteromeric complex (Benton et al. 2006) and play a central role in odorant detection and the activation of downstream transduction machinery. Insect ORNs generally express a conventional OR conferring its odorant-binding specificity together with a highly conserved non-conventional OR belonging to the OR83b sub-family of proteins (Jones et al. 2005, Krieger et al. 2003, Larsson et al. 2004, Pitts et al. 2004). Pioneering experiments in heterologous expression systems have demonstrated that heterodimeric Drosophila ORs (DORs) elicit cellular responses following odorant stimulation (Neuhaus et al. 2005, Wetzel et al. 2001), effectively defining their function as bona fide ORs. This functionality has been repeatedly validated with $A n$. gambiae (Ditzen et al. 2008, Hallem et al. 2004a, Kwon et al. 2006, Lu et al. 2007, Sato et al. 2008, Xia et al. 2008), Bombyx mori Linnaeus (Nakagawa et al. 2005, Syed et al. 2006), Heliothis virescens (Fabricius) (Grosse-Wilde et al. 2007) and Apis mellifera Linnaeus ORs (Wanner et al. 2007). Not surprisingly, these data indicate that the expression of a given Or gene in insect ORNs determines their functional specificity (Dobritsa et al. 2003, Hallem et al. 2004a, Hallem et al. 2004b, Jones et al. 2005).

Whereas the functional role of insect ORs is well established, the molecular events preceding and following their activation remain decidedly unclear although our understanding of the processes has dramatically improved of late. Extracellular events associated with odorant detection involve soluble OBPs and ODEs (Figure 2), and SNMPs are thought to establish a molecular bridge specifically between ORs and pheromone ligands. While the precise biochemical processes leading to the activation of downstream molecular targets are not expressly defined, recent reports suggest that insect ORs exhibit either ionotropic or alternatively both iono- and metabotropic characteristics. On the one hand, the rapid response kinetics of odorant receptors observed in heterologous expression systems are consistent with the behaviour of ligand-gated ion channels that act independently of G-protein signaling (Sato et al. 2008). In contrast, another study indicates an additional metabotropic response requiring the recruitment of G-proteinmediated second-messenger pathways that is delayed and slower relative to the initial ionotropic response (Wicher et al. 2008). Although the question remains open, the incorporation of G-protein dependent pathways that presumably require the synthesis of second-messenger intermediates is also consistent with numerous studies linking ORs to GPCR signalling pathways (Kain et al. 2008, Kalidas and Smith 2002, Woodard et al. 1992). 


\section{Structural and evolutionary aspects of the mosquito olfactory gene families}

\section{Odorant-binding and chemosensory proteins}

Two classes of soluble proteins, the OBPs and chemosensory proteins (CSPs), have been found in the lumen of chemosensory sensilla of insects. Odorant-binding proteins were first discovered in 1981 in the silk moth Antheraea polyphemus (Cramer) (Vogt and Riddiford 1981) and in the cow (Pelosi et al. 1982). While sharing the same name, mammalian OBPs are characterised as lipocalin family proteins (Flower et al. 2000), and insect OBPs are not phylogenetically related and, not surprisingly, have entirely different structures. Classic insect Obp genes encode hexa-helical proteins (Kruse et al. 2003, Wogulis et al. 2006) whereas mammalian lipocalins exhibit a $\beta$-barrel motif (Bianchet et al. 1996). CSPs were first identified in Drosophila (McKenna et al. 1994). And both OBPs and CSPs have been identified in a plethora of insects' genera of both heterometabolous and holometabolous lineages and are presumed to perform similar functions in odour detection. Odorant-binding protein and Csp genes encode small, globular hydrophilic proteins (14-20 kDa and $11 \mathrm{kDa}$, respectively) that are excreted in significant amounts out of the cell into the peri-receptor space. As is the case for so many other components of olfactory signalling pathways, the role of these proteins remains very much a matter of debate. Nevertheless, the premise they do play important and perhaps essential roles is supported by numerous studies in all systems studied to date that demonstrate very high OBP and CSP expression levels in olfactory tissues, as well as a more limited set of in vitro binding assays (Pelosi et al. 2006, Wanner et al. 2004). Interestingly, several members of both OBP and CSP families are expressed outside chemosensory tissues and are therefore likely involved in other processes. Indeed, current evidence suggest that CSPs are not involved in olfactory processes even though they have been associated with olfactory tissues (Sabatier et al. 2003). Accordingly, without questioning their relevance, we will not discuss the potential olfactory involvement of CSPs in this context but rather will focus on OBPs where there are compelling data insofar as Drosophila pheromone detection is concerned.

Multiple OR functional studies in cell-based assays that are entirely devoid of OBPs have shown that OBPs are not strictly required for OR activation in these heterologous systems (Neuhaus et al. 2005, Wetzel et al. 2001), although the addition of OBPs to these systems enhances the response sensitivity to semiochemicals (Matsuo et al. 2007, Syed et al. 2006, Wang et al. 2007). The strongest evidence for an in vivo olfactory function of OBPs is typified by the studies on the LUSH-OBP/ OBP76a paradigm in Drosophila (Smith 2007). What is the role of LUSH and to what extent its mode of action represents the OBP family? The LUSH protein is present in the sensillum lymph of the pheromone sensitive T1 sensilla located on the Drosophila antenna. Elegant physiological (Xu et al. 2005) and more recent structure-function analyses (Laughlin et al. 2008) have demonstrated that LUSH itself is the ligand for the pheromone sensitive neurons and that the CVA pheromone, mediates the conversion of LUSH into its active conformation thereby interacting with SNMP1 and DOR67d. Several amino-acid residues in the LUSH protein have been implicated in this conformational change and are conserved among other insect LUSH homologs. It is important to note that other unknown factors are implicated in this process as all three proteins cited above are not sufficient to mimic the in vivo observations. Importantly, it is likely that this, and perhaps all pheromone detection pathways represents a distinct paradigm for odorant recognition that reflects a requirement for extreme sensitivity.

The release of the complete genomes of Drosophila (Adams et al. 2000), An. gambiae (Holt et al. 2002) and Ae. aegypti (Nene et al. 2007) has allowed a full accounting of the Obp gene repertoires in each of these species. The Drosophila genome carries 49 Obp genes while Ae. aegypti and An. 
gambiae possess 57 and 66 putative Obp members, respectively (Figure 3). Among individual species, OBPs share little overall amino-acid sequence identity, ranging from $13.3 \%, 16.6 \%$ to $21.7 \%$ in Drosophila, An. gambiae and Ae. aegypti, respectively (Zhou et al. 2008). Of the three Dipteran species, the overall amino-acid identity among the classic 6-cysteine-bearing OBPs is less than $5 \%$. It has been proposed that this elevated number and sequence diversity of OBPs is an adaptation to the variety of chemical cues that this system needs to interact with (Mohl et al. 2002).

The Dipteran OBP family consists of several distinct subgroups based on peptide length and more importantly based on the number of cysteine residues and their positions within the peptide primary structure. This arbitrary distinction, however, does not hold phylogenetic scrutiny (Pelosi et al. 2006). Historically, classic OBPs have been characterised by a 6-cysteine motif to which all other OBPs are gauged. Additional groups displaying a variation on the OBP archetype include the 'Plus- $C$ ' (8 cysteine residues), 'Minus- $C$ ' (4 cysteine residues) and 'Atypical' OBPs (elongated C-terminus). Interestingly, members of the atypical group have so far only been found in mosquitoes (Xu et al. 2003, Zhou et al. 2008). Their absence in the Drosophila (Xu et al. 2003) and honeybee (Foret et al. 2007) genomes suggests that this gene subfamily arose sometime after the fly/mosquito split approximately 250 million years ago (Gaunt and Miles 2002, Zdobnov et al. 2002). Moreover, at this time there is no hard evidence for the involvement of atypical OBPs in olfactory processes.

Molecular phylogenetic analyses underscore the high degree of sequence divergence among these Dipteran species including several instances of species-specific gene expansions (Bohbot and Vogt 2005, Zhou et al. 2008). By contrast, the genomes of Ae. aegypti, An. gambiae and Drosophila possess Obp gene members belonging to the OS-E/OS-F, LUSH and OBP19a groups suggesting that some level of functional conservation applies among these three species (Zhou et al. 2008). Obp genes tend to occur in clusters within insect genomes (Hekmat-Scafe et al. 2002, Xu et al. 2003) and within each cluster exhibit similar exon-intron structures (Zhou et al. 2006). Apart from the intron-less members of the atypical sub-class, Obp genes contain 1 to 4 introns (Vogt 2002) including one usually found immediately downstream of the sequence encoding the peptide secretion signal-sequence (Hekmat-Scafe et al. 2002, Xu et al. 2003, Zhou et al. 2008).

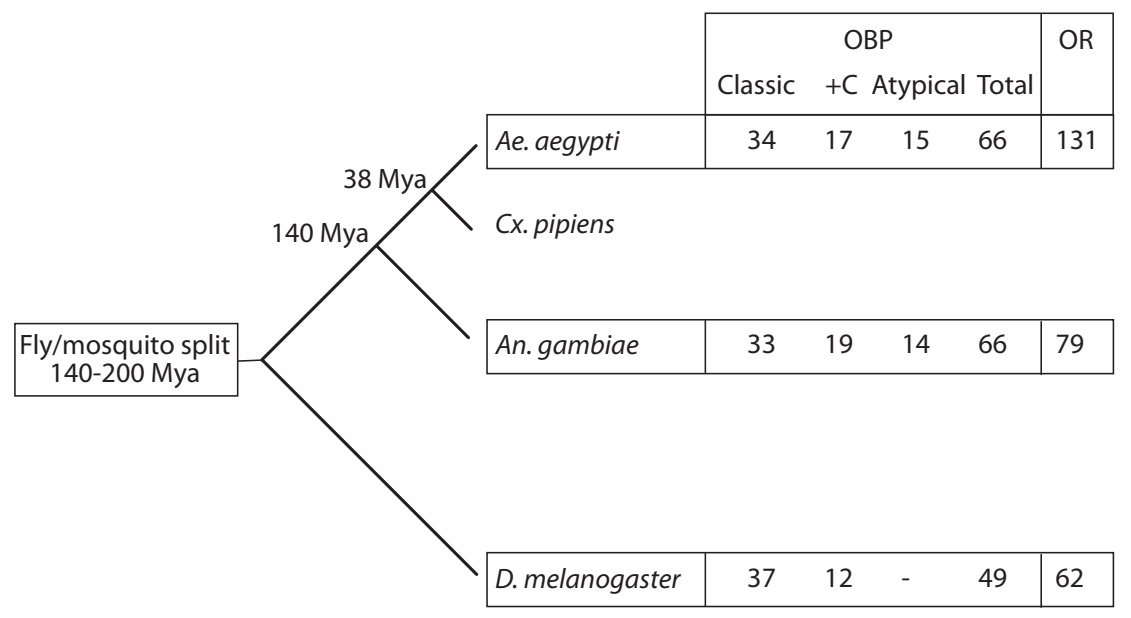

Figure 3. Odorant-Binding Proteins (OBPs) and Odorant Receptors (ORs) in the Dipteran genomes (Hekmat-Scafe et al. 2002, Wicher et al. 2008, Xu et al. 2003, Zhou et al. 2008). 
The combination of structural and genomic conservation of these genes, particularly between Ae. aegypti and An. gambiae, and their amino-acid sequence diversity suggest rapid evolutionary dynamics driven by gene duplication events. While several studies have shown the binding capability of these proteins in vitro, and despite their presence in the peri-receptor compartment of chemosensory sensilla, direct functional evidence for a precise role(s) in olfactory signalling remains elusive.

\section{Sensory neuron membrane proteins}

The SNMP was first described as a putative pheromone binding membrane protein in the antennae of the silk moth Antheraea polyphemus (Rogers et al. 1997, Vogt et al. 1988). Since these initial observations, highly conserved SNMPs have been identified in several insect families (Nichols and Vogt 2008). SNMPs are transmembrane receptors, and are directly related to the CD36 family fatty acid transporters/scavenger receptors which have been shown to bind a number of ligands in various biochemical pathways including lipid metabolism, homeostasis and others (Rac et al. 2007). Two closely related Snmp genes (Snmp1 and Snmp2) are found in Diptera (Nichols and Vogt 2008) and twelve to fourteen CD36-like genes have been identified in the genome of Drosophila, Ae. aegypti and An. gambiae.

In Drosophila, SNMP has recently been shown to be involved in pheromone detection (Benton et al. 2007). In this case, Snmp 1 is expressed in trichoid sensilla and is required for the electrophysiological response of DOr67d-expressing ORNs to the male pheromone cis-vaccenyl acetate. Furthermore, protein-fragment complementation assays indicate that SNMP1 and DOR83b are physically in close proximity on the surface of the cell membrane (Benton et al. 2007). The role of Snmp1 in pheromone sensitivity extends to Lepidoptera where HR13 (Grosse-Wilde et al. 2007), a pheromone receptor from Heliothis virescens, requires SNMP1 for function, suggesting that SNMPs may be generally involved in the recognition of the hydrocarbon chain common to the pheromones of both species (Benton et al. 2007). Lastly, Drosophila Snmp 1 is also expressed in the support cells surrounding pheromone-sensitive ORNs in several chemosensory sensilla distributed across a variety of appendages where their biological role has yet to be determined (Benton et al. 2007, Forstner et al. 2008, Nichols and Vogt 2008).

\section{Odorant receptors}

From a number of perspectives, ORs remain the central molecular players among olfactory proteins. Not surprisingly, the first identified insect ORs were found in Drosophila (Clyne et al. 1999, Gao and Chess 1999, Vosshall et al. 1999). Functional validation of the role of DORs as bona fide ORs came some time later through extensive use of numerous heterologous expression systems (Dobritsa et al. 2003, Goldman et al. 2005, Hallem et al. 2004b, Kreher et al. 2005, Wetzel et al. 2001). As was the case with the majority of gene families, these initial studies in Drosophila opened the door for similar studies in disease vector insects. Bioinformatics and molecular-based analyses of mosquito genomes have provided the identification of complete OR repertoire sets in An. gambiae (AgORs) (Hill et al. 2002) and more recently, in Ae. aegypti (AaORs) (Bohbot et al. 2007). In contrast to the 60 DOr genes, there are 79 (Hill et al. 2002) and 131 (Bohbot et al. 2007) Or genes in An. gambiae and $A$ e. aegypti, respectively (Figure 3).

Traditionally classified as G-protein coupled receptors (GPCRs), insect ORs, Aa/AgORs belong to a novel membrane protein family with no homology with vertebrate ORs. In addition to their characteristic lack of primary sequence conservation, insect OR proteins are recognised 
as functionally obligate heterodimers composed of a conventional and highly divergent OR and a highly conserved non-conventional co-receptor called OR83b in Drosophila and OR7 in mosquitoes ( $~ 80 \%$ amino-acid identity). In contrast, conventional ORs from flies and mosquitoes hardly share any significant sequence similarity outside relatively conserved ORs such as the DOR43a/OR2 sub-group (Figure 4). The similarity between the universal co-receptor DOR83b/ OR7 group is a reflection of their conserved function as chaperones involved in protein trafficking of conventional ORs and their participation in odorant detection (Benton et al. 2006, Jones et al. 2005, Larsson et al. 2004, Neuhaus et al. 2005). Interestingly, DOR43a and the mosquito OR2 homologs do not recognise the same odorants (Hallem et al. 2004a,b, Storkuhl and Kettler 2001, Wetzel et al. 2001). Similarly to OBPs (Vogt 2002), Or genes tend to occur in clusters. This feature is more pronounced in the mosquito genomes (Bohbot et al. 2007, Hill et al. 2002) relative to that observed in Drosophila (Robertson et al. 2003). The former exhibit gene clusters of 2 to 11 genes while DOrs occur for the most part as scattered singletons and in a few instances in clusters of up to 3 genes (Robertson et al. 2003). Genes within a given cluster typically display higher sequence similarity. The evolutionary history leading to these accrued gene duplication events in mosquitoes is unknown but indicates perhaps that these insects have relied on this mechanism to drive OR phenotypic diversity thereby increasing their odour space. The comparative molecular phylogenetic analyses of Aa/AgORs have confirmed the characteristic divergence of these ORs (generally less than 20\% overall amino-acid sequence identity). Within each repertoire are several OR-specific expansions that likely reflect the evolutionary divergence as well as the unique chemical ecology of these two mosquito species. It is remarkable that 11 homologous $\mathrm{Aa} / \mathrm{AgOR}$ groups can be nevertheless distinguished based on sequence similarity (Figure 4) and microsyntenic relationships (Bohbot et al. 2007), suggesting that at least in some cases, significant evolutionary constrains have been imposed upon this gene family.

\section{The olfactory apparatus of vector mosquitoes}

The olfactory apparatus of vector mosquitoes is comprised of ORNs and support cells that are spread over several adult and larval appendages that together provide an array of sensory capabilities that presumably reflects biological requirements (Figure 2). One method of marking ORNs is to assess the expression of Or genes. In that context a complete Or expression map of the larval and adult olfactory appendages is available for Ae. aegypti (Bohbot et al. 2007). The larval antennae and all three adult olfactory appendages in mosquitoes, the antenna, the proboscis, and the maxillary palp, are populated by a diversity of porous sensilla containing the sensory dendrites of ORNs (Lu et al. 2007, Pitts et al. 2004, Pitts and Zwiebel 2006, Xia et al. 2008). In An. gambiae detailed transcription profiles of the proboscis, the maxillary palp and the larval antenna have been established (Kwon et al. 2006, Lu et al. 2007, Xia et al. 2008), while the precise expression profile of olfactory genes in the adult antenna has yet to be fully elucidated.

\section{Olfactory properties of the mosquito antennae}

Paradoxically, and in large part as a result of its complexity, the mosquito antennae, which is the principal adult olfactory appendage, remains the least understood in so far as the molecular bases of olfactory physiology. That said, a significant body of physiological work has been completed in Ae. aegypti (Davis 1974) and other vector mosquitoes as reviewed in Davis (1996). More recently several important studies have begun to unravel the olfactory physiology of $A n$. gambiae antennae (Meijerink et al. 2001, Meijerink and van Loon 1999, Qiu et al. 2006). At the same time there is a growing appreciation of the antennal expression patterns of several genes that encode signal transduction components that have been linked in one degree or another to 
INTERspecific comparison

Specific ORs

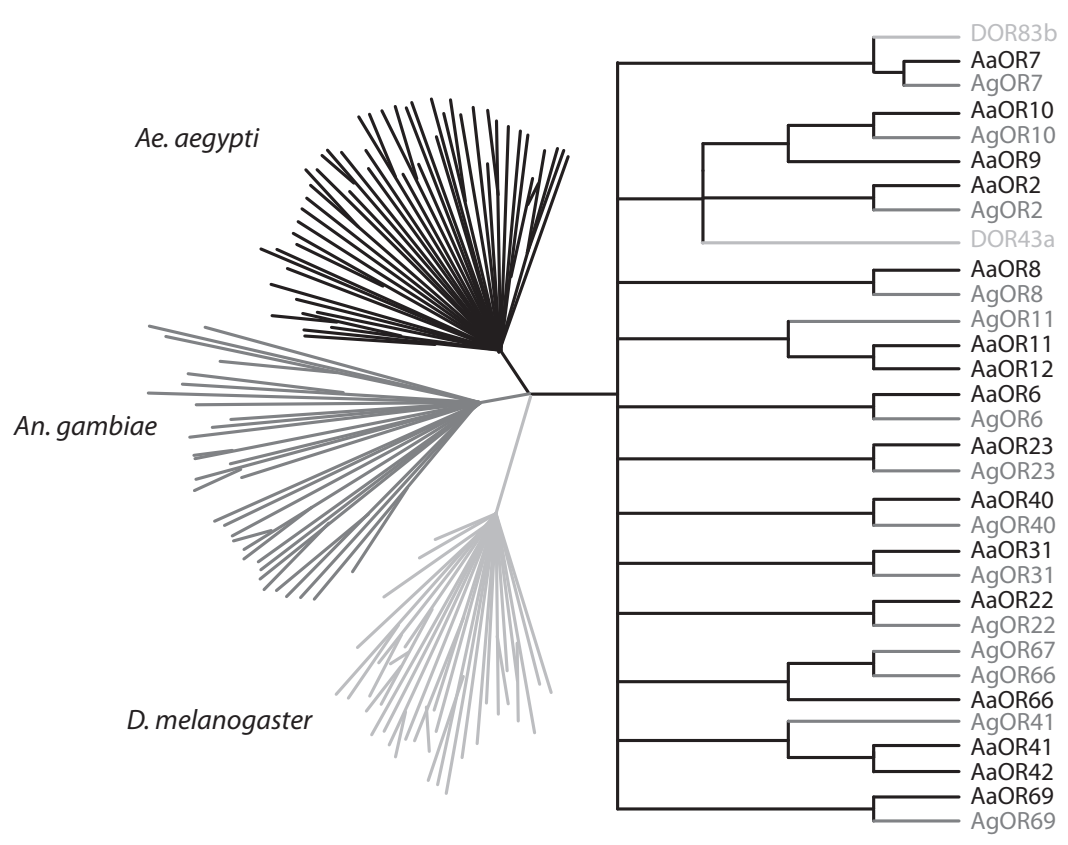

Figure 4. Phylogenetic comparison of Dipteran odorant receptor proteins (ORs). ORs are highly divergent at the protein level, the vast majority being species specific. A small subset of ORs display various levels of sequence conservation between Aedes and Anopheles mosquitoes. The mosquito OR7 and Drosophila OR83b are true orthologs and are defined as universal ORs ( 80\% amino-acid identity). The other similarity between mosquitoes and flies is restricted to the mosquito OR2 and DOR43a ( 36\% identity) (Bohbot et al. 2007, Hill et al. 2002).

olfaction. As discussed above, these include antennal arrestins (Merrill et al. 2003), G-proteins (Rutzler et al. 2006), OBPs (Biessmann et al. 2002, Xu et al. 2003) as well as an ever- increasing spectrum of ORs. These include representatives from An. gambiae (Fox et al. 2001, Hill et al. 2002), Ae. aegypti (Bohbot et al. 2007) and Cx. quinquefaciatus (Xia and Zwiebel 2006). Of these, at least one has been linked to human odorants (Hallem et al. 2004a) while several have been reported to show sexually biased expression patterns (Fox et al. 2001, latrou and Biessmann 2008). Current efforts in the field are doubtlessly focused on providing a detailed understanding of antennal odour coding mechanisms.

\section{Olfactory properties of the mosquito labellum}

The proboscis is a long slender organ housing the feeding stylets of the adult mosquito, harboring numerous chemosensory sensilla at its bulbous tip known as the labellum (Figure 5). Early studies on the mosquito labellum have focused largely on its gustatory function. In Culiseta inornata (Williston) three morphological and functional types of labellar sensilla have been defined (Mclver 1982, Pappas and Larsen 1976); the Type 1 (T1) and the Type 2 (T2) sensilla are distributed on the external surface of the labellum, while the Type 3 (T3) sensilla are located on the oral surface - all 
three types are sensitive to taste stimuli (Mclver 1982, Owen et al. 1974, Pappas and Larsen 1976). Recent electrophysiological studies, however, demonstrated that the labellum of An. gambiae is also sensitive to odorant stimuli including butylamine and several aliphatic carboxylic acids (Kwon et al. 2006). Here at least one sensillum that is reminiscent of the Cs. inornata T2 sensilla harbours at least two ORNs and responds to butylamine, aliphatic acids and several ketone compounds including acetophenone (Kwon et al. 2006). The presence of this cryptic olfactory capacity in the primary gustatory organ of An. gambiae is especially interesting inasmuch as butylamine has been identified in human skin emanations (Ellin et al. 1974). Furthermore, the odorants that elicit responses from labellar sensilla have relatively low volatility compared to other known mosquito attractants, raising the suggestion that the labellum of An. gambiae might be involved in the close-range odour detection at the penultimate stages of host-seeking or oviposition behaviour (Kwon et al. 2006).

In a decided departure from the Drosophila labellum which is devoid of olfactory function and putative OR expression, immuno-histochemical studies revealed that the non-conventional and obligate co-receptor AgOR7 is expressed in about 50 labellar T2 sensilla of An. gambiae (Pitts et

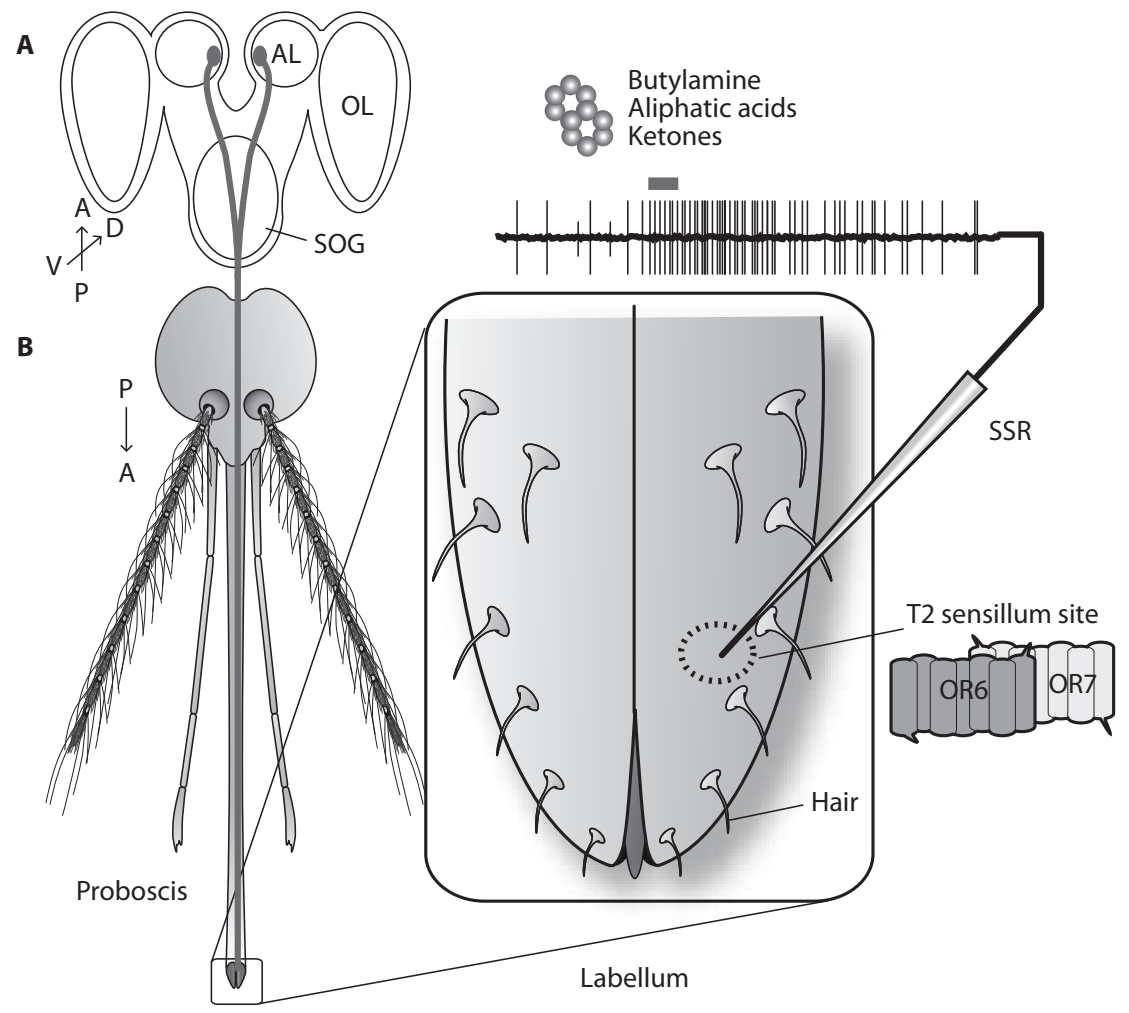

Figure 5. The labellum of An. gambiae exhibits olfactory responses. Schematic dorsal view of the mosquito head and brain, anterior $(A)$, posterior $(P)$, dorsal $(D)$ and ventral $(V)$ axes, as indicated. $(A)$ Neurons of the labellum project to a posteromedial region of the antennal lobes (AL) in the brain. (B) A subset of $T 2$ sensilla on the labellum respond to odorant stimuli in single sensillum recording (SSR) experiments. Coincidently, T2 sensilla express OR6 and OR7. SOG: sub-oesophageal ganglion, OL: optic lobe. 
al. 2004). Similarly AaOR7 and CqOR7 staining is localised to the labella of Ae. aegypti and CX. quinquefasciatus (Melo et al. 2004, Xia and Zwiebel 2006), respectively. Overall, more than 20 conventional AgOrs are expressed on the labellum of An. gambiae, and in situ hybridisation studies have thus far demonstrated that at least one of these AgOrs, AgOr6, is co-expressed with AgOR7 in a subset of labellar ORNs (Kwon et al. 2006). It is worthwhile to note that AgOR6, which has been associated with a $\mathrm{T} 2$ sensillum that respond in vivo to butylamine, aliphatic acids and several ketone compounds (Kwon et al. 2006), has recently been shown to specifically elicit responses to precisely those compounds when expressed in the Xenopus oocyte heterologous expression system (Xia et al. 2008).

Surprisingly, only one or two glomeruli in the antennal lobe receive projections from the labellar ORNs of An. gambiae and Ae. aegypti (Ghaninia et al. 2007, Kwon et al. 2006). These glomeruli are located in the medial region of the antennal lobe and might be innervated by both labellar and palpal ORNs (Ghaninia et al. 2007, Kwon et al. 2006). It is possible that the An. gambiae labellum harbours multiple functional types of ORNs, each expressing a different AgOR in addition to AgOR7, and this ensemble of ORNs expressing a combinatorial of over 20 AgORs converge on one or two medial glomeruli in the antennal lobe. This type of organisation represent a significant departure from established paradigms in the olfactory or gustatory system in insects where ORNs or gustatory receptor neurons (GRNs) with different receptor profiles will converge onto distinct regions of the antennal lobe or the suboesophageal ganglion (Rutzler and Zwiebel 2005, Vosshall and Stocker 2007).

\section{Olfactory properties of the mosquito maxillary palps}

In contrast to the antenna, which contains the largest quantity and variety of olfactory sensilla, the maxillary palp is considerably less complex, harboring a single type of chemosensory sensillum, the capitate peg (Figure 6). The maxillary palp of mosquitoes consists of five segments, although female anophelines have much longer palps than those found in culicines, where the $5^{\text {th }}$ segment is reduced to a knob. Furthermore, whereas in adult female anophelines, capitate pegs are distributed on palpal segments two, three and four, their distribution is restricted to the $4^{\text {th }}$ segment in female culicines (Mclver 1972).

Extensive single-sensillum electrophysiological recordings (SSRs) have been carried out in female An. gambiae, Ae. aegypti and Cx. quinquefasciatus (Lu et al. 2007, Syed et al. 2006). These experiments invariably revealed the existence of a single functional class of capitate pegs across the maxillary palp (Lu et al. 2007, Syed et al. 2006), although the number of capitate pegs per palp varies drastically among the three species (Mclver 1982). Furthermore, these structures consistently contain three ORNs that form stereotyped triads within each capitate peg sensillum (Lu et al. 2007, Syed et al. 2006). The capitate peg (cp) A and B neurons respond with high sensitivity to $\mathrm{CO}_{2}$ and 1-octen-3-ol, respectively, both of which are established mosquito attractants (Grant and O'Connell 1996, Lu et al. 2007, Syed et al. 2006). It is possible that An. gambiae and other mosquitoes use a complex host odour blend as a crucial host-seeking cue, of which $\mathrm{CO}_{2}$ and 1-octen-3-ol are two components. It has been hypothesised (Lu et al. 2007) that by pairing the $\mathrm{CO}_{2}$-sensitive $\mathrm{cpA}$ neuron with the 1-octen-3-ol-sensitive $\mathrm{cpB}$ neuron within the capitate peg sensilla, the mosquito might be able to perceive the ratio between these two compounds within the host odour blend with high fidelity and spatial resolution. Indeed this organisation is shared by several cases of insect pheromone-sensing sensilla, in which ORNs responsive to individual components of the pheromone blend are compartmentalised (Nakagawa et al. 2005, Wojtasek et al. 1998). Interestingly, the insect repellent DEET (N,N-diethyl-meta-toluamide) inhibits the 


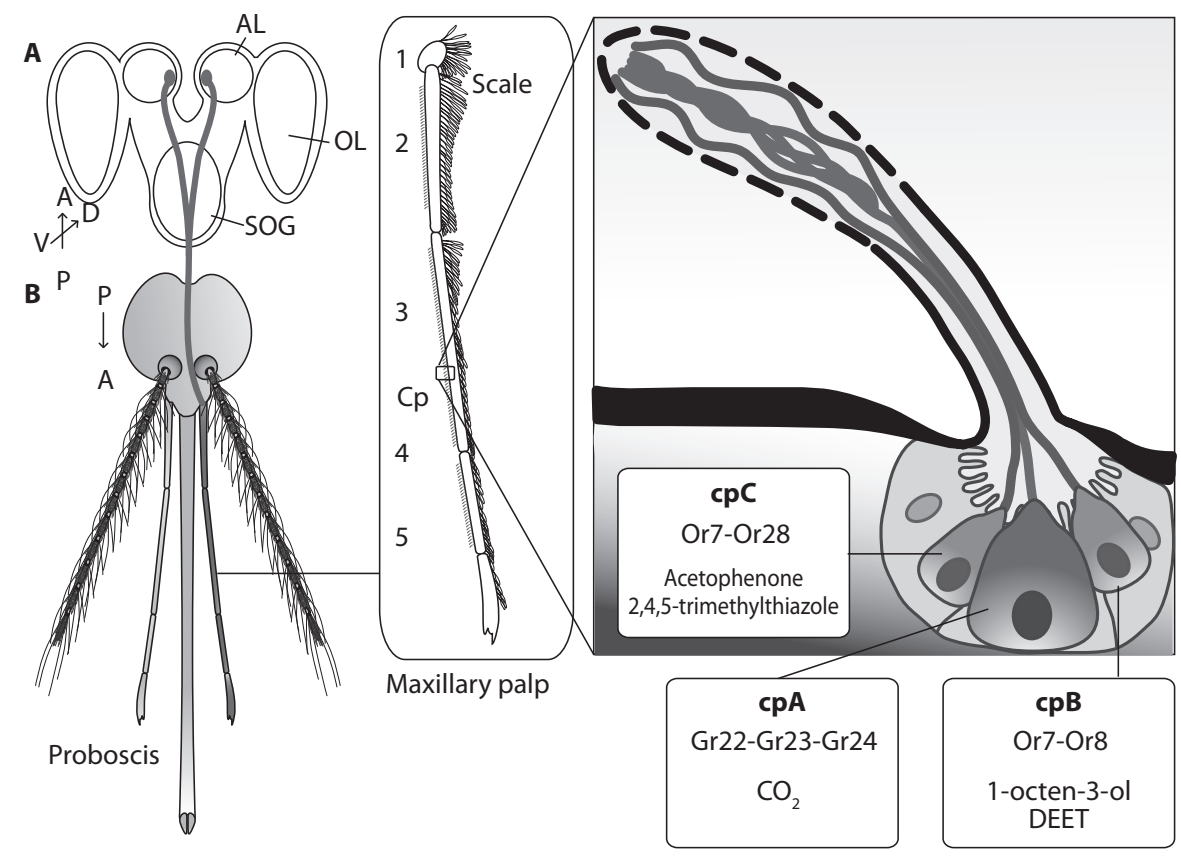

Figure 6. The maxillary palp of An. gambiae exhibits olfactory responses. Schematic view of the mosquito head and brain, anterior (A), posterior (P), dorsal (D) and ventral (V) axes, as indicated. (A) Palpal ORNs project to a posteromedial region of the antennal lobes $(A L)$ in the brain. (B) Capitate pegs $(C P)$ are located on the ventral side of the $2^{\text {nd }}$, $3^{\text {rd }}$ and $4^{\text {th }}$ segments of the maxillary palp. Each neuron in the $C p(C p A, C p B, C p C)$ responds to different odorant stimuli and expresses a different set of odorant receptors (Ors) or gustatory receptors (Grs). SOG, suboesophageal ganglion, OL, optic lobe.

spontaneous firing (Lu et al. 2007) and 1-octen-3-ol-evoked responses in the An. gambiae cpB neuron; whereas $\mathrm{CO}_{2}$-evoked responses of the $\mathrm{cpA}$ neuron are unaffected (Ditzen et al. 2008). The response profiles of the $\mathrm{cpC}$ neuron seem to differ between anophelines and culicines. Whereas the An. gambiae $\mathrm{cpC}$ neuron responds to a broad panel of odorants including 2,4,5-trimethylthiazole and acetophenone (Lu et al. 2007), the cpC neurons in Ae. aegypti and Cx. quinquefasciatus seem to be extremely narrowly-tuned and do not respond to any of the tested stimuli (Syed et al. 2006) (M. Ghaninia, B.S. Hansson and R. Ignell, personal communication), suggesting that they might be involved in the detection of mosquito pheromones or kairomones.

Localisation studies using in situ hybridisation indicate that a distinctive set of AgOrs and AgGrs are expressed in the triad of ORNs in the maxillary palp of An. gambiae (Jones et al. 2007, Lu et al. 2007). The cpA neuron co-expresses three $\mathrm{CO}_{2}$ receptors, AgGr22, AgGr23, and AgGr24; and the other two ORNs express AgOr7 along with AgOr8 or AgOr28, respectively (Jones et al. 2007, Lu et al. 2007) (Figure 6). However, additional AgOrs might also be expressed in the maxillary palp of An. gambiae, as a small fraction of AgOr7-positive ORNs is devoid of AgOr8 or AgOr28 signals in double in situ hybridisation assays (Lu et al. 2007). Indeed, a recent study showed that in addition to AgOr8 and AgOr28, several other AgOrs could be detected from palpal cDNAs, albeit only after 35 cycles of PCR amplification (latrou and Biessmann 2008). Two AaOrs are expressed in the maxillary palp 
of Ae. aegypti (Bohbot et al. 2007). Of these AaOr8 is a close ortholog of AgOr8 (with 70\% identity), whereas AaOr49 is not closely related to AgOr28 (Bohbot et al. 2007). Three AaGrs, AaGr1, AaGr2, and $A a G r 3$, are close homologs of $A g G r 22, A g G r 23$, and $A g G r 24$ (with 72-89\% identity), respectively (Kent et al. 2007), and are presumably expressed in the Ae. aegypti equivalent of the cpA neuron. It remains interesting to speculate as to what additional factors are expressed and contribute to the olfactory signal transduction in the capitate peg sensilla. In addition to these Ors, multiple Obp genes are expressed in the maxillary palp of the female An. gambiae mosquito although their role(s) in olfactory signalling remain to be elucidated (Biessmann et al. 2005).

Heterologous expression systems have successfully recapitulated the response properties of palpal AgORs and AgGRs and assigned them to the An. gambiae $\mathrm{cpA}, \mathrm{cpB}$, or $\mathrm{cpC}$ neurons (Lu et al. 2007). Specifically, the response spectra of AgOR8 and AgOR28, when co-expressed with AgOR7 and $\mathrm{G} \mathrm{Ga}_{15}$ subunit in Xenopus oocytes, closely mirror that of the native $\mathrm{cpB}$ and $\mathrm{cpC}$ neurons (Lu et al. 2007). Of these, AgOR8 manifests a highly sensitive response to 1-octen-3-ol, and this is in the absence of the native dendritic structure and environment (Lu et al. 2007). These data are consistent with the current paradigms that insect ORs provide the primary determinant of response spectra of ORNs (Hallem et al. 2004b, Nakagawa et al. 2005). AaOR8, the Ae. aegypti ortholog of AgOR8, also responds to 1-octen-3-ol with high sensitivity when expressed in Xenopus oocytes (G. Wang and L.J. Zwiebel, unpublished data), and this suggests that AaOR8 is likewise expressed in the Ae. aegypti cpB neuron. The three An. gambiae $\mathrm{CO}_{2}$ receptors, AgGR22, AgGR23, and AgGR24, which display high sequence homology to other insect $\mathrm{CO}_{2}$ receptors (Jones et al. 2007, Lu et al. 2007), are sufficient to drive a $\mathrm{CO}_{2}$ response when co-expressed in the Drosophila antennal basiconic type (ab) 3A ORN (Lu et al. 2007). Importantly, sensitivity to $\mathrm{CO}_{2}$ in this neuron does not require AgOR7 expression in An. gambiae or DOR83b in Drosophila. Indeed, it would seem that Drosophila has lost its ortholog of AgGR23 subsequent to the divergence of the two species (Lu et al. 2007) and ectopic expression of DmGR21a and DmGR63a, the fruit fly ortholog of AgGR22 and AgGR24, is sufficient to induce $\mathrm{CO}_{2}$ sensitivity in the otherwise $\mathrm{CO}_{2}$-insensitive ab3A neuron (Jones et al. 2007, Kwon et al. 2007). The function of AgGR23 in the cpA neuron remains uncharacterised. Whereas one copy of $A g G r 22$ and AgGr24 transgenes is not sufficient to induce $\mathrm{CO}_{2}$ sensitivity in the ab3A neuron, adding an AgGr23 transgene further to this ORN will make it $\mathrm{CO}_{2}$-sensitive (Lu et al. 2007). However, compared to the An. gambiae cpA neuron, the response of three $\mathrm{AgGr}$ transgenes to $\mathrm{CO}_{2}$ is weak and its sensitivity is low (Lu et al. 2007), and this limitation hampers further interpretation of the result.

Anterograde staining of maxillary palp axons revealed that mosquito palpal ORNs innervate a set of dorso-medial glomeruli in the antennal lobe (Distler and Boeckh 1997, Ghaninia et al. 2007). Three glomeruli in Ae. aegypti and five to six glomeruli in An. gambiae receive afferent input from the maxillary palp nerve (Ghaninia et al. 2007, Ignell et al. 2005); and in both species, one glomerulus is considerably larger than the others and has been suggested to receive projections from $\mathrm{CO}_{2}$-sensitive ORNs (Anton et al. 2003, Ignell et al. 2005). In the mosquito it would therefore appear that $\mathrm{CO}_{2}$-sensitive ORNs connect to a dorso-medial glomerulus in the antennal lobe and mediate attraction to $\mathrm{CO}_{2}$. In contrast, the Drosophila $\mathrm{CO}_{2}$-sensitive ORNs innervate a ventral glomerulus and mediate avoidance (Suh et al. 2007, Suh et al. 2004), suggesting that different locations of the $\mathrm{CO}_{2}$-receptive glomerulus in the antennal lobe may reflect divergent neural circuits that potentially mediate differential behavioural responses. Interestingly, it has recently been suggested this difference is partially accounted for by miR-279, a regulatory microRNA that is expressed in the Drosophila maxillary palp to prevent expression of Drosophila $\mathrm{CO}_{2}$ receptors and drive the projections of $\mathrm{CO}_{2}$-sensitive ORNs away from medial glomeruli (Cayirlioglu et al. 2008). It is possible that Drosophila has gained or started to utilise miR-279 after divergence from 
its common ancestor with the mosquito, and that this, along with additional mechanisms, has shifted expression of the $\mathrm{CO}_{2}$-sensitive ORNs from the maxillary palp to the antenna and modified their projections in the antennal lobe.

\section{Larval olfaction}

The study of larval olfaction in mosquitoes followed seminal work on the larval feeding behaviours and ecology as important elements in larval control strategies as well as basic studies in Drosophila that used molecular genetics to dissect sensory and behaviour pathways in the less complex larval neural system. More recently these approaches have started to merge together to illuminate several elements of olfactory-driven behaviours in the mosquito.

Contrary to aquatic mosquito larvae that typically develop in nutrient-poor environments, terrestrial-based Drosophila larvae have access to a virtually unlimited amount of food (Merritt et al. 1992) and not surprisingly, respond to a wide variety of odorants (Cobb and Domain 2000). Attractive and repellent responses are mediated by 23-25 ORs (Fishilevich et al. 2005, Kreher et al. 2005) that are distributed in 21 ORNs (Singh and Singh 1984) within the dorsal organ of the larva. Remarkably, while single ORNs can detect odorants, full behavioural responses seem to require the combinatorial activation of several different ORs probably enhancing the sensitivity of the olfactory system.

Until recently, little information was known on the molecular and cellular aspects of the larval chemosensory system of mosquitoes. The larval antenna as best studied in Culicidae, typically comprises the sensory cone and the peg organ (Figure 7), the latter housing the two sensilla considered to be the principal sites for larval chemoreception (Nicastro et al. 1998, Zacharuk et al. 1971). The sensory cone is a multiporous sensillum while the peg organ is considered a taste sensillum with a terminal pore on its distal end. A combination of in situ hybridisation and RT-PCR techniques have shown that $13 \mathrm{AgOrs}$ are expressed in the sensory cone of An. gambiae (Pitts et al. 2004, Xia et al. 2008). Interestingly almost twice as many AaOrs are expressed in the larval antenna of Ae. aegypti (Bohbot et al. 2007) (Figure 7). Thirty percent of the larval AgOrs are stage specific in An. gambiae (Xia et al. 2008), while in Ae. aegypti this proportion doubles (Bohbot et al. 2007). The reasons behind these qualitative and quantitative differences are unknown but certainly reflect the respective and specific evolutionary history of these insects. It certainly suggests that the Aedes larval odour space is more complex than its anopheline cousin.

There is behavioural evidence that An. gambiae larvae respond both positively and negatively to various compounds of different chemical structures and ablation of the An. gambiae larval antenna suppresses these olfactory-driven behaviours (Xia et al. 2008). Larval attractants include aromatic compounds such as isomers of methylphenol and indole, which are associated with food sources and are also known to trigger electrophysiological responses in adult female mosquitoes (Blackwell and Johnson 2000). 1-Octen-3-ol, in addition to activating the cpB neuron in adult female An. gambiae (Lu et al. 2007), is a component of mammalian body odours (Ramoni et al. 2001). Two compounds have been shown to induce repellent response in larvae: of particular significance is the commercial insect repellent DEET, which recently has been postulated to act by blocking the response activity of a subset of ORs (Ditzen et al. 2008). The fact that DEET is able to evoke repulsive behaviours in Anopheles larvae and in adult Cx. quinquefasciatus in the absence of any other sensory stimuli suggests that it is able to act as a direct excito-repellent (Syed and Leal 2008, Xia et al. 2008). The molecular mechanism responsible for these avoidance 


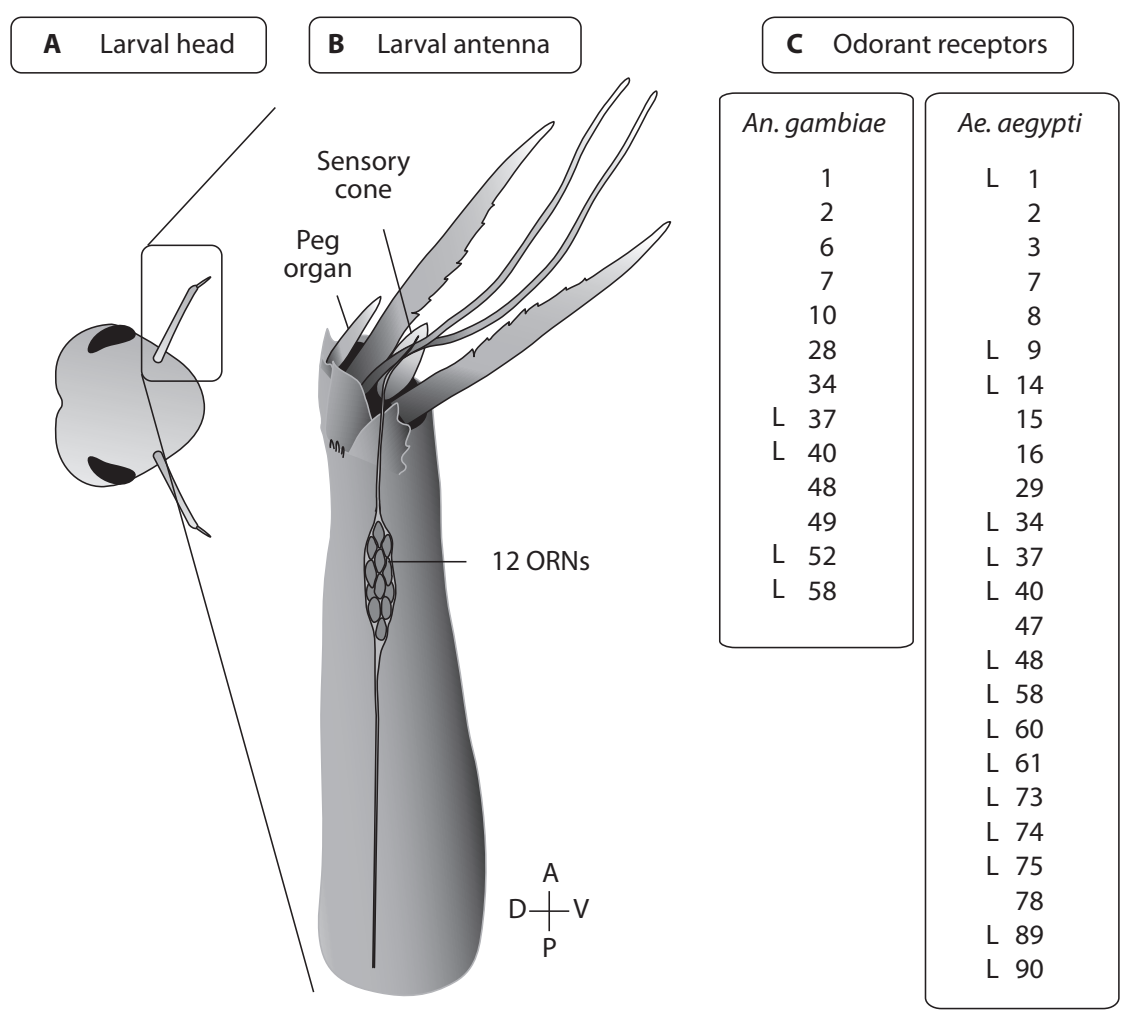

Figure 7. Larval antenna of mosquitoes exhibits olfactory responses. (A) Schematic dorsal view of a larval head. (B) Twelve odorant receptor neurons (ORNs) are located in the larval antenna of An. gambiae. Their dendrites project into the sensory cone. (C) Expression of Or genes in the larval antenna of Ae. aegypti and An. gambiae. L, larval specific expression.

effects is olfactory mediated as a DEET specific ORN located in the short trichoid sensilla on the Cx. quinquefasciatus antennae responds to DEET alone (Syed and Leal 2008).

Heterologous expression in Xenopus oocytes coupled with the two-electrode voltage clamp technique revealed that AgOR1/2/6/10/28/34/48 respond to two or more odorants while the larval specific AgOR40 is activated by DEET (Xia et al. 2008). Olfactory receptors of An. gambiae (AgORs) either display broad responses to various odorants or are narrowly tuned to a few compounds, suggesting redundancy at the level of odour coding at the peripheral level, a feature widely observed in Drosophila (Kreher et al. 2005). It has been proposed that the partial physiological convergence between larval and adult olfaction is a reflection of the shared subset of expressed Ors between the larval and adult stages of mosquitoes (Xia et al. 2008) and flies (Kreher et al. 2005).

Olfactory receptors of larval and adult stages of Drosophila project to discrete, non-overlapping areas in the antennal lobe. In addition, each fly larval ORN expresses one DOr. This molecular and cellular feature has been described as the one ORN-one OR-one glomerulus 'paradigm'. This linear organisation is also present in the olfactory system of the Anopheles larva, whereby based on the 
expression of $\mathrm{AgOr7}$, only twelve ORNs project their dendrites into the sensory cone of Anopheles (Xia et al. 2008) matching the number of conventional AgOrs identified by RT-PCR. In Aedes, the situation may be different as the Or/ORN ratio is close to two (Bohbot et al. 2007).

\section{Concluding remarks}

Our understanding of the molecular mechanisms underlying olfactory signal transduction in vector mosquitoes has dramatically improved. The diverse nature of the cellular and molecular processes involved in olfaction will most likely bring to light several parallel models to better explain pheromone as well as general odorant perceptions. Over and above these basic studies, OBP and OR proteins display several features including characteristic and species-specific molecular diversity, a central role in olfactory transduction and accessibility that make them potential targets that are amenable to large scale and high-throughput screening with an eye towards the development of novel tools for vector control through behavioural misdirection.

\section{References}

Adams MD, Celniker SE, Holt RA, Evans CA, Gocayne JD, Amanatides PG, Scherer SE, Li PW, Hoskins RA, Galle RF, George RA, Lewis SE, Richards S, Ashburner M, Henderson SN, Sutton GG, Wortman JR, Yandell MD, Zhang Q, Chen LX, Brandon RC, Rogers YH, Blazej RG, Champe M, Pfeiffer BD, Wan KH, Doyle C, Baxter EG, Helt G, Nelson CR, Gabor GL, Abril JF, Agbayani A, An HJ, Andrews-Pfannkoch C, Baldwin D, Ballew RM, Basu A, Baxendale J, Bayraktaroglu L, Beasley EM, Beeson KY, Benos PV, Berman BP, Bhandari D, Bolshakov S, Borkova D, Botchan MR, Bouck J, Brokstein P, Brottier P, Burtis KC, Busam DA, Butler H, Cadieu E, Center A, Chandra I, Cherry JM, Cawley S, Dahlke C, Davenport LB, Davies P, de Pablos B, Delcher A, Deng Z, Mays AD, Dew I, Dietz SM, Dodson K, Doup LE, Downes M, DuganRocha S, Dunkov BC, Dunn P, Durbin KJ, Evangelista CC, Ferraz C, Ferriera S, Fleischmann W, Fosler C, Gabrielian AE, Garg NS, Gelbart WM, Glasser K, Glodek A, Gong F, Gorrell JH, Gu Z, Guan P, Harris M, Harris NL, Harvey D, Heiman TJ, Hernandez JR, Houck J, Hostin D, Houston KA, Howland TJ, Wei MH, Ibegwam C, Jalali M, Kalush F, Karpen GH, Ke Z, Kennison JA, Ketchum KA, Kimmel BE, Kodira CD, Kraft C, Kravitz S, Kulp D, Lai Z, Lasko P, Lei Y, Levitsky AA, Li J, Li Z, Liang Y, Lin X, Liu X, Mattei B, McIntosh TC, McLeod MP, McPherson D, Merkulov G, Milshina NV, Mobarry C, Morris J, Moshrefi A, Mount SM, Moy M, Murphy B, Murphy L, Muzny DM, Nelson DL, Nelson DR, Nelson KA, Nixon K, Nusskern DR, Pacleb JM, Palazzolo M, Pittman GS, Pan S, Pollard J, Puri V, Reese MG, Reinert K, Remington K, Saunders RD, Scheeler F, Shen H, Shue BC, Sidén-Kiamos I, Simpson M, Skupski MP, Smith T, Spier E, Spradling AC, Stapleton M, Strong R, Sun E, Svirskas R, Tector C, Turner R, Venter E, Wang AH, Wang X, Wang ZY, Wassarman DA, Weinstock GM, Weissenbach J, Williams SM, WoodageT, Worley KC, Wu D, Yang S, Yao QA, Ye J, Yeh RF, Zaveri JS, Zhan M, Zhang G, Zhao Q, Zheng L, Zheng XH, Zhong FN, Zhong W, Zhou X, Zhu S, Zhu X, Smith HO, Gibbs RA, Myers EW, Rubin GM and Venter JC (2000) The genome sequence of Drosophila melanogaster. Science 287: 2185-2195.

Anton S, van Loon JJA, Meijerink J, Smid HM, Takken W and Rospars J-P (2003) Central projections of olfactory receptor neurons from single antennal and palpal sensilla in mosquitoes. Arthropod Structure \& Development 32: 319-327.

Benton R, Sachse S, Michnick SW and Vosshall LB (2006) Atypical membrane topology and heteromeric function of Drosophila odorant receptors in vivo. PLoS Biol 4: e20.

Benton R, Vannice KS and Vosshall LB (2007) An essential role for a CD36-related receptor in pheromone detection in Drosophila. Nature 450 (7167): 289-293.

Bianchet MA, Bains G, Pelosi P, Pevsner J, Snyder SH, Monaco HL and Amzel LM (1996) The three-dimensional structure of bovine odorant binding protein and its mechanism of odor recognition [see comments]. Nat Struct Biol 3: 934-939.

Biessmann H, Nguyen QK, Le D and Walter MF (2005) Microarray-based survey of a subset of putative olfactory genes in the mosquito Anopheles gambiae. Insect Mol Biol 14: 575-589.

Biessmann H, Walter MF, Dimitratos S and Woods D (2002) Isolation of CDNA clones encoding putative odourant binding proteins from the antennae of the malaria-transmitting mosquito, Anopheles gambiae. Insect Mol Biol 11: 123-132. 
Blackwell A and Johnson SN (2000) Electrophysiological investigation of larval water and potential oviposition chemoattractants for Anopheles gambiae s.s. Ann Trop Med Parasitol 94: 389-398.

Bohbot J, Pitts RJ, Kwon HW, Rutzler M, Robertson HM and Zwiebel LJ (2007) Molecular characterization of the Aedes aegypti odorant receptor gene family. Insect Mol Biol. 16: 525-537.

Bohbot J and Vogt RG (2005) Antennal expressed genes of the yellow fever mosquito (Aedes aegypti L.); characterization of odorant-binding protein 10 and takeout. Insect Biochem Mol Biol 35: 961-979.

Bosch OJ, Geier M and Boeckh J (2000) Contribution of fatty acids to olfactory host finding of female Aedes aegypti. Chem Senses 25: 323-330.

Cabrera M and Jaffe K (2007) An aggregation pheromone modulates lekking behavior in the vector mosquito Aedes aegypti (Diptera: Culicidae). J Am Mosq Control Assoc 23: 1-10.

Cayirlioglu P, Kadow IG, Zhan X, Okamura K, Suh GS, Gunning D, Lai EC and Zipursky SL (2008) Hybrid neurons in a microRNA mutant are putative evolutionary intermediates in insect $\mathrm{CO} 2$ sensory systems. Science 319: 1256-1260.

Clyne PJ, Warr CG, Freeman MR, Lessing D, Kim J and Carlson JR (1999) A novel family of divergent seven-transmembrane proteins: candidate odorant receptors in Drosophila. Neuron 22: 327-338.

Cobb M and Domain I (2000) Olfactory coding in a simple system: adaptation in Drosophila larvae. Proc Biol Sci 267: 2119-2125.

Davis EE (1974) Identification of antennal chemoreceptors of the mosquito, Aedes aegypti: a correction. Experientia 30: $1282-1283$.

Davis EE (1996) Olfactory control of mosquito behaviour. Ciba Found Symp 200: 48-53.

Dekker T, Steib B, Carde RT and Geier M (2002) L-lactic acid: a human-signifying host cue for the anthropophilic mosquito Anopheles gambiae. Med Vet Entomol 16: 91-98.

Distler P and Boeckh J (1997) Central projections of the maxillary and antennal nerves in the mosquito Aedes aegypti. J Exp Biol 200: 1873-1879.

Ditzen M, Pellegrino M and Vosshall LB (2008) Insect odorant receptors are molecular targets of the insect repellent DEET. Science 319: 1838-1842.

Dobritsa AA, van der Goes van Naters W, Warr CG, Steinbrecht RA and Carlson JR (2003) Integrating the molecular and cellular basis of odor coding in the Drosophila antenna. Neuron 37: 827-841.

Ellin RI, Farrand RL, Oberst FW, Crouse CL, Billups NB, Koon WS, Musselman NP and Sidell FR (1974) An apparatus for the detection and quantitation of volatile human effluents. J Chromatogr 100: 137-152.

Fishilevich E, Domingos Al, Asahina K, Naef F, Vosshall LB and Louis M (2005) Chemotaxis behavior mediated by single larval olfactory neurons in Drosophila. Curr Biol 15: 2086-2096.

Flower DR, North AC and Sansom CE (2000) The lipocalin protein family: structural and sequence overview. Biochim Biophys Acta 1482: 9-24.

Foret S, Wanner KW and Maleszka R (2007) Chemosensory proteins in the honey bee: Insights from the annotated genome, comparative analyses and expressional profiling. Insect Biochem Mol Biol 37: 19-28.

Forstner M, Gohl T, Gondesen I, Raming K, Breer H and Krieger J (2008) Differential expression of SNMP-1 and SNMP-2 proteins in pheromone-sensitive hairs of moths. Chem Senses 33: 291-299.

Foster WA (1995) Mosquito sugar feeding and reproductive energetics. Annu Rev Entomol 40, 443-474.

Fox AN, Pitts RJ, Robertson HM, Carlson JR and Zwiebel LJ (2001) Candidate odorant receptors from the malaria vector mosquito Anopheles gambiae and evidence of down-regulation in response to blood feeding. PNAS 98: 14693-14697.

Gao Q and Chess A (1999) Identification of candidate Drosophila olfactory receptors from genomic DNA sequence. Genomics 60: 31-39.

Gaunt MW and Miles MA (2002) An insect molecular clock dates the origin of the insects and accords with palaeontological and biogeographic landmarks. Mol Biol Evol 19: 748-761.

Geier M, Bosch OJ and Boeckh J (1999) Ammonia as an attractive component of host odour for the yellow fever mosquito, Aedes aegypti. Chem Senses 24: 647-653.

Ghaninia M, Hansson BS and Ignell R (2007) The antennal lobe of the African malaria mosquito, Anopheles gambiae innervation and three-dimensional reconstruction. Arthropod Struct Dev 36: 23-39. 
Goldman AL, Van der Goes van Naters W, Lessing D, Warr CG and Carlson JR (2005) Coexpression of two functional odor receptors in one neuron. Neuron 45: 661-666.

Grant A and O'Connell R, eds (1996) Electrophysiological responses from receptor neurons in mosquito maxillary palp sensilla. Wiley, Chichester, UK.

Grosse-Wilde E, Gohl T, Bouche E, Breer H and Krieger J (2007) Candidate pheromone receptors provide the basis for the response of distinct antennal neurons to pheromonal compounds. Eur J Neurosci 25: 2364-2373.

Hallem E, Fox AN, Zwiebel LJ and Carlson JR (2004a) A mosquito odorant receptor tuned to a component of human sweat. Nature 427: 212-213.

Hallem E, Ho MG and Carlson JR (2004b) The molecular basis of odor coding in the Drosophila antenna. Cell 117: 965-979.

Healy TP and Copland MJ (1995) Activation of Anopheles gambiae mosquitoes by carbon dioxide and human breath. Med Vet Entomol 9: 331-336.

Healy TP and Copland MJ (2000) Human sweat and 2-oxopentanoic acid elicit a landing response from Anopheles gambiae. Med Vet Entomol 14: 195-200.

Hekmat-Scafe DS, Scafe CR, McKinney AJ and Tanouye MA (2002) Genome-wide analysis of the odorant-binding protein gene family in Drosophila melanogaster. Genome Res 12: 1357-1369.

Hill CA, Fox AN, Pitts RJ, Kent LB, Tan PL, Chrystal MA, Cravchik A, Collins FH, Robertson HM and Zwiebel LJ (2002) G protein-coupled receptors in Anopheles gambiae. Science 298: 176-178.

Holt RA, Subramanian GM, Halpern A, Sutton GG, Charlab R, Nusskern DR, Wincker P, Clark AG, Ribeiro JM, Wides R, Salzberg SL, Loftus B, Yandell M, Majoros WH, Rusch DB, Lai Z, Kraft CL, Abril JF, Anthouard V, Arensburger P, Atkinson PW, Baden H, de Berardinis V, Baldwin D, Benes V, Biedler J, Blass C, Bolanos R, Boscus D, Barnstead M, Cai S, Center A, Chaturverdi K, Christophides GK, Chrystal MA, Clamp M, Cravchik A, Curwen V, Dana A, Delcher A, Dew I, Evans CA, Flanigan M, Grundschober-Freimoser A, Friedli L, Gu Z, Guan P, Guigo R, Hillenmeyer ME, Hladun SL, Hogan JR, Hong YS, Hoover J, Jaillon O, Ke Z, Kodira C, Kokoza E, Koutsos A, Letunic I, Levitsky A, Liang Y, Lin JJ, Lobo NF, Lopez JR, Malek JA, McIntosh TC, Meister S, Miller J, Mobarry C, Mongin E, Murphy SD, O'Brochta DA, Pfannkoch C, Qi R, Regier MA, Remington K, Shao H, Sharakhova MV, Sitter CD, Shetty J, Smith TJ, Strong R, Sun J, Thomasova D, Ton LQ, Topalis P, Tu Z, Unger MF, Walenz B, Wang A, Wang J, Wang M, Wang X, Woodford KJ, Wortman JR, Wu M, Yao A, Zdobnov EM, Zhang H, Zhao Q, Zhao S, Zhu SC, Zhimulev I, Coluzzi M, della Torre A, Roth CW, Louis C, Kalush F, Mural RJ, Myers EW, Adams MD, Smith HO, Broder S, Gardner MJ, Fraser CM, Birney E, Bork P, Brey PT, Venter JC, Weissenbach J, Kafatos FC, Collins FH and Hoffman SL (2002) The genome sequence of the malaria mosquito Anopheles gambiae. Science 298: 129-149.

latrou K and Biessmann $\mathrm{H}$ (2008) Sex-biased expression of odorant receptors in antennae and palps of the African malaria vector Anopheles gambiae. Insect Biochem Mol Biol 38: 268-274.

Ignell R, Dekker T, Ghaninia M and Hansson, BS (2005) Neuronal architecture of the mosquito deutocerebrum. J Comp Neurol 493: 207-240.

Jones WD, Cayirlioglu P, Kadow IG and Vosshall LB (2007) Two chemosensory receptors together mediate carbon dioxide detection in Drosophila. Nature 445: 86-90.

Jones WD, Nguyen TA, Kloss B, Lee KJ and Vosshall LB (2005) Functional conservation of an insect odorant receptor gene across 250 million years of evolution. Curr Biol 15: R119-121.

Kain P, Chakraborty TS, Sundaram S, Siddiqi O, Rodrigues V and Hasan G (2008) Reduced odor responses from antennal neurons of $\mathrm{G}(\mathrm{q})$ alpha, phospholipase Cbeta, and rdgA mutants in Drosophila support a role for a phospholipid intermediate in insect olfactory transduction. J Neurosci 28: 4745-4755.

Kalidas S and Smith DP (2002) Novel genomic cDNA hybrids produce effective RNA interference in adult Drosophila. Neuron 33: 177-184.

Kellogg FE (1970) Water vapour and carbon dioxide receptors in Aedes aegyptii. J Insect Physiology 16: 99-108.

Kent LB, Walden KK and Robertson HM (2007) The Gr family of candidate gustatory and olfactory receptors in the yellow-fever mosquito Aedes aegypti. Chem Senses 33: 79-93.

Knols BGJ (1996) Odour-mediated host seeking behavior of the Afro-tropical malaria vector Anopheles gambiae Giles. Thesis, Wageningen Agricultural University, Wageningen, the Netherlands.

Kreher SA, Kwon JY and Carlson JR (2005) The molecular basis of odor coding in the Drosophila larva. Neuron 46: 445-456. 
Krieger J, Klink O, Mohl C, Raming K and Breer H (2003) A candidate olfactory receptor subtype highly conserved across different insect orders. J Comp Physiol A Neuroethol Sens Neural Behav Physiol 189: 519-526.

Kruse SW, Zhao R, Smith DP and Jones DN (2003) Structure of a specific alcohol-binding site defined by the odorant binding protein LUSH from Drosophila melanogaster. Nat Struct Biol 10: 694-700.

Kwon HW, Lu T, Rutzler M and Zwiebel LJ (2006) Olfactory responses in a gustatory organ of the malaria vector mosquito Anopheles gambiae. PNAS 103: 13526-13531.

Kwon JY, Dahanukar A, Weiss LA and Carlson JR (2007) The molecular basis of CO2 reception in Drosophila. PNAS 104: 3574-3578.

Larsson MC, Domingos Al, Jones WD, Chiappe ME, Amrein H and Vosshall LB (2004) Or83b encodes a broadly expressed odorant receptor essential for Drosophila olfaction. Neuron 43: 703-714.

Laughlin JD, Ha TS, Jones DN and Smith DP (2008) Activation of pheromone-sensitive neurons is mediated by conformational activation of pheromone-binding protein. Cell 133: 1255-1265.

Lu T, Qiu YT, Wang G, Kwon JY, Rutzler M, Kwon HW, Pitts RJ, van Loon JJ, Takken W, Carlson JR and Zwiebel LJ (2007) Odor coding in the maxillary palp of the malaria vector mosquito Anopheles gambiae. Curr Biol 17: 1533-1544.

Matsuo T, Sugaya S, Yasukawa J, Aigaki T and Fuyama Y (2007) Odorant-binding proteins OBP57d and OBP57e affect taste perception and host-plant preference in Drosophila sechellia. PLoS Biology 5: e118 doi: 110.1371/journal. pbio.0050118.

Mclver SB (1972) Fine structure of pegs on the palps of female culicine mosquitoes. Can J Zool 50: 571-576.

Mclver SB (1982) Sensilla of mosquitoes (Diptera: Culicidae). J Med Entomol 19: 489-535.

McKenna MP, Hekmat Scafe DS, Gaines P and Carlson JR (1994) Putative Drosophila pheromone-binding proteins expressed in a subregion of the olfactory system. J Biol Chem 269: 16340-16347.

Meijerink J, Braks MA and Van Loon JJ (2001) Olfactory receptors on the antennae of the malaria mosquito Anopheles gambiae are sensitive to ammonia and other sweat-borne components. J Insect Physiol 47: 455-464.

Meijerink J and van Loon JJ (1999) Sensitivities of antennal olfactory neurons of the malaria mosquito, Anopheles gambiae, to carboxylic acids. J Insect Physiol 45: 365-373.

Melo AC, Rutzler M, Pitts RJ and Zwiebel LJ (2004) Identification of a chemosensory receptor from the yellow fever mosquito, Aedes aegypti, that is highly conserved and expressed in olfactory and gustatory organs. Chem Senses 29: 403-410.

Merrill CE, Pitts RJ and Zwiebel LJ (2003) Molecular characterization of arrestin family members in the malaria vector mosquito, Anopheles gambiae. Insect Molecular Biology 12: 641-650.

Merritt RW, Dadd RH and Walker ED (1992) Feeding behavior, natural food, and nutritional relationships of larval mosquitoes. Annu Rev Entomol 37: 349-376.

Mohl C, Breer H and Krieger J (2002) Species-specific pheromonal compounds induce distinct conformational changes of pheromone binding protein subtypes from Antheraea polyphemus. Invert Neurosci 4: 165-174.

Nakagawa T, Sakurai T, Nishioka T and Touhara K (2005) Insect sex-pheromone signals mediated by specific combinations of olfactory receptors. Science 307: 1638-1642.

Nene V, Wortman JR, Lawson D, Haas B, Kodira C, Tu ZJ, Loftus B, Xi Z, Megy K, Grabherr M, Ren Q, Zdobnov EM, Lobo NF, Campbell KS, Brown SE, Bonaldo MF, Zhu J, Sinkins SP, Hogenkamp DG, Amedeo P, Arensburger P, Atkinson PW, Bidwell S, Biedler J, Birney E, Bruggner RV, Costas J, Coy MR, Crabtree J, Crawford M, Debruyn B, Decaprio D, Eiglmeier K, Eisenstadt E, El-Dorry H, Gelbart WM, Gomes SL, Hammond M, Hannick LI, Hogan JR, Holmes MH, Jaffe D, Johnston JS, Kennedy RC, Koo H, Kravitz S, Kriventseva EV, Kulp D, Labutti K, Lee E, Li S, Lovin DD, Mao C, Mauceli E, Menck CF, Miller JR, Montgomery P, Mori A, Nascimento AL, Naveira HF, Nusbaum C, O'leary S, Orvis J, Pertea M, Quesneville H, Reidenbach KR, Rogers YH, Roth CW, Schneider JR, Schatz M, Shumway M, Stanke M, Stinson EO, Tubio JM, Vanzee JP, Verjovski-Almeida S, Werner D, White O, Wyder S, Zeng Q, Zhao Q, Zhao Y, Hill CA, Raikhel AS, Soares MB, Knudson DL, Lee NH, Galagan J, Salzberg SL, Paulsen IT, Dimopoulos G, Collins FH, Birren B, Fraser-Liggett CM and Severson DW (2007) Genome sequence of Aedes aegypti, a major arbovirus vector. Science 316: 1718-1723.

Neuhaus EM, Gisselmann G, Zhang W, Dooley R, Stortkuhl K and Hatt H (2005) Odorant receptor heterodimerization in the olfactory system of Drosophila melanogaster. Nat Neurosci 8: 15-17. 
Nicastro D, Melzer R, Hruschka $H$ and Smola U (1998) Evolution of small sense organs: sensilla on the larval antennae traced back to the origin of the Diptera. Naturwissenschaften 85: 501-505.

Nichols Z and Vogt RG (2008) The SNMP/CD36 gene family in Diptera, Hymenoptera and Coleoptera: Drosophila melanogaster, D. pseudoobscura, Anopheles gambiae, Aedes aegypti, Apis mellifera, and Tribolium castaneum. Insect Biochem Mol Biol 38: 398-415.

Nijhout HF and Craig GB (1971) Reproductive isolation in Stegomyia mosquitoes. III evidence for a sexual pheromone. Entomologia Experimentalis et Applicata 14: 399-412.

Owen WB, Larsen JR and Pappas LG (1974) Functional units in the labellar chemosensory hairs of the mosquito Culiseta inornata (Williston). J Exp Zool 188: 235-247.

Pappas LG and Larsen JR (1976) Gustatory hairs on the mosquito, Culiseta inornata. Journal of Experimantal Zoology 196: 351-360.

Pelosi P, Baldaccini NE and Pisanelli AM (1982) Identification of a specific olfactory receptor for 2-isobutyl-3methoxypyrazine. Biochem J 201: 245-248.

Pelosi P, Zhou JJ, Ban LP and Calvello M (2006) Soluble proteins in insect chemical communication. Cell Mol Life Sci 63: 1658-1676.

Pitts RJ, Fox AN and Zwiebel LJ (2004) A highly conserved candidate chemoreceptor expressed in both olfactory and gustatory tissues in the malaria vector, Anopheles gambiae. PNAS 101: 5058-5063.

Pitts RJ and Zwiebel LJ (2006) Antennal sensilla of two female anopheline sibling species with differing host ranges. Malar J 5: 26.

Qiu YT, van Loon JJ, Takken W, Meijerink J and Smid HM (2006) Olfactory Coding in Antennal Neurons of the Malaria Mosquito, Anopheles gambiae. Chem Senses 31, 845-863.

Rac ME, Safranow K and Poncyljusz W (2007) Molecular basis of human CD36 gene mutations. Mol Med 13: 288-296.

Ramoni R, Vincent F, Grolli S, Conti V, Malosse C, Boyer FD, Nagnan-Le Meillour P, Spinelli S, Cambillau C and Tegoni M (2001) The insect attractant 1-octen-3-ol is the natural ligand of bovine odorant-binding protein. J Biol Chem 276: 7150-7155.

Robertson HM, Warr CG and Carlson JR (2003) Molecular evolution of the insect chemoreceptor gene superfamily in Drosophila melanogaster. PNAS 100 Suppl 2: 14537-14542.

Rogers ME, Sun M, Lerner MR and Vogt RG (1997) Snmp-1, a novel membrane protein of olfactory neurons of the silk moth Antheraea polyphemus with homology to the CD36 family of membrane proteins. J Biol Chem 272: 14792-14799.

Rudolfs W (1922) Chemotropism of mosquitoes. Bulletin New Jersey Agr Exp St 367: 4-23.

Rutzler M, Lu T and Zwiebel LJ (2006) Galpha encoding gene family of the malaria vector mosquito Anopheles gambiae: expression analysis and immunolocalization of AGalphaq and AGalphao in female antennae. J Comp Neurol 499: 533-545.

Rutzler M and Zwiebel L (2005) Molecular biology of insect olfaction: recent progress and conceptual models. J Comp Physiol A Neuroethol Sens Neural Behav Physiol: 1-14.

Sabatier L, Jouanguy E, Dostert C, Zachary D, Dimarcq JL, Bulet P and Imler JL (2003) Pherokine-2 and -3. Eur J Biochem 270: 3398-3407.

Sato K, Pellegrino M, Nakagawa T, Nakagawa T, Vosshall LB and Touhara K (2008) Insect olfactory receptors are heteromeric ligand-gated ion channels. Nature 452 (7190): 1002-1006.

Singh RN and Singh K (1984) Fine structure of the sensory organs of Drosophila melanogaster Meigen larva (Diptera: Drosophilidae). Int J Insect Morp Embr 13: 255-273.

Smallegange RC, Qiu YT, van Loon JJ and Takken W (2005) Synergism between ammonia, lactic acid and carboxylic acids as kairomones in the host-seeking behaviour of the malaria mosquito Anopheles gambiae sensu stricto (Diptera: Culicidae). Chem Senses 30: 145-152.

Smith DP (2007) Odor and pheromone detection in Drosophila melanogaster. Pflugers Arch 454: 749-758.

Steib BM, Geier M and Boeckh J (2001) The effect of lactic acid on odour-related host preference of yellow fever mosquitoes. Chem Senses 26: 523-528.

Storkuhl KF and Kettler R (2001) Functional analysis of an olfactory receptor in Drosophila melanogater. PNAS 98: 9381-9385. 
Suh GS, Ben-Tabou de Leon S, Tanimoto H, Fiala A, Benzer S and Anderson DJ (2007) Light activation of an innate olfactory avoidance response in Drosophila. Curr Biol 17: 905-908.

Suh GS, Wong AM, Hergarden AC, Wang JW, Simon AF, Benzer S, Axel R and Anderson DJ (2004) A single population of olfactory sensory neurons mediates an innate avoidance behaviour in Drosophila. Nature 431: 854-859.

Syed Z, Ishida Y, Kimbrell DA and Leal WS (2006) Pheromone reception in fruit flies expressing a moth's odorant receptor. PNAS 103: 16538-16543.

Syed $Z$ and Leal WS (2008) Mosquitoes smell and avoid the insect repellent DEET. PNAS 105 (36): 13598-13603.

Takken W, Dekker T and Wijnholds YG (1997) Odor-mediated flight behavior of Anopheles gambiae Giles sensu stricto and $A$-stephensi Liston in response to $\mathrm{CO}_{2}$, acetone, and 1-octen-3-ol (Diptera:Culicidae). Journal of Insect Behavior 10: 395-407.

Takken W and Knols BG (1999) Odor-mediated behavior of Afrotropical malaria mosquitoes. Annu Rev Entomol 44: 131-157.

Vogt RG (2002) Odorant binding protein homologues of the malaria mosquito Anopheles gambiae; possible orthologues of the OS-E and OS-F OBPs OF Drosophila melanogaster. J Chem Ecol 28: 2371-2376.

Vogt RG, Prestwich G. D and Riddiford LM (1988) Sex pheromone receptor proteins. Visualization using a radiolabeled photoaffinity analog. J Biol Chem 263: 3952-3959.

Vogt RG and Riddiford LM (1981) Pheremone binding and inactivation by moth antennae. Nature 293: 161-163.

Vosshall LB, Amrein H, Morozov PS, Rzhetsky A and Axel R (1999) A spatial map of olfactory receptor expression in the Drosophila antenna. Cell 96: 725-736.

Vosshall LB and Stocker RF (2007) Molecular architecture of smell and taste in Drosophila. Annu Rev Neurosci 30: 505-533.

Wang P, Lyman RF, Shabalina SA, Mackay TF and Anholt RR (2007) Association of polymorphisms in odorant-binding protein genes with variation in olfactory response to benzaldehyde in Drosophila. Genetics 177: 1655-1665.

Wanner KW, Nichols AS, Walden KK, Brockmann A, Luetje CW and Robertson HM (2007) A honey bee odorant receptor for the queen substance 9-oxo-2-decenoic acid. PNAS 104: 14383-14388.

Wanner KW, Willis LG, Theilmann DA, Isman MB, Feng Q and Plettner E (2004) Analysis of the insect os-d-like gene family. J Chem Ecol 30: 889-911.

Wetzel CH, Behrendt HJ, Gisselmann G, Stortkuhl KF, Hovemann B and Hatt H (2001) Functional expression and characterization of a Drosophila odorant receptor in a heterologous cell system. PNAS 98: 9377-9380.

Wicher D, Schafer R, Bauernfeind R, Stensmyr MC, Heller R, Heinemann SH and Hansson BS. (2008) Drosophila odorant receptors are both ligand-gated and cyclic-nucleotide-activated cation channels. Nature 452 (7190): 1007-1011.

Wogulis M, Morgan T, Ishida Y, Leal WS and Wilson DK (2006) The crystal structure of an odorant binding protein from Anopheles gambiae: evidence for a common ligand release mechanism. Biochem Biophys Res Commun 339: 157-164.

Wojtasek H, Hansson BS and Leal WS (1998) Attracted or repelled? a matter of two neurons, one pheromone binding protein, and a chiral center. Biochem Biophys Res Commun 250: 217-222.

Woodard C, Alcorta E and Carlson J. (1992) The rdgB gene of Drosophila: a link between vision and olfaction. Journal of Neurogenetics 8: 17-31.

Xia Y, Wang G, Buscariollo D, Pitts J, R, Wenger H and Zwiebel L (2008) The molecular basis of olfactory-based behavior in Anopheles gambiae larvae. PNAS 105: 6433-6438.

Xia Y and Zwiebel LJ (2006) Identification and characterization of an odorant receptor from the West Nile Virus mosquito, Culex quinquefasciatus. Insect Biochemistry and Molecular Biology 36: 169-176.

$\mathrm{Xu}, \mathrm{P}$, Atkinson, R, Jones, DN and Smith, DP (2005) Drosophila OBP LUSH is required for activity of pheromone-sensitive neurons. Neuron 45: 193-200.

Xu, PX, Zwiebel, LJ and Smith, DP (2003) Identification of a distinct family of genes encoding atypical odorant-binding proteins in the malaria vector mosquito, Anopheles gambiae. Insect Molecular Biology 12: 549-560.

Zacharuk, RY, Yin, LR.-S and Blue, SG (1971) Fine structure of the antenna and its sensory cone in the larvae of Aedes aegypti (L.). J Morph 135: 273-298.

Zdobnov EM, von Mering C, Letunic I, Torrents D, Suyama M, Copley RR, Christophides GK, Thomasova D, Holt RA, Subramanian GM, Mueller HM, Dimopoulos G, Law JH, Wells MA, Birney E, Charlab R, Halpern AL, Kokoza E, Kraft CL, Lai Z, Lewis S, Louis C, Barillas-Mury C, Nusskern D, Rubin GM, Salzberg SL, Sutton GG, Topalis P, Wides R, Wincker 
P, Yandell M, Collins FH, Ribeiro J, Gelbart WM, Kafatos FC and Bork P (2002) Comparative genome and proteome analysis of Anopheles gambiae and Drosophila melanogaster. Science 298: 149-159.

Zhou, JJ, He, XL, Pickett, JA and Field, LM (2008) Identification of odorant-binding proteins of the yellow fever mosquito Aedes aegypti: genome annotation and comparative analyses. Insect Mol Biol 17: 147-163.

Zhou, JJ, Kan, Y, Antoniw, J, Pickett, JA and Field, LM (2006) Genome and EST analyses and expression of a gene family with putative functions in insect chemoreception. Chem Senses 31: 453-465. 


\title{
3. Olfactory physiology of blood-feeding vector mosquitoes
}

\author{
Yu Tong Qiu and Joop J.A. van Loon
}

\begin{abstract}
The olfactory organs of blood-feeding mosquitoes are the antennae, maxillary palps and proboscis. On each of these organs, several morphologically distinct types of multiporous sensilla are present that typically house two to four olfactory receptor neurons (ORNs). Electrophysiological studies of ORN responsiveness have allowed the identification of several functional ORN-classes within each sensillum type. Functional ORN-types are distinguished based on their unique spectrum of responses to a set of volatile organic compounds (VOCs). Each neuron expresses one or a few membrane-bound olfactory receptor $(\mathrm{OR})$ proteins that function as the molecular determinant of the response specificity of the ORN. Genomic analysis has identified 79 and 131 candidate OR-genes in the malaria mosquito Anopheles gambiae and the yellow fever mosquito Aedes aegypti, respectively. In the sensillum lymph surrounding the dendrites, water-soluble odorantbinding proteins (OBPs) fulfil roles in transport and inactivation of VOCs. In Anopheles gambiae 57 OBP-genes have been found and in Ae. aegypti this number is 66. The ORNs have been shown to encode odour quality, i.e. molecular structure, odour concentration as well as temporal changes in concentration and spatial distribution. Responses of ORNs belong to two major types: some odour stimuli elicit excitation whereas other odours cause inhibition relative to the pre-stimulus activity. Both response modes are controlled by the same OR. Both generalist ORNs and specialist ORNs are found. The responses of ORNs to odorants are concentration-dependent above a threshold concentration, and both absolute concentration and the change in concentration can be encoded. The ensemble of mosquito ORNs responds to odours emitted by hosts, plants and oviposition sites, which together harbour a substantial diversity of molecular structures. Yet, the number of VOCs tested on the full array of ORN functional types of any mosquito species is still far from exhaustive and rarely exceeds 100 VOCs. A substantial concerted effort of molecular biologists, electrophysiologists and ethologists lies ahead to further unravel the mosquito olfactory system. The information thus obtained is essential for the development of behavioural disruption methods that will contribute to the control of mosquito vector populations.
\end{abstract}

Keywords: sensillum, neuron coding, odour, receptor

\section{Introduction}

Blood feeding mosquitoes are disagreeable to humans not only due to their annoying biting habit but especially because many species transmit fatal human diseases such as malaria, yellow fever, dengue and West Nile virus. Olfaction is essential in the life of a mosquito to ensure survival and successful reproduction. Mosquitoes largely rely on the sense of smell to find a mate, nectar, blood and oviposition sites. Understanding the mechanisms of mosquito olfaction and odour coding is crucial to develop measures based on behavioural disruption to control mosquitoes. Because mosquito species that act as vectors of infectious diseases belong predominantly to the genera Anopheles, Aedes and Culex, we restrict ourselves mainly to these three mosquito genera in this chapter. 


\section{Location and morphology of olfactory organs in adult mosquitoes}

The main olfactory organs of insects are the antenna and mouthparts (Figure 1), which carry specialised cuticular extensions, the so-called sensilla that house olfactory receptor neurons (ORNs). The majority of olfactory sensilla of adult mosquitoes are situated on the antennae, yet a smaller number is located on the maxillary palps and the proboscis. Female mosquitoes possess

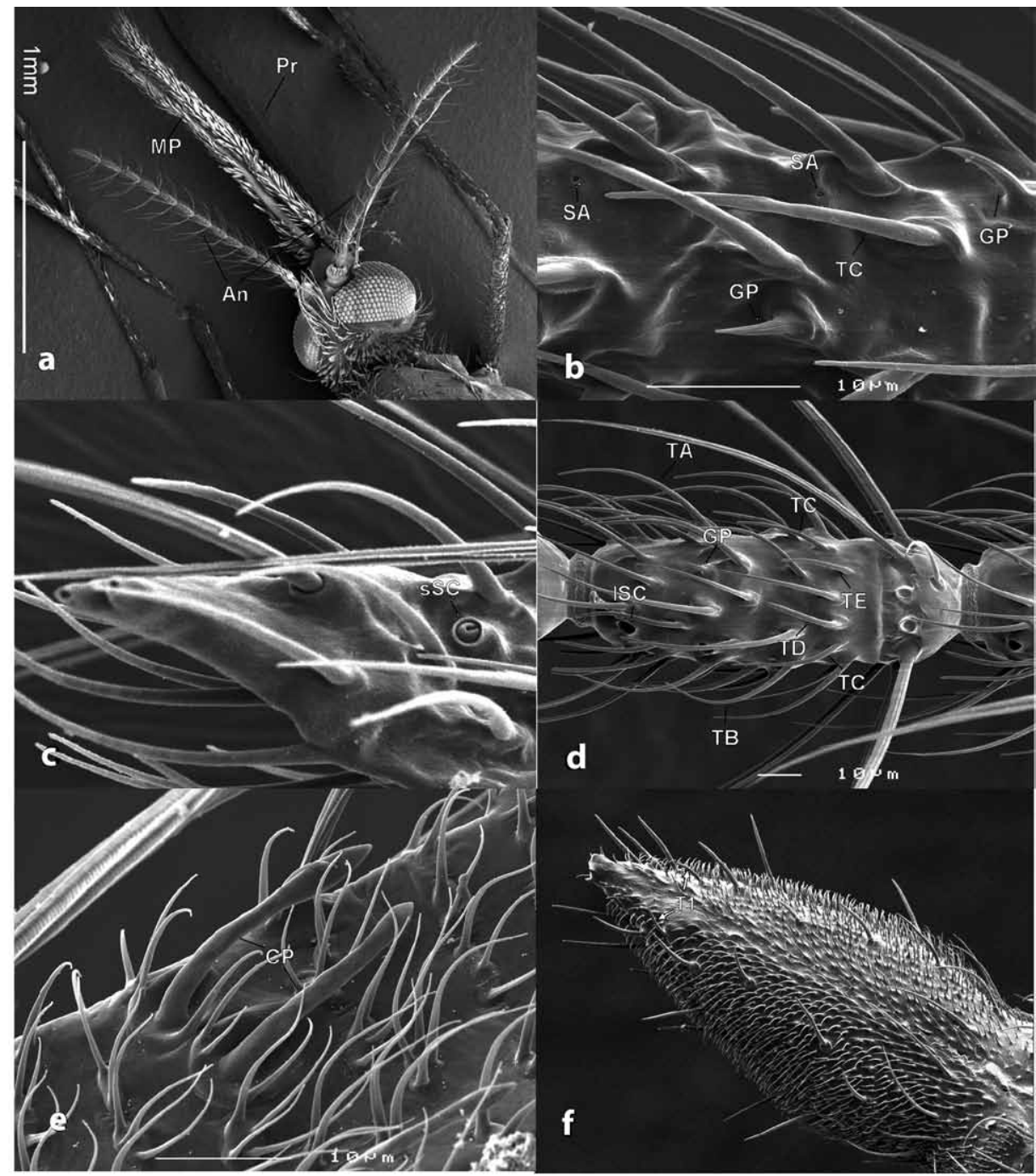

Figure 1. Scanning electron micrograph of olfactory organs of female Anopheles gambiae. (a) The head showing An: antenna; MP: maxillary palp; Pr: proboscis. (b) Sensilla on the antennae. SA: sensillum ampullacea; GP: grooved peg sensillum; TC: sensillum trichodea subtype $C$. (c) The $13^{\text {th }}$ segment of the antenna. sSC: small sensillum coeloconicum. (d) One segment of the antenna. ISC: large sensillum coeloconicum; TA, TB, TC, TD or TE: sensillum trichodea subtype $A, B, C, D$ or $E$. (e) Capitate peg sensilla (CP) on the maxillary palps. (f) The tip of the proboscis showing the olfactory sensilla T1 (SEM photos provided by Hans Smid). 
a much larger number of olfactory sensilla than their male counterparts (Mclver 1982, Table 1). Highly specialised in detecting the wing beat sounds from conspecific female mosquitoes (Cator et al. 2009), the antennae of male mosquitoes are inhabited by fibrillae on most of the antennal segments, only the proximal and the two most distal segments are populated with olfactory sensilla (Ismail 1962).

Olfactory sensilla are hair- or peg-shaped and have a multiporous wall structure. A typical insect olfactory sensillum is composed of two or more bipolar olfactory receptor neurons (ORNs), although sensilla containing one neuron have also been described (Mclver 1982), three auxiliary cells and the surrounding glia, epidermis and cuticle (Figure 2). The surface of the insect cuticle is hydrophobic, a property reducing water evaporation and increasing water repellency. Odour molecules, many of which are non-polar, first have to penetrate through the sensillum wall before reaching the ORNs. There are two types of sensillum walls: single-walled olfactory sensilla contain pore channels through which the non-polar components are excreted to the epicuticle during sensillum formation (Figure 2). Pore channels that remain open are later used for the transportation of the odour molecules into the sensillum lymph (Steinbrecht 1997). In contrast, a double-walled olfactory sensillum is composed of hollow cuticular finger-like structures, which are fused to each other and form at the fusion points, spoke-channels. It is likely that odour molecules get into the sensillum lumen of double-walled sensilla via these channels (Steinbrecht 1997).

\section{Antennal sensilla}

The antennae of adult mosquitoes bear five types of sensilla: chaetica, coeloconica, ampullacea, trichodea, and grooved pegs (Figure 1). The latter type is also referred to as sensilla basiconica in some references, which may be confused with the single-walled basiconic sensilla; therefore here we only use the name grooved peg. Sensilla chaetica are mechanosensilla with a thick wall and a socket at their base. Anophelines are the only mosquitoes in the family of Culicidae that have a large type of coeloconic sensilla. Although both large and small sensilla coeloconica have a 'peg in pit' structure, they differ from each other both morphologically and functionally. The small

Table 1. Numbers of olfactory sensilla of mosquito species located on the antennae and maxillary palps (Mclver 1982).

\begin{tabular}{|c|c|c|c|c|c|c|c|c|}
\hline \multirow[t]{3}{*}{ Species } & \multirow{2}{*}{\multicolumn{2}{|c|}{ Palpi }} & \multicolumn{6}{|c|}{ Antenna } \\
\hline & & & \multicolumn{2}{|c|}{ Trichodea } & \multicolumn{2}{|c|}{ Grooved peg } & \multicolumn{2}{|c|}{ Large coeloconica } \\
\hline & q & $\hat{0}$ & q & $\hat{0}$ & q & $\hat{0}$ & q & $\sigma^{\pi}$ \\
\hline Anopheles gambiae & 67 & 14 & 629 & 227 & 84 & 20 & 33 & 14 \\
\hline Anopheles maculipennis & 65 & 13 & 618 & 300 & 62 & 27 & 28 & 12 \\
\hline Anopheles stephensi & 98 & 9 & 573 & 194 & 107 & 28 & 32 & 8 \\
\hline Aedes aegypti & 29 & 17 & 532 & 175 & 105 & 36 & 0 & 0 \\
\hline Culex pipiens & 78 & 15 & 901 & 239 & 265 & 95 & 0 & 0 \\
\hline Culex territans & 39 & 15 & 537 & 216 & 275 & 174 & 0 & 0 \\
\hline Mansonia africana & 68 & 21 & 623 & 147 & 208 & 36 & 0 & 0 \\
\hline Mansonia uniformis & 75 & 17 & 571 & 164 & 212 & 39 & 0 & 0 \\
\hline Wyomia smithii & 34 & 20 & 218 & 189 & 36 & 30 & 0 & 0 \\
\hline
\end{tabular}




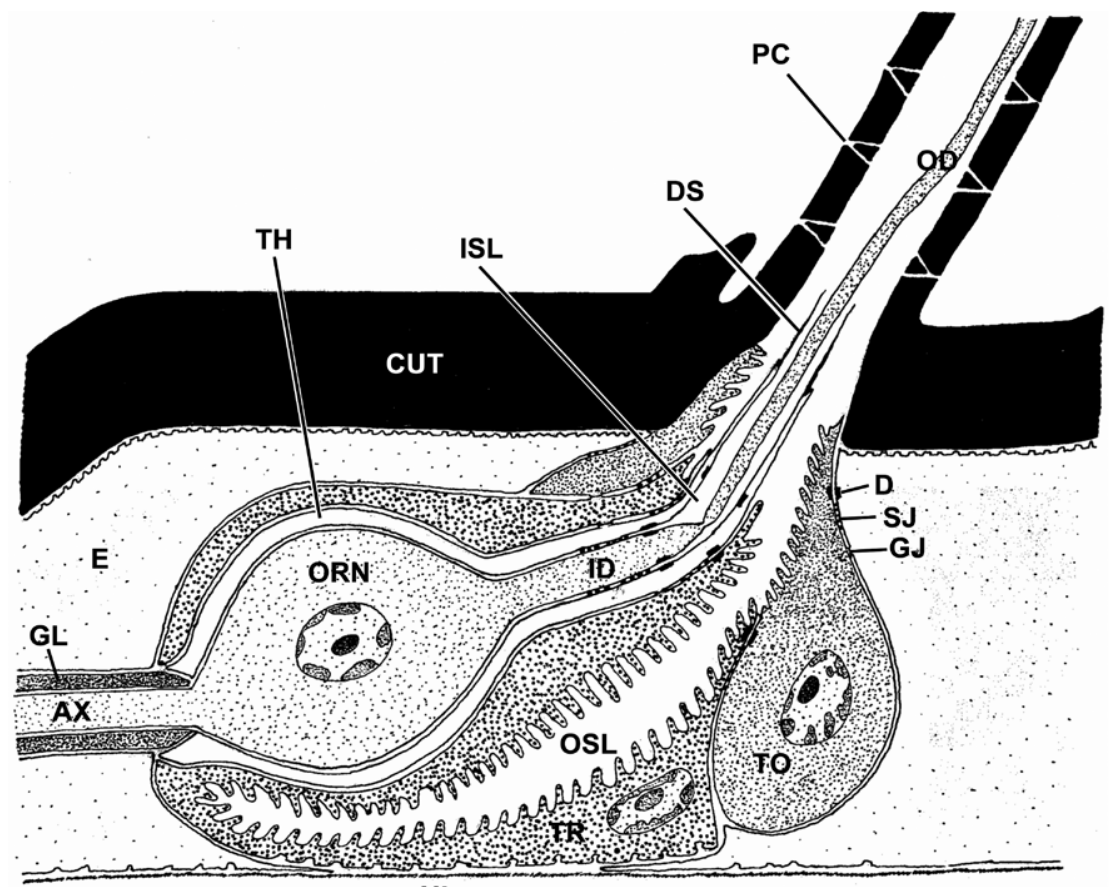

HL

Figure 2. Schematic drawing of the structure of typical olfactory sensilla. AX: axon; CUT: cuticle; D: desmosomes; DS: dendrite sheath; E: epidermis; GJ: gap junctions; GL: glia; HL: haemolymph; ID: inner dendritic segment; ISL: inner sensillum lymph space; OD: outer dendritic segment = ciliary dendrite; ORN: olfactory receptor neuron; OSL: outer sensillum lymph space; PC: pore channel; SJ: septate junction; TH: thecogen cell; TO: tormogen cell; TR: trichogen cell (from Qiu 2005 after Keil and Steinbrecht 1987).

coeloconica contain a single-walled peg, with possibly one or a few pores at the tip (Clements 1999, Mclver 1982). A pair of small coeloconic sensilla is located the tip of mosquito antennae, occasionally these sensilla are found on other antennal segments (Mclver 1982, Pitts and Zwiebel 2006). Two of the three neurons innervating these small sensilla coeloconica were found to be sensitive to convection heat, whereas none of the three neurons are olfactory (Davis and Sokolove 1975, Gingl et al. 2005, Wang et al. 2009). Sensilla ampullacea are thought to function as convection heat detectors because they have a structure similar to that of small sensilla coeloconica (Mclver 1982). Only sensilla trichodea, grooved peg sensilla and large coeloconica are innervated by olfactory neurons.

\section{Sensilla trichodea}

The most abundant type of antennal sensillum of mosquitoes are sensilla trichodea (Figure 1b, 1c), which are single-walled olfactory sensilla normally containing dendrites of two ORNs, occasionally sensilla trichodea innervated by a single neuron can be found on the most distal antennal flagella (Boo 1980a, Mclver 1982) (Figure 3a, 3c). The average number of sensilla trichodea in a species ranges from 200 to 1000 (Table 1, based on Mclver (1982). Mosquito antennae carry several different shapes of sensilla trichodea, each of which has distinct functional characteristics. Culicines have 

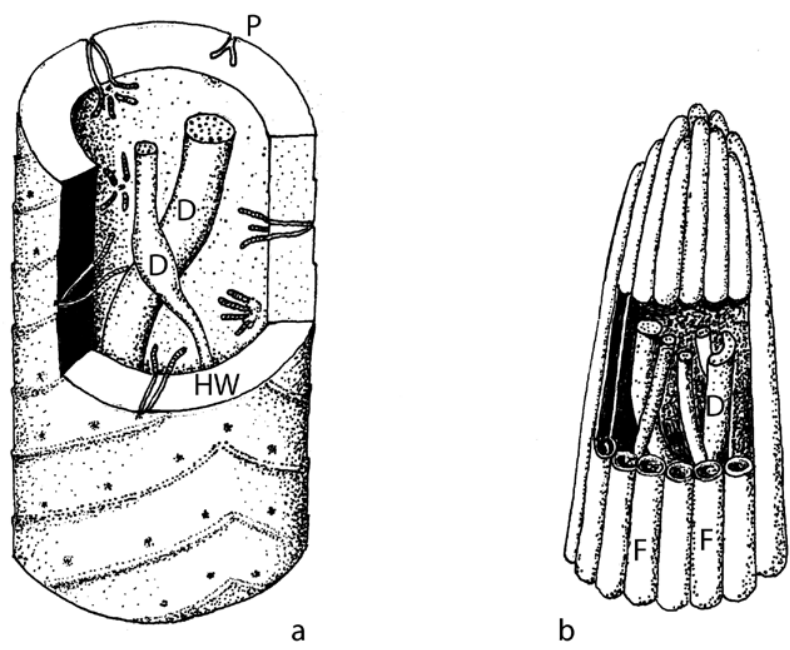

b
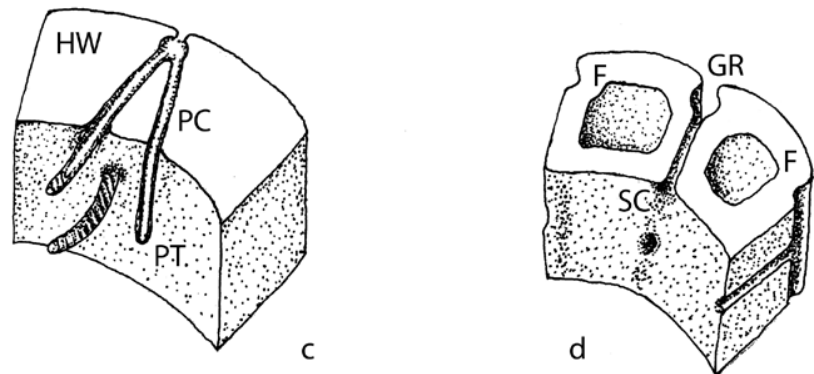

Figure 3 Schematic diagrams of single- and double-wall structure of olfactory sensilla. (a) Section through a sensillum trichodeum (single-walled) and pore tubules. HW: hair wall; D: dendrite; P: pores. (b) Coeloconic sensillum (double-walled), opened to show dendrites therein. The cuticular fingers (F) are hollow and partly fused. (c) Detail of wall of sensillum trichodeum. PC: pore channel; PT: pore tubules. (d) Detail of the double wall of a coeloconic sensillum. SC: spoke channels; F: fingers; GR: groove (from Qiu 2005 after Keil 1999).

basically four types of sensilla trichodea: T1, T2, T3 and T4, classified according to their length, wall thickness and whether the tip is sharp or blunt (reviewed by Mclver 1982). This classification does not fit well for anophelines. Transmission microscope studies by Boo $(1980 \mathrm{a}, \mathrm{b})$ identified five types of sensilla trichodea, designated $A-E$, on the antennae of Anopheles stephensi Liston according to hair length, shape, diameter, wall thickness, neuron branching and pore channel density.

Van den Broek and Den Otter (1999) compared the sensitivities of ORNs in four anopheline species with host preferences ranging from zoophilic to anthropophilic and found differences in the number and sensitivity of ORNs in sensilla trichodea responding to carboxylic acids and 1-octen-3-ol. 


\section{Grooved pegs}

Grooved peg sensilla are double-walled sensilla containing 2 to 5 neurons one of which is assumed to be a hygroreceptor (Figure 3b, 3d). In Aedes and Culex species, grooved peg sensilla show great variation in peg length and it was suggested that two types exist and that the groove length correlated with the sensitivity of innervating neurons to lactic acid (Bowen 1995). However, large overlap occurs between length distributions of pegs with different lactic acid sensitivity.

\section{Large coeloconic sensilla}

The large coeloconic sensilla are only found on the antennae of anopheline mosquitoes (Table 1, Mclver 1982). Double-walled pegs are contained in sunken pits. The number of large coeloconic sensilla on each antenna of female mosquitoes range from 28 for An. maculipennis Meigen to 50 for An. ziemanni Grünberg and these are innervated by four or five neurons, the function of which has not been studied.

\section{Maxillary palps and proboscis}

On the maxillary palps the only sensilla innervated by ORNs are the capitate peg sensilla, which in female anophelines are located on the ventral side of palp segments 2-4 and in male anophelines on segment 4 (Mclver and Siemicki 1975). In both sexes of Culex and Aedes mosquitoes capitate pegs are located on segment 4 (reviewed by Mclver 1982). Three neurons are co-compartmentalised in each capitate peg sensillum. The dendrite of one neuron forms numerous lamellae (reviewed by Mclver 1982).

At the tip of the labellar lobe there are three types of trichoid sensilla, two of which (T1 and T2) are located externally and one (T3) internally (Mclver 1982, Pappas and Larsen 1976). These sensilla were thought to contain mechanical and gustatory neurons, however, a recent study revealed that T2 sensilla contain olfactory receptor proteins and have an olfactory function (Kwon et al. 2006).

\section{Olfactory transduction}

Transduction is the process by which quality (molecular structure) and quantity (concentration) of odorants are converted to the neural code contained in the frequency and temporal patterns of action potentials (see Chapter 4, this volume). The nature of this process is considered to be common to all insects, although most information is derived from studies on Lepidoptera and the fruit fly, Drosophila melanogaster Meigen (Rutzler and Zwiebel 2005). The sensillum lymph of olfactory sensilla contains water soluble proteins, called odorant binding proteins (OBPs) that bind odour molecules and transport them to receptor molecules on the dendritic membrane (Klein 1987, Vogt and Riddiford 1981). The possible functions of OBPs include: selective binding with odour molecules; binding with irrelevant or harmful compounds to reduce their chance of coming into contact with the dendritic membrane (Park et al. 2000); selective transport of odour molecules to specific receptor molecules on the dendritic membrane and selective inactivation of odour molecules (Steinbrecht 1998). Based on the presence of six conserved cystein residues and a conserved spacing between the cysteins, 57 putative OBP-genes in An. gambiae Giles and 66 in Aedes aegypti (L.) have been identified (Xu et al. 2003, Zhou et al. 2008).

On the plasma membrane of the olfactory neuron dendrite one to three ligand-binding olfactory receptor (OR) genes are co-expressed with a conservative co-receptor gene, the Or83b family 
in Drosophila and AgOR7 in An. gambiae. Both OR protein and the co-receptor protein have a seven-transmembrane domain, but unlike the vertebrate G-protein-coupled receptors (GPCRs) their amino termini locate intracellularly. From the genome of An. gambiae, 276 G-proteincoupled receptor homologous genes were identified and 79 of these are considered as putative OR-genes (Hill et al. 2002). Recently, 131 putative odorant receptor genes have been identified from the genome sequence of Ae. aegypti (Bohbot et al. 2007; Chapter 2, this Volume). In An. gambiae conventional AgORs are co-expressed in ORNs with a highly conserved OR gene, AgOR7, and form a heterodimer (Pitts et al. 2004). Recent studies suggest that insect ORs respond to odour ligands along two distinct pathways (Sato et al. 2008, Wicher et al. 2008). Firstly, insect OR heterodimers function as a ligand-gated ion channel. Secondly, the OR heterodimers also follow a metabotropic pathway by forming a non-selective cation channel activated by cyclic nucleotides and odorant-sensing units. When the depolarisation of the membrane resulting from the respective ion flows reaches a threshold, action potentials are generated at the point where the axon exits the perikaryon and travel along the axon, which projects to the antennal lobe in the brain (see Chapter 4, this volume).

\section{Peripheral odour coding}

\section{Properties of odour coding}

By using the single sensillum recording (SSR) method, the action potentials of ORNs can be recorded in situ; their specificity and sensitivity in response to odour stimuli can be studied by quantifying the frequency of action potentials over defined post-stimulus time intervals. ORNs have been shown to encode odour quality, concentration as well as temporal changes in odour concentration and spatial distribution (De Bruyne et al. 2001, Heinbockel and Kaissling 1996, Mustaparta 2002).

ORNs respond selectively to odour compounds with certain structural features such as chain length, electron cloud distribution, the position of double bonds and functional groups of the odour molecule (Boeckh and Ernst 1983, Liliefors et al. 1985, 1987, Shields and Hildebrand 2001). The specificity range or odour tuning width of an ORN is largely determined by the olfactory receptor that is expressed therein. An elegant method to study the function of an single OR-gene is to delete the endogenous OR gene expressed in a specified neuron in the D. melanogaster antenna and to replace it by a single exogenous OR gene (Dobritsa et al. 2003, Hallem et al. 2004a). Not only the differences but also the overlap of the response spectra between different ORNs is important information for the central nervous system to discriminate the quality of an odour compound (De Bruyne et al. 2001, Den Otter and Van der Goes van Naters 1993, Sass 1978). These ORN properties allow several degrees of freedom for odour coding. Based on the response spectra of ORNs to a panel of odorant stimuli, ORNs and the sensilla containing them can be classified into different functional groups (De Bruyne et al. 2001, Ghaninia et al. 2007, Qiu et al. 2006).

ORN responses belong to two major types: some odour stimuli elicit excitation whereas other odours cause inhibition relative to the pre-stimulus activity (De Bruyne et al. 2001, Ghaninia et al. 2007, Qiu et al. 2006, Shields and Hildebrand 2001). This process is controlled by the same OR and can be explained by a simple model (Hallem et al. 2004b). Without odour stimulus, ORs are present as two forms in homeostasis: active and inactive. Once an OR binds with a ligand that elicits an excitation response, the active form is stabilised and the firing frequency is increased; in case an OR binds with a ligand that elicits an inhibition-type response, the inactive form is stabilised and the firing frequency is decreased. 
The responses of ORNs to odorants are concentration-dependent above a threshold concentration, and both absolute concentration and the change in concentration can be detected, provided that the quality of the odorant is coded independently (Todd et al. 1992). It seems that pheromonesensitive-ORNs in lepidopterans are only sensitive to the concentration change (Kaissling 1998). Carbon dioxide-sensitive receptor neurons of some insect species can detect both absolute and relative concentrations (Bogner 1992, Bogner et al. 1986, Den Otter and Van der Goes van Naters 1992, Grant et al. 1995). More ORs are activated when exposed to higher concentrations of odours, which might be a mechanism for insects to detect the absolute odour concentrations (Hallem et al. 2004a).

In most cases two or more ORNs are co-compartmentalised in one sensillum providing the nervous system with one more dimension for odour coding (Todd and Baker 1999). Such an arrangement might be particularly important in the detection of odorant mixtures. The fact that sex pheromonesensitive receptor neurons in moths, which respond behaviourally to a species-specific ratio of often two major components, are commonly housed in the same sensillum, implies a function of co-compartmentalisation in discrimination of ratios between components of odour blends (Baker et al. 1988, Cossé et al. 1995, Hansson et al. 1987, O' Connell 1975).

Temporal characteristics of ORN responses to odours are another feature of odour coding, which is especially important to enable odour discrimination while flying or walking upwind (Baker 1985, Kennedy et al. 1981, Kramer 1992). A response is called phasic if the frequency of firing action potentials decreases abruptly shortly after the onset of the excitation response. A tonic response is characterised by an increase in firing frequency that outlasts the duration of stimulation. The temporal response characteristics of an ORN to a certain stimulus seem largely independent of dosage (De Bruyne et al. 2001, Qiu et al. 2006).

\section{Natural odours utilised by mosquitoes}

\section{Flower- and honeydew-associated odours}

To meet their energetic needs, both female and male mosquitoes of all physiological stages need carbohydrates, obtained mainly from floral and extra-floral nectar and honeydew (Foster 1995 2008). Flower extracts and synthetic plant odours were shown to attract mosquitoes (Foster 2008, Foster and Hancock 1994, Hancock and Foster 1993, Healy and Jepson 1988, Jepson and Healy 1988, Mauer et al. 1999). Information on the compounds in floral fragrances attractive to mosquitoes is scarce (Jhumur et al. 2008). Floral fragrances are composed of a rich diversity of compounds among which terpenes, phenols, aldehydes, fatty acid derivatives and benzenoids are most likely attractants of mosquitoes (Foster 1995, Jhumur et al. 2008)

\section{Human odours}

Female mosquitoes are guided to their blood hosts by the physical and chemical cues emanating from these hosts. Heat, moisture and visual cues from blood hosts are perceived by searching female mosquitoes at a close range. Gillies and Wilkes (1968) reported that carbon dioxide could attract mosquitoes at distances of 18-36 m and natural odours from a calf at distances of 54-73 m.

Human odour is composed of a complex of volatiles released from human skin and exhaled in human breath. GC-MS analysis of human skin emanations collected on glass beads revealed 346 compound peaks (Bernier et al. 2000). More than 100 compounds were identified from exhaled 
human breath (Krotoszynski et al. 1977). Ellin et al. (1974) analysed the composition of total human body effluvia and identified 135 out of more than 300 compounds detected.

Human skin secretes higher amounts of lactic acid compared to other vertebrates, which compound proved to be a mosquito attractant (Dekker et al. 2002, Smith et al. 1970). Lactic acid is attractive by itself for Ae. aegypti (Smith et al. 1970) but not for An. gambiae, whereas a synergistic effect was found between lactic acid, ammonia and a mixture of aliphatic carboxylic acids (Smallegange et al. 2002). Braks and Takken (1999) reported that incubated human sweat was attractive to An. gambiae and that most of this effect could be ascribed to the emission of ammonia produced by microbial activity in sweat. When freshly produced sweat was incubated, the abundance of other components, such as indole, 1-dodecanol, 6-methyl-5-hepten-2-one and geranyl acetone, changed. Incubated sweat was found to be highly attractive whereas the attraction to fresh sweat was weak (Meijerink et al. 2000).

Female mosquitoes were strongly attracted to nylon stockings worn by a human (Pates et al. 2001). GC-MS analysis of the extracts of Limburger cheese, which resembles, to a human nose, the smell of unwashed human feet, revealed the major components to be carboxylic acids (Knols et al. 1997). Forty compounds were identified from a diethyl-ether extract of human sweat, the major components being aliphatic carboxylic acids (Healy and Copland 2000). Oxocarboxylic acids were found in human blood and urine (Chalmers and Lawson 1982). Six oxocarboxylic acids were reported to stimulate landing responses of An. gambiae (Healy et al. 2002). Chemical analysis revealed that the typical human axillary odour is composed of $C_{6}$ to $C_{11}$ straight-chain, branched and unsaturated acids (Zeng et al. 1991, 1996).

\section{Oviposition-site related odours}

Mosquitoes rely on olfactory, optical and tactile cues for locating oviposition sites (Beehler et al. 1993, Dhileepan 1997). Substances originating from mosquito larvae, pupae and eggs have been found attractive to gravid females (reviewed by Bentley and Day 1989, Blackwell and Johnson 2000, Chadee 1993, Zahiri et al. 1997).

Mosquito breeding sites produce semiochemicals, mainly of microbial origin, that stimulate mosquito oviposition (Beehler et al. 1994a, Hasselschwert and Rockett 1988, Hazard et al. 1967). Infusions from decaying wood were found attractive to gravid Ae. triseriatus (Say) and an active component was identified as $p$-cresol (4-methylphenol) (Bentley et al. 1979, 1981). Grass infusions were shown to contain oviposition stimuli for Culex mosquitoes, the attractive compounds include, among others, 3-methylindole, 4-methylphenol and indole (Du and Millar 1999, Millar et al. 1992, Mboera et al. 2000, Lindh et al. 2008). The behavioural responses of mosquitoes to olfactory cues is reviewed in detail in the laboratory (Chapter 7), and in the (semi-)field (Chapter 8) elsewhere in this Volume.

\section{Response spectra of mosquito ORNs}

The response characteristics of ORNs are determined by the ORs expressed therein (Hallem et al. 2004b). Normally ORNs in morphologically different sensilla have different response spectra indicating that they express different ORs. Moreover, sensilla that appear morphologically similar may house ORNs with different response spectra. 
ORNs can be classified according to their spectrum of response specificity to a panel of odour stimulants. The selection of the odour panel has a direct bearing on the classification. An ideal odour panel should contain the best stimulant of each ORN under investigation. Unfortunately, which stimulants are the best for the ORNs is unknown beforehand and in fact it is the central research question that first needs to be answered. In most studies, odours that are known to elicit behavioural activities and odours that typically occur in volatile blends emitted by nectar, hosts or oviposition sites are included in the test panel. However, these odours are not necessarily the best stimulants for the ORNs. For example, the most potent stimulant of the $\mathrm{C}$ neuron innervating capitate peg sensilla on the maxillary palps of female An. gambiae is 2,4,5-trimethylthiazole which is a flavour compound and has not previously been identified as a mosquito infochemical (Lu et al. 2007).

Anopheline and culicine mosquitoes show similarities as well as differences in the subtypes of olfactory sensilla and the function of ORNs. We summarised the results of several electrophysiological studies on different morphological subtypes of sensilla trichodea and grooved peg sensilla in both mosquito families in an attempt to make a comparison (Table 2). For examples,

Table 2. Function of ORNs in sensilla trichodea and grooved peg sensilla in the mosquito Subfamilies Culicinae and Anophelinae.

\begin{tabular}{llll}
\hline Mosquito subfamily & Sensilla & Subtype & Functional types
\end{tabular}

trichodea

short sharp-tipped sst1

sst2

sst3

sst4

long sharp-tipped

blunt I (long)

sbtl1

sbtl2

blunt II (short)

sbtll1

sbtll2

grooved peg lactic acid excited

lactic acid non-responding

lactic acid inhibited 
ORNs sensitive to ammonia and lactic acid are found in grooved peg sensilla in both families; indole-sensitive ORNs are found in short sharp-tipped sensilla trichodea; four functional types have been found in the short sharp-tipped ST in Ae. aegypti, whereas two were found in a similar morphological type of ST in An. gambiae. This comparison is necessarily limited at this time because (1) not all sensillar sub-types have been studied; (2) in anophelines three additional morphological ST-subtypes are present; (3) disparate odour panels have been used in different studies.

\section{Sensilla trichodea (ST)}

Among the five subtypes of ST (A-E) of anophelines only the medium-length sharp-tipped subtype $C$ and short sharp-tipped subtype $E$ have been well studied for their function. By testing the response of olfactory sensilla to a panel of 44 compounds that are components of human odour, plant volatile blends or derived from oviposition sites, Qiu et al. (2006) identified four functional types among ST subtype $C$ and two functional types among subtype $E$ in female $A n$. gambiae. 'Generalist' ORNs that are tuned to a broad range of odours were found in ST subtype $\mathrm{E}$, whereas 'moderate specialist' ORNs that are tuned to a narrow range of odours were found

Inhibition

ethyl propanoate, 4-methylcyclohexanol Kuthiala et al. 1992

4-methylcyclohexanol; 2-butoxyethanol; indole; $\quad 2-3 \mathrm{C}$ acids Ghaninia et al. 2007 ethyl lactate

2-butoxyethanol; indole Ghaninia et al. 2007

4-methylcyclohexanol; 2-butoxyethanol; a-thujone; 3C, 6C acids Ghaninia et al. 2007 ethyl butarate; ethyl propionate 4-methylcyclohexanol; 2-butoxyethanol; ethyl-Lseveral fatty acids and esters Ghaninia et al. 2007 lactate 2-10C acids of fatty acids

7-10C acids; volatile oils; eugenol; citronellol volatile oils Lacher 1967

2-5C acids; eugenol; Lacher 1967
citronellol

4-methylcyclohexanol; 2-butoxyethanol; a-thujone; Ghaninia et al. 2007 a-pinene; acetic acid; ethyl lactate; methyl-\& ethyl butyrate ethyl butyrate; ethyl-L-lactate; ethyl propionate; 2-butoxyethanol esters of 2-4C acids; 1-octen-3-ol; 2-butoxyethanol; 2-3C acids 4methylphenol; a-pinene

lactic acid; 2-3C acids; fluoro-lactate; ammonia pyruvic acid; ammonia 2-3C acids; indole Ghaninia et al. 2007 propionic acid Ghaninia et al. 2007 DEET; fluoro-lactate Davis and Sokolove 1976 2-3C acids; pyruvic acid; ethyl Davis and Sokolove 1976 lactate; 2-butoxyethanol

ammonia; water; pyruvic acid; acetic acid lactic acid; DEET; fluro-lactate; Davis and Sokolove 1976 pyruvic acid; 2-3C acids 
Table 2. Continued.

\begin{tabular}{llll}
\hline Mosquito subfamily & Sensilla & Subtype & Functional types
\end{tabular}

\begin{tabular}{|c|c|c|c|}
\hline \multirow[t]{9}{*}{ Anophelinae } & trichodea & $\begin{array}{l}\mathrm{C} \text { (medium-length sharp- } \\
\text { tipped) }\end{array}$ & $\begin{array}{l}\text { TC1 } \\
\text { TC2 } \\
\text { TC3 } \\
\text { TC4 }\end{array}$ \\
\hline & & E (short sharp-tipped) & $\overline{\mathrm{TE} 1}$ \\
\hline & & & TE2 \\
\hline & & Cor $E$ & \\
\hline & grooved peg & GP1 & \\
\hline & & GP2 & \\
\hline & & GP3 & \\
\hline & & GP4 & \\
\hline & & $\overline{\mathrm{GP5}}$ & \\
\hline
\end{tabular}

in subtype $C$, with one 'specialist' ORN tuned to only geranyl acetone. Phenols were among the most effective stimulants for several neuron types belonging to different functional classes in both subtype $C$ and $E$. Subtype $E$, but not subtype $C$, also houses neurons responding to aliphatic carboxylic acids, oxocarboxylic acids as well as 1-octen-3-ol and its homologs. ORNs responding to 4-methylcyclohexanol and 4-methylphenol were found in short sharp-tipped ST of An. stephensi (Bentley et al. 1982).

The function of ORNs in ST with various shapes was reported for several species of culicines (Bentley et al. 1979, Bowen 1990, Davis 1976, Klowden and Blackmer 1987, Kuthiala et al. 1992, Lacher 1967) (Table 2). A recent study by Ghaninia et al. (2007) classified 11 functional groups within ST using a panel of 16 compounds from six different chemical groups (Table 2). The short sharp-tipped ST contains at least one neuron that is sensitive to 2-butoxy ethanol, which is a component of plant volatiles and attracts Culex quinquefasciatus Say (http: //www.pherobase. net/database/species/species-Culex-.php, accessed 25 August 2009). ORNs in the short sharptipped ST respond strongly to indole, 4-methylcyclohexanol and esters of short-chain carboxylic acids; these compounds stimulate oviposition by gravid culicine mosquitoes (Allan and Kline 1995, Beehler et al. 1994b, Bentley et al. 1982, Perry and Fay 1967), whereas indole is also a major 
Compounds eliciting

References

Excitation

Inhibition

ammonia; phenols

Qiu et al. 2006

geranyl acetone

Qiu et al. 2006

indole; phenols

Qiu et al. 2006

ammonia

Qiu et al. 2006

4-methylcyclohexanol; 4-methylphenol

Bentley et al. 1982

indole; phenols; 1-hexen-3-ol; 5-7C acids; 3-methyl- 3-4C acids

Qiu et al. 2006

1-butanol, ammonia

4-methylphenol; 4-ethylphenol; geranyl acetone;

Qiu et al. 2006

2-nonanone; 1-hexen-3-ol; 1-hepten-3-ol; 1-octen-

3-ol; 7-octenoic acid; 3-methyl-2-hexenoic acid;

3C, 3M4C,6C, 9C, 10C, $12 \mathrm{C}$ acids; oxo-4-5C acids;

ammonia

geranyl acetone, 3-methyl-1-butanol, sulcatone,

2-6C acids

ammonia, 2-6C acids; 1-octen-3-ol; 3- \& 4-methyl

phenol

Meijerink and van Loon

1999, Meijerink et al.

2001, Van den Broek and

Den Otter 1999

ammonia; 4-5C amines

Qiu et al. 2006

ammonia; 4-5C amines; oxo-4-5C acids

Qiu et al. 2006

ammonia; 1-butylamine; 2-oxo-butanoic acid; lactic

Qiu et al. 2006

acid

ammonia; 1-butylamine; 2-oxo-butanoic acid; lactic $3-6 \mathrm{C}$ and $9 \mathrm{C}$ acids

Qiu et al. 2006

acid

ammonia; 4-5C amines; 4-6C acids

Qiu et al. 2006

component of aged human sweat (Meijerink et al. 2000). Short blunt-tipped ST contain neurons sensitive to esters of short-chain carboxylic acids, 4-methylphenol and alcohols, the former two types of compounds were reported to attract gravid mosquitoes and considered to be oviposition stimulants (Bentley et al. 1979, Davis 1976, Klowden and Blackmer 1987). An ORN strongly tuned to the plant volatile, a-thujone, is also found in the short blunt-tipped ST (Ghaninia et al. 2007). The long blunt-tipped STs were found responding to C2-C5 carboxylic acids by inhibition, but to C7$\mathrm{C} 10$ by excitation; they showed excitatory responses to terpineol but were inhibited by eugenol and citronellol, the latter three being terpenoids of plant origin (Lacher 1967). Except for some responses to C2-C10 carboxylic acids recorded by Lacher (1967), long sharp-tipped STs were not responsive to the compounds that have been tested in the study by Ghaninia et al. (2007).

\section{Grooved peg sensilla}

Grooved peg sensilla in An. gambiae and An. quadriannulatus Theobald were reported to respond to polar compounds such as ammonia, lactic acid, acetone, butylamine as well as to complex natural odour mixtures such as incubated sweat, cow odour and Limburger cheese odour, which 
is perceived by the human nose to resemble human foot odour (Meijerink et al. 2001, Van den Broek and Den Otter 1999).

Qiu et al. (2006) found that all sensilla they recorded from in An. gambiae contained at least one neuron that responded to ammonia and 1-butylamine. ORNs in GP sensilla were found responding to oxocarboxylic acids, short-chain carboxylic acids and lactic acid, compounds that are shown to attract mosquitoes or to enhance landing responses (Braks et al. 2001, Healy and Copland 2000, Knols et al. 1997, Smallegange et al. 2005).

Similar as for anophelines, grooved peg sensilla in culicine mosquitoes have also been found responding to short-chain carboxylic acids but hardly to plant and oviposition site-associated volatiles (Davis 1988, Davis and Sokolove 1976). However, the responses of the GP sensilla in Ae. aegypti to ammonia and lactic acid are strikingly different from the responses recorded from the homologous sensilla in An. gambiae. Almost all the GP sensilla in An. gambiae contain ORNs that were excited by ammonia, whereas only $8 \%$ of those in Ae. aegypti responded to ammonia (Davis and Sokolove 1976, Qiu et al. 2006). In contrast, the LA-excited neuron population in Ae. aegypti was present in a higher proportion of grooved peg sensilla (58.8\%) than that in An. gambiae (40\%) (Qiu et al. 2006). The difference in sensitivity of peripheral neurons to ammonia and lactic acid reflects the different roles of these two compounds in the behaviour of these two mosquito species: for An. gambiae ammonia alone is attractive to the females, but lactic acid alone has a negligible effect; while for Ae. aegypti the relative importance of the two compounds in attraction appears reversed (Steib et al. 2001, Geier et al. 1999, Smallegange et al. 2005).

\section{Labellar sensilla}

Because the characteristic co-receptor AgOR7 was found to be expressed in T2 sensilla on the proboscis of An. gambiae and Ae. aegypti, it has been hypothesised that the proboscis of these mosquitoes also functions as an olfactory organ (Melo et al. 2004, Pitts et al. 2004). Subsequent studies by Kwon et al. (2006) tested the response of ORNs in T2 sensilla on the proboscis of female An. gambiae to a range of compounds and found that these neurons were responding to butylamine, acetic acid, oxo-carboxylic acids and ketones with a heterocyclic ring, including acetothiophene, acetylpyridine, acetylthiazole, and acetylphenone. In situ hybridisation revealed the co-expression of the conventional AgOR6 localised in sensilla T2.

\section{Capitate peg sensilla}

For blood feeding mosquitoes and biting midges (Diptera: Ceratopogonidae), a receptor neuron sensitive to $\mathrm{CO}_{2}$ was found in sensilla basiconica on the maxillary palps (Grant and Kline 2003, Grant and O'Connell 1996, Grant et al. 1995, Kellogg 1970). Because almost all the sensilla containing $\mathrm{CO}_{2}$ receptors are innervated by one neuron with an expanded lamellar structure, it has been postulated that this is the neuron that is sensitive to $\mathrm{CO}_{2}$. The capitate peg sensilla of mosquitoes also house a neuron that is highly sensitive to 1-octen-3-ol (Grant and O'Connell 1996).

The function of the three neurons innervating capitate sensilla on the maxillary palps of An. gambiae was studied by Lu et al. (2007). Activity of three neurons has been identified. A neuron from which the largest spike amplitude was recorded responded to $\mathrm{CO}_{2}$ over a narrow concentration range. This neuron showed excitatory responses to several heterocyclic compounds and inhibitory responses to several compounds including indole. The B-neuron was extremely sensitive to 1-octen-3-ol, it also responded to several analogues of 1-octen-3-ol, but with lower 
sensitivity, and several ketones including 6-methyl-5-hepten-2-one. The $\mathrm{C}$ neuron in the capitate sensilla was broadly tuned to heterocyclic compounds, ketones and alcohols. In situ hybridisation indicated the coexpression of three AgGRs (AgGR22, 23 and 24) in neuron A and the co-receptor AgOR7 was expressed together with AgOR8 in the B neuron and with AgOR28 in the C neuron. Expression of these AgORs in heterologous systems verified that the response spectra of the individual receptors matched the in vivo responses of the three neurons.

\section{Effects of physiological state}

The behaviour of a mosquito is determined by the prevalent physiological state. For example, a female mosquito does not take her first blood meal before she reaches a certain age; after a blood meal has been taken, a female mosquito will not respond to host-derived cues for the next 48 to 72 hours until the eggs have matured, in due time she needs to find a suitable oviposition site to deposit the eggs. Does the peripheral olfactory system respond to changes in physiological state? Would mating, age, blood meal, gonotrophic cycle, body size and infection state affect the response of ORNs to odours? Several studies addressed these questions and provided some answers, whereas most of the questions remain unanswered.

\section{Age}

Davis (Davis 1984a) reported that the sensitivity of grooved peg sensilla in Ae. aegypti increased with increasing post-emergence time, which was in accordance with increased host-seeking activity. By comparing the response of the $\mathrm{CO}_{2}$ receptor neuron in capitate peg sensilla of Ae. aegypti, Grant and O'Connell (2007) found that newly emerged female mosquitoes were much less sensitive to $\mathrm{CO}_{2}$ than older females, whereas for males no such pronounced difference was found. The $\mathrm{CO}_{2}$ sensitivity was in accordance with the host-seeking activity.

\section{Blood meal and oviposition}

Lactic acid sensitivity was found to be down-regulated after female mosquitoes took a blood meal, suggesting an involvement of the peripheral olfactory system in the modulation of foraging behaviour of Ae. aegypti (Davis 1984b). After oviposition, the lactic acid sensitivity returns to the pre-blood-fed level. Davis and Takahashi (1980) found that ORNs in blunt-tipped type II sensilla of gravid females were more sensitive to oviposition site-related compounds, namely methyl- and ethyl-esters of C2-C4 carboxylic acids, than the non-gravid females.

After a blood meal, a new functional type of sensilla trichodea E was found for An. gambiae, which was highly sensitive to indole and 3-methyl indole, and sensitive to C5-C9 carboxylic acids as well as 7-octenoic acid, which is a component of human axillary odours (Qiu et al. 2006, Zeng et al. 1991). Increasing sensitivity to these compounds might facilitate female mosquitoes to locate oviposition sites and hosts in the next gonotrophic cycle more efficiently. The overall sensitivity of ORNs in gravid mosquitoes to ammonia and phenols was lower (Qiu et al. 2006), supporting a role in the observed suppression of host-seeking behaviour. Up to 435 gene products are either up- or down-regulated after the ingestion of a blood meal by female An. gambiae (Holt et al. 2002, Ribeiro 2003). One of the putative OR genes (AgOR1) was strongly down regulated $12 \mathrm{~h}$ after blood feeding (Fox et al. 2001). When AgOR1 was expressed in a Drosophila ORN of which the original OR was deleted ('empty neuron'), it was found specifically responsive to 4-methylphenol (Hallem et al. 2004a). The overall decrease of sensitivity to phenols found by Qiu et al. (2006) might be caused by the down-regulation of these and similar genes. 


\section{Diapause}

The lactic acid-sensitive neurons found in active females do not respond in the diapausing females of $C x$. pipiens Linnaeus, whereas neurons that are sensitive to putative oviposition stimulants are unaffected during diapause (Bowen et al. 1988). A subsequent study showed that in female $C X$. pipiens that had terminated diapause high sensitivity to lactic acid was restored.

\section{Translation of physiology to behaviour}

\section{Input-output relations}

As discussed above, mosquito ORNs have varied specificity spectra and sensitivity ranges. Acrossfibre patterning is the most plausible coding principle operating in the mosquito olfactory system (Ghaninia et al. 2007, Qiu et al. 2006). What conclusions can we draw from the electrophysiological studies in terms of their behavioural relevance? Can we predict which compound is attractive or repellent to mosquitoes based on our electrophysiological data? Can we say anything at all about the behavioural relevance of a compound or compound mixture after the electrophysiological activity these elicit has been recorded? Does the response intensity of ORNs correlate with behavioural response? Has one compound a higher behavioural impact when more ORNs are tuned to it or when it is tuned to only by few specific neurons? Do excitatory and inhibitory responses have a different behavioural effect? We must establish that we have only preliminary answers to some of these questions. Correlations between ORN specificity and sensitivity to a compound and behavioural significance of this compound have been found in several cases, but a full picture is still out of reach. Several examples are given below.

In all the tested species $\mathrm{CO}_{2}$ receptors are found in the capitate peg sensilla on the maxillary palps (Grant et al. 1997, Grant and O' Connell 1996, Lu et al. 2007). As expected, $\mathrm{CO}_{2}$ is indeed generally attractive to mosquitoes (Dekker et al. 2001, Gillies 1980, Mboera and Takken 1997, Qiu et al. 2007). It is noteworthy that the $\mathrm{CO}_{2}$-sensitive neurons respond within a narrow concentration range and the response intensity is not particularly high. An ORN responding to 1-octen-3-ol is co-compartmentalised in the same capitate pegs as the $\mathrm{CO}_{2}$ receptor neurons. The sensitivity to 1-octen-3-ol is exceptionally high compared to other ligands, and the response intensity is much higher than observed for the $\mathrm{CO}_{2}$-sensitive neuron. Yet in behavioural tests, 1-octen-3-ol showed much more variable effects to various mosquito species and on its own is rarely attractive to mosquitoes (Kline 1994).

For ammonia, an abundant component of incubated sweat, a better match between electrophysiology and behaviour has been found. Ammonia elicits excitatory responses in all functional types of grooved peg sensilla and some types of sensilla trichodea in An. gambiae (Meijerink et al. 2001, Qiu et al. 2006), which finding nicely correlates with the reliable attraction of female An. gambiae to sources of this compound (Braks et al. 2001, Smallegange et al. 2005). Lactic acid has a similar behavioural effect on female Ae. aegypti as ammonia on female An. gambiae, and neurons that are sensitive to this compound are found in the majority of the grooved peg sensilla, as mentioned before.

$\mathrm{N}, \mathrm{N}$-diethyl-3-methylbenzamide (DEET) is a strong repellent for many species of mosquitoes and several electrophysiological studies attempted to reveal its mode of action. It was found in Ae. aegypti that blunt-tipped type I ST contain neurons that can be excited by DEET (Lacher 1967). Other studies suggested that DEET makes female mosquitoes anosmic to lactic acid (Davis 
1985, Davis and Sokolove 1976) and oviposition stimulants (Kuthiala et al. 1992). A recent study showed that DEET reduced the sensitivity of a neuron expressing AgOR8 responsive to 1-octen-3ol (Ditzen et al. 2008). More recently, however, Syed and Leal (Syed and Leal 2008) showed that the decrease of 1-octen-3-ol activity is due to the adsorbing properties of DEET, as deduced from the observation that when the two compounds were delivered separately the response to 1-octen3-ol was unaffected. These authors also found DEET sensitive neurons on the short sharp-tipped ST in CX. quinquefasciatus.

\section{Conclusions and outlook}

Understanding the chemical ecology of natural odours mediating mosquito behaviours is extremely important. Many natural odours from different sources share the same chemical components. It is the combination of different components and the ratios between the amounts of these components that determine characteristic profiles of natural odours. For example, carboxylic acids, indoles and phenols are found among plant volatiles, human emanations and oviposition site odours alike. From behavioural studies it has become clear that not a single VOC, but a subset of VOCs (the most effective composition of which is as yet unknown) as occurring in the complex compound mixtures that natural sources provide, form an ensemble code that allows the olfactory system to discriminate between a nectar source, a host body or an oviposition site. Components in a mixture may synergise or antagonise the activity of each other already at the peripheral level (Ochieng et al. 2002). As an example, it will be of considerable interest to analyse the possible peripheral interactions occurring between ammonia, lactic acid and carboxylic acids when these are offered as different blends of these three components that have been shown to cause behavioural synergism (Smallegange et al. 2005). Electrophysiological studies on volatile blends are rare, as a strong bias for single compound stimulations has been noted. Subsequent processing of all olfactory input takes place in the olfactory lobe of the brain to either generate or suppress a behavioural response (Chapter 4, this volume).

The amount of electrophysiological data on ORN-functional types has grown over the past decade but is still modest and indeed far from exhaustive. The large number of sensilla (Table 1) and even more so, ORNs, on mosquito antennae and the absence of regionalised sensillar distributions make it difficult to carry out an exhaustive inventory. It is to be expected that a larger sample size of the total ORNs studied enhances the chance of identifying additional functional classes. Thus, increasing this sample size is still a first and foremost but also tedious task, yet essential to increase the likelihood that the sample attains a size representative for the total ORN population. The availability of cloned OR-genes allows for screening of their ligand-specificity spectra gene by gene using heterologous platforms. This necessitates close collaboration between molecular biologists and electrophysiologists as for verification of the data thus obtained in the donor mosquito species itself, the use of SSR-techniques remains a crucial final step.

Looking at mosquito olfaction from the perspective of the complex chemistry of natural VOCmixtures released by hosts, oviposition sites and nectar- and honeydew sources, the number of VOCs tested on the full array of ORN functional types of any mosquito species is still far from exhaustive and rarely exceeds 100 VOCs. Increasing the panel of VOCs is likely to reveal new functional ORN-types. Expanding this panel to the 300 or so VOCs that are produced by the human body is one goal, but including non-natural analogs and indeed, synthetic compounds, is bound to aid our insight in ORN specificity and olfactory coding in blood feeding vector mosquitoes. The predictability of olfaction-mediated behavioural responses based on quantified olfactory input is 
a real scientific challenge for any sensory physiologist and bears particular societal relevance in case of mosquito species that are such threatening disease vectors.

\section{References}

Allan SA and Kline DL (1995) Evaluation of organic infusions and synthetic compounds mediating oviposition in Aedes albopictus and Aedes aegypti (Diptera: Culicidae). J Chem Ecol 21: 1847-1860.

Baker TC (1985) Chemical control of behavior. Pergamon Press, Oxford, UK.

Baker TC, Hansson BS, Löfstedt C and Löfqvist J (1988) Adaptation of antennal neurons in moths is associated with cessation of pheromone-mediated upwind flight. PNAS 85: 9826-9830.

Beehler JW, Millar JG and Mulla MS (1993) Synergism between chemical attractants and visual cues influencing oviposition of the mosquito, Culex quinquefasciatus (Diptera: Culicidae). J Chem Ecol 19: 635-644.

Beehler JW, Millar JG and Mulla MS (1994a) Protein hydrolysates and associated bacterial contaminants as oviposition attractants for the mosquito Culex quinquefasciatus. Med Vet Entomol 8: 381-385.

Beehler JW, Millar JG and Mulla MS (1994b) Field evaluation of synthetic compounds mediating oviposition in Culex mosquitos (Diptera, Culicidae). J Chem Ecol 20: 281-291.

Bentley MD, McDaniel IN, Yatagai M, Lee H-P and Maynard R (1979) $p$-Cresol: an oviposition attractant of Aedes triseriatus. Environ Entomol 8: 206-209.

Bentley MD, McDaniel IN, Yatagai M, Lee HP and Maynard R (1981) Oviposition attractants and stimulants of Aedes triseriatus (Say) (Diptera: Culicidae). Environ Entomol 10: 186-189.

Bentley MD, McDaniel IN and Davis EE (1982) Studies of 4-methylcyclohexanol: an Aedes triseriatus (Say)(Diptera: Culicidae) oviposition attractant. Environ Entomol 19: 589-592.

Bentley MD and Day JF (1989) Chemical ecology and behavioral aspects of mosquito oviposition. Annu Rev Entomol 34: 401-421.

Bernier UR, Kline DL, Barnard DR, Schreck CE and Yost RA (2000) Analysis of human skin emanations by gas chromatography/mass spectrometry. 2 . Identification of volatile compounds that are candidate attractants for yellow fever mosquito (Aedes aegypti). Anal Chem 72: 747-756.

Blackwell A and Johnson SN (2000) Electrophysiological investigation of larval water and potential oviposition chemoattractants for Anopheles gambiae sensu stricto. Ann Trop Med Parasitol 94 389-398.

Boeckh J and Ernst K-D (1983) Olfactory food and mate recognition. Springer, Berlin, Heidelberg, Germany.

Bogner F, Boppré M, Ernst K-D and Boeckh J (1986) $\mathrm{CO}_{2}$ sensitive receptors on labial palps of Rhodogastria moths (Lepidoptera: Arctiidae): physiology, fine structure and central projection. J Comp Physiol [A] 158: 741-749.

Bogner $\mathrm{F}$ (1992) Response properties of $\mathrm{CO}_{2}$-sensitive receptors in tsetse flies (Diptera: Glossina palpalis). Physiol Entomol 17: 19-24.

Bohbot J, Pitts RJ, Kwon HW, Rutzler M, Robertson HM and Zwiebel LJ (2007) Molecular characterization of the Aedes aegypti odorant receptor gene family. Insect Mol Biol 16: 525-537.

Boo KS (1980a) Fine structure of the antennal sensory hairs in female Anopheles stephensi. Z Parasitenkd 61: 161-171.

Boo KS (1980b) Antennal sensory receptors of the male mosquito, Anopheles stephensi. Z Parasitenkd 61: 249-264.

Bowen MF, Davis EE and Haggart DA (1988) A behavioral and sensory analysis of host-seeking behavior in the diapausing mosquito Culex pipiens. J Insect Physiol 34: 805-813.

Bowen MF (1990) Post-diapause sensory responsiveness in Culex pipiens. J Insect Physiol 36: 923-929.

Bowen MF (1995) Sensilla basiconica (grooved pegs) on the antennae of female mosquitos - electrophysiology and morphology. Entomol Exp Appl 77: 233-238.

Braks MAH and Takken W (1999) Incubated human sweat but not fresh sweat attracts the malaria mosquito Anopheles gambiae sensu stricto. J Chem Ecol 25: 663-672.

Braks MAH, Meijerink J and Takken W (2001) The response of the malaria mosquito, Anopheles gambiae, to two components of human sweat, ammonia and L-lactic acid, in an olfactometer. Physiol Entomol 26: 142-148.

Cator LJ, Arthur BJ, Harrington LC and Hoy RR (2009) Harmonic convergence in the love songs of the dengue vector mosquito. Science 323: 1077-1079. 
Chadee DD (1993) Oviposition response of Aedes aegypti (L.) to the presence of conspecific eggs in the field in Trinidad, W.I. J Am Mosq Control Assoc 64: 63-66.

Chalmers RA and Lawson AM (1982) Organic acids in man. Chapman \& Hall, London, UK.

Clements AN (1999) Adult integumental sensilla: their structure, physiology and connections with the brain. In: Clements AN (ed) The biology of mosquitoes. Vol. 2: Sensory reception and behaviour. CAB International, Wallingford, UK, pp 8-54.

Cossé AA, Campbell MG, Glover TJ, Linn CEJ, Todd JL, Baker TC and Roelofs WL (1995) Pheromone behavioral responses in unusual male European corn borer hybrid progeny not correlated to electrophysiological phenotypes of their pheromone-specific antennal neurons. Experientia 51: 809-816.

Davis EE and Sokolove PG (1975) Temperature responses of antennal receptors of the mosquito Aedes aegypti. J Comp Physiol [A] 96: 223-236.

Davis EE (1976) Receptor sensitive to oviposition site attractants on antennae of mosquito, Aedes aegypti. J Insect Physiol 22: 1371-1376.

Davis EE and Sokolove PG (1976) Lactic acid-sensitive receptors on antennae of mosquito, Aedes aegypti. J Comp Physiol [A] 105: 43-54.

Davis EE and Takahashi FT (1980) Hormonal modification of chemoreceptor sensitivity in an insect. Am Zool 20: 936-936.

Davis EE (1984a) Development of lactic acid receptor sensitivity and host-seeking behavior in newly emerged female Aedes aegypti mosquitoes. J Insect Physiol 30: 211-215.

Davis EE (1984b) Regulation of sensitivity in the peripheral chemoreceptor systems for host-seeking behavior by a hemolymph-borne factor in Aedes aegypti. J Insect Physiol 30: 179-183.

Davis EE (1985) Insect repellents - concepts of their mode of action relative to potential sensory mechanisms in mosquitos (Diptera, Culicidae). J Med Entomol 22: 237-243.

Davis EE (1988) Structure-response relationship of the lactic acid-excited neurones in the antennal grooved-peg sensilla of the mosquito Aedes aegypti. J Insect Physiol 34: 443-449.

De Bruyne M, Foster K and Carlson JR (2001) Odor coding in the Drosophila antenna. Neuron 30: 537-552.

Dekker T, Takken W and Cardé RT (2001) Structure of host-odour plumes influences catch of Anopheles gambiae s.s. and Aedes aegypti in a dual-choice olfactomer. Physiol Entomol 26: 124-134.

Dekker T, Steib B, Cardé RT and Geier M (2002) L-Lactic acid, a human-signifying host cue for the anthropophilic mosquito Anopheles gambiae sensu stricto. Med Vet Entomol 16: 91-98.

Den Otter CJ and van der Goes van Naters WM (1992) Single cell recordings from tsetse (Glossina m. morsitans) antennae reveal olfactory, mechano- and cold receptors. Physiol Entomol 17: 33-42.

Den Otter CJ and Van der Goes van Naters WM (1993) Responses of individual antennal olfactory cells of tsetse flies (Glossina m. morsitans) to phenols from cattle urine. Physiol Entomol 18: 43-49.

Dhileepan K (1997) Physical factors and chemical cues in the oviposition behavior of arboviral vectors Cules annulirostris and Culex molestus (Diptera: Culicidae). Environ Entomol 26: 318-326.

Ditzen M, Pellegrino M and Vosshall LB (2008) Insect odorant receptors are molecular targets of the insect repellent DEET. Science 319: 1838-1842.

Dobritsa AA, van der Goes van Naters W, Warr CG, Steinbrecht RA and Carlson JR (2003) Integrating the molecular and cellular basis of odor coding in the Drosophila antenna. Neuron 37: 827-841.

Du YJ and Millar JG (1999) Electroantennogram and oviposition bioassy responses of Culex quinquefasciatus and Culex tarsalis to chemicals in odors from bermuda grass infusions. J Med Entomol 36: 158-166.

Ellin RI, Farrand RL, Oberst FW, Crouse CL, Billups NB, Koon WS, Musselman NP and Sidell FR (1974) An apparatus for the detection and quantitation of volatile human effluents. J Chromatogr 100: 137-152.

Foster WA and Hancock RG (1994) Nectar-related olfactory and visual attractants for mosquitoes. J Am Mosq Control Assoc 10: 288-296.

Foster WF (1995) Mosquito sugar feeding and reproductive energetics. Annu Rev Entomol 40: 443-447.

Foster WA (2008) Phytochemicals as population sampling lures. J Am Mosq Control Assoc 24: 138-146.

Fox AN, Pitts RJ, Robertson HM, Carlson JR and Zwiebel LJ (2001) Candidate odorant receptors from the malaria vector mosquito Anopheles gambiae and evidence of downregulation in response to blood feeding. PNAS 98: 14693-14697. 
Geier M, Bosch OJ and Boeckh J (1999) Ammonia as an attractive component of host odour for the Yellow fever mosquito Aedes aegypti. Chem Senses 24: 647-653.

Ghaninia M, Ignell R and Hansson BS (2007) Functional classification and central nervous projections of olfactory receptor neurons housed in antennal trichoid sensilla of female yellow fever mosquitoes, Aedes aegypti. Eur J Neurosci 26: 1611-1623.

Gillies MT and Wilkes TJ (1968) A comparison of the range of attraction of animal baits and of carbon dioxide for some West African mosquitoes. Bull Entomol Res 59: 441-456.

Gillies MT (1980) The role of carbon dioxide in host-finding by mosquitoes (Diptera: Culicidae): a review. Bull Entomol Res 70: 525-532.

Gingl E, Hinterwirth A and Tichy $\mathrm{H}$ (2005) Sensory representation of temperature in mosquito warm and cold cells. J Neurophysiol 94: 176-185.

Grant AJ, Wigton BE and Aghajanian JG (1995) Electrophysiological responses of receptor neurons in mosquito maxillary palp sensilla to carbon dioxide. J Comp Physiol [A] 177: 389-396.

Grant AJ and O' Connell RJ (1996) Electrophysiological responses from receptor neurons in mosquito maxillary palp sensilla. In: Bock GR and Cardew G (eds), Olfaction in Mosquito Host Interactions Ciba Foundation Symposium 200, Wiley, New York, USA, pp 233-248.

Grant AJ, Borroni PF and O' Connell RJ (1997) Pulsed pheromone stimuli affect the temporal response of antennal receptor neurones of the adult cabbage looper moth. Physiol Entomol 22: 123-130.

Grant AJ and Kline DL (2003) Electrophysiological responses from Culicoides (Diptera: Ceratopogonidae) to stimulation with carbon dioxide. J Med Entomol 40: 284-293.

Hallem EA, Fox AN, Zwiebel LJ and Carlson JR (2004a) Mosquito receptor for human-sweat odorant. Nature 427: 212-213.

Hallem EA, Ho MG and Carlson JR (2004b) The molecular basis of odor coding in the Drosophila antenna. Cell 117: 965-979.

Hancock RG and Foster WA (1993) Effect of preblood-meal sugar on sugar seeking and upwind flight by gravid and parous Aedes aegypti (Diptera: Culicidae). J Med Entomol 30: 353-359.

Hansson BS, Lofstedt C and Roelofs WL (1987) Inheritance of olfactory response to sex pheromone components in Ostrinia nubilalis. Naturwissenschaften 74: 497-499.

Hasselschwert D and Rockett CL (1988) Bacteria as ovipositional attractants for Aedes aegypti (Diptera: Culicidae). The Great Lakes Entomologist 21: 163-168.

Hazard El, Mayer MS and Savage KE (1967) Attraction and oviposition stimulation of gravid female mosquitoes by bacteria isolated from hay infusions. Mosquito News 27: 133-136.

Healy TP and Jepson PC (1988) The location of floral nectar sources by mosquitoes: the long-range responses of Anopheles arabiensis Patton (Diptera: Culicidae) to Achillea millefolium flowers and isolated floral odour. Bull Entomol Res 78: 651-657.

Healy TP and Copland MJW (2000) Human sweat and 2-oxopentanoic acid elicit a landing response from Anopheles gambiae. Med Vet Entomol 14: 195-200.

Healy TP, Copland MJW and Cork A (2002) Landing responses of Anopheles gambiae elicited by oxocarboxylic acids. Med Vet Entomol 16: 126-132.

Heinbockel T and Kaissling KE (1996) Variability of olfactory receptor neuron responses of female silkmoths (Bombyx mori L.) to benzoic acid and ( \pm )-linalool. J Insect Physiol 42: 565-578.

Hill CA, Fox AN, Pitts RJ, Kent LB, Tan PL, Chrystal MA, Cravchik A, Collins FH, Robertson HM and Zwiebel LJ, (2002)G protein-coupled receptors in Anopheles gambiae. Science 298(5591): 176-178.

Holt RA, Subramanian GM, Halpern A, Sutton GG, Charlab R, Nusskern DR, Wincker P, Clark AG, Ribeiro JM, Wides R, Salzberg SL, Loftus B, Yandell M, Majoros WH, Rusch DB, Lai Z, Kraft CL, Abril JF, Anthouard V, Arensburger P, Atkinson PW, Baden H, de B, V, Baldwin D, Benes V, Biedler J, Blass C, Bolanos R, Boscus D, Barnstead M, Cai S, Center A, Chaturverdi K, Christophides GK, Chrystal MA, Clamp M, Cravchik A, Curwen V, Dana A, Delcher A, Dew I, Evans CA, Flanigan M, Grundschober-Freimoser A, Friedli L, Gu Z, Guan P, Guigo R, Hillenmeyer ME, Hladun SL, Hogan JR, Hong YS, Hoover J, Jaillon O, Ke Z, Kodira C, Kokoza E, Koutsos A, Letunic I, Levitsky A, Liang Y, Lin JJ, Lobo NF, Lopez JR, Malek JA, McIntosh TC, Meister S, Miller J, Mobarry C, Mongin E, Murphy SD, O'Brochta DA, Pfannkoch C, Qi R, 
Regier MA, Remington K, Shao H, Sharakhova MV, Sitter CD, Shetty J, Smith TJ, Strong R, Sun J, Thomasova D, Ton LQ, Topalis P, Tu Z, Unger MF, Walenz B, Wang A, Wang J, Wang M, Wang X, Woodford KJ, Wortman JR, Wu M, Yao A, Zdobnov EM, Zhang H, Zhao Q, Zhao S, Zhu SC, Zhimulev I, Coluzzi M, della TA, Roth CW, Louis C, Kalush F, Mural RJ, Myers EW, Adams MD, Smith HO, Broder S, Gardner MJ, Fraser CM, Birney E, Bork P, Brey PT, Venter JC, Weissenbach J, Kafatos FC, Collins FH and Hoffman SL, (2002)The genome sequence of the malaria mosquito Anopheles gambiae. Science 298(5591): 129-149.

Ismail IAH (1962) Sense organs in the antennae of Anopheles maculipennis atroparvus (V.Thiel) and their possible function in relation to the attraction of female mosquito to man. Acta Trop 19: 1-58.

Jepson PC and Healy TP (1988) The location of floral nectar sources by mosquitoes: an advanced bioassay for volatile plant odours and initial studies with Aedes aegypti (L.) (Diptera: Culicidae). Bull Entomol Res 78: 641-650.

Jhumur US, Dötterl S and Jürgens A (2008) Floral odors of Silene otites: their variability and attractiveness to mosquitoes. J Chem Ecol 34: 14-25.

Kaissling KE (1998) Flux detectors versus concentration detectors: two types of chemoreceptors. Chem Senses 23: 99-111.

Keil TA and Steinbrecht RA (1987) Diffusion barriers in silkmoth sensory epithelia: application of lanthanum tracer to olfactory sensilla of Antheraea polyphemus and Bombyx mori. Tissue Cell 19: 119-134.

Keil TA (1999) Morphology and development of the peripheral olfactory organs. In: Hansson BS (ed) Insect olfaction. Springer-Verlag, Berlin, Germany, pp 5-47.

Kellogg FE (1970) Water vapour and carbon dioxide receptors in Aedes aegypti. J Insect Physiol 16: 99-108.

Kennedy JS, Ludlow AR and Sanders CJ (1981) Guidance of flying male moths by wind-borne sex pheromone. Physiol Entomol 6: 395-412.

Klein U (1987) Sensillum-lymph proteins from antennal olfactory hairs of the moth (Saturniidae). Insect Biochemistry 17: 1193-1204.

Kline DL (1994) Olfactory attractants for mosquito surveillance and control: 1-octen-3-ol. J Am Mosq Control Assoc 10: 280-287.

Klowden MJ and Blackmer JL (1987) Humoral control of preoviposition behavior in the mosquito, Aedes aegypti. J Insect Physiol 33: 689-692.

Knols BGJ, van Loon JJA, Cork A, Robinson RD, Adam W, Meijerink J, de Jong R and Takken W (1997) Behavioural and electrophysiological responses of the female malaria mosquito Anopheles gambiae (Diptera: Culicidae) to Limburger cheese volatiles. Bull Entomol Res 87: 151-159.

Kramer $E$ (1992) Attractivity of pheromone surpassed by time-patterned application of two nonpheromone compounds. J Insect Behav 5: 83-97.

Krotoszynski B, Gabriel G and O'Neill H (1977) Characterization of human expired air: A promising investigative and diagnostic technique. J Chromatogr Sci 15: 239-244.

Kuthiala A, Gupta RK and Davis EE (1992) Effect of the repellent deet on the antennal chemoreceptors for oviposition in Aedes aegypti (Diptera: Culicidae). J Med Entomol 29: 639-643.

Kwon HW, Lu T, Rutzler M and Zwiebel LJ (2006) Olfactory responses in a gustatory organ of the malaria vector mosquito Anopheles gambiae. PNAS 103: 13526-13531.

Lacher V (1967) Elektrophysiologische Untersuchungen an einzelnen Geruchsrezeptoren auf den Antennen weiblicher Moskitos (Aedes aegypti L.). J Insect Physiol 13: 1461-1470.

Liliefors T, Thelin B and Van der Pers JNC (1985) Chain-elongated analogues of a pheromone component of the turnip moth, Agrotis segetum. A structure-activity study using molecular mechanics. J Royal Chem Soc, Perkin Transactions II 1957-1962.

Liliefors T, Bengtsson BO and Hansson BS (1987) Effects of double-bond configuration on interaction between a moth sex pheromone component and its receptor: a receptor-interaction model based on molecular mechanics. J Chem Ecol 13: 2023-2040.

Lindh JM, Kannaste A, Knols BGJ, Faye I and Borg-Karlson AK (2008) Oviposition responses of Anopheles gambiae s.s. (Diptera: Culicidae) and identification of volatiles from bacteria-containing solutions. J Med Entomol 45: 1039-1049. 
Lu T, Qiu YT, Wang G, Kwon JY, Rutzler M, Kwon HW, Pitts RJ, van Loon JJA, Takken W, Carlson JR and Zwiebel LJ (2007) Odor coding in the maxillary palp of the malaria vector mosquito Anopheles gambiae. Curr Biol 17: 1533-1544.

Mboera LEG and Takken W (1997) Carbon dioxide chemotropism in mosquitoes (Diptera: Culicidae) and its potential in vector surveillance and management programmes. Med Vet Entomol 85: 355-368.

Mboera LEG, Takken W, Mdira KY, Pickett JA (2000) Sampling gravid Culex quinquefasciatus (Diptera: Culicidae) in Tanzania with traps baited with synthetic oviposition pheromone and grass infusions. J Med Entomol 37: 172-176.

Mauer DJ and Rowley WA (1999) Attraction of Culex pipiens pipiens (Diptera: Culicidae) to flower volatiles. J Med Entomol 36: 503-507.

Mclver S and Siemicki R (1975) Palpal sensilla of selected Anopheline mosquitoes. J Parasitol 61: 535-538.

Mclver S (1982) Review article: Sensilla of mosquitoes (Diptera: Culicidae). J Med Entomol 19: 489-535.

Meijerink J and van Loon JJA (1999) Sensitivity of antennal olfactory neurons of the malaria mosquito, Anopheles gambiae, to carboxylic acids. J Insect Physiol 45: 365-373.

Meijerink J, Braks MAH, Brack AA, Adam W, Dekker T, Posthumus MA, van Beek TA and van Loon JJA (2000) Identification of olfactory stimulants for Anopheles gambiae from human sweat samples. J Chem Ecol 26: 1367-1382.

Meijerink J, Braks MAH and van Loon JJA (2001) Olfactory receptors on the antennae of the malaria mosquito Anopheles gambiae are sensitive to ammonia and other sweat-borne components. J Insect Physiol 47: 455-464.

Melo ACA, Rutzler M, Pitts RJ and Zwiebel LJ (2004) Identification of a chemosensory receptor from the yellow fever mosquito, Aedes aegypti, that is highly conserved and expressed in olfactory and gustatory organs. Chem Senses 29: 403-410.

Millar JG, Chaney JD and Mulla MS (1992) Identification of oviposition attractants for Culex quinquefasciatus from fermented bermuda grass infusions. J Am Mosq Control Assoc 8: 11-17.

Mustaparta H (2002) Encoding of plant odour information in insects: peripheral and central mechanisms. Entomol Exp Appl 104: 1-13.

O' Connell RJ (1975) Olfactory receptor responses to sex pheromone components in the red-banded leafroller moth. J Gen Physiol 65: 179-205.

Ochieng SA, Park KC and Baker TC (2002) Host plant volatiles synergize responses of sex pheromone-specific olfactory receptor neurons in male Helicoverpa zea. J Comp Physiol [A] 188: 325.

Pappas LG and Larsen JR (1976) Gustatory hairs on mosquito, Culiseta inornata. J Exp Zool 196: 351-360.

Park SK, Shanbhag SR, Wang Q, Hasan G, Steinbrecht RA and Pikielny CW (2000) Expression patterns of two putative odorant-binding proteins in the olfactory organs of Drosophila melanogaster have different implications for their functions. Cell Tissue Res 300: 181-192.

Pates HV, Takken W, Stuke K and Curtis CF (2001) Differential behaviour of Anopheles gambiae sensu stricto (Diptera: Culicidae) to human and cow odours in the laboratory. Bull Entomol Res 91: 289-296.

Perry AS and Fay RW (1967) Correlation of chemical constitution and physical properties of fatty acid esters with oviposition response of Aedes aegypti. Mosquito News 27: 175-183.

Pitts RJ, Fox AN and Zwiebel LJ (2004) A highly conserved candidate chemoreceptor expressed in both olfactory and gustatory tissues in the malaria vector Anopheles gambiae. PNAS 101: 5058-5063.

Pitts RJ and Zwiebel LJ (2006) Antennal sensilla of two female anopheline sibling species with differing host ranges. Malaria Journal 5: 26.

Qiu YT 2005. Sensory and behavioural responses of the malaria mosquito Anopheles gambiae to human odours. PhD Thesis,Wageningen University, Wageningen, pp 208.

Qiu YT, Van Loon JJA, Takken W, Meijerink J and Smid HM (2006) Olfactory coding in antennal neurons of the malaria mosquito, Anopheles gambiae. Chem Senses 31: 845-863.

Qiu YT, Smallegange RC, Ter Braak CJF, Spitzen J, van Loon JJA, Jawara M, Milligan P, Galimard AM, Van Beek TA, Knols BGJ and Takken W (2007) Attractiveness of MM-X traps baited with human or synthetic odor to mosquitoes (Diptera: Culicidae) in The Gambia. J Med Entomol 44: 970-983.

Ribeiro JMC (2003) A catalogue of Anopheles gambiae transcripts significantly more or less expressed following a blood meal. Insect Biochem Mol Biol 33: 865-882. 
Rutzler M and Zwiebel LJ (2005) Molecular biology of insect olfaction: recent progress and conceptual models. J Comp Physiol [A] 191: 777-790.

Sass H (1978) Olfactory receptors on the antenna of Periplaneta: response constellations that encode food odors. J Comp Physiol [A] 128: 227-233.

Sato K, Pellegrino M, Nakagawa T, Nakagawa T, Vosshall LB and Touhara K (2008) Insect olfactory receptors are heteromeric ligand-gated ion channels. Nature 452(7190): 1002-1006.

Shields VDC and Hildebrand JG (2001) Responses of a population of antennal olfactory receptor cells in the female sphinx moth Manduca sexta to plant-associated volatile organic compounds. J Comp Physiol [A] 186: 1135-1151.

Smallegange RC, Geier M and Takken W 2002. Behavioural responses of Anopheles gambiae to ammonia, lactic acid and a fatty acid in a y-tube olfactometer. In: Proceedings of Experimental \& Applied Entomology, N.E.V. Amsterdam, the Netherlands, pp 147-152.

Smallegange RC, Qiu YT, Van Loon JJA and Takken W (2005) Synergism between ammonia, lactic acid and carboxylic acids as kairomones in the host-seeking behaviour of the malaria mosquito Anopheles gambiae sensu stricto (Diptera: Culicidae). Chem Senses 30: 145-152.

Smith CN, Smith N, Gouck HK, Weidhaas DE, Gilbert IH, Mayer MS, Smittle BJ and Hofbauer A (1970) L-Lactic acid as a factor in the attraction of Aedes aegypti (Diptera: Culicidae) to human hosts. Ann Entomol Soc Am 63: 760-770.

Steib BM, Geier M and Boeckh J (2001) The effect of lactic acid on odour-related host preference of yellow fever mosquitoes. Chem Senses 26: 523-528.

Steinbrecht RA (1997) Pore structures in insect olfactory sensilla: a review of data and concepts. Int J Insect Morphol Embryol 26: 3-4.

Steinbrecht RA (1998) Odorant-binding proteins: expression and function. Ann N Y Acad Sci 855: 323-332.

Syed Z and Leal WS (2008) Mosquito smell and avoid the insect repellent DEET. PNAS 105: 13598-13603.

Todd JL, Haynes KF and Baker TC (1992) Antennal neurons specific for redundant pheromone components in normal and mutant Trichoplusia ni males. Physiol Entomol 17: 183-192.

Todd JL and Baker TC (1999) Function of peripheral olfactory organs. Springer-Verlag, Heidelberg, Germany.

Van den Broek IVF and Den Otter CJ (1999) Olfactory sensitivities of mosquitoes with different host preferences (Anopheles gambiae s.s., An. arabiensis, An. quadriannulatus, An. m. atroparvus) to synthetic host odours. J Insect Physiol 45: 1001-1010.

Vogt RG and Riddiford LM (1981) Pheromone binding and inactivation by moth antennae. Nature 293: 161-163.

Wang GR, Qiu YT, Lu T, Kwon HW, Pitts RJ, Van Loon JJA, Takken W and Zwiebel LJ (2009) Anopheles gambiae TRPA1 is a heat-activated channel expressed in thermosensitive sensilla of female antennae. Eur J Neurosci 30: 967-974.

Wicher D, Schäfer R, Bauernfeind R et al. (2008) Drosophila odorant receptors are both ligand-gated and cyclicnucleotide-activated cation channels. Nature 24: 1007-1011.

Xu PX, Zwiebel LJ and Smith DP (2003) Identification of a distinct family of genes encoding atypical odorant-binding proteins in the malaria vector mosquito, Anopheles gambiae. Insect Mol Biol 12: 549-560.

Zahiri N, Rau ME and Lewis DJ (1997) Oviposition responses of Aedes aegypti and Ae. atropalpus (Diptera: Culicidae) females to waters from conspecific and heterospecific normal larvae and from larvae with Plagiochis elegans (Trematoda: Plagiorchiidae). J Med Entomol 35: 565-568.

Zeng XN, Leyden JJ, Lawley HJ, Sawano K, Nohara I and Preti G (1991) Analysis of characteristic odors from human male axillae. J Chem Ecol 17: 1469-1493.

Zeng XN, Leyden JJ, Spielman Al and Preti G (1996) Analysis of characteristic human female axillary odors: qualitative comparison to males. J Chem Ecol 22: 237-257.

Zhou JJ, He XL, Pickett JA and Field LM (2008) Identification of odorant-binding proteins of the yellow fever mosquito Aedes aegypti: genome annotation and comparative analyses. Insect Mol Biol 17: 147-163. 



\title{
4. Odour coding and neural connections
}

\author{
Rickard Ignell, Meryem S. Sengul, Sharon R. Hill and Bill S. Hansson
}

\begin{abstract}
In this chapter, we provide a general overview over the structure and function of the peripheral and central olfactory systems of insects, with special emphasis on the olfactory system of mosquitoes. We give a short presentation of what currently is known about the functional genomics of odorant-binding proteins as well as odorant receptors. Emphasis is, however, put on the structure and functional characteristics of olfactory sensilla to bring the reader up to date of what has been accomplished within this field over the last couple of years. Furthermore, readers are given an in depth overview of how the primary olfactory centre, the antennal lobe, of insects is structured as well as how it deals with input olfactory information. Finally, we give a description of the structure and function of higher olfactory centres.
\end{abstract}

Keywords: olfactory signal transduction, olfactory receptor neuron, local interneuron, projection neuron, coding

\section{Introduction}

Disease-vector insects, like most other insects, rely heavily on olfactory cues for survival and reproduction. Thus, to gain insight into the biology of a particular insect species, it is often of paramount importance to study its olfactory system, including the structure and function of both its peripheral and central nervous systems. Our understanding of the neural and peri-neural aspects of the mosquito olfactory system have significantly expanded in recent years due to the genomic sequencing of some of the most important disease vectors, the African malaria mosquito, Anopheles gambiae Giles (Holt et al. 2002), the yellow fever mosquito, Aedes aegypti (L.) (Nene et al. 2007), and the southern house mosquito, Culex quinquefasciatus Say (http: //cpipiens.vectorbase. org). As a result, we have gained an increased knowledge about the molecular components of odour detection that ultimately regulate the function of the peripheral olfactory system.

In this chapter, we provide a general description of what is currently known about the molecular basis of olfactory signal transduction in insects, providing a brief summary on what is known in mosquitoes [a more thorough presentation is given in Chapter 2 of this volume]. The knowledge on the initial steps of signal transduction improves our understanding of the functional anatomical organisation of the olfactory system in insects in general, and in mosquitoes specifically. Functional anatomical studies in mosquitoes have primarily been conducted on the peripheral olfactory system. In the next two sections, we provide a general overview of what is known about the system in insects, and provide some specific data on mosquitoes obtained from our own ongoing research [a more detailed description on the peripheral system in mosquitoes is given in Chapter 3 of this volume]. To date, very little is known about central nervous integration of olfactory information in mosquitoes. However, thorough analyses have been made on the neural connectivity in the primary olfactory system, the antennal lobe (AL) of both An. gambiae and Ae. aegypti (Ghaninia et al. 2007a,b, Ignell et al. 2005). These studies emphasise that the central olfactory system of mosquitoes display great structural resemblance with that of other insects, suggesting that the coding mechanisms might be similar to that observed in these species. Furthermore, we provide an overview of what is currently known about odour coding in the $\mathrm{AL}$ 
and higher olfactory centres. We conclude by highlighting topics that need to be addressed in future studies to better grasp the neuroethology of these important vector species.

\section{Morphology of the peripheral olfactory system}

The olfactory organs of insects include the antennae, and most often a pair of mouthparts acting as secondary olfactory organs. In arthropods that do not have antennae, e.g. ticks, either a pair of legs or mouthparts take over this function. Irrespective of the location of the organs, they are often covered by hair-shaped sensilla, i.e. small cuticular sensory organs. These organs consist of three structural elements: a cuticular outer sheath structure, sensory cells and enveloping support cells. Based on the cuticular structure of a sensillum, we are able to discriminate different types of sensilla through microscopic analysis. According to a system suggested by Altner (1977), these types are: (a) wall-pore sensilla, (b) tip-pore sensilla, and (c) aporous sensilla; in which wall-pore sensilla are mainly olfactory and the most abundant type found on the olfactory organs. Wall-pore sensilla may be further divided, based on cuticular structure, into single-walled (including e.g. sensilla basiconica and trichodea) and double-walled sensilla (including sensilla coeloconica and grooved pegs) (Hallberg and Hansson 1999, Keil 1999). The number of bipolar sensory neurons, in this case olfactory receptor neurons (ORNs) innervating a particular sensillum type varies from one to approximately fifty (Keil 1999). Surrounding these neurons are often three, but sometimes four, enveloping support cells (the tormogen, trichogen and thecogen cells), each of which have a particular role in the development of the sensillum and take an active part in the mature sensillum (reviewed by Keil 1999).

\section{Olfactory sensilla in mosquitoes}

Olfactory sensilla on both antennae and maxillary palps of mosquitoes (Figure 1) have been described previously for several species (reviewed by Clements 1999, Mclver 1982, Sutcliffe 1994). Also recently, olfactory sensilla were described on the labellar lobes of the proboscis (Figure 1A, Kwon et al. 2006), previously recognised only as a gustatory organ. Apart from the large coeloconic sensilla that are absent in culicine mosquitoes, the types of sensilla found on the olfactory organs of mosquitoes are generally well conserved, also compared with other insects [see Chapter 3, this volume for more information].

\section{Perireceptor events in insect olfaction}

In the last few decades, biochemical, molecular and genomic studies have greatly improved our understanding of the mechanisms underlying insect olfaction. It is now evident that detection of chemical cues in insects occurs through a series of events, several of which are mediated by different protein families in the sensillum lymph (Figure 2). In order to reach the odorant receptors expressed on the dendrites of the ORNs (see below), odour molecules need to enter through sensillar pores and pass through an aqueous environment (Figure 2). This transport has been proposed to be mediated by soluble proteins, referred to as odorant-binding proteins (OBPs), in the sensillum lymph (Figure 2). Comparison among insect OBPs have revealed common structural properties such that they are small, water-soluble, extracellular proteins with a signal peptide sequence cleaved during secretion. Odorant-binding proteins also share the conservation of six cysteins that is important for the stability of the protein structure. The abundance of OBPs in the sensillum lymph, their differential distribution in various sensillum types and the specificity of their ligand-binding properties suggest that they are important players in the first step of odour 

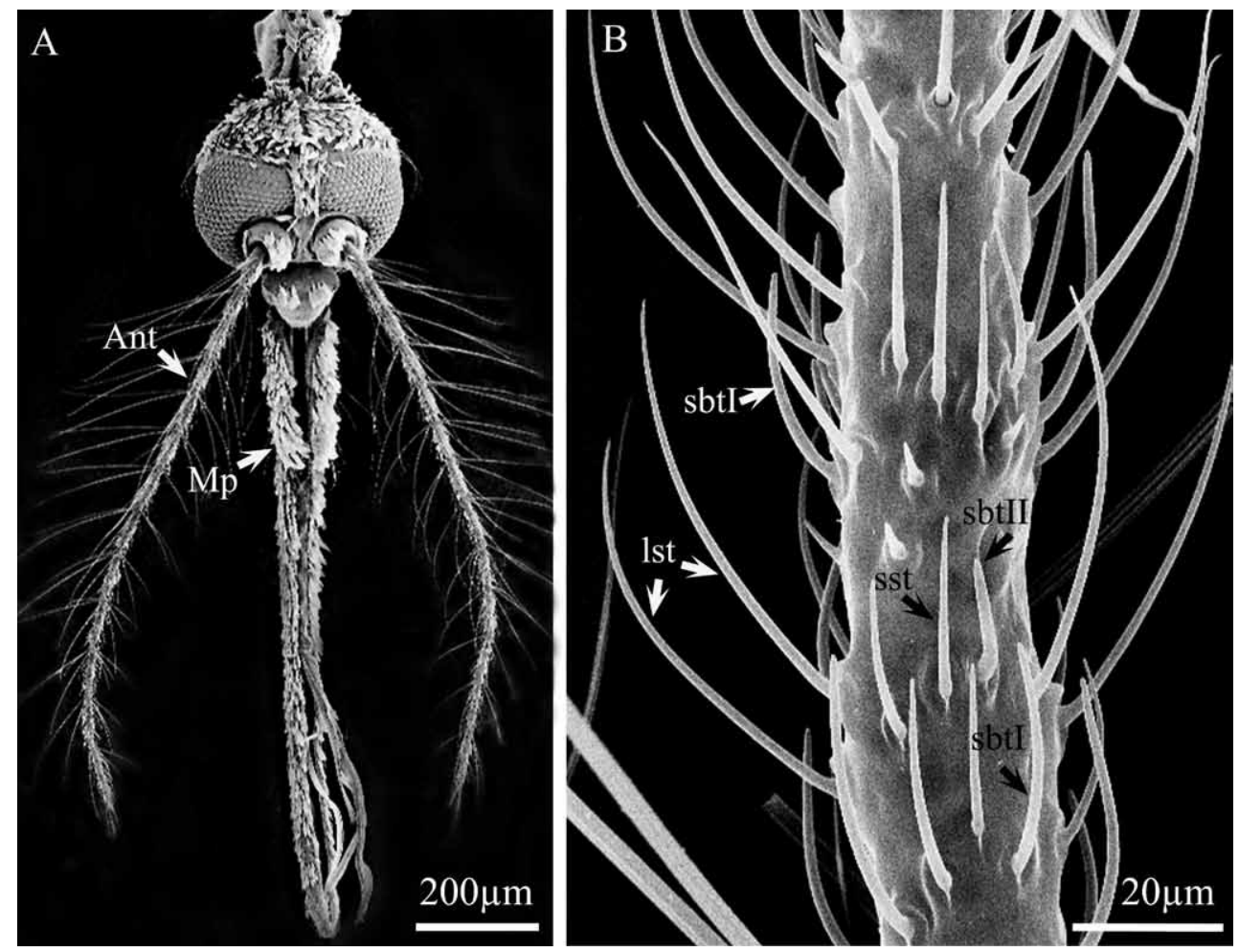

Figure 1. (A) Scanning electron micrograph of the head of a female Aedes aegypti shows the olfactory organs, the antennae (Ant), maxillary palps (Mp) and labellar lobes (LL) of the proboscis. (B) Scanning electron micrograph of an individual segment of the antenna of the same female shows grooved peg sensilla and four sub-types of olfactory sensilla trichodea: short sharp tipped (sst), short blunt-tipped I (sbtl), short blunt-tipped II (sbtII), and long sharp-tipped (Ist) (redrawn, with permission, from Ghaninia et al. 2007b).

coding in insects. Here we will focus on the general aspects of insect OBPs and recent findings that indicate their possible roles in olfactory-driven behaviour of insects.

\section{Odorant-binding proteins}

The first insect odorant-binding protein was identified in the male antennae of the moth Antheraea polyphemus (Cramer) using the radioactively labelled pheromone ( $E, Z)-6,11$-hexadecadienyl acetate as a probe in ligand-binding experiments. This odorant-binding protein was referred to as a pheromone-binding protein (PBP) (Vogt and Riddiford 1981). Subsequently, additional OBP sequences were identified in Lepidoptera (Györgyi et al. 1988, Raming et al. 1989, Vogt et al. 1989) that showed differential expression pattern and distinct binding properties to different classes of odorant molecules (Krieger et al. 1996, Pelosi 1998, Vogt and Lerner 1989, Vogt et al. 1991a,b). Accordingly, insect OBPs are now classified into three groups: PBPs, general odorant-binding proteins (GOBPs) and antennal binding protein X (ABPX). 
A

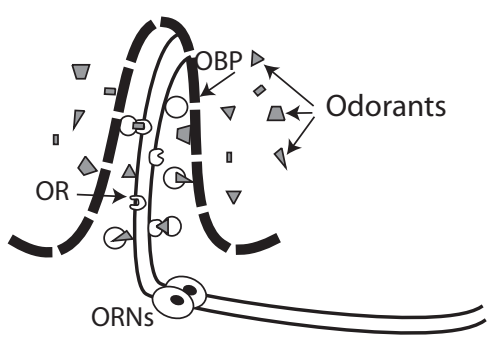

B

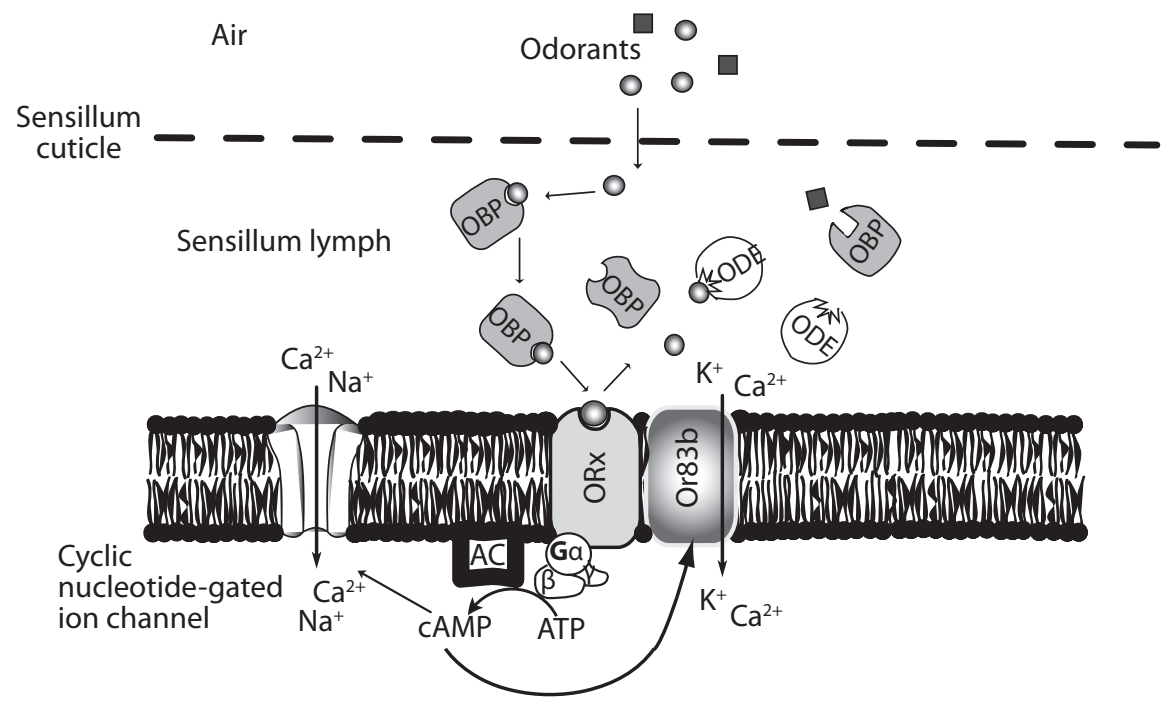

Figure 2. Schematic representation of the components of olfactory signal transduction in insects. (A) Odorants enter the aqueous olfactory sensillum lymph via pores of the cuticular hair wall of the sensillum. In the sensillum lymph, hydrophilic OBPs are believed to bind and transport the odorants to conventional OR (ORx) located at the dendritic membrane of the ORNs. (B) Olfactory signal transduction is initiated as the odorant bind to the ORx, which is associated with the membrane-bound heterotrimeric G-protein. Odorants that stimulate the ORx are removed from the vicinity of the receptor and degraded by ODEs. Conventional ORx forms a heterodimer with the Or83b family OR that is required for the proper localisation of the ORx to the dendritic membrane in order to trigger signal transduction. Upon binding of the odorant molecule to the ORx, a conformational change forms a ORxOr83b duplex activating a Ga-protein that, in turn, stimulates AC, resulting in the generation of CAMP. This triggers cyclic nucleotide-gated ion channel that increases intracellular $\mathrm{Ca}^{2+}$ and $\mathrm{Na}^{+}$and membrane depolarisation. Two models for odorant-induced production of cyclic nucleotide signalling have been proposed recently. Wicher et al. (2008), the model showed here, observed a G-protein coupled cyclic nucleotide-gated ion channel stimulation and activation of Or83b that allows inward flow of ions. Sato et al. (2008), however, showed the stimulation of the ORx-Or83b duplex, and influx of cations through ion channels, in the absence of a second messenger system. These data provide an important evidence for the mechanism of signal transduction in insect olfactory systems; while, the roles of the second messengers in the signalling pathway are needed to be investigated more in detail. $A C$, adenylyl cyclase; ATP, adenosine triphosphate; CAMP, cyclic adenosine monophosphate; Ga, G-protein a subunit; $G \beta$, G-protein $\beta$ subunit; $G$, G-protein $\gamma$ subunit; $O B P$, odorant-binding protein; $O D E$, odorant-degrading enzyme; OR, odorant receptor; ORNs, olfactory receptor neurons. 
Immunocytochemical localisation of OBPs has been determined in Lepidoptera where pheromone-sensitive sensilla trichodea express PBPs (Steinbrecht et al. 1992), whereas sensilla basiconica mainly express GOBPs (Laue et al. 1994). No co-localisation of PBPs and GOBPs in the same sensillum has been observed (Steinbrecht et al. 1995). Differential expression of OBPs has also been found in Diptera (Hekmat-Scafe et al. 1997, Park et al. 2000). However, co-localisation of closely related OBPs (i.e. OS-E and OS-F) in the same sensillum has been shown in Drosophila melanogaster Meigen (Hekmat-Scafe et al. 1997). This finding shows that more than one OBP can be expressed in an individual sensillum.

In the post-genomic era, a large number of Obp genes have been identified in Dipteran insects as well. In D. melanogaster, 51 Obp genes have been identified (Galindo and Smith 2001, Graham and Davies 2002, Hekmat-Scafe et al. 2002). In An. gambiae and Ae. aegypti, 57 and 66 genes have been found to encode OBPs, respectively (Biessmann et al. 2005, Xu et al. 2003, Zhou et al. 2008). In D. melanogaster and An. gambiae, most of these genes have been characterised through reversetranscriptase polymerase chain reaction (RT-PCR), in situ hybridisation and GAL4/UAS expression systems. Comparisons of OBPs among these species have revealed conserved gene homologues as well as gene expansions (Xu et al. 2003, 2008), which may reflect species-specific functions (see also Chapter 2, this volume).

Expression of Obp genes is not restricted to olfactory tissues, such as antennae, maxillary palps and the proboscis. There is a considerable overlapping expression of $O b p$ genes in olfactory and gustatory tissues of mosquitoes (Biessmann et al. 2005, Li et al. 2005, Sengul and Tu 2008) and fruit flies (Hekmat-Scafe et al. 2002). It is thus plausible that OBPs are involved in both types of chemoreception, or alternatively, that both olfactory and gustatory tissues share some common functions. These findings may contribute significantly to understand the possible roles of OBPs in olfactory processes (discussed below).

\section{Physiological roles of OBPs in olfaction}

Since the discovery of the first insect OBP in moth antennae (Vogt and Riddiford 1981), intensive efforts have been made to determine the structures of OBPs in various insect species to reveal the mechanisms behind OBP function. Structural information is so far only available for six insect OBPs, including the D. melanogaster LUSH protein (Kruse et al. 2003) and OBP1 in An. gambiae (Wogulis et al. 2006). These studies found some common features, e.g. that OBPs contain a-helices that form a hydrophobic binding pocket for the ligand and that OBPs undergo a pH-dependent conformational change that determines ligand binding and release.

Despite a wealth of biochemical and structural information, the exact physiological function of OBPs still remains elusive. However, the advantage of genetic tools in D. melanogaster has contributed significantly to the understanding of the function of OBPs in insect olfaction. Xu et al. (2005) found that a D. melanogaster mutant for an OBP, named LUSH, showed defects in its perception of the aggregation pheromone, 11-cis vaccenyl acetate (cVA). The expression of the lush transgene in the mutants restored the LUSH phenotype, indicating that LUSH is required to bind CVA and, in turn, stimulate the CVA-sensitive neurons to elicit a pheromone-induced behaviour. More recently, it was shown that LUSH is required for CVA-sensitive receptors (Or67d) to respond to CVA in vivo ( $\mathrm{Ha}$ and Smith 2006). Other D. melanogaster OBPs, such as OBP57d and OBP57e, have also been shown to regulate behavioural differentiation in members of the D. melanogaster species complex (Matsuo et al. 2007). Flies having the mutant gene Obp57d/e 
showed altered responses to the host plant odorants, hexanoic acid and octanoic acid, which influenced the behaviour related to oviposition site preference.

The availability of the Ae. aegypti and An. gambiae genomes (Holt et al. 2002, Nene et al. 2007) has allowed for the identification of a substantial number of putative Obp genes and their subsequent tissue localisation in these species. More interestingly, some of these genes have been shown to be down-regulated after a blood meal (Biessmann et al. 2005, Justice et al. 2003a, Sengul and Tu 2008). This may indicate that these Obp genes play an important role in host-seeking behaviours. Consequently, future research will have to focus on the understanding of how these genes influence the behaviour of mosquitoes. Experiments to establish transformed mosquitoes to produce Obp knock-out mutants are highly demanding as it is difficult to assess the phenotype. However, a recent RNA interference strategy has shown a significant decrease (8-fold and $>100$ fold reduction) in the mRNA levels of the two candidate Obp genes in the olfactory tissues of female Ae. aegypti mosquitoes (Sengul 2008). This finding is very encouraging and shows that it is possible to establish an efficient method to knockdown Obp genes in olfactory tissues of mosquitoes. Such analysis opens doors to future systematic analysis of the molecular players involved in mosquito olfaction.

\section{Transduction mechanisms}

When odour molecules have travelled through the sensillum lymph, they reach the surface of the olfactory receptor neuron dendrite (Figure 2). Here, olfactory receptor (OR) proteins reside inside the membrane. These proteins are the main key to the specificity of the ORNs. Insect ORs were first identified in D. melanogaster (Clyne et al. 1999, Gao and Chess 1999, Vosshall et al. 1999). They were a new family of putative seven-transmembrane G-protein coupled receptors (GPCR). It is downstream from these receptors that the translation from a chemical signal to an electrical one, the transduction cascade, takes place (Figure 2B). In vertebrates this cascade has been well characterised. The binding of a ligand to the OR activates a G-protein coupled to the receptor. The G-protein in turn activates an adenylyl cyclase, which converts ATP to cAMP. The cAMP is a well-known signalling molecule in various cell processes, and here acts on the intracellular part of a cyclic nucleotide-gated ion channel, and changes its conductance. This change in turn opens chloride channels and thereby changes the charge of the inside of the cell vs. the outside. If this process proceeds long enough, an action potential is formed. In crustaceans similar processes have been observed, but here two second messengers, CAMP and $\mathrm{IP}_{3}$, are involved.

It was long considered that insect olfactory transduction processes were very similar to what had been observed in vertebrates and crustaceans. Recent progress does, however, challenge these views. Several factors of the insect system seem different. One peculiarity of the insect system was reported by Benton et al. (2006), who showed that insect ORs are inserted 'inside-out' as compared to the vertebrate system. The insect ORs have an extracellular $\mathrm{N}$-terminus and an intracellular C-terminus. The functional implications of this architecture are so far more or less unknown.

Vertebrate ORNs always express a single type of OR only. However, when the putative insect ORs were discovered, it was demonstrated that the ORN-type-specific OR in D. melanogaster was more or less always co-expressed with a ubiquitous, second OR, OR83b (Figure 2B, Neuhaus et al. 2005). Later experiments showed that this protein is vital for ORN function and that in its absence the type-specific OR does not even reach the dendrite during development (Larsson et al. 2004). In Manduca moths, Nakagawa et al. (2005) showed that expression of a pheromone receptor and the OR83b homolog was sufficient to obtain channel activation after ligand stimulation. The OR83b 
proteins heterodimerise with the ORN-type-specific ORs (Figure 2B). The type-specific ORs thus form a complex allowing direct interactions between the two proteins. The OR83b-type of protein has been found in all insects investigated so far, and seems to be a general insect feature in insect olfactory systems. In mosquitoes, AgOR7, AaOR7, and CqOR7 represent the possible orthologs of the D. melanogastor OR83b in An. gambiae, Ae. aegypti and Cx. quinquefasciatus, respectively (Bobhot et al. 1997, Hill et al. 2002, Melo et al. 2004, Xia and Zwiebel 2006).

Recent studies, including the ubiquitous OR83b receptor, have shown that this receptor is in fact a non-specific cation channel, under both ionotropic and metabotropic control (Figure 2B, Sato et al. 2008, Wicher et al. 2008). The initial and very fast response is cAMP-independent, while a second and much more sensitive response is $\mathrm{G}_{\mathrm{s}}$-mediated and CAMP-dependent. More or less simultaneously Kain et al. (2008) showed very strong evidence for a $\mathrm{G}_{\mathrm{q}}$ and a phospholipase $C$ involvement. The transduction system is not clearly understood, and contains a number of different transduction pathways. The function of each of these components still remains to be elucidated.

\section{Peripheral olfactory system}

The ORNs represent the first neuronal filter in olfactory signalling. Arthropod vectors find their hosts' particular odour blend amidst a varied and complex odour environment. It is this identification of signature volatile blends from the background noise that is decoded by the vector chemosensory system, and translated into host-seeking behaviours. The first level of neuronal discrimination occurs in the ORNs in which the electrical signals are coded using odorant selectivity, volatile sensitivity and timing of the response.

\section{Selectivity}

An ORN is the first component in a signal processing channel conveying information concerning a constrained number of volatile compounds to the AL. This constraint on an individual ORN's responses to specific compounds, or families of compounds, is referred to as 'tuning' (Hallem et al. 2004, Hamana et al. 2003, Stensmyr et al. 2003). Tuning is largely, possibly exclusively, dependant on OR-ligand affinity (see previous section on transduction mechanisms) and the perireceptor events in the aqueous environment surrounding the ORN dendrites (see Figure 2).

The affinity of volatile compounds for their cognate receptors has been determined in many ways: in vivo functional characterisation of sensilla with single sensillum recording (SSR) describes ORN tuning, whether it is narrowly tuned, e.g. pheromone-receptive neurons in a multitude of species (e.g. Bengtsson et al. 1990, Liljefors et al. 1984, 1985, 1987); or broadly tuned, e.g. many kairomonereceptive antennal trichoid sensilla in mosquitoes (Figure 3, Ghaninia et al. 2007b, Hill et al. 2009, Qiu et al. 2006a,b). Only a few volatile pheromones have been identified in disease vectors, such as oviposition pheromones (Davis and Bowen 1994, Du and Millar 1999, Pickett and Woodcock 1996), and in fact for many years, it has been claimed that there was little or no volatile pheromone communication between arthropod vectors (e.g. Schofield and Moreman 1976). More recently, however, there have been both identifications and other strong indications of sex/aggregationtype pheromone communication in a variety of vectors, such as ticks, triatomines, sandflies and mosquitoes (Brazil and Hamilton 2002, Cabrera and Jaffe 2007, Grenacher et al. 2001, Guerenstein and Guerin 2004, Vitta et al. 2007)(see also elsewhere in this Volume). The narrow tuning of pheromone-receptive ORNs has been extensively investigated in many species, and probably most widely studied in Lepidoptera (e.g. Baker et al. 2004, Domingue et al. 2007). Studies which subtly 


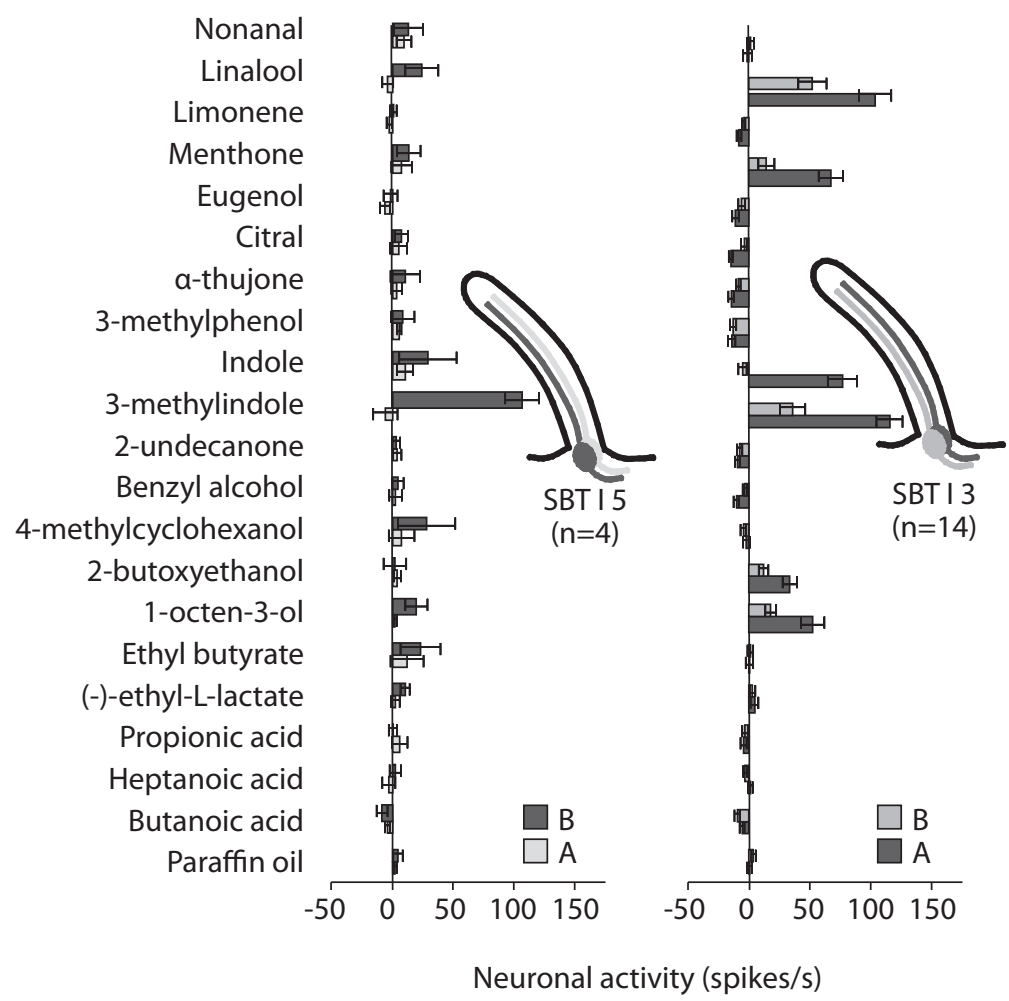

Figure 3. An example of odorant receptor neuron (ORN) selectivity (tuning) from the mosquito Culex quinquefasciatus. The short blunt type I trichoid sensillum $5 B$ (SBT I 5B) is narrowly tuned, responding maximally to a single compound, 3-methylindole, whereas SBT I 3A responds to linalool, menthone, indole, 3-methylindole, 2-butoxyethanol and 1-octen-3-ol, demonstrating a more broad pattern of tuning. Two spontaneously active neurons are found in all trichoid types ( $A$ and $B$ ). The neuronal responses of $A$ and $B$ (See legend) are shown as an average over (n) replicates (reproduced with permission from Hill et al. 2009).

manipulated the structure of one pheromone component in the moth Agrotis segetum (Denis and Schiffermüller) revealed that the optimal tuning of a pheromone receptor is dependent on the molecular shape, chirality and electron distribution of the pheromone component (Bestman 1981, Bestman and Vostrowsky 1982, Larsson et al. 1999, Priesner 1979, Priesner et al. 1975, Wojtasek et al. 1998).

The ORN responses of disease vectors to kairomones have come under intense scrutiny in recent years, due to the colossal potential for kairomones to be used as trap attractants and as repellents for personal protection (e.g. Ditzen et al. 2008, Justice et al. 2003b, Mason et al. 1993, Pickett and Woodcock 1996, Schmidt 2005). Small panel SSR screens ( $<100$ compounds) have described generalised tuning curves for approximately $80 \%$ of the ORNs in three mosquito species: An. gambiae (Kwon et al. 2006, Lu et al. 2007, Qiu et al. 2006b), Ae. aegypti (Ghaninia et al. 2007b, Siju et al. 2010) and CX. quinquefasciatus (Hill et al. 2009, Syed and Leal 2007). 
Historically, kairomone-responding ORNs have been considered, with a few exceptions, to be broadly tuned (e.g. Behan and Schoonhoven 1978, Hansson et al. 1989). Many recent studies, however, have clarified that broad or general tuning, as indicated by previous studies, is a result of two common experimental practices: the lack of screens identifying the actual key ligands for these ORNs; and the concentrations at which kairomones were applied were many hundreds of times higher than environmentally and physiologically relevant (e.g. Anderson et al. 1995, Dickens 1990, Hansson et al. 1999, Heinbockel and Kaissling 1996, Larsson et al. 2001, Priesner 1979, Shields and Hildebrand 2001, Skiri et al. 2004). Kazama and Wilson (2008), working with D. melanogaster, have determined that high volatile concentrations, which result in high ORN spike frequencies, dampen $A L$ input receptivity and thus, high frequency ORN responses are not the optimal characteristics by which to identify key ligands. In contrast, a better method to gauge ORN selectivity is to identify those compounds which elicit threshold responses at low concentrations.

In vitro heterologous expression of ORs in mammalian cell-lines and ectopic expression of ORs in the $D$. melanogaster empty neuron system have been largely able to reconstruct the in vivo tuning profiles, with the bonus of identifying the OR responsible for the ligand binding pattern (e.g. Hallem et al. 2004, Kwon et al. 2007, Rospars et al. 1996, Syed et al. 2007, Carrey et al. 2010). The high correlation between the in vivo and in vitro response profiles indicates that the binding between odorant and receptor is chiefly responsible for the selective nature of ORNs. As a result, the heterologous expression system is well-placed as a technique to identify potential key ligands through large scale screening methods akin to those used in pharmaceutical-type screens (Hallem et al. 2004, Malnic 2007, Touhara 2007). Moreover, putative key ligands can be validated in vivo using SSR.

\section{Sensitivity}

For the most part, the olfactory system identifies odours not by the activation of a single processing channel but through comparisons among all active channels (see below). In this configuration, it is necessary to know the precise tuning curves of each ORN type before the organism can identify a complex odour blend (for review see Vosshall and Stocker 2007).

As mentioned above, ORNs are to a greater or lesser degree promiscuous in their responses to volatiles, e.g. one ORN responds to changes in the flux of more than one volatile compound; and more than one ORN responds to the same compound, but to varying degrees (Figure 4). It is the comparison of these differentially tuned curves in the CNS that leads to the identification of relevant odour plumes (for review see Todd and Baker 1999). Comparisons among the three mosquito species' ORN response profiles reveal that these organisms, which have maintained a similar host choice during evolutionary separation, employ distinct patterns of odour tuning in the ORNs. Although many similar volatiles are recognised by all three species, there appears to be little conservation in the individual ORN tuning curves (Ghaninia et al. 2007a, Hill et al. 2009, Qiu et al. 2006b, Siju et al. 2010).

Olfactory receptor neurons can respond to odorants in extremely low amounts: e.g. sensilla generally respond to a threshold dose sex-pheromones between $10^{-7} \mathrm{~g}$ and $10^{-12} \mathrm{~g}$ (Angioy et al. 2003, Boeckh and Selsam 1984, Kaissling 1971, Kaissling and Priesner 1970) and to kairomones at amounts often 10 to 100 fold higher (Hansson et al. 1999, Hill et al. 2009, Jönsson and Anderson 1999, Larsson et al. 2001). It is important to mention that these experimentally derived threshold values are all relative, and should not be taken as absolute values; since volatility differs greatly amongst various compounds, and experimental factors such as the speed of airflow and the 


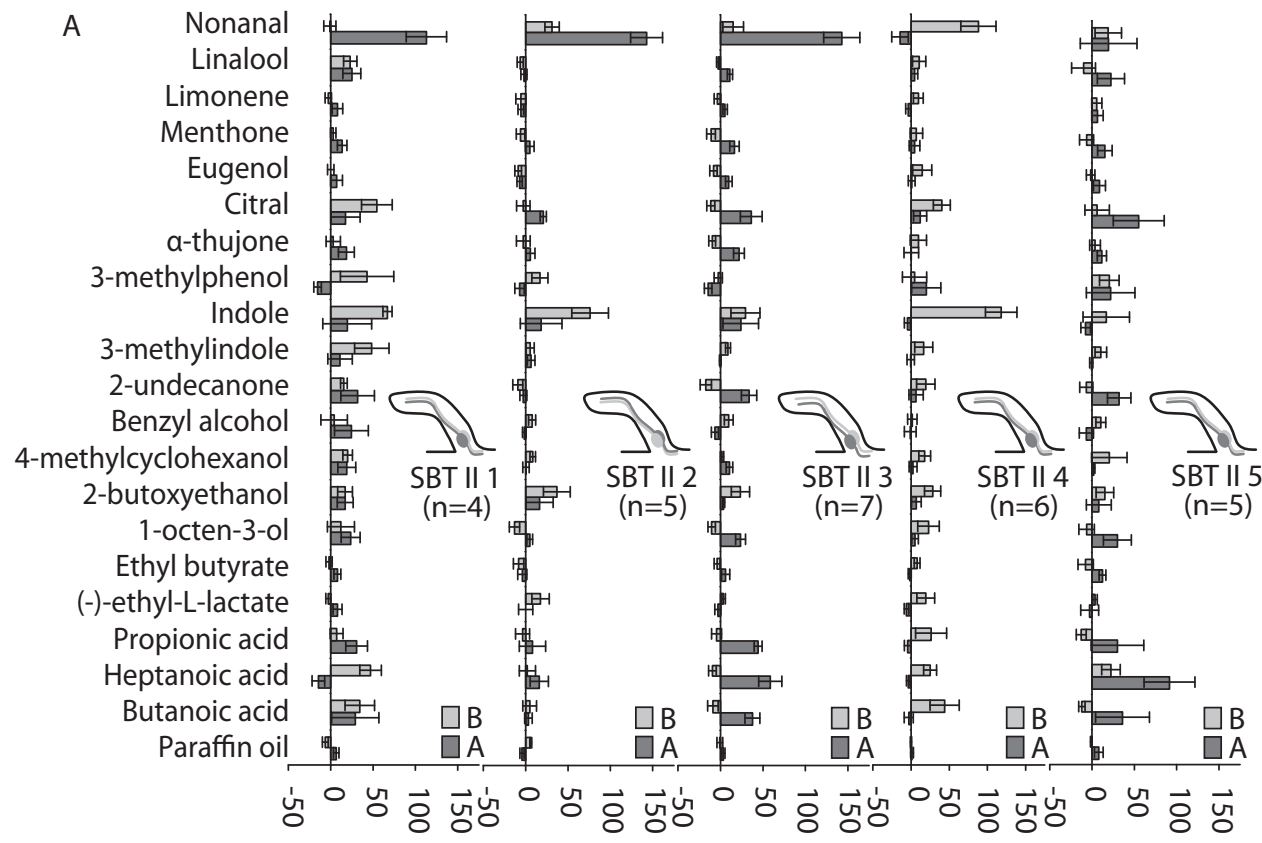

Neuronal activity (spikes/s)

B

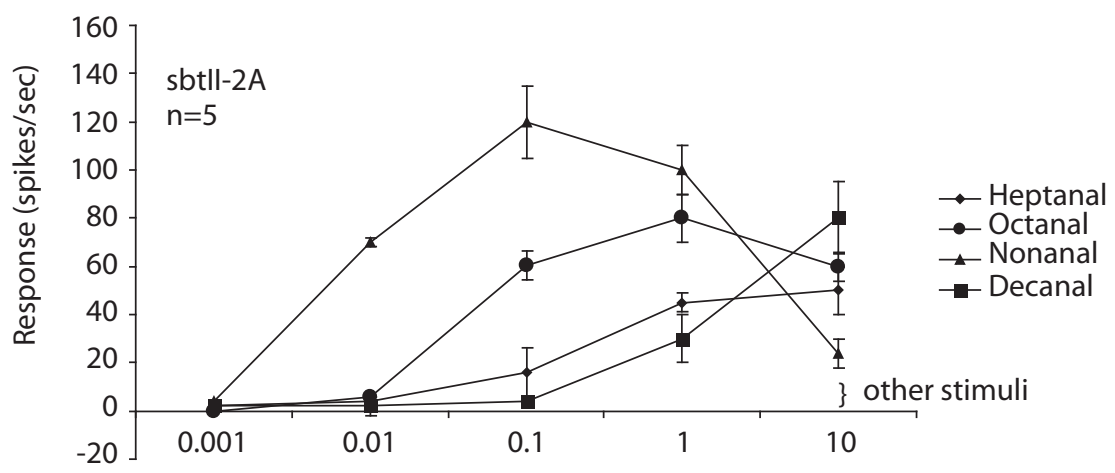

Concentration (\%)

Figure 4. An example of odorant receptor neuron (ORN) sensitivity from the mosquitoes ( $A$ ) Culex quinquefasciatus in which four of the five short blunt type II trichoid sensilla (SBT II 1-4) contain ORNs responding to varying degrees to nonanal and another four SBT II (1,3-5) sensilla respond differentially to the carboxylic acids (butanoic, heptanoic and propionic acid). Two spontaneously active neurons are found in all trichoid types ( $A$ and $B$ ). The neuronal responses of $A$ and $B$ (See legend) are shown as an average over ( $n$ ) replicates; and (B) Aedes aegypti in which the sbt II 2A ORN responds with differing sensitivity to four short chain aldehydes (C7-C10) (reproduced with permission from Hill et al. 2009 and Ghaninia et al. 2008). 
duration of stimulus can greatly affect the response of ORNs (Ignell and Hansson 2005). New techniques such as SSR coupled to a gas chromatograph (GC-SSR) are being used to identify novel key ligands directly from natural odour collections in physiologically and environmentally relevant quantities (Ghaninia et al. 2008). Additional analyses such as raster plots, peristimulus histograms and inter-spike interval superimposition should be employed if more absolute quantification of ORN threshold response is being sought (Ignell and Hansson 2005).

\section{Temporal coding and stimulus frequency}

As mentioned briefly above, the frequency and duration of ORN response is a critical component of signal coding in the peripheral olfactory nervous system (Hallem et al. 2004, Kazama and Wilson 2008). An intermittent frequency of odour stimulus is often essential in order to elicit a behavioural response (Baker et al. 1985, Dekker et al. 2005, Geier and Boeckh 1999, Justus et al. 2002, Kennedy et al. 1981). For example, a homogeneous plume of $\mathrm{CO}_{2}$ is sufficient to activate upwind flight behaviours in Ae. aegypti, but is insufficient to enable host source-finding; whereas, a filamentous plume of $\mathrm{CO}_{2}$ with an average pulse frequency of $\sim 6 \mathrm{~Hz}$, which incidentally is much more akin to a natural host odour plume structures (Mafra-Neto and Cardé 1998, Murlis and Jones 1981), will substantially enhance source-finding behaviours (Dekker et al. 2005, Geier and Boeckh 1999). At the level of ORN activity in response to intermittent stimuli, neuronal response in moths has been shown to mimic the stimulus pulses from 3-12 Hz (Almaas et al. 1991, Baker et al. 1988, Kaissling 1986, Marion-Poll and Tobin 1992) and even up to a staggering frequency of $33 \mathrm{~Hz}$ (Bau et al. 2002).

Tracking the temporal characteristics of an odour plume gives the vector the ability to track the structure of the plume, e.g. size of plume quanta and distance between these quanta; and the ability to trace the plume back to its source, the ultimate goal of any seeking behaviour (e.g. Dekker et al. 2005, Lemon and Getz 1998, Moore 1994, Vergassola et al. 2007, Vickers 2006, Willis and Arbas 1991). The two main temporal coding regimes used in the periphery are phasic and tonic spiking response patterns (Figure 5). Phasic responses are generated by those neurons that are able to track stimuli at high pulsed frequencies, thus describing the plume quanta size

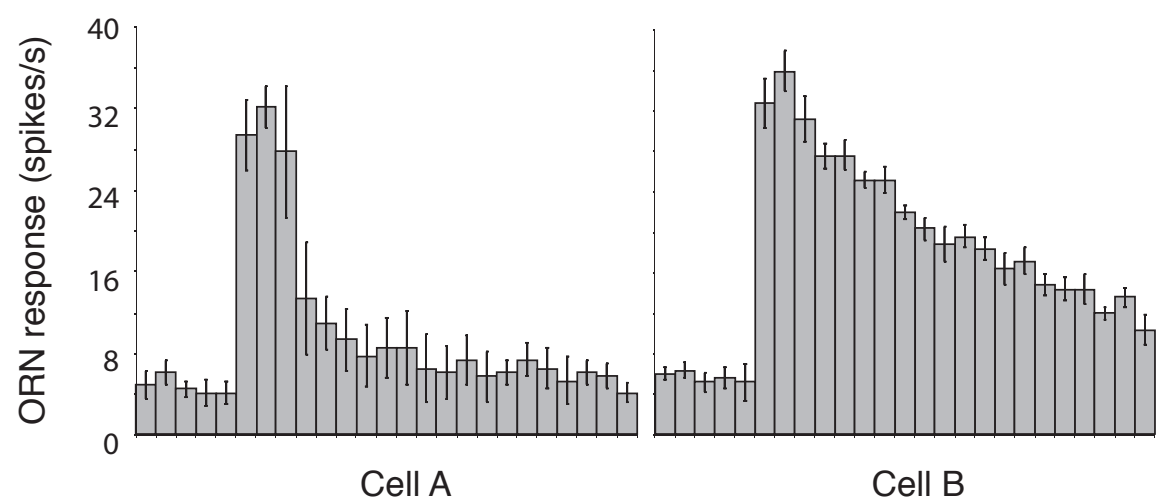

Figure 5. An example of different temporal characteristics of the odorant receptor neurons (ORNs) from the mosquito Culex quinquefasciatus. The short blunt type I trichoid sensillum 3 (SBTI 3) is depicted responding to a 0.5 s stimulus of linalool (10-2 v/v). Each bar represents the response from each of the ORNs contained within the SBT I 3 sensillum ( $A$ and B neuron) during $100 \mathrm{~ms}$ (reproduced with permission from Hill et al. 2009). 
and spacing (Figure 5A). Those ORNs responding with the longer lasting tonic spiking patterns are believed to make up the short term memory component of the peripheral olfactory system (Figure 5B), thereby providing the recent stimulus history for direct temporal comparison with current ORN stimulus response (Almaas and Mustaparta 1991, Den Otter and Van der Goes van Naters 1993). As many ORNs respond to the same compound with different sensitivities, they also respond to the same volatile with different temporal patterns (e.g. Ghaninia et al. 2007b, Hill et al. 2009, Qiu et al. 2006b). In Cx. quinquefasciatus, two ORNs within the same sensillum, i.e. SBT I 3, respond to the green leaf volatile linalool with apparently similar threshold sensitivities, but with different temporal characteristics (Figure 5). The 'A neuron' responds in a fast phasic manner, while the 'B neuron' responds with an initial phasic component followed by a long lasting tonic response (Figure 5, Hill et al. 2009). This coupling of the long and short term responses to individual stimuli is not, however, universal. There are many examples of sensilla, both morphologically distinct and indistinct, which exhibit sensitivity to the same compounds and yet display a continuum of temporal characteristics (Almaas et al. 1991, Ghaninia et al. 2007b, Heinbockel and Kaissling 1996, Hill et al. 2009). The diversity with which the arthropods code chemosensory information in the periphery and transmit these complex signals to the higher olfactory centres through neuronal selectivity, sensitivity and temporal response patterns, identifies the peripheral olfactory system as an essential first processing centre in transforming chemical cues into complex and finely tuned vector behaviours.

\section{Anatomy and function of the primary olfactory centre: the antennal lobe}

\section{Antennal lobe glomeruli - the first relay stations in the central nervous system}

In insects, ORNs from the antennae (Figure 6A), and in some cases from the mouthparts (Figure $6 \mathrm{~B})$, convey chemical information into the primary olfactory centre of the insect brain, the $\mathrm{AL}$ (Figure 6C, Anton and Homberg 1999, Schachtner et al. 2005), producing internal representations of the odour signals detected at the peripheral level. In ticks, olfactory afferents from Haller's organ project into an orthologous region in the central nervous system called the olfactory lobe (Van Wijk et al. 2006), suggesting that the organisation of the olfactory systems is highly conserved in the different arthropod lineages. In insects, the formation of these representations has been shown to involve progressive transformations of the olfactory information between layers of pre- and postsynaptic neurons in a hierarchical manner (Ache and Young 2005, Hansson and Christensen 1999, Ignell and Hansson 2005, Urban 2002). The initial step in this transformation is a reorganisation of the seemingly heterogeneous peripheral olfactory system into a highly organised chemotopic map in spherical neuropil, glomeruli, of the AL (Hansson and Christensen 1999) that house the synaptic contacts between ORN terminals and AL interneurons. The number, size and localisation of AL glomeruli is species specific as revealed by mapping and 3-dimensional reconstructions in various species (reviewed by Schachtner et al. 2005), including the mosquitoes Ae. aegypti (Figures 6E, 6F and 6G, Ignell et al. 2005) and An. gambiae (Ghaninia et al. 2007a). Data from the spider mite, Phytoseiulus persimilis Athias-Henriot, also suggest that the olfactory lobe of mites have a glomerular organisation (Van Wijk et al. 2006). Sexual dimorphism on the basis of particular specialised glomeruli and on varying glomeruli numbers has been described in species belonging to Dictyoptera, Hymenoptera, Lepidoptera, and Diptera (Anton and Homberg 1999, Hansson and Anton 2000, Rospars 1988). The functional significance of the observed sexual dimorphism in mosquitoes, including differences in number, shape and size of particular glomeruli (Figure 6D, Ghaninia et al. 2007a, Ignell et al. 2005), is still to be revealed. However, examples of the functional significance of sexual dimorphism abound. For example, in all cockroaches and moths that use female-produced, long distance sex attractants, a pronounced sexual dimorphism 

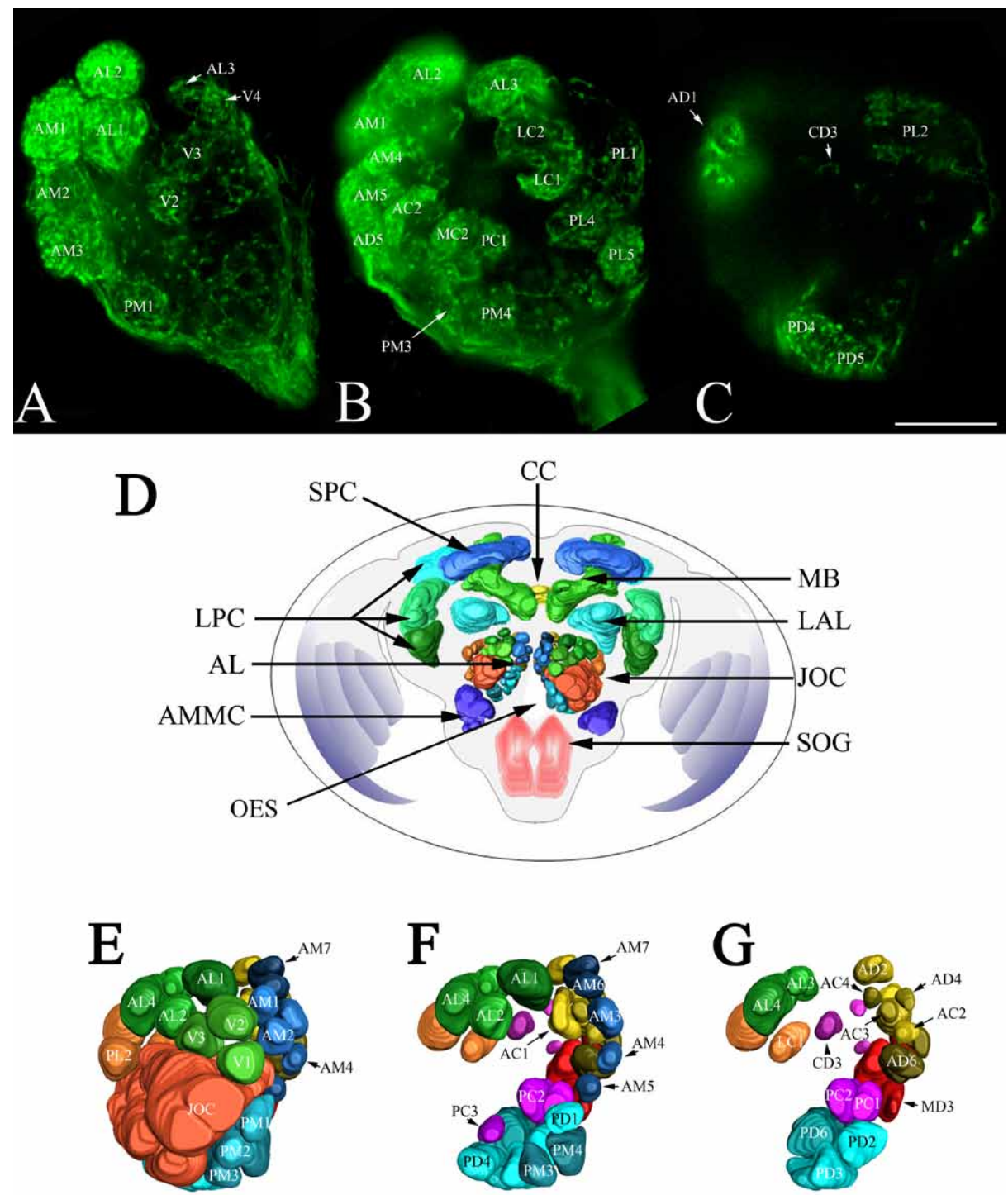

Figure 6. Confocal images of an AL of a female Aedes aegypti after anterograde staining of the ipsilateral antennal nerve $(A-C)$. Scale bar represents $25 \mu \mathrm{m}$. Individual glomeruli are labeled as in $E-G$. Note that the perimeter of individual glomeruli, in most cases, can be clearly seen. (D) Three-dimensional reconstruction of the brain of an androgynous Ae. aegypti, with surface reconstructions of male (right) and female (left) AL glomeruli as well as neuropil structures directly or indirectly involved in the integration of olfactory and mechanosensory information. AL: antennal lobe, AMMC: antennal motor and mechanosensory center, CC: central complex, JOC: Johnston's organ center, LAL: lateral accessory lobe, LPC: lateral protocerebrum, MB: mushroom body, OES: esophagus, SOG: subesophageal ganglion, SPC: superior protocerebrum. (E-G) Three-dimensional model of the AL of a male Ae. aegypti as seen from a ventral perspective. a: anterior, p: posterior, l: lateral, m: medial, v: ventral, d: dorsal, c: central. Ventral glomeruli are subsequently removed from the complete model to uncover the dorsal glomeruli. A total of 49 glomeruli are identified (reproduced with permission from Ignell et al. 2005). 
is present in the form of a macroglomerulus or a macroglomerular complex in the males (Hansson and Christensen 1999).

The reorganisation and convergence of broadly distributed ORNs into AL glomeruli appears to be a general rule in various insects' olfactory systems, as revealed through tracing studies of ORNs, originally in moths (e.g. Christensen et al. 1995, Hansson et al. 1992, Ochieng' et al. 1995) and now also reiterated in vector insects like mosquitoes (Figures 6A and 6B, Anton et al. 2003, Ghaninia et al. 2007a), and through molecular and anatomical mapping of ORNs in the model species $D$. melanogaster (Couto et al. 2005, Vosshall et al. 2000). Studies on D. melanogaster have allowed us to confirm the general validity of the one ORN - one glomerulus hypothesis put forward in the pre-genomic studies; stating that functionally distinct ORNs converge into distinct glomeruli in the AL (Couto et al. 2005, Vosshall et al. 2000). The systematic survey of D. melanogaster odorant receptor (OR) expression at neural resolution have further revealed that although ORNs in distinct sensillum types project to distinct glomeruli, their neighbour relations are not preserved (Couto et al. 2005), i.e. they display a non-parallel topography unlike that observed in the visual, auditory and somatosensory systems. Olfactory receptor neurons that express similar ORs, however, tend to map to closely appositioned glomeruli in the AL (Couto et al. 2005). This type of organisation ensures a clustered odour representation of similar odorants at the central nervous level, which is supported by in vivo imaging studies of ORN ensembles on insects like the honeybee, Apis mellifera Linnaeus (Galizia and Menzel 2000, Joerges et al. 1997), the moths, Spodoptera littoralis Boisduval (Carlsson et al. 2002, Carlsson and Hansson 2003) and Manduca sexta Linnaeus (Hansson et al. 2001), and the fly, D. melanogaster (Ng et al. 2002, Wang et al. 2003). This suggests that odours, and specifically odour quality, ultimately are encoded by spatial patterns of activation in defined sets of glomeruli before higher order brain centres are able to recognise and discriminate the different molecular features that are relayed via second-order neurons, the so-called projection neurons (PNs) (Galizia and Menzel 2000, Hildebrand and Shepherd 1997, Vosshall et al. 2000).

\section{Intra- and interglomerular synaptic interactions in the antennal lobe and quality coding}

The ORNs and the different interneuronal elements that arborise within the AL glomeruli are synaptically interconnected and taken together make up a complex neural network (Figure 6). The synaptic organisation of the AL has been studied at the ultrastructural level in the cockroach, Periplaneta americana Linnaeus, and the moth, M. sexta (Boeckh and Tolbert 1993, Distler and Boeckh 1996, 1997a,b, Malun et al. 1991a,b). The synaptic wiring within single glomeruli provides anatomical evidence for discrete processing channels within individual $A L$ glomeruli, in which functionally distinct ORNs converge and make direct synaptic contacts with PNs. Single neuron tracing of PNs in D. melanogaster along with the stereotypic mapping of ORNs in AL glomeruli also provide anatomical support for these types of channels (Figure 7B, Couto et al. 2005, Marin et al. 2002). As mentioned above, an odorant stimulus will typically elicit activity in several ORN classes (Figure 4A), and consequently several processing channels will be activated by any given stimulus (see above). If no transformation occurs through intercalated local interneurons (LNs, Figure 7A) within or between these channels, a direct feed-forward excitation of the PNs by the ORNs takes place, i.e. the olfactory information will faithfully be transmitted to higher brain centres, as suggested by $\mathrm{Ng}$ et al. (2003), Root et al. (2007) and Wang et al. (2003). Accumulating evidence over the last few years from different insects, however, emphasises that LNs that tend to have broad multiglomerular ramifications (Figure 7A), and thus provide a scaffold for crosstalk between channels, play a major role in shaping the output response of the processing channels (reviewed by Ignell and Hansson 2005, see also Bhandawat et al. 2007, Olsen and Wilson 2008, Olsen et al. 2007, Root et al. 2007, Shang et al. 2007, Wilson and Laurent 2005). In addition, intraglomerular events 
appear to have a significant influence on these processes (Bhandawat et al. 2007, Christensen et al. 2000, Kazama and Wilson 2008, Olsen and Wilson 2008, Wilson et al. 2004).

In recent years, the $D$. melanogaster AL has been the model of choice for investigating how intraand interglomerular events shape the processing channels. Although results emanating from these studies have expanded our knowledge about the neural mechanisms that shape the PN odour responses, it is important to acknowledge that these results may not directly transfer to the olfactory systems of other insects (reviewed by lgnell and Hansson 2005). A growing body of evidence, however, favours an interaction of interglomerular, or lateral, inhibitory connections between the processing channels in all insect species studied (Christensen et al. 1998, Olsen and Wilson 2008, Sachse and Galizia 2002, Waldrop et al. 1987). Lateral inhibition, through GABAergic LNs acting on both ORNs and PNs, is supported by ultrastructural analysis of their synaptic wiring (Boeckh and Tolbert 1993, Distler and Boeckh 1996, 1997a,b, Malun et al. 1991a,b). Recent evidence from $D$. melanogaster suggests that a significant component of the lateral inhibition is directed towards ORNs (Olsen and Wilson 2008). The functional significance of this is that lateral inhibition mediates a gain control in the olfactory system by suppressing strong and redundant responses. As a consequence, this damping of excessive input decreases cross-correlation between the outputs of the different processing channels. This in turn is believed to promote a more efficient neural code for odours that promote quality coding. In A. mellifera and $M$. sexta, however, physiological evidence supports a different model in which a substantial component of the lateral inhibition seems to act to sharpen the tuning curve of PNs rather than the ORN output, creating a more restricted spatial pattern of output from the odour-activated AL glomeruli (Christensen et al. 1998, Sachse and Galizia 2002, Waldrop et al. 1987). Evidence for a similar, but minor, component in $D$. melanogaster is available, but the functional implication of this arrangement is yet unknown (Olsen and Wilson 2008).

In addition to the role in regulating patterns of activity in the $A L, G A B A e r g i c ~ L N s$ are also involved in generating temporal patterns of activity within the $A L$, which has been suggested to be critical for strengthening the neural representation of odours in other regions of the central nervous system (reviewed by Christensen and White 2000, Laurent 2002). Disruption of lateral inhibition in the olfactory network through GABA receptor antagonists results in an overall increase in the output from the processing channels (Christensen et al. 1998, Sachse and Galizia 2002, Wilson et al. 2004). As a result, detection and discrimination thresholds are increased as evidenced by psychophysical data from A. mellifera and M. sexta indicating that the salient odour signal is lost (Hosler et al. 2000, Mwilaria et al. 2008). In D. melanogaster, the increased output from the processing channels has been linked to the activity of excitatory cholinergic LN connections between glomeruli (Olsen et al. 2007, Root et al. 2007, Shang et al. 2007), driven by direct monosynaptic input from ORNs (Kazama and Wilson 2008). This type of cholinergic connection has, so far, neither been supported physiologically nor immunohistologically in other insect olfactory systems (Homberg and Müller 1999). Instead, available data support alternative pathways for the observed phenomenon (Christensen et al. 1998). In D. melanogaster, cholinergic LNs are believed to broaden the tuning of PNs resulting in an increased separation between odour representations in PN coding space (Bhandawat et al. 2007, Wilson et al. 2004). As a consequence, PNs are believed to use their dynamic range more efficiently by protecting the sensory representations from noise contamination added at later stages in the olfactory processing. Note that this model is in stark contrast to that which is believed to occur in the olfactory network of insects like moths and honeybees, where lateral inhibition is acting to decrease the spatial pattern of output. Irrespective of the transformation process used, the general consensus among olfactory neurophysiologists is that discrimination of odours, i.e. quality coding, seems to depend on a combinatorial coding process involving the 
ensemble activity of a diverse array of PNs in the AL (reviewed by Christensen and White 2000, Hansson and Christensen 1999).

\section{Additional features of the olfactory signals relayed to higher brain centres}

The intra- and interglomerular interactions within the AL provides the olfactory system with a second level of neuronal signal filtering, with ORNs being the first level, allowing several features of the olfactory signals - their quality, quantity and temporal characteristics - to filter through and to be conveyed to higher brain centres (Figure 7C). As mentioned above, a substantial amount of data is available for quality coding in the AL. In this section, we will present how the olfactory system relays information about the quantity as well as the temporal characteristics of an odorant signal.

An increase in odour quantity, or concentration, at both the ORN and the PN level, is generally encoded as an increase in the response frequency of these neurons. Odour-evoked ORN signals generally seem to be amplified in the postsynaptic PN (Bhandawat et al. 2007, Boeckh and Ernst 1987, Christensen and Hildebrand 1994, Hansson et al. 1991, Hartlieb et al. 1997). This increase may be partly explained by the spatial summation of convergent ORNs onto PNs (reviewed by Hansson and Christensen 1999, see also Kazama and Wilson 2008). An additional factor dictating the sensitivity of PNs appears to be the size of the innervated glomerulus (Kazama and Wilson 2008). Projection neurons with the highest sensitivity often innervate large glomeruli, like the sexually dimorphic macroglomeruli and macroglomerular complexes observed in male moths and cockroaches (Hansson and Christensen 1999, Kazama and Wilson 2008). Results from D. melanogaster indicate that the odour-evoked ORN signals are transformed non-linearly, in which weak ORN responses are more significantly amplified in PNs compared to strong ORN responses (Bhandawat et al. 2007). The non-linear transformation seems to be dependent on the strength of the ORN-PN synapses, in which high concentrations of odour stimuli tend to produce a shortterm depression at the synapse, triggered by vesicular depletion or presynaptic inhibition via GABAergic LNs (Bhandawat et al. 2007, Kazama and Wilson 2008). So far, presynaptic inhibition has only been shown in D. melanogaster (Kazama and Wilson 2008). This transience promotes adaptation but also appears to be a major reason for the more broadly tuned PNs compared to ORNs at high concentrations (Kazama and Wilson 2008). The non-linear transformation emphasises that synapses in the olfactory network are optimised for high sensitivity near odour detection thresholds (Kazama and Wilson 2008). This is supported by behavioural experiments on insects other than D. melanogaster (Angioy et al. 2003, Kaissling 1971, Kaissling and Priesner 1970).

Although results from $D$. melanogaster shed novel light on the functional characteristics of the olfactory system, it is important to acknowledge that other insects seem to have solved concentration coding differently. In A. mellifera, odour coding seems to be performed by two parallel neuronal pathways, an intensity-coding channel and a concentration-invariant channel, that independently extract information about odour quality and concentration (Sachse and Galizia 2003). Psychophysical studies also support this type of coding pathways (Getz and Smith 1991, Kramer 1976, Sakura et al. 2002). Morphological evidence indicating similar pathways is present in other insects, including mosquitoes (Anton and Homberg 1999, Ignell et al. 2005).

In addition to extracting information about odour quality and quantity, the olfactory system of an insect must be able to detect and extract temporal information of a given odour stimulus signal. This can be explained by the fact that the temporal intermittency of an odour signal is important for driving the orientation behaviour of an insect (Baker 1990, Baker et al. 1985, Kennedy et al. 1981, Mafra-Neto and Cardé 1994, Vickers and Baker 1994). In all insects studied so far, PNs are able 
to follow stimulus pulses at frequencies between 1 and $10 \mathrm{~Hz}$ (Christensen and Hildebrand 1988, Heinbockel et al. 1999, Kazama and Wilson 2008, Lei and Hansson 1999, Lemon and Getz 1998). The ability of a PN to follow a pulsed stimulus may be linked to a short-term synaptic depression, likely presynaptic vesicle depletion, at the ORN-PN synapse as revealed in D. melanogaster (Kazama and Wilson 2008). Alternatively, or perhaps additionally, stimulus tracking of PNs may be regulated by intercalated inhibitory LNs that trigger characteristic pulse-tracking capabilities of certain PNs, as observed in moths (Christensen and Hildebrand 1997, Lei and Hansson 1999, Waldrop et al. 1987).

\section{Anatomy and function of higher olfactory centres}

The topographic map in the antennal lobe, of at least $D$. melanogaster, is retained in the higher olfactory centres, the mushroom body and the lateral horn of the protocerebrum (Jefferies et al. 2007, Lin et al. 2007, Marin et al. 2002, Wong et al. 2002). Projection neurons that innervate a given glomerulus display stereotypic branching patterns in these centres, whereas projection neurons innervating different glomeruli exhibit very different arborisation patterns. It is plausible that olfactory information relayed from the $\mathrm{AL}$ of mosquitoes and other haematophagous insects also is retained in a similar way considering the conserved anatomy of the higher olfactory system in the insect lineage (Strausfeld et al. 1998).

A central question in olfaction is how the brain discriminates different odours to elicit an appropriate behavioural response. Stereotypic connectivity maps of odorant-to-OR (Hallem and Carlson 2006), ORN-to-PN (Couto et al. 2005, Fishilevich and Vosshall 2005), and PN-to-Kenyon cell, the principal neuron type of the mushroom body (Jefferies et al. 2007, Lin et al. 2007) have allowed the construction of neural computation of odour discrimination in the $D$. melanogaster brain. Stereotypic PN-to-KC connectivity and functional imaging suggest that differential representation of the odours in the $A L$ is maintained in the $M B$ calyx and possibly further processed in the different MB neurons (Jefferies et al. 2007, Lin et al. 2007). The distribution of odour responses across different classes of Kenyon cells and the imposition of odor-sensitive excitatory and inhibitory responses both appear to enhance distinct neural representations of different odours. Such complexity of odour representations greatly reduces the possibility of overlap between spatiotemporal patterns elicited by two different odorants making them easier to discriminate.

Classical lesion experiments (Erber et al. 1980, Heisenberg et al. 1985) and genetic blockade of KC synaptic output have also shown that mushroom bodies are essential for memory formation and retrieval in fruit flies (Dubnau et al. 2001, Krashes et al. 2007, McGuire et al. 2001, Schwaerzel et al. 2002). By determining how odours are represented across KCs in comparison to the tuning profile of ORNs, Turner et al. (2008) found that representations were significantly better separated in the mushroom body than in the receptor layer. This combination of sparseness and representation divisibility is desirable of memory systems (Garcia-Sanchez and Huerta 2003, Huerta et al. 2004, Kanerva 1988, Olshausen and Field 2004, Tsodyks and Feigelman 1988, Willshaw and LonquetHigging 1969) and consistent with the functional role assigned to the mushroom bodies (De Belle and Heisenberg 1994, Dubnau et al. 2001, Hammer and Menzel 1998, Krashes et al. 2007, McGuire et al. 2001, Schwaerzel et al. 2002). With the increasing knowledge concerning the anatomy and physiology of the mushroom body, we are making good progress towards an understanding of memory formation and retrieval. Recent functional imaging studies of the mushroom body of $D$. melanogaster, have, for example, revealed memory traces, manifested as $\mathrm{Ca}^{2+}$ activity, in specific subtypes of Kenyon cells (Wang et al. 2008, Yu et al. 2006). If the predictions by Turner et al. (2008) are correct, i.e. that there is a strong selective pressure to generate sparse representations in the 
mushroom bodies, it may be proposed that this 'sparsening' is an intrinsic requirement of any memory system.

Anatomical analyses of PN branching patterns in the lateral horn of the protocerebrum (e.g. Figure $7 \mathrm{~B}$ ) in $D$. melanogaster reveals a segregation of putative pheromone representing PNs and most other PNs (Jefferis et al. 2007). Interestingly, these authors found a high degree of sexual dimorphism in the lateral horn region that emphasise sex-specific integration in the lateral horn that may underlie sex-specific behaviours. The spatial segregation of pheromone representation contrasts with the representation of glomeruli that receive input from non-pheromonal PNs. Many of these PN classes display extensive overlap, which makes the representations of different odorants overlapping. The findings by Jefferis et al. (2008) thus suggest that olfactory information is highly intermixed at the LH level compared to the glomerular organisation of the antennal lobe, but that rather discrete channels are retained for pheromones all the way from the sensory periphery to the LH. They further propose that the LH is globally organised according to biological values rather than chemical nature of the odorant information. This finding is reminiscent of the male silkworm moth, Bombyx mori, where PNs from the macroglomerular complex representing sex pheromones send axon projections to a discrete area in the lateral protocerebrum (Seki et al. 2005). Spatial segregation of the pheromone representation in higher olfactory centres may therefore be a conserved feature in insects.

\section{Future outlook}

Investigation of the olfactory systems, including morphological and physiological studies in the periphery, the $A L$ and the higher brain centres, within a wider variety of vectors is of fundamental importance in the development of the olfaction-based methods of trapping and repelling vectors, demonstrated so successfully in other insect species. With the inclusion of descriptions of more olfactory systems from haematophagous arthropods, there is the increased probability that common features of olfactory signally processing will emerge, providing further insight into processes mediating vector-related olfactory behaviours. Such information will ultimately help identify novel tools to protect human hosts through the target interference with vector-related behaviours.

With the recent genome sequencing of some of the most important disease vectors, an accumulation of information about the perireceptor and transduction mechanisms involving olfactory coding of volatile signals that drive important behaviours like mating, host finding and oviposition is expected. Access to the genome of, at present, three species of mosquitoes allow for comparative genome analysis such that the identification of the complete repertoire of OBPs and ORs provide rich resources for linking molecular, evolutionary and ecological processes. Systematic physiological analysis of the olfactory system of these species is important to link in vitro and in vivo data. To better grasp olfactory coding in mosquitoes, central olfactory processes are needed to be investigated more in detail. For this purpose, previous studies on other insects, where anterograde staining and optical imaging of ORNs have been efficiently used, can be applied for a better understanding of odour coding in mosquitoes.

\section{Acknowledgements}

We would like to thank the Swedish research council, Formas. 


\section{References}

Ache BW and Young JM (2005) Olfaction: diverse species, conserved principles. Neuron 48: 417-430.

Almaas TJ and Mustaparta H (1991) Heliothis virescens: response characteristics of receptor neurons in sensilla trichodea Type 1 and Type 2. J Chem Ecol 17: 953-972.

Almaas TJ, Christensen TA and Mustaparta H (1991) Chemical communication in heliothine moths. I. Antennal receptor neurons encode several features of intra- and interspecific odorants in the male corn earworm moth Helicoverpa zea. J Comp Physiol A 169: 249-258.

Altner H (1977) Insect sensillum specificity and structure: an approach to a new typology. Olfaction Taste 6: 295-303.

Anderson P, Hansson BS and Löfkvist J (1995) Plant-odour-specific receptor neurones on the antennae of female and male Spodoptera littoralis. Physiol Entomol 20: 189-198.

Angioy AM, Desogus A, Barbarossa IT, Anderson P and Hansson BS (2003) Extreme sensitivity in an olfactory system. Chem Senses 28: 279-284.

Anton S and Homberg U (1999) Antennal lobe structure. In: Hansson BS (ed) Insect olfaction. Springer, Berlin, Germany, pp 97-124.

Anton S, Van Loon JJ, Meijerink J, Smid HM, Takken W and Rospars JP (2003) Central projections of olfactory receptor neurons from single antennal and palpal sensilla in mosquitoes. Arthropod Struct Dev 32: 319-327.

Baker TC (1990) Upwind flight and casting flight: complementary phasic and tonic systems used for location of sex pheromone sources by male moths. In: Doving K (ed) ISOT X, Proc. 10th Int. Symp. Olfaction Taste, GCS/AS, Oslo, Norway, pp 18-25.

Baker TC, Willis MA, Haynes KF and Phelan PL (1985) A pulsed cloud of sex pheromone elicits upwind flight in male moths. Physiol Entomol 10: 257-265.

Baker TC, Hansson BS, Löfstedt C and Löfqvist J (1988) Adaptation of antennal neurons in moths is associated with cessation of pheromone mediated upwind flight. PNAS 85: 9826-9830.

Baker TC, Ochieng' SA, Cossé AA, Lee SG, Todd JL, Quero C and Vickers NJ (2004) A comparison of responses from olfactory receptor neurons of Heliothis subflexa and Heliothis virescens to components of their sex pheromone. J Comp Physiol A 190: 155-165.

Bau J, Justus KA and Cardé RT (2002) Antennal resolution of pulsed pheromone plumes in three moth species. J Insect Physiol 48: 433-442.

Behan M and Schoonhoven LM (1978) Chemoreception of an oviposition deterrent associated with eggs in Pieris brassicae. Entomol Exp Appl 24: 163-179.

Bengtsson M, Liljefors T, Hansson BS, Löfstedt C and Copaja SV (1990) Structure-activity relationships for chainshortened analogs of (Z)-5-decenyl acetate, a pheromone component of the turnip moth, Agrotis segetum. J Chem Ecol 16: 667-684.

Benton R, Sachse S, Michnick SW and Vosshall LB (2006) Atypical membrane topology and heteromeric function of Drosophila odorant receptors in vivo. PlosBiol 4: 240-257.

Bestmann HJ (1981) Pheromon Rezeptor-Wechsilwirkung bei Insekten. Mitt Dt Ges Allg Angew Ent 2: 242-247.

Bestmann HJ and Vostrowsky O (1982) Peripheral aspects of olfacto-endocrine interactions. Structure-activity. In: Breipohl W (ed) Olfaction and endocrine regulation. IRL Press, London, UK, pp 253-265.

Bhandawat V, Olsen SR, Gouwens NW, Schlief ML and Wilson RI (2007) Sensory processing in the Drosophila antennal lobe increases reliability and separability of ensemble odor representations. Nat Neurosci 10: 1474-1482.

Biessmann H, Nguyen QK, Le D and Walter MF (2005) Microarray-based survey of a subset of putative olfactory genes in the mosquito Anopheles gambiae. Insect Mol Biol 14: 575-589.

Bohbot J, Pitts RJ and Kwon HW (2007) Molecular characterization of the Aedes aegypti odorant receptor gene family. Insect Mol Biol 16: 525-537.

Boeckh J and Selsam P (1984) Quantitative investigation of the odour specificity of central olfactory neurones in the American cockroach. Chem Senses 9: 369-380.

Boeckh J and Ernst KD (1987) Contribution of single unit analysis in insects to an understanding of olfactory function. J Comp Physiol A 161: 549-565. 
Boeckh J and Tolbert LP (1993) Synaptic organization and development of the antennal lobe in insects. Microsc Res Tech 24: 260-280.

Brazil RP and Hamilton JGC (2002) Isolation and identification of 9-methylgermacrene-B as the putative sex pheromone of Lutzomyia cruzi (Mangabeira, 1938) (Diptera: Psychodidae). Memorias Do Instituto Oswaldo Cruz 97: 435-436.

Cabrera M and Jaffe K (2007) An aggregation pheromone modulates lekking behaviour in the vector mosquito Aedes aegypti (Diptera: Culicidae). J Am Mosq Control Assoc 23: 1-10.

Carey AF, Wang G, Su C-Y, Zwiebel LJ and Carlson JR (2010) Odorant reception in the malaira mosquito, Anopheles gambiae. Nature 464: 66-71.

Carlsson MA and Hansson BS (2003) Dose-response characteristics of glomerular activity in the moth antennal lobe. Chem Senses 28: 269-278.

Carlsson MA, Galizia G and Hansson BS (2002) Spatial representation of odours in the antennal lobe of the moth Spodoptera littoralis (Lepidoptera: Noctuidae). Chem Senses 27: 231-244.

Christensen TA and Hildebrand JG (1988) Frequency coding by central olfactory neurons in the sphinx moth Manduca sexta. Chem. Senses 13: 123-130.

Christensen TA and Hildebrand JG (1994) Neuroethology of sexual attraction and inhibition in heliothine moths. In: Schildberger $\mathrm{K}$ and Elsner N (eds) Neural basis of behavioural adaptations. Progress in Zoology, vol 39. Fischer, Stuttgart, Germany, pp 37-46.

Christensen TA and White J (2000) Representation of olfactory information in the brain. In: Finger TE, Silver WL and Restrepo D (eds) The neurobiology of taste and smell. Wiley-Liss Inc, New York, USA, pp 201-232.

Christensen TA, Harrow ID, Cuzzocrea C, Randolph PW and Hildebrand JG (1995) Distinct projections of two populations of olfactory receptor axons in the antennal lobe of the sphinx moth Manduca sexta. Chem. Senses 20: 313-323.

Christensen TA, Waldrop B and Hildebrand JG (1998) GABAergic mechanisms that shape the temporal response to odors in moth olfactory projection neurons. Ann NY Acad Sci 855: 475-481.

Christensen TA, Pawlowski VM, Lei H and Hildebrand JG (2000) Multi-unit recordings reveal context dependent modulation of synchrony in odor-specific neural ensembles. Nat Neurosci 3: 927-991.

Clements AN (1999) The biology of mosquitoes, vol. 2. Sensory reception and behaviour. CABI Publishing, Wallingford, UK.

Clyne PJ, Warr CG, Freeman MR, Lessing D, Kim J and Carlson JR (1999) A novel family of divergent seven-transmembrane proteins: candidate odorant receptors in Drosophila. Neuron 22: 327-338.

Couto A, Alenius M and Dickson BJ (2005) Molecular, anatomical, and functional organization of the Drosophila olfactory system. Curr Biol 15: 1535-1547.

Davis EE and Bowen MF (1994) Sensory physiological basis for attraction in mosquitoes. J Am Mosq Control Assoc 10: 316-325.

De Belle JS and Heisenberg M (1994) Associative odor learning in Drosophila abolished by chemical ablation of mushroom bodies. Science 263: 692-695.

Dekker T, Geier M and Cardé RT (2005) Carbon dioxide instantly sensitizes female yellow fever mosquitoes to human skin odours. J Exp Biol 208: 2963-2972.

Den Otter CJ and Van der Goes van Naters W (1993) Responses of individual antennal olfactory cells of tsetse flies (Glossina m. morsitans) to phenols from cattle urine. Physiol Entomol 18: 43-49.

Dickens JC (1990) Specialized receptor neurons for pheromone and host plant odors in the boll weevil, Anthonomus grandis Boh (Coleoptera: Curculionidae). Chem Senses 15: 311-331.

Distler PG and Boeckh J (1996) Synaptic connection between olfactory receptor cells and uniglomerular projection neurons in the antennal lobe of the American cockroach, Periplaneta americana. J Comp Neurol 370: 35-46.

Distler PG and Boeckh J (1997a) Synaptic connection between identified neuron types in the antennal lobe glomeruli of the cockroach, Periplaneta americana: II. Local multiglomerular interneurons. J Comp Neurol 378: 307-319.

Distler PG and Boeckh J (1997b) Synaptic connections between identified neuron types in the antennal lobe glomeruli of the cockroach, Periplaneta americana: II. Local multiglomerular interneurons. J Comp Neurol 383: 529-540.

Ditzen M, Pellegrino M and Vosshall LB (2008) Insect odorant receptors are molecular targets of the insect repellent DEET. Science 319: 1838-1842. 
Domingue MJ, Musto CJ, Linn CE Jr, Roelofs WL and Baker TC (2007) Altered olfactory receptor neuron responsiveness in rare Ostrinia nubilalis males attracted to the O-furnacalis pheromone blend. J Insect Physiol 53: 1063-1071.

Du YJ and Millar JG (1999) Electroantennogram and oviposition bioassay responses of Culex quinquefasciatus and Culex tarsalis (Diptera: Culicidae) to chemicals in odours from Bermuda grass infusions. J Med Entomol 36: 158-166.

Dubnau J, Grady L, Kitamoto T and Tully T (2001) Disruption of neurotransmission in Drosophila mushroom body blocks retrieval but not acquisition of memory. Nature 411: 476-480.

Erber J, Masuhr T and Menzel R (1980) Localization of short-term memory in the brain of the bee, Apis mellifera. Physiol Entomol 5: 343-358.

Fishilevich E and Vosshall LB (2005). Genetic and functional subdivision of the Drosophila antennal lobe. Curr Biol 15: 1548-1553.

Galindo K and Smith DP (2001) A large family of divergent Drosophila odorant-binding proteins expressed in gustatory and olfactory sensilla. Genetics 159: 1059-1072.

Galizia CG and Menzel R (2000) Odour perception in honeybees: coding information in glomerular patterns. Curr Opin Neurobiol 10: 504-510.

Gao Q and Chess A (1999) Identification of candidate Drosophila olfactory receptors from genomic DNA sequence. Genomics 60: 31-39.

Garcia-Sanchez M and Huerta R (2003) Design parameters of the fan-out phase of sensory systems. J Comput Neurosci 15: 5-17.

Geier M and Boeckh J (1999) A new Y-tube olfactometer for mosquitoes to measure the attractiveness of host odours. Entomol Exp Appl 92: 9-19.

Getz WM and Smith KB (1991) Olfactory perception in honeybees: Concatenated and mixed odorant stimuli, concentration and exposure effects. J Comp Physiol A 169: 215-230.

Ghaninia M, Hansson BS and Ignell R (2007a) The antennal lobe of the African malaria mosquito, Anopheles gambiae innervation and three-dimensional reconstruction. Arthropod Struct Dev 36: 23-39.

Ghaninia M, Ignell R and Hansson BS (2007b) Functional classification and central nervous projections of olfactory receptor neurons housed in antennal trichoid sensilla of female yellow fever mosquitoes, Aedes aegypti. Eur J Neurosci 26: 1611-1623.

Ghaninia M, Larsson MC, Hansson BS and Ignell R (2008) Natural odor ligands for olfactory receptor neurons of female mosquitoes, Aedes aegypti, using gas chromatography- single sensillum recordings. J Exp Biol 211: 3020-3027.

Graham LA and Davies PL (2002) The odorant-binding proteins of Drosophila melanogaster: annotation and characterization of a divergent gene family. Gene 292: 43-55.

Grenacher S, Kröber T, Guerin PM and Vlimant M (2001) Behavioural and chemoreceptor cell responses of the tick, Ixodes ricinus, to its own faeces and faecal constituents. Exp Appl Acarol 25: 641-660.

Guerenstein PG and Guerin PM (2004) A comparison of volatiles emitted by adults of three triatomine species. Entomol Exp Applic 111: 151-155.

Györgyi TK, Roby-Shemkovitz AJ and Lerner MR (1988) Characterization and cDNA cloning of the pheromone-binding protein from the tobacco hornworm, Manduca sexta: a tissue-specific developmentally regulated protein. PNAS 85: 9851-9855.

Ha TS and Smith DP (2006) A pheromone receptor mediates 11-cis-vacenyl acetate-induced responses in Drosophila. J Neurosci 26: 8727-8733.

Hallberg E and Hansson BS (1999) Arthropod sensilla: morphology and phylogenetic considerations. Microsc Res Tech 47: 428-439.

Hallem EA and Carlson JR (2006). Coding of odors by a receptor repertoire. Cell 125: 143-160.

Hallem EA, Ho MG, Carlson JR (2004) The molecular basis of odour coding in the Drosophila antenna. Cell 117: 965-979.

Hamana H, Hirono J, Kizumi M and Sato T (2003) Sensitivity-dependent hierarchical receptor codes for odours. Chem Senses 28: 87-104.

Hammer M and Menzel R (1998) Multiple sites of associative odor learning as revealed by local brain microinjections of octopamine in honeybees. Learn Mem 5: 146-156. 
Hansson BS and Christensen TA (1999) Functional characteristics of the antennal lobe. In: Hansson BS (ed) Insect olfaction. Springer, Berlin, Germany, pp 125-161.

Hansson BS and Anton S (2000) Function and morphology of the antennal lobe: new developments. Ann Rev Entomol 45: 203-231.

Hansson BS, Löfstedt C and Foster SP (1989) Z-linked inheritance of male olfactory response to sex pheromone components in two species of tortricid moths, Ctenopseustis obliquana and Ctenopseustis sp. Entomol Exp Appl 53: $137-145$.

Hansson BS, Christensen TA and Hildebrand JG (1991) Functionally distinct subdivisions of the macroglomerular complex in the antennal lobe of the male sphinx moth Manduca sexta. J Comp Neurol 312: 264-278.

Hansson BS, Ljungberg H, Hallberg E and Löfstedt C (1992) Functional specialization of olfactory glomeruli in a moth. Science 256: 1313-1315.

Hansson BS, Larsson M and Leal WS (1999) Green leaf volatile-detecting olfactory receptor neurones display very high sensitivity and specificity in a scarab beetle. Physiol Entomol 24: 121-126.

Hansson BS, Carlsson MA and Kalinovà B (2001) Olfactory activation patterns in the antennal lobe of the sphinx moth, Manduca sexta. J Comp Physiol A 189: 301-308.

Hartlieb E, Anton S and Hansson BS (1997) Dose-dependent response characteristics of antennal lobe neurons in the male moth Agrotis segetum (Lepidoptera: Noctuidae). J Comp Physiol A 181: 469-476.

Heinbockel T and Kaissling K-E (1996) Variability of olfactory receptor neuron responses of female silkmoths (Bombyx mori L.) to benzoic acid and ( \pm -linalool. J Insect Physiol 42: 565-578.

Heinbockel T, Christensen T and Hildebrand JG (1999) Temporal tuning of odor responses in pheromone-responsive projection neurons in the brain of the sphinx moth Manduca sexta. J Comp Neurol 409: 1-12.

Heisenberg M, Borst A, Wagner S and Byers D (1985) Drosophila mushroom body mutants are deficient in olfactory learning. J Neurogenet 2: 1-30.

Hekmat-Scafe DS, Steinbrecht RA and Carlson JR (1997) Coexpression of two odorant-binding protein homologs in Drosophila: implications for olfactory coding. J Neurosci 17: 1616-1624.

Hekmat-Scafe DS, Scafe CR, McKinney AJ and Tanouye MA (2002) Genome-wide analysis of the odorant-binding protein gene family in Drosophila melanogaster. Genome Res 12: 1357-1369.

Hildebrand JG and Shepherd GM (1997) Mechanisms of olfactory discrimination: converging evidence for common principles across phyla. Ann Rev Neurosci 20: 595-631.

Hill CA, Fox AN, Pitts RJ, Kent LB, Tan PL, Chrystal MA, Cravchik A, Collins FH, Robertson HM and Zwiebel LJ (2002) G protein-coupled receptors in Anopheles gambiae. Science 298: 176-178.

Hill SR, Hansson BS and Ignell R (2009) Characterisation of antennal trichoid sensilla from female southern house mosquito, Culex quinquefasciatus Say. Chemical Senses 34: 231-252.

Holt RA, Subramanian GM, Halpern A, Sutton GG, Charlab R, Nusskern DR, Wincker P, Clark AG, Ribeiro JM, Wides R, Salzberg SL, Loftus B, Yandell M, Majoros WH, Rusch DB, Lai Z, Kraft CL, Abril JF, Anthouard V, Arensburger P, Atkinson PW, Baden H, de Berardinis V, Baldwin D, Benes V, Biedler J, Blass C, Bolanos R, Boscus D, Barnstead M, Cai S, Center A, Chaturverdi K, Christophides GK, Chrystal MA, Clamp M, Cravchik A, Curwen V, Dana A, Delcher A, Dew I, Evans CA, Flanigan M, Grundschober-Freimoser A, Friedli L, Gu Z, Guan P, Guigo R, Hillenmeyer ME, Hladun SL, Hogan JR, Hong YS, Hoover J, Jaillon O, Ke Z, Kodira C, Kokoza E, Koutsos A, Letunic I, Levitsky A, Liang Y, Lin JJ, Lobo NF, Lopez JR, Malek JA, McIntosh TC, Meister S, Miller J, Mobarry C, Mongin E, Murphy SD, O'Brochta DA, Pfannkoch C, Qi R, Regier MA, Remington K, Shao H, Sharakhova MV, Sitter CD, Shetty J, Smith TJ, Strong R, Sun J, Thomasova D, Ton LQ, Topalis P, Tu Z, Unger MF, Walenz B, Wang A, Wang J, Wang M, Wang X, Woodford KJ, Wortman JR, Wu M, Yao A, Zdobnov EM, Zhang H, Zhao Q, Zhao S, Zhu SC, Zhimulev I, Coluzzi M, della Torre A, Roth CW, Louis C, Kalush F, Mural RJ, Myers EW, Adams MD, Smith HO, Broder S, Gardner MJ, Fraser CM, Birney E, Bork P, Brey PT, Venter JC, Weissenbach J, Kafatos FC, Collins FH and Hoffman SL (2002) The genome sequence of the malaria mosquito Anopheles gambiae. Science 298: 129-149.

Homberg U and Müller U (1999) Neuroactive substances in the antennal lobe. In: Hansson BS (ed) Insect Olfaction. Springer, Berlin, Germany, pp 182-204. 
Hosler JS, Buxton KL and Smith BH (2000) Impairment of olfactory discrimination by blockade of GABA and nitric oxide activity in the honey bee antennal lobes. Behav Neurosci 114: 514-525.

Huerta R, Nowotny T, García-Sanchez M, Abarbanel HD and Rabinovich MI (2004) Learning classification in the olfactory system of insects. Neural Comput 16: 1601-1640.

Ignell R and Hansson BS (2005) Insect olfactory neuroethology - an electrophysiological perspective. In: Christensen TA (ed) Methods in insect sensory neuroscience. CRC Press, Boca Raton, USA, pp 319-347.

Ignell R, Dekker T, Ghaninia M and Hansson BS (2005) Neuronal architecture of the mosquito deutocerebrum. J Comp Neurol 493: 207-240.

Jefferis GS, Potter CJ, Chan AM, Marin EC, Rohlfing T, Maurer CR Jr and Luo L (2007) Comprehensive mapsof Drosophila higher olfactory centers: spatially segregated fruit and pheromone representation. Cell 128: 1187-1203.

Jönsson M and Anderson P (1999) Electrophysiological response to herbivore-induced host plant volatiles in the moth Spodoptera littoralis. Physiol Entomol 24:377-385.

Joerges J, Küttner A, Galizia G and Menzel R (1997) Representations of odours and odour mixtures visualized in the honeybee brain. Nature 387: 285-288.

Justice RW, Dimitratos S, Walter MF, Woods DF and Biessmann H (2003a) Sexual dimorphic expression of putative antennal carrier protein genes in the malaria vector Anopheles gambiae. Insect Mol Biol 12: 581-594.

Justice RW, Biessmann H, Walter MF, Dimitratos SD and Woods DF (2003b) Genomics spawns novel approaches to mosquito control. Bioessays 25: 1011-1020.

Justus K, Murlis J, Jones C and Carde R (2002). Measurement of odor-plume structure in a wind tunnel using a photoionization detector and a tracer gas. Environ Fluid Mech 2: 115-142.

Kain P, Chakraborty TS, Sundaram S, Siddiqi O, Rodrigues V and Hasan G (2008) Reduced odor responses from antennal neurons of $\mathrm{G}(\mathrm{q})$ alpha, phospholipase Cbeta, and rdgA mutants in Drosophila support a role for a phospholipid intermediate in insect olfactory transduction. J Neurosci 30: 4745-4755.

Kaissling K-E (1971) Insect olfaction. In: Beidler LM (ed) Handbook of sensory physiology, Vol.IV; Chem senses. SpringerVerlag, Berlin, Germany, pp 351-431.

Kaissling K-E (1986) Chemo-electrical transduction in insect olfactory receptors. Ann Rev Neurosci 9: 121-145.

Kaissling K-E and Priesner E (1970) Die Riechschwelle des Seidenspinners. Naturwissenschaften 57: 23-28.

Kanerva P (1988) Sparse distributed memory. MIT Press, Cambridge, MA, USA.

Kazama $\mathrm{H}$ and Wilson RI (2008) Homeostatic matching and nonlinear amplification at identified central synapses. Neuron 58: 401-413.

Keil TA (1999) Morphology and development of the peripheral olfactory organs. In: Hansson BS (ed) Insect olfaction. Springer, Berlin, pp 5-47.

Kennedy JS, Ludlow AR and Sanders CJ (1981) Guidance of flying male moths by wind-borne sex pheromone. Physiol Entomol 6: 395-412.

Kramer E (1976) The orientation of walking honeybees in odour fields with small concentration gradients. Physiol Entomol 1:27-37.

Krashes MJ, Keene AC, Leung B, Armstrong JD and Waddell S (2007) Sequential use of mushroom body neuron subsets during Drosophila odor memory processing. Neuron 53: 103-115.

Krieger J, von Nickisch-Rosenegk E, Mameli M, Pelosi P and Breer H (1996) Binding proteins from the antennae of Bombyx mori. Insect Biochem Mol Biol 26: 297-307.

Kruse SW, Zhao R, Smith DP and Jones DN (2003) Structure of a specific alcohol-binding site defined by the odorant binding protein LUSH from Drosophila melanogaster. Nat Struct Biol 10: 694-700.

Kwon HW, Lu T, Rützler M and Zwiebel LJ (2006) Olfactory responses in a gustatory organ of the malaria vector mosquito Anopheles gambiae. PNAS 103: 13526-13531.

Kwon JY, Dahanukar A, Weiss LA and Carlson JR (2007) The molecular basis of $\mathrm{CO}_{2}$ reception in Drosophila. PNAS 104: 3574-3578.

Larsson MC, Leal WS and Hansson BS (1999) Olfactory receptor neurons specific to chiral sex pheromone components in male and female Anomala cuprea beatles (Coleoptera: Scarabaeidae). J Comp Physiol A 184: 353-359. 
Larsson MC, Leal WS and Hansson BS (2001) Olfactory receptor neurons detecting plant odours and male volatiles in Anomala cuprea beetles (Coleoptera: Scarabaeidae). J Insect Physiol 47: 1065-1076.

Larsson MC, Domingos Al, Jones WD, Chiappe ME, Amrein H and Vosshall LB (2004) Or83b encodes a broadly expressed odorant receptor essential for Drosophila olfaction. Neuron 43: 703-714.

Laue MR, Steinbrecht RA and Ziegelberger G (1994) Immunocytochemical localization of general odorant-binding protein in olfactory sensilla of the silkmoth Antheraea polyphemus. Naturwissenschaften 81: 178-180.

Laurent G (2002) Olfactory network dynamics and the coding of multidimensional signals. Nat Rev Neurosci 3: 885-895.

Lei $\mathrm{H}$ and Hansson BS (1999) Central processing of pulsed pheromone signals by antennal lobe neurons in the male moth Agrotis segetum. J Neurophysiol 81: 1113-1122.

Lemon WC and Getz WM (1998) Responses of cockroach antennal lobe projection neurons to pulsatile stimuli. Ann NY Acad Sci 855: 517-520.

Li ZX, Pickett JA, Field LM and Zhou JJ (2005) Identification and expression of odorant-binding proteins of the malariacarrying mosquitoes Anopheles gambiae and Anopheles arabiensis. Arch Insect Biochem Physiol 58: 175-189.

Liljefors T, Thelin B and Van der Pers JNC (1984) Structure-activity relationships between stimulus molecules and response of a pheromone receptor cell in turnip moth, Agrotis segetum: modifications of the acetate group. J Chem Ecol 10: 1661-1675.

Liljefors T, Thelin B, Van der Pers, JNC and Lofstedt C (1985) Chain-elongated analogues of a pheromone component of the turnip moth, Agrotis segetum. A structure-activity study using molecular mechanics. J Chem Soc Perkin Trans II 1957-1962.

Liljefors T, Bengtsson M and Hansson BS (1987) Effects of double-bond configuration on interaction between a moth sex pheromone component and its receptor: a receptor-interaction model based on molecular mechanics. J Chem Ecol 13: 2023-2040.

Lin HH, Lai JS, Chin AL, Chen YC and Chiang AS (2007) A map of olfactory representation in the Drosophila mushroom body. Cell 128: 1205-1217.

Lu T, Qiu YT, Wang G, Kwon JY, Rutzler M, Kwon HW, Pitts RJ, van Loon JJ, Takken W, Carlson JR and Zwiebel LJ (2007) Odor coding in the maxillary palp of the malaria vector mosquito Anopheles gambiae. Curr Biol 17: 1533-1544.

Malnic B (2007) Searching for the ligands of odorant receptors. Mol Neurobiol 35: 175-181.

Malun D (1991a) Inventory and distribution of synapses of identified uniglomerular projection neurons in the antennal lobe of Periplaneta americana. J Comp Neurol 305: 348-360.

Malun D (1991b) Synaptic relationship between GABA-immunoreactive neurons and an identified uniglomerular projection neuron in the antennal lobe of Periplaneta americana: A double-labeling electron microscopic study. Histochemistry 96: 197-207.

Mafra-Neto A and Cardé RT (1994) Fine-scale structure of pheromone plumes modulates upwind orientation of flying moths. Nature 369: 142-144.

Mafra-Neto A and Cardé RT (1998) Rate of realized interception of pheromone pulses in different wind speeds modulates almond moth orientation. J Comp Physiol A 182: 563-572.

Marin EC, Jefferis GS, Komiyama T, Zhu H and Luo L (2002) Representation of the glomerular olfactory map in the Drosophila brain. Cell 109: 243-255.

Marion-Poll F and Tobin TR (1992) Temporal coding of pheromone pulses and trains in Manduca sexta. J Comp Physiol A 171: 505-512.

Mason JR, Bean NJ and Clark L (1993) Development of chemosensory attractants for white-tailed deer (Odocoileus virginianus). Crop Protection 12: 448-452.

Matsuo T, Sugaya S, Yasukawa J, Aigaki T and Fuyama Y (2007) Odorant-binding proteins OBP57d and OBP57e affect taste perception and host-plant preference in Drosophila sechellia. PLoS Biol 5: 985-996.

Mclver SB (1982) Sensilla of mosquitoes (Diptera: Culicidae). J Med Entomol 19: 489-535.

McGuire SE, Le PT and Davis RL (2001) The role of Drosophila mushroom body signaling in olfactory memory. Science 293: 1330-1333. 
Melo AC, Rützler M, Pitts RJ and Zwiebel LJ (2004) Identification of a chemosensory receptor from the yellow fever mosquito, Aedes aegypti, that is highly conserved and expressed in olfactory and gustatory organs. Chem Senses 29: 403-410.

Moore PA (1994) A model of the role of adaptation and disadaptation in olfactory receptor neurons: implications for the coding of temporal and intensity patterns in odor signals. Chem Senses 19:71-86.

Murlis J, Jones CD (1981) Fine-scale structure of odour plumes in relation to distant pheromone and other attractant sources. Physiol Entomol 6: 71-86.

Mwilaria EK, Ghatak C and Daly KC (2008) Disruption of GABAA in the insect antennal lobe generally increases odor detection and discrimination thresholds. Chem Senses 33: 267-281.

Nakagawa T, Sakurai T, Nishioka T and Touhara K (2005) Insect sex pheromone signals mediated by specific combinations of olfactory receptors. Science 307: 1638-1642.

Neuhaus EM, Gisselmann G, Zhang W, Dooley R, Störtkuhl K and Hatt H (2005) Odorant receptor heterodimerization in the olfactory system of Drosophila melanogaster. Nat Neurosci 8: 15-17.

Nene V, Wortman JR, Lawson D, Haas B, Kodira C, Tu ZJ, Loftus B, Xi Z, Megy K, Grabherr M, Ren Q, Zdobnov EM, Lobo NF, Campbell KS, Brown SE, Bonaldo MF, Zhu J, Sinkins SP, Hogenkamp DG, Amedeo P, Arensburger P, Atkinson PW, Bidwell S, Biedler J, Birney E, Bruggner RV, Costas J, Coy MR, Crabtree J, Crawford M, Debruyn B, Decaprio D, Eiglmeier K, Eisenstadt E, El-Dorry H, Gelbart WM, Gomes SL, Hammond M, Hannick LI, Hogan JR, Holmes MH, Jaffe D, Johnston JS, Kennedy RC, Koo H, Kravitz S, Kriventseva EV, Kulp D, Labutti K, Lee E, Li S, Lovin DD, Mao C, Mauceli E, Menck CF, Miller JR, Montgomery P, Mori A, Nascimento AL, Naveira HF, Nusbaum C, O'leary S, Orvis J, Pertea M, Quesneville H, Reidenbach KR, Rogers YH, Roth CW, Schneider JR, Schatz M, Shumway M, Stanke M, Stinson EO, Tubio JM, Vanzee JP, Verjovski-Almeida S, Werner D, White O, Wyder S, Zeng Q, Zhao Q, Zhao Y, Hill CA, Raikhel AS, Soares MB, Knudson DL, Lee NH, Galagan J, Salzberg SL, Paulsen IT, Dimopoulos G, Collins FH, Birren B, Fraser-Liggett CM and Severson DW (2007) Genome sequence of Aedes aegypti, a major arbovirus vector. Nature 316: 1718-1723.

Ng M, Roorda RD, Lima SQ, Zemelman BV, Morcillo P and Miesenböck G (2002) Transmission of olfactory information between three populations of neurons in the antennal lobe of the fly. Neuron 36: 463-474.

Ochieng' SA, Anderson P and Hansson BS (1995) Antennal lobe projection patterns of olfactory receptor neurons involved in sex pheromone detection in Spodoptera littoralis (Lepidoptera: Noctuidae). Tissue Cell 27: 221-232.

Olsen SR and Wilson RI (2008) Lateral presynaptic inhibition mediates gain control in an olfactory circuit. Nature 452: 956-960.

Olsen SR, Bhandawat V and Wilson RI (2007) Excitatory interactions between olfactory processing channels in the Drosophila antennal lobe. Neuron 54: 89-103.

Olshausen BA and Field DJ (2004) Sparse coding of sensory inputs. Curr Opin Neurobiol 14: 481-487.

Park SK, Shanbhag SR, Wang Q, Hasan G, Steinbrecht RA and Pikielny CW (2000) Expression patterns of two putative odorant-binding proteins in the olfactory organs of Drosophila melanogaster have different implications for their functions. Cell Tissue Res 300: 181-192.

Pelosi P (1998) Odorant-binding proteins: Structural aspects. Ann NY Acad Sci 30: 281-293.

Pickett JA and Woodcock CM (1996) The role of mosquito olfaction in oviposition site location and in the avoidance of unsuitable hosts. Ciba Found Symp 200, pp 109-119; discussion pp 119-123, pp 178-183.

Priesner E (1979) Progress in the analysis of pheromone receptor systems. Ann Zool Ecol Anim 11: 533-546.

Priesner E, Jacobson M and Bestmann HJ (1975) Structure-response relationships in noctuid sex pheromone reception. Z Naturf 30: 283-293.

Qiu YT, Smallegange RC, Van Loon JJ, Ter Braak CJ and Takken W (2006a) Interindividual variation in the attractiveness of human odours to the malaria mosquito Anopheles gambiae s. s. Med Vet Entomol 20: 280-287.

Qiu YT, Van Loon JJ, Takken W, Meijerink J and Smid HM (2006b) Olfactory coding in antennal neurons of the malaria mosquito, Anopheles gambiae. Chem Senses 31: 845-863.

Raming K, Krieger J, Breer H (1989) Molecular cloning of an insect pheromone-binding protein. FEBS Lett 256: 215-218.

Root CM, Semmelhack JL, Wong AM, Flores J, Wang JW (2007) Propagation of olfactory information in Drosophila. PNAS 104: 11826-11831. 
Rospars JP (1988) Structure and development of the insect antenno deutocerebral system. Int J Insect Morphol Embryol 17: 243-294.

Sachse S and Galizia CG (2002) Role of inhibition for temporal and spatial odor representation in olfactory output neurons: A calcium imaging study. J Neurophysiol 87: 1106-1117.

Sachse S and Galizia CG (2003) The coding of odour-intensity in the honey bee antennal lobe: local computation optimizes odour representation. Eur J Neurosci 18: 2119-2132.

Sakura M, Okada R and Mizunami M (2002) Olfactory discrimination of structurally similar alcohols by cockroaches. J Comp Physiol A 188: 787-797.

Rospars JP, Lánský P, Tuckwell HC and Vermeulen A (1996) Coding of odour intensity in a steady-state deterministic model of an olfactory receptor neuron. J Comput Neurosci 3: 51-72.

Sato K, Pellegrino M, Nakagawa T, Nakagawa T, Vosshall LB and Touhara K (2008) Insect olfactory receptors are heteromeric ligand-gated ion channels. Nature 24: 1002-1006.

Schachtner J, Schmidt M and Homberg U (2005) Organization and evolutionary trends of primary olfactory brain centres in Tetraconata (Crustacea + Hexapoda). Arthropod Struct Dev 34: 257-299.

Schmidt CW (2005) Outsmarting olfaction: the next generation of mosquito repellents. Environ Health Perspect 113: 468-471.

Schofield CJ and Moreman K (1976) Letter: Apparent absence of a sex attractant in adult Triatoma infestans (Klug), vector of Chagas' disease. Trans R Soc Trop Med Hyg 70: 165-166.

Schwaerzel M, Heisenberg M and Zars T (2002) Extinction antagonizes olfactory memory at the subcellular level. Neuron 35: 951-960.

Seki, Y, Aonuma H and Kanzaki R (2005). Pheromone processing center in the protocerebrum of Bombyx mori revealed by nitric oxide-induced anti-cGMP immunocytochemistry. J Comp Neurol 481: 340-351.

Sengul MS (2008) Two odorant-binding protein genes in mosquitoes: Comparative genomics, expression and function. Dissertation, Virginia Polytechnic Institute and State University, USA.

Sengul MS and Tu Z (2008) Characterization and expression of the odorant-binding protein 7 gene in Anopheles stephensi and comparative analysis among five mosquito species. Insect Mol Biol 17: 631-645.

Shang Y, Claridge-Chang A, Sjulson L, Pypaert M and Miesenböck G (2007) Excitatory local circuits and their implications for olfactory processing in the fly antennal lobe. Cell 128:601-612.

Shields VDC and Hildebrand JG (2001) Recent advances in insect olfaction, specifically regarding the morphology and sensory physiology of antennal sensilla of the female sphinx moth Manduca sexta. Micros Res Techniq 55: 307-329.

Siju, KP, Hill SR, Hansson BS and Ignell R (2010) Influence of blood meal on the responsiveness of olfactory receptor neurons in antennal sensilla trichodea of the yellow fever mosquito, Aedes aegypti. Journal of Insect Physiology 56: 659-665

Skiri HT, Galizia CG and Mustaparta H (2004) Representation of primary plant odorants in the antennal lobe of the moth Heliothis virescens using calcium imaging. Chem Senses 29: 253-267.

Steinbrecht RA, Ozaki M and Ziegelberger G (1992) Immunocytochemical localization of pheromone-binding protein in moth antenna. Cell Tissue Res 270: 287-302.

Steinbrecht RA, Laue MR and Ziegelberger G (1995) Immunolocalization of pheromone-binding protein and general odorant-binding protein in olfactory sensilla of the silkmoths Antheraea and Bombyx. Cell Tissue Res 282: 203-217.

Stensmyr MC, Dekker T and Hansson BS (2003) Evolution of the olfactory code in the Drosophila melanogaster subgroup. Proc R Soc Lond B Biol Sci 270: 2333-2340.

Strausfeld NJ, Hansen L, Li Y, Gomez RS and Ito K (1998) Evolution, discovery, and interpretations of arthropod mushroom bodies. Learning Memory 5: 11-37.

Sutcliffe JF (1994) Sensory bases of attractancy: morphology of mosquito olfactory sensilla - a review. J Am Mosq Control Assoc 10: 309-315.

Syed Z and Leal WS (2007) Maxillary palps are broad spectrum odorant detectors in Culex quinquefasciatus. Chem Senses 32: 727-738.

Syed Z, Ishida Y, Taylor K, Kimbrell DA and Leal WS (2007) Pheromone reception in fruit flies expressing a moth's odorant receptor. PNAS 103: 16538-16543. 
Todd JL and Baker TC (1999) Function of peripheral olfactory organs. In: Hansson BS (ed) Insect olfaction. SpringerVerlag, Berlin, Germany, pp 67-96.

Touhara K (2007) Deorphanizing vertebrate olfactory receptors: recent advances in odorant-response assays. Neurochem Int 51: 132-139.

Tsodyks MV and Feigelman MV (1988) The enhanced storage capacity in neural networks with low activity level. Europhys Lett 6: 101-105.

Turner GC, Bazhenov M and Laurent G (2008) Olfactory representations by Drosophila mushroom body neurons. J Neurophysiol 99: 734-746.

Urban NN (2002) Lateral inhibition in the olfactory bulb and in olfaction. Physiology and Behavior 77: 607-612.

Vergassola ME. Villermaux and Shraiman BI (2007), Infotaxis' as a strategy for searching without gradients. Nature 445: 406-409.

Vickers NJ (2006) Winging it: moth flight behavior and responses of olfactory neurons are shaped by pheromone plume dynamics. Chem Senses 31: 155-166.

Vickers NJ and Baker TC (1994) Reiterative responses to single strands of odor promote sustained upwind flight and odor source location by moths. PNAS 91: 5756-5760.

Vitta AC, Mota TR, Diotaiuti L and Lorenzo MG (2007) The use of aggregation signals by Triatoma brasiliensis (Heteroptera: Reduviidae). Acta Tropica 101: 147-152.

Vogt RG and Riddiford LM (1981) Pheromone binding and inactivation by moth antennae. Nature 293: 161-163.

Vogt RG and Lerner MR (1989) Two groups of odorant binding proteins in insects suggest specific and general olfactory pathways. Neurosci Abstr 15: 1290.

Vogt RG, Köhne AC, Dubnau JT and Prestwich GD (1989) Expression of pheromone binding proteins during antennal development in the gypsy moth Lymantria dispar. J Neurosci 9: 3332-3346.

Vogt RG, Prestwich GD and Lerner MR (1991a) Odorant-binding-protein subfamilies associate with distinct classes of olfactory receptor neurons in insects. J Neurobiol 22: 74-84.

Vogt RG, Rybczynski R and Lerner MR (1991b) Molecular cloning and sequencing of general odorant-binding proteins GOBP1 and GOBP2 from tobacco hawk moth Manduca sexta: comparisons with other insect OBPs and their signal peptides. J Neurosci 11: 2972-2984.

Vosshall LB and Stocker RF (2007) Molecular architecture of smell and taste in Drosophila. Annu Rev Neurosci 30: 505-533.

Vosshall LB, Amrein H, Morozov PS, Rzhetsky A and Axel R (1999) A spatial map of olfactory receptor expression in the Drosophila antenna. Cell 96: 725-736.

Vosshall LB, Wong AM and Axel R (2000). An olfactory sensory map in the fly brain. Cell 102: 147-159.

Waldrop B, Christensen TA and Hildebrand JG (1987) GABA-mediated synaptic inhibition of projection neurons in the antennal lobes of the sphinx moth, Manduca sexta. J Comp Physiol A 161:23-32.

Wang JW, Wong AM, Flores J, Vosshall LB and Axel R (2003) Two-photon calcium imaging reveals an odor-evoked map of activity in the fly brain. Cell 112: 271-282.

Wang Y, Mamiya A, Chiang AS and Zhong Y (2008) Imaging of an early memory trace in the Drosophila mushroom body. J Neurosci 28: 4368-4376.

Wicher D, SchÃufer R, Bauernfeind R, Stensmyr MC, Heller R, Heinemann SH and Hansson BS (2008) Drosophila odorant receptors are both ligand-gated and cyclic-nucleotide-activated cation channels. Nature 24: 1007-1011.

Van Wijk M, Wadman WJ, Sabelis MW (2006) Morphology of the olfactory system in the predatory mite Phytoseiulus persimilis. Exp Appl Acarol 40: 217-229.

Willis MA and Arbas EA (1991) Odor-modulated upwind flight of the sphinx moth, Manduca sexta L. J Comp Physiol A 169: 427-440.

Willshaw D and Longuet-Higgins HC (1970) Associative memory model. In: Meltzer B, Michie O (eds) Machine intelligence Vol 5. University of Edinburgh Press, Edinburgh, UK, pp 351-359.

Wilson RI and Laurent G (2005) Role of GABAergic inhibition in shaping odor-evoked spatiotemporal patterns in the Drosophila antennal lobe. J Neurosci 25: 9069-9079.

Wilson RI, Turner GC and Laurent G (2004) Transformation of olfactory representations in the Drosophila antennal lobe. Science 303: 366-370. 
Wogulis M, Morgan T, Ishida Y, Leal WS and Wilson DK (2006) The crystal structure of an odorant binding protein from Anopheles gambiae: Evidence for a common ligand release mechanism. Biochem Biophysics Res Commun 339: 157-164.

Wojtasek H, Hansson BS and Leal WS (1998) Attracted or repelled? A matter of two neurons, one pheromone binding protein, and a chiral centre. Biochem Biophys Res Commun 250: 217-222.

Wong AM, Wang JW and Axel R (2002). Spatial representation of the glomerular map in the Drosophila protocerebrum. Cell 109: 229-241.

Xia Y and Zwiebel LJ (2006) Identification and characterization of an odorant receptor from the West Nile virus mosquito, Culex quinquefasciatus. Insect Biochem Mol Biol 36: 169-176.

Xu PX, Zwiebel LJ and Smith DP (2003) Identification of a distinct family of genes encoding atypical odorant-binding proteins in the malaria vector mosquito, Anopheles gambiae. Insect Mol Biol 12: 549-560.

Xu P, Atkinson R, Jones DN and Smith DP (2005) Drosophila OBP LUSH is required for activity of pheromone-sensitive neurons. Neuron 45: 193-200.

Yu D, Akalal DB and Davis RL (2006) Drosophila alpha/beta mushroom body neurons form a branch-specific, long-term cellular memory trace after spaced olfactory conditioning. Neuron 52: 845-855.

Zhou JJ, He XL, Pickett JA and Field LM (2008) Identification of odorant-binding proteins of the yellow fever mosquito Aedes aegypti: genome annotation and comparative analyses. Insect Mol Biol 17: 147-163. 


\title{
5. The detection of carbon dioxide and its role in the orientation to hosts by haematophagous insects
}

\author{
Alan J. Grant and Robert J. O'Connell
}

\begin{abstract}
Host orientation behaviours of mosquitoes and other blood-seeking insects are controlled to a large degree by signals released by the host, including heat, moisture and sound, as well as visual and olfactory cues. Clearly olfactory signals play a primary role in mediating such behaviours. Although other olfactory signals such as lactic acid, 1-octen-3-ol and ammonia have been implicated in orientation, perhaps the most important of these volatiles is carbon dioxide $\left(\mathrm{CO}_{2}\right)$. Carbon dioxide is a by-product of cellular respiration that is released in large amounts by potential hosts and has been associated with mosquito behaviour since the early 1920s. In this report we focus on aspects of carbon dioxide detection including the morphological and physiological characteristics of sensory structures located on the maxillary palps which contain receptor neurons responsive to $\mathrm{CO}_{2}$. The relevant characteristics of these receptor neurons include the threshold and slope of the concentration response functions, the temporal pattern of the discharge, the effects of different background $\mathrm{CO}_{2}$ concentrations and the physiological condition of the insect. Among these conditions are age, diapause status and whether the species is autogenous or anautogenous. The physiological characteristics of $\mathrm{CO}_{2}$ detection in female mosquitoes are contrasted with similar characteristic in male mosquitoes as well as in other insects such as biting midges (Ceratopogonidae). The recent advances involving molecular research to further our understanding of the cellular processes of $\mathrm{CO}_{2}$ detection and the role of deterrents and repellants on such processes are discussed. Finally, the uses of $\mathrm{CO}_{2}$ as a tool in the design of control strategies including the development of traps for monitoring and potential control are discussed.
\end{abstract}

Keywords: carbon dioxide, mosquitoes, biting midges, behaviour, sensory physiology, blood feeding

\section{Introduction}

Each year, there are approximately three hundred million new cases involving the major diseases transmitted by mosquitoes. One and a half million fatalities can be attributed to these diseases or their complications each year (Breman 2001, Mayor 2008). For decades, efforts have been made to better understand the mechanisms that underlie host attraction, feeding and mating of haematophagous insects (Friend and Smith 1977, Galun 1977). In particular, many have attempted to describe and synthesise the complex of signals which control host-seeking behaviour in mosquitoes (Davis and Bowen 1994, Daykin et al. 1965, Takken 1991, Zwiebel and Takken 2004). A number of factors including: moisture, heat, (Clements 1963, 1999); host volatiles such as: lactic acid (Acree, Jr. et al. 1968, Smith et al. 1969), carbon dioxide $\left(\mathrm{CO}_{2}\right)$ (Gillies 1980, Mayer and James 1969) and other compounds (Kline et al. 1990, 1991) appear to be important stimuli for mosquito orientation.

Many biting insects, including anautogenous mosquitoes, require a blood meal before oogenesis. For example, a female Aedes aegypti (L.) mates soon after emergence and sometime later she blood feeds. Within several days of blood feeding, she lays her eggs. The blood serves as a primary 
source of energy for egg development. To acquire this blood meal, the female mosquito must locate a host for feeding. One of the sensory cues involved in this host-seeking behaviour is $\mathrm{CO}_{2}$; a primary byproduct of cellular respiration, which is released in large quantities by all potential hosts. Carbon dioxide had been implicated in mosquito orientation as early as the 1920s (Rudolfs 1922, Crumb 1922) and continues to be seen as one of the important chemical signals utilised by mosquitoes and other haematophagous insects in their host-locating and feeding behaviours (Bowen 1991, Galun 1977, Gillies 1980). To serve that end, female mosquitoes, as well as many other biting insects, prove to be equipped with an array of sensors that are highly sensitive and specifically tuned to respond to behaviourally relevant doses of $\mathrm{CO}_{2}$ (Grant and Kline 2003, Grant and O'Connell 1996a, Grant et al. 1995, Kellogg 1970). Carbon dioxide-sensitive receptor neurons have been identified and studied morphologically in mosquitoes (Mclver 1972b), and their physiological properties (Grant et al. 1995, Grant and O'Connell 1996b) have been explored in several other arthropods, including Lepidoptera (Bogner 1990, Bogner et al. 1986), Hymenoptera (Lacher 1964, Stange and Diesendorf 1973), other Diptera (Bogner 1992, Grant and Kline 2003) and Arachnida (Steullet and Guerin 1992). It is clear from all these studies that attraction to hosts is a complex behaviour that can be modified by a large number of environmental effectors many of which may be unique to a particular species (Davis 1996).

Since the early extirpation experiments of Roth and others (Jones and Madhukar 1976, Omer and Gillies 1971, Roth 1951), it has been clear that chemoreceptor neurons in sensilla located on the maxillary palps together with those on the antenna, play an important role in the detection and processing of the chemical stimuli implicated in initiating and modulating host location and feeding behaviours in insects (Bowen 1991, Gillies 1980, Kline et al. 1991, Takken and Kline 1989). Early physiological studies of the peripheral sensory system in mosquitoes had focused largely on the responses of olfactory receptor neurons in antennal sensilla. The antenna does contain a highly sensitive L-lactic acid receptor neuron, whose activity can be modulated by the behavioural repellent N,N-diethyl-m-toluamide (DEET) (Davis 1977, 1985, Davis and Sokolove 1976) The response characteristics of other antennal sensilla will be discussed by other author's in this volume and will not be addressed further in this review. A single early report (Kellogg 1970) described electrophysiological responses to $\mathrm{CO}_{2}$ in neurons housed in sensilla basiconica on the maxillary palp of female mosquitoes. We now know that one of the three sensory neurons normally found in sensilla basiconica responds to small increments in $\mathrm{CO}_{2}$ concentration and is sensitive to concentrations that are appropriate for orientation behaviour (Grant et al. 1995). In addition to the $\mathrm{CO}_{2}$-sensitive neuron, the maxillary palp sensillum is innervated by two other spontaneously active receptor neurons. The neuron producing the smallest amplitude action potential is sensitive to stimulation with low concentrations of 1-octen-3-ol (octenol) (Grant and O'Connell 1996b), another mosquito attractant (Takken and Kline 1989). The neuron producing the intermediate-sized action potential is sensitive to yet another compound (unpublished data) and all three of these cells have axons that project directly to the brain. These three cells are developmentally related to each other so it is possible that they all could share similar functional purposes guiding host-locating behaviours.

\section{Morphology of the maxillary palp and sensilla}

Like many female mosquitoes, Ae. aegypti have specialised sensilla on the ventral lateral surface of the fourth subsegment from the base of each maxillary palp (Mclver 1972a, 1982). This overall morphology is shared by many species of mosquitoes. For illustrative purposes we show in Figure $1 \mathrm{~A}-\mathrm{B}$ scanning electron micrographs (SEMs) from a female Culex pipiens Linnaeus. Male mosquitoes also possess morphologically similar sensilla that are distributed along the distal half of the third 

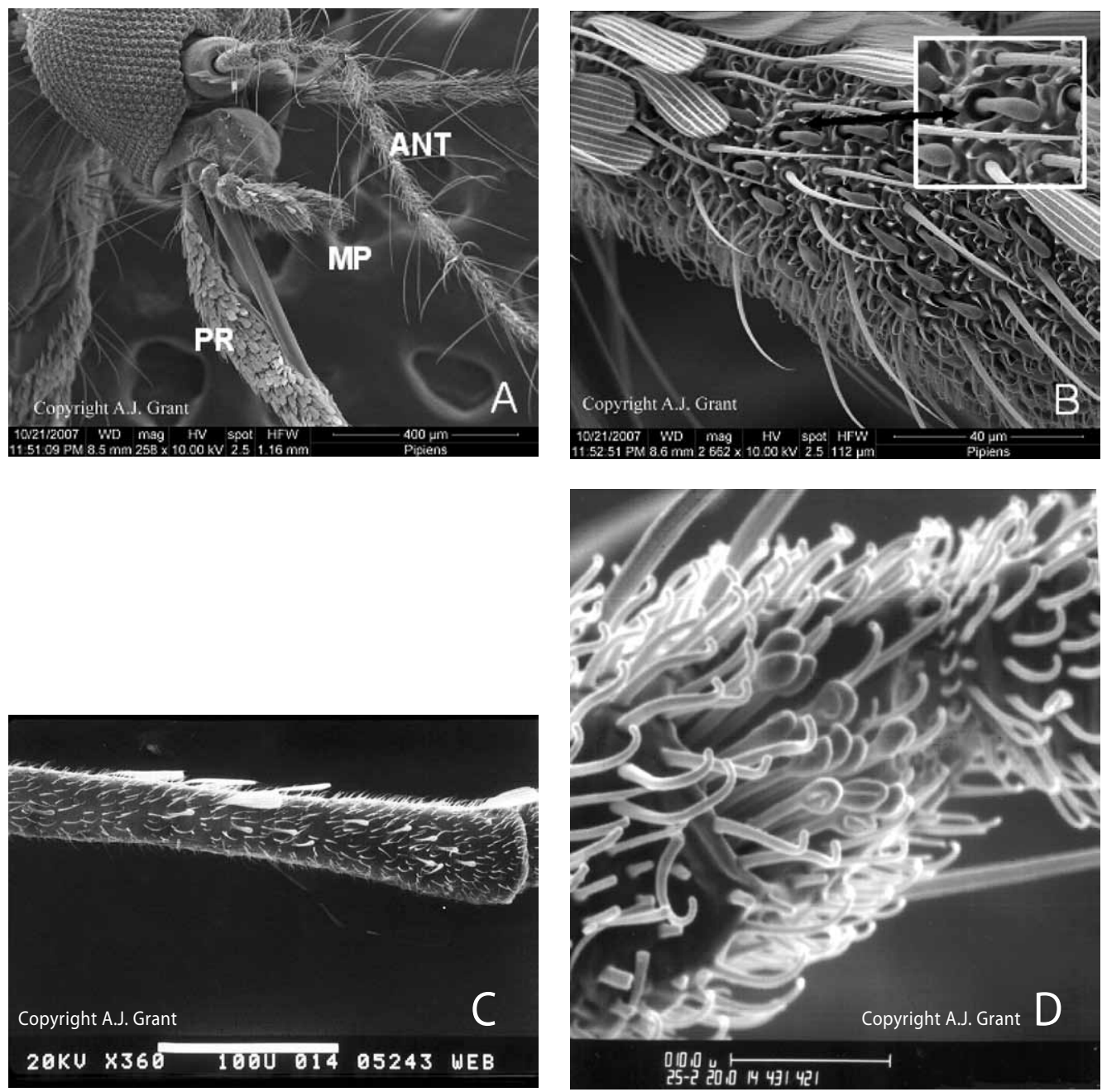

Figure 1. Scanning electron micrographs (SEMs) of maxillary palps of mosquito species $(A-C)$ and biting midges (D). (A) Portion of the head of a 21-22 day old female Cx. pipiens showing the maxillary palps (MP), antennae $(A N T)$, and proboscis (PR). (B) Fourth subsegment of a maxillary palp of a 21-22 day old female Cx. pipiens showing individual sensilla. Inset: individual sensillum containing a neuron sensitive to $\mathrm{CO}_{2}$. (C) Portion of the maxillary palp of a male Ae. aegypti depicting the basiconic sensilla containing neurons sensitive to $\mathrm{CO}_{2}$. (D) Portion of the maxillary palp of a female C. furens (Poey) showing a cluster of $\mathrm{CO}_{2}$-sensitive basiconic sensilla situated in an enlarged depression on the distal portion of the third subsegment from the base.

subsegment (Figure 1C) (Mclver 1972a, 1982). The presence of these sensilla is prevalent among mosquitoes, but their numbers and distribution varies from species to species (Braverman and Hulley 1979). Female Ae. aegypti possess approximately 25 sensilla on each palp. The sensilla basiconica in both Ae. aegypti and Cx. quinquefasciatus Say are restricted to the fourth subsegment from the head, whereas Anopheles stephensi Liston have sensilla basiconica distributed along the terminal three subsegments of the palp. It has been suggested that differences in the number 
and distribution of particular chemoreceptors on the antennae and maxillary palps of various species of mosquito are related to differences in the insect's relative preferences for particular hosts (Braverman and Hulley 1979) or in their landing and probing responses to them. In addition to the variation in distribution of sensilla, the overall length of the maxillary palps varies. The palps of An. stephensi are significantly longer (about 1,450 $\mu \mathrm{m}$ ) than the palps of either Ae. aegypti (about $350 \mu \mathrm{m}$ ) or $C x$. quinquefasciatus (about $300 \mu \mathrm{m}$ ). Although there is considerable variation in the external morphology of the palps and the distribution of sensilla along them the physiological characteristics of the neurons that innervate them show strong similarities (Figure 2); particularly with regards to the response of the neuron producing the largest amplitude action potential (up to $300 \mu \mathrm{V}$ peak to peak), which is reliably sensitive to stimulation with $\mathrm{CO}_{2}$ (Grant et al. 1995, Kellogg 1970). However, our recent unpublished studies suggest that several Anopheline species have reduced sensitivity. Lu et al. reported that An. gambiae Giles showed an elevated threshold and a diminished dose response functions (Lu et al. 2007).

The presence of these $\mathrm{CO}_{2}$-sensitive sensilla is often correlated with blood feeding behaviour. In other arthropods, sensilla with similar morphological features are found to contain neurons which also respond to $\mathrm{CO}_{2}$. For example, biting midges possess similar sensilla with neurons that respond to $\mathrm{CO}_{2}$. Although the Phantom Midge (Family Choaboridae) is taxonomically related to the mosquito, it had long been thought to be a non-biting insect. Subsequent investigations revealed the presence of blood in the digestive tract and an anatomical study revealed that these animals have mouthparts appropriate for biting and that they also possess basiconic sensilla on the palps which are morphologically similar to those examined here (McKeever and Pound 1979). To date, this correlation only holds for females of the species.

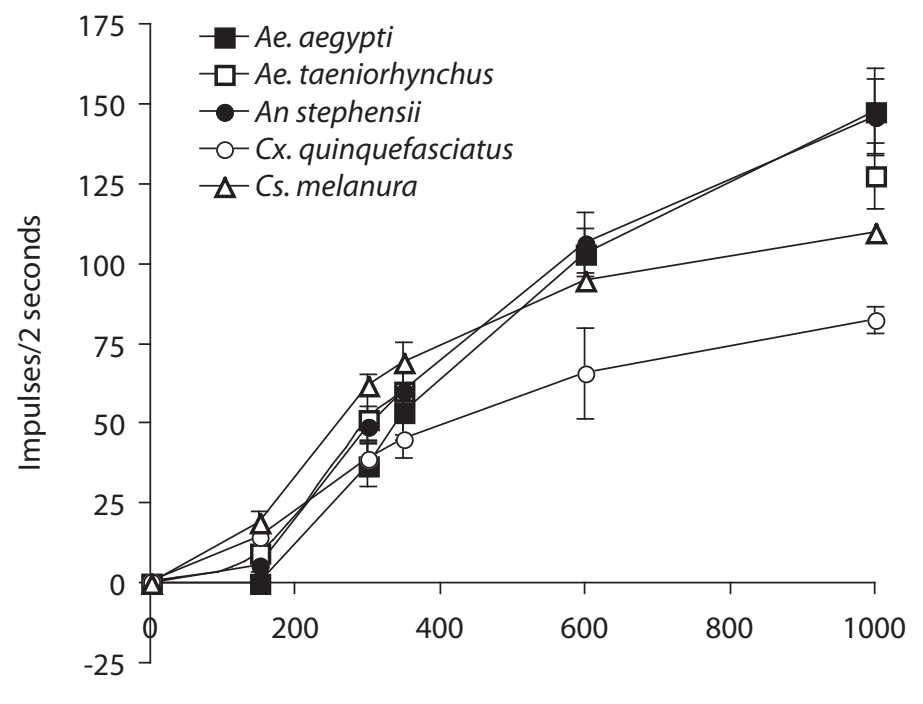

Carbon dioxide (PPM)

Figure 2. Mean \pm SE number of action potentials during $2 \mathrm{~s}$ stimulus pulses of the indicated concentrations of $\mathrm{CO}_{2}$ from s. basiconica on the maxillary palps of individuals from five different species of female mosquito (Ae. aegypti, An. stephensi, Cx. quinquefasciatus, Cs. melanura (Coquillett), and Ae. taeniorhynchus (Wiedemann)). All of these functions were established in a background environment containing 0 ppm $\mathrm{CO}_{2}$. 


\section{Methodology in electrophysiological studies}

Standard electrophysiological techniques can be used to record extracellular responses from the receptor neurons located in the sensilla found on insects. For $\mathrm{CO}_{2}$-sensitive neurons in biting insects such as mosquitoes and biting midges, details of the recording methodology have been described fully in previous reports (Grant and O'Connell 1986, O'Connell 1972) and are summarised below. To insure stable recordings, the insect must be carefully immobilised with the target structure positioned in a manner that allows unobstructed access of the recording microelectrode to the selected sensillum. Whole adults are secured on a glass plate with minute strips of sticky tape or fibre threads to secure the thorax, abdomen, legs and wings. Once the insect is secured, the preparation is positioned under the objectives of a compound light microscope with an effective magnification of approximately $750 \mathrm{X}$.

The recording microelectrodes are hand-made from 125- $\mu \mathrm{m}$ diameter straightened tungsten wire. They are electrolytically sharpened to a tip diameter less than $1 \mu \mathrm{m}$ in a $10 \%$ electrolyte solution (Galbreath and Galbreath 1977, Hubel 1957). An indifferent electrode of similar design is inserted into the eye of the insect and the recording electrode is inserted through the cuticle at the base of an individual sensillum. The microelectrodes are mounted in micromanipulators for positioning. Following placement of the recording electrode in the fluid space of the sensillum the electrical signals obtained from the neurons within the sensillum are band-passed filtered, amplified and sent in parallel to an audio monitor and a computer for subsequent data acquisition, action potential discrimination, analysis and storage. The basic work station, including the microscope, manipulators, preparation stage and amplifier are all mounted on a vibration isolation table in a Faraday cage. The electrical circuits required for this apparatus are isolated and independently earthed through a dedicated ground.

Data acquisition and analysis are accomplished using a software programme Autospike, developed by the late Dr. Jan N.C. van der Pers (www.syntech.nl/s). This programme is similar in principle to one used earlier (O'Connell et al. 1973) and is designed to record and analyse electrophysiological information from insect chemosensilla. This programme runs on a Windows platform with an analog-to-digital/digital-to-analog (AD/DA) interface via an IDAC controller. Discrimination and sorting of action potentials arising from the several neurons usually found in a single sensillum are easily accomplished with Autospike. Electrophysiological preparations from mosquitoes and other insects typically last for several hours before there are detectable changes in their response properties.

Chemical stimulation of olfactory receptor neurons in insects is usually accomplished with two opposing gas streams directed towards the sensory structure (Borroni and O'Connell 1992). One of these is normally on and carries the desired background stream (550 $\mathrm{mls} / \mathrm{min})$. The second stream is normally off and carries the desired stimulus (440 $\mathrm{mls} / \mathrm{min}$ ) stream. An independent controller activates the valves controlling these two streams and also initiates data collection by the software. To reliably manipulate $\mathrm{CO}_{2}$ stimulus concentration the flow dilution olfactometer design (O'Connell and Mozell 1969) normally used in olfactory studies should not be used. In this basic design a stock concentration of $\mathrm{CO}_{2}$ is diluted to the desired stimulus concentration by adding diluent flow from a pure air stream and adjusting the ratio of flow in the two gas streams to produce the final desired concentration. Although the bulk concentration of $\mathrm{CO}_{2} \mathrm{Can}$ be adjusted in this fashion, its use reveals a considerable variation in neuronal response. This appears to be due to inconsistent mixing within the stimulus stream. As we shall discuss later, $\mathrm{CO}_{2}$ receptor neurons appear to have an enhanced ability to follow rapid changes in concentration 
especially when compared to the more tonic pheromone receptor neurons normally found in insect antennae (Borroni and O'Connell 1992). Because of this factor, reliable $\mathrm{CO}_{2}$ stimuli should be delivered without dilution from certified formulated gas cylinders, each containing a calibrated concentration of $\mathrm{CO}_{2}$. For our studies we chose $0,150,300,350,600$ and $1000 \mathrm{ppm} \mathrm{CO}_{2}$, each containing $20 \%$ purified oxygen, and the remainder made up with purified nitrogen. Between $\mathrm{CO}_{2}$ stimulations, the preparation is bathed in a stream of carbon dioxide-free synthetic air $(0 \mathrm{ppm}$ $\mathrm{CO}_{2}$ ). For each preparation $2 \mathrm{~s}$ pulses of $\mathrm{CO}_{2}$ are sequentially presented at each of the certified concentrations. The interval between individual stimuli is normally 10-20 s. Following a brief rest period of approximately one to five minutes the whole protocol is repeated. Reproducibility of the response to a particular stimulus concentration is very high and individual responses at a particular concentration rarely differ from each other by more than a few impulses. Three to five repetitions of this protocol are made for each preparation to form the average. T-tests are then performed for the grand average response across all preparations at each dose to determine if there are any statistical differences between treatments.

\section{Temporal pattern of response}

The maxillary palp sensilla of both mosquitoes and biting midges (Diptera: Ceratopogonidae) contain neurons that are responsive to stimulation with low concentrations of $\mathrm{CO}_{2}$. Stimulation of these neurons with pulses of $\mathrm{CO}_{2}$ elicits typical biphasic action potentials with peak-to peak amplitudes of several $100 \mu \mathrm{Vs}$ (Figure 3). This neural signal is sent to the insect brain where

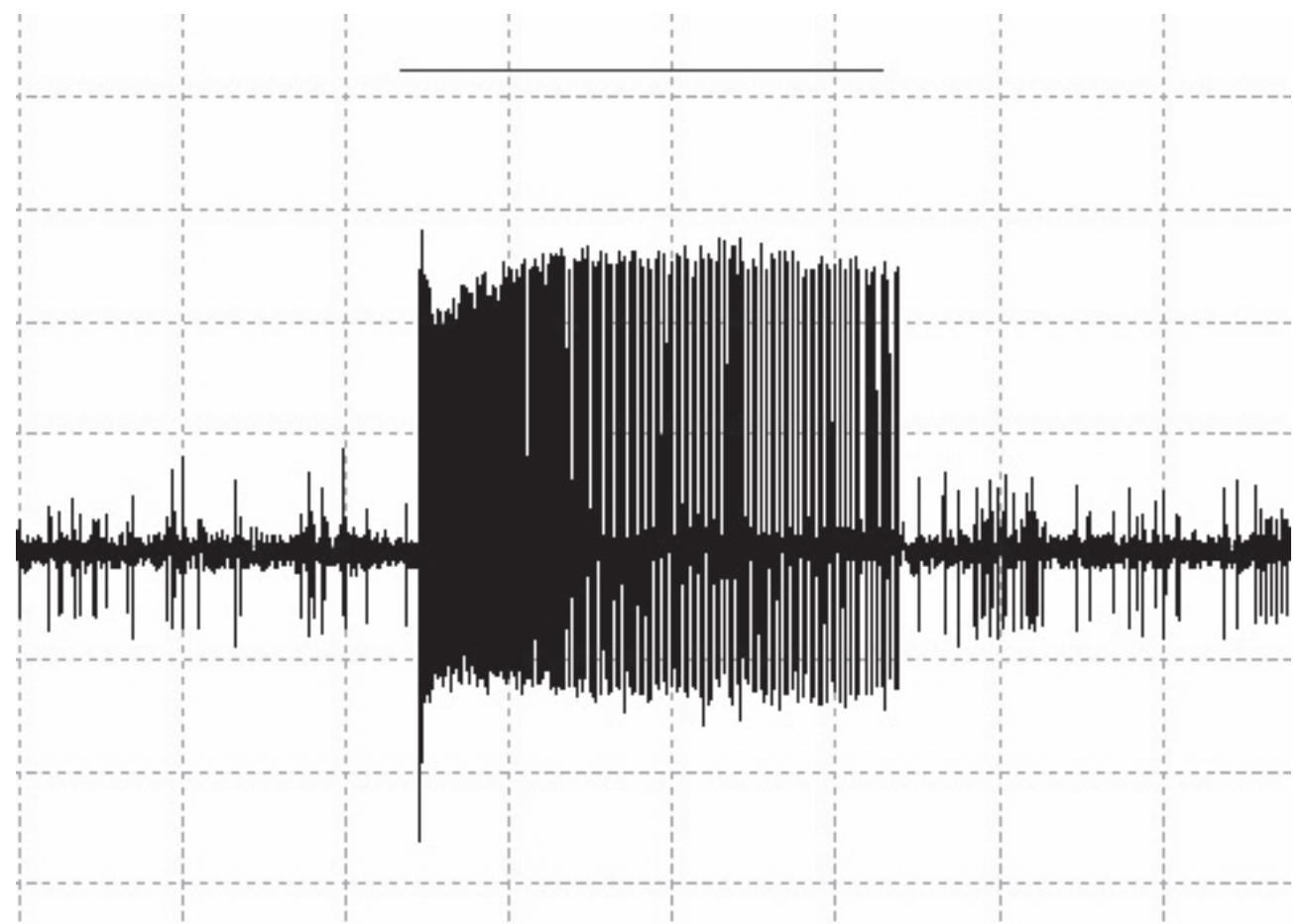

Figure 3. Typical electrophysiological response from a female Cx. pipiens in response to a 2 s stimulation of 600 ppm $\mathrm{CO}_{2}$ in a background of 0 ppm $\mathrm{CO}_{2}$. 
environmental $\mathrm{CO}_{2}$ information is processed. Processing in the central nervous system probably is dependent not only on the presence or absence of impulse activity in particular afferents, but also on the pattern of action potentials generated in them during the odour stimulus. Generally these neurons do not produce nerve impulses in environments lacking $\mathrm{CO}_{2}$. To evaluate the temporal pattern of activity for the $\mathrm{CO}_{2}$-sensitive neurons in the maxillary palp sensilla, we can evaluate the instantaneous frequency of action potentials generated by these neurons during the stimulus pulse (Figure 4). Both mosquito and biting midge $\mathrm{CO}_{2}$-sensitive cells respond with a sharp 'phasic' burst of activity, lasting 100-200 ms, followed by a lower tonic level of response whose duration and magnitude is dependent on stimulus concentration and the length of the stimulus pulse. Step increases in stimulus concentration elicit phasic increases in activity in both mosquito and biting midge receptor neurons, step decreases in concentration elicit reciprocal phasic reductions in activity followed by a slower increase in activity as the response returns to a level proportional to the new background concentration of $\mathrm{CO}_{2}$. This latter pattern can only be observed when steps in stimulus concentration are given from a background level of $\mathrm{CO}_{2}$. This pattern of discharge is widely believed to provide a sharpening mechanism (Dionne 1992) that allows the nervous system to detect the small changes in concentration that likely signals the presence of potential hosts (Grant and O'Connell 1996a).

The difference in the temporal pattern of discharge may also reflect intrinsic differences in the way in which the respective chemical signals in the environment are processed. The response properties of $\mathrm{CO}_{2}$-sensitive receptor neurons to step changes in concentration suggest the presence of at least two different mechanisms. On one hand, there appears to be a tonic response mechanism that simply reads out a response rate proportional to the steady state concentration of $\mathrm{CO}_{2}$ with relatively little desensitisation over time. On the other hand, there is also a rate-sensitive mechanism whose output rapidly follows both the direction and the rate of change of $\mathrm{CO}_{2}$ concentration. As is the case with cold receptor neurons in vertebrates (Darian-Smith et al. 1973) and invertebrates

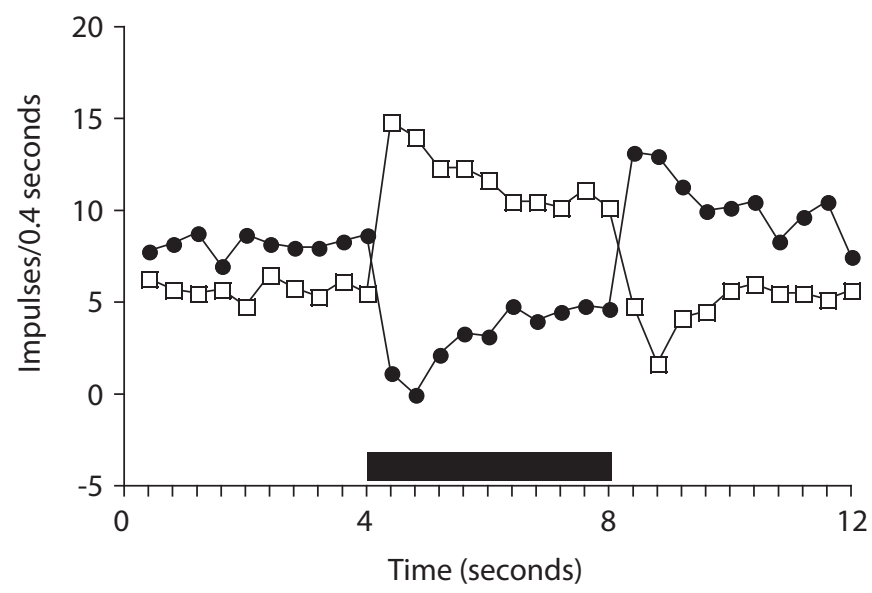

Figure 4. The temporal pattern of response from a $\mathrm{CO}_{2}$-sensitive receptor neuron in a female Ae. aegypti. The open symbols represent the average response to a $4 \mathrm{~s}$ stimulus pulse stepping up to $350 \mathrm{ppm} \mathrm{CO}_{2}$ from a background concentration of $300 \mathrm{ppm}$. The solid symbols represent the average response to a $4 \mathrm{~s}$ stimulus pulse stepping down to $300 \mathrm{ppm}$ from a level of $350 \mathrm{ppm} \mathrm{CO}$. In both cases the number of action potentials was averaged in $400 \mathrm{~ms}$ time bins and represents the mean from a single sensillum stimulated 10 times with each pulse protocol. 
(Altner and Loftus 1985) these phasic properties provide a sharpening mechanism that greatly exaggerates the response output of the receptor neuron as stimulus intensity changes rapidly. For example, the average tonic levels of activity elicited by $\mathrm{CO}_{2}$ backgrounds of 300 and $350 \mathrm{ppm}$ differ from each other by only about 6 impulses/s (Figure 4). However, a rapid step from 300 to 350 and back to $300 \mathrm{ppm}$ elicits peak phasic responses that differ from each other by approximately $38 \mathrm{impulses} / \mathrm{s}$. Although we do not yet know the 'following' capabilities of the $\mathrm{CO}_{2}$ receptor, the ability of the neuron to produce phasic bursts whose magnitude and sign are a function of the magnitude and sign of the change in $\mathrm{CO}_{2}$ concentration should have the effect of enhancing the sensory signal elicited and transmitted to the central nervous system by rapid changes in concentration. It is likely that this ability of the system to follow rapid changes in concentration accounts for the difficulty associated with standard dilution techniques of stimulation referenced earlier.

Depending on the nature of the central processing associated with these temporal inputs, the ability of the sensory system to follow rapid changes in $\mathrm{CO}_{2}$ concentration might be enhanced by these characteristics. This pattern of response would also be particularly appropriate if mosquitoes oriented to hosts by following the rapidly changing increments in $\mathrm{CO}_{2}$ concentration that are likely to be encountered in nature (Elkinton et al. 1984), as opposed to simply flying upwind toward a source in a steadily increasing gradient as might be imagined from a point source release of an odorant. It should be clear then that receptor neurons in insect olfactory sense organs can vary in both their chemical sensitivity and in their temporal characteristics (see Chapter 4, this volume).

\section{Responses in different background concentrations of $\mathrm{CO}_{2}$}

For ease of comparison, most electrophysiological tests reported here were to step increases in $\mathrm{CO}_{2}$ concentration from a background environment lacking $\mathrm{CO}_{2}\left(0 \mathrm{ppm} \mathrm{CO}_{2}\right)$. The resulting response magnitude fits a log function dependent simply on $\mathrm{CO}_{2}$ concentration. However, biting insects in nature never encounter environments without $\mathrm{CO}_{2}$. Therefore, when we tested the same set of $\mathrm{CO}_{2}$ concentrations applied from different background levels of $\mathrm{CO}_{2}$ (Figure 5), we obtain some useful additional information about the sensory neuron. Here too, we see the same basic pattern of response to stimulation in different background concentrations of $\mathrm{CO}_{2}$ in mosquitoes and biting midges. The average numbers of impulses generated in different background concentrations during a $2 \mathrm{~s}$ stimulus containing a given $\mathrm{CO}_{2}$ concentration are similar or slightly less as long as the background concentration is lower than the stimulus concentration. However, if the background concentration is higher than the stimulus concentration, the average number of impulses generated to the stimulus step is greatly diminished. For example, in backgrounds containing 0,150 or $300 \mathrm{ppm} \mathrm{CO}$ the magnitude of responses elicited by a stimulus containing $600 \mathrm{ppm} \mathrm{CO}_{2}$, are comparable to each other. However, in a background concentration of $1000 \mathrm{ppm}$ $\mathrm{CO}_{2}$, the magnitude of response to a similar stimulus containing $600 \mathrm{ppm} \mathrm{CO}$ is greatly reduced.

One implication of this observation is straightforward. One can imagine that the reduced sensory signal may impede the animal's ability to find hosts in high backgrounds. Conversely, the animal is more likely to only find hosts that emit $\mathrm{CO}_{2}$ concentrations higher than the background. From this we can surmise that continued increases in the environmental concentrations of $\mathrm{CO}_{2}$ might result in large shifts in host-seeking behaviour. However, without additional information on the response of hosts to these changes it is impossible to predict how these shifts could alter host detection and subsequent disease transmission. 


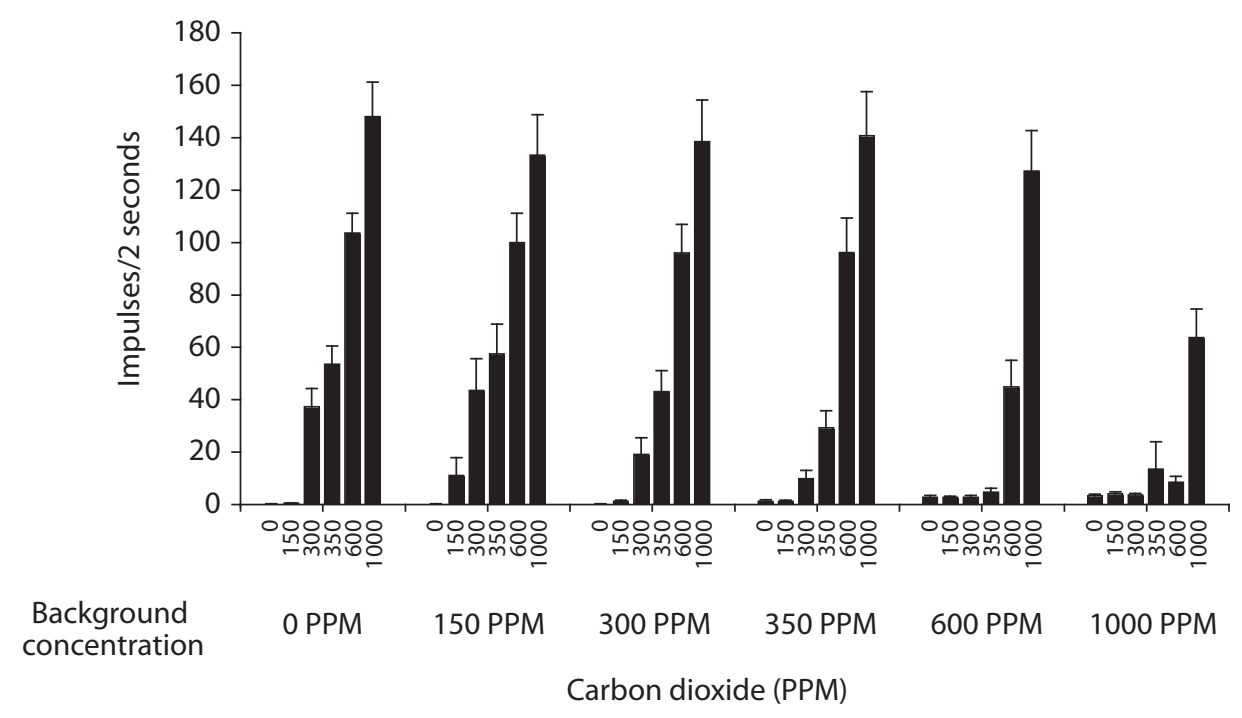

Figure 5. Mean + SE responses of $\mathrm{CO}_{2}$-sensitive receptor neurons in female Ae. aegypti. The stimuli consisted of 2 $s$ pulses of different concentrations of $\mathrm{CO}_{2}$ delivered from each of the indicated background levels of $\mathrm{CO}_{2}$.

\section{Detection of $\mathrm{CO}_{2}$ by male mosquitoes}

Male mosquitoes possess morphologically similar sensilla to those structures found on the maxillary palps of females (Mclver 1972a, 1980). They prove to contain $\mathrm{CO}_{2}$-sensitive receptor neurons as well (Grant et al. 1995). The spatial distribution of these sensilla basiconica on the male palp is not restricted to the fourth sub-segment from the head, as in the female of many species. Instead, the distribution includes the distal half of the third subsegment from the head (Figure 1C). Neurons that innervate these male sensilla have thresholds and sensitivities to $\mathrm{CO}_{2}$ that are similar to those in females (Figure 6, Grant and O'Connell 2007).

The apparent ability of males to detect $\mathrm{CO}_{2}$ raises questions about the potential function of this chemical stimulus in their biology. Male mosquitoes are not known to blood feed, as their mouthparts are not adapted for piercing (Matheson 1945). But males do feed on plant nectars. Perhaps their ability to detect $\mathrm{CO}_{2}$ is utilised in their search for these food sources. Depending on when during the day they are active this input may be useful in detecting either sources or sinks of $\mathrm{CO}_{2}$ involved with the photosynthetic activity of plants. Alternatively, male Ae. aegypti have been observed near human hosts where mating occurs as the females seek a blood meal (Hartberg 1971). This suggests that males of some species may use $\mathrm{CO}_{2}$ as a secondary cue to orient to places where females are likely to be more abundant and available for mating.

\section{Effect of age on $\mathrm{CO}_{2}$ responses}

Following emergence, female mosquitoes typically perform a series of behaviours which includes mating, feeding and oviposition. For an anautogenous mosquito such as Ae. aegypti, a blood meal is required to obtain the protein needed for egg production. To acquire this blood, the female engages in periods of host-seeking. Host seeking is governed by sensory cues associated 


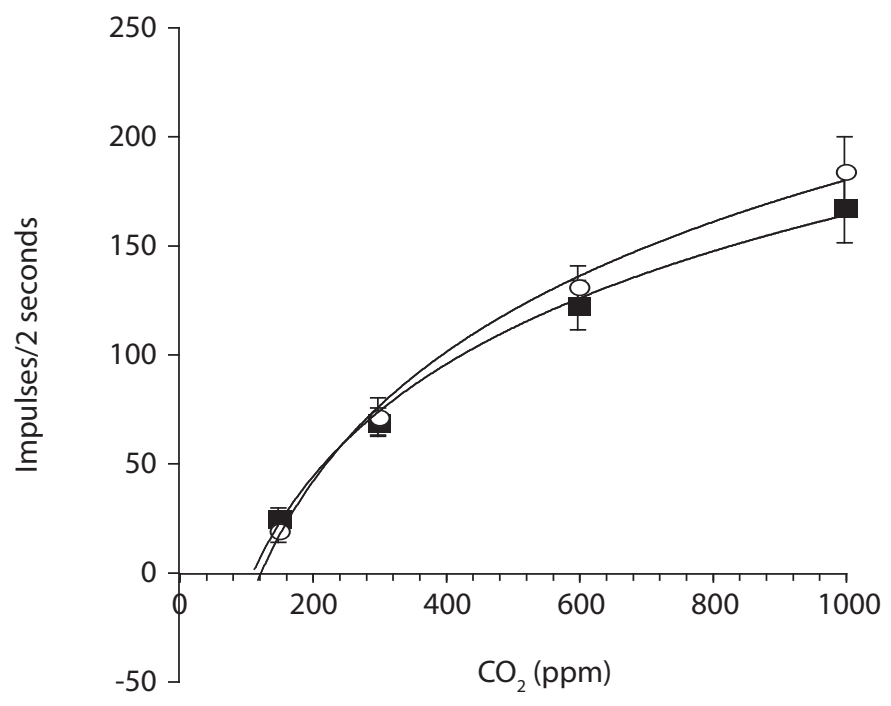

Figure 6. Mean $\pm S E$ concentration-response function from male (open circles; $n=20$ ) and female (solid square; $n=30)$. $\mathrm{CO}_{2}$-sensitive receptor neurons in female Ae. aegypti in response to a $2 \mathrm{~s}$ stimulation of the indicated concentration of $\mathrm{CO}_{2}$. Stimulation with the control concentration of $0 \mathrm{ppm} \mathrm{CO}_{2}$ elicited no response in any mosquito. Male responses were not statistically different from female responses at any concentration tested.

with host-produced odours (Takken 1991) including $\mathrm{CO}_{2}$. Mating generally occurs within the first few days following emergence (Christopher 1960, Clements 1999). Blood feeding is not required for copulation to occur. Numerous behavioural reports suggest that for most anautogenous mosquitoes relatively little feeding activity occurs during the first few days of adult life and hostseeking and feeding activities increase dramatically from then to $96 \mathrm{~h}$ after emergence (Christopher 1960, Davis 1984a, Seaton and Lumsden 1941). This enhanced host-seeking level is maintained for several weeks. Since host-seeking behaviour changes as the insect ages, it is interesting to evaluate the dynamic sensitivity of the $\mathrm{CO}_{2}$ receptor neurons across this timeframe. To that end, we recorded electrophysiological responses from sensory neurons to stimulation with various concentrations of $\mathrm{CO}_{2}$ in newly emerged adults ( $0-2 \mathrm{~d}$ old $)$ when host orientation is reduced, and in older insects (4-7 d old) when host orientation reaches a maximum. Female Ae. aegypti show pronounced differences in the sensitivity of their $\mathrm{CO}_{2}$ receptor neurons across these young and older age classes (Figure 7, Grant and O'Connell 2007). Young females have a significantly lower overall response rate to stimulation with 300,600 and 1000 ppm $\mathrm{CO}_{2}$ when compared with the same stimuli in older females. Recording from male Ae. aegypti did not show a comparable age dependent difference for any of the $\mathrm{CO}_{2}$ concentrations tested (Grant and O'Connell 2007).This difference in sensitivity as a function of age was also observed for female $C x$. pipiens (unpublished results). Similar differences in the rates of development of sensory systems have been studied electrophysiologically and reported in other insects as well (Blaney et al. 1986, Crnjar et al. 1990, Davis 1984b, Schweitzer et al. 1976).

In addition to the variation in overall sensitivity between young and older female mosquitoes, the temporal pattern of discharge to a continuous stimulus $\left(1000 \mathrm{ppm} \mathrm{CO}_{2}\right)$ also differed between the two ages (Grant and O'Connell 2007). As we have argued before (Grant and O'Connell 1996b) 


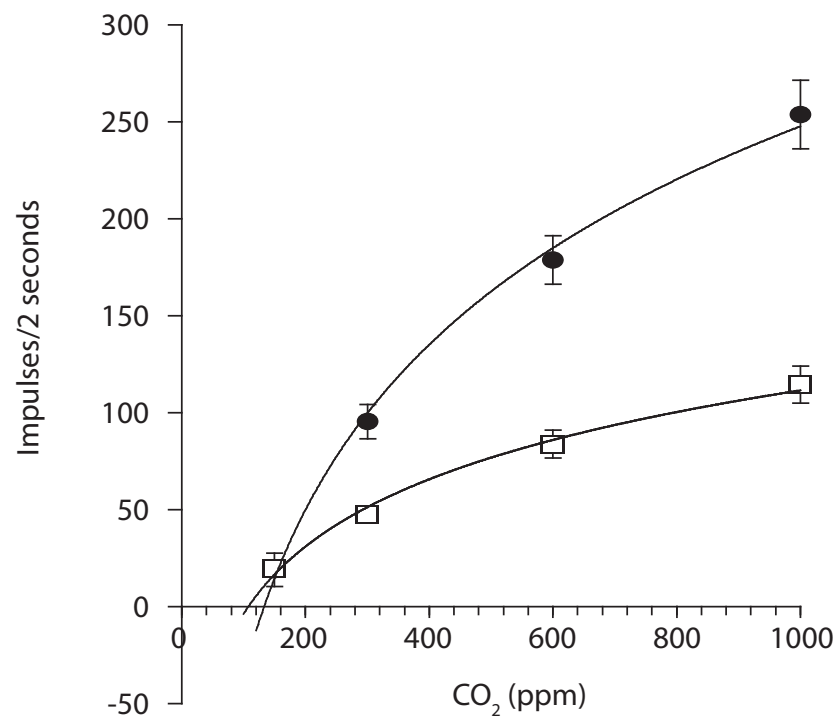

Figure 7. Mean $\pm S E$ concentration-response functions from young (open square; $n=15$ ) and older (solid circles; $n=15) \mathrm{CO}_{2}$-sensitive receptor neurons in female Ae. aegypti in response to a 2 second stimulation of the indicated concentration of $\mathrm{CO}_{2}$. Stimulation with the control concentration of $0 \mathrm{ppm} \mathrm{CO}_{2}$ elicited no response in any mosquito. Responses were statistically different from each other at concentrations of 300,600 and $1000 \mathrm{ppm}$. The solid circles and open squares represent data points and the solid lines represent functions fitted to these points.

the temporal discharge pattern may enhance the neuron's ability to discriminate rapid changes in $\mathrm{CO}_{2}$ concentration that are presumed to occur in the downwind stimulus plume emanating from a potential host. Thus, enhancing the capability of the peripheral sensory system to detect $\mathrm{CO}_{2}$ changes as the female mosquito matures should enhance host-seeking in older females. On the other hand, these changes in sensitivity may be accidental and simply reflect the normal physiological maturation of the sensory system or the changes in the periphery may be timed by other more central processes in the mosquito which are designed to optimise the sensory capabilities of the system when it is required to mediate host-seeking behaviour. It is not possible to state the causal relationships between these host-related sensory capabilities and the development of host-seeking behaviours. However, one is tempted to speculate that the maturation we have demonstrated in the sensory system is responsible for the age-related shift in host-seeking behaviour.

\section{$\mathrm{CO}_{2}$ detection in biting midges}

In addition to mosquitoes, other biting insects detect $\mathrm{CO}_{2}$ including but not limited to Tabanidae, Simuliidae, some Muscidae and Ceratopogonidae. Among the Ceratopogonids or biting midges, Culicoides is the most important genus with respect to the health and comfort of humans and animals (Kettle 1965, Linley et al. 1983). Annoyance from adult female biting midges often may become serious enough to affect outdoor recreational and work-related activities. Their presence also has an adverse effect on tourism and land development, especially in coastal areas of the United States (Linley and Davies 1971) and in parts of Europe. Animal and human health 
are affected by disease organisms transmitted by adult female biting midges, which include protozoans, filarial worms and viruses (Blanton and Wirth 1979, Kettle 1965, Linley et al. 1983).

Sensilla basiconica on the maxillary palps of female C. furens (Poey), C. stellifer (Coquillett) and C. mississippiensis (Hoffman) all contain a receptor neuron which responds to stimulation with low levels of $\mathrm{CO}_{2}$ (Figure 1D). These neurons produce typical biphasic action potentials with peak-to-peak amplitudes of up to $350 \mu \mathrm{V}$. As in our mosquito work, we recorded responses to stimulation with $0,150,300,600$ and $1000 \mathrm{ppm} \mathrm{CO}_{2}$. The Culicoides $\mathrm{CO}_{2}$-sensitive neurons have basic physiological characteristics that are similar to those in the mosquito $\mathrm{CO}_{2}$-sensitive neurons (Grant and O'Connell 1996b, 2007, Grant et al. 1995). Both mosquito and biting midge receptor neurons respond with a phasic-tonic discharge pattern to increases or decreases in $\mathrm{CO}_{2}$ concentration. The neurons of both are silent in environments without $\mathrm{CO}_{2}$. However, there are several interesting differences between biting midges and mosquito response properties. First, in most cases, their response thresholds appear different. Stimulation with 150 ppm $\mathrm{CO}_{2}$ elicited a response in $C$. furens, which is statistically larger than the activity seen in response to stimulation with 0 ppm CO $\mathrm{CO}_{2}$ (Figure 8). Aedes aegypti, on the other hand, often require higher concentrations to elicit a significant increase in response (Grant et al. 1995). This indicates that the threshold of detection is lower for $C$. furens and as a consequence their neurons are more likely to be active at ambient levels. Second, the slopes of the concentration response functions are considerably different. The response function for Ae. aegypti is steeper than for Culicoides. This indicates that a small change in stimulus concentration will elicit a larger change in impulse activity in Aedes as

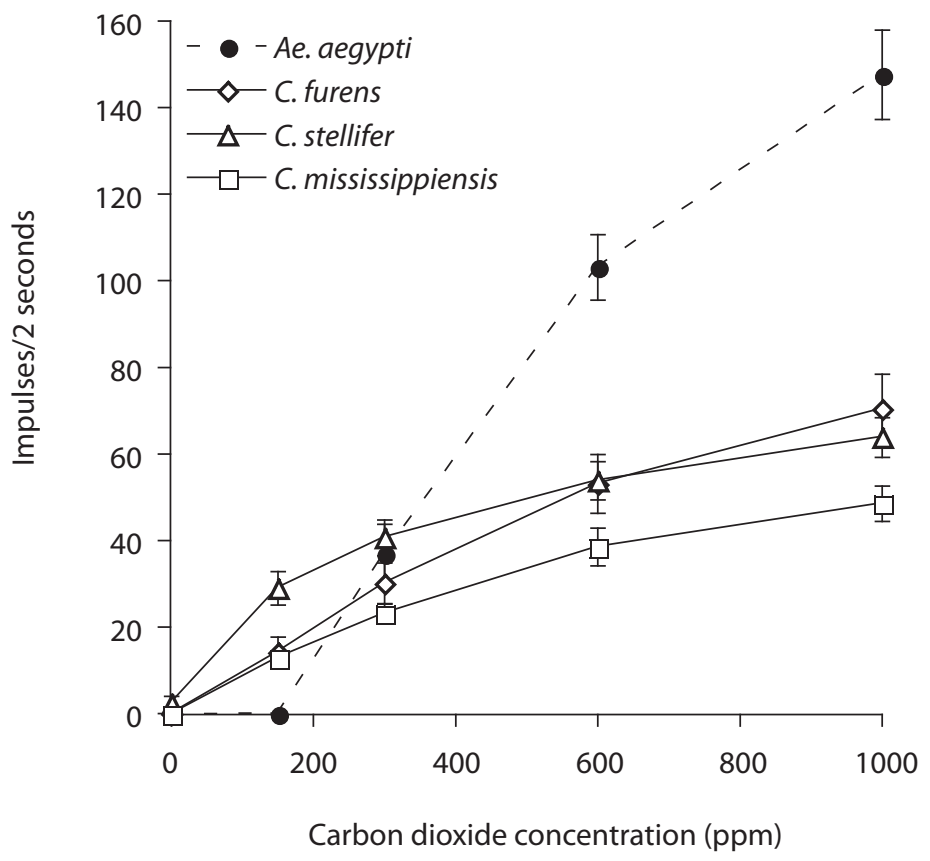

Figure 8. Concentration-response functions from three species of Culicoides [C. furens ( $n=15), C$. mississippiensis $(n=3)$ and $C$. stellifer (Coquillett) $(n=6)$ ] in response to graded stimulations of $\mathrm{CO}_{2}$. For comparison, also included is the concentration response function for Ae. aegypti ( $n=13)$ (Grant et al. 1995). 
compared to Culicoides suggesting that the mosquito would be able to detect a smaller change in $\mathrm{CO}_{2}$ concentration. Although the threshold and slope of the concentration functions were different, they do overlap with each other near ambient levels $\left(350 \mathrm{ppm} \mathrm{CO}_{2}\right)$.

The significance of a lower threshold of sensitivity to $\mathrm{CO}_{2}$ in Culicoides is unclear. Ambient atmospheric levels of $\mathrm{CO}_{2}$ are currently about $350 \mathrm{ppm}$. So, it seems unlikely that having the ability to detect lower $\mathrm{CO}_{2}$ concentrations than ambient would enhance the insect's host-seeking ability. Many nematoceran insects are autogenous and utilise sugar feeding for survival (Stewart and Kline 1999). The environment contains many sources and sinks for $\mathrm{CO}_{2}$. Actively photosynthesising plants may produce local reductions in the concentration of $\mathrm{CO}_{2}$. Thus, it is possible that these insects are using their enhanced $\mathrm{CO}_{2}$ sensitivity to find $\mathrm{CO}_{2}$ levels below ambient where plants that contain nectar for sugar feeding are located. Culicoides mississippiensis is known to sugar feed from the plant Yaupon Holly, llex vornitoria Aiton, during the day and possibly during the night (Stewart and Kline 1999). Therefore, it is possible that low concentrations of $\mathrm{CO}_{2}$ in conjunction with other volatiles may serve as a plant attractant for sugar feeding. In a similar manner, higher concentrations of $\mathrm{CO}_{2}$ may serve as a host-seeking attractant for blood feeding.

Sensitivity to low concentrations of $\mathrm{CO}_{2}$ also may have no behavioural significance. It has been suggested (Stange 1997, Stange and Wong 1993) that certain species of moths, which also have $\mathrm{CO}_{2}$-sensitive neurons, actually are adapted to lower, pre-industrialisation levels of $\mathrm{CO}_{2}$. Although our methods differed from those of Stange in characterising $\mathrm{CO}_{2}$ sensitivity, perhaps the sensitivity to lower concentrations of $\mathrm{CO}_{2}$ in Culicoides simply reflects an earlier sensitivity, which has not yet been genetically adapted to fit the dramatic increase caused by human-based emissions of $\mathrm{CO}_{2}$.

\section{Diapause and $\mathrm{CO}_{2}$ detection}

Culex pipiens is a vector of several medically important viruses in the United States including West Nile. Under environmental conditions of short day length and low ambient temperatures during late larval and early pupal stages, the mosquitoes are induced to enter diapause (Spielman and Wong 1973). Diapausing adults demonstrate a slower rate of development of primary ovarian follicles. In addition, host-seeking and feeding behaviours are reduced. Because of these differences we sought to determine if the $\mathrm{CO}_{2}$-sensitivity of palp receptor neurons was affected by diapausing status. To test this, we recorded responses from the $\mathrm{CO}_{2}$-sensitive peripheral sensory neurons in the maxillary palps of adult female mosquitoes to graded stimulation with $\mathrm{CO}_{2}$ in diapausing and non-diapausing populations. Non-diapausing $C x$. pipiens were reared from pools of egg rafts to adults in environmental chambers at $18{ }^{\circ} \mathrm{C}, 15: 9 \mathrm{~h}$ light-dark cycle, and $75 \% \mathrm{RH}$. To induce diapause, alternate groups selected from the same raft pools were reared with short days 7:17 h light-dark cycle. To confirm the effectiveness of our rearing protocols, primary ovarian follicle and germarium lengths were measured in a portion of each cohort to determine diapause status, and the stage of ovarian development was determined according to Christopher (1911).

Our electrophysiological data (Figure 9) indicate that the diapause state does not appear to affect the sensitivity of these sensory neurons. Diapausing and non-diapausing female $C x$. pipiens have similar electrophysiological responses to stimulation with $0,150,300,600$, and $1000 \mathrm{ppm} \mathrm{CO}_{2}$. These finding suggest that the reduction in host-seeking behaviour observed in diapausing $C_{X}$. pipiens is not determined by changes in the $\mathrm{CO}_{2}$ sensitivity of peripheral sensory neurons. We note that these data suggest that the changes in behaviour that occur with diapause must arise because of factors that affect some other stage of central nervous system processing (see Chapter 4 , this volume). 


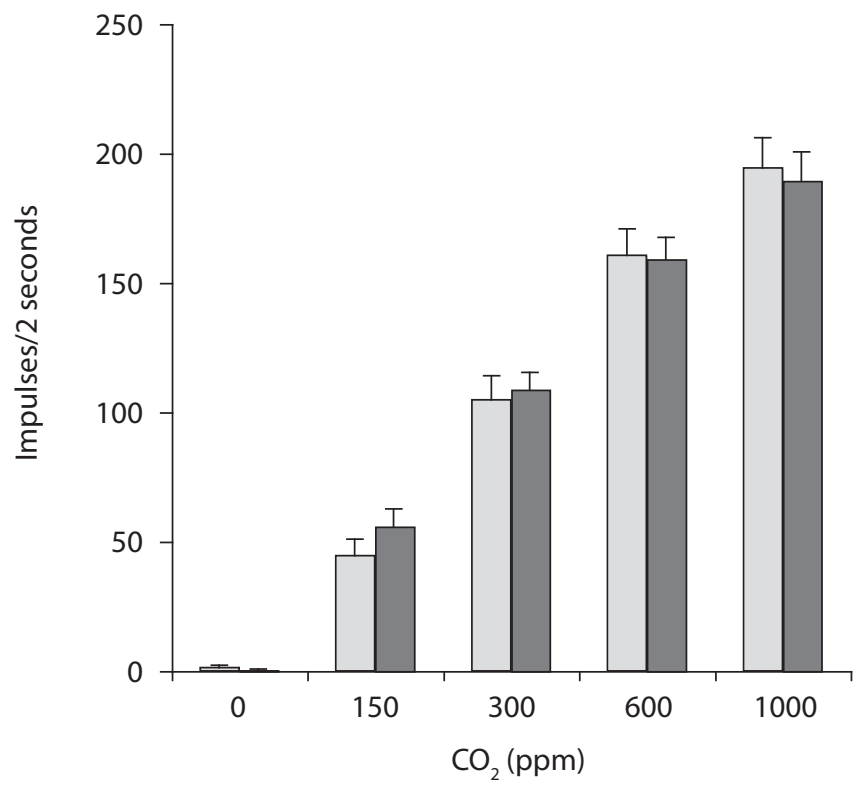

Figure 9. Mean + SE concentration-response from $\mathrm{CO}_{2}$-sensitive neurons on female $\mathrm{Cx}$. pipiens that were reared under temperature and light conditions to produce non-diapausing adults (light grey; $n=35$ ) and diapausing adults (dark grey; $n=26$ ). Those adults reared under diapausing condition were not statistically different from those reared under non-diapausing conditions at any concentrations tested.

\section{$\mathrm{CO}_{2}$ responses from receptor neurons in autogenous mosquitoes}

Culex pipiens forms a complex of mosquitoes whose taxonomic status is still unclear. Culex pipiens was first described by Linneaus in 1758 and Cx. molestus by Forskal in 1775 (Vinogradova 2000). Morphologically these two mosquitoes are very similar. Physical differences such as comparative measures of maxillary palp to proboscis length in males have been suggested as a potential diagnostic trait. However, such lengths appear quite variable from population to population, greatly reducing their predictive value. In our hands, this metric discriminated potential $C x$. molestus Forskal from CX. pipiens with approximately $85 \%$ accuracy. Although considered by some a distinct species or subspecies, many are inclined to consider $C x$. molestus as an 'intraspecific category, i.e. a variety, eco-physiological race or ecotype' (Vinogradova 2000). Whatever the taxonomic classification, $C x$. molestus are autogenous and are able to produce eggs without taking a blood meal, whereas $C x$. pipiens are anautogenous and require a blood meal for egg production. Both $C_{X}$. pipiens and $C_{X}$. molestus produce an egg raft, however, the size of the rafts differ considerably. Culex molestus (sugar-fed) rafts contained 78.9 $\pm 7.0(\mathrm{SE}, \mathrm{n}=13)$ eggs, whereas an average blood-fed $C x$. pipiens raft contained 190.7 $\pm 7.7(S E, n=3)$ eggs.

Since the life cycle of the autogenous $C x$. molestus does not require blood feeding, we were interested in characterising the $\mathrm{CO}_{2}$-sensitive receptor neurons in their palps and comparing their sensitivity with those found in Cx. pipiens. The females of both groups of mosquitoes possess maxillary palps with similar basiconic sensilla. Although no detailed study of fine structure of female maxillary palps has been done, $C x$. molestus and $C x$. pipiens palps and sensilla appear 
very similar. However, recordings from the $\mathrm{CO}_{2}$-sensitive neurons revealed some interesting differences in their overall sensitivity to $\mathrm{CO}_{2}$ stimulation. Culex molestus are significantly less sensitive as compared to $C x$. pipiens (Figure 10). Extrapolating from the dose-response curves; both $\mathrm{Cx}_{x}$. molestus and $\mathrm{Cx}$. pipiens have similar thresholds of slightly over $100 \mathrm{ppm} \mathrm{CO}_{2}$; however the slopes of the concentration response functions are different. These results are interesting because as was the case with the difference between young and older Ae. aegypti and Cx. pipiens, the group that shows reduced or no host-seeking behaviours possessed sensory neurons less sensitive to $\mathrm{CO}_{2}$. Culex molestus can be induced to blood feed and we are currently conducting experiments to determine if the nutrient source (sugar or blood) affects the receptor neurons of the resulting offspring. Preliminary data from $\mathrm{Cx}$. molestus suggest that aspects of the $\mathrm{CO}_{2}$ sensitivity is dependent on diet.

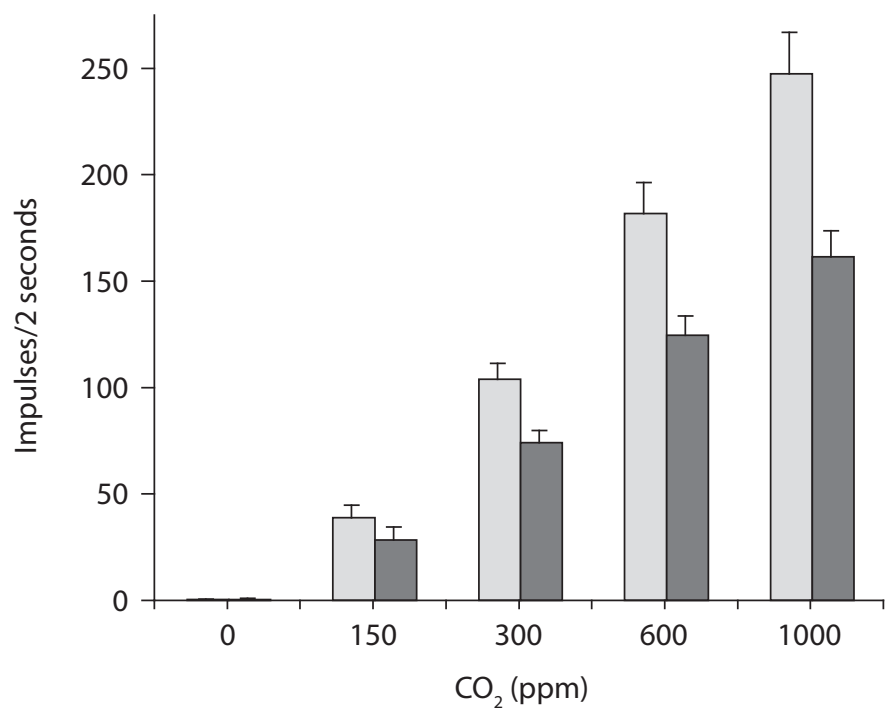

Figure 10. Mean + SE concentration-response relationships for female Cx. p. pipiens (light grey; $n=15)$ and Cx. p. molestus (dark grey; $n=20$ ). All recording were from insects 5-8 days post emergence. Values were statistically different at 150, 300, 600 and 1000 ppm $\mathrm{CO}_{2}$ (t-test, two tailed; $\mathrm{P}<0.01$ ).

\section{Potential effects of changes in atmospheric levels of $\mathrm{CO}_{2}$}

We are becoming increasingly convinced that our environment is changing (Gore 2006). Pressures associated with post-industrial development and methods of environmental management have led to large changes in the levels of chemicals in our environment. One of these chemicals is $\mathrm{CO}_{2}$, the ambient level of which has been increasing for several centuries. Pre-industrial revolution environmental levels of $\mathrm{CO}_{2}$ were estimated at or below $200 \mathrm{ppm}$. Current ambient atmospheric levels range between 330 and $350 \mathrm{ppm}$. Predictions of future levels have been as high as 500 ppm. Increasing levels of environmental $\mathrm{CO}_{2}$ as well as other greenhouse gasses are believed by some to contribute to an elevation in global temperatures. There are many consequences of global warming including sea level elevation and wide-spread regional climate change. Some have speculated that such environmental changes in temperature could lead to a shifting of 
habitat range for many species including mosquitoes (Eisen et al. 2008). Such a change in range could potentially affect the transmission of vector-borne diseases. Diseases that are prevalent in tropical climates might become more prevalent in temperate areas with significantly more public health impact.

Given that $\mathrm{CO}_{2}$ is one of the primary activators-attractants for biting insects and that many biting insects possess highly specialised sensory neurons to detect $\mathrm{CO}_{2}$, we suggest that alterations in $\mathrm{CO}_{2}$ levels alone could also have effects on host-seeking behaviour. We know that the threshold of response of these sensory neurons lies around 100-120 ppm $\mathrm{CO}_{2}$. Based on the response characteristics of these neurons, we question how potentially increasing environmental levels of $\mathrm{CO}_{2}$ could affect the ability of the sensory neurons to process $\mathrm{CO}_{2}$ changes and consequently how they will modulate orientation behaviour. We know that when these neurons are challenged with graded stimulation with increasing doses of $\mathrm{CO}_{2}$, the background $\mathrm{CO}_{2}$ concentration is important. In other words, a stimulus pulse of $1000 \mathrm{ppm} \mathrm{CO}$ in a background of $150 \mathrm{ppm} \mathrm{CO}_{2}$ elicits a significantly different response compared to a similar stimulus pulse of $1000 \mathrm{ppm} \mathrm{CO}_{2}$ from a background of $600 \mathrm{ppm} \mathrm{CO}$ (Figure 5). The signal received by the CNS is different. At present we do not know how this change in signal will be interpreted by the mosquito CNS and what behavioural consequences may result. In addition, little is understood about the effects of changes in long-term stimulation of these sensory neurons. The $\mathrm{CO}_{2}$-sensitive system seems to be rather plastic and it is likely that the overall consequences for behaviour will not be obvious. However, it is clear now that a more thorough understanding of the system would be useful.

\section{Molecular studies of $\mathrm{CO}_{2}$ detection}

With the advent of molecular methods of study there have been many exciting advances in our understanding of the cellular mechanism underlying olfactory detection in insects. Early and continuing studies have been conducted with the model systems Drosophila (Dobritsa et al. 2003, Hallem et al. 2006, Jones et al. 2007, Rutzler and Zwiebel 2005). With the sequencing of the Anopheles genome in 2002 (Holt et al. 2002) much work has focused on An. gambiae (Goldman et al. 2005, Hallem et al. 2004, Hill et al. 2002, Kwon et al. 2007, Lu et al. 2007). Recent published work has begun to unravel and identify the molecular nature of the receptor proteins involved in $\mathrm{CO}_{2}$ detection. Vosshall and her colleagues (Jones et al. 2007) report that receptor neurons in Drosophila co-express two receptor proteins necessary for $\mathrm{CO}_{2}$ detection. These proteins $\mathrm{Gr} 21$ and Gr63a have analogues in mosquitoes, GPRGR22 and GPRGR24. Both of these are expressed in olfactory neurons found in maxillary palp sensilla of An. gambiae (Jones et al. 2007). This work also indicates that the expression of these two proteins in $\mathrm{CO}_{2}$-insensitive cells confers sensitivity and the removal of Gr63a causes mutant flies to lose this sensitivity both at a behavioural and sensory level. Work from other labs has implicated a third receptor (AgGR23) whose addition clearly augments $\mathrm{CO}_{2}$ responsiveness (Lu et al. 2007). The roles of each of these proteins remains to be determined in the presumed cascade of events that leads to the detection and processing of this important environmental cue. It is tempting to speculate that these proteins will have differential effects on the sensitivity and overall rate of response to $\mathrm{CO}_{2}$.

Work by Ditzen et al. (2008) has utilised the molecular understanding of receptor protein systems to understand the mode of action of the repellent DEET (N,N-diethyl-meta-toluamide). They report that DEET strongly inhibits the 1-octen-3-ol response of An. gambiae and Drosophilia melanogaster Meigen by mediating a response in the 0 R $83 \mathrm{~b}$ receptor protein and the mosquito analogue GPROR7 receptor complex. However, using electrophysiological techniques the detection and sensitivity of the $\mathrm{CO}_{2}$ receptor neuron appears to be unaffected. These responses confirm that the 
addition of DEET strongly inhibited the octenol response but did not affect the $\mathrm{CO}_{2}$ sensitivity. In a similar fashion we also observed that DEET did not modulate the $\mathrm{CO}_{2}$ response in An. gambiae Giles (Figure 11). More recent work in $C x$. quinquefasciatus has suggested an alternative mechanism for DEET action on the octenol response. Syed and Leal (2008) have proposed that DEET acts through another channel by directly stimulating receptor neurons found in a specific antennal trichoid sensillum (Pickett et al. 2008, Syed and Leal 2008). Molecular understanding of receptor mechanisms will lead to further elucidation of the mode of action of attractants and repellents. Such understanding should enhance the development of semiochemicals and ultimately lead to the development of novel control strategies.

A

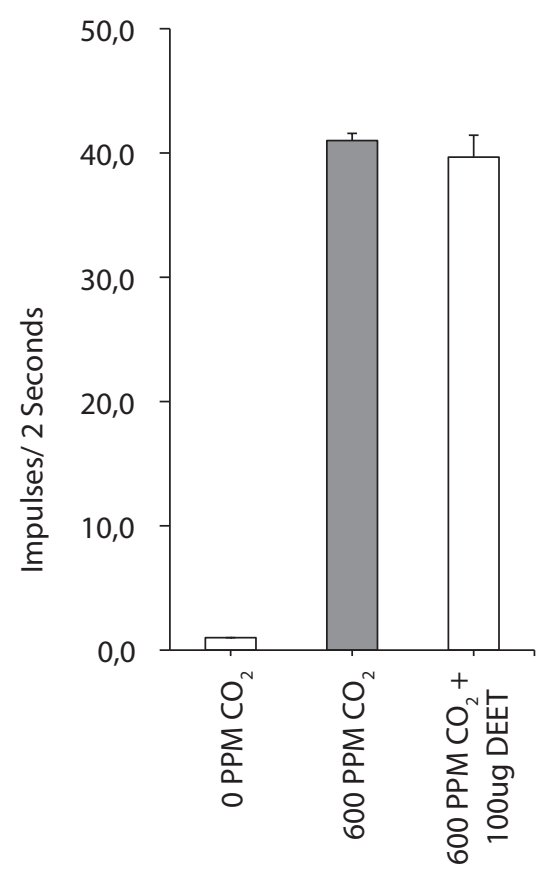

B $\quad 0$ ppm
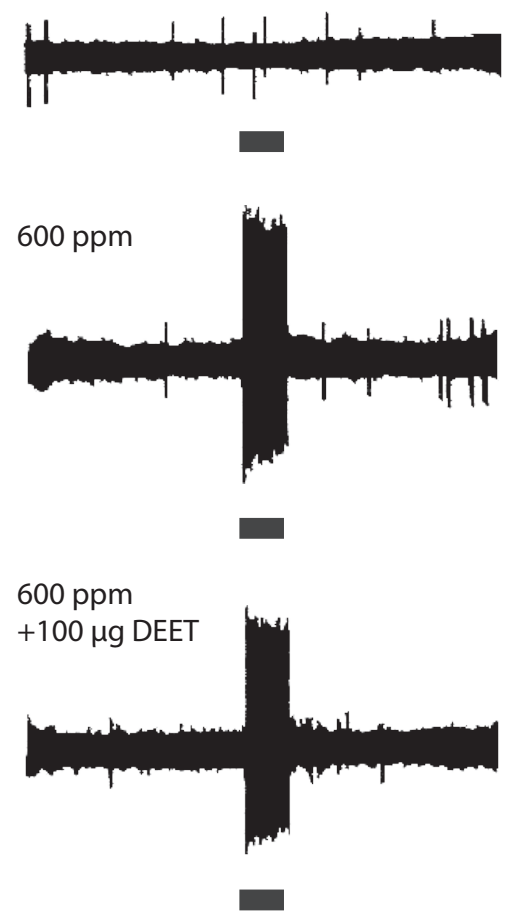

Figure 11. Electrophysiological responses from basiconic sensilla on the maxillary palps of female An. gambiae. (A) Mean $+\mathrm{SE}$ responses $(n=3)$ to $2 \mathrm{~s}$ stimulations of $0 \mathrm{ppm} \mathrm{CO}_{2}, 600 \mathrm{ppm} \mathrm{CO}$ and $600 \mathrm{ppm} \mathrm{CO}_{2}+100 \mu \mathrm{g}$ of DEET. Preparations were held in a background environment of $0 \mathrm{ppm} \mathrm{CO}_{2}$ (these data were presented at the 1998 Association for Chemoreception Sciences meetings).

\section{The use of $\mathrm{CO}_{2}$ in insect trapping}

Carbon dioxide has long been used as an attractant for female mosquitoes and other biting insects in trapping technology. For most, but not all species of biting insects, $\mathrm{CO}_{2}$ serves to selectively attract female biting insects in a concentration dependent manner (Figure 12A-B). For several species, a significant but smaller number of males are also attracted. Reports in the 1960s 
A

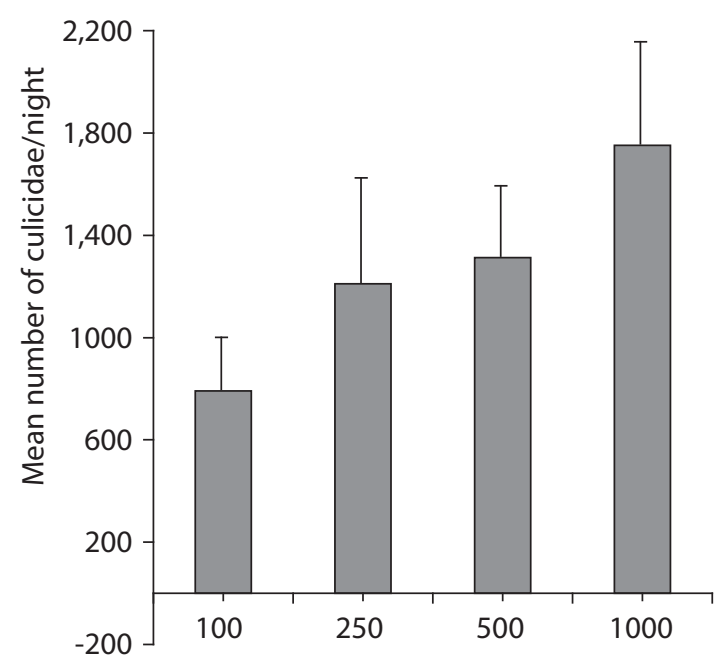

B

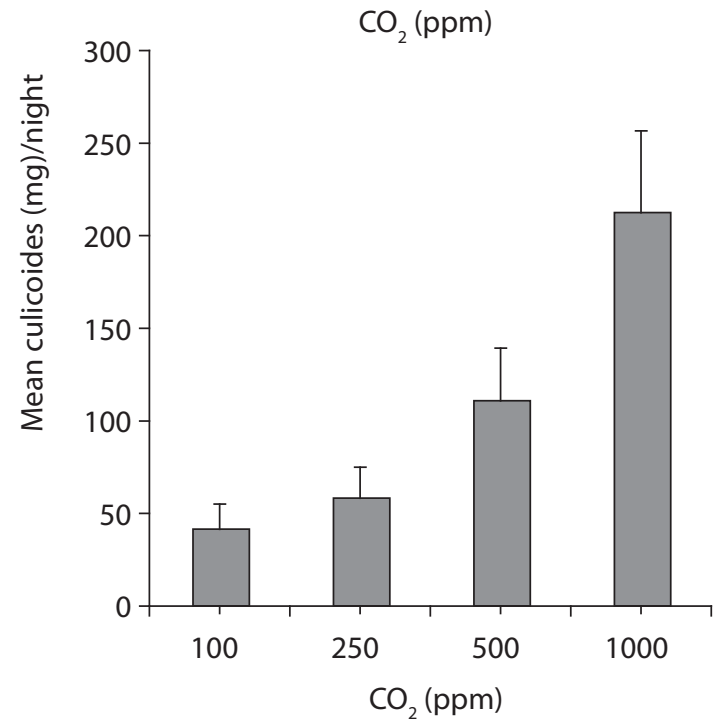

Figure 12. Mean + SE concentration-response relationship of trap catch of (A) mosquitoes and (B) biting midges as a function of $\mathrm{CO}_{2}$ release in field experiments conducted in Cedar Key, FL, USA. The trap was a passive counter-flow trap whose $\mathrm{CO}_{2}$ source was a compressed tank. Release concentration amounts were determined by restrictive line orifices and regulated pressure. Each point represents eight nights of trapping.

demonstrated the utility of adding $\mathrm{CO}_{2}$ to surveillance traps (Carestia and Savage 1967, Newhouse et al. 1966, Vickery, Jr. et al. 1966, Wright 1967). Often $\mathrm{CO}_{2}$ was added in the form of dry ice that sublimates to gas. In CDC style traps, blocks of dry ice are added above the traps in an insulated chamber with a port releasing $\mathrm{CO}_{2}$ into the trap stream. In some instances, a bag of dry ice could be simply hung in the vicinity of the trap attracting insects to the general area. Several pounds of dry ice are often sufficient to generate $\mathrm{CO}_{2}$ for 12-14 hours. Alternately, regulated cylinders 
containing compressed $\mathrm{CO}_{2}$ could be used to release the gas at a standard rate (often $500 \mathrm{mls} /$ minute). This rate could be adjusted to allow a standard 20 pound tank to last for days to weeks. This works well for surveillance system where trapping periods are relatively short. To further enhance catch, other attractants such as 1-octen-3-ol, lactic acid or ammonia are added. These supplemental attractants are often used to 'tailor' catches to specific problem species, such as anthropophilic day-biters.

In the 1990s, newer technology for commercial traps appeared on the market. They were designed to operate continuously for longer periods of time. These traps generated $\mathrm{CO}_{2}$ by catalytically combusting propane on a bed of coated beads. Heat generated from this burn process is converted to electricity, which is used to power fans that dispersed the $\mathrm{CO}_{2}$ attractant plume and vacuumed approaching insects into nets. An added advantage to this technology was the co-generation of heat and moisture, two addition attractants for many biting insects (Kline 2002). The advantages of such traps are several-fold. First, the trap is able to generate attractants in the form of $\mathrm{CO}_{2}$, heat and moisture and collect insects continuously for over one month on a standard 20-lb 'barbeque grill' sized propane tank. Second, by generating its own power, the trap was not reliant on battery or line voltage. Consequently this allowed traps to be placed at some distance from living areas. The rational is that proper placement of the trap at some distance from commonly used areas can result in biting insects being drawn away from areas where they would most likely encounter a host for a blood meal. Third, these traps tended to be very specific usually attracting few nontarget insects.

The release of $\mathrm{CO}_{2}$ from traps can be modulated in several ways to tune attractiveness. First, the overall concentration released can be modified. Generally larger amounts of $\mathrm{CO}_{2}$ will result in larger catches. Second, the strategy of release can affect catch. For example, counter flow release systems, where the plume dispensing the $\mathrm{CO}_{2}$ stream is countercurrent to the aspiration stream, enhances species diversity and numbers in the catch. Third, the temporal nature of the $\mathrm{CO}_{2}$ release can affect catch rate. Pulsing the attractant can be used to release discrete amounts of $\mathrm{CO}_{2}$ at specific intervals or times of day. This aspect of release is complicated by the fact that even a continuous release of $\mathrm{CO}_{2}$ is easily and quickly broken into pockets by a turbulent stream of wind. The importance of such discontinuous signals has been studied and discussed for field (Murlis and Jones 1981); bioassay (Dekker et al. 2001) and sensory (Grant et al. 1997) systems. In pulsing systems, several variables can be modulated including: concentration of $\mathrm{CO}_{2}$ release, duration of release and interval between pulses. All these must be evaluated to determine optimal release conditions. Clearly traps can serve as an important tool to monitor population fluctuations and to assay for potential health risks by collecting and testing a variety of hematophagous insects for potentially dangerous viruses and other pathogens.

\section{Conclusion}

In this brief review we summarised our current understanding of the properties and role of $\mathrm{CO}_{2}$ sensitive chemosensory systems in modulating host-seeking behaviours in mosquitoes. We see that these orientation behaviours are complicated and potentially plastic. They represent a dynamic system whose sensitivity may be modulated by environmental factors. Under what conditions does the system's sensitivity change? We have shown here that the age of the insect as well as the blood feeding tendencies affect $\mathrm{CO}_{2}$ sensitivity, but there are many other developmental and environmental conditions that may also modulate the response. For example, does the infected state of an individual mosquito change peripheral sensitivity? 
There are many steps in the chain of events that lead from the detection of an environmental cue to a change in animal behaviour. Each provides a potential point of attack where treatment may alter the behavioural outcome. A complete understanding of this chain of events in disease vector organisms can provide new approaches to mechanisms that may be effective in decreasing disease transmission. We suggest that a failure to appreciate this fact supports another cautionary tale with widespread impact.

\section{References}

Acree F, Jr., Turner RB, Gouck HK, Beroza M and Smith N (1968) L-lactic acid: a mosquito attractant isolated from humans. Science 161: 1346-1347.

Altner $\mathrm{H}$ and Loftus R (1985) Ultrastructure and function of insect thermo- and hygroreceptors. Annu Rev Entomol 30: 273-295.

Blaney WM, Schoonhoven LM and Simmonds MSJ (1986) Sensitivity variations in insect chemoreceptors; a review. Experientia 42: 13-19.

Blanton FS and Wirth WW (1979) The sand flies (Culicoides) of Florida (Diptera: Ceratopogonidae): Florida Department of Agriculture and Consumer Services, USA.

Bogner F (1990) Sensory physiological investigation of carbon dioxide receptors in Lepidoptera. J Insect Physiol 36: 951-957.

Bogner $\mathrm{F}$ (1992) Response properties of $\mathrm{CO}_{2}$-sensitive receptors in tsetse flies (Diptera: Glossina palpalis. Physiol Entomol 17: 19-24.

Bogner F, Boppré M, Ernst K-D and Boeckh J (1986) $\mathrm{CO}_{2}$ sensitive receptors on labial palps of Rhodogastria moths (Lepidoptera: Arctiidae): physiology, fine structure and central projections. J Comp Physiol [A] 158: 741-749.

Borroni PF and O'Connell RJ (1992) Temporal analysis of adaptation in moth (Trichoplusia ni) pheromone receptor neurons. J Comp Physiol [A] 170: 691-700.

Bowen MF (1991) The sensory physiology of host-seeking behavior in mosquitoes. Annu Rev Entomol 36: 139-158.

Braverman Y and Hulley PE (1979) The relationship between the numbers and distribution of some antennal and palpal sense organs and host preference in some Culicoides (Diptera: Ceratopogonidae) from southern Africa. J Med Entomol 15: 419-424.

Breman JG (2001) The ears of the hippopotamus: manifestations, determinants, and estimates of the malaria burden. Am J Trop Med Hyg 64 (1-2 Suppl): 1-11.

Carestia RR and Savage LB (1967) Effectiveness of carbon dioxide as a mosquito attractant in the CDC minature light trap. Mosquito News 27: 90-92.

Christopher SR (1911) The development of the egg follicle in anophelines. Paludism 2: 73-88.

Christopher SSR (1960) Aedes aegypti (L), the yellow fever mosquito: its life history, bionomics and structue. Cambridge University Press, Cambridge, UK.

Clements AN (1963) The physiology of mosquitoes. Pergamon Press, Oxford, UK.

Clements AN (1999) The biology of mosquitoes. Volume 2 Sensory reception and behaviour. CABI Publishing, Wallingford, UK.

Crnjar R,Yin C-M, Stoffolanojr.JG, Barbarossa IT, Liscia A and Angioy AM (1990) Influence of age on the electroantennogram response of the female blowfly (Phormia regina) (Diptera: Calliphoridae). J Insect Physiol 36: 917-921.

Crumb SE (1922) A mosquito attractant. Science 55(1426): 446-447.

Darian-Smith I, Johnson KO and Dykes R (1973) 'Cold' fiber population innervating palmar and digital skin of the monkey: responses to cooling pulses. J Neurophysiol 36: 325-346.

Davis EE (1977) Response of the antennal receptors of the male Aedes aegypti mosquito. J Insect Physiol 23 613-617.

Davis EE (1984a) Development of Lactic acid-receptor sensitivity and host-seeking behavior in newly emerged female Aedes aegypti mosquitoes. J Insect Physiol 30: 211-215.

Davis EE (1984b) Regulation of sensitivity in the peripheral chemoreceptor systems for host-seeking behaviour by a haemolymph-borne factor in Aedes aegypti. J Insect Physiol 30: 179-183. 
Davis EE (1985) Insect repellents: concepts of their mode of action relative to potential sensory mechanisms in mosquitoes (Diptera: Culicidae). J Med Entomol 22: 237-243.

Davis EE (1996) Olfactory control of mosquito behaviour. In: Bock GR and Cardew G (eds) Olfaction in mosquito-host interactions. John Wiley \& Sons, Chichester, UK, pp 48-53.

Davis EE and Bowen MF (1994) Sensory physiological basis for attraction in mosquitoes. J Mosquito Control Assoc 10: 316-325.

Davis EE and Sokolove PG (1976) Lactic acid-sensitive receptors on the antennae of the mosquito, Aedes aegypti. J Comp Physiol [A] 105: 43-54.

Daykin PN, Kellogg FE and Wright RH (1965) Host-finding and repulsion of Aedes aegypti. Can Ento 97: 239-263.

Dekker T, Takken W and Carde RT (2001) Structure of host-odour plumes influences catch of Anopheles gambiae s.s. and Aedes aegypti in a dual-choice olfactometer. Physio Entomology 26: 124-134.

Dionne VE (1992) Chemosensory responses in isolated olfactory receptor neurons from Necturus maculosus. J Gen. Physiol 99: 415-433.

Ditzen M, Pellegrino M and Vosshall LB (2008) Insect odorant receptors are molecular targets of the insect repellent DEET. Science 319(5871): 1838-1842.

Dobritsa AA, Van der Goes van Naters, Warr CG, Steinbrecht RA and Carlson JR (2003) Integrating the molecular and cellular basis of odor coding in the Drosophila antenna. Neuron 37: 827-841.

Eisen L, Bolling BG, Blair CD, Beaty BJ and Moore CG (2008) Mosquito species richness, composition, and abundance along habitat-climate-elevation gradients in the northern Colorado Front Range. J Med Entomol 45: 800-811.

Elkinton JS, Cardé RT and Mason CJ (1984) Evaluation of time-average dispersion models for estimating pheromone concentration in a deciduous forest. J Chem Ecol 10: 1081-1108.

Friend WG and Smith JJB (1977) Factors affecting feeding by bloodsucking insects. Ann Rev Ento 22: 309-331.

Galbreath RA and Galbreath NH (1977) A simple method for making fine dissecting needles. Ent News 88: 143-1447.

Galun R (1977) Responses of blood-sucking arthropods to vertebrate hosts. In: Shorey HH and McKelvey JrJJ (eds) Chemical control of insect behavior theory and application. John Wiley \& Sons, New York, USA, pp 103-115.

Gillies MT (1980) The role of carbon dioxide in host-finding by mosquitoes (Diptera: Culicidae): a review. Bull Ent Res 70: 525-532.

Goldman AL, Van der Goes van Naters, Lessing D, Warr CG and Carlson JR (2005) Coexpression of two functional odor receptors in one neuron. Neuron 45: 661-666.

Gore A (2006) An inconvenient truth: the planetary emergency of global warming and what we can do about it. Rodale Books, Emmaus, PA, USA. 328 p.

Grant AJ and O'Connell RJ (1996a) Electrophysiological responses from receptor neurons in mosquito maxillary palp sensilla. In: Bock GR and Cardew G (eds) Olfaction in mosquito-host interactions. John Wiley \& Sons, Chichester, UK, pp 233-248.

Grant AJ and O'Connell RJ (2007) Age-related changes in female mosquito carbon dioxide detection. J Med Entomol 44: 617-623.

Grant AJ, Borroni PF and O'Connell RJ (1997) Pused pheromone stimuli affect the temporal response of antennal receptor neurones of the adult cabbage looper moth. Physiol Entomol 22: 123-130.

Grant AJ and Kline DL (2003) Electrophysiological responses from Culicoides (Diptera: Ceratopogonidae) to stimulation with carbon dioxide. J Med Entomol 40: 284-293.

Grant AJ and O'Connell RJ (1986) Neurophysiological and morphological investigations of pheromone-sensitive sensilla on the antenna of male Trichoplusia ni. J Insect Physiol 32: 503-515.

Grant AJ and O'Connell RJ (1996b) Electrophysiological responses from receptor neurons in mosquito maxillary palp sensilla. Wiley, Chichester, UK.

Grant AJ, Wigton BE, Aghajanian JG and O'Connell RJ (1995) Electrophysiological responses of receptor neurons in mosquito maxillary palp sensilla to carbon dioxide. J Comp Physiol [A] 177: 389-396.

Hallem EA, Dahanukar A and Carlson JR (2006) Insect odor and taste receptors. Annu Rev Entomol 51: 113-135.

Hallem EA, Nicole FA, Zwiebel LJ and Carlson JR (2004) Olfaction: mosquito receptor for human-sweat odorant. Nature 427(6971): 212-213. 
Hartberg WK (1971) Observations on the mating behaviour of Aedes aegypti in nature. Bull WHO 45: 847-850.

Hill CA, Fox AN, Pitts RJ, Kent LB, Tan PL, Chrystal MA, Cravchik A, Collins FH, Robertson HM and Zwiebel LJ (2002) G protein-coupled receptors in Anopheles gambiae. Science 298(5591): 176-178.

Holt RA, Subramanian GM, Halpern A, Sutton GG, Charlab R, Nusskern DR, Wincker P, Clark AG, Ribeiro JM, Wides R, Salzberg SL, Loftus B, Yandell M, Majoros WH, Rusch DB, Lai Z, Kraft CL, Abril JF, Anthouard V, Arensburger P, Atkinson PW, Baden H, de B, V, Baldwin D, Benes V, Biedler J, Blass C, Bolanos R, Boscus D, Barnstead M, Cai S, Center A, Chaturverdi K, Christophides GK, Chrystal MA, Clamp M, Cravchik A, Curwen V, Dana A, Delcher A, Dew I, Evans CA, Flanigan M, Grundschober-Freimoser A, Friedli L, Gu Z, Guan P, Guigo R, Hillenmeyer ME, Hladun SL, Hogan JR, Hong YS, Hoover J, Jaillon O, Ke Z, Kodira C, Kokoza E, Koutsos A, Letunic I, Levitsky A, Liang Y, Lin JJ, Lobo NF, Lopez JR, Malek JA, McIntosh TC, Meister S, Miller J, Mobarry C, Mongin E, Murphy SD, O'Brochta DA, Pfannkoch C, Qi R, Regier MA, Remington K, Shao H, Sharakhova MV, Sitter CD, Shetty J, Smith TJ, Strong R, Sun J, Thomasova D, Ton LQ, Topalis P, Tu Z, Unger MF, Walenz B, Wang A, Wang J, Wang M, Wang X, Woodford KJ, Wortman JR, Wu M, Yao A, Zdobnov EM, Zhang H, Zhao Q, Zhao S, Zhu SC, Zhimulev I, Coluzzi M, della TA, Roth CW, Louis C, Kalush F, Mural RJ, Myers EW, Adams MD, Smith HO, Broder S, Gardner MJ, Fraser CM, Birney E, Bork P, Brey PT, Venter JC, Weissenbach J, Kafatos FC, Collins FH and Hoffman SL (2002) The genome sequence of the malaria mosquito Anopheles gambiae. Science 298(5591): 129-149.

Hubel DH (1957) Tungsten microelectrodes for recording from single units. Science 125(549): 550.

Jones JC and Madhukar BV (1976) Effects of antennectomy on blood-feeding behavior of Aedes aegypti mosquitoes. Ent Exp \& Appl 19: 19-22.

Jones WD, Cayirlioglu P, Kadow IG and Vosshall LB (2007) Two chemosensory receptors together mediate carbon dioxide detection in Drosophila. Nature 445(7123): 86-90.

Kellogg FE (1970) Water vapour and carbon dioxide receptors in Aedes aegypti. J Insect Physiol 16: 99-108.

Kettle DS (1965) Biting ceratopogonids as vectors of human and animal diseases. Acta Tropica 22: 356-362.

Kline DL (2002) Evaluation of various models of propane-powered mosquito traps. J Vector Ecology 27: 1-7.

Kline DL, Dame DA and Meisch MV (1991) Evaluation of 1-octen-3-ol and carbon dioxide as attractants for mosquitoes associated with irrigation rice fields in Arkansas. J Am Mosquito Control Ass 7: 165-169.

Kline DL, Takken W, Wood JR and Carlson DA (1990) Field studies on the potential of butanone, carbon dioxide, honey extract, 1-octen-3-ol, L-Lactic acid and phenols as attractant for mosquitoes. Med Vet Ent 4: 383-391.

Kwon JY, Dahanukar A, Weiss LA and Carlson JR (2007) The molecular basis of $\mathrm{CO}_{2}$ reception in Drosophila. PNAS 104: 3574-3578.

Lacher V (1964) Elektrophysiologische untersuchungen an einzelnen rezeptoren für geruch, kohlendioxyd, luftfeuchtigkeit und temperatur auf den antennn der arbeitsbiene und der drohne (Apis mellifera L.). Zeitschrift für vergleichende Physiologie 48(587): 623.

Linley JR, Hoch AL and Pinheiro FP (1983) Biting midges (Diptera: Ceratopogonidae) and human health. J Med Entomol 20:347-364.

Linley JR and Davies JB (1971) Sandflies and tourism in Florida and the Bahamas and Caribbean Area. J Econ Ento 6410: 264-278.

Lu T, Qiu YT, Wang G, Kwon JY, Rutzler M, Kwon HW, Pitts RJ, van Loon JJ, Takken W, Carlson JR and Zwiebel LJ (2007) Odor coding in the maxillary palp of the malaria vector mosquito Anopheles gambiae. Curr Biol 17: 1533-1544.

Matheson R (1945) Guide to the insects of connecticut part VI. the Diptera or true flies of connecticut. State of Connecticut State Geological and Natural History Survey, USA.

Mayer MS and James JD (1969) Attraction of Aedes aegypti (L.): Responses to human arms, carbon dioxide, and air currents in a new type of olfactometer. Bull Ent Res 58: 629-642.

Mayor S (2008) WHO report shows progress in efforts to reduce malaria incidence. BMJ 337: a1678.

Mclver SB (1972a) Fine structure of pegs on the palps of female culicine mosquitoes. Can J Zoo 50: 571-576.

Mclver SB (1972b) Fine structure of the sensilla chaetica on the antennae of Aedes aegypti (Diptera: Culicidae). Ann Entomol Soc Amer 65: 1390-1397.

Mclver SB (1980) Sensory aspects of mate-finding behavior in male mosquitoes (Diptera: Culicidae). J Med Entomol 17: 54-57. 
Mclver SB (1982) Sensilla of mosquitoes (Diptera: Culicidae). J Med Entomol 19: 489-535.

McKeever S and Pound JM (1979) The mouthparts of female Corethrella brakeleyi and C. wirthi (Diptera: Chaoboridae). J.Morph. 161: 157-168.

Murlis J and Jones CD (1981) Fine-scale structure of odour plumes in relation to insect orientation to distant pheromone and other attractant sources. Physiol Entomol 6: 71-86.

Newhouse VF, Chamberlain RW, Johnston JG and Sudia WD (1966) Use of dry ice to increase mosquito catches of the CDC miniature light trap. Mosquito News 26: 30-35.

O'Connell RJ and Mozell MM (1969) Quantitative stimulation of frog olfactory receptors. J Neurophysiol 32 51-63.

O'Connell RJ (1972) Responses of olfactory receptors to the sex attractant, its synergist and inhibitor in the red-banded leaf roller, Argyrotaenia velutinana. In: Schneider D (ed) Olfaction and taste IV. Wissenschaftliche Verlagsgesellschaft MBH, Stuttgart, Germany, pp 180-186.

O'Connell RJ, Kocsis WA and Schoenfeld RL (1973) Minicomputer identification and timing of nerve impulses mixed in a single recording channel. Proc IEEE 61: 1615-1621.

Omer SM and Gillies MT (1971) Loss of response to carbon dioxide in palpectomized female mosquitoes. Ent Exp \& Appl 14:251-252.

Pickett JA, Birkett MA and Logan JG (2008) DEET repels ORNery mosquitoes. PNAS 105: 13195-13196.

Roth LM (1951) Loci of sensory end-organs used by mosquitoes (Aedes aegypti (L.) and Anopheles quadrimaculatus Say) in receiving host stimuli. Ann Entomol Soc Amer 44: 59-74.

Rudolfs W (1922) Chemotropism of mosquitoes. New Jersey Agricultural Experimienrt Stations Bulletin 367: 5-23.

Rutzler M and Zwiebel LJ (2005) Molecular biology of insect olfaction: recent progress and conceptual models. J Comp Physiol [A] 191: 777-790.

Schweitzer ES, Sanes JR and Hildebrand JG (1976) Ontogeny of electroantennogram responses in the moth, Manduca sexta. J Insect Physiol 22: 955-960.

Seaton DR and Lumsden WHR (1941) Observations on the effects of age, fertilization and light on biting by Aëdes aegypti (L.) in a controlled microclimate. AnnTrop Med Parasit 35: 23-36.

Smith K, Thompson G and Koster HD (1969) Sweat in schizophrenic patients: identification of the odorous substance. Science 166(3903): 398-399.

Spielman A and Wong J (1973) Environmental control of ovarian diapause in Culex pipiens. Ann Entomol Soc Amer 66: 905-907.

Stange G (1997) Effects of changes in atmospheric carbon dioxide on the location of hosts by the moth Cactoblastis cactorum. Oecologia 110: 539-545.

Stange $\mathrm{G}$ and Diesendorf $\mathrm{M}$ (1973) The response of the honeybee antennal $\mathrm{CO}_{2}$-receptors to $\mathrm{N}_{2} \mathrm{O}$ and Xe. J Comp Physiol 86: 139-158.

Stange $\mathrm{G}$ and Wong C (1993) Moth response to climate. Nature 365699.

Steullet P and Guerin PM (1992) Perception of breath components by the tropical bont tick, Amblyomma variegatum Fabricus (Ixodidae) I. $\mathrm{CO}_{2}$-excited and $\mathrm{CO}_{2}$-inhibited receptors. J Comp Physiol [A] 170: 665-676.

Stewart RG and Kline DL (1999) Sugar feeding by Culicoides mississippiensis (Diptera: Ceratopogonidae) on the Yaupon Holly, llex vomitoria. J Med Entomol 36: 268-271.

Syed $Z$ and Leal WS (2008) Mosquitoes smell and avoid the insect repellent DEET. PNAS 105: 13598-13603.

Takken W and Kline DL (1989) Carbon dioxide and 1-octen-3-ol as mosquito attractants. J Am Mosquito Control Assoc 5:311-316.

Takken W (1991) The role of olfaction in host-seeking of mosquitoes: a review. Insect Sci Applic 12: 287-295.

Vickery CA, Jr., Meadows KE and Baughman IE (1966) Synergism of carbon dioxide and chick as bait for Culex nigripalpus. Mosquito News 26: 507-508.

Vinogradova EB (2000) Culex pipiens pipiens mosquitoes: taxonomy, distribution, ecology, hysiology, genetics, applied importance and control. Pensoft Publishers, Sofia, Bulgaria.

Wright RH (1967) A mosquito trap. J Econ Ento 60: 876-877.

Zwiebel LJ and Takken W (2004) Olfactory regulation of mosquito-host interactions. Insect Biochem Mol Biol 34: 645652. 



\title{
6. Host finding by female mosquitoes: mechanisms of orientation to host odours and other cues ${ }^{1}$
}

\author{
Ring T. Cardé and Gabriella Gibson
}

\begin{abstract}
The mechanisms by which mosquitoes locate their blood hosts are difficult to observe and analyse under natural conditions, given the small size of these insects, the crepuscular/nocturnal activity rhythms of most medically important species, and the distances of less than ten to hundreds of metres travelled to obtain a blood meal. Aspects of these mechanisms can be characterised from observations of the responses of mosquitoes in the laboratory and field to host cues at each stage in the process of host location. The main stages are typically described as activation, orientation to wind direction and odour-plume following, and arrestment near the source. Volatile chemicals emitted by the host extend the range over which it can be detected much further than any other cue, such as visual features or body temperature. Once a mosquito detects airborne odour cues, the evidence indicates that it relies primarily on an interaction between olfactory and visual inputs to move upwind toward the odour source by optomotor-guided anemotaxis, utilising contact with the host odour plume that is carried by the airstream to update its heading with respect to wind direction by visual cues, until it arrives near the odour source, when upwind progress is arrested and its responses to other host stimuli, such as heat, moisture and visual features of the host are thought to bring the mosquito into contact with the host. Knowledge of the sensory systems and physiological factors that control behaviour provide clues as to how mosquitoes locate hosts. For example, (1) the daily timing of quiescence and activity is mainly controlled by an endogenous circadian clock that determines the times of day, and hence the environmental conditions, such as light intensities, at which mosquitoes are most active, (2) mosquito eyes are highly specialised to detect high contrast features at low light levels at the expense of resolution, and, therefore, they can probably see hosts only $<5-10$ metres away, whereas they can be attracted by host odours originating tens of metres upwind, (3) the concentration and spatial structure of the plume of semiochemicals affect the type of response elicited in mosquitoes, and (4) responsiveness to particular odours is influenced by various hormones released on adult emergence, as a result of mating and throughout the gonotrophic cycle.
\end{abstract}

Keywords: carbon dioxide, odour plume, kairomone, upwind anemotaxis, klinokinesis, visual cues, odour salience

\section{Introduction}

A female mosquito's successful location of a host for feeding is under considerable selective pressure for reliability, energetic efficiency, and stealth to avoid predation, as only through securing a blood meal can the females of most species reproduce. When the host is beyond visual range, orientation manoeuvres modulating host finding are mainly related to contacting a plume of host-emitted odour and following it upwind, although other kinetic strategies are possible, such as 'aim and shoot,' whereby mosquitoes at rest on a surface detect the direction of air currents by mechanoreception and take-off upwind when stimulated by host odours. Plume following strategies ought to be sufficiently flexible to enable a mosquito to reach a prospective host under

\footnotetext{
${ }^{1}$ Both authors contributed equally to this review.
} 


\section{Box 1. Glossary of terms used in this chapter, emphasising their use in orientation to odours in wind.}

\begin{abstract}
Anautogenous
Anemotaxis

Autogenous

Crepuscular

Klinokinesis

Longitudinal klinokinesis

Mechanoreception

Optomotor

Spontaneous activity

Transverse klinokinesis

Female mosquitoes that require a blood meal to produce eggs. Maintaining a set angle to wind flow; positive anemotaxis (usually what is meant by 'anemotaxis') is orientation upwind. Female mosquitoes that produce their first ovarian cycle without a blood meal.

Activity at twilight, either dawn or dusk.

Orientation using successively sensed changes in stimulus concentration.

Orientation using successively sensed changes in stimulus concentration in the direction toward the origin of the stimulus, e.g. changes in odour concentration sensed within the plume as the organism moves towards its origin.

Sensing displacement of air movement by mechanical deflection of hair or body extensions, e.g. Johnston's organ at the base of the antennae.

Use of visual feedback to regulate manoeuvres, e.g. sensing and directing displacement by visual monitoring of changes of the organism's position in space.

When kept in constant environmental conditions (sound-proof and light-proof chambers), mosquitoes fly spontaneously at certain phases of the circadian day, even though there are no external stimuli to trigger such a response. This pattern of spontaneous activity is thought to reveal the underlying pattern of daily cycles of various behaviours under natural conditions.

Orientation using successively sensed changes in stimulus concentration lateral to the direction towards the origin of the stimulus, e.g. orienting by using differences in concentration of the odour plume sensed during lateral (across plume) movement, which may include detection of the plume's edge.
\end{abstract}

the naturally varying conditions of the field, where turbulent wind flow creates a spatially and temporally intermittent odour plume of fluctuating directionality. The sensory inputs available to modulate this process vary enormously with mosquito species, because of wide variance in the breadth of acceptable hosts and the odour cues they present, in the structure of habitat and its influence on plume dispersion, and environmental conditions, particularly those of wind speed, wind turbulence and light levels (Gibson and Torr 1999).

This review focuses mainly on those orientation manoeuvres that are presumed to operate at long range - that is, when the female mosquito is many metres or more away from a prospective host and often beyond visual range. However, as mosquitoes reach the host's immediate vicinity other cues, such as visual aspects of the host, heat and humidity may add to the suite of sensory inputs, and together these can influence continued orientation towards the host, hovering near the host, landing on the host, exploration of the host's surface, and biting. In some mosquitoes, there appear to be two orientation steps: flight to the host habitat, followed by pre-attack'resting', 
and then flight to the host. For those species most closely associated with human habitats, this resting phase has been observed most often to occur just before or after house entry (Clements 1999), a stage in host location that we know even less about than odour-plume following.

As mosquitoes approach a host, the overall plume structure, concentration of odours and nature of visual stimuli generally change, possibly providing mosquitoes with some information as to the proximity of the host. In the case of house-entering, apart from the effects of an aperture (i.e. open windows or eaves) on air flow, plume structure, and visual stimuli, the olfactory inputs available to a mosquito are likely to change as it approaches a prospective host. Indoors the concentration of odours generally increases, and some 'minor' odour components may play an increasingly greater role as these reach threshold concentrations. At close range, a mosquito may lose contact with the plume of carbon dioxide when its flight path allows exploration of body areas removed from exhalations. Then odours from specific regions of the host's body may become more important for close-range orientation and landing. Little is known, however, about the spatial and temporal pattern of host odours in the relatively stagnant conditions of habitations, nor about the neurophysiological effects of relatively continuous exposure to host emanations on mosquito olfactory systems (e.g. sensory adaptation or habituation?). In a stagnant environment it is possible that there are remnants of exhalations and odours from surfaces with prior host contact everywhere (e.g. worn clothing, bedding material). The profile of odours (the behaviourally relevant constituents, as well as their ratios and concentrations) emitted by individuals of a given host species also can differ among individuals, with distinctive chemical signatures postulated to influence individual variability in host attractiveness.

Much of what has been learned about the mechanisms of insect flight along odour plumes is based not only on work with mosquitoes, but with other insects, particularly moths, tsetse and Drosophila. Together these model systems have been used to establish the kinds of manoeuvres that flying insects use to orient along plumes in wind, and, in several moth species, how orientation toward an odour source may occur in still air (reviewed by Cardé and Willis 2008, Hardie et al. 2001). How mosquitoes orient to a host under windless conditions remains a largely unexplored question. Many of the laboratory and field assays that have contributed to our understanding of mosquito orientation have been designed, not to define orientation mechanisms, but either to improve the efficacy of odour-baited mosquito traps or to aid in the identification of the semiochemicals mediating orientation. Notwithstanding, some of these studies have added directly to our understanding of orientation, in part by helping define the probable role of different odours in long-distance and close-range orientation. Some of the field-based methods used to explore long-range responses of tsetse to host odours been applied to mosquitoes, with promising results (Knols et al. 1998, Torr et al. 2008).

This review will emphasise what is known about plume-orientated behaviours of female mosquitoes in host finding, identify gaps in our understanding, and suggest areas for future exploration. We will give particular attention to how changes in plume structure may influence orientation strategies. We also will consider how internal cues (e.g. circadian rhythms and physiological state) and environmental cues (e.g. visual cues related to wind direction and speed and thermal cues related to convection currents) interact with odour in influencing host finding. Important reviews of this topic include those by Clements (1999), Gibson and Torr (1999), Sutcliffe (1987), Takken (1991) and Takken and Knols (1999). 


\section{Activation}

The circadian rhythms of spontaneous activity for several species of medically important species have been established (Clements 1999), demonstrating that the daily timing of active and quiescent phases in mosquitoes is controlled mainly endogenously, thereby restricting most mosquito flight to species-specific times of day (e.g. nocturnal, diurnal, crepuscular). Accordingly, species-specific host-location strategies have evolved in the context of a specific range of general environmental conditions, e.g. a range of light intensities, patterns of wind dynamics, atmospheric carbon dioxide concentrations and activity rhythms of potential hosts and predators (Gibson and Torr 1999).

The pattern of spontaneous activity obtained under constant environmental conditions is perhaps best thought of as an indication of the fluctuating threshold of sensitivity to external and internal cues (Brady 1975). Both the daily timing of maximum sensitivity to stimuli and the type of stimuli that give rise to a response change throughout a mosquito's life, particularly for females. These behavioural changes are generally triggered by changes in hormone levels and physiological states brought on by significant events, such as mating, the intake of either blood or plant juices, and oviposition, and more gradual processes such as changes in nutritional status (Clements 1999). For example, it has been shown for several species that the patterns of circadian activity of newly emerged uninseminated females are similar to those of conspecific males (Anopheles gambiae Giles s.S., Jones and Gubbins 1978; An. stephensi Liston, Rowland 1989; Culex quinquefasciatus Say, Jones and Gubbins 1979, Clements 1999), and coincides with the timing of swarming and mating behaviour in the field. Uninseminated females and males aggregate at swarms sites, and hence they must respond similarly to swarm-formation stimuli since they arrive at specific swarming sites at about the same time. There is evidence, however, that males join swarms slightly in advance of females; the onset of spontaneous activity in uninseminated female $C x$. quinquefasciatus is $\sim 10$ min earlier than in males of the same age (Gibson 1983). Once mated, however, the pattern of female circadian activity changes to coincide with the timing of blood feeding observed in the field (Gillies 1988, Clements 1999) and females become refractory to mating (Jones and Gubbins 1979, Shutt et al. 2010). These changes in pattern and type of activity have been shown for several Culex species to be induced by male accessory gland (MAG) proteins, which are normally passed from male to female during mating (Chiba et al. 1992, Clements 1999, Jones and Gubbins 1979).

In contrast, Aedes aegypti (L.) females are active for the first few and last few hours of daylight, irrespective of their insemination status, and respond to host odours even before they mate. Aedes aegypti males are also attracted to host animals (see also Chapter 5, this volume), intercepting females as they approach the host, hence mating and blood feeding occur during the same circadian phase and without any obvious post-mating change in the stimuli to which females respond (Corbet and Smith 1974), although MAG proteins cause them to become refractory to reinsemination once mated (Craig 1967, Shutt et al. 2010).

Once females are in the active phase of their circadian rhythm, laboratory studies have shown that they 'take-off' in response to activating cues, such as carbon dioxide (Eiras and Jepson 1991, Gillies 1980, Healy and Copeland 1995), or spontaneously, without any particular environmental stimulus required. Little is known about what stimulates mosquitoes to take flight from their 'resting sites' in the field. In one of the few experimental observations of activation in the field, Gillies (1988) reported that 10,000 An. gambiae s.l. released inside an experimental hut earlier in the day, left the hut in a mass exodus within 10-20 min of sunset. In a study of tsetse behaviour in the field, Torr (1988) concluded that that the initial activation of resting flies is primarily mediated through endogenous, 
rather than host stimuli: no more than $35 \%$ of resting flies responded to a range of visual and olfactory cues known to be highly attractive to flying tsetse. The rest gradually left the resting-site refuge in a stochastic pattern over the course of their 'active phase.'Thus, both 'spontaneous activity' and responsiveness to particular environmental cues are likely to activate mosquitoes, causing them to leave sheltered resting sites which brings them into contact with a wider range of more accessible environmental stimuli, which evoke subsequent behaviour, depending on their physiological state (i.e. stage in the gonotrophic cycle of blood feeding and egg laying) and the nature of the stimulus (Clements 1999, Jones and Gubbins 1978, Rowland 1989).

\section{Strategies for locating a plume of host odour}

A mosquito's first contact with host odour may arise through activation, followed by 'ranging flight' - to put it teleologically - flight that occurs 'in search of' a host. Little is known about the orientation of ranging flights, but there remains some debate (reviewed by Cardé and Willis 2008) about the optimal strategy for discovering an odour plume: should a mosquito head crosswind, upwind or downwind? Alternatively, is a random heading with respect to the current wind flow optimal? Two key issues governing the likely heading of a particular direction of the flight path with respect to wind flow are: the balance between energy expenditure that wind direction and the mosquito's airspeed dictate and the rapidity and probability of plume contact. Ranging strategies in wind have been debated from theoretical perspectives, with the predictions on optimality generally contingent on how frequently the wind shifts direction and an insect's ability, if any, to keep track of its position relative to recent changes in wind direction (Figure 1).

Insects that are strong fliers, and/or fly significantly faster than the wind speed can fly at an angle to the wind, and still make progress against it. Tsetse typically fly at $6-8 \mathrm{~m} / \mathrm{s}$ in winds of $\sim 0.5 \mathrm{~m} / \mathrm{s}$. A field study on tsetse in Zimbabwe showed that the orientation to wind direction when no obvious visual or olfactory cues were present was predominantly downwind, and significantly crosswind when they left a visual target they had been circling, again with no odour present (Gibson et al. 1991).

Smaller, weaker fliers, such as mosquitoes, that fly at $\sim 20 \mathrm{~cm} / \mathrm{s}$ in still air (Gibson 1995) are more at the mercy of the wind; they can be blown off-course easily by a wind of $0.5 \mathrm{~m} / \mathrm{s}$. Therefore, we might expect them to adopt an orientation to the wind that is most stable, i.e. to fly as directly upwind as possible, correcting for drift across their visual flow-field to maintain an even flow of images from front to back in all areas of the eye. This may be why some species of mosquitoes fly upwind in wind tunnels, even in the evident absence of olfactory cues (Costantini et al. 2001, Takken et al. 1997). At wind speeds of less than their preferred flight speed, however, they would be more able to maintain a course at a fixed angle to the wind. Therefore, within the range of wind speeds wherein flight is feasible, the 'search' strategy may, to some extent at least, be wind-speed dependent.

In the case of mosquitoes, we have little field evidence to bear on this question. Gillies and Wilkes (1974) proposed that mosquitoes tended to head downwind (in accord with an optimal expenditure of energy model). They compared rates of mosquito capture with and without a large (57 m long, $2.9 \mathrm{~m}$ high) semi-circular screen, permeable to air flow but not mosquitoes, set $18 \mathrm{~m}$ away from human bait and oriented on nights of relatively uniform wind direction to form a barrier to upwind movement. As rates of capture were comparable in the two situations, mosquitoes were assumed to generally head downwind before plume contact. Gillies and Wilkes (1978) later retracted this interpretation, because they determined that screen fences had an 


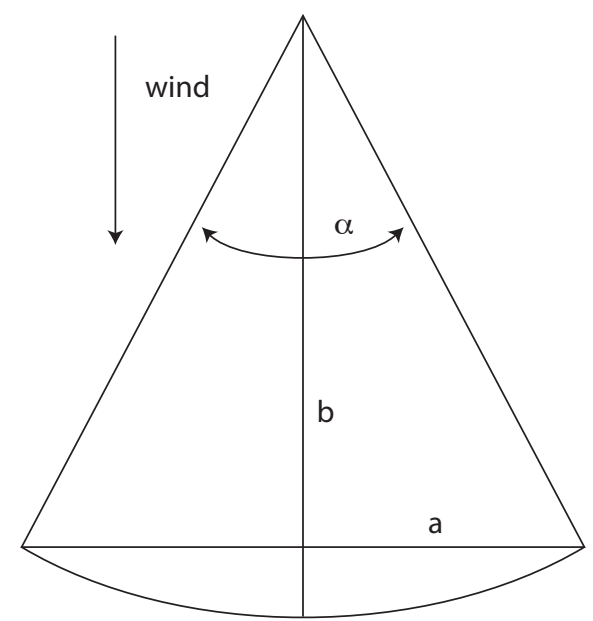

Figure 1. Possible consequences of a shifting wind direction on optimal strategies for foraging for an odour plume (see Sabelis and Schippers 1984). When the wind shifts over $60^{\circ}$ (a), it would appear more advantageous to head downwind rather than crosswind, because the plume's width (a) will exceed its downwind length (b), and then it is more efficient energetically to head with the mean wind flow (shown by the arrow). Most important, the probability of plume contact would be increased (Sabelis and Schippers 1984). Depending on the insect's flight capabilities and the wind speed, heading upwind might also be more efficient than a crosswind preference for rapidly contacting the plume. However, there are several implicit assumptions in this model (see Cardé and Willis 2008 for a full discussion). If the insect is to maintain a course that is on average headed downwind (or upwind) along the mean wind direction over the time interval of a sweep exceeding $60^{\circ}$ of wind direction, then it would need to keep track of its heading in the environment (its cardinal direction) over the time interval during which the wind direction shifted over $60^{\circ}$. Moreover, the insect would need to somehow 'calculate' the mean wind direction over this time interval, which of course is not a constant. These requirements would appear beyond an insect's capabilities. Likely, if upwind, downwind, or crosswind ranging strategies are adopted (as opposed to a random heading with respect to wind flow), the insect would make such a navigational decision based on the then current wind direction. This model also does not consider that because of the forces of turbulent diffusion, the instantaneous distribution of odour within the sweep would be patchy; instead the model assumes an above threshold concentration throughout the sweep (Modified from Sabelis and Schippers (1984) and used with permission of Springer).

insignificant effect on the mosquitoes' eventual direction of movement. Mosquitoes approaching such nets generally ascended the net and then continued onwards with the same heading, so that all captured mosquitoes could have simply headed upwind to reach the human bait.

Wind tunnel studies have produced conflicting evidence. Anopheles gambiae s.s. mosquitoes in evident absence of host odours (other than ambient carbon dioxide) generally head upwind (Costantini et al. 2001, Takken et al. 1997). Aedes aegypti mosquitoes, on the other hand, generally take flight and head upwind only after contacting a plume of either carbon dioxide or human skin odour (Dekker et al. 2005), possibly because they are diurnal, and have more visual information about wind direction, they are more able to maintain diverse orientations to wind. Wind-tunnel trials may be able to provide some information useful in establishing strategies of ranging flight with respect to wind flow, although natural mosquito behaviour may be affected by spatial constraints of the wind tunnel structure. In this artificial environment, mosquitoes can readily fly upwind or downwind and the duration of such flights can be extended for many minutes 
by moving a visual pattern below on the tunnel's floor in the direction appropriate to simulate the visual effect of the mosquito being displaced along its intended course (e.g. Kennedy 1940). Crosswind flight in such tunnels, however, rapidly carries the insect to the wind tunnel's wall and so this assay method can only document short bouts of crosswind ranging. Specialised wind tunnels that permit wind direction to shift or that mimic wind shifts by manipulation of the visual surround may be useful in establishing instantaneous strategies for dealing with fluctuations in wind direction (Drosophila, Zanen et al. 1994; tsetse, Colvin et al. 1989), but again they do not allow simulation of a directional preference over even moderate distances (less than a meter) or sustained time intervals. Therefore such assays are of limited value in establishing possible ranging strategies in the field.

Rather than engage in ranging flight, some mosquitoes may perch on foliage, waiting for a host to pass nearby. If the host is mobile, a sit-and-wait strategy may be useful, especially for day-active species, as then the visual cues will be most prominent (Gibson and Torr 1999). Visual cues from the host may serve to initiate flight (activation), and collimate flight toward the host, even after encounter of the odour plume. Although visual cues would be expected to be useful for day-active mosquitoes, they may also aid nocturnal orientation. However, it is important to bear in mind that even diurnal species, such as Ae. aegypti, have eyes that are designed to maximise their light gathering potential at the expense of resolution. In this respect their eye design is more similar to nocturnal species, such as An. gambiae s.s., than to true diurnal species, such as Toxorhynchites brevipalpis Theobald or Sabethes cayaneus (Fabricius) (Land et al. 1999). In practical terms, this means that the light sensitivity of An. gambiae s.s. enables orientation to high-contrast images at light intensities equivalent to only 1 log unit of starlight (Gibson 1995), i.e. typical of a moonless starlit night, but their resolution is so poor $\left(\sim 8^{\circ}\right.$ angle of resolution) they would have difficulties resolving an image the width of a person from a distance of $5 \mathrm{~m}$, even in bright light, as compared to $\sim 50 \mathrm{~m}$ for a tsetse. If a host is moving, the range of detection would increase, although for nocturnal species that feed on quiescent hosts, movement would be of relatively little importance. The visual resolution of Ae. aegypti, which is generally referred to as diurnal even though it is most active in the daylight hours nearest dawn and dusk, is only $\sim 6^{\circ}$, as compared to $3^{\circ}$ for a fully diurnal mosquito such as Tx. brevipalpis, and 1-4 for higher flies, such as tabanids and tsetse (Gibson and Young 1991). Nonetheless, by being active during daylight hours, the visual capabilities of Ae. aegypti are greater than for strictly nocturnal species; the extra light enables it to detect and respond to moving objects that it could probably not resolve at night (Muir et al. 1992).

Field studies at night are consistent with what we might expect from these physiological measurements. Bidlingmayer and Hem $(1979,1980)$ used arrays of suction traps with and without an associated dark visual cue approximately the height of a human, finding that several mosquito species were capable of orienting to such a silhouette over a distance of at least $15 \mathrm{~m}$ in the absence of any host-odour cues.

Bidlingmayer (1971) and Bidlingmayer and Hem (1981) also showed that mosquito species could be characterised as 'open grassland' species or 'woodland' species, by their responsiveness to visual features in the environment; grassland species tended to move out of woodland at dusk, remaining close to the woodland edge at night, i.e. possibly following the sharp visual 'edge' of the dark treeline against the lighter sky, whereas woodland species were only caught in the open field at night if they were trapped in the vicinity of tall shrubs, which suggests they may fly from tree to tree by attraction to the visual stimulus of the tree against the background of lighter vegetation. 
Correlated with their response to visual features, grassland and woodland species may also differ in their response to the types of odour plumes that occur in these two habitats. The behaviour of wind, and hence odour plumes, is greatly affected by obstacles such as trees (see above). Studies of the plume structure of pheromones released by moths (Willis et al. 1994) and bark beetles (Wyatt et al. 1993) have shown that trees create turbulence in the pheromone-bearing odour plumes, which causes odours to move vertically up and down the leeward side of the tree. The flight paths of male moths and beetles in these plumes are quite different to plumes from pointsources generated in open air. Similarly, the odour plumes of hosts in trees, such as nesting birds and small mammals, are likely to be quite different to odour plumes emitted by animals in the open. Accordingly, the flight pattern of woodland mosquito species may have evolved in response to both visual features of the woodland and the plume-structure of woodland-dwelling hosts.

\section{Pre-attack resting}

Another useful strategy may be to find a host's habitat and remain there either until a host appears or until the time for biting. It has been proposed that ornithophagous Culex mosquitoes use odours from bird droppings to find their hosts' nocturnal roosting sites (Cooperband et al. 2008). Highly anthropophilic species such as An. gambiae s.s. and Ae. aegypti enter human dwellings where they may sit on interior surfaces, until they respond to sedentary hosts or to mobile hosts that enter their dwellings during the mosquito's normal blood-feeding time (Clements 1999). In eastern Kenya, the propensity of Ae. aegypti to enter houses appears to be a heritable trait, based on crosses of so-called domestic and feral strains. In mark-release-recapture trials with human bait, the domestic strain had good fidelity for house-entering, the feral strain rarely entered houses, and hybrids showed intermediate levels of house-entering (Trpis and Hausermann 1978).

Clements (1999) cites several intriguing examples of Anopheles spp. moving to human dwellings or thorn bush cattle enclosures early in the evening and then host feeding at a later time. In Anopheles gambiae s. I. (likely An. arabiensis Patton), there was a 4-hr interval between moving to pre-attack resting sites near cattle hosts and biting (Smith 1958). Unresolved is whether the same suite of host odours governs orientation to pre-attack resting sites near hosts and subsequent orientation to the host. The act of 'resting' and its duration may be influenced by the same odour cues that induced movement to the pre-attack site. Of interest is the evident differential daily timing of these two orientation events and their different endpoints; our traditional laboratory bioassay methods may be inadequate for capturing these differences. Indeed, many laboratorybased bioassay studies report about a $50 \%$ response rate over several minutes (usually 15 min), which may be due to a natural stochastic rate at which individual mosquitoes become responsive during the course of their 'active' phase. The duration of an assay also can colour its outcome (Kennedy 1977); some host odours evoke a relatively rapid attraction, whereas others that induce little response within the first few minutes become attractive, given sufficient assay time (e.g. 15 min, Dekker et al. 2001).

\section{Following an odour plume in wind}

Kennedy (1940) was the first to establish how an airborne insect orients upwind along an odour plume, and, satisfyingly, his experimental subject was a mosquito! Using a small wind tunnel (38 cm long, $10 \mathrm{~cm}$ wide and $7.5 \mathrm{~cm}$ high), Kennedy induced upwind flight in Ae. aegypti by introducing his breath (thereby increasing the concentration of carbon dioxide, and other host volatiles) into the airflow. By moving a pattern that was projected onto the tunnel's floor, it was clear that the mosquito adjusted its trajectory and ground speed by detecting the flow of its visual 
surround. The optomotor response induces the subject to turn so as to maintain an even flow of images across the visual field from front to back, which leads a flying insect to correct for wind drift and maintain a stable orientation to the wind under field conditions (Gilbert and Kuenen 2008). This mechanism is termed odour-mediated optomotor-guided, positive anemotaxis and its role in plume following behaviour has been verified in many other insect groups (moth spp., Kennedy and Marsh 1974, Willis and Cardé 2008; tsetse, Colvin et al. 1989). Indeed, it is the only verified mechanism by which an airborne organism can gauge the direction of wind flow and then set and maintain a heading relative to wind direction.

David et al. (1982) demonstrated that under general conditions of fluctuating wind direction with a relatively constant velocity over an open space, at any point in an odour plume where odours would be detected, the instantaneous wind direction in the immediate area points directly towards the odour source (Figure 2B), even if the wind direction at the source has changed since the odour packet left the source. In this general case, flying insects should set a steady course directly upwind whenever they detect a host odour. This strategy works well when the distance between the responding insect and the odour source is small (a few tens of metres or less) and the wind direction does not vary much. When wind speed varies considerably (see below) and in more complex landscapes, however, simply heading straight upwind after having detected odour, is a less reliable strategy for locating an odour source. As a plume of odour is transported downwind,

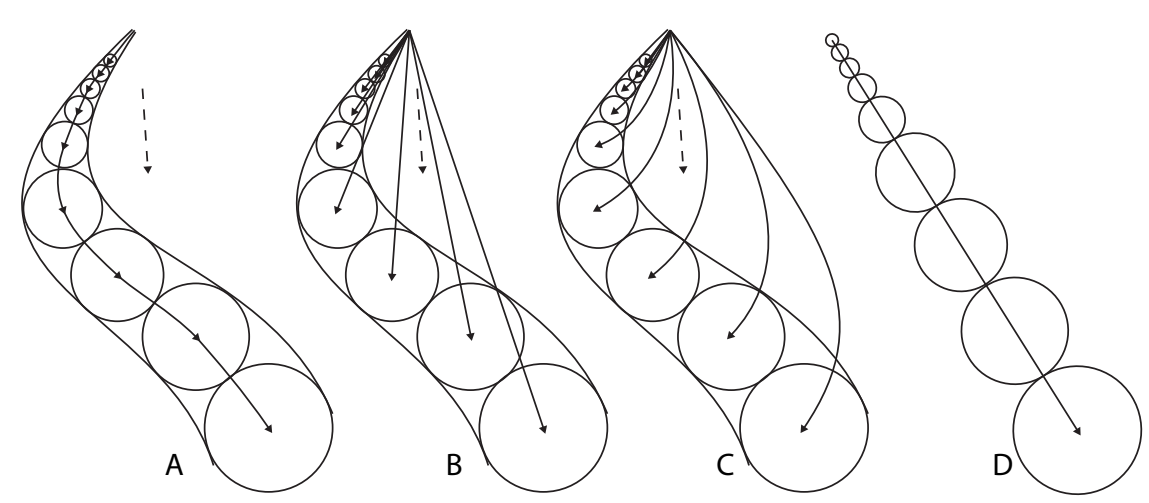

Figure 2. Schematic of the effects of a shifting wind direction on the path of meandering odour plumes, in this case of sex pheromones, as viewed from above. Pheromone is shown as a series of puffs (circles) that expand by turbulent diffusion over time. The paths of the puffs are depicted by the lines leading from the source along the centre of each puff; the arrow tips designate the instantaneous wind direction within each puff. The dashed lines and arrow tips represent the mean direction of wind flow at the source over the time course of each plume's meandering. (A) The instantaneous wind directions within the plume assumed by behavioural biologists before David et al. (1982). The plume's centreline and the instantaneous wind direction at any point are assumed to be aligned and so simply would keep the responding insect within the plume's boundaries and this course would lead directly to the odour's source. (B) Conforms with the experiments of David et al. (1982) in an open field with a varying wind direction but a relatively constant wind speed. Heading upwind in the downwind sector of the plume often leads the responder out of the plume. (C) Matches observations of Elkinton et al. (1987) beneath a forest canopy and Brady et al. (1989) in an open savannah; this effect is caused by changes in wind direction and an increase in velocity. Again, heading upwind can lead out of the plume. (D) Shows a steady wind direction - during such 'favourable' and typically brief alignments of wind direction and the plume's centreline, insects can make rapid progress toward the source (reprinted with permission of Wiley-Blackwell from Elkinton et al. 1987, p. 400). 
turbulent forces, especially those created by obstacles such as vegetation or environmental features on a landscape scale, fragment the plume into filaments and create odour-free (or below odour threshold of detection) gaps which can be large enough to impair orientation. Even in a turbulent flow, however, filaments of relatively undiluted odour can persist over many metres of transport, even as the average concentration of odours within the plume's boundaries decreases (Murlis and Jones 1981, Murlis et al. 1992). The presence of relatively undiluted odour filaments permits detection of the odour plume farther downwind of the odour source than would be the case if the plume was thoroughly mixed. In this case, flying insects may need to alter their heading and 'cast' across the odour plume (as is the case in moths, Kuenen and Cardé 1994) or even back track to some degree (as for tsetse; Torr and Mangwiro 1996) to re-establish contact with the odour plume.

A second challenge for insects flying toward an odour source is that within an odour plume the instantaneous direction upwind may lead, not along the plume's long axis, but instead to the plume's boundaries and beyond. Shifts in wind direction realign the plume's long axis away from due upwind, even within a few metres of the odour source (Elkinton et al. 1987, Brady et al. 1989). Moths following a pheromone plume upwind and then entering an odour-free area (caused either by turbulent diffusion or by wind shifts) have a strategy for re-contacting the plume: they 'cast' back and forth across the wind without making progress upwind. If this manoeuvre enables them to recontact the plume, upwind flight along the plume resumes; if not, after several seconds of casting, they shift to ranging flight. This strategy is most well-understood for moths, which have a relatively low flight speed in relation to wind speed, so that by casting they more-or-less hold station with respect to the ground, and hence with respect to the odour source, while they continue to fly crosswind to increase their chances of re-encountering the odour stimulus (Kuenen and Cardé 1994, Cardé and Willis 2008). Tsetse, however, which generally fly many times faster than the mean wind speed, respond quite differently to losing odour by inadvertently leaving an odour plume. They slow down and make a smooth turn of $\sim 120^{\circ}$ upwind, which takes them roughly back to where they had last detected host odour, but further upwind and hence closer to the odour source (Gibson and Brady 1985, 1988). In the case of tsetse and other blood feeding insects, the odour source may not be stationary, so it may pay to be less precise in flying directly upwind to where a host might have been (Williams 1994) than it is for moths to calling females.

The strategies used by plume-following mosquitoes to recontact the lost scent of a host are unknown. It is clear, however, that a mosquito's detection of a plume of host odour does not ensure that she will be able to follow the odour plume to its source. Mosquitoes fly at about the same ground speed in relation to wind speed as moths, and we might expect to find a strategy of casting more likely than the smooth turns associated with tsetse. In wind-tunnel trials, Dekker (2002) found that Ae. aegypti surged upwind upon contact with a carbon dioxide plume and headed crosswind upon loss of contact - suggestive of moth casting upon loss of contact with of pheromone plume.

Given that there is a tendency for wind speeds to cycle daily, with the highest speeds tending to occur midday and the lowest speeds in the middle of night, wind direction should be more readily detectable during the day when its speed is on average highest. Of course, there can be lulls in wind flow at anytime, and even during the day the wind speed may fall below an insect's threshold of detection by the optomotor response. Detection of wind flow at night generally is more difficult, however, because wind speed is on average reduced and low light levels impair the visual detection of drift. Low wind speeds can be an advantage for weak fliers, such as mosquitoes, sandflies and biting midges, however, which are easily blown off course at 
higher wind speeds. Although mosquito eyes are certainly well-adapted for detecting strongly contrasting visual features at low light intensities (Land et al. 1999), trap catches and landing catches can be significantly higher on moonlit nights than on moonless overcast nights (Clements 1999), indicating that mosquitoes may well be operating near the limit of their visual abilities under natural lighting.

It is difficult to generalise about the comparative challenges for mosquitoes navigating along odour plumes in daytime, twilight, or night time conditions. Higher wind speeds typical of daytime are correlated with less variability in the magnitude and degree of shifts in wind direction, although turbulence levels also increase, causing odour gaps of larger size. Thus, at night plume following should, in principle, be more challenging than in daytime, because at night there is not only a reduction in wind velocity, but the low light level poses an increased difficulty in detection of wind-induced drift, a prerequisite to determination of the direction of airflow. However, the navigational capabilities of each species may be attuned to the characteristics of its habitat and the meteorological conditions typical of the daily time it forages for hosts. In daytime, the structures of odour plumes in open forests and in grassy fields differ markedly (Elkinton et al. 1987) and in daytime the wind speed may be too high to permit plume following. However, the wind flow at night in certain structured environments, such as valleys, can aid host-seeking behaviour; catabatic winds produced by cool air moving down the valley floor under nocturnal atmospheric inversions can give rise to a non-turbulent air current that flows gently downhill, thereby producing a relatively steady stream of olfactory cues.

There are two other proposed strategies for flying upwind along an odour plume, neither of which involves optomotor feedback. As wind velocity increases with distance from the ground, Gillett (1979) suggested that mosquitoes dipping toward ground level might sense differences in their acceleration within the wind shear via changes in pressure on mechanoreceptors and use this feedback to gauge the direction upwind. If the mosquito was headed upwind, for example, a dip would produce an increased acceleration because the mosquito would encounter a decrease in airflow. Successive comparisons of accelerations versus decelerations might provide the needed directional information. Mechanoreceptive feedback could be provided by sensory hairs, the halteres or by the Johnston's organ at the base of the antennae. This mechanism has not been tested experimentally, and it assumes in part that mosquitoes continue to orient upwind in light levels that are too dim to permit conventional optomotor response.

The second alternative mechanism is 'aim-then-shoot', wherein orientation over a distance consists of a sequence of short flights. A stationary organism detects odour by olfactory sensory organs and wind flow direction by mechanoreceptors while at rest on a substrate and then flies upwind in a straight line, guided by visual cues that are set upon takeoff. Landing occurs if contact with odour is broken and flight is reinitiated if odour is again sensed. Such a series of steps may eventually lead to the odour source. This is a mechanoreceptive anemotaxis (Kennedy 1986) and there is some evidence that it occurs in cabbage root flies and tsetse (see Cardé and Willis 2008), although optomotor anemotaxis is also employed by tsetse (Gibson et al. 1991). The occurrence of aim then shoot in mosquito host seeking remains speculative.

\section{Orientation to odour in still air}

Mechanisms for finding an odour source under either windless conditions or when airflow is undetectable by visual means has been studied in depth only in moths. In wind-tunnel assays, moths orientating along a pheromone plume formed in wind usually continue along the plume in 
a zigzag path when the wind flow is quickly stopped (e.g. Kuenen and Baker 1983, Willis and Cardé 1990). These observations established that moths are capable of using a form of klinokinesis, that is, orientation using successively sensed changes in pheromone concentration. In these wind-tunnel manipulations, moths travelled about a metre along the plume in still air, apparently having already set a course while in the wind using visual cues, which appears to have been sufficient to enable them to continue in the same direction, albeit without wind. Changes in odour concentration near the boundaries of a wind-formed odour plume could guide a flying insect toward the source by 'transverse klinokinesis'. If this mechanism is employed by mosquitoes, it likely occurs when wind forms an odour plume, and then ceases or falls below a speed that can be detected visually.

Mosquitoes could use longitudinal klinokinesis to self-steer a course toward an odour source. The collimating cue would be increases in odour concentration detected during an approach to the odour source. In still air the odour gradient would be caused by molecular diffusion and, if this mechanism applies to mosquito orientation, it most likely occurs only very near (within decimetres or less) of a host, particularly indoors on a still night when the inhabitants of the dwelling are asleep. Such a gradient also can exist within a wind-formed odour plume, but changes in concentration are steep enough only within decimetres of the source to be of potential value in orientation (Murlis et al. 1992), and this mechanism would be redundant to simply using anemotaxis.

Another orientation strategy useful close to a host would be to follow plumes formed by heatinduced convention currents from a warm-blooded host. Such a strategy would enable orientation to the body surface in still air. Odour plumes that are formed in this way would rise and orientation downward toward the host could involve a range of cues, including; an optomotor response, gravitational cues, sensing of a vertical heat gradient, sensing of a vertical odour gradient, plume edges, or some combination of these cues. It is even conceivable that entirely 'non-directional' orientation, for example, by changes in concentration governing the rate of turning (klinokinesis) or speed of flight (orthokinesis), could bring the mosquito close to the host (Williams 1994). Convection currents appear to guide the selection of biting sites in members of the An. gambiae complex (De Jong and Knols 1996, Dekker et al. 1998). Vertical flight has been shown in Ae. aegypti to be influenced by optomotor cues (Daykin 1967). In a wind tunnel with vertical airflow, moving stripes were used to simulate the visual effect of displacement due to an airstream; mosquitoes tended to fly with respect to the stripes as though they were flying toward the odour source. Once again, the optomotor response could account for downward descent toward a host along a convective airstream.

Mosquitoes also may approach a host by following an increasing temperature gradient formed by convection currents by klinokinesis, in a manner similar to following increasing chemical gradients in still air. Their antennae are known to be particularly sensitive to small changes in temperature. In Ae. aegypti, Davis and Sokolove (1975) found one set of thermoreceptors to be sensitive to increases in temperature and a second set responded to sudden decreases in temperature. Together, these receptors were capable of sensing changes in temperature as minute as $\pm 0.2{ }^{\circ} \mathrm{C}$ (Davis and Sokolove 1975).

Because convection currents are created by a differential between body temperature and ambient temperature, with the warmer air rising, the speed of the air current generated by convection currents will depend on the magnitude of the difference between these temperatures. In the tropics at night, there is often less than a $10{ }^{\circ} \mathrm{C}$ difference and therefore convection currents may 
not move quickly enough or extend far enough from the source before the air reaches ambient temperature. Nonetheless, with a sensitivity to temperature differences of as little as $\pm 0.2{ }^{\circ} \mathrm{C}$, mosquitoes may be capable of following convection currents by following a temperature gradient that varies by at least this much. Alternatively, they could close in on a host via klinokinesis and/ or orthokinesis, as described earlier in the case of movement along a vertical odour gradient (Warnes 1995).

Eiras and Jepson (1994) found that some Ae. aegypti mosquitoes flew downward along heat convention currents in the absence of any host cues in a small bioassay chamber; orientation was enhanced by addition of water vapour and even more so by extracted human sweat. A human hand, however, produced the highest proportion of source location - likely because of the suite of natural host odours emitted. In such assays when the stimulus is a human hand it is difficult to disentangle the effects of a temperature gradient from the potential effects of changes in odour concentration. Aedes aegypti mosquitoes descended along a heat gradient presented alone, but human skin emanations alone induce upwind flight (e.g. Dekker et al. 2005) and, in the absence of wind or other cues such as heat, skin odours may induce downward flight. These kinds of assays establish the cues contributing to downward movement in a natural convention current that is laden with human skin odour. Just how the mosquito determines a downward direction is not firmly established.

\section{Which odours induce orientation?}

In addition to being an 'activator', carbon dioxide seems to be a universal 'attractant' for female mosquitoes (Gillies 1980, Reeves 1953, Rudolfs 1922, see also Chapter 4, this volume) and generally all haematophagous arthropods, and it is widely employed as 'bait' in traps used for mosquito surveillance. Carbon dioxide is of course ubiquitous as a natural constituent of the atmosphere (at $\sim 0.04 \%$ ), and it comprises approximately $4 \%$ of vertebrate exhalations. A host-generated plume of carbon dioxide may be detectable over background for a considerable distance downwind. Zöllner et al. (2004) released carbon dioxide at a rate equivalent to two cattle $(20 \mathrm{l} / \mathrm{min})$ in a riparian habitat in daytime and detected the plume with a carbon dioxide sensor $64 \mathrm{~m}$ downwind (the maximum distance sampled) as fluctuations of $300 \mathrm{ppm}$ over background, albeit with frequency of contact inversely proportional to distance from the source. Of course, the rate of release of carbon dioxide from smaller vertebrate hosts would be less - chickens produce about 30-40 ml/min (Barott and Pringle 1946, Clements 1999). The distances over which carbon dioxide alone is effective as an attractant have been measured with ramp traps arrayed in a radial pattern from a central release of carbon dioxide at a rate equivalent to two calves (Gillies and Wilkes 1969, 1970, 1972). In these experiments the distances of 'attraction' of Aedes, Anopheles, Culex and Mansonia species ranged from about 8 to $18 \mathrm{~m}$. It is worth bearing in mind that traps are not perfectly efficient at capturing all mosquitoes in the area, so the numbers caught may fall below detectability when few mosquitoes approach it, and therefore this estimate of range of attraction is likely to be an underestimate.

A wind-borne plume of carbon dioxide fluctuates in intensity against a background concentration and so to detect a plume of carbon dioxide mosquitoes could rely on sensing its intermittent structure, an idea dating to Wright and Kellogg (1962). Mosquitoes detect carbon dioxide by receptors on the maxillary palps and an increase or decrease in concentration of as little as $50 \mathrm{ppm}$ from ambient clearly modifies receptor firing rates in Aedes, Anopheles and Culex mosquitoes (Grant and O'Connell 1996). In assays with odour plumes filling an $8 \mathrm{~cm}$-diameter Y-tube olfactometer, a fluctuating intensity of carbon dioxide induced a higher proportion of net upwind flight than 
a homogeneous cloud of carbon dioxide in Ae. aegypti (Geier et al. 1999a). In a wind tunnel, Ae. aegypti turned upwind and accelerated its flight speed after a brief encounter with an individual filament of carbon dioxide (Dekker et al. 2005). These two observations suggest that in Ae. aegypti plume intermittency is associated with sustained upwind movement, stimulated by repetitive encounters with carbon dioxide filaments, similar to the mechanism proposed for upwind orientation of male moths along a turbulent pheromone plume (Mafra-Neto and Cardé 1994, Vickers and Baker 1994). These findings with Ae. aegypti suggest that it is the fluctuating intensity of carbon dioxide over background that induces sustained upwind flight. The applicability of this principle to the upwind orientation other mosquitoes remains to be investigated.

\section{Odour salience}

In a wind tunnel assay with Ae. aegypti, a single, transient ( $<100 \mathrm{~ms})$ exposure to a filament of carbon dioxide lowered the threshold of response to human skin odour by a factor of more than ten (Dekker et al. 2005). This finding could mean that for Ae. aegypti the 'long-distance' attractant is carbon dioxide and not human skin odour, and/or that carbon dioxide lowers the threshold for attraction to a human skin odour, acting at some distance from the host. Alternatively, as this species is highly anthropophilic and would be expected to be in or near human dwellings before the final stages of host orientation, the presence of a fluctuating intensity of carbon dioxide could signal that a potential host is nearby and initiate further orientation mediated by both carbon dioxide and skin odours.

Odours other than carbon dioxide influence orientation and it has been proposed that for some mosquito species with narrow host ranges, such as some highly anthropophilic species, carbon dioxide alone may not induce orientation. Instead, at long-range, mosquitoes would sense specific host odours in addition to the carbon dioxide plume and carbon dioxide alone would be of diminished importance to the induction of plume following (e.g. Mboera and Takken 1997, Mboera et al. 1998, Takken and Knols 1999, Pates et al. 2001). Whether host odours other than carbon dioxide induce plume following at long range in such species is an issue that remains unresolved and which will be difficult to verify experimentally.

Traditionally, mosquitoes have been characterised by their 'host preferences', which is mainly based on the outcome of their responsiveness to host cues: the origin of the blood imbibed by individual mosquitoes. Even after taking into account biases due to relative host availability (e.g. as measured by various indices, including relative number or biomass of hosts present, but not protected from mosquitoes), it is clear that even amongst species that transmit human pathogens, the profiles of 'host preference' are highly variable between species, and even within species over their geographic range of distribution (Clements 1999). At first sight, one might expect differences in host preference to be due to differences in the profiles of host odours to which each species is attracted. There may be host-specific odours, or ratios of blends to which particular species are most responsive, but so far, no combination of odours has been found to be as attractive as a live host with carbon dioxide at biologically relevant doses), or to mimic a specific type of host (Chapter 7, this volume). However, we do know that the pattern of responsiveness to whole body odours and carbon dioxide vary considerably among species and even within a species. 'Host preference' may reflect differences between species in the response to particular chemicals. In addition, differences between host preferences as striking as that noted between members of the An. gambiae species complex (An. gambiae s.s. is one of the most anthropophilic species in the world, An. arabiensis is also highly anthropophilic, but rather more opportunistic, often feeding on cattle and An. quadriannulatus (Theobald) feeds almost entirely on bovids) may be due to 
behaviours associated with host location, such as house entry, that are confounded with plume following. Current methods of assaying the response to odours in the field, such as odour-baited traps of all types, cannot distinguish between these two kinds of orientation.

To investigate further the relative responsiveness of mosquitoes to carbon dioxide and other host volatiles, Costantini et al. (1996) undertook a series of dose-response assays in the field using odour-baited entry traps (OBETs) to test the relative importance of carbon dioxide to a range of mosquito species, and found that for each species the catch increased with dose of carbon dioxide in a similar log-linear fashion. If the dose-response data are considered in relation to a standard human-landing catch, however, the behaviour of each species was quite different. At one extreme, even the highest dose of carbon dioxide did not catch more An. gambiae s.l. (highly anthropophilic) than a single human landing catch, whereas for Mansonia uniformis (Theobald) (zoophilic, with generalist host preference) the three highest doses of carbon dioxide caught significantly more mosquitoes than a human bait, and An. funestus Giles (also anthropophilic) did not respond to carbon dioxide alone in sufficient numbers to assess a dose response. These findings demonstrate that the response to carbon dioxide and other human volatiles is strikingly species specific, and, therefore, we should expect to discover a variety of mechanisms by which species effectively 'choose' a host. With respect to An. gambiae s.s., these results suggest that although increasing doses of carbon dioxide increased the catch, the responsiveness to hostspecific cues may take over in importance after an initial attraction to carbon dioxide; in the absence of human-specific cues, it appears that An. gambiae s.s. was either less attracted to enter the OBET (proxy for house entry) and/or did not stay in the vicinity of the trap long enough to be caught in high numbers as the relative abundance of human odours declined. For Ma. uniformis, carbon dioxide alone appears to be sufficient to arrest females near the source and elicit 'entry' behaviour even in the absence of other host cues.

\section{Still-air bioassays}

The precise role assigned to carbon dioxide in mediating orientation may be contingent on the type of assay used. Because An. gambiae s.s. fly upwind in unscented air in a wind tunnel (Costantini et al. 2001, Takken et al. 1997), this assay method is difficult to use in orientation studies with this species, although upwind flight centred within a discrete odour plume may signify attraction. Much of what we know about its odour-mediated behaviour and the behaviour of other An. gambiae s.l. complex species stems from a series of two-port, still air olfactometer studies comparing the highly anthropophilic An. gambiae s.s. with zoophilic species such as An. quadriannulatus. A large, still-air olfactometer determines 'attraction' by capture in odouremitting ports; it does not allow upwind flight along a wind-borne plume, except very close to the port entrance. This bioassay method was not designed to explore how odour plumes modulate orientation, however, but to identify potential host-emitted attractants.

In one study, port entrance of An. gambiae s.s. was induced by odour sources previously in contact with human skin, whereas carbon dioxide was ineffective in inducing port entrance (Pates et al. 2001), leading to the conclusion that carbon dioxide alone played a minor role in orientation. Other studies, however, found that An. gambiae s.s. were intercepted by an electrocution net placed $1 \mathrm{~cm}$ in front of carbon dioxide sources (either homogeneous or turbulent presentations), even though these odour sources of carbon dioxide were not effective for port entry (Dekker et al. 2001). When the carbon dioxide source was positioned $5 \mathrm{~cm}$ downwind of the port entrance, the capture rate for human skin emanations was elevated (Spitzen et al. 2008), further confirming that carbon dioxide could play a role in guiding An. gambiae s.s. to a host. 
Anopheles quadriannulatus, however, was not captured by carbon dioxide alone in this assay, but cow odour plus carbon dioxide was efficacious, and, unexpectedly, human odour alone was even more effective (Pates et al. 2005). The port-entry, still-air bioassay has produced a wealth of basic information on mosquito odour preference (and repellency) on species in the An. gambiae s.l. complex, but this method does not provide substantive insight into orientation per se. Rather this bioassay measures the culmination of what is presumed to be a complex repertoire of behaviours, and this method may not be helpful in determining specific orientation manoeuvres that occur along odour plumes meters or more downwind of the source.

In the case of Ae. aegypti, human skin odour alone also induces upwind flight and it seems to induce the highest proportion of rapid source location when it is presented in a relatively homogeneous rather than an intermittent plume, the opposite effect of plume structure with carbon dioxide (Dekker et al. 2005, Geier et al. 1999a). A relatively homogeneous plume of skin odour may occur very close to the host. Numerous human odours potentially involved in attraction have been identified from skin head space (e.g. Bernier et al. 2000) and sweat (Braks et al. 2001, Cork and Park 1996), but relatively few compounds have proved 'attractive', that is, induce upwind movement over short distances in laboratory assays. L-lactic acid, short-chain fatty acids and ammonia are attractive for Aedes and Anopheles (e.g. Acree et al. 1968, Bosch et al. 2000, Braks et al. 2001, Dekker et al. 2002, Geier et al. 1999b). Carboxylic acids, alcohols and aldehydes are attractive for Culex (Puri et al. 2006). Generally these assays measure either displacement in an upwind direction, movement upwind over relatively short distances or into an entrance port of a still-air bioassay chamber, often over many minutes. It is unclear if these odour-induced movements correlate with host-finding manoeuvres in the field or if the airborne concentrations of odours are presented at levels comparable to those that would occur naturally (see Chapter 7 and Chapter 8 , this volume).

None of these compounds has been tested in an assay that would verify that these compounds (or some combination) lure mosquitoes upwind over long (multi-metre) distances. These compounds may combine with the carbon dioxide plume to act additively or synergistically to induce attraction from a distance. Alternatively, these odours may modulate only late-stage orientation manoeuvres such as hovering near or landing on the host (e.g. Healy and Copeland 2000, Healy et al. 2002), contributing little to orientation at the 10 to 20 metre range documented as the range of attraction of carbon dioxide (Gillies and Wilkes 1969, 1970, 1972).

Hovering and landing entail very different visual feedback than generated by upwind flight. Recent studies of the heading of Drosophila tethered in flight simulators (Fry et al. 2009) have provided confirmation of the role that visual feedback plays in regulating upwind flight to an odour source. In this setup, the flow of the visual surround from computer-controlled banks of LEDs was set to pass from ahead to around each side of the fly. This simulated what the fly would experience during forward flight in wind and indeed flies directed their heading towards the visually simulated upwind direction. Forward flight also requires input from mechanosensory cues, provided at least in part by the Johnston's organ (Budick et al. 2007). Drosophila also orient towards vertical silhouettes, which in the flight simulator is represented by a vertical band of LEDs that are turned off; this visual stimulus presumably mimics a suitable landing or feeding site (Maimon et al. 2008). In nature, such a visual stimulus directly ahead would appear to expand during approach, causing the fly to slow its progress before landing. When upwind flight is stimulated, however, flies generally avoid objects ahead, turning either right or left. (A fly in free flight heading upwind would crash into a rapidly looming visual stimulus if it did not treat the object as an aversive visual cue.) 
As a mosquito approaches a host, perhaps first hovering nearby before landing, it also must alter the pattern of visual feedback from a flow of the visual surround signifying upwind movement to frontal object expansion. It is tempting to speculate that there are specific stimuli, such as particular host odours perceived at close range, that trigger this change, as suggested by the work of Healy and Copeland (2000) and Healy et al. (2002).

\section{Field traps baited with carbon dioxide and other host odours}

Carbon dioxide has long been used as attractive bait in traps used in surveillance programmes (e.g. CDC-type traps, see Qiu et al. 2007a). The most commonly used trap configurations capture mosquitoes by drawing them upwind along a carbon dioxide plume leading to the vicinity of suction near the trap entrance. Three-dimensional video observations of $C x$. quinquefasciatus and Culex tarsalis Coquillett mosquitoes orienting to such traps in a large field wind tunnel (Cooperband and Cardé 2006a,b) have shown that of the mosquitoes reaching the origin of the carbon dioxide plume, relatively few are actually captured (Figure 3). The reason for such a low proportion of capture is not certain, but it appears that reaching the origin of the plume does not routinely place the mosquito within an area of suction and that the most mosquitoes do not engage in localised 'search' near the origin of the carbon dioxide plume long enough to enter an area of suction. Most mosquitoes simply depart after a few to tens of seconds in the vicinity of the
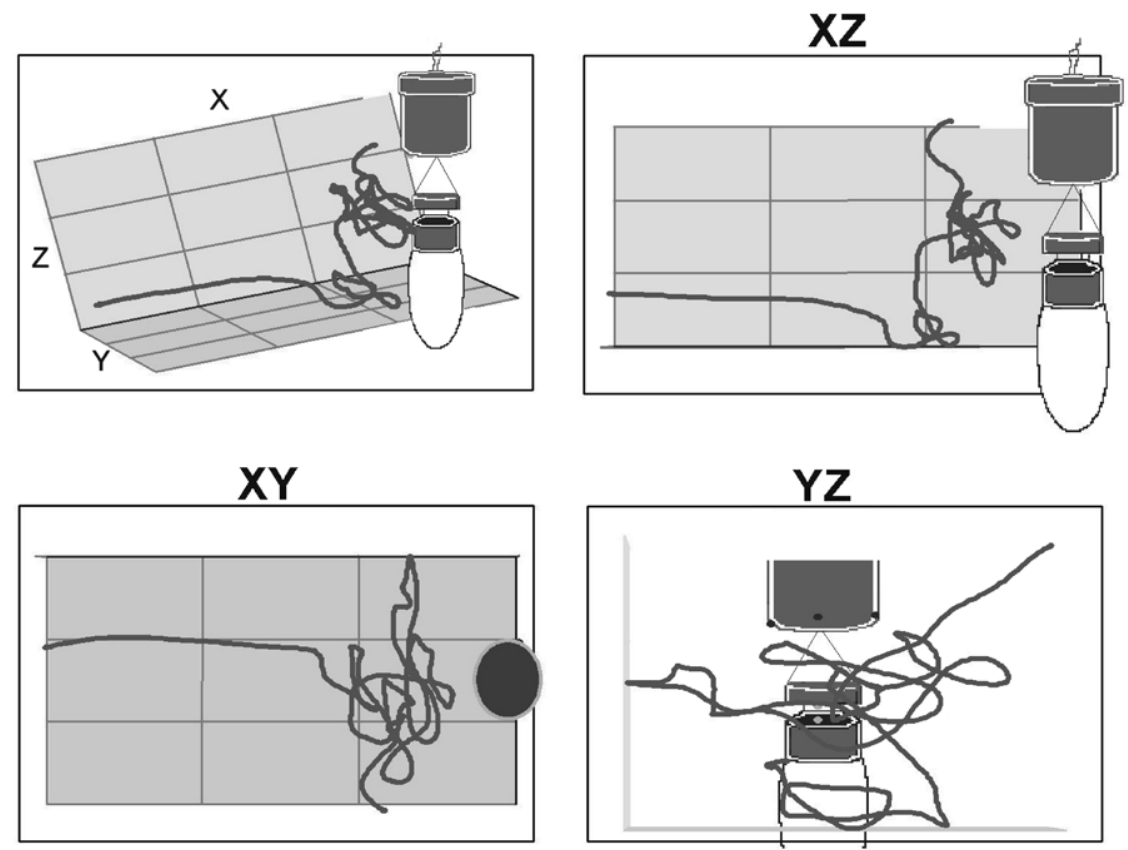

Figure 3. The track of a female Culex tarsalis flying in the vicinity of a suction trap baited with carbon dioxide (dry ice) shown from three perspectives. This mosquito was not captured and eventually departed the trap's vicinity. This track was video recorded in 3-D in a field wind tunnel. $X Z$ is a side view with the air flowing from right to left, $X Y$ is an overhead perspective, and $Y Z$ is a view from upwind of the trap (reprinted with permission of WileyBlackwell from Cooperband and Cardé 2006b, p. 24). 
trap. It seems likely that capture rates are contingent in part on how long a given mosquito spends in the vicinity of the trap's effective area of suction: the longer it lingers, the greater the chance its meandering flight will take it into the region of 'no return'. Similar capture inefficiencies apply to other mosquito species with different types of traps, as shown by Torr et al. (2008), described in the next section, although much remains to be determined.

Adding additional odours such as 1-octen-3-ol to carbon dioxide can improve trap catch for some mosquitoes (e.g. Takken and Kline 1989), but the reasons for this are speculative. Such compounds may lower the threshold for attraction, thereby extending the downwind reach (active space) of the plume. These compounds also may act additively or synergistically with carbon dioxide, evoking a response in a higher proportion of mosquitoes. Both of these effects would act at a distance and increase the number of mosquitoes lured to the trap. Alternatively, these compounds may add to time spent near the trap, thereby increasing the chance that a circuitous flight path will intersect the area of suction, and so elevate the probability of capture. At our present state of understanding it is difficult to know which of these alternatives may apply to compounds that can augment trap catch over carbon dioxide alone. It is also noteworthy that, with few exceptions, these chemicals typically provide only a modest increase in catch when added to carbon dioxide: 1-octen-3-ol, for example, doubled or tripled catches of Aedes species, for example, whereas for some Culex species it can depress the catch (Kemme et al. 1993, Van Essen et al. 1994). Curiously, unbaited suction traps captured no more Culex species than traps baited with 1-octen-3-ol (Mboera et al. 2000, Qiu et al. 2007b).

\section{Field trapping with electric nets (E-nets) and odour-baited entry traps (OBETs)}

One approach to establishing which odours mediate attraction at long range and close to the odour source is to use electric nets set a distances of interest, as has been used by Vale and colleagues with great success in dissecting the orientation responses of tsetse to odours and visual cues (e.g. Vale 1982). E-nets are designed to intercept tsetse passively, and can be used to establish details of the approach to an odour source and the relative numbers of flies arriving at different olfactory and/or visual baits without the need for any additional behavioural responses that are normally required to catch tsetse as they arrive at an odour source, such as flying into a trap, or landing on a target. E-nets are proving to be useful for similar purposes with mosquitoes (Knols et al. 1998, Torr 1994, Torr et al. 2008).

Another device that is useful for assaying the responses of anthropophilic mosquitoes to host cues is the odour-baited entry trap (OBET), which is intended to simulate the odour plume emanating from a human dwelling and permits mosquitoes to enter the trap via a window-sized port (Costantini et al. 1993). Entering an OBET is assumed to be equivalent to house-entering behaviour and it serves as a proxy for an open window. OBET entry differs, however, in one respect from house entry: the odour plume from an OBET would be relatively homogeneous in structure, because it is driven by a fan, exiting at a velocity of about $0.5 \mathrm{~m} / \mathrm{s}$ from the port. Artificial 'smoke' was used to establish the patterns of odour dispersing from experimental 'huts' that were designed to typify entry points of human dwellings in West Africa. The issuing of this visual surrogate for odour suggested a more intermittent plume structure (Snow 1987) than the fan-generated, homogeneous outflow from OBETs.

Torr et al. (2008) have combined the E-net and OBET techniques to compare the orientation of An. arabiensis and An. quadriannulatus in the field. Such a comparative approach measures two successive anthropophilic behaviours involved in orientation: attraction and house-entering. It 
can be used to compare odour sources such as carbon dioxide with or without added odours and also natural baits such as cattle or humans. In these comparisons it was clear that the propensity to be attracted to the vicinity of an odour source did not always correlate with house entering. For example, An. arabiensis was equally attracted to the vicinity of human and cattle baits, but was far more apt to enter an OBET baited with human odour. This finding indicates that there may be an interaction between the response to a particular host odour and the house-entry response. Although this observation has not been fully explored, it goes some way to explaining why this species is paradoxically the second most important vector of malaria in sub-Sahara Africa and yet is considered to be significantly less anthropophilic than its sibling species, An. gambiae s.s.; the human blood index (proportion of blood meals of human origin) is virtually $100 \%$ for $A n$. gambiae s.s in most of areas, but varies for An. arabiensis across its distribution, from $80-100 \%$ in western Africa, to $7-60 \%$ in eastern Africa and $<10 \%$ in Madagascar (Garrett-Jones et al. 1980). In field studies in Ethiopia, Tirados et al. (2006) found the An. arabiensis population inherently to be anthropophagic, but this was counterbalanced by its tendency to blood feed outdoors and remain outdoors after feeding, where it mainly came into contact with cattle at night. The disparity is presumably indicative of differential odour suites mediating attraction from a distance and houseentering. Of course, this also reveals that we do not have the observational tools necessary to fully understand what mediates the full sequence of host-finding behaviours.

\section{How far do mosquitoes travel to locate hosts?}

'Dispersal studies', generally conducted by mark-release-recapture experiments, provide some insights into how far mosquitoes travel during the course of their 'search' for resources such as blood-meal hosts and oviposition sites. Obviously, mark-release-recapture studies can only assess the summation of all distances travelled from the time of release to capture, and provide no information about the route taken by individuals that are captured or the cues that guided this movement. 'Dispersion' in the sense used here refers to flight that carries mosquitoes away from a release point, either guided by olfactory cues, wind direction and environmental features or non-directed flight (i.e. random with respect to environmental stimuli). Across the range of species and habitats that have been investigated, species fall into several categories with respect to measured patterns of dispersal. At one extreme, salt marsh breeding species are known to fly kilometres away from breeding sites. There is evidence that this may be true migratory flight; they appear to be unresponsive to host stimuli, flying too high to detect host-odour plumes and they are generally carried by the wind (Silver 2008). Anopheles gambiae has been shown to disperse from breeding sites for hundreds of metres to kilometres, depending on environmental conditions. Where breeding sites are closely associated with human dwellings, they disperse less far than in areas where they breed in large irrigated fields at a distance from villages (Gillies 1988, Takken et al. 1998). It is not known to what extent these flights are migratory vs. directed flight in response to host stimuli. Finally, the maximum distance that Ae. aegypti appears to disperse is on the order of hundreds of metres, probably because they tend to breed in relatively high density urban habitats, where the main resources required for blood feeding and breeding occur in high density (Maciel-de-Freitas 2010, Reiter et al. 1995, Scott et al. 2000) Rather than providing much insight into the mechanism by which mosquitoes locate hosts, dispersal studies may be best used to establish the density of species-specific resources in localised areas, and to indicate something about how far and in what direction with respect to environmental features mosquitoes need to fly before they encounter a resource-rich area that arrests further flight. 


\section{Discussion}

The mechanism of host location in mosquitoes over distances in the range of tens of metres (beyond visual range) is thought to be confined to tracking a plume of wind-borne host odour upwind toward the odour source by optomotor anemotaxis. At close range in still or nearly still air (as would occur in a human dwelling) a mosquito might orient by klinokinesis, using changes in odour concentration, or following convection current temperature gradients to steer toward the host, but these mechanisms are so far unverified. Gross visual cues from the host also could serve as collimating cues at ranges of ten or fewer metres and if the mosquito enters the host odour plume, both cues may be used in orientation. Near the host, the final stage of orientation and then landing may be governed by visual and odour cues combined with host-produced heat and humidity. In still air, orientation may involve downward flight along heat-convected odour plumes. The probable and presumed most common navigational mechanisms for host orientation, categorised by distance from the host and the presumed sensory inputs governing these manoeuvres, are summarised in Table 1.

There is enormous variation in conditions under which orientation occurs, contingent on habitat, how wind disperses the plume, time of day or night and associated light levels, availability of visual cues from the host, and host movement. The rate of carbon dioxide emission varies with host size and the odour profiles of acceptable hosts vary among individuals of the same species. In humans there is considerable individual variation in odour-based attractiveness (e.g. Lindsay et al. 1993, Logan et al. 2008), but how such variability affects mosquito orientation is not well understood.

Table 1. A simplified overview of the orientation mechanisms and their probable sensory inputs used by female mosquitoes for location of a human-sized host. At distances of $\approx 1$ to $10 \mathrm{~m}$ from the host, orientation to hovering range can be mediated by solely by visual cues (a gradual expansion of the host fixed in the frontal visual field) and the mosquito need not be in contact with the plume of host odour. Hovering near the host may include orientation to specific body regions that are particularly favoured by given species. These simplifications do not account for other sequences, such as orientation to host odour in still air or orientation to the host's habitat (such as a human dwelling) with a delay in biting. Mechanosensory input is also important to maintenance of upwind movement.

\begin{tabular}{|c|c|c|c|c|}
\hline & \multicolumn{4}{|l|}{ Distance } \\
\hline & $>10 \mathrm{~m}$ & $\approx 10$ to $1 \mathrm{~m}$ & $\approx 1 \mathrm{~m}$ & host contact \\
\hline Odour cues & fluctuating $\mathrm{CO}_{2}$ & $\begin{array}{l}\text { fluctuating } \mathrm{CO}_{2}+ \\
\text { other kairomones }\end{array}$ & $\begin{array}{l}\text { fluctuating } \mathrm{CO}_{2} \text { ? + } \\
\text { other kairomones } \\
\text { heat? humidity? }\end{array}$ & $\begin{array}{l}\text { fluctuating } \mathrm{CO}_{2} \text { ? } \\
\text { kairomones inducing } \\
\text { landing? } \\
\text { heat? humidity? }\end{array}$ \\
\hline Behavioural output & upwind anemotaxis & upwind anemotaxis & hovering near host & landing on host \\
\hline Visual input & $\begin{array}{l}\text { front-to-rear flow } \\
\text { of downward visual } \\
\text { field }\end{array}$ & $\begin{array}{l}\text { front-to-rear flow } \\
\text { of downward visual } \\
\text { field }\end{array}$ & $\begin{array}{l}\text { steady size of large } \\
\text { frontal visual field }\end{array}$ & $\begin{array}{l}\text { rapid expansion of } \\
\text { frontal visual field }\end{array}$ \\
\hline Visual cues & visual surround & host body & host body & host body \\
\hline
\end{tabular}


Much of what is known about the plume-following manoeuvres of female mosquitoes has been inferred from assays aimed, not at defining orientation mechanisms, but rather designed to aid in the identification of the host-emitted volatiles mediating attraction. Do these bioassays monitor responses involved in orientation? If not, are behaviourally active compounds being overlooked? As responses may be contingent on aspects of the physiological state of the mosquitoes used in the assay which have not been fully explored, such as distance flown and duration of exposure to host cues, and characteristics of stimuli presentation, such as concentration, odour plume structure, presence of carbon dioxide, particular odour mixtures and their ratios, devising diagnostic bioassays is likely to remain a difficult task.

In other odour-based orientation systems in insects, some of these questions are answered with comparative ease. The pheromone blend emitted by female moths is, for example, relatively simple, comprising one to four constituents in nearly all species. All stages of response, from the initial detection of the pheromone plume at a distance to contact with the female appear governed by the entire pheromone blend, which is assumed to vary little because of stabilising selection. In mosquitoes, the initial detection of the host odour plume may involve only carbon dioxide or it may involve carbon dioxide plus additional host-emitted odours. Close to the host, these added odours may be more significant in evoking specific behaviours, such as habitat finding (pre-attack resting sites), house-entering, and landing, as was found to be the case with the variability in response of tsetse to different ratios and concentrations of known attractants in field studies (Brady and Griffiths 1993). In contrast to moths where the pheromone signal is relatively simple and invariant, the odour profile of mosquito hosts is spectacularly variable and involves hundreds of potential components, adding to the difficulty of establishing which odour constituents contribute to orientation.

\section{Future studies}

Much of what we know about mosquito orientation to hosts and the odours they emit is based on studies of a handful of model species, often from colonies reared in the laboratory for decades, and mainly of species that vector human pathogens. Future studies should validate behavioural findings with wild mosquitoes to be sure we are not misguided by behavioural artefacts of colonisation. A wide range of approaches to exploring host location can be undertaken in the field and in the laboratory. First, in wind-tunnel and still-air bioassays of flight along odour plumes, it is possible to control features of plume structure and air movement while monitoring mosquito movement in 3-D with video. With such bioassays it is also possible to introduce additional hostrelated cues thought to contribute to orientation: heated surfaces, humidity gradients, and visual objects. Although the characteristics of plume structure can be measured at fixed points in a wind tunnel (e.g. Justus et al. 2002, Dekker et al. 2005), because odour intensity fluctuates at any given spatial position, it is not possible to specify how instantaneous odour input matches a particular orientation manoeuvre. Nonetheless, it is possible to experimentally control the overall structure of the odour plume and then determine when the mosquito is clearly within or outside it, although the plume's ragged boundary will remain an area of uncertainty. When a mosquito enters an odour plume, its change in orientation may be attributed to odour contact.

Identifying the odour stimuli evoking close approach to the host and alighting may require new, specialised bioassays (e.g. Healy and Copeland 2000, Healy et al. 2002). Different mosquito species appear to select favoured body regions of humans for biting (De Jong and Knols 1996), possibly cueing in on region-specific odours or their interaction with the carbon dioxide plume and other breath constituents. Many kinds of sensory input are available in close proximity to the host - 
increased humidity, heat, and visual cues, adding to the challenge of bioassay design. All of these factors appear to be enough to guide mosquitoes to a host in virtually still air, apart from the air currents generated by convection, which also appears to be important to selection of a biting site (Dekker et al. 1998). Further studies are warranted on the behaviour of mosquitoes in still air in the presence of this suite of stimuli to understand more fully the mechanisms of host orientation within enclosed spaces.

A second approach is to document manoeuvres in the field. This has the obvious advantage of observing mosquitoes behaving under natural circumstances. This technique is technically challenging, however, because mosquitoes are small and it is difficult to accurately monitor their manoeuvres, in part because in the field the background is visually heterogeneous. If more than one species is present, it may be impossible to know which species is being observed. Large, field wind tunnels (Cooperband et al. 2006a,b) offer a bridge to observing freely flying mosquitoes in a relatively natural environment, but the relatively laminar flow of wind and the plume structure that is generated may not be fully reflective of the patterns that are encountered under natural conditions, and of course, the distance to be travelled is limited by the size of the tunnel. Moreover, whereas it is possible in a wind tunnel setting to specify where the odour plume's boundaries are relative to a manoeuvring mosquito, in the field, it is difficult to establish precisely when a mosquito is in contact with the odour plume. Nonetheless, plume structure is clearly a relevant cue and one that future work should emphasise.

Another approach is to monitor the orientation manoeuvres in field by E-nets and OBETs (Torr et al. 2008). These techniques allow documentation of orientated responses ('attraction') to a live host, to odour sources of carbon dioxide, or to synthetic kairomones. Attraction can be compared to entry into dwellings baited with odours or hosts, as measured with OBETs. These responses can differ dependent on how the odour source is presented. In other words, for a given species, what appears to 'attractive' with host as the odour source may prove less so when measured with an OBET. One unresolved concern with OBETs is that the odour plumes they generate may not mimic the structure of naturally fenestrated odour plumes.

Identification of the suite of natural host odours inducing attraction and other host-directed behaviours would greatly facilitate our ability to carry out studies of the mechanisms of orientation. For most of their lives female mosquitoes cycle between responding to host cues, resting-site cues while they digest blood and eggs develop, and oviposition cues. Now that the genomes of $A n$. gambiae s.S, Ae. aegypti and Culex pipiens L. have been sequenced, it will be possible to investigate correlations between gene expression and behaviour throughout the gonotrophic cycle, enabling the identification of the proteins involved with odour detection, which may in turn help to identify particular odorants to which mosquito sensory systems are primed to be most responsive to during each physiological stage (see Chapter 2, this volume).

\section{Acknowledgments}

We are very grateful to Steve Schofield for his comments on this review.

\section{References}

Acree, F, Turner R, Grouck HK, Beroza M and Smith N (1968) L-lactic acid: a mosquito attractant isolated from humans. Science 161: 1346-1347. 
Barott HG and Pringle EM (1946) Energy and gaseous metabolism of the chicken from hatch to maturity as affected by temperature. J Nutr 31: 35-50.

Bernier UR, Kline DL, Barnard DR, Schreck CE and Yost RA (2000) Analysis of human skin emanations by gas chromatography/mass spetrometry. 2. Identification of volatile compounds that are candidate attractants for the yellow fever mosquito (Aedes aegypti). Anal Chem 72: 747-756.

Bidlingmayer WL (1971) Mosquito flight paths in relation to the environment. 1. Illumination levels, orientation, and resting areas. Ann Entomol Soc Am 64: 1121-1131.

Bidlingmayer WL and Hem DG (1979) Mosquito (Diptera: Culicidae) flight behaviour near conspicuous objects. Bull Entomol Res 69: 691-700.

Bidlingmayer WL and Hem DG (1980) The range of visual attraction and the effect of competitive visual attractants upon mosquito (Diptera: Culicidae) flight. Bull Entomol Res 70: 321-342.

Bidlingmayer WL and Hem DG (1981) Mosquito flight paths in relation to the environment. Effect of the forest edge upon trap catches in the field. Mosq News 41: 55-59.

Bosch OJ, Geier M and Boeckh J (2000) Contribution of fatty acids to olfactory host finding of female Aedes aegypti. Chem Senses 25: 323-330.

Brady JN (1975) Circadian changes in central excitability - The origin of behavioural rhythms in tsetse flies and other animals? J Entomol (A) 50: 79-95.

Brady J and Griffiths N (1993) Upwind flight responses of tsetse flies (Glossina spp.) (Diptera: Culicidae) to acetone, octenol and phenols in nature: a video study. Bull Entomol Res 83: 329-333.

Brady J, Gibson G and Packer MJ (1989) Odour movement, wind direction and the problem of host-finding by tsetse flies. Physiol Entomol 14:369-380.

Braks MAH, Meijerink J and Takken W (2001) The response of the malaria mosquito, Anopheles gambiae, to two components of human sweat, ammonia and L-lactic acid, in an olfactometer. Physiol Entomol 26: 142-148.

Budick SA, Reiser MB and Dickinson MH (2007) The role of visual and mechanosensory cues in structuring forward flight in Drosophila melanogaster. J Exp Bio 210: 4092-4103.

Cardé RT and Willis MA (2008) Navigational strategies used by insects to find distant, wind-borne sources of odor. J Chem Ecol 34: 854-866.

Chiba Y, Shinkawa Y, Yoshii M, Matsumoto A, Tomioka K and Takahashi SY (1992) A comparative study on insemination dependency of circadian activity pattern in mosquitoes. Physiol Entomol 17: 213-218.

Clements AN (1999) The biology of mosquitoes, vol 2. CAB International, Wallingford, UK.

Colvin J, Brady J and Gibson G (1989) Visually guided, upwind turning behaviour of free-flying tsetse flies in odour-laden wind: a wind-tunnel study. Physiol Entomol 14,: 31-39.

Cooperband MF and Cardé RT (2006a) Comparison of plume structures of carbon dioxide emitted from different mosquito traps. Med Vet Entomol 26: 1-10.

Cooperband MF and Cardé RT (2006b) Orientation of Culex mosquitoes to carbon dioxide-baited traps: flight manoeuvres and trapping efficiency. Med Vet Entomol 26: 11-26.

Cooperband MF, McElfresh JS, Millar JG and Cardé RT (2008) Attraction of Culex quinquefasciatus Say (Diptera: Culicidae) to odors from chicken feces. J Insect Physiol 54: 1182-1194.

Corbet PS and Smith SM (1974) Diel periodicities of landing of nulliparous and parous Aedes aegypti (L.) at Dar es Salaam, Tanzania (Diptera: Culicidae) Bull Entomol Res 64: 111-121.

Cork A and Park KC (1996). Identification of electrophysiologically-active compounds for the malaria mosquito, Anopheles gambiae, in human sweat extracts. Med Vet Entomol 10: 269-276.

Costantini C, Gibson G, Brady J, Merzagora L and Coluzzi M (1993) A new odour-baited trap to collect host-seeking mosquitoes. Parassitologia 35: 5-9.

Costantini C, Gibson G, Sagnon N'F, della Torre A, Brady J and Coluzzi M. (1996) Mosquito responses to carbon dioxide in a West African Sudan savanna village. Med Vet Entomol 10:220-227.

Costantini C, Birkett MA, Gibson G, Ziesmann J, Sagnon N'F, Mohammed HA, Coluzzi M and Pickett JA (2001) Electroantennogram and behavioural responses of the malaria vector Anopheles gambiae to human-specific sweat components. Med Vet Entomol. 15: 259-266. 
Craig GB (1967) Mosquitoes: female monogamy induced by male accessory gland substances. Science 156: 1499-1501. David CT, Kennedy JS, Ludlow AR, Perry JN and Wall C (1982) A reappraisal of insect flight toward distant point source of wind-borne odor. J Chem Ecol 8: 1207-1215.

Davis EE and Sokolove PG (1975) Temperature responses of antennal receptors of the mosquito, Aedes aegypti. J Comp Physiol 96: 223-236.

Daykin PN (1967) Orientation of Aedes aegypti in vertical air currents. Can Entomol 99: 303-308.

De Jong R and Knols BGJ (1996) Selection of biting sites by mosquitoes. In: Block GR and Cardew G (eds) Olfaction in mosquito-host interactions. Ciba Foundation Symposium 200, John Wiley and Sons, Chichester, UK, pp 89-103.

Dekker T (2002) Influence of plume structure, composition and concentration on orientation behavior of mosquitoes to host odors. PhD thesis, University of California, Riverside, USA.

Dekker T, Takken W, Knols BGJ, Bouman E, van de Laak S, de Bever A and Huisman PWT (1998) Selection of biting sites on a human by Anopheles gambiae s.s., An. arabiensis and An. quadriannulatus. Entomol Exp Appl 87: 295-300.

Dekker T, Takken W and Cardé RT (2001) Structure of host-odour plumes influences catch of Anopheles gambiae s.s. and Aedes aegypti in a dual-choice olfactometer. Physiol Entomol 26: 124-134.

Dekker T, Steib B, Cardé RT and Geier M (2002). L-Lactic acid: a human-signifying host cue for the anthropophilic mosquito Anopheles gambiae. Med Vet Entomol 16: 91-98.

Dekker T, Geier M and Cardé RT (2005) Carbon dioxide instantly sensitizes female yellow fever mosquitoes to human skin odours. J Exp Biol 208: 2963-2972.

Eiras AE and Jepson PC (1991) Host location by Aedes aegypti (Diptera: Culicidae): a wind tunnel study of chemical cues. Bull Entomol Res 81: 151-160.

Eiras AE and Jepson PC (1994) Responses of Aedes aegypti (Diptera: Culicidae) to host odours and convection currents using an olfactometer bioassay. Bull Entomol Res 84: 207-211.

Elkinton JS, Schal C, Ono T and Cardé RT (1987) Pheromone puff trajectory and upwind flight of male gypsy moths in a forest. Physiol Entomol 12: 399-406.

Fry SN, Rohrseitz N, Straw AD and Dickinson MH (2009) Visual control of flight speed in Drosophila melanogaster. J Exp Biol 212: 1120-1130.

Garrett-Jones C, Boreham PFL and Pant CP (1980) Feeding habits of anophelines (Diptera: Culicidae) in 1971-78, with reference to the human blood index: a review. Bull Entomol Res 70: 165-185.

Geier M, Bosch OJ and Boeckh J (1999a) Influence of odour plume structure on upwind flight of mosquitoes towards hosts. J Exp Biol 202: 1639-1648.

Geier M, Bosch OJ and Boeckh J (1999b) Ammonia as an attractive component of host odour for the yellow fever mosquito, Aedes aegypti. Chem Senses 24: 1-7.

Gibson, G. (1983) The swarming behaviour of a mosquito. PhD thesis, University of Sussex, UK.

Gibson $G$ (1995) A behavioural test of the sensitivity of a nocturnal mosquito, Anopheles gambiae, to dim white, red and infra-red light. Physiol Entomol 20: 224-228.

Gibson G and Brady J (1985) 'Anemotactic' flight paths of tsetse flies in relation to host odour: a preliminary video study in nature of the response to loss of odour. Physiol Entomol 10:395-406.

Gibson G and Brady J (1988) Flight behaviour of tsetse flies in host odour plumes: the initial response to leaving or entering odour. Physiol Entomol 13: 29-42.

Gibson G and Torr SJ (1999) Visual and olfactory responses of haematophagous Diptera to host stimuli. Med Vet Entomol 13: 2-23.

Gibson G and Young S (1991) The optics of tsetse fly eyes in relation to their behaviour and ecology. Physiol Entomol 16: 273-282.

Gibson G, Packer M, Steullet P and Brady J (1991) Orientation of tsetse flies to wind within and outside host odour plumes in the field. Physiol Entomol 16: 47-56.

Gilbert C and Kuenen LPS (2008) Multimodal integration: visual cues help odor-seeking flies. Curr Biol 18: R295-R297.

Gillett JD (1979) Out for blood: flight orientation up-wind in absence of visual clues. Mosq News 39: 221-229.

Gillies MT (1980) The role of carbon dioxide in host-finding by mosquitoes (Diptera: Culicidae): a review. Bull Entomol Res 80: 525-532. 
Gillies MT (1988) Anopheline mosquitoes: vector behaviour and bionomics. In: Wernsdorfer WH and Gregor IM (eds) Malaria: principles and practice of malariology. Churchill and Livingstone, Edinburgh, UK, pp 453-485.

Gillies MT and Wilkes TJ (1969) A comparison of the range of attraction of animal baits and of carbon dioxide for some West African mosquitoes. Bull Entomol Res 59: 441-456.

Gillies MT and Wilkes TJ (1970) The range of attraction of single baits for some West African mosquitoes. Bull Entomol Res 60: 225-235.

Gillies MT and Wilkes TJ (1972) The range of attraction of animal baits and carbon dioxide for mosquitoes. Bull Entomol Res 61: 389-404.

Gillies MT and Wilkes TJ (1974) Evidence for downwind flight by host-seeking mosquitoes. Nature 252: 388-389.

Gillies MT and Wilkes TJ (1978) The effect of high fences on the dispersal of some West African mosquitoes (Diptera: Culicidae). Bull Entomol Res 68: 401-408.

Grant AJ and O'Connell RJ (1996) Electrophysiological responses from receptor neurons in mosquito maxillary palp sensilla. In: Block GR and Cardew G (eds) Olfaction in mosquito-host interactions. Ciba Foundation Symposium 200, John Wiley and Sons, Chichester, UK, pp 233-248.

Hardie J, Gibson G and Wyatt TD (2001) Insect behaviours associated with resource finding. In: Woiwod IP, Reynolds $\mathrm{DR}$ and Tomas CD (eds) Insect movement: mechanisms and consequences. CAB International, Wallingford, UK, pp 87-109.

Healy TP and Copeland MJW (1995) Activation of Anopheles gambiae mosquitoes by carbon dioxide and human breath. Med Vet Entomol 9: 331-336.

Healy TP and Copeland MJW (2000) Human sweat and 2-oxopentanoic acid elict a landing response from Anopheles gambiae. Med Vet Entomol 14: 195-200.

Healy TP, Copeland MJW, Cork A, Przyborowska A and Halket JM (2002) Landing responses of Anopheles gambiae elicited by oxocarboxylic acids. Med Vet Entomol 16: 126-132.

Jones MDR and Gubbins SJ (1978) Changes in the circadian flight activity of the mosquito Anopheles gambiae in relation to insemination, feeding and oviposition. Physiol Entomol 3: 213-220.

Jones MDR and Gubbins SJ (1979) Modification of female flight activity by a male accessory gland pheromone in the mosquito Culex pipiens quinquefasciatus. Physiol Entomol 4, 345-351.

Justus KA, Murlis J, Jones C and Cardé RT (2002) Measurement of odor-plume structure in a wind tunnel using a photoionization detector and a tracer gas. Environ Fluid Mech 2: 115-142.

Kemme JA, Van Essen PHA, Ritchie SA and Kay BH (1993) Response of mosquitoes to carbon dioxide and 1-octen-3-ol in Southeast Queensland, Australia. J Am Mosq Control Assoc 9: 431-435.

Kennedy JS (1940) The visual responses of flying mosquitoes. Proc Zool Soc Lond Ser A 109, 221-242.

Kennedy JS (1977). Behaviorally discriminating assays of attractants and repellents. In: Shorey HH and McKelvey JJ, Jr (eds) Chemical control of insect behavior. Wiley Interscience, New York, USA, pp 215-229.

Kennedy JS (1986) Some current issues in orientation to odour sources. In: Payne TL, Birch MC, and Kennedy CEJ (eds) Mechanisms in insect olfaction. Clarendon Press, Oxford, UK, pp 11-25.

Kennedy JS and Marsh D (1974) Pheromone-regulated anemotaxis in flying moths. Science 184: 999-1001.

Knols, BGJ, Mboera LEG and Takken W (1998) Electric nets for studying odour-mediated host-seeking behaviour of mosquitoes. Med Vet Entomol 12: 116-120.

Kuenen LPS and Baker TC (1983) A non-anemotactic mechanism used in pheromone source location by flying moths. Physiol Entomol 8: 277-289.

Kuenen LPS and Cardé RT (1994) Strategies for contacting a lost pheromone plume: casting and upwind flight in the male gypsy moth. Physiol Entomol 19: 15-29.

Land MF, Gibson G, Horwood J and Zeil J (1999) Fundamental differences in the optical structure of the eyes of nocturnal and diurnal mosquitoes. J Comp Physiol [A] 185: 91-103.

Lindsay SW, Adiamah H, Miller JE, Pleass RJ and Armstrong JRM (1993) Variation in attractiveness of human-subjects to malaria mosquitoes (Diptera: Culicidae) in The Gambia. J Med Entomol 30: 368-373. 
Logan JG, Birkett MA, Clark SJ, Powers S, Seal NJ, Wadhams LJ, Mordue Luntz AJ and Pickett JA (2008) Identification of human-derived volatile chemicals that interfere with attraction of Aedes aegypti mosquitoes. J Chem Ecol 34: 308-322.

Maciel-de-Freitas R, Souza-Santos R, Codeco, CT and Lourenco-de-Oliveira R (2010) Influence of the spatial distribution of human hosts and large size containers on the dispersal of the mosquito Aedes aegypti within the first gonotrophic cycle. Med Vet Entomol 24: 24:74-82.

Mafra-Neto A and Cardé RT (1994) Fine-scale structure of pheromone plumes modulates upwind orientation of flying moths. Nature 369: 142-144.

Maimon G, Straw AD and Dickinson MH (2008) A simple vision-based algorithm for decision making in flying Drosophila. Curr Biol 18: 463-470.

Mboera LEG and Takken W (1997) Carbon dioxide chemotrophism in mosquitoes (Diptera: Culicidae) and its potential in vector surveillance and management programmes. Rev Med Vet Entomol 85: 355-368.

Mboera LEG, Knols BGJ, Takken W and Huisman PWT (1998) Olfactory responses of female Culex quinquefasciatus Say (Diptera: Culicidae) in a dual-choice olfactometer. J. Vector Ecol 23: 107-113.

Mboera LEG, Takken W and Sambu EZ (2000) The response of Culex quinquefasciatus (Diptera: Culicidae) to traps baited with carbon dioxide, 1-octen-3-ol, acetone, butyric acid and human foot odour in Tanzania. Bull Entomol Res 90: 155-159.

Muir LE, Kay BH and Thorne MJ (1992) Aedes aegypti (Diptera: Culicidae) vision: response to stimuli from the optical environment. J Med Entomol 29: 455-450.

Murlis J and Jones CD (1981) Fine-scale structure of odour plumes in relation to distant pheromone and other attractant sources. Physiol Entomol 6: 71-86.

Murlis J, Elkinton JS and Cardé RT (1992) Odor plumes and how insects use them. Annu Rev Entomol 37: 505-532.

Pates HV, Takken W, Stuke K and Curtis CF (2001) Differential behaviour of Anopheles gambiae sensu stricto (Diptera Culicidae) to human and cow odours in the laboratory. Bull Entomol Res 91: 289-296.

Pates HV, Takken W and Curtis CF (2005) Laboratory studies on the olfactory behaviour of Anopheles quadriannulatus. Entomol Exp Appl 114: 153-159.

Puri SN, Mendki MJ, Sukumaran D, Ganesan K, Prakash S and Sekhar K (2006) Electroantennogram and behavioral responses of Culex quinquefasciatus (Diptera: Culicidae) females to chemicals found in human skin emanations. J Med Entomol 43: 207-213.

Qiu YT, Spitzen J, Smallegange RC and Knols BGJ (2007a) Monitoring systems for adult insect pests and disease vectors. In: Takken W and Knols BGJ (eds) Emerging pests and vector-borne diseases in Europe. Ecology and control of vector-borne diseases, vol 1. Wageningen Academic Publishers, Wageningen, the Netherlands, pp 329-353.

Qiu YT, Smallegange RC, Ter BC, Spitzen J, Van Loon JJ, Jawara M, Milligan P, Galimard AM, Van Beek TA, Knols BG and Takken W (2007b) Attractiveness of MM-X traps baited with human or synthetic odor to mosquitoes.(Diptera: Culicidae) in The Gambia. J Med Entomol 44: 970-983.

Reeves WC (1953) Quantitative field studies on a carbon dioxide chemotrophism of mosquitoes. Am J Trop Med Hyg 2: 325-331.

Reiter P, Amador MA, Anderson RA and Clark GG (1995) Dispersal of Aedes aegypti in an urban area after blood-feeding as demonstrated by rubidium-marked eggs. Am J Trop Med Hyg 52: 177-179.

Rowland M (1989) Changes in the circadian flight activity of the mosquito Anopheles stephensi associated with insemination, blood-feeding, oviposition and nocturnal light intensity. Physiol Entomol 14:77-84.

Rudolfs W (1922) Chemotrophism of mosquitoes. Bull NJ Agric Exp Stn 367: 4-23.

Sabelis MW and Schippers P (1984) Variable wind directions and anemotactic strategies of searching for an odour plume. Oecologica 63: 225-228.

Scott TW, Morrison AC. Lorenz LH, Clark GG, Strickman D, Kittayapong P, Zhou H and Edman JD (2000) Longitudinal studies of Aedes aegypti (Diptera: Culicidae) in Thailand and Puerto Rico: population dynamics. J. Med. Entomol 37: 77-88.

Shutt B, Stables L, Aboagye-Antwi F, Moran J and Tripet F (2010) Male accessory gland proteins induce female monogamy in anopheline mosquitoes. Med Vet Entomol 24: 91-94. 
Silver JB (2008) Mosquito Ecology, $3^{\text {rd }}$ edn. Springer Science+Business Media B.V., Dordrecht, The Netherlands.

Smith A (1958) Outdoor cattle feeding and resting of A. gambiae Giles and A. pharoensis Theo. in the Pare-Taveta area of East Africa. East African Med J 35: 559-567.

Snow WF (1987) Studies of house-entering habits of mosquitoes in The Gambia, West Africa: experiments with prefabricated huts with varied wall apertures. Med Vet Entomol 1: 9-21.

Spitzen J, Smallegange RC and Takken W (2008) Effect of human odours and positioning of $\mathrm{CO}_{2}$ release point on trap catches of the malaria mosquito Anopheles gambiae sensu stricto in an olfactometer. Physiol Entomol 33: 116-122.

Sutcliffe JF (1987) Distance orientation of biting flies to their hosts. Insect Sci Applic 8: 611-616

Takken W (1991) The role of olfaction in host-seeking of mosquitoes: a review. Insect Sci Applic 12: 287-295.

Takken W and Kline DL (1989) Carbon dioxide and 1-octen-3-ol as mosquito attractants. J Am Mosq Contr Assoc 5: 311-316.

Takken W, Charlwood DJ, Billingsley PF and Gort G (1998) Dispersal and survival of Anopheles funestus and A.gambiae s.l. (Diptera: Culicidae) during the rainy season in southeast Tanzania. Bull Entomol Res 88: 561-566.

Takken W and Knols BGJ (1999) Odor-mediated behavior of Afrotropical malaria mosquitoes. Annu Rev Entomol 44: 131-157.

Takken W, Dekker T and Wijnholds YG (1997) Odor-mediated flight behavior of Anopheles gambiae Giles sensu stricto and An. stephensi Liston in response to $\mathrm{CO}_{2}$, acetone, and 1-octen-3-ol. J Insect Behav 10: 395-407.

Tirados I, Costantini C, Gibson G and Torr SJ (2006) Blood-feeding behaviour of the malarial mosquito Anopheles arabiensis: implications for vector control. Med Vet Entomol 20: 425-437.

Torr SJ (1988) The activation of resting tsetse flies (Glossina) in response to visual and olfactory stimuli in the field. Physiol Entomol 13: 315-325.

Torr SJ (1994) The tsetse (Diptera: Glossinidae) story: implications for mosquitoes. J Am Mosq Contr Assoc 10: 258-265.

Torr SJ and Mangwiro TNC (1996) Upwind flight of tsetse (Glossina spp) in response to natural and synthetic host odour in the field. Physiol Entomol 21: 143-150.

Torr SJ, Della Torre A, Calzetta M, Costantini C and Vale GA (2008) Towards a fuller understanding of mosquito behaviour: use of electrocuting grids to compare the odour-orientated responses of Anopheles arabiensis and An, quadriannulatus in the field. Med Vet Entomol 22: 93-108.

Trpis M and Hausermann W (1978) Genetics of house-entering behaviour in East African populations of Aedes aegypti (L.) (Diptera: Culicidae) and its relevance to speciation. Bull Entomol Res 68: 521-532.

Vale GA (1982) The trap orientated behaviour of tsetse flies (Glossinidae) and other Diptera. Bull Entomol Res 72: 71-93.

Van Essen PHA, Kemme JA, Ritchie SA and Kay BH (1994) Differential responses of Aedes and Culex mosquitoes to octenol or light in combination with carbon dioxide in Queensland, Australia. Med Vet Entomol 8: 63-67.

Vickers NJ and Baker TC (1994) Reiterative responses to single strands of odor promote sustained upwind flight and odor source location by moths. PNAS 91: 5756-5760.

Warnes ML (1995) Field studies on the effects of cattle skin secretion on the behaviour of tsetse. Med Vet Entomol 9: 284-288.

Williams B (1994) Models of trap seeking by tsetse flies: anemotaxis, klinotaxis and edge detection. J Theor Biol 168: 105-115.

Willis MA and Cardé RT (1990) Pheromone-modulated optomotor response in male gypsy moths, Lymantria dispar L.: upwind flight in a pheromone plume in different wind speeds. J Comp Physiol A 167: 699-706.

Willis MA, David CT, Murlis J and Cardé RT (1994) Effects of pheromone plume structure and visual stimuli on the pheromone-modulated upwind flight of male gypsy moths (Lymantria dispar L.) in a forest. J Insect Behav 7: 385-409.

Wright RH and Kellogg FE (1962) Responses of Aedes aegypti to moist convection currents. Nature 194: 402-403.

Wyatt TD, Phillips ADG and Grégoire J-C (1993) Turbulence, trees and semiochemicals: wind-tunnel orientation of the predator, Rhizophagus grandis to its barkbeetle prey, Dendroctonus micans. Physiol Entomol 18: 204-210.

Zanen PO, Sabelis MW, Buonaccorsi JP and Cardé RT (1994) Search strategies of fruit flies in steady and shifting winds in the absence of food odours. Physiol Entomol 19: 335-341.

Zöllner GE, Torr SJ, Ammann C and Meixner FX (2004) Dispersion of carbon dioxide plumes in African woodland: implication for host-finding by tsetse flies. Physiol Entomol 29: 381-394. 



\title{
7. Host-seeking behaviour of mosquitoes: responses to olfactory stimuli in the laboratory
}

\author{
Renate C. Smallegange and Willem Takken
}

\begin{abstract}
This chapter reviews the current understanding of odour-mediated host-seeking behaviour in mosquitoes based on laboratory (indoor) studies. Most recent studies have focused on Aedes aegypti L., Anopheles gambiae sensu stricto Giles and Culex quinquefasciatus Say, as these species are all strongly associated with human biting and disease transmission. Where relevant, reference to other mosquito species is provided. Host seeking in mosquitoes is mediated primarily by chemical cues, and therefore most studies focus on the response to host odours and identification of specific compounds or odour blends. Laboratory tools for the study of odour-mediated behaviour include Y-tube and dual-choice (or dual-port) olfactometers, wind tunnels and roomsize arenas in which mosquitoes can fly freely. Mosquitoes are observed individually or in groups, usually at a specified age or range of ages, at a pre-determined time of the day when they are considered to be naturally responsive to host cues. Aedes aegypti and An. gambiae respond strongly to natural human skin emanations, whereas $C x$. quinquefasciatus shows variation in this behaviour dependent on its geographic origin. Carbon dioxide acts as a principal stimulus for each of these mosquito species although with a variable species-specific effect. Several hostderived compounds have been identified that cause behavioural responses in these mosquito species, including ammonia, L(+)-lactic acid and aliphatic carboxylic acids. In addition, acetone and dimethyl disulfide enhance the attraction of blends of these compounds to Ae. aegypti. Some other ketones, such as 6-methyl-5-hepten-2-one and geranyl acetone disrupt the host-seeking process of Ae. aegypti and An. gambiae. Several aldehydes, such as octanal and nonanal, are attractive to $C_{x}$. quinquefasciatus, and reduce upwind flight and the total number of landings in Ae. aegypti. Based on these data odour blends have been identified that are attractive to these mosquito species, albeit less so than natural human odour, indicating that additional, hitherto unidentified, semiochemicals are involved in host-seeking behaviour that are likely to improve the efficacy of blends. Behavioural research combined with studies on molecular olfaction is ongoing to discover compounds that affect the host-seeking process, and further research under semi-field and field conditions is required to explore the effectiveness of putative attractants and repellents in natural circumstances.
\end{abstract}

Keywords: bioassay, host-odours, kairomone, odour baits, olfaction, repellent, synthetic blend, vector-borne disease

\section{Introduction}

Some species of mosquitoes are vectors of pathogens that give rise to several important infectious diseases of humans and animals. Several of these diseases thrive in tropical climates, where the abundance of vectors, aided by favourable environmental conditions, allows for high levels of transmission. Approximately half of the world's population, especially in less developed countries, are at risk (Jones et al. 2008, WHO 2008, 2009). Although new progress has recently been made in the prevention and control of vector-borne diseases, current methods appear insufficient to prevent many cases of illnesses and deaths due to various factors (e.g. societal, economical, political, mosquito and parasite resistance) (Greenwood et al. 2008, Morrison et al. 2008, Takken 
and Knols 2009). In addition, the recent expansion of vector-borne diseases into more temperate, industrialised countries, such as the occurrence of West Nile virus and Chikungunya in the USA and Europe, respectively (Gould 2003, Gould and Fikrig 2004, Takken and Knols 2007, Vazeille et al. 2008), demonstrate the need for further development and implementation of measures to protect people at risk (e.g. with bednets, vaccines) and to control mosquito vectors (e.g. with insecticides, biological control agents, treated surface and materials, the sterile insect technique, monitoring tools).

In tropical areas, An. gambiae sensu stricto (hereafter termed An. gambiae), Ae. aegypti and CX. quinquefasciatus are medically important mosquito vectors of infectious pathogens such as malaria, dengue and bancroftian filariasis, respectively (Cook 1996). The females of these mosquito species are considered to be anthropophilic (Takken and Knols 1999) and often live in close association with humans. Since host seeking by mosquitoes is mainly mediated by olfactory stimuli (Takken 1991, Takken and Knols 1999), it is likely that the odour complex of the blood-host contains hostspecific and non-host-specific chemicals to which these mosquito species respond (Gillies 1988). It is important to note that the semiochemicals involved in the attraction of mosquitoes to hosts may include both repellents and attractants, and the effects of some chemicals are likely to be dose-dependent. Once these semiochemicals have been identified, it is expected that they can be incorporated into technologies that can be used to trap, kill or repel mosquitoes (Cook et al. 2007, Day and Sjogren 1994, Hassanali et al. 2008, Kline 2006, 2007, Logan and Birkett 2007, Takken and Knols 2009) as has been successfully demonstrated for tsetse flies (see Chapter 12, this volume; also see Chapter 17, this volume). Ultimately, some of these products may improve or even replace current control technologies or sampling tools in epidemiological studies of mosquito-borne diseases. Improvement of present methods could be achieved by adding an odour bait to an existing mosquito trap, by replacing a bait with a better performing one, or by combining a newly developed odour trap with current mosquito control tools.

Crumb (1922) was the first to describe the role of host-derived semiochemicals in mosquito behaviour. In addition, Rudolfs (1922) and Van Thiel (1937) discovered the unique and important role of carbon dioxide $\left(\mathrm{CO}_{2}\right)$ (see review of Mboera and Takken 1997). Since then many mosquito attractants have been discovered, but there is little evidence to explain how these compounds act as olfactory mediators of behaviour and none are as attractive as natural odour blends (Bernier et al. 2007, Bosch et al. 2000, Bowen 1991, Okumu et al. 2010, Spitzen et al. 2008, Smallegange et al. in press, Takken and Knols 1999). Only a few odorants are used as baits for mosquito surveillance or control, in contrast to the extensive use of host-derived baits for other insects of medical or veterinary importance (e.g. tsetse flies, screwworm, biting midges, triatomine bugs) (Cork and Hall 2007, Cruz-Lopez et al. 2001, Logan and Birkett 2007, Qiu et al. 2007b, Vale 1993, see also Chapters 10 and 12 of this volume).

Since the review of Takken and Knols (1999), which summarised our knowledge on the role of semiochemicals in the life history of African malaria mosquitoes at the close of the last century, new techniques and relevant information have become available. In this chapter, we describe recent results of laboratory studies on the identification of semiochemicals that play a role in the host-seeking behaviour of mosquitoes, with emphasis on An. gambiae, Ae. aegypti and Cx. quinquefasciatus, the species that have been studied in most detail over the last decade. It is not the purpose of this chapter to go into details of each step involved in the host-seeking process (Takken 1996, see also Chapter 1 of this volume) or of the strategies employed by mosquitoes to find distant odour sources (the latter has recently been reviewed by Cardé and Willis (2008) for insects in general). Instead, we will provide an overview of the individual chemicals and 
synthetic odour blends that have been tested for their possible effect on the behaviour of host-seeking mosquitoes. We also briefly describe methods and bioassays deployed to identify candidate semiochemicals. Subsequently, we will discuss problems and knowledge gaps that are encountered in this research area. Studies on anopheline host-seeking behaviour performed in semi-field and field situations are discussed in Chapter 8 (this volume).

\section{Identification of candidate semiochemicals}

Anopheles gambiae and Ae. aegypti are considered to be highly anthropophilic mosquito species, i.e. the females express a preference for biting humans to get their blood meal (Gillies 1964, Harrington 2001, Takken and Knols 1999, White 1974). Several hundred volatile compounds emitted by humans have been identified, especially in the last twenty years (Bernier et al. 1999, 2000, 2002, Cork and Park 1996, Curran et al. 2005, 2007, Deng et al. 2004, Ellin et al. 1974, Gallagher et al. 2008, Hasegawa et al. 2004, Haze et al. 2001, Healy and Copland 2000, Healy et al. 2002, Krotoszynski et al. 1997, Meijerink et al. 2000, Natsch et al. 2006, Penn et al. 2007, Perry et al. 1970, Philips 1997, Zeng et al. 1991, 1996). It is assumed that the host-seeking behaviour of mosquitoes is mediated by a blend of specific compounds, and not by the complete assemblage of compounds present in host odour (Zwiebel and Takken 2004). Theoretically, knowledge about the compounds present in the human odour profile can be used to test which compounds play a role in the host-seeking behaviour of anthropophilic mosquito species. However, testing the behavioural effect of each compound in different concentrations and in all possible combinations is not practical because of the large number of compounds present in human emanations (Bernier et al. 2000, Curran et al. 2005, Penn et al. 2007, Philips 1997). Here we outline some techniques that can be used to narrow down the selection of candidate compounds (Logan and Birkett 2007, Smallegange et al. 2003, Van der Goes van Naters and Carlson 2006).

It has been shown that some persons are bitten by mosquitoes more often than others (Curtis 1986, Lindsay et al. 1993, Scott et al. 2006). This may be the result of a differential attractiveness of the body odours of human individuals to mosquitoes (Bernier et al. 2002, Knols et al. 1995, Logan et al. 2008, Lindsay et al. 1993, Mukabana et al. 2002, Qiu et al. 2004a, 2006a, Schreck et al. 1990). Comparing the odour profiles of highly and poorly attractive individuals can reveal compounds that play a role in host selection. Gas-chromatography (GC) and mass-spectrometry (MS) are used to study the differences in the odour profiles of individuals and to identify the compounds involved (Bernier et al. 1999, 2000, 2002, Logan et al. 2008). These differences may be qualitative and/or quantitative, which makes it difficult to identify the compounds involved in host selection. The discovery that human skin emanations collected on glass beads lose their attractiveness after a certain period of time suggests that volatile compounds decreasing in relative abundance during this period play a role in the attractiveness of the emanations (Bernier et al. 2000, 2002, Qiu et al. 2004a, Schreck et al. 1981, Smallegange et al. 2003). Comparing odour profiles of differential attractive individuals resulted in weak attractants and putative repellents for Ae. aegypti (Bernier et al. 2002, Logan et al. 2008).

Skin microbiota plays a role in the production of human body odour (Leyden et al. 1981, Shelley et al. 1953). Blood agar incubated with microbiota from human feet or Staphylococcus epidermidis, a bacteria species commonly found on human skin, is attractive to An. gambiae. Identifying volatile compounds produced by microorganisms present on human skin revealed 10 putative attractants for this mosquito species (Verhulst et al. 2009). 
Although not a new technology we should add here that, in addition to identifying volatile organic compounds emanating from the human skin and comparing odour profiles of individuals, electrophysiological methods, such as electroantennography (EAG) and single sensillum recording (SSR) techniques, either without or in combination with GC and MS, can be applied to identify which compounds in the human odour profile stimulate the olfactory sensilla on mosquito antennae or maxillary palps (see Chapter 3 in this volume for more details, as well as Logan et al. 2008, Lu et al. 2007, Qiu et al. 2004b). Ultimately, the effects of odours on mosquitoes must be investigated directly, with behavioural assays. The sensory systems of a mosquito may be able to detect a volatile compound, but this does not necessarily mean that a motor response is triggered, or that the response is necessarily related to the behaviour under investigation. Behavioural observations are required to establish the mechanisms by which odorants guide mosquitoes to a host, e.g. they may act as attractants, repellents, activators or arrestants (Costantini et al. 2001, Logan et al. 2008, Mukabana et al. 2004).

The publication of the full gene sequence of An. gambiae (Holt et al. 2002) and Ae. aegypti (Nene et al. 2007) has enabled rapid progress in molecular genetics. The sequencing of the $C x$. quinquefasciatus genome has almost been completed (Pelletier and Leal 2009, Waterhouse et al. 2008). A large family of olfactory receptor genes (ORs) and gustatory receptor genes (GRs) has been identified (Van der Goes van Naters and Carlson 2006). These genes play a crucial role in the binding of olfactory molecules to neural membranes (see Chapter 2, this volume). The molecular regulation of olfaction appears highly conserved in the animal kingdom, although the OR and GR families are highly specific to species (Robertson and Wanner 2006). This would explain why some insects express a highly selective host preference, as found in An. gambiae and Ae. aegypti. Work on the molecular regulation of olfaction in Drosophila and Xenopus has elucidated the processes by which small molecules control physiological activity in insects (Hallem et al. 2006, Rutzler and Zwiebel 2005). This knowledge is currently being used for the identification of semiochemicals to which mosquitoes respond (Carey et al. 2010, Hallem et al. 2004, Justice et al. 2003, Leal et al. 2008, Wang et al. in press and Chapter 2, this volume).

Combined with powerful electrophysiological techniques (Chapter 3, this volume), molecular genetics can provide new insights into the olfactory behaviour of mosquitoes and can be used as a tool for the rapid identification of candidate semiochemicals. Behavioural studies, however, remain essential for the final assessment of the role of these chemicals in the insect's life-history. The olfactory receptor neurons may be able to detect certain chemical stimuli, the brain will associate them with a specific behavioural response (Klowden 1995, see also De Bruyne and Baker 2008).

Similar strategies are used to discover olfactory cues that affect mosquitoes with more variable host preferences, such as $C x$. quinquefasciatus. The host-preference of $C x$. quinquefasciatus appears to be region-dependent, as the following examples illustrate. Field experiments in Tanzania showed that more $C x$. quinquefasciatus were caught in human-odour baited traps than in traps baited with goat or calf odour (Mboera and Takken 1999). By contrast, blood meal analyses have shown that in Kenya this species has a preference for livestock over human hosts (Muturi et al. 2008). In a study in Tucson, USA, blood meal analysis revealed that $50 \%$ of $C x$. quinquefasciatus females fed on humans and $32 \%$ on birds, respectively (and therefore this species is considered to be a potential West Nile virus vector; Zinser et al. 2004), whereas in Tenessee, USA, and Mexico they were found to feed less on humans than on birds (Eilizondo-Quiroga et al. 2006, Savage et al. 2007). In addition, a greater percentage of $C x$. quinquefasciatus females, from a colony originating in Florida, USA, responded to chicken than to human odour in an olfactometer study (Allan et al. 
2006b). These latter results indicate that in the USA CX. quinquefasciatus is less anthropophilic and more ornithophilic than in Tanzania. Similarly, in Senegal and Madagascar An. gambiae populations were found to feed predominantly on cattle (Diatta et al. 1998, Duchemin et al. 2001). In addition, Ae. aegypti responds not only to human odour, but also to mouse and chicken odour (Allan et al. 2006b, McCall et al. 1996, Mclver 1968), suggesting that this species is actually an opportunistic feeder. The reliability of results obtained in host preference studies, however, depends on the method used (field and laboratory), the geographic area covered (field) or geographic origin of the mosquitoes (laboratory) and the availability of host species (field and laboratory), which demands for caution in using labels such as 'anthropophilic' and to confine research to analysing only human odours (Kilpatrick et al. 2006, Lefèvre et al. 2009, Torr et al. 2008).

A wider host-range broadens the search for volatile organic compounds mediating host-seeking behaviour. Overlap in odour profiles of different blood-host species of a mosquito species, such as human and chicken, may reveal attractive compounds such as nonanal (Syed and Leal 2009). However, differences in these odour profiles may explain differences in relative attraction to these hosts due to allomones or additional kairomones (Dicke and Sabelis 1988) emitted by the host.

Compounds present in bovine emanations have been thoroughly examined in relation to the development of surveillance and control tools for tsetse, cattle and stable flies using the techniques described above and have been published (e.g. Birkett et al. 2004, Bursell et al. 1988, Schofield and Brady 1997, Schofield et al. 1995, 1997, Spinhirne et al. 2003, 2004, Torr et al. 1995, Vale 1980, Vale and Hall 1985a,b, Vale et al. 1988 and Chapter 12, this volume). Also, several compounds emitted by chickens have been identified, for example by solvent extraction and volatile trapping of faeces, feathers, feet or skin, and include alcohols, aldehydes, carboxylic acids, diones and ketones (Bernier et al. 2008, Cooperband et al. 2008, Haathi and Fales 1967, Williams et al. 2003).

Although in nature mosquitoes do not feed on blood directly, females of both $C x$. quinquefasciatus and Ae. aegypti were found to be attracted to the odour of bovine and avian blood. Culex females landed more on membranes filled with avian than with bovine blood, whereas Aedes mosquitoes showed no landing preference (Allan et al. 2006a). The volatile chemicals emanating from blood have been identified (Ashley et al. 1992, Issachar et al. 1982, Sastry et al. 1980) but few of these have been tested for their effects on mosquitoes in the laboratory, although components found in exhaled breath and skin emanations may be similar to those present in blood (Sastry et al. 1980).

An initial screening of compounds for their effect on the host-seeking behaviour of mosquitoes in a laboratory before testing them under field conditions has the advantage that the environmental circumstances during the bioassays and physiological state (e.g. age, time since sugar or bloodmeal, stage in gonotrophic cycle) of the mosquitoes can be controlled during the experiments. Before the results of a bioassay are put into practical use, they should first be validated in semi-field and/or field experiments (Knols et al. 2002, see also Chapter 8 in this volume). (Semi-)field studies provide information about the effects of natural temperature, humidity, wind, the presence of competitive odours, etc. on the responses to odours, and can be used to test the effectiveness of an odour bait over larger distances than can be tested in the laboratory (Njiru et al. 2006, Okumu et al. 2010, Williams et al. 2006a). Field evaluations can also reveal simultaneously whether other vectors are attracted or repelled by the odour compounds tested (Jawara et al. 2009, Qiu et al. 2007a). 


\section{Bioassay techniques in the laboratory}

In the laboratory, host-seeking behaviour in response to odour can be evaluated by observing individual insects. This can be done by direct observation (human landing catches) in a cage (De Jong and Knols 1995, Dekker et al. 1998, Knols et al. 1994a) or wind tunnel (Takken et al. 1997) or by video recording (Beeuwkes et al. 2008, Cooperband and Cardé 2006, Dekker et al. 2005, Healy and Copland 1995, Young et al. 1993).

Y-tube and dual-port (or dual-choice) olfactometers are used to assess the response of mosquitoes to a choice between an odour and clean air or two different odours (Geier and Boeckh 1999, Knols et al. 1994b, Posey et al. 1998). Y-tube olfactometers (Figure 1A) are conveniently small devices for rapid screening of odours. Aedes aegypti in particular responds well in Y-tubes (Geier and Boeckh 1999, Logan et al. 2008). Dual-port olfactometers (Figure 1B) are more suitable for mosquito species that perform better in larger arenas, such as An. gambiae (Knols et al. 1994b).

Mosquito responses to odours can also be studied in large indoor cages, for instance inside a bednet or a closed room. These tools have been used for studies on the selection of biting sites on volunteers (De Jong and Knols 1995, Dekker et al. 1998, Knols et al. 1994a) and for studies with insect repellents (Curtis and Hill 1988). By placing mosquito traps in indoor cages (Figure 1C), the effect of different odorants on trapping efficacy can be investigated (Silva et al. 2005, Smallegange et al. 2009).

Several methods have been developed for testing putative repellents. The most commonly used methods to test putative repellents for employment directly on the human skin have recently been reviewed by Barnard et al. (2007). Various types of olfactometers have been used to test compounds for their efficacy as spatial repellents (Bernier et al. 2005, Dogan and Rossignol 1999, Grieco et al. 2005, Kline et al. 2003, Logan et al. 2008, Waka et al. 2006).

Laboratory-based studies have been done with individual mosquitoes or with groups of up to 50 mosquitoes per test. In wind tunnels, experiments are usually done with one insect at a time (Healy and Copland 1995, Takken et al. 1997), whereas in olfactometers most studies are based on the results of groups of 15 to 50 insects. Because of the possibility of learning (Alonso and SchuckPaim 2006, McCall and Eaton 2001, Tomberlin et al. 2006), individual mosquitoes should not be tested more than once, and each test should be performed with naïve insects.

\section{Effects of physiology on behaviour}

It should be taken into account that the host-seeking process of mosquitoes includes different phases. After an inactive period during which reproductive maturation (Lehane 1991) and development of the antennal sensitivity takes place (Davis 1984), female activity increases (influenced by circadian rhythm; see Klowden 1996). This increase makes it more likely for the females to encounter host-derived stimuli. Activation takes place as soon as these stimuli have been detected. This phase is followed by the orientation process that will bring the mosquitoes in the immediate vicinity of the host. As soon as the host has been located, the female will land and start blood feeding (Takken 1996). The mosquitoes will respond to different stimuli, among which olfactory, within each phase. Therefore, the choice for a certain bioassay may influence the discovery of semiochemicals that play a role in a specific phase of the mosquito host-seeking process. 
A

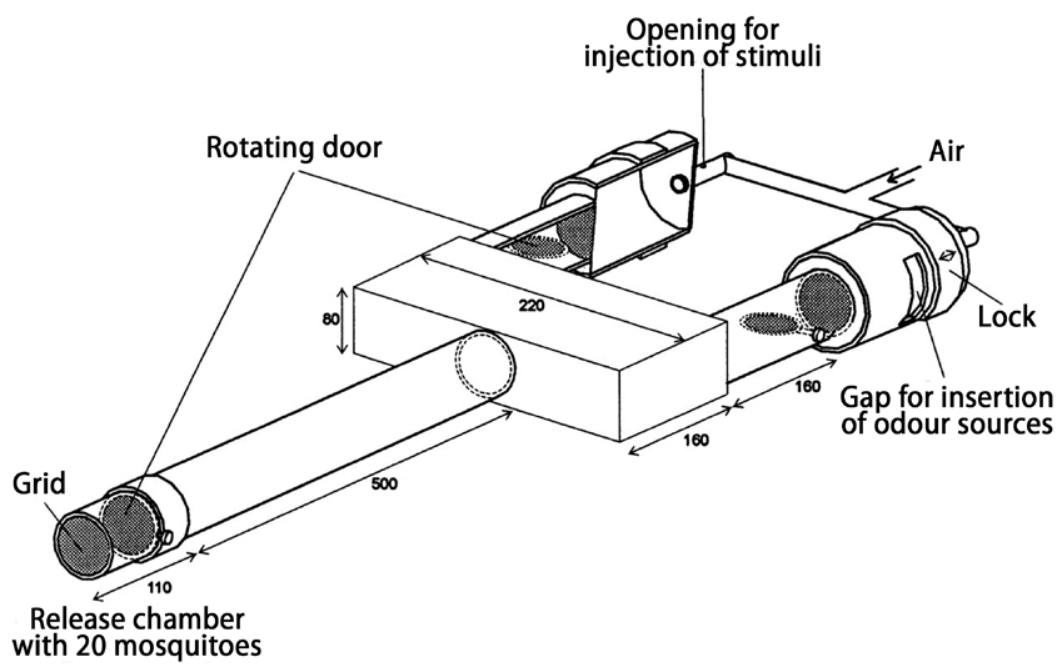

B

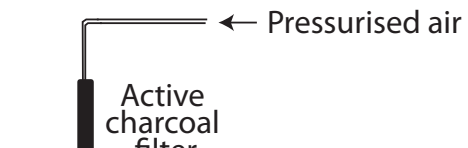

Active

filter

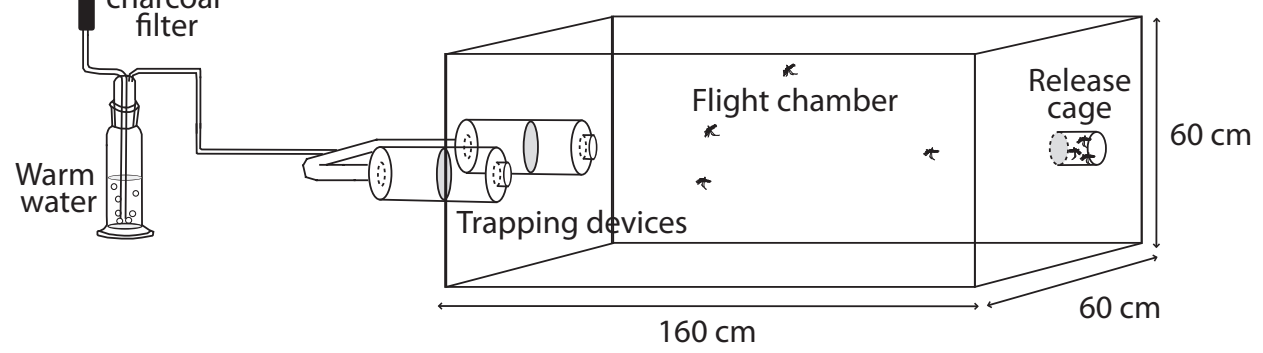

C

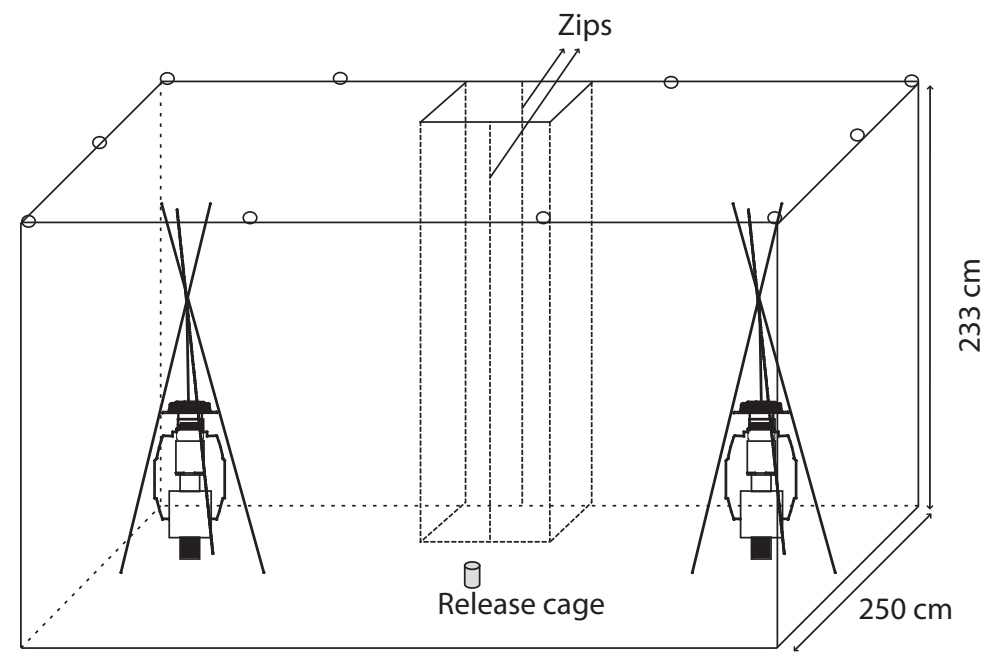

$330 \mathrm{~cm}$

Figure 1. Schematic drawings of (A) an Y-tube olfactometer (from Geier et al. 1999a), (B) a dual-port olfactometer (modified after Spitzen et al. 2008) and (C) two MM-X traps in an indoor netted cage. 
Olfactory cues are important external factors affecting host-seeking behaviour from a distance, whereas visual and physical cues, such as heat and moisture, play a role in close vicinity of the blood-host (Takken and Knols 1999). However, even when host-stimuli are present, hostseeking behaviour may not be expressed as a result of endogenous, physiological factors. These physiological factors include the circadian rhythm, age, nutritional state, mating condition, and presence of eggs, and should be considered in any study on insect behaviour (Klowden 1995, 1996).

Aedes aegypti is a diurnal mosquito and its host-seeking behaviour should therefore be studied during daytime under illuminated conditions (Geier and Boeckh 1999, Klowden 1995). Both An. gambiae and $C x$. quinquefasciatus are nocturnal. Biting activity of the latter species was observed throughout the night (Chandra 1995), allowing olfactometer experiments during the scotophase (Mboera et al. 1998). Puri et al. (2006), however, observed that the mosquitoes were most active during a period of one hour after dusk, and therefore performed experiments in dim light during this short period of time only. Experiments examining the host-seeking behaviour of An. gambiae are usually performed, in the dark or under moonlight conditions (Knols et al. 1994b), during the last 4 hours of the scotophase, when this species has frequently been reported to be naturally active (Haddow and Ssenkubuge 1973, Killeen et al. 2006, Maxwell et al. 1998). Shifts in timing of host-seeking activity, however, have occasionally been observed, chiefly because of vectorcontrol interventions (Braimah et al. 2005).

Host-seeking behaviour of Ae. aegypti increases from no response to host cues directly after adult emergence to more than $90 \%$ of the females responding to host-odours 102 hours post emergence (Davis 1984). Therefore, the best results can be obtained with females of 4-days-old or older. Generally, adults are tested within a specified range of ages, which varies overall from 3-10 days, to control for the effects of aging.

A blood meal changes the mosquito's physiology and behaviour, by affecting the sensitivity of the olfactory receptor organs (Davis 1984, Qiu et al. 2006b, see also Chapter 3 in this volume). After a full blood meal, An. gambiae is no longer attracted to host stimuli for 24 hours, with hostseeking behaviour returning to pre-blood meal levels 72 hours post feeding (Takken et al. 2001). In mated Ae. aegypti, the inhibition begins 30 hours after ingestion of the blood and reaches its maximum between 36 and 72 hours post feeding. The inhibition is less pronounced in unmated Ae. aegypti females (Klowden and Lea 1979). Nutritional status also affects the intensity of the inhibition (Klowden 1986). Test insects may be starved for up to 18 hours prior to testing. This can be done by replacing the carbohydrate food source with water before the experiment. Infection with pathogens/parasites may alter the host-seeking behaviour as well (Hurd 2003, Schaub 2006).

\section{Bioassay results with semiochemicals in laboratory studies}

In this section an overview is given of semiochemicals identified to play a role in the host-seeking process of Ae. aegypti, An. gambiae, Cx. quinquefasciatus, and some other mosquito species. The compounds, the bioassay in which they were tested and the effect that has been found are summarised in Tables 1-3. As some bioassays cannot differentiate between an inhibitory and a repellent effect, both terms are used interchangeably. Although, as Kennedy (1978) pointed out, this term does not reveal the many processes and underlying mechanisms involved, we use the term 'attractant' to refer to a chemical or blend that has been found to induce mosquitoes to orientate towards the odour source. 


\section{Aedes aegypti}

A human hand as the odour source stimulates Ae. aegypti females to fly upwind in a Y-tube olfactometer. A similar result was achieved by using high doses of heated or unheated ethanol skin extracts (Geier and Boeckh 1999, Geier et al. 1996). Liquid chromatography of the skin extract revealed active fractions of which one contained lactic acid and another probably ammonia (Geier et al. 1996, 1999a).

In humans, L-(+)-lactic acid (hereafter lactic acid) is present in breath and on the skin. Human skin rubbing extracts contain lactic acid in significantly higher quantities than skin extracts of 12 other mammals and chickens (Dekker et al. 2002) and have, presumably for that reason, received specific attention as a kairomone for anthropophilic mosquitoes. More interestingly, lactic acid was found in higher amounts on glass beads that were handled by individuals that were more attractive to Ae. aegypti than individuals with lower amounts of the compound in their skin emanations. From numerous studies it was shown that lactic acid plays a major role in the host-seeking behaviour of Ae. aegypti (Table 1) (Acree et al. 1968, Bernier et al. 2002, Eiras and Jepson 1991, Geier et al. 1996, Smith et al. 1970). Lactic acid is a weak attractant on its own but acts synergistically with carbon dioxide $\left(\mathrm{CO}_{2}\right)$, a major component of exhaled breath, and other volatiles emanating from human skin (Acree et al. 1968, Allan et al. 2006a, Bernier et al. 2002, Bosch et al. 2000, Eiras and Jepson 1991, Geier and Boeckh 1999, Geier et al. 1996, 1999a,b, Smith et al. 1970, Williams et al. 2006a). An ethanol extract of human skin was no longer attractive to Ae. aegypti females, presumably because the lactic acid had been removed from the extract (Geier et al. 1996).

Carbon dioxide on its own is an activator and weak attractant for Ae. aegypti, increasing take-off, flight velocity and flight duration, although homogeneous $\mathrm{CO}_{2}$ plumes decrease upwind flight and trap catches (Allan et al. 2006b, Bernier et al. 2007, Bosch et al. 2000, Dekker et al. 2001 b, 2005, Eiras and Jepson 1991, Geier and Boeckh 1999, Geier et al. 1999a,b, Kline et al. 2006). An encounter with a single $\mathrm{CO}_{2}$ filament increases the responsiveness to human skin odours (Dekker et al. 2005).

The presence of carboxylic acids on the skin is unique to humans compared to other mammals, birds and rodents (Nicolaides et al. 1968). Saturated aliphatic carboxylic acids with chain length $\mathrm{C} 1-\mathrm{C} 3, \mathrm{C} 5-\mathrm{C} 8$, and $\mathrm{C} 13-\mathrm{C} 18$ were found to increase the attractiveness of lactic acid at certain doses to Ae. aegypti females. The attractiveness increased even further when a second carboxylic acid was added, as long as one is a short chain (C1-3) and the other a medium length (C5-8) carboxylic acid. Nonanoic and undecanoic acid reduced the effect of lactic acid, whereas butanoic, decanoic and dodecanoic acid had no effect (Bosch et al. 2000). Acetic acid on its own was found to be attractive to host-seeking Ae. aegypti females and to elicit a landing response. Several other carboxylic acids, including two aromatics, induced landing responses (Table 1) (Allan et al. 2006a). In contrast, hexanoic acid reduced the number of landings (Douglas et al. 2005), probably due to the high doses used. Propanoic, butanoic, 3-methylbutanoic, pentanoic, heptanoic, decanoic, dodecanoic, tetradecanoic, pentadecanoic, hexadecanoic, heptadecanoic, and octadecanoic acid did not attract $A$ e. aegypti females, even though some of these acids were present at higher levels in the skin emanations of highly attractive persons (Allan et al. 2006a, Bernier et al. 2002, Bosch et al. 2000). Propanoic and pentanoic acid did not influence the attractiveness of $\mathrm{CO}_{2}$ (Bosch et al. 2000).

Ammonia was also found to enhance the attractiveness of lactic acid, although it is not attractive on its own or in combination with $\mathrm{CO}_{2}$ (Geier et al. 1999a). Propanoic and pentanoic acid enhanced the effect of a lactic acid and ammonia blend. A blend of ammonia, lactic acid, and two carboxylic 
Table 1. Overview of chemical compounds that have been tested for their impact on the host-seeking behaviour of Aedes aegypti using behavioural assays in the laboratory. (Details about concentrations of compounds are omitted).

\begin{tabular}{|c|c|c|c|}
\hline Compound & Response & Behavioural assay & Reference \\
\hline \multirow[t]{10}{*}{ carbon dioxide $\left(\mathrm{CO}_{2}\right)$} & (Weakly) attractive on its own; & Wind tunnel & Eiras and Jepson (1991) \\
\hline & flight activating effect; synergism & Wind tunnel tube & Geier et al. (1999a) \\
\hline & with lactic acid but not ammonia & Y-tube & Geier et al. (1999b) \\
\hline & & Y-tube & Geier and Boeckh (1999) \\
\hline & & Y-tube & Bosch et al. (2000) \\
\hline & & Dual-port olfactometer & Allan et al. (2006b) \\
\hline & & Dual-port olfactometer & Kline et al. (2006) \\
\hline & & Dual-port olfactometer & Bernier et al. (2007) \\
\hline & $\begin{array}{l}\text { Homogeneous } \mathrm{CO}_{2} \text { reduced trap } \\
\text { catch }\end{array}$ & Dual-port olfactometer & Dekker et al. (2001b) \\
\hline & Sensitizes to skin odours & Wind tunnel (3D) & Dekker et al. (2005) \\
\hline \multirow[t]{2}{*}{ ammonia $\left(\mathrm{NH}_{3}\right)$} & Not attractive on its own & Y-tube & Geier et al. (1999b) \\
\hline & $\begin{array}{l}\text { Enhanced the effect of lactic acid } \\
\mathrm{NH}_{3} \text { +hexanoic acid improved } \\
\text { blend of lactic acid + acetone + } \\
\text { dimethyl disulfide }\end{array}$ & Y-tube & Williams et al. (2006a) \\
\hline \multicolumn{4}{|l|}{ Alcohols } \\
\hline 4-hexen-1-ol & Not attractive on its own & Dual-port olfactometer & Bernier et al. (2002) \\
\hline 1-hepten-3-ol & Not attractive on its own & Dual-port olfactometer & Bernier et al. (2002) \\
\hline glycerol & Not attractive on its own & Dual-port olfactometer & Bernier et al. (2002) \\
\hline \multicolumn{4}{|c|}{ 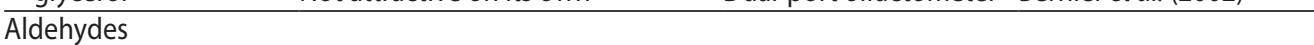 } \\
\hline hexanal & Reduced the number of landings & Landing assay & Douglas et al. (2005) \\
\hline heptanal & Not attractive on its own & Dual-port olfactometer & Bernier et al. (2002) \\
\hline \multirow[t]{2}{*}{ octanal } & Reduced the number of landings & Landing assay & Douglas et al. (2005) \\
\hline & Reduced upwind flight & Y-tube & Logan et al. (2008) \\
\hline \multirow[t]{2}{*}{ nonanal } & Not attractive & Dual-port olfactometer & Bernier et al. (2002) \\
\hline & Reduction of upwind flight & Y-tube & Logan et al. (2008) \\
\hline decanal & Reduced upwind flight & Y-tube & Logan et al. (2008) \\
\hline z-4-decanal & Reduced the number of landings & Landing assay & Douglas et al. (2005) \\
\hline \multicolumn{4}{|c|}{ Aliphatic carboxylic acids } \\
\hline \multirow[t]{12}{*}{ lactic acid (LA) } & Weakly attractive on its own; & Dual-port olfactometer & Acree et al. (1968) \\
\hline & Synergism with $\mathrm{CO}_{2}$ and other skin & Dual-port olfactometer & Smith et al. (1970) \\
\hline & components & Wind tunnel & Eiras and Jepson (1991) \\
\hline & & Y-tube & Geier et al. (1996) \\
\hline & & Y-tube & Geier and Boeck (1999) \\
\hline & & Wind tunnel tube & Geier et al. (1999a) \\
\hline & & Y-tube & Geier et al. (1999b) \\
\hline & & Y-tube & Bosch et al. (2000) \\
\hline & & Dual-port olfactometer & Bernier et al. (2002) \\
\hline & & Y-tube & Williams et al. (2006a) \\
\hline & & Dual-port olfactometer & Allan et al. (2006a) \\
\hline & Induced landing response & Landing assay & Allan et al. (2006a) \\
\hline methanoic acid & Enhanced the effect of LA & Y-tube & Bosch et al. (2000) \\
\hline
\end{tabular}


Table 1. Continued

\begin{tabular}{|c|c|c|c|}
\hline Compound & Response & Behavioural assay & Reference \\
\hline \multirow[t]{3}{*}{ acetic acid } & Enhanced the effect of LA & Y-tube & Bosch et al. (2000) \\
\hline & Attractive on its own & Dual-port olfactometer & Allan et al. (2006a) \\
\hline & Induced landing response & Landing assay & Allan et al. (2006a) \\
\hline \multirow[t]{5}{*}{ propanoic acid } & Not attractive on its own & Y-tube & Bosch et al. (2000) \\
\hline & & Dual-port olfactometer & Allan et al. (2006a) \\
\hline & Enhanced the effect of LA & Y-tube & Bosch et al. (2000) \\
\hline & Enhanced the effect of $\mathrm{LA}+\mathrm{NH}_{3}$ & Y-tube & Bosch et al. (2000) \\
\hline & No landing response & Landing assay & Allan et al. (2006a) \\
\hline \multirow[t]{3}{*}{ butanoic acid } & No effect on attractiveness of LA & Y-tube & Bosch et al. (2000) \\
\hline & Not attractive on its own & Dual-port olfactometer & Allan et al. (2006a) \\
\hline & Induced landing response & Landing assay & Allan et al. (2006a) \\
\hline \multirow[t]{2}{*}{ 3-methyl butanoic acid } & Not attractive on its own & Dual-port olfactometer & Allan et al. (2006a) \\
\hline & Induced landing response & Landing assay & Allan et al. (2006a) \\
\hline \multirow[t]{3}{*}{ pentanoic acid } & Not attractive on its own & Y-tube & Bosch et al. (2000) \\
\hline & Enhanced the effect of LA & & \\
\hline & Enhanced the effect of $\mathrm{LA}+\mathrm{NH}_{3}$ & & \\
\hline \multirow[t]{5}{*}{ hexanoic acid } & Enhanced the effect of LA & Y-tube & Bosch et al. (2000) \\
\hline & Attractive when combined with & Y-tube & Williams et al. (2006a) \\
\hline & $\mathrm{LA}+\mathrm{NH}_{3}$ & & \\
\hline & $\begin{array}{l}\text { Hexanoic acid }+\mathrm{NH}_{3} \text { improved } \\
\text { blend of lactic acid }+ \text { acetone }+ \\
\text { dimethyl disulfide }\end{array}$ & Y-tube & Williams et al. (2006a) \\
\hline & Reduced number of landings & Landing assay & Douglas et al. (2005) \\
\hline \multirow[t]{3}{*}{ heptanoic acid } & Enhanced the effect of LA & Y-tube & Bosch et al. (2000) \\
\hline & Not attractive on its own & Dual-port olfactometer & Allan et al. (2006a) \\
\hline & Induced landing response & Landing assay & Allan et al. (2006a) \\
\hline octanoic acid & Enhanced the effect of LA & Y-tube & Bosch et al. (2000) \\
\hline nonanoic acid & Reduced the attractive effect of $L A$ & Y-tube & Bosch et al. (2000) \\
\hline \multirow[t]{2}{*}{ decanoic acid } & No effect on the attractiveness of $L A$ & Y-tube & Bosch et al. (2000) \\
\hline & Not attractive on its own & Dual-port olfactometer & Bernier et al. (2002) \\
\hline undecanoic acid & Reduced the attractive effect of $L A$ & Y-tube & Bosch et al. (2000) \\
\hline \multirow[t]{2}{*}{ dodecanoic acid } & No effect on the attractiveness of $L A$ & Y-tube & Bosch et al. (2000) \\
\hline & Not attractive on its own & Dual-port olfactometer & Bernier et al. (2002) \\
\hline tridecanoic acid & Enhanced the effect of LA & Y-tube & Bosch et al. (2000) \\
\hline \multirow[t]{3}{*}{ tetradecanoic acid } & Enhanced the effect of LA & Y-tube & Bosch et al. (2000) \\
\hline & Not attractive on its own & Dual-port olfactometer & Allan et al. (2006a) \\
\hline & Induced landing response & Landing assay & Allan et al. (2006a) \\
\hline \multirow[t]{2}{*}{ pentadecanoic acid } & Enhanced the effect of LA & Y-tube & Bosch et al. (2000) \\
\hline & Not attractive on its own & Dual-port olfactometer & Bernier et al. (2002) \\
\hline \multirow[t]{4}{*}{ hexadecanoic acid } & Enhanced the effect of LA & Y-tube & Bosch et al. (2000) \\
\hline & Not attractive on its own & Dual-port olfactometer & Bernier et al. (2002) \\
\hline & & Dual-port olfactometer & Allan et al. (2006a) \\
\hline & Induced landing response & Landing assay & Allan et al. (2006a) \\
\hline
\end{tabular}


Table 1. Continued

\begin{tabular}{|c|c|c|c|}
\hline Compound & Response & Behavioural assay & Reference \\
\hline \multirow[t]{2}{*}{ heptadecanoic acid } & Enhanced the effect of LA & Y-tube & Bosch et al. (2000) \\
\hline & Not attractive on its own & Dual-port olfactometer & Bernier et al. (2002) \\
\hline \multirow[t]{4}{*}{ octadecanoic acid } & Enhanced the effect of LA & Y-tube & Bosch et al. (2000) \\
\hline & Not attractive on its own & Dual-port olfactometer & Bernier et al. (2002) \\
\hline & & Dual-port olfactometer & Allan et al. (2006a) \\
\hline & Induced landing response & Landing assay & Allan et al. (2006a) \\
\hline $\begin{array}{l}\text { Blend of lactic acid, } \\
\text { ammonia and two } \\
\text { carboxylic acids (C1- } \\
\text { C3 and C5-C8) }\end{array}$ & Attractive & Y-tube & Bosch et al. (2000) \\
\hline $\begin{array}{l}\text { Blend of octanal, } \\
\text { hexanal, Z-4 decenal, } \\
\text { decanal, hexanoic } \\
\text { and octanoic acid } \\
\end{array}$ & Reduced number of landings & Landing assay & Douglas et al. (2005) \\
\hline \multicolumn{4}{|c|}{ Aromatic carboxylic acids } \\
\hline benzoic acid & Induced landing response & Landing assay & Allan et al. (2006a) \\
\hline 2-hydroxybenzoic acid & I Induced landing response & Landing assay & Allan et al. (2006a) \\
\hline \multicolumn{4}{|c|}{ Ketones } \\
\hline \multirow[t]{3}{*}{ acetone } & Attractive on its own; Enhanced & Dual-port olfactometer & Bernier et al. (2003) \\
\hline & the effect of LA & Dual-port olfactometer & Bernier et al. (2007) \\
\hline & $\begin{array}{l}\text { Improved blend of } \mathrm{LA}+\mathrm{NH}_{3}+ \\
\text { hexanoic acid }\end{array}$ & Y-tube & Williams et al. (2006a) \\
\hline butanone & Weakly attractive & Dual-port olfactometer & Bernier et al. (2002) \\
\hline 2-pentanone & Weakly attractive & Dual-port olfactometer & Bernier et al. (2002) \\
\hline 3-pentanone & Weakly attractive & Dual-port olfactometer & Bernier et al. (2002) \\
\hline 6-methyl-5-hepten- & Weakly attractive & Dual-port olfactometer & Bernier et al. (2002) \\
\hline 2-one & Reduced upwind flight & Y-tube olfactometer & Logan et al. (2008) \\
\hline \multirow{2}{*}{$\begin{array}{l}\text { 2-decanone } \\
\text { geranyl acetone }\end{array}$} & Not attractive on its own & Dual-port olfactometer & Bernier et al. (2002) \\
\hline & Reduced upwind flight & Y-tube olfactometer & Logan et al. (2008) \\
\hline \multicolumn{4}{|l|}{ Sulfides } \\
\hline \multirow[t]{2}{*}{ methyl sulfide } & Attractive on its own & Dual-port olfactometer & Allan et al. (2006a) \\
\hline & Induced landing response & Landing assay & Allan et al. (2006a) \\
\hline \multirow[t]{2}{*}{ carbon disulfide } & Attractive on its own & Dual-port olfactometer & Allan et al. (2006a) \\
\hline & Induced landing response & Landing assay & Allan et al. (2006a) \\
\hline \multirow[t]{7}{*}{ dimethyl disulfide } & Attractive on its own & Dual-port olfactometer & Bernier et al. $(2003,2007)$ \\
\hline & & Dual-port olfactometer & Allan et al. (2006a) \\
\hline & No landing response & Landing assay & Allan et al. (2006a) \\
\hline & Enhanced the effect of LA & Dual-port olfactometer & Bernier et al. $(2003,2007)$ \\
\hline & Enhanced the effect of lactic acid & Lab. trapping exp's & Silva et al. (2005) \\
\hline & + acetone & Y-tube & Williams et al. (2006a) \\
\hline & & Dual-port olfactometer & Bernier et al. (2007) \\
\hline \multirow[t]{2}{*}{ ethyl disulfide } & Not attractive on its own & Dual-port olfactometer & Allan et al. (2006a) \\
\hline & No landing response & Landing assay & Allan et al. (2006a) \\
\hline
\end{tabular}


Table 1. Continued

\begin{tabular}{|c|c|c|c|}
\hline Compound & Response & Behavioural assay & Reference \\
\hline \multicolumn{2}{|c|}{ methyl propyl disulfide Not attractive } & Dual-port olfactometer & Allan et al. (2006a) \\
\hline & Induced landing response & Landing assay & Allan et al. (2006a) \\
\hline \multirow[t]{2}{*}{ dimethyl trisulfide } & Not attractive on its own & Dual-port olfactometer & Allan et al. (2006a) \\
\hline & No landing response & Landing assay & Allan et al. (2006a) \\
\hline \multirow{2}{*}{$\begin{array}{l}\text { Miscellaneous } \\
\text { dichloromethane }\end{array}$} & & & \\
\hline & $\begin{array}{l}\text { Attractive on its own } \\
\text { Enhanced the effect of } L A\end{array}$ & Dual-port olfactometer & Bernier et al. (2003) \\
\hline pyridine & Not attractive on its own & Dual-port olfactometer & Bernier et al. (2002) \\
\hline 4-pyridinamine & Not attractive on its own & Dual-port olfactometer & Bernier et al. (2002) \\
\hline benzene & Not attractive on its own & Dual-port olfactometer & Bernier et al. (2002) \\
\hline toluene & Not attractive on its own & Dual-port olfactometer & Bernier et al. (2002) \\
\hline heptane & Not attractive on its own & Dual-port olfactometer & Bernier et al. (2002) \\
\hline $1 H$-indole & Not attractive on its own & Dual-port olfactometer & Bernier et al. (2002) \\
\hline 2-octene & Not attractive on its own & Dual-port olfactometer & Bernier et al. (2002) \\
\hline styrene & Not attractive on its own & Dual-port olfactometer & Bernier et al. (2002) \\
\hline 2-nonene & Not attractive on its own & Dual-port olfactometer & Bernier et al. (2002) \\
\hline 1,3-butanediamine & Not attractive on its own & Dual-port olfactometer & Bernier et al. (2002) \\
\hline pentacosane & Not attractive on its own & Dual-port olfactometer & Bernier et al. (2002) \\
\hline cholesterol & Not attractive on its own & Dual-port olfactometer & Bernier et al. (2002) \\
\hline squalene & Not attractive on its own & Dual-port olfactometer & Bernier et al. (2002) \\
\hline
\end{tabular}

acids from the $\mathrm{C} 1-\mathrm{C} 3$ and $\mathrm{C} 5-\mathrm{C} 8$ groups was almost as attractive as an extract of human skin residues (attracting $68.1 \%$ and $77.9 \%$ of the tested mosquitoes, respectively). The difference in attractiveness may have been caused by 'missing' compounds in the synthetic blend or because the concentrations and proportions of the compounds in the blend were not optimal (Bosch et al. 2000).

Another promising attractive blend is the combination of lactic acid, acetone, and dimethyl disulfide (Bernier et al. 2007, Silva et al. 2005, Williams et al. 2006a). The latter two compounds have been detected in human breath and in human skin emanations (Bernier et al. 2000, Philips 1997). MM-X traps (Woodstream, Lititz, PA, USA; obtained originally from American Biophysics, East Greenwich, RI, USA) baited with this synthetic blend were attractive to Ae. aegypti females under controlled laboratory conditions (Silva et al. 2005). However, natural human odour was significantly more attractive than the triple blend in two-choice olfactometer experiments (Bernier et al. 2007). The addition of ammonia and hexanoic acid improved the attractiveness of the blend, and the addition of acetone to a blend of lactic acid, ammonia and hexanoic acid increased attraction to the latter blend (Williams et al. 2006a). BG-Sentinel traps (BioGents AG, Germany) baited with the four blends mentioned above (i.e. combinations of ammonia, hexanoic acid, lactic acid, acetone and dimethyl disulfide) appeared to be highly effective and Ae. aegyptispecific in field experiments in Australia (Williams et al. 2006a,b). However, this seemed to be due to visual more than olfactory cues (Williams et al. 2006a). 
Acetone, dimethyl disulfide and dichloromethane, attractants on their own, were found to act synergistically when combined in a binary blend with lactic acid (Allan et al. 2006a, Bernier et al. 2003, 2007). The binary blends were less attractive than the triple blend mentioned above (Bernier et al. 2007). Aedes aegypti females also responded to other sulfides that are found on human skin, in human breath or may be present in human blood (Ashley et al. 1992, Bernier et al. 2000, Krotoszynski et al. 1977, Philips 1997); carbon disulfide, methyl propyl disulfide and methyl sulfide, either in a dual-choice olfactometer and/or in landing assays were found to be attractive, but dimethyl trisulfide and ethyl disulfide were not (Allan et al. 2006a) (Table 1).

Several ketones, which were found to be present in higher amounts on glass beads handled by a person that was more attractive to Ae. aegypti, proved to be weak attractants in a dual-port olfactometer (Table 1). Two of these compounds, butanone and 2-pentanone, decreased after the skin sample had aged for 8 hours. In contrast, one of the ketones that was found to be a weak attractant, 6-methyl-5-heptene-2-one, was also found in lower quantities on a day when a person was more attractive (Bernier et al. 2002). Similarly, Logan et al. (2008) showed that 6-methyl-5heptene-2-one and especially geranyl acetone may be responsible for the decreased attraction of certain individuals. The former inhibited flight activation and probing activity but not relative attraction, whereas the latter reduced flight activity over all doses tested as well as relative attraction and probing activity.

The aldehydes octanal, nonanal and decanal, which are present at high levels in the skin emanations of less attractive individuals, caused a significant reduction in upwind flight of Ae. aegypti females and reduced attraction to a human hand at certain concentrations (Bernier et al. 2002, Logan et al. 2008). This indicates that compounds that are present on human skin may act as repellents or 'mask' the attractive effects of other skin compounds. The abundance of these compounds may explain the relative attractiveness of different individuals to this mosquito species.

Hexanal, octanal and z-4-decanal, all aldehydes produced by the seabird crested auklet (Aethia cristatella Pallas), were shown to decrease the number of landings on a human hand by Ae. aegypti mosquitoes at doses similar to concentrations produced by this bird species (Douglas et al. 2001, 2005). Octanal (2.5\%) was as repellent as a mixture of four aldehydes, including octanal and two carboxylic acids mimicking the auklet odorant in its natural composition. Both $2 \%$ hexanal and $2 \%$ hexanoic acid reduced the number of mosquito landings to the same extent (Douglas et al. 2005).

Several other compounds, which were interesting because they were more abundant in the skin emanations of a less attractive individual or were found in higher or lesser amounts when the skin emanations of a certain person were more attractive on some days than on other days, have been screened (Bernier et al. 2002). These compounds, which include two alcohols, 4-hexen-1-ol and 1-hepten-3-ol, with structural similarity to 1-octen-3-ol, an attractant for some mosquito and haematophagous fly species (e.g. Takken and Kline 1989, Schofield et al. 1997, Vale and Hall $1985 a, b)$, were not attractive when tested individually (Table 1).

Aedes albopictus (Skuse), a competent vector for many arboviruses, which exhibits an opportunistic host-feeding pattern and is extending its range around the world (Gratz 2004, Richards et al. 2006, Turell et al. 2005) has been shown to be activated (in a Y-tube olfactometer) to a greater degree by hexanoic acid, ethyl butyrate and dimethyl disulfide than by lactic acid. These compounds were also shown to be attractive to this mosquito species (Wang et al. 2006). In contrast, Shirai et al. (2001) found a repellent, or inhibitory, effect of lactic acid for this mosquito species when applied on normally attractive human or mouse skin. 


\section{Anopheles gambiae s.s.}

Experiments in an African village conducted by Haddow (1942) indicated that the host-seeking behaviour of An. gambiae females is mediated primarily by human odour. This has been proven by many studies since (reviewed by Takken and Knols 1999). In the laboratory, An. gambiae is strongly attracted to human emanations, in particular skin emanations. A single finger (Dekker et al. 2001b, Smallegange et al. 2002), ethanol washings of human hands and feet (Braks 1999), human sweat (Braks and Takken 1999, Meijerink et al. 2000, Smallegange et al. in press), and skin emanations of human hands or feet transferred to fabric (Dekker et al. 2001a, Pates et al. 2001b, Qiu et al. 2004b, Smallegange et al. in press, Spitzen et al. 2008) or glass beads (Qiu et al. 2004a, 2006a) are highly attractive when tested in wind tunnels, Y-tube or dual-choice olfactometers or indoor experiments with mosquito traps.

Several compounds present in human sweat or on human skin have been tested individually or in blends under laboratory conditions to identify their effect on An. gambiae females (Table 2). Ammonia is an important kairomone for this mosquito species; it is attractive over a wide range of concentrations and suppresses the inhibitory or repellent effect of a carboxylic acid mixture (Braks et al. 2001, Smallegange et al. 2005). However, at high doses ammonia has a repellent, or inhibitory, effect (Smallegange et al. 2005).

The role of lactic acid in the host-orientation phase seems to be different in An. gambiae compared to Ae. aegypti; on its own it is either not an attractant, or only a weak attractant (Braks et al. 2001, Dekker et al. 2002, Healy and Copland 2000, Smallegange et al. 2002, 2005). However, it has been found to augment the attractiveness of natural human skin odour, carbon dioxide, and synthetic odour blends, indicating that lactic acid is an essential compound in the orientation of An. gambiae to human hosts (Dekker et al. 2002, Qiu 2005, Smallegange et al. 2005, 2009).

Variable results have been obtained in field studies with carbon dioxide (Costantini et al. 1996, Knols et al. 1998, Mboera and Takken 1997, Qiu et al. 2007a). For this species the compound is considered to indicate the presence of a potential host while host-selection is accomplished by host-specific cues (Takken and Knols 1999). Dual-port olfactometer studies have shown that $\mathrm{CO}_{2}$ is a poor kairomone for An. gambiae that can even have a repellent, or inhibitory, effect depending on the structure of the plume and the positioning of the release point (Dekker et al. 2001b, Spitzen et al. 2008). The compound, however, activates An. gambiae, guiding them towards the release point of $\mathrm{CO}_{2}$ after which human skin emanations attract the mosquitoes into a trapping device (Dekker et al. 2001b, Healy and Copland 1995, Knols et al. 1994b, Spitzen et al. 2008). This was demonstrated by the fact that $\mathrm{CO}_{2}$ released in a turbulent plume in front of the trap entrance enhanced the attractiveness of skin emanations. This combination was more attractive than the synthetic blend of ammonia, lactic acid and $\mathrm{CO}_{2}$ (although the former two compounds increased the effect of $\mathrm{CO}_{2}$ ), indicating that additional compounds are involved in the host-seeking behaviour of this mosquito species (Spitzen et al. 2008). Recently, (semi-)field studies confirmed that the addition of $\mathrm{CO}_{2}$ to human emanations increases the number of An. gambiae caught in odour baited mosquito traps (Jawara et al. 2009, Njiru et al. 2006, Schmied et al. 2008).

Human sweat has been shown to stimulate An. gambiae females to land at the odour source, whereas a mixture of 22 aliphatic carboxylic acids, which have been identified from human sweat, did not elicit a landing response (Healy and Copland 2000). However, a blend of 12 aliphatic carboxylic acids was found to be attractive when highly diluted (Knols et al. 1997). A similar blend, although repellent or inhibitory on its own, was attractive when combined with ammonia and 
Table 2. Overview of chemical compounds that have been tested for their impact on the host-seeking behaviour of Anopheles gambiae s.s. using behavioural assays in the laboratory. (Details about concentrations of compounds are omitted).

\begin{tabular}{|c|c|c|c|}
\hline Compound & Response & Behavioural assay & Reference \\
\hline carbon dioxide $\left(\mathrm{CO}_{2}\right)$ & $\begin{array}{l}\text { Activation and upwind flight } \\
\text { (Weakly) attractive on its own } \\
\text { Homogeneous } \mathrm{CO}_{2} \text { reduced trap } \\
\text { catch; Turbulent } \mathrm{CO}_{2} \text { caused } \\
\text { activation and attractive synergism } \\
\text { with skin emanations }\end{array}$ & $\begin{array}{l}\text { Wind tunnel } \\
\text { Dual-port olfactometer } \\
\text { Dual-port olfactometer } \\
\text { Dual-port olfactometer }\end{array}$ & $\begin{array}{l}\text { Healy and Copland (1995) } \\
\text { Knols et al. (1994b) } \\
\text { Dekker et al. (2001b) } \\
\text { Spitzen et al. (2008) }\end{array}$ \\
\hline ammonia $\left(\mathrm{NH}_{3}\right)$ & $\begin{array}{l}\text { Attractive or repellent / inhibitory } \\
\text { effect depending on doses }\end{array}$ & $\begin{array}{l}\text { Dual-port olfactometer } \\
\text { Dual-port olfactometer } \\
\text { Y-tube }\end{array}$ & $\begin{array}{l}\text { Braks et al. (2001) } \\
\text { Smallegange et al. (2005) } \\
\text { Smallegange et al. (2002) }\end{array}$ \\
\hline \multicolumn{4}{|c|}{ (1) } \\
\hline 3-methyl-1-butanol & $\begin{array}{l}\text { Repellent / inhibitory effect when } \\
\text { combined with } \mathrm{NH}_{3}+\mathrm{LA}\end{array}$ & Dual-port olfactometer & Qiu (2005) \\
\hline 4-ethylphenol & $\begin{array}{l}\text { Repellent / inhibitory effect when } \\
\text { combined with } \mathrm{NH}_{3}+\mathrm{LA}\end{array}$ & Dual-port olfactometer & Qiu (2005) \\
\hline 1-dodecanol & $\begin{array}{l}\text { No effect when combined with } \\
\mathrm{NH}_{3}+\mathrm{LA}\end{array}$ & Dual-port olfactometer & Qiu (2005) \\
\hline \multicolumn{4}{|c|}{ Aliphatic carboxylic acids } \\
\hline \multirow[t]{5}{*}{ lactic acid (LA) } & No landing response & Wind tunnel & Healy and Copland (2000) \\
\hline & Not or weakly attractive on its own & $\begin{array}{l}\text { Dual-port olfactometer } \\
\text { Y-tube }\end{array}$ & $\begin{array}{l}\text { Braks et al. (2001) } \\
\text { Smallegange et al. (2002) }\end{array}$ \\
\hline & $\begin{array}{l}\text { Augmented attractiveness of } \mathrm{CO}_{2} \\
\text { and human odour }\end{array}$ & Y-tube & Dekker et al. (2002) \\
\hline & Synergism when combined with & Dual-port olfactometer & Smallegange et al. (2005) \\
\hline & $\mathrm{NH}_{3}$ and carboxylic acids & Dual-port olfactometer & Smallegange et al. (2009) \\
\hline acetic acid & $\begin{array}{l}\text { No effect in combination with } \mathrm{NH}_{3} \\
+\mathrm{LA}\end{array}$ & Dual-port olfactometer & Smallegange et al. (2009) \\
\hline propanoic acid & $\begin{array}{l}\text { Synergism when combined with } \\
\mathrm{NH}_{3}+\mathrm{LA}\end{array}$ & Dual-port olfactometer & Smallegange et al. (2009) \\
\hline $\begin{array}{l}\text { 2-methylpropanoic } \\
\text { acid }\end{array}$ & $\begin{array}{l}\text { No effect in combination with } \mathrm{NH}_{3} \\
+\mathrm{LA}\end{array}$ & Dual-port olfactometer & Smallegange et al. (2009) \\
\hline butanoic acid & $\begin{array}{l}\text { Synergism when combined with } \\
\mathrm{NH}_{3}+\mathrm{LA}\end{array}$ & Dual-port olfactometer & Smallegange et al. (2009) \\
\hline $\begin{array}{l}\text { 3-methyl butanoic } \\
\text { acid }\end{array}$ & $\begin{array}{l}\text { Synergism when combined with } \\
\mathrm{NH}_{3}+\mathrm{LA}\end{array}$ & Dual-port olfactometer & Smallegange et al. (2009) \\
\hline pentanoic acid & $\begin{array}{l}\text { Synergism when combined with } \\
\mathrm{NH}_{3}+\mathrm{LA}\end{array}$ & Dual-port olfactometer & Smallegange et al. (2009) \\
\hline \multirow[t]{2}{*}{ hexanoic acid } & No effect on its own & Y-tube & Smallegange et al. (2002) \\
\hline & $\begin{array}{l}\text { Synergism when combined with } \\
\mathrm{NH}_{3}+\mathrm{LA} \text { or repellent / inhibitory } \\
\text { effect depending on doses }\end{array}$ & Dual-port olfactometer & Smallegange et al. (2009) \\
\hline
\end{tabular}


Table 2. Continued.

\begin{tabular}{|c|c|c|c|}
\hline Compound & Response & Behavioural assay & Reference \\
\hline heptanoic acid & $\begin{array}{l}\text { Synergism when combined with } \\
\mathrm{NH}_{3}+\mathrm{LA} \text { or repellent / inhibitory } \\
\text { effect depending on doses }\end{array}$ & Dual-port olfactometer & Smallegange et al. (2009) \\
\hline octanoic acid & $\begin{array}{l}\text { Synergism when combined with } \\
\mathrm{NH}_{3}+\mathrm{LA}\end{array}$ & Dual-port olfactometer & Smallegange et al. (2009) \\
\hline nonanoic acid & $\begin{array}{l}\text { No effect when combined with } \\
\mathrm{NH}_{3}+\mathrm{LA}\end{array}$ & Dual-port olfactometer & Smallegange et al. (2009) \\
\hline decanoic acid & $\begin{array}{l}\text { No effect when combined with } \\
\mathrm{NH}_{3} \text { and LA }\end{array}$ & Dual-port olfactometer & Smallegange et al. (2009) \\
\hline dodecanoic acid & $\begin{array}{l}\text { No effect when combined with } \\
+ \text { LA }\end{array}$ & Dual-port olfactometer & Smallegange et al. (2009) \\
\hline tridecanoic acid & $\begin{array}{l}\text { No effect when combined with } \\
\mathrm{NH}_{3}+\mathrm{LA}\end{array}$ & Dual-port olfactometer & Smallegange et al. (2009) \\
\hline \multirow[t]{2}{*}{ tetradecanoic acid } & $\begin{array}{l}\text { Synergism when combined with } \\
\mathrm{NH}_{3}+\mathrm{LA}\end{array}$ & Dual-port olfactometer & Smallegange et al. (2009) \\
\hline & $\begin{array}{l}\text { Attractive when combined with } \\
\mathrm{NH}_{3}+\mathrm{LA}\end{array}$ & $\begin{array}{l}\text { Indoor trapping } \\
\text { experiment }\end{array}$ & Smallegange et al. (2009) \\
\hline hexadecanoic acid & $\begin{array}{l}\text { No effect in combination with } \mathrm{NH}_{3} \\
+\mathrm{LA}\end{array}$ & Dual-port olfactometer & Smallegange et al. (2009) \\
\hline blend of 12 & Attractive & Dual-port olfactometer & Knols et al. (1997) \\
\hline \multirow[t]{2}{*}{ carboxylic acids } & Repellent / inhibitory effect & Dual-port olfactometer & Smallegange et al. (2005) \\
\hline & $\begin{array}{l}\text { Synergism when combined with } \\
\mathrm{NH}_{3}+\mathrm{LA}\end{array}$ & Dual-port olfactometer & Smallegange et al. (2005) \\
\hline $\begin{array}{l}\text { blend of } 22 \\
\text { carboxylic acids }\end{array}$ & No landing response & Wind tunnel & $\begin{array}{l}\text { Healy and Copland } \\
(2000)\end{array}$ \\
\hline blend of ammonia, & Attractive & Dual-port olfactometer & Smallegange et al. (2009) \\
\hline $\begin{array}{l}\text { lactic acid and } 7 \\
\text { carboxylic acids }\end{array}$ & & $\begin{array}{l}\text { Indoor trapping } \\
\text { experiment }\end{array}$ & Smallegange et al. (2009) \\
\hline \multicolumn{4}{|c|}{ Unsaturated carboxylic acids } \\
\hline 7-octenoic acid & $\begin{array}{l}\text { Attractive when combined with } \\
\mathrm{NH}_{3}+\mathrm{LA}\end{array}$ & Dual-port olfactometer & Qiu (2005) \\
\hline $\begin{array}{l}\text { combination of (E/Z)- } \\
\text { 3-methyl-2-hexenoic } \\
\text { acid isomer mixture } \\
\text { and 7-octenoic acid }\end{array}$ & Reduced response to $\mathrm{CO}_{2}$ & Dual-port olfactometer & Costantini et al. (2001) \\
\hline \multicolumn{4}{|c|}{ Oxocarboxylic acids } \\
\hline 2-oxopropanoic acid & No landing response & Wind tunnel & Healy et al. (2002) \\
\hline 2-oxobutanoic acid & Induced landing response & Wind tunnel & Healy et al. (2002) \\
\hline $\begin{array}{l}\text { 2-oxo-3- } \\
\text { methylbutanoic acid }\end{array}$ & Induced landing response & Wind tunnel & Healy et al. (2002) \\
\hline \multirow[t]{2}{*}{ 2-oxopentanoic acid } & Induced landing response & Wind tunnel & $\begin{array}{l}\text { Healy and Copland } \\
\text { (2000) }\end{array}$ \\
\hline & & Wind tunnel & Healy et al. (2002) \\
\hline
\end{tabular}


Table 2. Continued.

\begin{tabular}{|c|c|c|c|}
\hline Compound & Response & Behavioural assay & Reference \\
\hline $\begin{array}{l}\text { 2-oxo-3- } \\
\text { methylpentanoic } \\
\text { acid }\end{array}$ & Induced landing response & Wind tunnel & Healy et al. (2002) \\
\hline $\begin{array}{l}\text { 2-oxo-4- } \\
\text { methylpentanoic } \\
\text { acid }\end{array}$ & Induced landing response & Wind tunnel & Healy et al. (2002) \\
\hline 2-oxohexanoic acid & Induced landing response & Wind tunnel & Healy et al. (2002) \\
\hline 2-oxooctanoic acid & No landing response & Wind tunnel & Healy et al. (2002) \\
\hline $\begin{array}{l}\text { 2-hydroxypentanoic } \\
\text { acid }\end{array}$ & No landing response & Wind tunnel & Healy et al. (2002) \\
\hline \multicolumn{4}{|l|}{ Ketones } \\
\hline \multirow[t]{3}{*}{ acetone } & $\begin{array}{l}\text { Activating effect when combined } \\
\text { with } \mathrm{CO}_{2}\end{array}$ & Wind tunnel & Takken et al. (1997) \\
\hline & $\begin{array}{l}\text { Enhanced the effect of } \mathrm{LA} \\
\text { decreased effect of } \mathrm{NH}_{3}\end{array}$ & Dual-port olfactometer & Qiu (2005) \\
\hline & $\begin{array}{l}\text { No aditional attractiviness when } \\
\text { combined with } \mathrm{NH}_{3}+\mathrm{LA}\end{array}$ & Dual-port olfactometer & Qiu (2005) \\
\hline $\begin{array}{l}\text { 6-methyl-5-hepten- } \\
\text { 2-one }\end{array}$ & $\begin{array}{l}\text { Repellent / inhibitory effect when } \\
\text { combined with } \mathrm{NH}_{3}+\mathrm{LA}\end{array}$ & Dual-port olfactometer & Qiu (2005) \\
\hline geranyl acetone & $\begin{array}{l}\text { Repellent / inhibitory effect when } \\
\text { combined with } \mathrm{NH}_{3}+\mathrm{LA}\end{array}$ & Dual-port olfactometer & Qiu (2005) \\
\hline \multicolumn{4}{|l|}{ Miscellaneous } \\
\hline benzothiazole & Repellent / inhibitory effect & Dual-port olfactometer & Qiu et al. (2004a) \\
\hline indole & $\begin{array}{l}\text { Repellent / inhibitory effect when } \\
\text { tested alone and when combined } \\
\text { with } \mathrm{NH}_{3}+\mathrm{LA}\end{array}$ & Dual-port olfactometer & Qiu (2005) \\
\hline blend of 1-butanol, & Attractive & Indoor trapping & Verhulst et al. (2009) \\
\hline 2,3-butanedione, & & experiment & \\
\hline $\begin{array}{l}\text { 2-methyl-1-butanol, } \\
\text { 2-methylbutanal, }\end{array}$ & & & \\
\hline 2-methylbutanoic acid & & & \\
\hline 3-hydroxy-2-butanone, & & & \\
\hline 3-methyl-1-butanol, & & & \\
\hline 3-methylbutanal, & & & \\
\hline $\begin{array}{l}\text { 3-methylbutanoic acid, } \\
\text { benzeneethanol }\end{array}$ & & & \\
\hline
\end{tabular}

lactic acid (Smallegange et al. 2005). In dual-choice olfactometer experiments, this blend was less attractive than a worn sock, suggesting that more volatiles play a role in the host-orientation of An. gambiae (Smallegange et al., in press).

In addition, seven individual saturated aliphatic carboxylic acids (propanoic, butanoic, 3-methylbutanoic, pentanoic, heptanoic, octanoic and tetradecanoic acid) and an unsaturated carboxylic 
acid (7-octenoic acid) increased the attractiveness of ammonia when combined in a tripartite blend together with lactic acid (Qiu 2005, Smallegange et al. 2009). A blend consisting of ammonia, lactic acid and the seven saturated carboxylic acids was attractive both in a dual-choice olfactometer and in indoor trapping experiments in a netted cage (Smallegange et al. 2009). Moreover, this synthetic blend, combined with $\mathrm{CO}_{2}$, attracted more mosquitoes than humans when present in different experimental huts, whereas it was equally or less attractive than humans when present within the same hut (Okumu et al. 2010).

Hexanoic and heptanoic acid were found to have inhibitory/repellent effects at certain doses (Smallegange et al. 2002, 2009). A range of other carboxylic acids (acetic, 2-methylpropanoic, nonanoic, decanoic, dodecanoic, tridecanoic and hexadecanoic acid) had no effect on the attractiveness of ammonia combined with lactic acid (Smallegange et al. 2009). The combination of 7-octenoic acid and the isomer mixture of (E/Z)-3-methyl-2-hexenoic acid reduced the response to $\mathrm{CO}_{2}$ in a dual-choice olfactometer (Costantini et al. 2001). The latter is a major component in human axillary odour, the former a minor component (Zeng et al. 1991, 1996).

Six oxocarboxylic acids (2-oxobutanoic, 2-oxo-3-methylbutanoic, 2-oxopentanoic, 2-oxo-3methylpentanoic, 2-oxo-4-methylpentanoic and 2-oxohexanoic acid), three of which have been identified in human sweat, caused a landing response in An. gambiae females (Table 2). More mosquitoes landed in response to 2-oxopentanoic acid than to the other oxocarboxylic acids. The landing response to this compound was found to be temperature dependent and decreased to control levels within 10 minutes after application (Healy and Copland 2000, Healy et al. 2002).

Three alcohols (1-dodecanol, 4-ethyl phenol, 3-methyl-butanol) individually added to ammonia and lactic acid had no effect, inhibited or repelled An. gambiae females (Qiu 2005), although two of them (1-dodecanol, 3-methyl-butanol) were found at high levels in incubated sweat, which was found to be more attractive than fresh sweat (Braks and Takken 1999, Meijerink et al. 2000).

Mosquitoes preferred ammonia over a blend of ammonia, lactic acid and either geranyl acetone or 6-methyl-5-hepten-2-one, depending on the doses of the ketones (Qiu 2005). These two ketones were found in equal quantities in fresh and incubated sweat (Meijerink et al. 2000).

Acetone is well known as an attractive kairomone for tsetse flies. This compound has been found in blood, milk, urine and breath of cattle, which is the host of these vectors of trypanosomiasis (Jordan 1986, Torr et al. 1995, Vale 1980, Vale and Hall 1985a,b). It is also relatively abundant in human breath (Philips 1997) and was found in fresh but not in incubated sweat (Meijerink et al. 2000). A human-equivalent physiological concentration of acetone had an activating effect on $A n$. gambiae when added to $\mathrm{CO}_{2}$ (Takken et al. 1997). It was also found to increase the attractiveness of lactic acid, but decreased the attractiveness of ammonia and was not attractive on its own. Although the triple blend of ammonia, lactic acid and acetone was attractive, it was as attractive as ammonia alone (Qiu 2005).

When tested alone, indole, which is abundantly present in incubated sweat (Meijerink et al. 2000), caused either no effect, inhibition or repelled, depending on the concentration tested. A blend of ammonia, lactic acid and indole was significantly less attractive than ammonia alone (Qiu 2005). A compound found in the headspace of worn nylon stockings but suspected to be not specifically human, benzothiazole, repelled An. gambiae females at close range in a dual-port olfactometer (Qiu et al. 2004b). 
An. gambiae is attracted to volatiles produced by human skin microbiota both in dual-choice olfactometer and in indoor trapping experiments. Moreover, MM-X traps baited with a blend of 10 compounds present in the headspace of human feet microbiota (1-butanol, 2,3-butanedione, 2-methyl-1-butanol, 2-methylbutanal, 2-methylbutanoic acid, 3-hydroxy-2-butanone, 3-methyl-1butanol, 3-methylbutanal, 3-methylbutanoic acid, benzeneethanol) caught significantly more An. gambiae during indoor trapping experiments than unbaited traps (Verhulst et al. 2009).

Another member of the An. gambiae complex, An. quadriannulatus (Theobald), has long been considered to be zoophilic and therefore of no medical importance (Takken et al. 1999). Hence its host-seeking behaviour has rarely been studied. Pates et al. (2001a), however, showed that this species has an equal preference for humans and calves. In addition, An. quadriannulatus preferred human odour over a synergistic combination of cow odour with $\mathrm{CO}_{2}$ in a dual-port olfactometer. Carbon dioxide alone was not attractive, whereas $\mathrm{CO}_{2}$ with 1-octen-3-ol had a inhibitory or repellent effect. The effect of acetone was not clear (Pates et al. 2005). A recent field study with An. quadriannulatus confirmed the finding that this species is attracted to human and cattle odours (Torr et al. 2008).

\section{Culex quinquefasciatus}

Almost $60 \%$ of the $C x$. quinquefasciatus females from a colony that originated from Sri Lanka that were released individually in a dual-port olfactometer were attracted within three minutes to polyamide stockings that had been worn by a human volunteer for the preceding 48-60 hours (Mboera et al. 1998), whereas, in a separate study, less than $25 \%$ of the mosquitoes from a colony in the USA released in groups of 50-70 females were attracted to a human hand (Allan et al. 2006b). Skin rubbings and ethanol washings of human hands and feet or the back were attractive to groups or individual female mosquitoes (Braks et al. 1999). These results demonstrate that $C X$. quinquefasciatus from different parts of the world are attracted to sources of volatiles that are present on human skin.

In laboratory studies carbon dioxide has little or no attractive effect on this species. A high concentration was found to be repellent. Contrary to what has been found for other mosquito species, no synergistic effect of $\mathrm{CO}_{2}$ in combination with skin emanations has been observed (Allan et al. 2006b, Braks et al. 1999, Mboera et al. 1998).

Culex quinquefasciatus was attracted to lactic acid in dual-port olfactometer studies (Allan et al. 2006a, Braks et al. 1999). Ethanol washings from human hands and feet were significantly more attractive than lactic acid alone, demonstrating that lactic acid is not the only attractive component on human skin (Braks et al. 1999). Eighteen other compounds present in human skin emanations were found to be attractive at certain doses to this species when tested individually in a Y-tube olfactometer (Table 3). Some of these attracted over $75 \%$ of the mosquitoes released: heptanal, nonanal, ethylene glycol, benzyl alcohol, and eleven carboxylic acids (Puri et al. 2006). Several of these compounds, however, were not found to have an effect on mosquitoes in a dualport olfactometer study, possibly due to the concentrations at which these compounds were tested (Table 3) (Allan et al. 2006a,b). Other alcohols and carboxylic acids had either no effect or exhibited decreased behavioural responses compared to the control, depending on the doses applied in a Y-tube olfactometer (Table 3) (Puri et al. 2006).

Recent field experiments showed that not only $\mathrm{CO}_{2}$ enhances trap catches, but also nonanal. The latter compound is dominantly present in the odour profile of pigeons, chickens and humans from 
Table 3. Overview of chemical compounds that have been tested for their impact on the host-seeking behaviour of Culex quinquefasciatus using behavioural assays in the laboratory. (Details about concentrations of compounds are omitted).

\begin{tabular}{|c|c|c|c|}
\hline Compound & Response & Behavioural assay & Reference \\
\hline carbon dioxide $\left(\mathrm{CO}_{2}\right)$ & $\begin{array}{l}\text { Not or weakly attractive on its } \\
\text { own; repellent / inhibitory effect at } \\
\text { high concentration; no synergism } \\
\text { with host emanations }\end{array}$ & $\begin{array}{l}\text { Dual-port olfactometer } \\
\text { Dual-port olfactometer } \\
\text { Dual-port olfactometer }\end{array}$ & $\begin{array}{l}\text { Mboera et al. (1998) } \\
\text { Braks et al. (1999) } \\
\text { Allan et al. (2006b) }\end{array}$ \\
\hline \multicolumn{4}{|l|}{ Alcohols } \\
\hline ethylene glycol & Attractive & Y-tube & Puri et al. (2006) \\
\hline glycerol & Repellent / inhibitory effect & Y-tube & Puri et al. (2006) \\
\hline phenol & No response & Y-tube & Puri et al. (2006) \\
\hline benzyl alcohol & Attractive & Y-tube & Puri et al. (2006) \\
\hline decanol & $\begin{array}{l}\text { Attractive or repellent / inhibitory } \\
\text { effect depending on doses }\end{array}$ & Y-tube & Puri et al. (2006) \\
\hline cholesterol & Attractive & Y-tube & Puri et al. (2006) \\
\hline \multicolumn{4}{|l|}{ Aldehydes } \\
\hline propanal & Attractive & Y-tube & Puri et al. (2006) \\
\hline hexanal & No response & Dual-port olfactometer & Allan et al. (2006b) \\
\hline heptanal & Attractive & Y-tube & Puri et al. (2006) \\
\hline \multirow[t]{2}{*}{ benzaldehyde } & No response & Y-tube & Puri et al. (2006) \\
\hline & & Dual-port olfactometer & Allan et al. (2006b) \\
\hline \multirow[t]{2}{*}{ nonanal } & Attractive & Y-tube & Puri et al. (2006) \\
\hline & No response & Dual-port olfactometer & Allan et al. (2006b) \\
\hline \multicolumn{4}{|c|}{ Aliphatic carboxylic acids } \\
\hline \multirow{3}{*}{ lactic acid (LA) } & Attractive & Dual-port olfactometer & Allan et al. (2006a) \\
\hline & & Dual-port olfactometer & Braks et al. (1999) \\
\hline & No landing response & Landing assay & Allan et al. (2006a) \\
\hline \multirow[t]{2}{*}{ acetic acid } & Not attractive on its own & Dual-port olfactometer & Allan et al. (2006a) \\
\hline & Induced landing response & Landing assay & Allan et al. (2006a) \\
\hline \multirow[t]{3}{*}{ propanoic acid } & Attractive & Y-tube & Puri et al. (2006) \\
\hline & Not attractive on its own & Dual-port olfactometer & Allan et al. (2006a) \\
\hline & No landing response & Landing assay & Allan et al. (2006a) \\
\hline \multirow[t]{2}{*}{ butanoic acid } & Not attractive on its own & Dual-port olfactometer & Allan et al. (2006a) \\
\hline & No landing response & Landing assay & Allan et al. (2006a) \\
\hline \multirow{6}{*}{$\begin{array}{l}\text { 3-methyl butanoic } \\
\text { acid } \\
\text { hexanoic acid } \\
\text { heptanoic acid }\end{array}$} & Not attractive on its own & Dual-port olfactometer & Allan et al. (2006a) \\
\hline & No landing response & Landing assay & Allan et al. (2006a) \\
\hline & Attractive & Y-tube & Puri et al. (2006) \\
\hline & Attractive & Y-tube & Puri et al. (2006) \\
\hline & Not attractive on its own & Dual-port olfactometer & Allan et al. (2006a) \\
\hline & No landing response & Landing assay & Allan et al. (2006a) \\
\hline octanoic acid & Attractive & Y-tube & Puri et al. (2006) \\
\hline nonanoic acid & Attractive & Y-tube & Puri et al. (2006) \\
\hline decanoic acid & Attractive & Y-tube & Puri et al. (2006) \\
\hline undecanoic acid & Attractive & Y-tube & Puri et al. (2006) \\
\hline dodecanoic acid & Attractive & Y-tube & Puri et al. (2006) \\
\hline tridecanoic acid & Attractive & Y-tube & Puri et al. (2006) \\
\hline
\end{tabular}


Table 3. Continued.

\begin{tabular}{|c|c|c|c|}
\hline Compound & Response & Behavioural assay & Reference \\
\hline \multirow[t]{3}{*}{ tetradecanoic acid } & Attractive & Y-tube & Puri et al. (2006) \\
\hline & Not attractive on its own & Dual-port olfactometer & Allan et al. (2006a) \\
\hline & No landing response & Landing assay & Allan et al. (2006a) \\
\hline pentadecanoic acid & Repellent / inhibitory effect & Y-tube & Puri et al. (2006) \\
\hline \multirow[t]{3}{*}{ hexadecanoic acid } & Repellent / inhibitory effect & Y-tube & Puri et al. (2006) \\
\hline & Not attractive on its own & Dual-port olfactometer & Allan et al. (2006a) \\
\hline & Induced landing response & Landing assay & Allan et al. (2006a) \\
\hline \multirow{4}{*}{$\begin{array}{l}\text { heptadecanoic acid } \\
\text { octadecanoic acid }\end{array}$} & Attractive & Y-tube & Puri et al. (2006) \\
\hline & Repellent / inhibitory effect & Y-tube & Puri et al. (2006) \\
\hline & Not attractive on its own & Dual-port olfactometer & Allan et al. (2006a) \\
\hline & Induced landing response & Landing assay & Allan et al. (2006a) \\
\hline \multicolumn{4}{|c|}{ Aromatic carboxylic acids } \\
\hline benzoic acid & No landing response & Landing assay & Allan et al. (2006a) \\
\hline $\begin{array}{l}\text { 2-hydroxybenzoic } \\
\text { acid }\end{array}$ & No landing response & Landing assay & Allan et al. (2006a) \\
\hline \multicolumn{4}{|l|}{ Sulfides } \\
\hline \multirow[t]{2}{*}{ methyl sulfide } & Not attractive on its own & Dual-port olfactometer & Allan et al. (2006a) \\
\hline & No landing response & Landing assay & Allan et al. (2006a) \\
\hline \multirow[t]{2}{*}{ carbon disulfide } & Not attractive on its own & Dual-port olfactometer & Allan et al. (2006a) \\
\hline & No landing response & Landing assay & Allan et al. (2006a) \\
\hline \multirow[t]{2}{*}{ dimethyl disulfide } & Not attractive on its own & Dual-port olfactometer & Allan et al. (2006a) \\
\hline & No landing response & Landing assay & Allan et al. (2006a) \\
\hline \multirow[t]{2}{*}{ ethyl disulfide } & Not attractive on its own & Dual-port olfactometer & Allan et al. (2006a) \\
\hline & No landing response & Landing assay & Allan et al. (2006a) \\
\hline methyl propyl & Not attractive on its own & Dual-port olfactometer & Allan et al. (2006a) \\
\hline disulfide & Induced landing response & Landing assay & Allan et al. (2006a) \\
\hline \multirow[t]{2}{*}{ dimethyl trisulfide } & Not attractive on its own & Dual-port olfactometer & Allan et al. (2006a) \\
\hline & Induced landing response & Landing assay & Allan et al. (2006a) \\
\hline \multicolumn{4}{|c|}{ Miscellaneous } \\
\hline meso-2,3-butanediol & Not attractive on its own & Dual-port olfactometer & Allan et al. (2006b) \\
\hline 2,3-butanediol & Not attractive on its own & Dual-port olfactometer & Allan et al. (2006b) \\
\hline a-pinene & Not attractive on its own & Dual-port olfactometer & Allan et al. (2006b) \\
\hline$\beta$-myrcene & Not attractive on its own & Dual-port olfactometer & Allan et al. (2006b) \\
\hline 2,3-docosanediol & Not attractive on its own & Dual-port olfactometer & Allan et al. (2006b) \\
\hline $\begin{array}{l}\text { blend of hexanal, } \\
\text { a-pinene, } \\
\text { benzaldehyde, } \\
\beta \text {-myrcene, and } \\
\text { nonanal }\end{array}$ & Not attractive & Dual-port olfactometer & Allan et al. (2006b) \\
\hline
\end{tabular}


various ethnic backgrounds. Traps baited with both nonanal and $\mathrm{CO}_{2}$ caught the highest number of Cx. quinquefasciatus (Syed and Leal 2009).

Culex quinquefasciatus females assayed in a dual-port olfactometer responded to a different extent to odour sources of animal origin (bovine blood, avian blood, chickens, chicken feathers, chicken feathers combined with $\mathrm{CO}_{2}$, cotton balls rubbed over chicken feathers and hexane extracts of chicken feathers) (Allan et al. 2006a,b). However, there was no response to five compounds identified from feathers (Williams et al. 2003) individually or as a blend (hexanal, benzaldehyde, nonanal, a-pinene, and $\beta$-myrcene), or to three individual compounds (meso-2,3-butanediol, 2,3-butanediol and 2,3-docosanediol) that have been identified in the preen gland of hens (Allan et al. 2006b, Haathi and Fales 1967; Table 3).

In a Y-tube olfactometer, fresh chicken faeces was attractive to $C x$. quinquefasciatus. Eight aldehydes ((E)-2-decanal, undecanal, dodecanal, tetradecanal, pentadecanal, hexadecanal, heptadecanal and octadecanal) identified in the headspace of the faeces elicited electroantennogram responses, as well as eight unknown compounds (Cooperband et al. 2008), and should be tested for their effect on the host-seeking behaviour of $C x$. quinquefasciatus females.

Volatile compounds associated with blood (aliphatic and aromatic carboxylic acids and sulphides) showed no attractiveness in olfactometer assays, with the exception of lactic acid (Table 3). However, in cage assays, significantly more landings were observed on collagen sausage casings treated with acetic, hexadecanoic, and octadecanoic acid, dimethyl trisulfide or methyl propyl disulfide than on casings treated with water (Allan et al. 2006a).

Female Cx. nigripalpus Theobald, another mosquito species associated with West Nile virus transmission in the USA (Godsey et al. 2005, Sardelis et al. 2001), were attracted to tetradecanoic acid, dimethyl disulfide and methyl propyl disulfide and landed on casings treated with lactic acid, octadecanoic acid, dimethyl trisulfide, ethyl disulfide or methyl propyl disulfide, although responses to bovine blood were low (Allan et al. 2006a). Culex nigripalpus and Cx. tarsalis Coquillett did not respond to human odour, whereas they were attracted to chicken odour in a dual-port olfactometer. Culex tarsalis was significantly more responsive to $\mathrm{CO}_{2}$ than were Ae. aegypti, $\mathrm{Cx}$. quinquefasciatus and Cx. nigripalpus (Allan et al. 2006b).

\section{Discussion}

In the last decade much progress has been made in the identification of kairomones and allomones of Ae. aegypti, An. gambiae and Cx. quinquefasciatus. However, in the laboratory assays these compounds and blends are less attractive than natural odours and work should continue to identify the 'missing' compounds. Recent advances in molecular olfaction and neurophysiology are expected to contribute to a rapid identification of essential odorants that are lacking from the current blends.

Robust behavioural assays are available for screening olfactory cues to which mosquitoes respond, although some devices are not suitable for all species. For example, An. gambiae behaviour is erratic in Y-tube assays (Smallegange et al. 2002), whereas Ae. aegypti and Cx. quinquefasciatus behaviour is normal and consistent in this device (Geier and Boeckh 1999, Puri et al. 2006).

The main bottleneck of high-throughput testing of candidate semiochemicals is the relatively time-consuming operation of the behavioural bioassays, in contrast to neurophysiological studies. 
Three-layer olfactometers, in which three sets of mosquitoes can be tested simultaneously, as developed by the USDA research laboratory in Gainesville, Florida, USA (Posey et al. 1998), are a valuable means of increasing the rate at which mosquitoes can be tested. Nevertheless, each batch of novel odour blends needs to be tested in sufficient replicates, which slows down the bioassay evaluation. The development of tools that might accelerate the behavioural assays may be very useful so that new producers of semiochemicals will not be disappointed. These behavioural assays require large numbers of female mosquitoes, which can be a burden on the research budget as this requires the availability of climate-controlled insectaries and trained staff.

Progress in the development of digital recording techniques allows for the study of insect behaviour using 3D image analysers (see publications for details of products available). Patterns of flight behaviour in response to specific host-odours and blends of odours have been studied, revealing interesting behavioural changes during odour-mediated upwind anemotaxis (Beeuwkes et al. 2008, Braks et al. 2005, Cooperband and Cardé 2006, Chapter 6 in this volume). Thus, the specific role of a semiochemical in the mosquito behavioural repertoire can be established. This will facilitate the discovery of more effective odorant blends with which to modify or control mosquito behaviour.

Current techniques in semiochemistry allow for the rapid analysis of volatiles of human and animal origin (Bernier et al. 1999, Curran et al. 2007, Penn et al. 2007). This poses a potentially huge problem for the behavioural ecologist, as it is unlikely that all compounds present in host emanations are involved in host-seeking behaviour (Zwiebel and Takken 2004), and it is impractical to examine each compound in a behavioural assay. High-throughput testing of semiochemicals is possible at the neurophysiological level (Chapter 2 and 3, this volume), but encounters logistical obstructions at the behavioural level. Thus, chemical ecologists will probably rely on testing selected compounds and blends, based on results from neurophysiology and historical evidence. The exploration of chemical libraries for small molecules that might affect mosquito behaviour, as is currently in progress at different research facilities, will add a potentially large number of compounds that require behavioural testing (Justice et al. 2003, Zwiebel and Takken 2004).

At present, a novel and promising technique is under development by several research groups, in which differences in attractiveness to mosquitoes between human individuals is being exploited to study chemical profiles with the aim of identifying critical chemical groups or compounds that cause these differences (Bernier et al. 2002, Logan et al. 2008, Smallegange et al. 2003, Qiu et al. 2006a). Such compounds may be either kairomones or allomones (Dicke and Sabelis 1988). If the compounds that are significantly more abundant in highly attractive persons can be identified, it is likely to provide a rapid method for the development of attractive odour blends for mosquitoes, as it is expected that these compounds play a role in the attraction of these individuals. Alternatively, poorly attractive individuals may produce compounds that quench or even suppress the activity of other chemicals, so that the mosquito can no longer identify the source or, perhaps, is even repelled from it (Logan et al. 2008).

The relative concentrations of semiochemicals in odour blends is a fundamental factor in determining their attractiveness. For example, $C x$. quinquefasciatus was not attracted to certain synthetic volatiles of chicken origin (Allan et al. 2006b), whereas it did respond to natural bird odours. This may indicate that either critical volatiles were missing or that the relative concentrations of components in the blend were not appropriate. This example illustrates some of the difficulties inherent in the development of effective synthetic odour baits. Knowledge of the naturally occurring ratios of compounds in host volatiles is not necessarily useful; artificial 
baits for trapping and killing tsetse represent the most successful application of semiochemicals to control vectors in the world, and yet the blend of chemicals in the bait is only loosely related to the naturally occurring ratios and concentrations of compounds emitted by live hosts (Vale and Hall 1985a,b). It is also worth noting that compounds and blends of compounds that are attractive at a particular concentration, may be repellent or cause inhibition at higher concentrations (e.g. Knols et al. 1997, Smallegange et al. 2005, Vale and Hall 1985a). Therefore, single compounds and blends should be tested across a range of concentrations in a methodical way, similar to range testing doses of drugs or toxins. Differences in the concentrations of compounds tested may explain differences in the results of assays based on the same compounds (Allan et al. 2006a,b, Puri et al. 2006). Similarly, the type of assay used (Y-tube olfactometer, dual-port olfactometer, landing assay) may affect the results obtained.

A more directed approach may help to identify the most important compounds controlling host-seeking behaviour of particular species. For example, having found that hexane extracts of chicken feathers were attractive to $C x$. quinquefasciatus while ether extracts were not (Allan et al. 2006b), Bernier et al. (2008) examined these extracts and found that the ether extracts contained aldehydes, which were also present in the hexane extracts, whereas the hexane extracts also contained alcohols, ketones and diones. Subsequent behavioural assays can focus now on compounds of the latter three groups or can be preceded by either on-line (coupled with GC) or off-line EAG recordings to determine whether components present in the hexane extract are detected by the mosquito olfactory structures as Cooperband et al. (2008) did with acidified chicken faeces. A similar approach could be used to identify more active compounds, in addition to lactic acid and ammonia, which are present in the fractions of skin extracts attracting Ae. aegypti (Geier et al. 1996, 1999a). Unidentified compounds may yet be found that increase the attractiveness of the best blends available so far (Williams et al. 2006a).

It has been shown repeatedly that a compound may be more attractive in mixtures than when applied singly (Geier et al. 1999a, Smallegange et al. 2005). Aedes aegypti females are more attracted to triple blends of lactic acid, acetone, dimethyl disulfide or dichloromethane than to binary blends, whereas binary blends are more attractive than single compounds (Bernier et al. 2007). A similar observation was found for tsetse flies: a mixture of three chemicals is more attractive than individual chemicals and more tsetse flies were trapped when the mixture was applied at higher doses for all components, except octenol, which reduces trap catches at higher doses, showing again the importance of controlling concentrations (Vale and Hall 1985b).

Compounds that have no clear effect on behaviour when used individually may, however, play an important role in a mixture, as was shown for lactic acid; although it is not or only weakly attractive to An. gambiae when applied alone, it has a synergistic effect when presented with ammonia and carboxylic acids (Qiu 2005, Smallegange et al. 2005, 2009). Similarly, Ae. aegypti is not attracted to ammonia alone, whereas this compound enhances the effect of lactic acid (Geier et al. 1999a). This phenomenon makes it even harder to 'predict' which mixture of compounds will produce an effective synthetic blend that is highly attractive to mosquitoes.

For Ae. aegypti and An. gambiae particular blends of kairomones attract a reasonably large number of mosquitoes in bioassays when tested on their own. However, the natural odour complex of the human host was significantly more attractive than any synthetic blend when compared directly against each other (Bernier et al. 2007, Spitzen et al. 2008, Smallegange et al. in press). It is therefore likely that essential components are lacking in these, otherwise, attractive blends. 
Another complication in the identification of kairomones is that a chemical may be attractive when applied alone, but shows an inhibitory or a (spatial) repellent effect in combination with human odours. Such an effect has been found for the widely used mosquito repellent DEET (N,Ndiethyl-3-methylbenzamide), which was found to be (weakly) attractive to Ae. aegypti when used on its own (Bernier et al. 2005, Dogan et al. 1999, Kline et al. 2003, Mehr et al. 1990), but caused (spatial) repellence or inhibited attraction when combined with natural human odours (Bernier et al. 2005, Curtis 1992, Dogan et al. 1999, Kline et al. 2003). Recently, however, Syed and Leal (2008) demonstrated true behavioural repellency of $C x$. quinquefasciatus by DEET in the absence of other chemostimuli.

In addition, the method of testing putative repellents can conceal its possible effect. Based on the definition of a repellent as'a chemical which causes insects to make oriented movements away from its source' (Dethier et al. 1960), it remains unclear from most Y-tube and dual-choice olfactometer assays whether compounds that attract significantly fewer mosquitoes than the control are truly repellents (Dogan and Rossignol 1999), because in these devices the insects cannot move away from the source. However, when the term repellent is used to designate products that intent to reduce the biting-rate of haematophagous insects (see White 2007), this term can be used for chemicals that have been shown to reduce the number of mosquitoes attracted to a natural or synthetic odour source when tested in a Y-tube or dual-choice olfactometer. Especially area or spatial repellents, which prevent mosquitoes from reaching their blood-host and are thus effective at a distance from the source of application (see Strickman 2007), may be discovered with these kind of bioassays. Several methods have been developed to test chemicals for their ability to be applied as mosquito repellents, either as a spatial or topical repellent. Each method has its advantages and disadvantages (e.g. Barnard et al. 2007, Chareonviriyaphap et al. 2002, Dogan and Rossignol 1999, Grieco et al. 2005, Hao et al. 2008, Kline et al. 2003, Klun et al. 2005, Rutledge and Gupta 2004). Which bioassay should be used may depend on the research question and possible future application method and may depend on the mode of action of the repellent. For these reasons we suggest to name chemicals that show a inhibitory or repellent effect in laboratory bioassays 'putative repellents' until their effectiveness has been proven in the field.

The previous paragraphs show that the bioassay used to test semiochemicals as behaviourmediating compounds for mosquitoes may provide an indication that a certain compound plays a role in the host-seeking process. However, the result may not be conclusive of the exact role of this compound. For example, most Y-tube and dual-choice olfactometers cannot discriminate between an inhibitory and a repellent effect (Dogan and Rossignol 1999); additional, appropriate bioassays should be performed. Since the host-seeking process exists of different phases (Takken 1996), the choice for a certain type of bioassay determines which phase is examined. For example, the activating effect of a chemical may be missed in a landing assay or an olfactometer when it is not combined with a chemical that induces a landing response or that is an attractant, whereas it may be noticed when video recording in combination with a 3D image analyser is used. Therefore, when no response has been found in a specific bioassay care should be taken to conclude that a certain compound has no effect on the host-seeking behaviour of a certain mosquito species; the compound may play a role in another host-seeking phase. In an olfactometer, attraction may not be visible, even though the mosquitoes make oriented movements towards the source of a chemical (Dethier et al. 1960), when the insects do not fly into the trapping system with which the olfactometer is applied.

Although similarities have been found in the response to certain olfactory cues (e.g. ammonia, lactic acid, aliphatic carboxylic acids, some ketones and aldehydes in Ae. aegypti, An. gambiae 
and $C x$. quinquefasciatus), it is clear that different mosquito species use different odour cues to find their blood-host, and the role of the individual compounds varies between species, even for species that have the same host preference. Finally, much research is required to establish the range of variation in behaviour within wild populations of a given mosquito species across its geographical distribution, for variability within populations, and for the effects of colonisation on natural host-seeking behaviour, as indicated by some disparities between the results of studies based on different populations of all three species reviewed here (Lefèvre et al. 2009; Williams et al. 2003, 2006c). It is likely that different attractive blends, odour delivery systems and trap types have to be used to manage different mosquito populations (Kline 2007).

\section{Conclusions}

Several compounds found in human emanations have been shown to cause a behavioural response in the laboratory in at least one of the three mosquito species, Ae. aegypti, An. gambiae and CX. quinquefasciatus. Carbon dioxide is a universal kairomone, but with distinctly different effects on the three species. In the laboratory, Aedes aegypti is strongly activated by this compound, whereas for An. gambiae it is also a synergist and effective when released at a distance from the other kairomones. The behavioural response of $C x$. quinquefasciatus to $\mathrm{CO}_{2}$ is at best weak.

Composed odour blends are attractive to mosquitoes in the laboratory. These include a combination of ammonia, lactic acid, hexanoic acid, acetone and dimethyl disulfide for Ae. aegypti, and of ammonia, lactic acid and aliphatic carboxylic acids for An. gambiae. However, there is not yet a blend that is equally attractive or more so than natural human odours. Studies comparing (1) odours from poorly and highly attractive persons, (2) odours from a preferred host and a nonhost and (3) blood-host preferences of mosquitoes from different geographic regions are likely to reveal critical compounds responsible for the strong attractiveness of humans to the three mosquito species reviewed here.

Six-methyl-5-hepten-2-one and geranyl acetone are candidate repellents, as these cause inhibition or repellence in Ae. aegypti (Logan et al. 2008) and An. gambiae (Qiu 2005). Interestingly, the release of these compounds from a human arm has been found to decrease when treated with DEET (Syed and Leal 2008).

Synthetic blends can be exploited to lure mosquitoes into odour-baited traps, manipulate them away from human houses or cause general disruption to normal host-seeking behaviour by mass release of human odorants (Day and Sjogren 1994, Kline 2007). Chapter 8 of this volume discusses how laboratory-based results may be transferred to the (semi-)field for further testing prior to their application.

It is expected that the studies in progress by several research consortia will lead to novel classes of odorant blends with which mosquito disease vectors can be monitored or controlled. Recent results from trapping with odour-baited MM-X or BG-Sentinel traps (Kline 2007, Kröckel et al. 2006, Mboera et al. 2000, Njiru et al. 2006, Qiu et al. 2007a, Schmied et al. 2008, Williams et al. 2006b) suggest that the application of such traps, provided the odorant stimulus is highly competitive with natural host odour, are likely to become reality in the near future. Novel synthetic odorant blends that disrupt the natural behaviour of mosquito vectors may be used in addition to current control methods (Greenwood 2008, Takken and Knols 2009) to achieve a greater effect than is presently possible. 


\section{Acknowledgements}

We thank Prof Marc J. Klowden en Dr Gabriella Gibson for constructive comments and suggestions on an earlier version of this manuscript.

\section{References}

Acree F, Jr, Turner RB, Gouck HK, Beroza M and Smith N (1968) L-lactic acid: A mosquito attractant isolated from humans. Science 161: 1346-1347.

Allan SA, Bernier UR and Kline DL (2006a) Attraction of mosquitoes to volatiles associated with blood. J Vector Ecol 31: 71-78.

Allan SA, Bernier UR and Kline DL (2006b) Laboratory evaluation of avian odors for mosquito (Diptera: Culicidae) attraction. J Med Entomol 43: 225-231.

Alonso WJ and Schuck-Paim C (2006) The 'ghosts' that pester studies on learning in mosquitoes: guidelines to chase them off. Med Vet Entomol 20: 157-165.

Ashley DL, Bonin MA, Cardinali FL, McCraw JM, Holler JS, Needham LL and Patterson DG, Jr (1992) Determining volatile organic compounds in human blood from a large sample population by using purge and trap gas chromatography/ mass spectrometry. Analytical Chemistry 64: 1021-1029.

Barnard DR, Bernier UR, Xue R-D and Debboun M (2007) Standard methods for testing mosquito repellents. In: Debboun M, Frances SP and Strickman D (eds) Insect repellents. Principles, methods, and uses. CRC Press, Boca Raton, FL, USA, pp 103-110.

Beeuwkes J, Spitzen J, Spoor CW, Van Leeuwen JL and Takken W (2008) 3-D flight behaviour of the malaria mosquito Anopheles gambiae s.s. inside an odour plume. Proceedings of Experimental and Applied Entomology, N.E.V., Amsterdam 19: 137-146.

Bernier UR, Allan SA, Quinn BP, Kline DL, Barnard DR and Clark GG (2008) Volatile compounds from the integument of White Leghorn chickens (Gallus gallus domesticus L.): Candidate attractants of ornithophilic mosquito species. J Sep Sci 31: 1092-1099.

Bernier UR, Booth MM and Yost RA (1999) Analysis of human skin emanations by gas chromatography/mass spectrometry. 1.Thermal desorption of attractants for the yellow fever mosquito (Aedes aegypti) from handled glass beads. Anal Chem 71: 1-7.

Bernier UR, Furman KD, Kline DL, Allan SA and Barnard DR (2005) Comparison of contact and spatial repellency of catnip oil and N,N-diethyl-3-methylbenzamide (Deet) against mosquitoes. J Med Entomol 42:306-311.

Bernier UR, Kline DL, Allan SA and Barnard DR (2007) Laboratory comparison of Aedes aegypti attraction to human odors and to synthetic human odor compounds and blends. J Am Mosq Control Assoc 23: 288-293.

Bernier UR, Kline DL, Barnard DR, Schreck CE and Yost RA (2000) Analysis of human skin emanations by gas chromatography/mass spectrometry. 2. Identification of volatile compounds that are candidate attractants for yellow fever mosquito (Aedes aegypti). Anal Chem 72: 747-756.

Bernier UR, Kline DL, Posey KH, Booth MM, Yost RA and Barnard DR (2003) Synergistic attraction of Aedes aegypti (L.) to binary blends of L-lactic acid and acetone, dichloromethane, or dimethyl disulfide. J Med Entomol 40: 653-656.

Bernier UR, Kline DL, Schreck CE, Yost RA and Barnard DR (2002) Chemical analysis of human skin emanations: comparison of volatiles from humans that differ in attraction of Aedes aegypti (Diptera: Culicidae). J Am Mosq Control Assoc 18: 186-195.

Birkett MA, Agelopoulos N, Jensen K-MV, Jespersen JB, Pickett JA, Prijs HJ, Thomas G, Trapman JJ, Wadhams LJ and Woodcock CM (2004) The role of volatile semiochemicals in mediating host location and selection by nuisance and disease-transmitting cattle flies. Med Vet Entomol 18: 313-322.

Bosch OJ, Geier M and Boeckh J (2000) Contribution of fatty acids to olfactory host finding of female Aedes aegypti. Chem Senses 25: 323-330.

Bowen MF (1991) The sensory physiology of host-seeking behavior in mosquitoes. Annu Rev Entomol 36: $139-158$. 
Braimah N, Drakeley C, Kweka E, Mosha F, Helinski M, Pates H, Maxwell C, Massawe T, Kenward MG, Curtis C (2005) Tests of bednet traps (Mbita traps) for monitoring mosquito populations and time of biting in Tanzania and possible impact of prolonged insecticide treated net use. Int J Trop Insect Sci 25: 208-213.

Braks MAH (1999) Human skin emanations in the host-seeking behaviour of the malaria mosquito Anopheles gambiae. $\mathrm{PhD}$ thesis Wageningen University, Wageningen, the Netherlands. $123 \mathrm{pp}$.

Braks MAH, Cooperband MF and Cardé RT (2005) 3-D flight track analyses of Culex mosquito orientation to ovipositionrelated odors. In: Noldus LP (ed) Proceedings Measuring Behavior 2005, Wageningen, the Netherlands, pp 209-210.

Braks MAH, Koenraadt CJM, Briet OJT and Takken W (1999) Behavioural responses of Culex quinquefasciatus (Diptera: Culicidae) to human odour, carbon dioxide and lactic acid. Proceedings of Experimental and Applied Entomology, N.E.V., Amsterdam 10: 91-97.

Braks MAH, Meijerink J and Takken W (2001) The response of the malaria mosquito, Anopheles gambiae, to two components of human sweat, ammonia and L-lactic acid, in an olfactometer. Physiol Entomol 26: 142-148.

Braks MAH and Takken W (1999) Incubated human sweat but not fresh sweat attracts the malaria mosquito Anopheles gambiae sensu stricto. Journal of Chemical Ecology 25: 663-672.

Bursell E, Gough AJE, Beevor PS, Cork A, Hall DR and Vale GA (1988) Identification of components of cattle urine attractive to tsetse flies, Glossina spp. (Diptera: Glossinidae). Bull Entomol Res 78: 281-291.

Cardé RT and Willis MA (2008) Navigational strategies used by insects to find distant, wind-borne sources of odor. J Chem Ecol 34: 854-866.

Carey AF, Wang G, Su C-Y, Zwiebel LJ and Carlson JR (2010) Odorant reception in the malaria mosquito Anopheles gambiae. Nature (in press) doi:10.1038/nature08834.

Chandra G (1995) Short report: peak period of filarial transmission. Am J Trop Med Hyg 53: 378-379.

Chareonviriyaphap T, Prabaripai A and Sungvornyothrin S (2002) An improved excito-repellency test chamber for mosquito behavioral tests. J Vector Ecol 27: 250-252.

Cook GC (1996) Manson's tropical diseases. $20^{\text {th }}$ edn. WB Saunders Company Ltd, London, UK.

Cook SM, Khan ZR and Pickett JA (2007) The use of push-pull strategies in integrated pest management. Annu Rev Entomol 52: 375-400.

Cooperband MF and Cardé RT (2006) Orientation of Culex mosquitoes to carbon dioxide-baited traps: flight manoeuvres and trapping efficiency. Med Vet Entomol 20: 11-26.

Cooperband MF, McElfresh JS, Millar JG, Cardé RT (2008) Attraction of female Culex quinquefasciatus Say (Diptera: Culicidae) to odors from chicken feces. J Insect Physiol 54: 1184-1192.

Cork A and Hall MJR (2007) Development of an odour-baited target for female New World screwworm, Cochliomyia hominivorax: studies with host baits and synthetic wound fluids. Med Vet Entomol 21: 85-92.

Cork A and Park KC (1996) Identification of electrophysiologically-active compounds for the malaria mosquito, Anopheles gambiae, in human sweat extracts. Med Vet Entomol 10: 269-276.

Costantini C, Birkett MA, Gibson G, Ziesmann J, Sagnon NF, Mohammed HA, Coluzzi M and Pickett JA (2001) Electroantennogram and behavioural responses of the malaria vector Anopheles gambiae to human-specific sweat components. Med Vet Entomol 15, 259-266.

Costantini C, Gibson G, Sagnon N, Della Torre A, Brady J and Coluzzi M (1996) Mosquito responses to carbon dioxide in a West African Sudan savanna village. Med Vet Entomol 10: 220-227.

Crumb SE (1922) A mosquito attractant. Science 55: 446-447.

Cruz-Lopez L, Malo EA, Rojas JC and Morgan ED (2001) Chemical ecology of triatomine bugs: vectors of Chagas disease. Med Vet Entomol 15: 351-357.

Curran AM, Rabin SI, Prada PA and Furton KG (2005) Comparison of the volatile organic compounds present in human odor using SPME-GC/MS. J Chem Ecol 31: 1607-1619.

Curran AM, Ramirez CF, Schoon AA and Furton KG (2007) The frequency of occurrence and discriminatory power of compounds found in human scent across a population determined by SPME-GC/MS. J Chromatogr B 846: 86-97.

Curtis CF (1986) Fact and fiction in mosquito attraction and repulsion. Parasitology Today 11: 316-318.

Curtis CF (1992) Personal protection methods against vectors of disease. Rev Med Vet Entomol 80: 543-553.

Curtis CF and Hill N (1988) Comparison of methods of repelling mosquitoes. Entomol Exp Appl 49: 175-179. 
Davis EE (1984) Development of lactic acid-receptor sensitivity and host-seeking behaviour in newly emerged female Aedes aegypti mosquitoes. J Insect Physiol 30: 211-215.

Day JF and Sjogren RD (1994) Vectro control by removal trapping. Am J Trop Med Hyg 50: 126-133.

De Bruyne M and Baker TC (2008) Odor detection in insects: volatile codes. J Chem Ecol 34: 882-897.

De Jong R and Knols BGJ (1995) Selection of biting sites on man by two malaria mosquito species. Cellular and Molecular Life Sciences (formerly Experientia) 51: 80-84.

De Jong R and Knols BGJ (1996) Selection of biting sites by mosquitoes: In: Bock GR and Cardew G (eds) Olfaction in mosquito host interactions. John Wiley and Sons Ltd, Chichester, UK, pp 89-103.

Dekker T, Geier M and Cardé RT (2005) Carbon dioxide instantly sensitizes female yellow fever mosquitoes to human skin odours. J Exp Biol 208: 2963-2972.

Dekker T, Steib B, Cardé RT and Geier M (2002) L-lactic acid, a human-signifying host cue for the anthropophilic mosquito Anopheles gambiae. Med Vet Entomol 16: 91-98.

Dekker T, Takken W and Braks MAH (2001a) Innate preference for host-odor blends modulates degree of anthropophagy of Anopheles gambiae sensu lato (Diptera: Culicidae). J Med Entomol 38: 868-871.

Dekker T, Takken W and Cardé RT (2001b) Structure of host-odour plumes influences catch of Anopheles gambiae s.s. and Aedes aegypti in a dual-choice olfactomer. Physiol Entomol 26: 124-134.

Dekker T, Takken W, Knols BGJ, Bouman E, Van de Laak S, De Bever A and Huisman PWT (1998) Selection of biting sites on a human host by Anopheles gambiae s.s., An. arabiensis and An. quadriannulatus. Entomol Exp Appl 87: 295-300.

Deng C, Zhang X and Li N (2004) Investigation of volatile biomarkers in lung cancer blood using solid-phase microextraction and capillary gas chromatography-mass spectrometry. Journal of Chromatography B 808: 269277.

Dethier VG, Browne LB and Smith CN (1960) The designation of chemicals in terms of the responses they elicit from insects. J Econ Entomol 53: 134-136.

Diatta M, Spiegel A, Lochouarn L, and Fontenille D (1998) Similar feeding preferences of Anopheles gambiae and A. arabiensis in Senegal. Trans R Soc Trop Med Hyg 92: 270-272.

Dicke M and Sabelis MW (1988) Infochemical terminology: based on cost-benefit analysis rather than origin of compounds? Funct Ecol 2. 131-139

Dogan EB, Ayres JW and Rossignol PA (1999) Behavioural mode of action of deet: inhibition of lactic acid attraction. Med Vet Entomol 13: 97-100.

Dogan EB and Rossignol PA (1999) An olfactometer for discriminating between attraction, inhibition, and repellency in mosquitoes (Diptera: Culicidae). J. Med. Entomol. 36: 788-793.

Douglas HD, Co JE, Jones TH and Conner WE (2001) Heteropteran chemical repellents identified in the citrus odor of a seabird (crested auklet: Aethia cristatella): evolutionary convergence in chemical ecology. Naturwissenschaften 88: 330-332.

Douglas HD, Co JE, Jones TH, Conner WE and Day JF (2005) Chemical odorant of colonial seabird repels mosquitoes. J Med Entomol 42: 647-651.

Duchemin J. B., Leong Pock Tsy J. M., Rabarison P, Roux J, Coluzzi M, and Costantini C (2001) Zoophily of Anopheles arabiensis and An. gambiae in Madagascar demonstrated by odour-baited entry traps. Med Vet Entomol 15: 50-57.

Elizondo-Quiroga A, Flores-Suarez A, Elizondo-Quiroga D, Ponce-Garcia G, Blitvich BJ, Contreras-Cordero JF, Gonzalez-Rojas JI, Mercado-Hernandez R, Beaty BJ and Fernandez-Salas I (2006) Host-feeding preference of Culex quinquefasciatus in Monterrey, Northeastern Mexico. J Am Mosq Control Assoc 22: 654-661.

Eiras AE and Jepson PC (1991) Host location by Aedes aegypti (Diptera Culicidae): a wind tunnel study of chemical cues. Bull Entomol Res 81: 151-160.

Ellin RI, Farrand RL, Oberst FW, Crouse CL, Billups NB, Koon WS, Musselman NP and Sidell FR (1974) An apparatus for the detection and quantitation of volatile human effluents. J Chromatogr 100: 137-152.

Gallagher M, Wysocki CJ, Leyden JJ, Spielman Al, Sun X and Preti G (2008) Analyses of volatile organic compounds from human skin. Br J Dermatol 159:780-791.

Geier M and Boeckh J (1999) A new Y-tube olfactometer for mosquitoes to measure the attractiveness of host odours. Entomol Exp Appl 92: 9-19. 
Geier M, Bosch OJ and Boeckh J (1999a) Ammonia as an attractive component of host odour for the Yellow fever mosquito Aedes aegypti. Chem. Senses 24: 647-653.

Geier M, Bosch OJ and Boeckh J (1999b) Influence of odour plume structure on upwind flight of mosquitoes towards hosts. J Exp Biol 202: 1639-1648.

Geier M, Sass H and Boeckh J (1996) A search for components in human body odour that attract female Aedes aegypti. In: Bock GR and Cardew G (eds) Olfaction in mosquito host interactions. John Wiley and Sons Ltd, Chichester, UK, pp 132-148.

Gillies MT (1964) Selection for host preference in Anopheles gambiae. Nature 203: 852-854.

Gillies MT (1988) Anopheline mosquitos: vector behaviour and bionomics. In: Wernsdorfer WH and McGregor I (eds) Malaria - principles and practice of malariology. Churchill Livingstone, Edinburgh, UK, pp 453-485.

Godsey MS, Jr., Blackmore MS, Panella NA, Burkhalter K, Gottfried K, Halsey LA, Rutledge R, Langevin SA, Gates R, Lamonte KM, Lambert A, Lanciotti RS, Blackmore CG, Loyless T, Stark L, Oliveri R, Conti L and Komar N (2005) West Nile virus epizootiology in the southeastern United States, 2001. Vector Borne Zoonotic Dis 5: 82-89.

Gould EA (2003) Implications for Northern Europe of the emergence of West Nile virus in the USA. Epidemiology and Infection 131: 583-589.

Gould LH and Fikrig E (2004) West Nile virus: a growing concern? J Clin Invest 113:1102-1107.

Gratz NG (2004) Critical review of the vector status of Aedes albopictus. Med Vet Entomol 18: 215-227.

Greenwood BM (2008) Control to elimination: implications for malaria research. Trends in Parasitology 24: 449-454.

Greenwood BM, Fidock DA, Kyle DE, Kappe SHI, Alonso PL, Collins FH and Duffy PE (2008) Malaria: progress, perils, and prospects for eradication. J Clin Invest 118: 1266-1276.

Grieco JP, Achee NL, Sardelis MR, Chauhan KR and Roberts DR (2005) A novel high-throughput screening system to evaluate the behavioral response of adult mosquitoes to chemicals. J Am Mosq Control Assoc 21: 404-411.

Haathi EOA and Fales HM (1967) The uropygiols: identification of the unsaponifiable constituent of a diester wax from chicken preen glands. J Lipid Research 8: 131-137.

Haddow AJ (1942) The mosquito fauna and climate of native huts at Kisumu, Kenya. Bull Entomol Res 33: 91-142.

Haddow AJ and Ssenkubuge Y (1973) The mosquito of bwamba county, Uganda. IX. Further studies on the biting behaviour of an outdoor population of the Anopheles gambiae Giles complex. Bull Entomol Res 62:407-414.

Hallem EA, Dahanukar A and Carlson JR (2006) Insect odor and taste receptors. Annu Rev Entomol 51: 113-135.

Hallem EA, Fox AN, Zwiebel LJ and Carlson JR (2004) Olfaction - Mosquito receptor for human-sweat odorant. Nature 427: 212-213.

Hao H, Wei J, Dai J and Du J (2008) Host-seeking and blood-feeding behavior of Aedes albopictus (Diptera: Culicidae) exposed to vapors of geraniol, citral, citronellal, eugenol, or anisaldehyde. J Med Entomol 45: 533-539.

Harrington LC (2001) Why do female Aedes aegypti (Diptera: Culicidae) feed preferentially and frequently on human blood? J Med Entomol 38: 411-422.

Hasegawa Y, Yabuki M and Matsukane M (2004) Identification of new odoriferous compounds in human axillary sweat. Chem Biodivers 1: 2042-2050.

Hassanali A, Herren H, Khan ZR, Pickett JA and Woodcock CM (2008) Integrated pest management: the push-pull approach for controlling insect pests and weeds of cereals, and its potential for other agricultural systems including animal husbandry. Phil Trans R Soc B 363: 611-621.

Haze S, Gozu Y, Nakamura S, Khono Y, Sawano K, Ohta H and Yamzaki K (2001) 2-Nonenal newly found in human body odor tends to increase with aging. J Invest Dermatol 116: 520-524.

Healy TP, Copland MJ, Cork A, Przyborowska A and Halket JM (2002) Landing responses of Anopheles gambiae elicited by oxocarboxylic acids. Med Vet Entomol 16: 126-132.

Healy TP and Copland MJW (1995) Activation of Anopheles gambiae mosquitoes by carbon dioxide and human breath. Med Vet Entomol 9: 33 1-336.

Healy TP and Copland MJW (2000) Human sweat and 2-oxopentanoic acid elicid a landing response from Anopheles gambiae. Med Vet Entomol 14: 195-200.

Holt RA, Subramanian GM, Halpern A, Sutton GG, Charlab R, Nusskern DR, Wincker P, Clark AG, Ribeiro JM, Wides R, Salzberg SL, Loftus B, Yandell M, Majoros WH, Rusch DB, Lai Z, Kraft CL, Abril JF, Anthouard V, Arensburger P, 
Atkinson PW, Baden H, de Berardinis V, Baldwin D, Benes V, Biedler J, Blass C, Bolanos R, Boscus D, Barnstead M, Cai S, Center A, Chaturverdi K, Christophides GK, Chrystal MA, Clamp M, Cravchik A, Curwen V, Dana A, Delcher A, Dew I, Evans CA, Flanigan M, Grundschober-Freimoser A, Friedli L, Gu Z, Guan P, Guigo R, Hillenmeyer ME, Hladun SL, Hogan JR, Hong YS, Hoover J, Jaillon O, Ke Z, Kodira C, Kokoza E, Koutsos A, Letunic I, Levitsky A, Liang Y, Lin JJ, Lobo NF, Lopez JR, Malek JA, McIntosh TC, Meister S, Miller J, Mobarry C, Mongin E, Murphy SD, O'Brochta DA, Pfannkoch C, Qi R, Regier MA, Remington K, Shao H, Sharakhova MV, Sitter CD, Shetty J, Smith TJ, Strong R, Sun J, Thomasova D, Ton LQ, Topalis P, Tu Z, Unger MF, Walenz B, Wang A, Wang J, Wang M, Wang X, Woodford KJ, Wortman JR, Wu M, Yao A, Zdobnov EM, Zhang H, Zhao Q, Zhao S, Zhu SC, Zhimulev I, Coluzzi M, della Torre A, Roth CW, Louis C, Kalush F, Mural RJ, Myers EW, Adams MD, Smith HO, Broder S, Gardner MJ, Fraser CM, Birney E, Bork P, Brey PT, Venter JC, Weissenbach J, Kafatos FC, Collins FH and Hoffman SL (2002) The genome sequence of the malaria mosquito Anopheles gambiae. Science 298: 129-149.

Hurd H (2003) Manipulation of medically important insect vectors by their parasites. Annu Rev Entomol 48: 141-146.

Issachar D, Holland JF and Sweeley CC (1982) Metabolic profiles of organic acids from human plasma. Analytical Chemistry 54: 29-32.

Jawara M, Smallegange RC, Jeffries D, Nwakanma DC, Awolola TS, Knols BGJ, Takken W and Conway DJ (2009). Optimising odour baited trap methods for collecting mosquitoes during the malaria season in The Gambia. PLoS ONE 12: e8167.

Jones KE, Patel NG, Levy MA, Storeygard A, Balk D, Gittleman JL and Daszak P (2008) Global trends in emerging infectious diseases. Nature 451: 990-993.

Jordan AM (1986) Trypanosomiasis control and African rural development. Longman Group Ltd., New York, USA.

Justice RW, Biessmann H, Walter MF, Dimitratos SD and Woods DF (2003) Genomics spawns novel approaches to mosquito control; Bioassays 25: 1011-1020.

Kennedy JS (1978) The concepts of olfactory 'arrestment' and 'attraction'. Physiol. Entomol 3: 91-98.

Killeen G, Kihonda J, Lyimo E, Oketich F, Kotas M, Mathenge E, Schellenberg J, Lengeler C, Smith T and Drakeley C(2006) Quantifying behavioural interactions between humans and mosquitoes: evaluating the protective efficacy of insecticidal nets against malaria transmission in rural Tanzania. BMC Infect. Dis. 6:161.

Kilpatrick AM, Kramer LD, Jones MJ, Marra PP and Daszak P (2006) West Nile virus epidemics in North America are driven by shifts in mosquito feeding behavior. PLoS Biol 4: e82.

Kline DL, Allan SA, Bernier UR and Posey KH (2006) Olfactometer and large cage evaluation of a solid phase technology for the controlled production of CO2. J Am Mosq Control Assoc 22: 378-381.

Kline DL (2006) Traps and trapping techniques for adult mosquito control. J Am Mosq Control Assoc 22: 490-496.

Kline DL (2007) Semiochemicals, traps targets and mass trapping technology for mosquito management. J Am Mosq Control Assoc 23: 241-251.

Kline DL, Bernier UR, Posey KH, and Barnard DR (2003) Olfactometric evaluation of spatial repellents for Aedes aegypti. J Med Entomol 40: 463-467.

Klowden MJ (1986) Effects of sugar deprivation on the host-seeking behaviour of gravid Aedes aegypti mosquitoes. J Insect Physiol 32: 479-483.

Klowden MJ (1995) Blood, sex and mosquito. The mechanism that control mosquito blood feeding behavior. Bioscience 45: 326-331.

Klowden MJ (1996) Endogenous factors regulating mosquito host-seeking behaviour. In: Bock GR and Cardew G (eds) Olfaction in mosquito interactions. Wiley, Chichester, UK, pp 212-225.

Klowden and Lea (1979) Humoral inhibition of host-seeking in Aedes aegypti during oocyte maturation. J Insect Physiol 25: 231-235.

Klun JA, Kramer M, Debboun M: (2005) A new in vitro bioassay system for discovery of novel human-use mosquito repellents. J Am Mosq Control Assoc 21:64-70.

Knols BG, Njiru BN, Mathenge EM, Mukabana WR, Beier JC and Killeen GF (2002) MalariaSphere: A greenhouse-enclosed simulation of a natural Anopheles gambiae (Diptera: Culicidae) ecosystem in western Kenya. Malar J 1: 19.

Knols BG, Takken W and De Jong R (1994a) Influence of human breath on selection of biting sites by Anopheles albimanus. J Am Mosq Control Assoc 10: 423-426. 
Knols BGJ, De Jong R and Takken W (1994b) Trapping system for testing olfactory responses of the malaria mosquito Anopheles-gambiae in a wind-tunnel. Med Vet Entomol 8: 386-388.

Knols BGJ, Mboera LEG and Takken W (1998) Electric nets for studying odour-mediated host-seeking behaviour of mosquitoes. Med Vet Entomol 12: 116-120.

Knols BGJ, Takken W, Charlwood D and De Jong R (1995) Species-specific attraction of Anopheles mosquitoes (Diptera: Culicidae) to different humans in South Tanzania. Proceedings of Experimental and Applied Entomology, N.E.V., Amsterdam 6: 201-206.

Knols BGJ, Van Loon JJA, Cork A, Robinson RD, Adam W, Meijerink J, De Jong R and Takken W (1997) Behavioural and electrophysiological responses of the female malaria mosquito Anopheles gambiae (Diptera: Culicidae) to Limburger cheese volatiles. Bull Entomol Res 87: 151-159.

Kröckel U, Rose A, Eiras AE and Geier M (2006) New tools for surveillance of adult yellow fever mosquitoes: comparison of trap catches with human landing rates in an urban environment. J Am Mosq Control Assoc 22: 229-238.

Krotoszynski B, Gabriel G and O'Neill H (1977) Characterization of human expired air: A promising investigative and diagnostic technique. J Chromatogr Sci 15: 239-244.

Leal WS, Barbosa RMR, Xu W, Ishida Y, Syed Z, Latte N, Chen AM, Morgan TI, Cornel AJ and Furtado A (2008) Reverse and conventional chemical ecology approaches for the development of oviposition attractants for Culex mosquitoes. PLOS ONE 3: e3045.

Lefèvre T, Gouagna L-C, Dabire KR, Elguero E, Fontenille D, Costantini C and Thomas F (2009) Evolutionary lability of odour-mediated host preference by the malaria vector Anopheles gambiae. Trop Med Int Health 14:228-236.

Lehane MJ (1991) Biology of blood-sucking insects. Harper Collins Academic, London, UK. 288 pp.

Leyden JJ, McGinley KJ, Holzle E, Labows JN and Kligman AM (1981) The microbiology of the human axilla and its relationship to axillary odor. J Investig Dermatol 77: 413-416.

Lindsay SW, Adiamah JH, Miller JE, Pleass RJ and Armstrong JR (1993) Variation in attractiveness of human subjects to malaria mosquitoes (Diptera: Culicidae) in The Gambia. J Med Entomol 30: 368-373.

Logan JG and Birkett MA (2007) Semiochemicals for biting fly control: their identification and exploitation. Pest Manage Sci 63: 647-657.

Logan JG, Birkett MA, Clark SJ, Powers S, Seal NJ, Wadhams LJ, Mordue AJ and Pickett JA (2008) Identification of humanderived volatile chemicals that interfere with attraction of Aedes aegypti mosquitoes. J Chem Ecol 34: 308-322.

Lu T, Qiu YT, Wang G, Kwon JY, Rutzler M, Kwon HW, Pitts RJ, Van Loon JJA, Takken W, Carlson JR and Zwiebel LJ (2007) Odor coding in the maxillary palp of the malaria vector mosquito Anopheles gambiae. Current Biology 17: 15331544.

Maxwell CA, Wakibara J, Tho S and Curtis CF (1998) Malaria-infective biting at different hours of the night. Med Vet Entomol 12:325-327.

Mboera LEG, Knols BGJ, Braks MAH and Takken W (2000) Comparison of carbon dioxide-baited trapping systems for sampling outdoor mosquito populations in Tanzania. Med Vet Entomol 14: 257-263.

Mboera LEG, Knols BGJ, Takken W and Huisman PWT (1998) Olfactory responses of female Culex quinquefasciatus Say (Diptera: Culicidae) in a dual-choice olfactometer. J Vector Ecol 23: 107-113.

Mboera LEG and Takken W (1997) Carbon dioxide chemotropism in mosquitoes (Diptera: Culicidae) and its potential in vector surveillance and management programmes. Rev Med Vet Entomol 85: 355-368.

Mboera LEG and Takken W (1999) Odour-mediated host preference of Culex quinquefasciatus in Tanzania. Entomol Exp Appl 92: 83-88.

McCall PJ, Harding JR, Roberts J and Auty B (1996) Attraction and trapping of Aedes aegypti (Diptera: Culicidae) with host odors in the laboratory. J Med Entomol 33:177-179.

McCall PJ and Eaton G (2001) Olfactory memory in the mosquito Culex quinquefasciatus. Med Vet Entomol 15: 197-203.

Mclver SB: Host preferences and discrimination by the mosquitoes Aedes aegypti and Culex tarsalis (Diptera: culicidae). J Med Entomol 1968, 5:422-428.

Mehr ZA, Rutledge LC, Buescher MD, Gupta RK and Zakaria MM (1990) Attraction of mosquitoes to diethyl methylbenzamide and ethyl hexanediol. J Am Mosq Control Assoc 6: 469-476. 
Meijerink J, Braks MAH, Brack AA, Adam W, Dekker T, Posthumus MA, Van BeekTA and Van Loon JJA (2000) Identification of olfactory stimulants for Anopheles gambiae from human sweat samples. J Chem Ecol 26: 1367-1382.

Morrison AC, Zielinski-Gutierrez E, Scott TW and Rosenberg R (2008) Defining challenges and proposing solutions for control of the virus vector Aedes aegypti. Plos Med 5: e68.

Mukabana WR, Takken W, Coe R and Knols BG (2002) Host-specific cues cause differential attractiveness of Kenyan men to the African malaria vector Anopheles gambiae. Malar J 1: 17.

Mukabana WR, Takken W, Killeen GF and Knols BGJ (2004) Allomonal effect of breath contributes to differential attractiveness of humans to the African malaria vector Anopheles gambiae. Malar J 3: 8 .

Muturi EJ, Muriu S, Shililu J, Mwangangi JM, Jacob BG, Mbogo C, Githure J and Novak RJ (2008) Blood-feeding patterns of Culex quinquefasciatus and other culicines and implications for disease transmission in Mwea rice scheme, Kenya. Parasitol Res 102: 1329-1335.

Natsch A, Derrer S, Flachsmann F and Schmid J (2006) A broad diversity of volatile carboxylic acids, released by a bacterial aminoacylase from axilla secretions, as candidate molecules for the determination of human-body odor type. Chem Biodivers 3: 1-20.

Nene V, Wortman JR, Lawson D, Haas B, Kodira C, Tu ZJ, Loftus B, Xi Z, Megy K, Grabherr M, Ren Q, Zdobnov EM, Lobo NF, Campbell KS, Brown SE, Bonaldo MF, Zhu J, Sinkins SP, Hogenkamp DG, Amedeo P, Arensburger P, Atkinson PW, Bidwell S, Biedler J, Birney E, Bruggner RV, Costas J, Coy MR, Crabtree J, Crawford M, Debruyn B, Decaprio D, Eiglmeier K, Eisenstadt E, El-Dorry H, Gelbart WM, Gomes SL, Hammond M, Hannick LI, Hogan JR, Holmes MH, Jaffe D, Johnston JS, Kennedy RC, Koo H, Kravitz S, Kriventseva EV, Kulp D, Labutti K, Lee E, Li S, Lovin DD, Mao C, Mauceli E, Menck CF, Miller JR, Montgomery P, Mori A, Nascimento AL, Naveira HF, Nusbaum C, O'Leary S, Orvis J, Pertea M, Quesneville H, Reidenbach KR, Rogers YH, Roth CW, Schneider JR, Schatz M, Shumway M, Stanke M, Stinson EO, Tubio JM, Vanzee JP, Verjovski-Almeida S, Werner D, White O, Wyder S, Zeng Q, Zhao Q, Zhao Y, Hill CA, Raikhel AS, Soares MB, Knudson DL, Lee NH, Galagan J, Salzberg SL, Paulsen IT, Dimopoulos G, Collins FH, Birren B, Fraser-Liggett CM and Severson DW (2007) Genome sequence of Aedes aegypti, a major arbovirus vector. Science 316: 1718-1723.

Nicolaides N, C. FH and Rice GR (1968) The skin surface lipids of man compared with those of eighteen species of animals. J. Investigative Dermatology 51: 83-89.

Njiru BN, Mukabana WR, Takken W and Knols BG (2006) Trapping of the malaria vector Anopheles gambiae with odourbaited MM-X traps in semi-field conditions in western Kenya. Malar J 5: 39.

Okumu FO, Killeen GF, Ogoma S, Biswaro L, Smallegange RC, Mbeyela E, Titus E, Munk C, Ngonyani H, Takken W, Mshinda H, Mukabana WR and Moore SJ (2010) Development and field evaluation of a synthetic mosquito lure that is more attractive than humans. PLoS ONE 5: e8951.

Pates HV, Takken W and Curtis CF (2005) Laboratory studies on the olfactory behaviour of Anopheles quadriannulatus. Entomol Exp Appl 114: 153-159.

Pates HV, Takken W, Curtis CF, Huisman PW, Akinpelu O and Gill GS (2001a) Unexpected anthropophagic behaviour in Anopheles quadriannulatus. Med Vet Entomol 15: 293-298.

Pates HV, Takken W, Stuke K and Curtis CF (2001b) Differential behaviour of Anopheles gambiae sensu stricto (Diptera: Culicidae) to human and cow odours in the laboratory. Bull Entomol Res 91:289-296.

Pelletier J and Leal WS (2009) Genome analysis and expression patterns of odorant-binding proteins from the southern house mosquito Culex pipiens quinquefasciatus. PLoS ONE 4: e6237.

Penn DJ, Oberzaucher E, Grammer K, Fischer G, Soini HA, Wiesler D, Novotny MV, Dixon SJ, Xu Y and Brereton RG (2007) Individual and gender fingerprints in human body odour. J R Soc Interface 4: 331-340.

Perry TL, Hansen S, Diamond S, Bullis B, Mok C and Melançon SB (1970) Volatile fatty acids in normal human physiological fluids. Clin Chim Acta 29: 369-374.

Philips M (1997) Method for the collection and assay of volatile organic compounds in breath. Anal Biochem 247: 272-278.

Posey KH, Barbard DR and Schreck CE (1998) Triple cage olfactometer for evaluating mosquito (Diptera: Culicidae) attraction responses. J Med Entomol 35: 330-334. 
Puri SN, Mendki MJ, Sukumaran D, Ganesan K, Prakash S and Sekhar K (2006) Electroantennogram and behavioral responses of Culex quinquefasciatus (Diptera: Culicidae) females to chemicals found in human skin emanations. J Med Entomol 43: 207-213.

Qiu YT (2005) Sensory and behavioural responses of the malaria mosquito Anopheles gambiae to human odours. PhD thesis, Wageningen University, Wageningen, the Netherlands, $208 \mathrm{pp}$.

Qiu YT, Smallegange RC, Hoppe S, Van Loon JJA, Bakker EJ and Takken W (2004a) Behavioural and electrophysiological responses of the malaria mosquito Anopheles gambiae Giles sensu stricto (Diptera: Culicidae) to human skin emanations. Med Vet Entomol 18: 429-438.

Qiu YT, Smallegange RC, Smid H, Van Loon JJA, Galimard AMS, Posthumus MA, Beek TAv and Takken W (2004b) GC-EAG analysis of human odours that attract the malaria mosquito Anopheles gambiae sensu strict. Proc Exp Appl Ent, N.E.V., Amsterdam 15: 59-64.

Qiu YT, Smallegange RC, Ter Braak CJF, Spitzen J, Van Loon JJA, Jawara M, Milligan P, Galimard AM, Van Beek TA, Knols BGJ and Takken W (2007a) Attractiveness of MM-X traps baited with human or synthetic odor to mosquitoes (Diptera: Culicidae) in The Gambia. J Med Entomol 44: 970-983.

Qiu YT, Spitzen J, Smallegange RC and Knols BGJ. (2007b). Monitoring systems for adult insect pests and disease vectors. In: Takken W and Knols BGJ (eds) Emerging pests and vector-borne diseases in Europe. Ecology and control of vector-botne diseases, vol. 1. Wageningen Academic Publishers, Wageningen, the Netherlands, pp 329-354.

Qiu YT, Smallegange RC, Van Loon JJA, Ter Braak CJF and Takken W (2006a) Interindividual variation in the attractiveness of human odours to the malaria mosquito Anopheles gambiae s. s. Med Vet Entomol 20: 280-287.

Qiu YT, van Loon JJA, Takken W, Meijerink J and Smid HM. 2006b. Olfactory coding in antennal neurons of the malaria mosquito, Anopheles gambiae. Chem Senses 31:845-863.

Richards SL, Ponnusamy L, Unnasch TR, Hassan HK and Apperson CS (2006) Host-feeding patterns of Aedes albopictus (Diptera: Culicidae) in relation to availability of human and domestic animals in suburban landscapes of central North Carolina. J Med Entomol 43: 543-551.

Robertson HM and Wanner KW (2006) The chemoreceptor superfamily in the honey bee, Apis mellifera: expansion of the odorant, but not gustatory, receptor family. Genome Res 16: 1395-1403.

Rudolfs W (1922) Chemotropism of mosquitoes. Bull NJ Agric Exp Stn 367: 4-23.

Rutledge LC and Gupta RK (2004) Evaluation of an in vitro bloodfeeding system for testing mosquito repellents. J Am Mosq Control Assoc 20: 150-154.

Rutzler M and Zwiebel LJ (2005) Molecular biology of insect olfaction: recent progress and conceptual models. J Comp Physiol A Neuroethol Sens Neural Behav Physiol 191: 777-790.

Sardelis MR, Turell MJ, Dohm DJ and O'Guinn ML (2001) Vector competence of selected North American Culex and Coquillettidia mosquitoes for West Nile virus. Emerging Infectious Diseases 7: 1018-1022.

Sastry SD, Buck KT, Janak J, Dressler M and Preti G (1980) Volatiles emitted by humans. In: Waller GR and Dermer OC (eds) Biochemical applications of mass spectrometry. John Wiley and Sons, New York, USA, pp 1085-1127.

Savage HM, Aggarwal D, Apperson CS, Katholi CR, Gordon E, Hassan HK, Anderson M, Charnetzky D, McMillen L, Unnasch EA and Unnasch TR (2007) Host choice and West Nile virus infection rates in blood-fed mosquitoes, including members of the Culex pipiens complex, from Memphis and Shelby County, Tennessee, 2002-2003. Vector Borne Zoonotic Dis 7: 365-386.

Schaub GA (2006) Parasitogenic alterations of vector behaviour. Int J Med Microbiol 296: 37-40.

Schofield S and Brady J (1997) Effects of carbon dioxide, acetone and 1-octen-3-ol on the flight responses of the stable fly, Stomoxys calcitrans, in a windtunnel. Physiol Entomol 22: 380-386.

Schofield S, Witty C and Brady J (1997) Effects of carbon dioxide, acetone and 1-octen-3-ol on the activity of the stable fly, Stomoxys calcitrans. Physiol Entomol 22: 256-260.

Schofield S, Cork A and Brady J (1995) Electroantennogram responses of the stable fly, Stomoxys calcitrans, to components of host odor. Physiol Entomol 20: 273-280.

Schmied WH, Takken W, Killeen GF, Knols BGJ and Smallegange RC (2008) Evaluation of two counterflow traps for testing behaviour-mediating compounds for the malaria vector Anopheles gambiae s.s. under semi-field conditions in Tanzania. Malar J 7:230. 
Schreck CE, Kline DL and Carlson DA (1990) Mosquito attraction to substances from the skin of different humans. J Am Mosq Control Assoc 6: 406-410.

Schreck CE, Smith N, Carlson DA, Price GD, Haile D and Godwin DR (1981) A material isolated from human hands that attracts female mosquitoes. J Chem Ecol 2: 429-438.

Scott TW, Githeko AK, Fleisher A, Harrington LC and Yan GY (2006) DNA profiling of human blood in anophelines from lowland and highland sites in western Kenya. Am J Trop Med Hyg 75: 231-237.

Shelley WWB, Hurley HHJ Jr., Nichols AAC (1953) Axillary odor; experimental study of the role of bacteria, apocrine sweat, and deodorants. AMA Arch Derm Syphilol 68: 430-446.

Shirai Y, Kamimura K, Seki T and Morohashi M (2001) L-lactic acid as a mosquito (Diptera: Culicidae) repellent on human and mouse skin. J Med Entomol 38: 51-54.

Silva IM, Eiras AE, Kline DL and Bernier UR (2005) Laboratory evaluation of mosquito traps baited with a synthetic human odor blend to capture Aedes aegypti. J Am Mosq Control Assoc 21:229-233.

Smallegange RC, Geier M and Takken W (2002) Behavioural responses of Anopheles gambiae to ammonia, lactic acid and a fatty acid in a y-tube olfactometer. Proc Exp Appl Ent, N.E.V., Amsterdam 13: 147-152.

Smallegange RC, Knols BGJ and Takken W (2010) Effectiveness of synthetic versus natural human volatiles as attractants for Anopheles gambiae (Diptera: Culicidae) sensu stricto. J Med Entomol 47: 338-344.

Smallegange RC, Qiu YT, Bukovinszkiné-Kiss G, Van Loon JJA and Takken W (2009) The effect of aliphatic carboxylic acids on olfaction-based host-seeking of the malaria mosquito Anopheles gambiae sensu stricto. J Chem Ecol 35: 933-943.

Smallegange RC, Qiu YT, Galimard AMS, Posthumus MA, Van Beek TA, Van Loon JJA and Takken W (2003) Why humans are attractive to malaria mosquitoes. Entomol Berichten 63: 50-53.

Smallegange RC, Qiu YT, Van Loon JJA and Takken W (2005) Synergism between ammonia, lactic acid and carboxylic acids as kairomones in the host-seeking behaviour of the malaria mosquito Anopheles gambiae sensu stricto (Diptera: Culicidae). Chem Senses 30: 145-152.

Smith CN, Smith N, Gouck HK, Weidhaas DE, Gilbert IH, Mayer MS, Smittle BJ and Hofbauer A (1970) L-Lactic acid as a factor in the attraction of Aedes aegypti (Diptera: Culicidae) to human hosts. Ann Entomol Soc Am 63: 760-770.

Spinhirne JP, Koziel JA and Chirase NK (2003) A Device for Non-invasive On-site Sampling of Cattle Breath with SolidPhase Microextraction. Biosystems Engineering 84: 239-246.

Spinhirne JP, Koziel JA and Chirase NK (2004) Sampling and analysis of volatile organic compounds in bovine breath by solid-phase microextraction and gas chromatography-mass spectrometry. J Chromatogr A 1025: 63-69.

Spitzen J, Smallegange RC and Takken W (2008) Effect of human odours and positioning of CO2 release point on trap catches of the malaria mosquito Anopheles gambiae sensu stricto in an olfactometer. Physiol Entomol 33: 116-122.

Strickman D (2007) Area repellent products. In: Debboun M, Frances SP and Strickman D (eds) Insect repellents. Principles, methods, and uses. CRC Press, Boca Raton, FL, USA, pp 385-395.

Syed $Z$ and Leal WS (2008) Mosquitoes smell and avoid the insect repellent DEET. PNAS 105: 13598-13603.

Syed Z and Leal WS (2009) Acute olfactory response of Culex mosquitoes to a human- and bird-derived attractant. PNAS 106: 18809-18814

Takken W (1991) The role of olfaction in host-seeking of mosquitoes: a review. Insect Sci Appl 12: 287-295.

Takken W (1996) Synthesis and future challenges: the response of mosquitoes to host odours. In: Bock GR and Cardew $\mathrm{G}$ (eds) Olfaction in mosquito host interactions. John Wiley and Sons Ltd, Chichester, UK, pp 302-320.

Takken W, Dekker T and Wijnholds YG (1997) Odor-mediated flight behavior of Anopheles gambiae Giles sensu stricto and An. stephensi Liston in response to CO2, acetone, and 1-Octen-3-ol (Diptera: Culicidae). J Insect Behav 10: 395-407.

Takken W, Eling W, Hooghof JDT, Hunt R and Coetzee M (1999) Susceptibility of Anopheles quadriannulatus Theobald (Diptera: Culicidae) to Plasmodium falciparum. Trans R Soc Trop Med Hyg 93: 578-580.

Takken W and Kline DL (1989) Carbon dioxide and 1-octen-3-ol as mosquito attractants. J Am Mosq Control Assoc 5: 311-316.

Takken W and Knols BGJ (1999) Odor-mediated behavior of afrotropical malaria mosquitoes. Ann Rev Entomol 44: 131-157.

Takken W and Knols BGJ (2007) Emerging pests and vector-borne diseases in Europe. Ecology and control of vectorborne diseases Vol. 1. Wageningen Academic Publishers, Wageningen, the Netherlands, $499 \mathrm{pp}$. 
Takken W and Knols BGJ (2009) Malaria vector control: Current and future strategies. Trends Parasitol 25: 101-104.

Takken W, Van Loon JJA and Adam W (2001) Inhibition of host-seeking response and olfactory responsiveness in Anopheles gambiae following blood feeding. J Insect Physiol 47: 303-310.

Tomberlin J, Rains G, Allan S, Sanford M and Lewis W (2006) Associative learning of odor with food- or blood-meal by Culex quinquefasciatus Say (Diptera: Culicidae). Naturwissenschaften 93:551-556.

Torr S, Della Torre A, Calzetta M, Costantini C and Vale GA (2008) Towards a fuller understanding of mosquito behaviour: use of electrocuting grids to compare the odour-orientated responses of Anopheles arabiensis and An. quadriannulatus in the field. Med Vet Entomol 22: 93-108.

Torr SJ, Hall DR and Smith JL (1995) Responses of tsetse flies (Diptera: Glossinidae) to natural and synthetic ox odours. Bull Entomol Res 85: 157-166.

Turell MJ, Dohm DJ, Sardelis MR, guinn ML, Andreadis TG and Blow JA (2005) An update on the potential of North American mosquitoes (Diptera: Culicidae) to transmit West Nile virus. J Med Entomol 42: 57-62.

Vale GA (1980) Field studies of the responses of tsetse flies (Glossinidae) and other Diptera to carbon dioxide, acetone and other chemicals. Bull Entomol Res 70: 563-570.

Vale GA (1993) Development of baits for tsetse flies (Diptera: Glossinidae) in Zimbabwe. J Med Entomol 30: 831-842.

Vale GA and Hall DR (1985b) The use of 1-octen-3-ol, acetone and carbon dioxide to improve baits for tsetse flies, Glossina spp. (Diptera: Glossinidae). Bull Entomol Res 75: 219-231.

Vale GA and Hall DR 1985a The role of 1-octen-3-ol, acetone and carbon dioxide in the attraction of tsetse flies, Glossina spp. (Diptera: Glossinidae), to ox odour. Bull Entomol Res 75: 209-217.

Vale GA, Hall DR and Gough AJE (1988) The olfactory responses of tsetse flies, Glossina spp. (Diptera: Glossinidae), to phenols and urine in the field. Bull Entomol Res 78: 293-300.

Van der Goes van Naters W and Carlson JR (2006) Insects as chemosensors of humans and crops. Nature 444: 302-307.

Van Thiel P (1937) Quelles sont les excitations incitant l'Anopheles maculipennis atroparvus a visiter et a piquer I'homme ou le batail? Bulletin Soc Path Exot 30: 193-203.

Vazeille M, Jeannin C, Martin E, Schaffner F and Failloux AB (2008) Chikungunya: a risk for Mediterranean countries? Acta Trop 105: 200-202.

Verhulst NO, Beijleveld H, Knols BGJ, Takken W, Schraa G, Bouwmeester HJ and Smallegange RC (2009). Cultured skin microbiota attracts malaria mosquitoes. Malar J 8: 302.

Waka M, Hopkins RJ, Glinwood R and Curtis C (2006) The effect of repellents Ocimum forskolei and deet on the response of Anopheles stephensi to host odours. Med Vet Entomol 20:373-376.

Wang G, Carey AF, Carlson JR and Zwiebel LJ (in press) The molecular basis of odor coding in the malaria vector mosquito Anopheles gambiae. PNAS (in press).

Wang ZY, Mo JC and Zhang SM (2006) Laboratory and field evaluations of potential human host odors for Aedes albopictus Skuse (Diptera: Culicidae). J Agric Urban Entomol 23: 57-64.

Waterhouse RM, Wyder S and Zdobnov EM (2008) The Aedes aegypti genome: a comparative perspective. Insect Mol Biol 17: 1-8.

White GB (1974) Anopheles gambiae complex and disease transmission in Africa. Trans R Soc Trop Med Hyg 68: 278-301.

White GB (2007) Terminology of insect repellents. In: Debboun M, Frances SP and Strickman D (eds) Insect repellents. Principles, methods, and uses. CRC Press, Boca Raton, FL, USA, pp 31-46.

WHO (2009) Malaria. WHO factsheet No 94. World Heath Organisation, Geneva, Switzerland.

WHO (2008) Dengue and dengue haemorrhagic fever. WHO factsheet No 117. World Heath Organisation, Geneva, Switzerland.

Williams CR, Bergbauer R, Geier M, Kline DL, Bernier UR, Russell RC and Ritchie SA (2006a) Laboratory and field assessment of some kairomone blends for host-seeking Aedes aegypti. J Am Mosq Control Assoc 22: 641-647.

Williams CR, Kokkinn MJ and Smith BP (2003) Intraspecific Variation in odor-mediated host preference of the mosquito Culex annulirostris. J Chem Ecol 29: 1889-1903.

Williams CR, Long SA, Russell RC and Ritchie SA (2006b) Field efficacy of the BG-Sentinel compared with CDC Backpack Aspirators and CO2-baited EVS traps for collection of adult Aedes aegypti in Cairns, Queensland, Australia. J Am Mosq Control Assoc 22: 296-300. 
Williams CR, Ritchie SA, Russell RC, Eiras AE, Kline DL and Geier M (2006c) Geographic variation in attraction to human odor compounds by Aedes aegypti mosquitoes (Diptera: Culicidae): a laboratory study. J Chem Ecol 32: 1625-1634.

Young S, Hardie J and Gibson G (1993) Flying insects in the laboratory. In: Wratten SD (ed) Video techniques in animal ecology and behaviour. Chapman and Hall, London, UK, pp 17-32.

Zeng C, Leyden JJ, Spielman Al, and Preti G (1996) Analysis of characteristic human female axillary odors: qualitative comparison to males. J Chem Ecol 22: 237-257.

Zeng XN, Leyden JJ, Lawley HJ, Sawano K, Nohara I and Preti G (1991) Analysis of characteristic odors from human male axillae. J Chem Ecol 17: 1469-1493.

Zinser M, Ramberg F and Willott E (2004) Culex quinquefasciatus (Diptera: Culicidae) as a potential West Nile virus vector in Tucson, Arizona: blood meal analysis indicates feeding on both humans and birds. J Insect Sci 4: 20.

Zwiebel LJ and Takken W (2004) Olfactory regulation of mosquito-host interactions. Insect Biochem Mol Biol 34: 645652. 


\title{
8. Host-seeking behaviour of Afrotropical anophelines: field and semi-field studies
}

\author{
Wolfgang R. Mukabana, Evelyn A. Olanga and Bart G.J. Knols
}

\begin{abstract}
The search for a synthetic mosquito attractant based on one or more human-derived kairomones has been the goal of many laboratory studies. Besides alleviating the occupational risk to which volunteers participating in vector surveillance are subjected whilst performing landing catches, discovery of potent attractants also underpins the development and deployment of mass trapping devices for controlling transmission of mosquito-borne diseases. Whereas a few potential synthetic attractants have recently been developed and tested, not much has been done under open field conditions. Odour delivery methodologies are still needed and the potency of available attractants is too small at present to warrant full-scale application of this technology for malaria vector surveillance and control. Cheap traps and trapping devices that operate energy-free or utilise an affordable or renewable source of energy to power them, a cheap source of carbon dioxide, a cheap and easy method for attractant delivery, and discovery of generic attractants that can trap multiple vectors need to be developed. Only then shall the potential impact of this technology on incidence and prevalence of various vector-borne diseases come to fruition.
\end{abstract}

Keywords: Anopheles, malaria, kairomones, host-seeking behaviour, Africa

\section{Introduction}

About one million deaths and close to five hundred million clinical episodes of malaria occur throughout the world each year (Breman and Holloway 2007). Ninety percent of malaria-related deaths occur in sub-Saharan Africa (Boutin et al. 2005), mainly among pregnant women and in children below five years of age (Philips 2001). Malaria hampers socioeconomic development by interfering with savings and investment, lowering workforce productivity, promoting absenteeism, causing premature mortality and increasing medical costs (Sachs and Malaney 2002). This makes malaria-endemic countries not only poor but also associates them with lower and slower rates of economic growth. The global distribution of per-capita gross domestic product (GDP) shows a distinct correlation between malaria and poverty. The reverse is true for countries where malaria has been eradicated (Gallup and Sachs 2001).

Human malarias are transmitted by Anopheles mosquitoes and are caused by single or multiple infections of four species of Plasmodium parasites. These include $P$. falciparum, P. malariae, P. ovale and $P$. vivax. Recently, P. knowlesi has been reported to cause malaria in Southeast Asia (CoxSingh et al. 2007, Kim-Sung et al. 2009). While P. vivax is most widely distributed, P. falciparum, which is the most widespread Plasmodium species in sub-Saharan Africa, causes the most severe complications. The main vectors of malaria in Africa are mosquitoes belonging to the Anopheles gambiae and An. funestus complexes.

The An. gambiae complex consists of seven morphologically indistinguishable sibling species namely An. gambiae s.s., An. arabiensis, An. bwambae, An. merus, An. melas, An. quadriannulatus species A (Coetzee et al. 2000, White 1974) and An. quadriannulatus species B (Hunt et al. 1998). Anopheles gambiae, the nominal taxon, and An. arabiensis are the most important vector species 
in the complex (Coetzee et al. 2000, Gillies and De Meillon 1968, Gillies and Coetzee 1987, White 1974). The occurrence of $80 \%$ of the world's malaria in tropical Africa (WHO 1993) is due to the strong human biting habits (anthropophily) and relatively long lifespan of these two species (Collins and Besansky1994), besides An. funestus which has similar biological characteristics. Anopheles gambiae is a more efficient vector because of its endophilic and anthropophilic characteristics (Costantini et al. 1999). The vectorial capacity of An. arabiensis is slightly lower than that of Anopheles gambiae because of its ability to feed on other animals when human hosts are not available (Gillies and Coetzee 1987). Distinct chromosomal 'forms' of An. gambiae, which are strongly associated with specific habitats, exist in West Africa. Three of these so-called ecophenotypes (i.e. BAMAKO, MOPTI, and SAVANNAH) have been found to occur in sympatry at numerous sites (Coluzzi et al. 1979). Two other members of the complex, namely An. melas in West Africa and An. merus in East Africa, are localised vectors depending on their levels of contact with people. Of the other sibling species, An. bwambae is responsible for localised malaria transmission among the Bambute pygmies of Bwamba in Uganda whereas An. quadriannulatus is not considered a vector, in spite of its competence to transmit $P$. falciparum (Takken and Knols 1999). The Ethiopian population of An. quadriannulatus was recently recognised as being distinct (from its South African counterpart) and is designated An. quadriannulatus species B (Hunt et al. 1998).

The An. funestus complex consists of a group of nine species including An. funestus, An. rivulorum, An. parensis, An. vaneedeni, An. leesoni, An. fuscivenosus, An. aruni, An. brucei and An. confusus (Cohuet et al. 2004). Anopheles funestus is the main species within the An. funestus group that transmits malaria (Cohuet et al. 2004, Gillies and De Meillon 1968, Wilkes et al. 1996). It is endophilic and anthropophilic and is considered as one of the major vectors of malaria in Africa (Cohuet et al. 2004). Whereas An. funestus, An. rivulorum, and An. leesoni are widely distributed throughout subSaharan Africa, the other members of the group are more locally distributed: An. parensis and An. confusus are found in Eastern Africa, An. vaneedeni in the northern areas of South Africa, An. aruni in Zanzibar, An. fuscivenosus in Zimbabwe, and An. brucei in Nigeria. The distribution, ecological and behavioural aspects of the main vectors of malaria in Africa are presented in Table 1.

African malaria mosquitoes locate their blood meal hosts largely based on olfactory cues (Takken and Knols 1999). Physical cues, encompassing heat and moisture, also play a role that is hitherto not well understood (e.g. Mukabana et al. 2004, Olanga et al. 2010, Takken et al. 1997). Dissecting and analysing the broad spectrum of human emanations (Costantini et al. 1993) can provide an important basis for developing synthetic compounds or blends with desirable attractant (Logan and Birkett 2007), repellent or attractant 'masking' properties (Logan et al. 2008). Several compounds identified from human emanations have been demonstrated to exhibit attractant properties under varying experimental conditions. In this chapter we explore the extent to which odour baits have been employed to assess the host-seeking behaviour of African malaria mosquitoes under semi-field and field conditions. Emphasis is placed on studies conducted in the last decade, as earlier work is reviewed by Takken and Knols (1999).

\section{Odour-mediated host-seeking behaviour of African malaria vectors in the field}

Haddow (1942) was among the first to recognise the importance of host odours in the behaviour of African malaria vectors. He demonstrated that human body odour attracted anophelines to a house, and that the attraction was proportional to body mass. Since then numerous studies demonstrated the role of human odour in the attraction of malaria vectors (reviewed in Takken and Knols 1999). Few studies attempted to unravel the role of individual chemicals constituting 


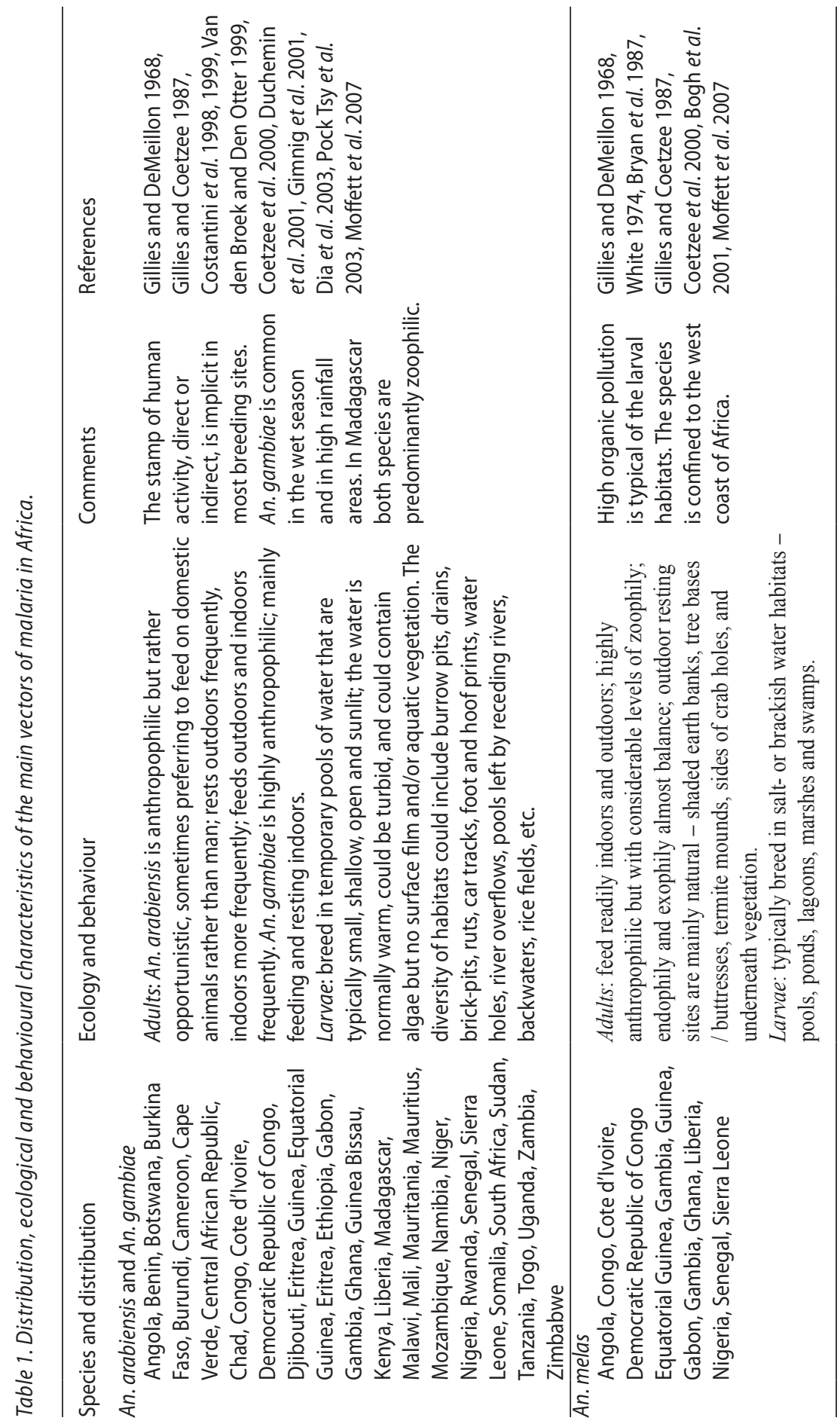




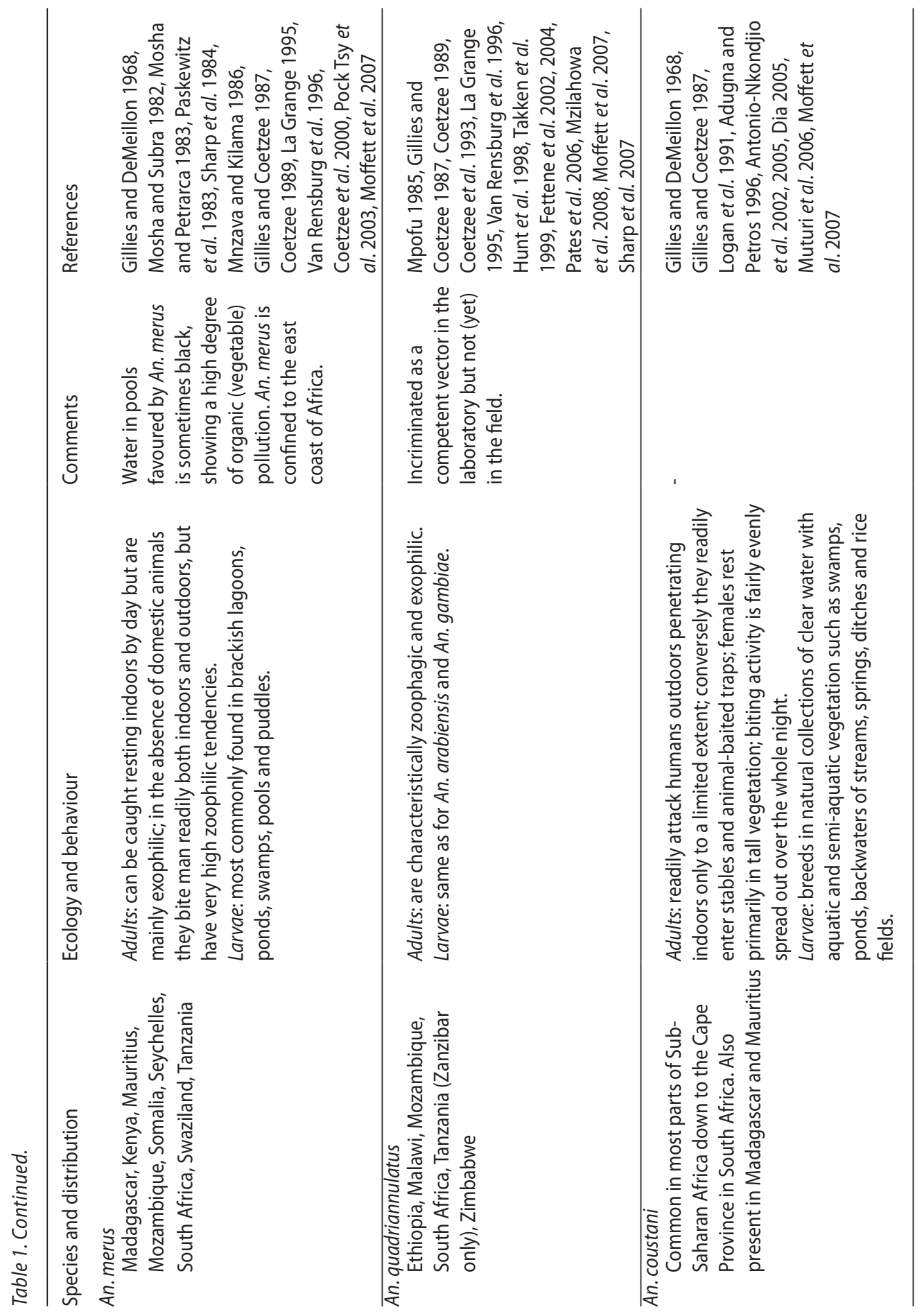




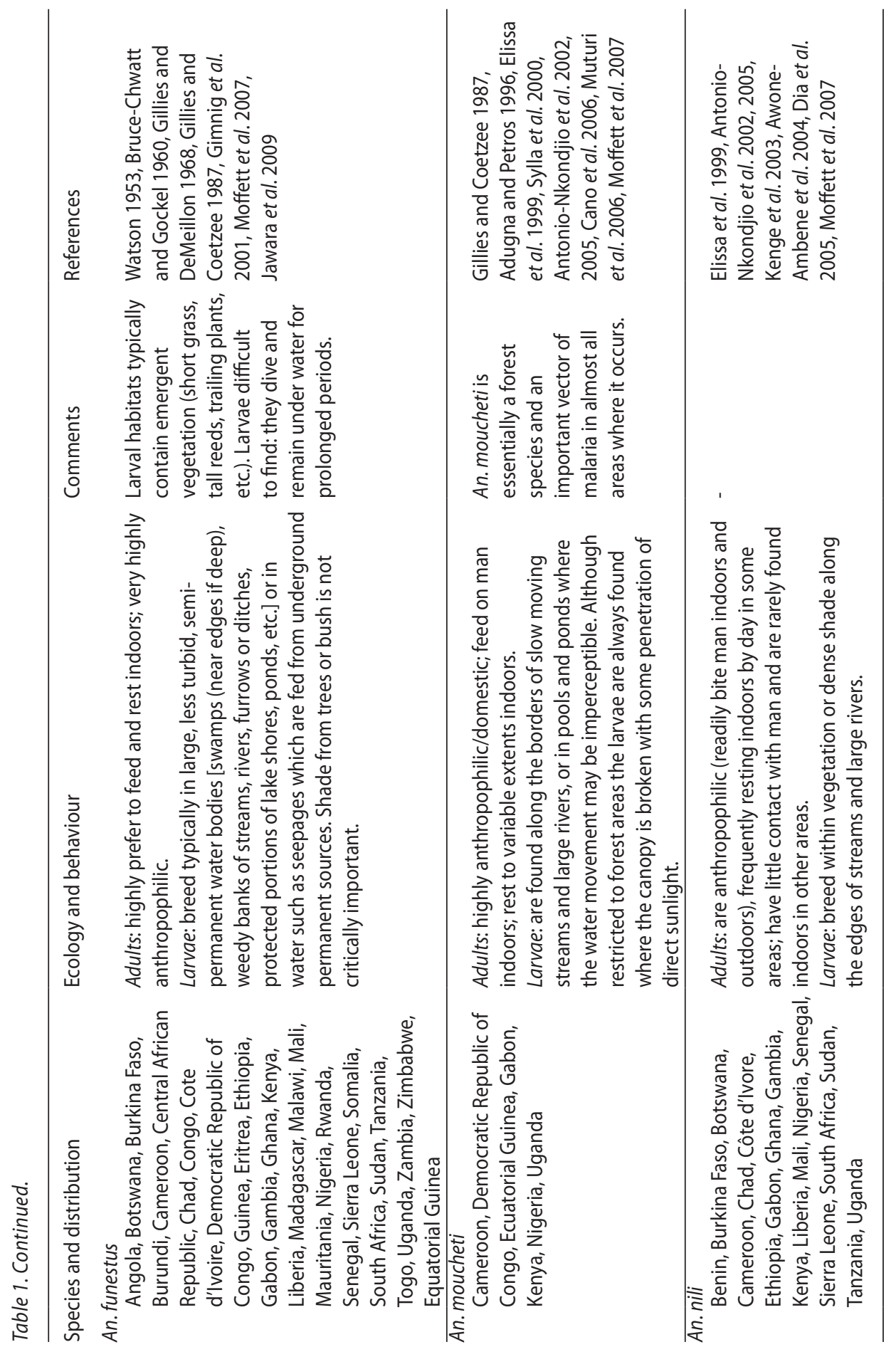




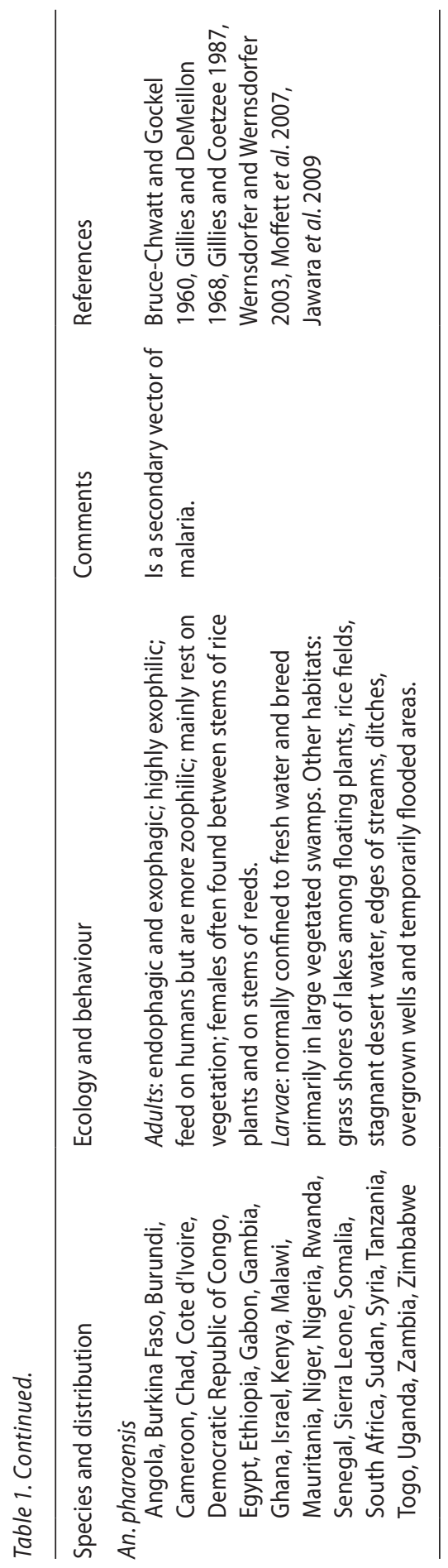


attractive odours. Carbon dioxide $\left(\mathrm{CO}_{2}\right)$ was recognised as a universal mosquito attractant, including African anophelines (Mboera and Takken 1997). Apart from the studies that focused on $\mathrm{CO}_{2}$ only, the number of semi-field and field studies that have been undertaken between 1993 and 2010 to further our understanding of odour-mediated host-seeking behaviour of African malaria vectors are limited (Table 2). These largely centered on understanding mosquito behavioural responses to crude, unrefined odour samples, mostly whole human odour (Table 2). Other unrefined odour bait sources that have been investigated within this period include human foot odour (Jawara et al. 2009, Murphy et al. 2001, Njiru et al. 2006, Olanga et al. 2010, Okumu et al. 2010a, Schmied et al. 2008), human breath (Knols et al. 1998, Mukabana et al. 2004) and sheep, goat, pig (Mahande et al. 2007), cattle (Costantini et al. 1993, 1998, Dekker and Takken 1998, Duchemin et al. 2001, Kweka and Mahande 2009, Kweka et al. 2009, Mahande et al. 2007, Tirados et al. 2006) and monkey odours (Costantini and Diallo 2001). A handful of these studies have been carried out in West Africa where malaria vector species, characterised by certain peculiarities (Costantini et al. 1999), differ in some ways from those found in eastern Africa, including the islands of the Indian Ocean (Duchemin et al. 2001).

Specific objectives of the studies conducted are diverse but can be grouped into four. These included: (1) development of mosquito sampling and surveillance tools (Costantini et al. 1993, Dia et al. 2005, Govella et al. 2009, Jawara et al. 2009, Knols et al. 1998, Laganier et al. 2003, Mathenge et al. 2002, 2004, 2005), (2) investigation of the basis of differences of attractiveness of humans to mosquitoes (Brady et al. 1997, Knols et al. 1995, Lacroix et al. 2005, Lindsay et al. 1993, Mboera et al. 1997, Mukabana et al. 2002, 2004); (3) determination of the interspecific host preferences of different mosquitoes species (Costantini and Diallo 2001, Costantini et al. 1998, Dekker and Takken 1998, Duchemin et al. 2001, Kweka and Mahande 2009, Kweka et al. 2009, Mahande et al. 2007, Tirados et al. 2006, Torr et al. 2008) and (4) identification of chemical attractants for Afrotropical anophelines (Beavers et al. 1998, Costantini et al. 1996, 2001, Gibson et al. 1997, Murphy et al. 2001, Njiru et al. 2006, Okumu et al. 2010a, Qiu et al. 2007). To date, nothing has been reported on the actual deployment of chemical attractants for operational mosquito control or surveillance in Africa.

The species of African Anopheles mosquitoes mentioned in the published studies are diverse and include Anopheles gambiae s.s., An. arabiensis, An. coustani, An. funestus, An. nili, An. pharoensis, An. quadriannulatus, An. sergenti, An. squamosus and An. ziemanni. In terms of host preference $A n$. gambiae s.s. (Costantini and Diallo 2001, Costantini et al. 1993, 1998), An. funestus (Duchemin et al. 2001) and An. pharoensis (Costantini et al. 1998) preferred to enter human-baited over animalodour baited traps in dual-choice assays. This was also the case for An. arabiensis (Kweka et al. 2009, Torr et al. 2008). However, in some cases for An. gambiae, An. arabiensis (Duchemin et al. 2001, Kweka and Mahande 2009) and An. funestus (Costantini et al. 1998) this was the reverse. Carbon dioxide was identified as a cause for differences in human attractiveness to mosquitoes (Brady et al. 1997) and the chemical compounds 1-octen-3-ol $+\mathrm{CO}_{2}$ (Beavers et al. 1998), L-lactic acid $+\mathrm{CO}_{2}$ (Murphy et al. 2001), 7-octenoic acid (Costantini et al. 2001), and a blend containing ammonia + L-lactic acid $+\mathrm{CO}_{2}+3$-methylbutanoic acid (Qiu et al. 1997) attracted various African mosquito species in the field. 


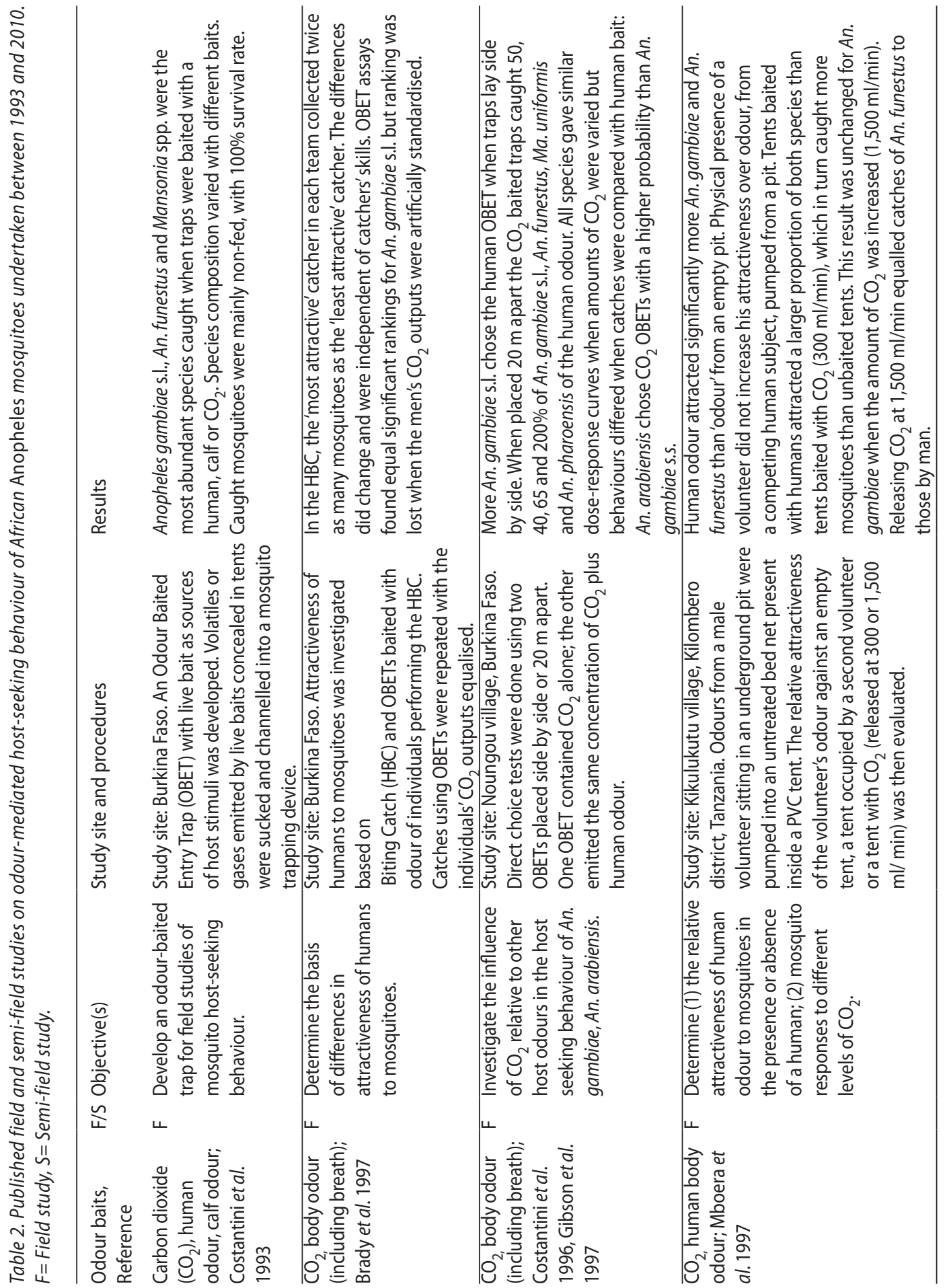




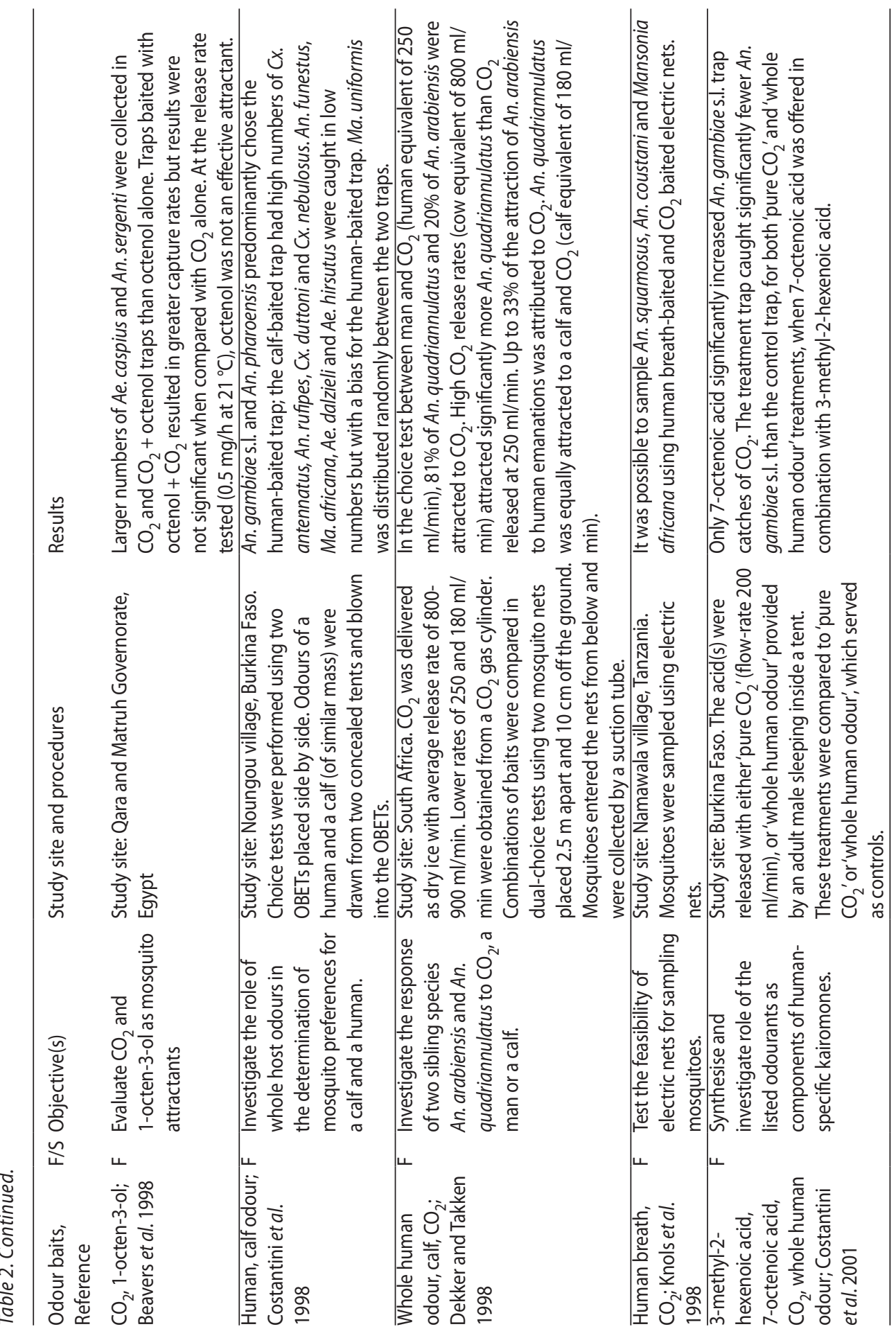




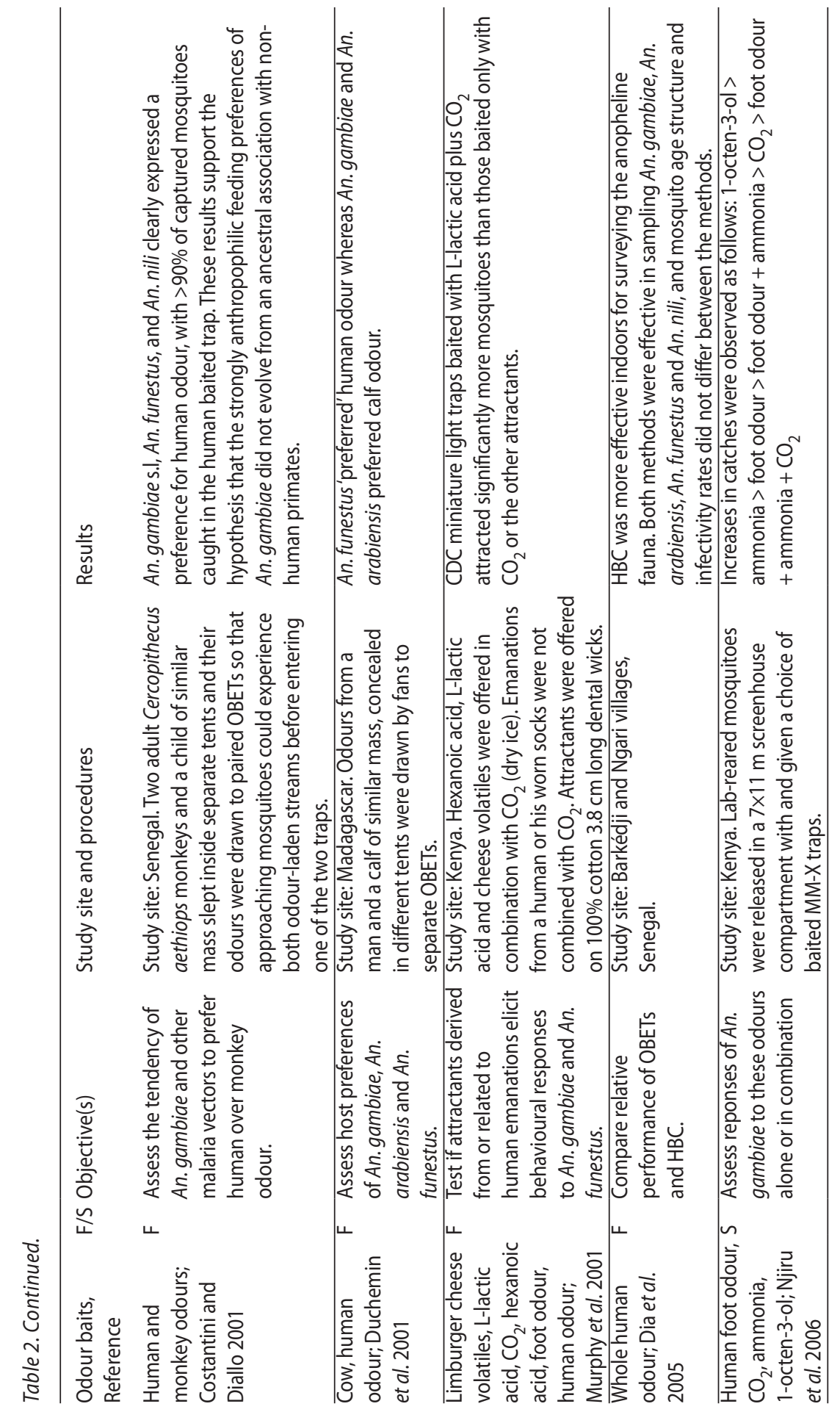




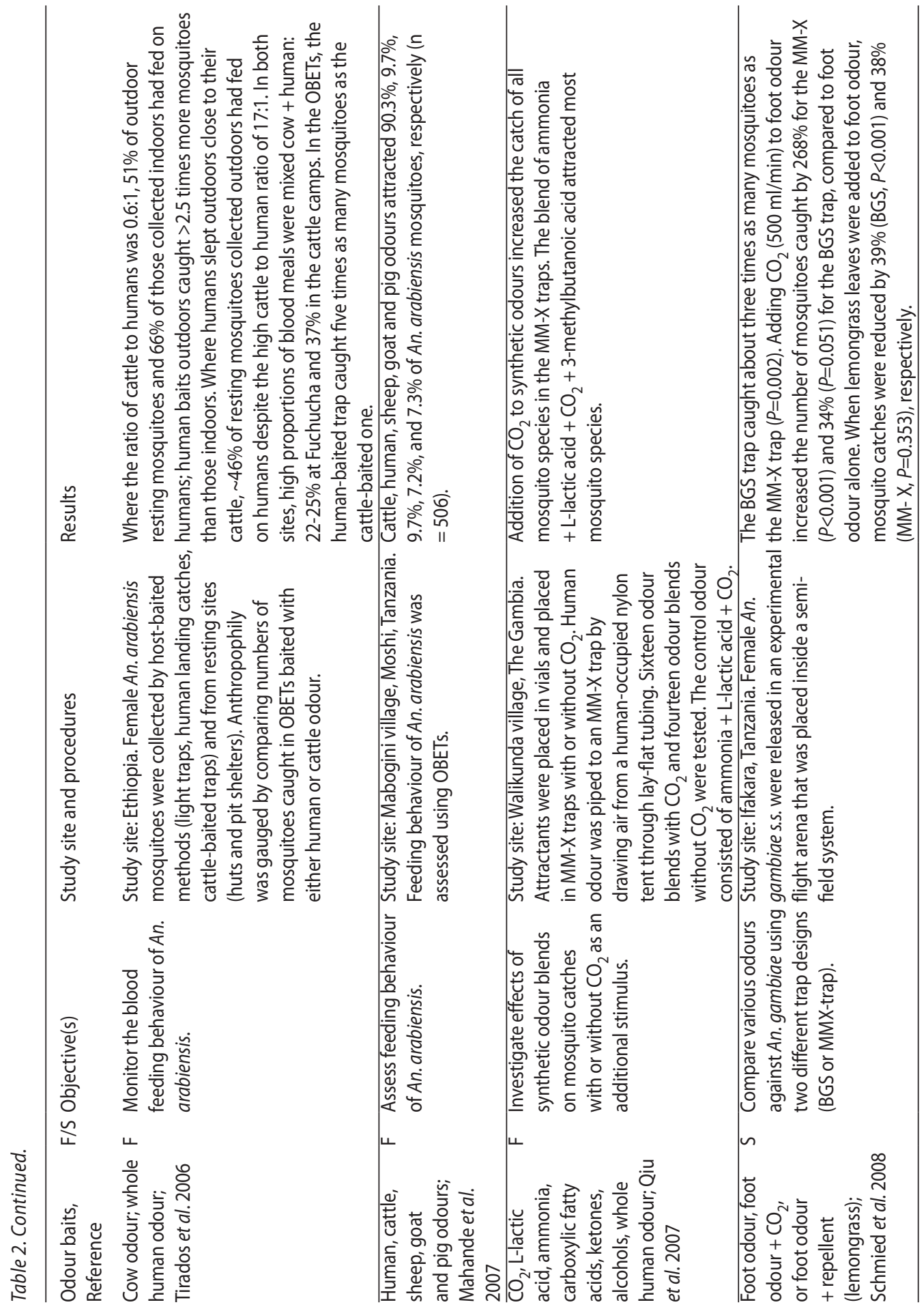




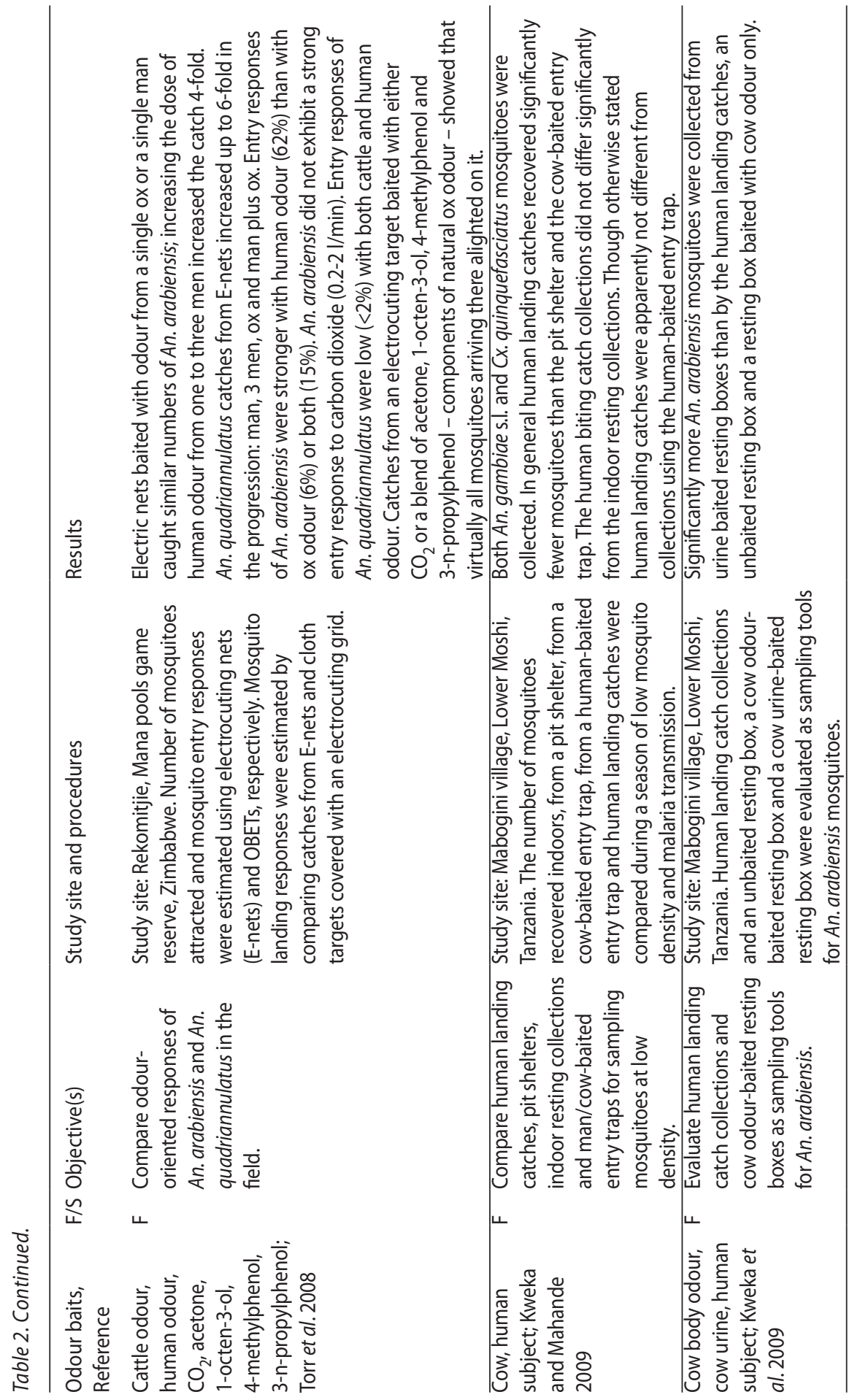




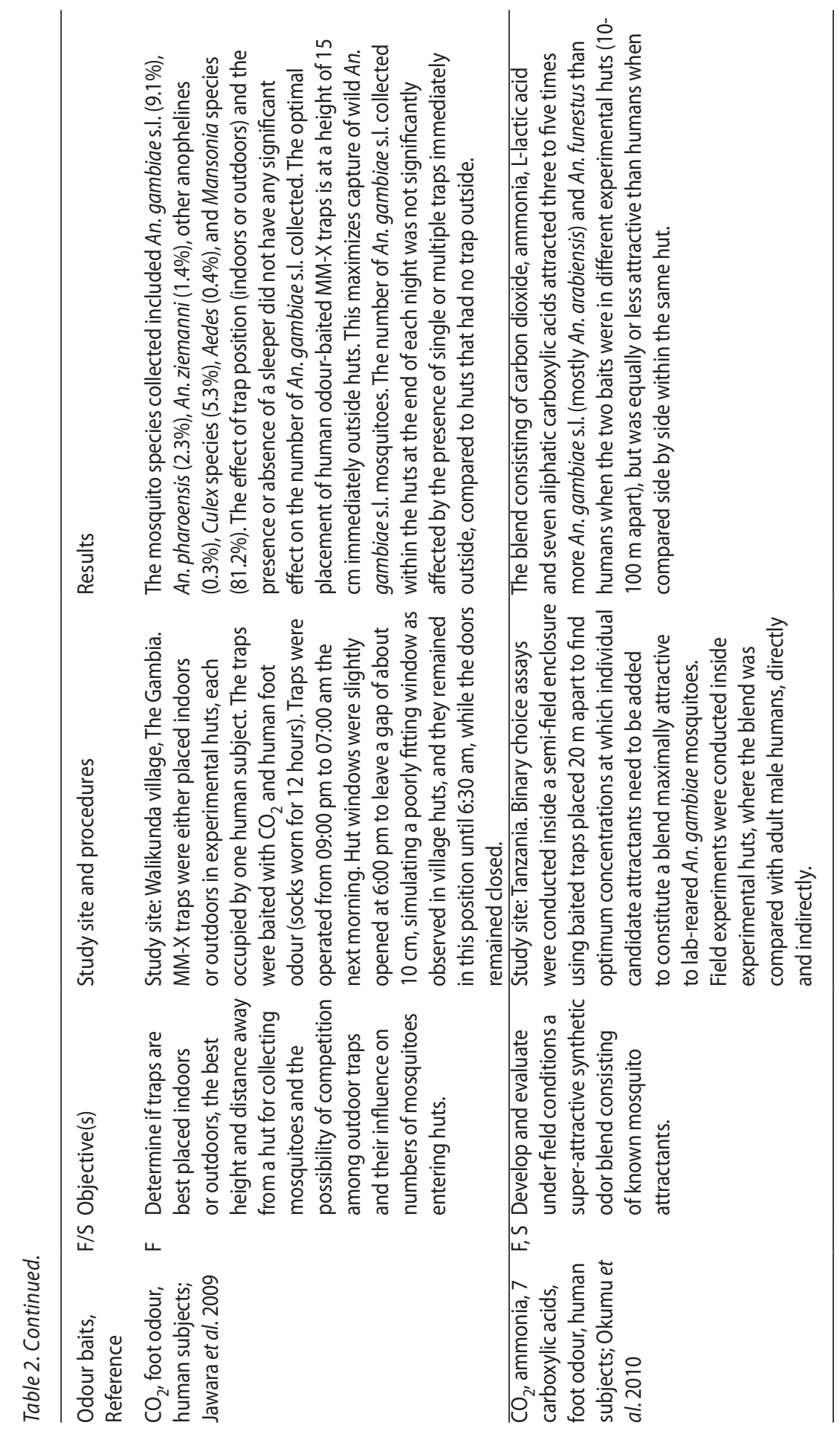




\section{Synthetic odour baits attractive to African anophelines under semi-field and field conditions}

The discovery of synthetic odour baits that are capable of attracting mosquitoes much the same as a human being (Brady et al. 1997) can enhance the development of powerful tools for vector surveillance (Takken and Knols 1999) and control (Day and Sjogren 1994, Kline 2007, Logan and Birkett 2007, Takken and Knols 2009). Individual chemical compounds and blends thereof have been shown to attract African Anopheles mosquitoes under semi-field and field environments. The chemicals, which comprise of commonly known kairomones like $\mathrm{CO}_{2}$, carboxylic acids, ketones, phenols, L-lactic acid, and ammonia, are described in subsequent paragraphs. This chapter is restricted to semi-field and field studies on African anophelines where $\mathrm{CO}_{2}$ was tested in combination with crude odourants or at least one synthetic attractant (Table 2). The selection of candidate attractants for these studies was in many cases informed by laboratory studies, described in Chapter 7.

Carbon dioxide, a major constituent of human exhaled air (300-500 $\mathrm{ml} / \mathrm{min}$.), has been identified as an attractant for many mosquito species including the main vectors of malaria in Africa (Gillies 1980, Mboera and Takken 1997, Mboera et al. 1997, 2000, Takken 1991, Takken and Knols 1999). Gillies (1980) suggested that this compound acts as an activator, initiating flight responses, as well as being an attractant. There is strong evidence that $\mathrm{CO}_{2}$ acts synergistically with other chemical compounds to attract host-seeking mosquitoes (Dekker et al. 2002, Kline et al. 1990, Murphy et al. 2001, Njiru et al. 2006, Takken and Kline 1989). Indeed, addition of $\mathrm{CO}_{2}$ to traps baited with various synthetic compounds significantly increased catches of African anophelines including An. gambiae s.s. (Costantini et al. 2001, Murphy et al. 2001), An. arabiensis (Costantini et al. 2001, Murphy et al. 2001, Torr et al. 2008), An. quadriannulatus (Torr et al. 2008), An. funestus (Murphy et al. 2001), An. sergenti (Beavers et al. 1998), An. pharoensis (Qiu et al. 2007) and An. ziemanni (Qiu et al. 2007). In a field study in Burkina Faso, $\mathrm{CO}_{2}$ added to 7-octenoic acid significantly increased the number of An. gambiae s.s. and An. arabiensis attracted to odour-baited entry traps. In The Gambia, MM-X traps baited with a synthetic odour blend consisting of ammonia, lactic acid, $\mathrm{CO}_{2}$ and 3-methylbutanoic acid attracted large numbers of mosquitoes belonging to the genera Mansonia, Anopheles and Culex (Qiu et al. 2007). Field studies in Egypt demonstrated that $\mathrm{CO}_{2}$ plus 1-octen-3-ol attracted similar numbers of An. sergenti as $\mathrm{CO}_{2}$ alone (Beavers et al. 1998).

Limburger cheese, the smell of which is reminiscent of human foot odour, is known to attract African anopheline mosquitoes under laboratory conditions (De Jong and Knols 1995, Knols and De Jong 1996). One field study carried out in western Kenya, using Limburger cheese in its original form, also demonstrated this (Owino 2006) while another, carried out in the same region using a synthetic analogue of the cheese, did not (Murphy et al. 2001). In these studies Limburger cheese odours were delivered using either MM-X counter flow geometry traps (Owino 2006) or CDC miniature light traps (Murphy et al. 2001). Species of African malaria mosquitoes that were successfully trapped using Limburger cheese in its original form included An. gambiae s.S., An. arabiensis and An. funestus (Owino 2006). The difference in results may be attributed to usage of synthetic versus authentic forms of the cheese and differences in odour delivery methodologies. In the case of Murphy et al. (2001), a combination of lactic acid and $\mathrm{CO}_{2}$ was the only treatment that significantly attracted more An. gambiae s.l. and An. funestus mosquitoes, although numbers caught were very low. This may be attributed to the difficulty of baiting CDC light traps, the fan of which disperses odours widely. 
Synthetic blends consisting of acetone, 1-octen-3-ol, 4-methylphenol and 3-n-propylphenol, which are components of cattle odour, have recently been used to bait mosquito electrocuting nets (Torr et al. 2008). Anopheles arabiensis did not exhibit a strong entry response to $\mathrm{CO}_{2}(0.2-2 \mathrm{l} /$ $\mathrm{min}$ ) baited entry traps. Catches from an electrocuting target baited with either $\mathrm{CO}_{2}$ or a blend of acetone, 1-octen-3-ol, 4-methylphenol and 3-n-propylphenol induced overwhelmingly high An. gambiae s.l. landing rates (Torr et al. 2008). In a recent study, an odour blend consisting of known mosquito attractants namely $\mathrm{CO}_{2}$, ammonia and carboxylic acids was evaluated (Okumu et al. 2010a; see Table 2 for compound names). Field experiments conducted inside experimental huts found the blend to attract three to five times more An. arabiensis than humans when the two baits were in different experimental huts (10-100 meters apart). However, the blend was equally or less attractive than humans when compared side by side within the same huts (Okumu et al. 2010a).

From the foregoing it is clear that one or more synthetic analogues of human sweat combined with $\mathrm{CO}_{2}$ (Costantini et al. 2001, Qiu et al. 2007) or $\mathrm{CO}_{2}$ combined with various aliphatic carboxylic acids (Okumu et al. 2010a) are currently the best attractants for host-seeking Afrotropical malaria mosquitoes under field conditions. Further research on the development of potent attractants needs to be carried out with the aim of (1) identifying additional candidate human specific odours (2) determine their optimum concentrations to attract host-seeking mosquitoes and (3) optimising existing synthetic odour blends.

\section{Semi-field research on odour bait development}

Historically, semi-field systems, which in essence are large outdoor screened cages, have been used for mosquito research in several countries and for several mosquito species (reviewed by Ferguson et al. 2008). Only since the late 1990s have such systems become a research tool to identify important kairomones for African anophelines. A major advantage of such systems is the fact that a fixed and controllable number of mosquitoes can be introduced in them, which delivers constant and comparable results. Moreover, by placing such systems directly in areas where malaria vectors occur in nature it is possible to rear offspring of field-collected gravid females, thereby ensuring that the genetic background of test mosquitoes is similar to that of the field population. Availability of efficient traps that can easily be baited with $\mathrm{CO}_{2}$ and other attractants, such as the MM-X trap (see Njiru et al. 2006) and the BGS trap (see Schmied et al. 2008) has further strengthened semi-field studies on anopheline host-seeking behaviour. When using two such traps in simple cross-over designs to account for positional effects, rapid progress can be made to improve baits. For instance, the recent step-wise and incremental improvement of a powerful blend to attract An. gambiae s.s. by Okumu et al. (2010a) started with a basic blend of $\mathrm{CO}_{2}$ and ammonia, and was then augmented with various concentrations of L-lactic acid, until an optimal concentration was found. Thereafter, each of seven aliphatic carboxylic acids was added to this base blend, at varying concentrations, until again the optimal increase in catch was reached. This approach culminated in the development of a blend that attracted 3-5 times as many mosquitoes than a human volunteer, when tested in experimental huts in a field setting. These developments clearly demonstrate the power of semi-field research, and we advocate the use of such systems not only for further attractant development, but also for the development of low-cost trapping devices, an aspect of bait-trap technology that has not received enough attention to date. 


\section{The promise of using odour baits for malaria vector surveillance and control in Africa}

Although the goals of developing potent insect attractants are diverse, the central goal lies in vector control and surveillance (Kline 2007, Logan and Birkett 2007). With respect to mosquitoes, impact on target populations can be achieved through mass trapping (Anonymous 2005, Kline and Lemire 1998), lure and kill technology (Day and Sjogren 1994, Kline 2007) or lure and contaminate technology (e.g. by using biopesticides like entomopathogenic fungi). The efficacy of mass trapping, despite its underlying conceptual, technical, logistical and financial limitations, has been successfully demonstrated for mosquito population reduction in the USA (Anonymous 2005, Kline 2007, Kline and Lemire 1998). However, this strategy is still being developed, with current efforts being centered on searching for new attractants and attractant formulations (Healy and Copland 2000, Healy et al. 2002, Okumu et al. 2010a, Qui et al. 2007), improving on existing ones (Smallegange et al. 2005), and developing trapping devices (Kline 2006, Kröckel et al. 2006). Efficacy trials of candidate synthetic attractants under field (Qiu et al. 2007) and semi-field conditions (Njiru et al. 2006, Okumu et al. 2010a, Olanga et al. 2010) are also underway.

In terms of surveillance the need of replacing the risk prone human landing catch for measuring the degree of contact between humans and mosquitoes (Service 1993) is urgent. The key challenge is to find synthetic attractants, which by acting as human surrogates can be used in sampling devices for malaria vectors (Brady et al. 1997). Whereas identification of a potent synthetic attractant will go a long way in helping to develop malaria vector surveillance tools and mass trapping devices, other challenges relate to suitable ways and means of delivering attractant odours. In the studies carried out thus far delivery methods of synthetic attractants included use of wicks (Murphy et al. 2001), glass vials (Costantini et al. 2001, Qiu et al. 2007), sealed polythene sachets (Torr et al. 2008), and nylon strips (Okumu et al. 2010b). The use of wicks, glass vials and nylon strips has the major disadvantage that whereas release rates of synthetic odours can be measured by weighing before and after placement in the field, measurement may not be as precise, especially for compounds which tend to absorb water such as octenol. This is in contrast to sealed polythene sachets, which guarantee measurable constant release rates of synthetic attractants. However, the use of pressurised cylinders (Costantini et al. 2001, Qiu et al. 2007, Torr et al. 2008) or dry ice (Murphy et al. 2001) as delivery methods of $\mathrm{CO}_{2}$ is disadvantageous as the methods are not logistically sound. They are expensive and laborious for use in the field. Handy sources and more effective methods of delivering $\mathrm{CO}_{2}$ and other gaseous kairomones should be sought.

Besides the disadvantages associated with odour delivery technologies, the physical gadgets useful for handling and dispensing attractant-impregnated materials are limiting. Field experiments have so far relied on using odour-baited entry traps (Brady et al. 1997, Costantini at Diallo 2001, Costantini et al. 1993, 1996, 1998, Dia et al. 2005, Gibson et al. 1997, Kweka and Mahande 2009, Kweka et al. 2009, Mahande et al. 2007, Tirados et al. 2006, Torr et al. 2008); mosquito magnet model X (MM-X) traps (Jawara et al. 2009, Okumu et al. 2010a, Qiu et al. 2007), CDC miniature light traps (Murphy et al. 2001), BGS traps (Schmied et al. 2008), electric nets (Knols et al. 1998, Torr et al. 2008) and resting boxes (Kweka et al. 2009). Apart from the MM-X and BGS trap none of the other gadgets is well suited at present for delivering attractants in a desirable manner.

In general the bottlenecks that might impede the use of synthetic attractants for malaria vector surveillance and control in Africa include (1) lack of cheap traps and trapping devices, (2) lack of affordable sources of energy to power traps, (3) lack of cheaper sources of $\mathrm{CO}_{2}$, this being a key kairomone for many malaria vectors and may be a chemical that will remain an integral 
component of attractive blends, (4) lack of cheap and easy methods for delivering mosquito synthetic attractants, (5) low diversity of synthetic attractants, and (6) lack of a generic attractant that can trap multiple vectors.

\section{Concluding remarks}

This chapter has focused on odour baits that have been employed to assess the host-seeking behaviour of African Anopheles mosquitoes under field and semi-field conditions. The reported findings on mosquito responses to synthetic attractants provide optimism that the search for a surrogate human is possible. A potent synthetic human odour will enhance the development of powerful mosquito trapping devices that can be exploited to increase the success of control programmes through forecasting epidemics accurately and for formulating, planning and rolling out control activities.

\section{References}

Adugna N and Petros B (1996) Determination of the human blood index of some anopheline mosquitoes by using ELISA. Ethiop Med J 34: 1-10.

Anonymous, 2005. Collier Mosquito Control District - completed projects. Stevens' Landing. Available at: http: //www. collier-mosquito.org/stevens_landing.php (accessed November 30, 2009).

Antonio-Nkondjio C, Awono-Ambene P, Toto JC, Meunier JY, Zebaze-Kemleu S, Nyambam R, Wondji CS, Tchuinkam T and Fontenille D (2002) High malaria transmission intensity in a village close to Yaounde the capital city of Cameroon. J Med Entomol 39: 350-355.

Antonio-Nkondjio C, Simard F, Awono-Ambene P, Ngassam P, Toto J, Tchuinkam T and Fontenille D (2005) Malaria vectors and urbanization in the equatorial forest region of south Cameroon. Trans R Soc Trop Med Hyg 99:347-354.

Awone-Ambene HP, Kengne P, Simard F, Antonio-Nkondjio C and Fontenille D (2004) Description and bionomics of Anopheles (Cellia) ovengensis (Diptera: Culicidae) a new malaria vector species of the Anopheles nili group from South Cameroon. J Med Entomology 41: 561-568.

Beavers GM, Hanifa HA and Tetreault GE (1998) Response of mosquitoes (Diptera: Culicidae) to carbon dioxide and octenol in Egypt. J Egypt Soc Parasitol 28(2): 303-312.

Bøgh C, Clarke SE, Pinder M, Sanyang F and Lindsay SW (2001) Effect of passive zooprophylaxis on malaria transmission in The Gambia. J Med Entomol 38: 822-828.

Boutin JP, Pradines B, Pages F, Legros F, Rogier C and Migliani R (2005) Epidemiology of Malaria. Rev Prat 55(8): 833-840.

Brady J, Costantini C, Sagnon N, Gibson G and Coluzzi M (1997) The role of body odours in the relative attractiveness of different men to malarial vectors in Burkina Faso. Annal of Trop Med Parasitol 91: 121-122.

Breman JG and Holloway CN (2007) Malaria Surveillance Counts. Am J Trop Med Hyg, 77 (Suppl 6): 36-47.

Bruce-Chwatt LJ and Göckel CW (1960) A study of the blood-feeding patterns of Anopheles mosquitoes through precipitin tests. Bull WHO 22: 685-720.

Bryan, JH, Petrarca V, Di Deco MA and Coluzzi M (1987) Adult behaviour of members of the Anopheles gambiae complex in the Gambia with special reference to An. melas and its chromosomal variants. Parasitologia 29: 221-249.

Cano J, Angel Desalzo M, Moreno M, Chen Z, Nzambo S, Bobuakasi L, Buatiche JN, Ondo M, Micha F and Benito A (2006) Spatial variability in the density distribution and vectorial capacity of anopheline species in a high transmission village (Equatorial Guinea). Malar J 5: 21.

Coetzee M (1989) Comparative morphology and multivariate analysis for the discrimination of four members of the Anopheles gambiae group in Southern Africa. Mosq Sys 21: 100-116.

Coetzee M (2004) Distribution of the African malaria vectors of the Anopheles gambiae complex. Am J Trop Med Hyg 70: 103-104.

Coetzee M, Craig M and Le Sueur D (2000) Distribution of African malaria mosquitoes belonging to the Anopheles gambiae complex. Parasitology Today 16: 74-77. 
Coetzee M, Hunt RH, Baack L and Davidson G (1993) Distribution of mosquitoes belonging to the Anopheles gambiae complex, including malaria vectors, south of latitude 15uS. S Afr J Sci 89: 227-231.

Cohuet A, Simard F, Wondji CS, Antonio-Nkondjio C, Awono-Ambene P and Fontenille D (2004) High malaria transmission intensity due to Anopheles funestus (Diptera: culicidae) in a village of Savannah-forest transition area in Cameroon. J Med entomol 41: 901-905.

Collins FH and Besansky NJ (1994) Vector biology and the control of malaria in Africa. Science 264: 1874-1875.

Coluzzi M, Sabatini A, Petrarca V and di Deco MA (1979) Chromosomal differentiation and adaptation to human environments in the Anopheles gambiae complex. Trans R Soc Trop Med Hyg, 73: 483-497.

Costantini C and Diallo M (2001) Preliminary lack of evidence for simian odour preferences of savanna populations of Anopheles gambiae and other malaria vectors. Parassitologia 43: 179-182.

Costantini C, Birkett MA, Gibson G, Ziesmann J, Sagnon NF, Mohammed HA, Coluzzi M and Pickett JA (2001) Electroantennogram and behavioural responses of the malaria vector Anopheles gambiae to human-specific sweat components. Med Vet Entomol 15: 259-266.

Costantini C, Gibson G, Brady J, Merzagora L and Coluzzi M (1993) A new odour-baited trap to collect host-seeking mosquitoes. Parassitologia 35: 5-9.

Costantini C, Gibson G, Sagnon N, della Torre A, Brady J and Coluzzi M (1996) Mosquito responses to carbon dioxide in a West African Sudan savanna village. Med Vet Entomol 10: 220-227.

Costantini C, Sagnon N, Della TA, Diallo M, Brady J, Gibson G and Coluzzi M (1998) Odour-mediated host preferences of West African mosquitoes, with particular reference to malaria vectors. Am J Trop Med Hyg 58: 56-63.

Costantini C, Sagnon NF, Torre AD and Coluzzi M (1999) Mosquito behavioural aspects of vector-human interactions in the Anopheles gambiae complex. Parassitologia 41:209-217.

Cox-Singh J, Davis TME, Lee K, Shamsul SG, Matusop A, Ratnam S, Rahman HA, Conway DJ and Singh B (2007) Plasmodium knowlesi malaria in humans is widely distributed and potentially life threatening. Clinical infectious Diseases 46: 16-171.

Day JF and Sjogren RD (1994) Vector control by removal trapping. Am J Trop Med Hyg 50: 126-133.

De Jong R and Knols BGJ (1995) Olfactory responses of host-seeking Anopheles gambiae s.s. Giles (Diptera: Culicidae). Acta Tropica, 59: 333-335.

Dekker T and Takken W (1998) Differential responses of mosquito sibling species Anopheles arabiensis and An. quadriannulatus to carbon dioxide, a man or a calf. Med Vet Entomol 12: 136-140.

Dekker T, Steib B, Cardé RT and Geier M (2002) L-lactic acid: a human-signifying host cue for the anthropophilic mosquito Anopheles gambiae. Med Vet Entomol 16: 91-98.

Dia I, Diallo D, Duchemin J, Ba Y, Konate L, Costantini C and Diallo M (2005) Comparisons of human-landing catches and odour-baited entry traps for sampling malaria vectors in Senegal. J Med Entomol 42(2): 104-109.

Duchemin JB, Pocktsy JM, Rabarison P, Roux J, Coluzzi M and Costantini C (2001) Zoophily of Anopheles arabiensis and An. gambiae in Madagascar demonstrated by odour-baited entry traps. Med Vet Entomol 15: 50-57.

Elissa N, Karch S, Bureau P, Ollomo B, Lawoko M, Yangari P, Ebang B and Georges AJ (1999) Malaria transmission in a region of savanna-forest mosaic Haut-Ogooue Gabon. J Am Mosq Control Assoc 15: 15-23.

Fettene M, Hunt RH, Coetzee M and Tessema F (2004) Behaviour of Anopheles arabiensis and Anopheles quadriannulatus sp. B mosquitoes and malaria transmission in Southwestern Ethiopia. Afr Entomol 12: 83-87.

Fettene M, Koekemoer LL, Hunt RH and Coetzee M (2002) PCR assay for identification of Anopheles quadriannulatus species B from Ethiopia and other sibling species of the Anopheles gambiae complex. Med Vet Entomol 16:214-217.

Gallup JL and Sachs JD (2001) The economic burden of malaria. Am J Trop Med Hyg 64: 85-96.

Gibson G, Constantini C, Sagnon F, Torre A and Coluzzi M (1997) The responses of Anopheles gambiae and other mosquitoes in Burkina Faso to $\mathrm{CO}_{2}$-the start of a search for synthetic human odor. Annal Trop Med Parasitol 91: 123-124.

Gillies MT (1980) The role of carbon dioxide in host finding by mosquitoes (Diptera: Culicidae): a review. Bull Entomol Res 70: 525-532.

Gillies MT and Coetzee M (1987) A supplement to the Anophelinae of African South of the Sahara. South African Institute of Medical Research, Johannesburg, South Africa. 
Gillies MT and De Meillon B (1968) The Anophelinae of Africa South of the Sahara. South African Institute of Medical Research, Johannesburg, South Africa.

Gimnig JE, Ombok M, Kamau L and Hawley WA (2001) Characteristics of larval anopheline (Diptera: Culicidae) habitats in western Kenya. J Med Entomol 38: 282-288.

Govella NJ, Chaki PP, Geissbuehler Y, Kannady K, Okumu F, Charlwood JD, Anderson RA and Killeen GF (2009) A new tent trap for sampling exophagic and endophagic members of the Anopheles gambiae complex. Malar J 8: 157.

Haddow AJ (1942) The mosquito fauna and climate of native huts at Kisumu, Kenya. Bull Entomol Res 33: 91-142.

Healy TP and Copland MJW (2000) Human sweat and 2-oxopentanoic acid elicit a landing response from Anopheles gambiae. Med Vet Entomol 14: 195-200.

Healy TP, Copland MJW, Cork A, Przyborowska A and Halket JM (2002) Landing responses of Anopheles gambiae elicited by oxocarboxylic acids. Med Vet Entomol 16: 126-132.

Hunt RH, Coetzee M and Fettene M (1998) The Anopheles gambiae complex: a new species from Ethiopia. Trans R Soc Trop Med Hyg 92: 231-235.

Jawara M, Smallegange RC, Jeffries D, Nwakanma DC, Awolola TS, Knols BGJ, Takken W and Conway DJ (2009) Optimizing odor-baited trap methods for collecting mosquitoes during the malaria season in The Gambia. PLoS One 4: e8167.

Kenge P, Awono-Ambene P, Antonio-Nkondjio C, Simard F and Fontenille D (2003) Molecular identification of the Anopheles nili group of African malaria vectors. Med Vet Entomol 17: 67-74.

Kim-Sung L, Cox-Singh J, Brooke G, Matusop A and Singh B (2009) Plasmodium knowlesi from archival blood films: further evidence that human infections are widely distributed and not newly emergent in Malaysian Borneo. Int J Parasitol. 2009 August; 39(10): 1125-1128.

Kline L (2006) Traps and trapping techniques for adult mosquito control. J Am Mosq Control Assoc 22(3): 490-496.

Kline DL (2007) Semiochemicals, traps/targets and mass trapping technology for mosquito management. J Am Mosq Control Assoc 23: 241-251.

Kline DL and Lemire GF (1998) Evaluation of attractant baited traps/targets for mosquito management on Key Island, Florida, USA. J Vect Ecol 23: 171-185.

Kline DL, Takken W, Wood JR and Carlson D (1990) Field studies on the potential of butanone, carbon dioxide, honey extract, I-octen-3-ol, lactic acid, and phenols as extracts for mosquitoes. Med Vet Entomol 4: 383-391.

Knols BGJ and De Jong R (1996) Limburger cheese as an attractant for the malaria mosquito Anopheles gambiae s.s. Parasitol Today, 12: 159-160.

Knols BGJ, De Jong R, Takken W (1995) Differential attractiveness of isolated humans to mosquitoes in Tanzania. Trans Roy Soc Trop Med Hyg 89: 604-606.

Knols BGJ, Mboera LEG and Takken W (1998) Electric nets for studying odour-mediated host seeking behavior of mosquitoes. Med Vet Entomol 12: 116-120.

Kröckel U, Rose A, Eiras A and Geier M (2006) New tools for surveillance of adult Aedes aegypti: comparison of trap catches with human landing collections in an urban environment. J Am Mosq Control Assoc 22: 229-238.

Kweka EJ and Mahande AM (2009) Comparative evaluation of four mosquitoes sampling methods in rice irrigation schemes of lower Moshi, northern Tanzania. Malar J 8: 149

Kweka EJ, Mwang'onde BJ, Kimaro E, Msangi S, Massenga CP and Mahande AM (2009) A resting box for outdoor sampling of adult Anopheles arabiensis in rice irrigation schemes of lower Moshi, northern Tanzania. Malar J 8: 82

La Grange JJP (1995) Survey of anopheline mosquitoes (Diptera: Culicidae) in a malarious area of Swaziland. Afr Entomol 3:217-219.

Laganier R, Randimby FM, Rajaonarivelo V and Robert V (2003) Is the Mbita trap a reliable tool for evaluating the density of anopheline vectors in the highlands of Madagascar? Malar J 2: 42.

Lacroix R, Mukabana WR, Gouagna LC and Koella JC (2005) Malaria infection increases attractiveness of humans to mosquitoes. PLoS Biol 3: 1590-1593.

Lindsay SW, Adiamah JH, Miller JE, Pleass RJ and Armstrong JRM (1993) Variation in attractiveness of human subjects malaria mosquitoes (Diptera: Culicidae) in The Gambia. J Med Entomol 30: 368-373.

Logan JG and Birkett MA (2007) Semiochemicals for biting fly control: their identification and exploitation. Pest Manag Sci, 63: 647-657. 
Logan JG, Birkett MA, Clark SJ, Powers S, Seal NJ, Wadhams LJ, Mordue AJ and Pickett JA (2008) Identification of humanderived volatile chemicals that interfere with attraction of Aedes aegypti mosquitoes. J Chem Ecol 34: 308-322.

Logan TM, Linthicum KJ, Thande PC, Wagateh JN and Roberts CR (1991) Mosquito species collected from a marsh in western Kenya during the long rains. J Am Mosq Control Assoc 7: 395-399

Mahande A, Mosha F, Mahande J and Kweka E (2007) Feeding and resting behaviour of malaria vector, Anopheles arabiensis with reference to zooprophylaxis. Malaria J 6: 100.

Mathenge EM, Killeen GF, Oulo DO, Irungu LW, Ndegwa PN and Knols BGJ (2002) Development of an exposure-free bednet trap for sampling Afrotropical malaria vectors. Med Vet Entomol 16: 67-74.

Mathenge EM, Misiani GO, Oulo DO, Irungu LW, Ndegwa PN, Smith TA, Killeen GF and Knols BG (2005) Comparative performance of the Mbita trap, CDC light trap and the human landing catch in the sampling of Anopheles arabiensis, An. funestus and culicine species in a rice irrigation in western Kenya. Malar J 4: 7.

Mathenge EM, Omweri GO, Irungu LW, Ndegwa PN, Walczak E, Smith TA, Killeen GF and Knols BG (2004) Comparative field evaluation of the Mbita trap, the Centers for Disease Control light trap, and the human landing catch for sampling of malaria vectors in western Kenya. Am J Trop Med Hyg 70: 33-37.

Mboera LEG and Takken W (1997) Carbon dioxide chemotropism in mosquitoes (Diptera: Culicidae) and its potential in vector surveillance and management programmes. Annu Rev Entomol 85: 355-368.

Mboera LEG, Knols BGJ, Braks MAH and Takken W (2000) Comparison of carbon dioxide-baited trapping systems for sampling outdoor mosquito populations in Tanzania. Med Vet Entomol 14:257-263.

Mboera LEG, Knols BGJ, Takken W and Della Torre A (1997) The response of Anopheles gambiae s.l. and An. funestus (Diptera: Culicidae) to tents baited with human odour or carbon dioxide in Tanzania. Bull Entomol Res 87: 173-178.

Mnzava AE and Kilama WL (1986) Observations on the distribution of the Anopheles gambiae complex in Tanzania. Acta Trop 43: 277-282.

Moffett A, Shackelford N and Sarkar S (2007) Malaria in Africa: vector species' niche models and relative risk maps. PLoS One 2: e824.

Mosha FW and Petrarca V (1983) Ecological studies on Anopheles gambiae complex sibling species on the Kenya coast. Trans R Soc Trop Med Hyg 77: 344-345.

Mosha FW and Subra R (1982) Ecological studies on Anopheles gambiae complex sibling species in Kenya 1 preliminary observations on their geographical distribution and chromosomal polymorphic inversions. Document WHO/ VBC/82867. World Health Organization, Geneva, Switserland.

Mpofu SM (1985) Seasonal vector density and disease incidence patterns of malaria in an area of Zimbabwe. Trans $R$ Soc Trop Med Hyg 79: 169-175.

Mukabana WR, Takken W, Coe R and Knols BGJ (2002) Host-specific cues cause differential attractiveness of Kenyan men to the malaria mosquito Anopheles gambiae. Malar J 1: 17.

Mukabana WR, Takken W, Killeen GF and Knols BGJ (2004) Allomonal effect of breath contributes to differential attractiveness of humans to the African malaria vector Anopheles gambiae. Malar J 3: 1.

Murphy MW, Dunton RF, Perich MJ and Rowley WA (2001) Attraction of Anopheles (Diptera: Culicidae) to volatile chemicals in Western Kenya. J Med Entomol 38: 242-244.

Muturi E, Shililu J, Jacob B, Gu W, Githure J and Novak R (2006) Mosquito species diversity and abundance in relation to land use in a riceland agroecosystem in Mwea, Kenya. J Vector Ecol 31: 129-137.

Mzilahowa T, Ball AJ, Bass C, Morgan JC, Nyoni B, Steen K, Donnelly MJ and Wilding CS (2008) Reduced susceptibility to DDT in field populations of Anopheles quadriannulatus and Anopheles arabiensis in Malawi: evidence for larval selection. Med Vet Entomol 22: 258-263

Njiru BN, Mukabana WR, Takken W and Knols BGJ (2006) Trapping of the malaria vector Anopheles gambiae with odourbaited MM-X traps in semi-field conditions in western Kenya. Malaria J 5: 39.

Okumu FO, Killeen GF, Ogoma S, Biswaro L, Smallegange RC, Mbeyela E, Titus E, Munk C, Ngonyani H, Takken W, Mshinda H, Mukabana WR and Moore SJ (2010a) Development and field evaluation of a synthetic mosquito lure that is more attractive than humans. PLoS One 5: e8951.

Okumu FO, Biswaro L, Mbeyela E, Killeen GF, Mukabana WR and Moore SJ (2010b) Using nylon strips to dispense mosquito attractants for sampling the malaria vector Anopheles gambiae s.s. J Med Entomol (in press). 
Olanga EA, Okal M, Mbadi PA, Kokwaro E and Mukabana WR (2010) Attraction of An. gambiae to odour baits augmented with heat and moisture. Malar J 9: 6.

Owino EA (2006) Field evaluation of Limburger cheese as an odour bait source for sampling afrotropical malaria vectors. MSc Thesis, University of Nairobi, Nairobi, Kenya.

Paskewitz SM, Ng K, Coetzee M and Hunt RH (1993) Evaluation of the polymerase chain reaction method for identifying members of the Anopheles gambiae (Diptera: Culicidae) complex in southern Africa. J Med Entomol 30: 953-957.

Pates HV, Takken W, Curtis CF and Jamet H (2006) Zoophilic Anopheles quadriannulatus species B found in a human habitation in Ethiopia. Ann Trop Med Parasitol 100(2): 177-179.

Phillips RS (2001) Current status of malaria and potential for control. Clin Microbiol Rev 14: 208-226.

Pock Tsy JM, Duchemin JB, Marrama L, Rabarison P, Le Goff G, Rajaonarivelo V and Robert V (2003) Distribution of the species of the Anopheles gambiae complex and first evidence of Anopheles merus as a malaria vector in Madagascar. Malar J 8: 33.

Qiu YT, Smallegange RC, Ter BC, Spitzen J, Van Loon JJ, Jawara M, Milligan P, Galimard AM, Van Beek TA, Knols BG and Takken W (2007) Attractiveness of MM-X traps baited with human or synthetic odor to mosquitoes (Diptera: Culicidae) in The Gambia. J Med Entomol 44: 970-983.

Sachs J and Malaney P (2002) The economic and social burden of malaria. Nature 11:681-685.

Schmied WH, Takken W, Killeen GF, Knols BG and Smallegange RC (2008) Evaluation of two counterflow traps for testing behaviour-mediating compounds for the malaria vector Anopheles gambiae s.s. under semi-field conditions in Tanzania. Malar J 7: 230.

Service MW (1993). Mosquito ecology, field sampling methods, 2nd ed. Elsevier Applied Science, London, UK.

Sharp BL, Kleinschmidt I, Streat E, Maharaj R, Barnes KI, Durrheim DN, Ridl FC, Morris N, Seocharan I, Kunene S, La Grange JJP, Mthembu JD, Maartens F, Martin CL and Barreto A (2007) Seven years of regional malaria control collaboration - Mozambique, South Africa, and Swaziland. Am. J. Trop. Med. Hyg. 76: 42-47.

Smallegange RC, Qiu YT, van Loon JJ and Takken W (2005) Synergism between ammonia, lactic acid and carboxylic acids as kairomones in the host-seeking behaviour of the malaria mosquito Anopheles gambiae sensu stricto (Diptera: Culicidae). Chem Senses 30: 145-152.

Sylla EHK, Kun JFJ and Kremsner PG (2000) Mosquito distribution and entomological inoculation rates in three malariaendemic areas in Gabon. Trans R Soc Trop Med Hyg 94: 652-656.

Takken W (1991) The role of olfaction in host-seeking of mosquitoes: a review. Insect Sci Appl 12: 287-295.

Takken W and Kline DL (1989) Carbon dioxide and I-octen-3-ol as mosquito attractants. J Am Mosq Control Assoc 5: 311-316.

Takken W and Knols BGJ (1999) Odor-mediated behaviour of Afrotropical malaria mosquitoes. Annu Rev Entomol 44: 131-157.

Takken W and Knols BGJ (2009) Malaria vector control: current and future strategies. Trends Parasitol 25: 101-104.

Takken W, Knols BGJ and Otten H (1997) Interactions between physical and olfactory cues in the host-seeking behaviour of mosquitoes: the role of relative humidity. Ann Trop Med Parasitol 91: 119-120.

Tirados I, Costantini C, Gibson G and Torr SJ (2006) Blood-feeding behaviour of the malarial mosquito Anopheles arabiensis: implications for vector control. Med Vet Entomol 20: 425-437.

Torr S, Della Torre A, Calzetta M, Costantini C and Vale GA (2008) Towards a fuller understanding of mosquito behaviour: use of electrocuting grids to compare the odour-orientated responses of Anopheles arabiensis and An. quadriannulatus in the field. Med Vet Entomol 22: 93-108.

Van den Broek IVF and Den Otter CJ (1999) Olfactory sensitivities of mosquitoes with different host preferences (Anopheles gambiae s.S., An. arabiensis, An. quadriannulatus, An. m. atroparvus) to synthetic host odours. J Insect Physiol 45: 1001-1010.

Van Rensburg AJ, Hunt RH, Koekemoer LL, Coetzee M, Shiff CJ and Minjas J (1996) The polymerase chain reaction method as a tool for identifying members of the Anopheles gambiae complex (Diptera: Culicidae) in northeastern Tanzania. J Am Mosq Control Assoc 12: 271-274.

Watson M (1953) African highway. John Murray, London, UK. 
Wernsdorfer $\mathrm{G}$ and Wernsdorfer WH (2003) Malaria at the turn from the 2 nd to the 3rd millenium. Wien klin Wochenschr 115 Suppl 3: 2-9.

Wilkes TJ, Matola YG and Charlwood JD (1996) Anopheles rivulorum, a vector of human malaria in Africa. Med Vet Entomol 10: 108-110.

White GB (1974) Anopheles gambiae complex and disease transmission in Africa. Trans R Soc Trop Med Hyg 68: 278-301. World Health Organization (1993) World malaria situation in 1991. Part I. Weekly Epidemiol Rec 34: 246. 


\title{
9. The chemical ecology of sandflies
}

\author{
Daniel P. Bray, Richard D. Ward and James G.C. Hamilton
}

\begin{abstract}
In the 25 years in which chemical ecology of sandflies (Diptera: Psychodidae) has been investigated, most work has focussed on Lutzomyia longipalpis, the primary vector of Leishmania chagasi in South and Central America. Studies of sex pheromones produced by male L. longipalpis have aided in understanding the population structure of this species complex, found to consist of a number of reproductively isolated sibling members, which may differ in their vectorial capacity. Furthermore, identification and synthesis of these pheromones is leading to the development of new monitoring and control devices, which aim to supplement and improve existing vector control strategies. Complementary behavioural studies have also explored the role of sex pheromones in mate choice, and the complex interactions that occur between males and females during courtship. Studies of oviposition pheromones, kairomones and the effects of Leishmania infection on host attractiveness have also contributed to our understanding of the intricacy of chemical ecology in L. longipalpis, with broad implications relevant to the development of control strategies in this and other insect vectors. In comparison, the chemical ecology of other sandfly species, many of which are also important disease vectors, has largely been ignored, in part because of the cost and difficulty of maintaining colonies for laboratory studies. Although much work has been done, many challenges remain, and considerable research effort will be required to understand the chemical ecology of sandflies in general, and the molecular basis of their intricate communication systems.
\end{abstract}

Keywords: 3-methyl-a-himachalene, 9-methylgermacrene, 9-methylgermacrene-B, attractant, cembrene, Lutzomyia longipalpis, oviposition pheromone, sex pheromone

\section{Introduction}

The economic and public health significance of the Diptera to man and his domestic animals cannot be overstated. Their ability to transmit viral and parasitic diseases results in a very significant human and animal disease burden and consequent economic cost. The phlebotomine sandflies are important vectors of single cell protozoan parasites of the genus Leishmania and the World Health Organization estimates that the disease burden in disability-adjusted life years (DALYs) is second only to that caused by malaria and that burden is carried disproportionately by the World's poor and disadvantaged peoples.

Chemical ecology in the Diptera involves a wide range of inter and intraspecies interactions and is employed in conjunction with tactile, visual and auditory signals. In particular, chemical signals have a key role in both courtship and sexual behaviour in general. Indications of pheromonal activity in the family Psychodidae was initially reported by Fuerborn (1922) who first described an eversible mesothoracic 'scent gland' in the moth fly Ulomyia fuliginosa (Meigen). In the haematophagous sub-family Phlebotominae, Lane and Ward (1984) published the first description of a pheromone releasing papule in Lutzomyia longipalpis s.l. (Lutz and Neiva), although the underlying secretory cell was drawn by Barth (1961) nearly 30 years earlier and described as an 'odiferous gland'. 
It is eight years since we last reviewed the progress that had been made in our understanding of the chemical ecology of sandflies (Ward and Hamilton 2002). Since then, significant advances have been made in our appreciation of their chemical communication systems, but much further research is still needed to understand basic biological aspects of chemical signalling, and to exploit our developing knowledge in creating new approaches to vector control. For example, having established the structures of the male sex pheromone in two populations of Lu. longipalpis s.l. (Hamilton et al. 1996a,b, 1999a,b), we have developed a cheap and efficient synthesis of the sex pheromone S-9-methylgermacrene-B and its analogue, 9-methylgermacrene (Hamilton and Krishnakumari 2004, Krishnakumari et al. 2004) to enable the initiation of field feasibility trials in Brazil.

Apart from the genus Lutzomyia there has recently been some progress in our investigations into the chemical ecology of two other phlebotomine genera; Sergentomyia and Phlebotomus. Results from laboratory-bioassays indicate that the members of the genus Phlebotomus use chemical communication between the sexes, the precise nature of which is under investigation (I Chelbi and JGC Hamilton, unpublished observations). Some Sergentomyia species have been found to produce compounds closely related in structure to the sex pheromones of the Lu. longipalpis species complex, but their role has not been confirmed (JGC Hamilton unpublished data).

Progress in other areas of sandfly chemical ecology has included studies of attractants and pheromones relating to oviposition behaviour, and limited investigations into defensive responses of immatures. Host odour work has continued and some new insights gained into the role of carbon dioxide obtained and other attractants in host-seeking behaviour. We also have evidence that indicates that animals infected with Leishmania infantum Nicolle, the causative agent of visceral leishmaniasis in the New World and southern Europe are more attractive to blood seeking female sandflies than uninfected animals (Nevatte 2006, O'Shea et al. 2002).

There have also been some significant advances in our understanding of the Lu. longipalpis s.l. species complex and we are now in a position to attempt to determine its significance in relation to disease transmission (Bauzer et al. 2007, Maingon et al. 2008). Finally, new insights have been gained through detailed analysis of courtship interactions, the results of which indicate that contact pheromones, along with audio and long range chemical signals, may also have a role to play in sexual behaviour (Bray and Hamilton 2007a).

\section{Oviposition in response to chemical cues}

Our laboratory has been active in examining the factors driving oviposition, concentrating on the New World vector of visceral leishmaniasis Lu. longipalpis s.l. We showed that eggs were attractive to conspecific ovipositing females (Dougherty et al. 1992, El Naiem and Ward 1991, 1992). Although the age of the eggs appeared not to be an important factor, the numbers of eggs involved appeared to be critical to produce significant attraction. The origin of the pheromone was traced to the accessory glands of the post bloodfed female and the substance was eventually identified as n-dodecanoic acid, a C12 fatty acid (Dougherty and Hamilton 1997). Other work showed that the green leaf volatiles hexanal and 2-methyl-2-butanol synergise the attraction of the oviposition pheromone (Dougherty et al. 1995) and a combination of synthetic green leaf volatiles and the oviposition pheromone mimicked the natural attraction of sandfly eggs (Dougherty and Hamilton 1997). 
Recently we have returned to this topic and in preliminary work have shown that the Old World vector of Leishmania donovani Laveran and Mesnil in the Indian sub-continent Phlebotomus argentipes Annandale and Brunetti also lays eggs that are attractive to conspecific gravid females and stimulate oviposition (SF Kinsmore, RD Ward, PJ Taylor, JGC Hamilton 2008, unpublished observations). Work to precisely describe the oviposition behaviour and determine the chemical nature of the attraction is underway.

\section{Sandfly responses to host odour}

Host odours act as important signals to many species of blood-feeding Diptera, facilitating both host location and discrimination (Colvin and Gibson 1992, Takken 1991). Understanding how sandflies choose and locate their hosts could aid in developing odour-baited traps for monitoring and control (Kelly and Dye 1997, Pinto et al. 2001) and help explain variation between individuals in attractiveness and subsequent biting risk (Hamilton and Ramsoondar 1994, Rebollar-Tellez et al. 1999). Despite considerable progress in recent years, there is still less known about sandfly responses to host odours than those of many other biting insects, including mosquitoes. Nevertheless, a number of studies have highlighted the importance of kairomones in sandfly host-seeking, both by demonstrated attraction to odours produced by animal baits in the field (Campbell-Lendrum et al. 1999, Christensen and Herrer 1973, Montoya-Lerma and Lane 1996, Pinto et al. 2001, Quinnell et al. 1992), and to whole host odour and its constituents in the laboratory (Bray and Hamilton 2007b, Morton and Ward 1989, Oshaghi et al. 1994, Quinnell et al. 1992).

Many sandflies (including the New World vectors of zoonotic leishmaniasises) are opportunistic feeders, and may be attracted by ubiquitous cues that indicate the presence of a living vertebrate, but provide little information as to the species of a potential host (Pinto et al. 2001, Quinnell et al. 1992). Carbon dioxide is a major constituent of host breath, and is a near universal activator and attractant of mosquitoes (Gillies 1980, Takken 1991). In the field, attraction of sandflies to $\mathrm{CO}_{2}$ released from traps has been recorded in Malaysia (Knudson et al. 1979) and more rigorously demonstrated in both Panama (Chaniotis 1983) and Brazil (Pinto et al. 2001). In the latter study, an approximate linear relationship was found between concentration of carbon dioxide released and numbers of sandflies (Lu. intermedia (Lutz and Neiva) and Lu. whitmani (Antunes and Coutinho)) captured. Increased attraction towards sources of greater $\mathrm{CO}_{2}$ release has been proposed as an explanation for the observed preference of several sandfly species for hosts of a larger size, or greater host density (Lu. longipalpis, Quinnell et al. 1992; Lu. evansi (Nuñez-Tovar) Montoya-Lerma and Lane 1996; Lu. whitmani Campbell-Lendrum et al. 1999). However, although many species of sandfly will respond to carbon dioxide to some extent, it is unlikely to be the sole mediator of attraction. In field experiments in Brazil, carbon dioxide attracted less than half the number of sandflies caught using whole human odour (Pinto et al. 2001). In addition, results of laboratory experiments have been inconclusive regarding the response of $\mathrm{Lu}$. longipalpis to $\mathrm{CO}_{2}$ : while host breath can activate flies to begin probing (Ready 1978) removal of carbon dioxide from preparations of host odour and pheromone did not decrease their attractiveness in bioassays (Nigam and Ward 1991).

Volatile molecules, emanating from breath and skin, may provide more specific information regarding a potential host than carbon dioxide alone. In particular, chemicals produced as a consequence of bacterial metabolism on the surface of the skin are likely to vary both between and within host species (Takken 1991). As a consequence, theses chemicals may be important both in attracting sandflies, and facilitating discrimination between potential sources of a blood meal. That sandflies respond to host volatiles was first demonstrated with laboratory hamsters, 
with males and female Lu. longipalpis attracted to volatile extracts presented in the absence of any other host cue, including $\mathrm{CO}_{2}$ (Oshaghi et al. 1994).

Similarly, both sexes of Lu. longipalpis are attracted to Petri dishes recently held in human hands (Hamilton and Ramsoondar 1994). That chemicals emanating from skin cause the observed landing response was confirmed by transferring the attractive odours from handled to un-handled dishes in non-polar solvents (Rebollar-Tellez et al. 1999). In both studies using humans, emanations taken from different test subjects differed significantly in their attractiveness, suggesting variation in the extracted odours does contribute to differences in individual attractiveness. In addition, flies from different Lu. longipalpis sibling-species (colonies originating from Jacobina and Lapinha in Brazil) were found to respond differently in trials, with flies from Jacobina more strongly attracted to human odour (Hamilton and Ramsoondar 1994). Differences between sibling species in their attraction to animals and human hosts may contribute to the uneven distribution of leishmaniasis in Brazil (Ward et al. 1983). Determining which chemicals are responsible for this attraction, and how sandflies of different species (or sibling-species) respond to them, would be a major step forward in understanding the ecology of the disease.

While relatively simple bioassays can aid in measuring attraction of sandflies to host-produced odours, more sophisticated techniques may be required to identify the individual components responsible. Host odour is comprised of a large number of different chemicals, only a few of which may elicit any biological response in host-seeking insects. Dougherty et al. (1999) used gas-chromatography-linked single sensillum recording (GC-SSR) to identify some of the components of fox odour (Vulpes vulpes Linnaeus) that can be perceived by Lu. longipalpis. Using electrophysiological readings taken from the ascoid sensillum on the flies' antennae, females were found to possess specific neurones perceptive to 16 chemicals in fox gland extract, which were identified by mass spectrometry. While none of the individual compounds were found to possess the same attractive qualities as whole fox odour, a synthetic blend of four or more of these chemicals did attract flies upwind. It appears female Lu. longipalpis may therefore use a number of volatile kairomones to locate a source of a blood meal, the ability to perceive a range of chemicals perhaps facilitating their wide host range (Dougherty et al. 1999). Variation in response to kairomones produced by different animals may be one of the mechanisms through which Lu. longipalpis sibling-species develop preferences for particular hosts. Electrophysiology may therefore be one method by which anthropophagic populations, and those likely to be important leishmaniasis vectors, can be identified.

Although only female sandflies bloodfeed, males of some species are also attracted to hosts as sites of mating aggregations (Lane et al. 1990, Miles et al. 1976, Quinnell and Dye 1994). Both field and laboratory studies indicate there are differences in how the sexes respond to components of host odour (Lutzomyia spp. (Chaniotis 1983); Lu. whitmani (Campbell-Lendrum et al. 1999, Pinto et al. 2001); Lu. intermedia (Pinto et al. 2001), Lu. longipalpis, (Bray and Hamilton 2007b, Hamilton and Ramsoondar 1994, Oshaghi et al. 1994), which presumably originate from differences in both the reason for seeking a host and reproductive strategy (Kelly and Dye 1997, Pinto et al. 2001). In the case of Lu. longipalpis, males arrive earlier and stay longer at the host, presumably to maximise reproductive success through number of matings, while females are only present for the time required to mate once and blood feed (Kelly and Dye 1997). In laboratory experiments, however, males have been found to be less attracted to both human volatiles (Hamilton and Ramsoondar 1994) and whole hamster odour (Bray and Hamilton 2007b), and more likely to land on control traps in trials with hamster volatile lures (Oshaghi et al. 1994). Although pheromones play an important part in the mating system of Lu. longipalpis, initial attraction to lekking sites is 
presumably mediated by host odour. It is possible therefore that males are attracted to cues not present in laboratory experiments, or that male behaviour is not adequately described by simple measures of attraction, such as number of flies captured, or number of landings made. Although males do not transmit leishmaniasis, understanding their responses to host odour in more detail could aid in both predicting where mating aggregations are likely to occur, and controlling populations through disruption of normal reproductive behaviour (Bray and Hamilton 2007b).

Identifying components of host odour which are attractive in the laboratory is only the first step in developing an effective lure for use in the field. To date, the only kairomone found to catch sandflies in the wild is carbon dioxide. Traps which release $\mathrm{CO}_{2}$ in combination with 1-octen-3-ol have been produced commercially for controlling other biting insects (Mands et al. 2004), but the cost of buying and maintaining traps that require gas and power supplies may render this option uneconomical for use against sandflies, particularly in poor areas where leishmaniasis is prevalent. Use of volatile blends, which could be manufactured artificially and more easily transported and sold as formulated lures, may have more potential. However, to date Lu. longipalpis is the only species for which attraction to volatile chemicals alone has been demonstrated: 1-octen-3-ol (Hall et al. 1984), one of the components of cattle odour known to attract tsetse flies, failed to increase attraction of $P$. argentipes to traps in Sri Lanka (Cameron et al. 1991). More work is needed to identify those chemicals present in host odour that can attract other important vector species, and to test these potential lures under field conditions for their efficacy in capturing both males and females. For those insects known to produce pheromones, combinations of the mating attractant and kairomones may be the only effective solution.

\section{Sandfly sex pheromones}

The sex pheromones of male Lu. longipalpis s.l. are produced by glandular tissue underlying cuticular papules on tergite 4 or tergites 3 and 4 . The underlying glandular tissue and papules give the appearance of spots of lighter coloured cuticle, these were first observed by Mangabeira (1969). Because of these males have been classified as one spot (1S) or two spot (2S). Live males, extracts of males and extracts made in organic solvent from the dissected tergites were found to be attractive on their own and in combination with host odour to unfed virgin females (Lane and Ward 1984, Lane et al. 1985, Morton and Ward 1989). Chemical fractionation and behavioural analysis subsequently showed that the most abundant chemical within the extracts was responsible for the observed attraction (Hamilton et al. 1994).

Coupled gas chromatography mass spectrometry has shown that the active compounds are terpenes, a class of natural products constructed from three or four five-carbon (C5) isoprene units. These are a common class of compounds in nature where they are used mainly as defensive chemicals in plants and termites. However, the Lu. longipalpis compounds are unusual in that the compounds found in two of the members of the species complex have an additional methyl group probably added in the early biosynthesis of the molecule (Hamilton et al. 1999c) i.e. they have 16 carbons $(\mathrm{C} 16, \mathrm{mw} 218)$. Thus the sex pheromone of one member of the complex has been shown to be the novel $\mathrm{C} 16\left(3 \times \mathrm{C}_{5}+\mathrm{CH}_{3}\right)$ monocyclic terpene (S)-9-methylgermacrene-B (Hamilton et al. 1996a, 1999a) and another, the novel C16 bicyclic terpene, 3-methyl-a-himachalene (Hamilton et al. 1996b, 1999b). The sex pheromones of another two members of the species complex have been shown to be different cembrene isomers i.e. they are C20 (mw 272) monocyclic diterpenes $(4 \times C 5)$. Work to fully characterise these compounds is continuing. 
Studies to determine if terpenes are present in other New World sandfly species have been undertaken and show that although present in other Lutzomyia species they are not ubiquitous. It appears that Lutzomyia spp. can be subdivided into three groups; those whose males produce terpenes and have cuticular papules e.g. Lu. longipalpis, Lu. lenti (Mangabeira), and Lu. carmelinoi Ryan; those that do not produce terpenes but still have the associated papules e.g. Lu. evandroi Costa Lima and Antunes, Lu. tejadai Galati and Caceres and Lu. migonei (França) and those that have neither terpenes nor papules e.g. Lu. whitmani and Lu. neivai (Pinto) (Hamilton et al. 2002). In some cases the compounds are oxygenated methylsesquiterpenes but more commonly they are diterpenes (Hamilton and Ward 1994, Hamilton et al. 1999c).

An investigation revealed that two Old World species Sergentomyia minuta Rondani and S. fallax Parrot also produce a range of terpenes. Both species have cuticular papules similar to those found on Lu. longipalpis. Interestingly both species produced a much greater range of sesquiterpenes and methyl sesquiterpenes than Lutzomyia spp. Sergentomyia fallax was found to produce eight known sesquiterpenes (C15, mw 204) the most abundant being allo-aroma-dendr9 -ene $(53 \%)$ and $\beta$-barbatene (23\%). Sergentomyia minuta produces a novel unsaturated tricyclic homosesquiterpene $(\mathrm{C} 16, \mathrm{mw} 216)$ as the major terpene $(68 \%)$ with $(\mathrm{E})$ - $\beta$-caryophyllene $(\mathrm{C} 15, \mathrm{mw}$ 204 ) as the next most abundant terpene (15\%). The remaining five compounds that were present in amounts greater than $1 \%$ of the total terpene content are novel predominantly unsaturated homosesquiterpene hydrocarbons. Although the biological function of terpenes in species other than Lu. longipalpis has not been confirmed it is likely that they are sex pheromones. It may also be possible to infer that the evolution of terpenes in the Phlebotominae is from highly complex tricyclic C16 compounds to the monocyclic C20 compounds found in Lutzomyia spp. (J.G.C.H. 2008, unpublished observations).

The presence of sex pheromones in Old World Phlebotomus spp. is an open question. Terpenes such as we have found in some Lutzomyia and Sergentomyia spp. appear to be absent e.g. $P$. argentipes, P. papatasi Scopoli, P. orientalis Parrot and P. duboscqi Leveu-Lemaire. However we have recently carried out extensive behavioural studies with $P$. papatasi from Tunisia and the results indicate that females are attracted in an olfactometer to live male headspace volatiles (I Chelbi and JGC Hamilton 2008 unpublished observations). This would suggest that sex pheromones may be more widely distributed in sandflies than previously recognised.

\section{Exploitation of sandfly pheromones}

Oviposition pheromones and stimulants may offer potential for exploitation through monitoring or control traps. However, to date little interest has been shown in their development, with most effort focussing on the potential application of male pheromones in vector management. Interestingly, because male pheromones attract females a significantly greater impact could be made on the fecundity of the population than if a female pheromone, attractive to males, was used. We have synthesised the sex pheromone 9-methylgermacrene-B, and an analogue 9-methylgermacrene (Krishnakumari et al. 2004). These compounds can be synthesised from a readily available plant intermediate, the analogue is more stable than the natural sex pheromone and has similar activity in laboratory bioassays (JGC Hamilton and K Krishnakumari, unpublished results). We are currently formulating these chemicals and carrying out laboratory and field experiments to determine their attractiveness. Our preliminary results indicate that the synthetic compounds are attractive to both male and female Lu. longipalpis (DP Bray, RP Brazil, K Krishnakumari and JGC Hamilton, unpublished results). 
We have also found in laboratory studies that although sex pheromone is attractive to female sandflies, sex pheromone presented in combination with host odour is significantly more attractive. This strong synergistic interaction between host odour and male pheromone suggests that synthetic pheromone lures should be positioned near host animals until the attractive elements of host odour have been identified and incorporated into the sex pheromone lure.

\section{Chemical communication and mating behaviour in sandflies}

Identifying semiochemicals that are attractive to sandflies, and which could therefore function as lures, represents an important stage in developing novel methods of control effective against vectors of leishmaniasis. In agriculture, integrated pest management strategies, which use insecticides in conjunction with pheromone lures and mating disruption techniques, have proven effective against a number of agricultural pests (Shani 2000). A similar approach applied to sandflies could mean cheaper, more efficient control, less reliant on spraying insecticides (Alexander et al. 2003, Kelly and Dye 1997) However, such strategies have at their foundation a thorough knowledge of the mating system of the target insect (Girling and Cardé 2006). For many species of sandfly, our current data may be insufficient: unless we understand how different chemical signals are used, and their function in mating and courtship behaviour, developing effective methods of pheromone-based control is likely to be difficult.

Few studies have explored the mating system of sandflies in any detail, with Lu. longipalpis the most well studied. Most attention in this species had focussed on the ability of male-produced pheromones to attract female flies from a distance. As these chemical signals only attract potential mates from the same pheromone-producing sibling species, they are likely to be important in maintaining reproductive isolation between different members of the Lu. longipalpis complex (Ward and Morton 1991). While these chemicals may function over considerable distances, their role in the small-scale interactions which occur between courting pairs remains unclear.

Laboratory studies of mating behaviour in Lu. longipalpis have shown that males establish territories on a host animal, which they defend through wing-flapping displays that escalate into occasional fights (Jarvis and Rutledge 1992). When a visiting female is detected, males approach them while wing-flapping, and continue to flap during copulation (Jarvis and Rutledge 1992, Jones and Hamilton 1998, Ward et al. 1988). The importance of male wing-flapping in courtship has been demonstrated through mating trials: the occurrence of male wing-flapping directed at females is a predictor of eventual copulation (Bray and Hamilton 2007a) and males which display for longer are more likely to be chosen by females (Jones and Hamilton 1998). However, the methods through which these behaviours signal to females are unknown. These displays may have a chemical component, and it has been suggested that the movement of the wings could help to disperse sex pheromones towards both males and females (Jones and Hamilton 1998, Lane et al. 1985, Ward et al. 1989), though this remains to be confirmed experimentally. In mate choice experiments, females were found to be more likely to choose males that had more pheromone remaining in their glands after the experiment, but there was no correlation between time spent wing flapping and amount of pheromone stored (Jones and Hamilton 1998).

Young male Lu. longipalpis can achieve mating prior to onset of pheromone production, and are occasionally chosen by females over older, pheromone producing males (Boufana et al. 1986, Jones 1997, Jones and Hamilton 1998). This suggests that the currently identified male pheromone is not the only signal involved in Lu. longipalpis mating behaviour. Sound recordings of courtship in this species have demonstrated that wing-flapping also produces audio signals that resemble 
the love songs integral to mating behaviour in Drosophila (Souza et al. 2002, 2004, Talyn and Dowse 2004, Ward et al. 1988). Furthermore, the audio patterns produced during copulation differ between sibling species of $L$. longipalpis, suggesting they may play a role in reproductive isolation (Ward et al. 1988). Females also perform short wing-flapping displays, which appears to signal receptivity to a potential mate (Jones and Hamilton 1998), and the occurrence of this behaviour during courtship is a strong predictor of eventual copulation (Bray and Hamilton 2007a). As no female pheromone has been identified, it is assumed that these displays produce audio signals only. Playback experiments, similar to those conducted with Drosophila, are needed to isolate the audio and chemical components of wing-flapping displays, in order to determine their relative importance in reproductive isolation and female mate choice Bray and Hamilton 2007a, Souza et al. 2004).

Recently, Bray and Hamilton (2007a) conducted more detailed studies of the small-scale interactions that occur between male and female Lu. longipalpis prior to copulation. They found that touching was a common occurrence during courtship, initiated by both males and females, with physical contact most often taking place between the tips of the legs and the antennae. This behaviour may represent the transfer and detection of cuticular hydrocarbons, important signals in courtship of many insect species (Howard and Blomquist 2005), but previously overlooked both in Lu. longipalpis and sandflies in general. In Drosophila, these signals can provide a range of information to a female about a potential mate prior to copulation, including age and sexual experience (Ferveur 2005). Chemical analyses are required to determine whether similar compounds are present in both Lu. longipalpis and other species of sandfly. If so, behavioural studies will be needed to determine their role in courtship, and their potential for exploitation in control programmes.

Interest in sandfly chemical ecology extends beyond developing novel methods of vector management, to the study of broader questions regarding the role of chemical communication in mate choice and reproductive isolation. Within a Lu. longipalpis lek, a small number of males will receive the majority of matings, a product more of female choice than male competition (Jones et al. 1998). How females discriminate between males, and the advantage of doing so are unclear, though there may be benefits in terms of the viability and attractiveness of offspring produced. In the laboratory, females prefer middle-aged males ( 4 to $6 \mathrm{~d}$ old), producing a greater proportion of hatching eggs when mated with males less than $8 \mathrm{~d}$ old, and attractive males tend to have attractive sons, demonstrating that there is a heritable component to male reproductive fitness (Jones et al. 1998). There is an intriguing possibility that differences in female preferences and associated male traits may have led to the reproductive isolation now observed between sibling species. Clearly, this proposal is worthy of attention for those interested in speciation, and the current population structure of Lu. longipalpis.

Descriptions of sandfly courtship behaviour in species other than Lu. longipalpis have been limited to qualitative descriptions of behaviour carried out in the laboratory, or notes based on field observations. However, the information that has been collected suggests that there are aspects of courtship that are common across species. Wing-flapping, similar to that described in $L u$. longipalpis, has been observed to occur in Lu. lichyi Floch et Abonnenc (Hamilton et al. 1999c), Lu. vexator Shannon (Chaniotis 1967), P. argentipes (Lane et al. 1990), P. orientalis (Ashford 1974), P. duboscai (Valenta et al. 2000), P. longipes Parrot and Martin (Gemetchu 1976) and P. martini Parrot (Beach et al. 1983). Males of several species also sway their abdomen from side to side when in proximity with a female (Lu. vexator (Chaniotis 1967), P. longipes (Gemetchu 1976), P. martini (Beach et al. 1983)). Both wing-flapping and abdomen swaying could act to disperse hitherto unidentified 
pheromones. If so, there may be considerable potential in developing semiochemical-based control measures effective against a range of sandfly species. It is hoped the progress being made with Lu. longipalpis will encourage further research into the mating systems of other important leishmaniasis vectors, in both the New and Old World.

\section{Future directions}

An enormous amount of basic investigation into the chemical ecology of sandflies remains to be carried out. Although the majority of our knowledge so far is based upon the 'Drosophila' of the sandfly world, Lu. longipalpis, there are at least 1000 species of sandfly where we know virtually nothing of their chemically moderated inter and intraspecific interactions. From a phylogenetic basis alone there is fundamental and useful information to be gained from identifying pheromones, to say nothing of the potential for their application in vector control.

Furthermore, studies of basic mating behaviour are required to determine how sandflies employ chemical signals (critical to developing semiochemicals as tools for vector management), and to understand the ecological forces driving evolution of pheromones in the Phlebotominae.

In the longer term, there is also the potential to develop new, innovative molecular approaches to controlling haematophagous insects through identifying those genes responsible for pheromone synthesis. For example, Chertemps et al. (2006) recently demonstrated that RNAi-knock-down of the desaturase gene desatF in Drosophila melanogaster Meigen led females to produce more male contact pheromone and less female pheromone than normal, resulting in reducing courtship and mating behaviour. Using newly available gene sequence data in conjunction with modern molecular techniques, it may similarly be possible to examine the relative importance of different chemical signals in the mating behaviour of the Psychodidae, through targeting of homologous genes with a role in pheromone production and to manipulate them for vector control.

While research continues into identifying semiochemicals attractive to sandflies, there remains little information on the receptors responsible for their detection. So far studies have concentrated only on the ascoid sensillae, which contain several sex pheromone receptors (Dougherty et al. 1999). However, at least eight other sensillae types are present on the antennal segments of blood feeding sandflies (Boufana 1990) whose function is unknown. Similarly, we need to understand how haematophagous insects perceive semiochemicals at the molecular level, presumably through use of odour-binding proteins (see Chapter 2, this volume). The genes for such proteins and the cDNAs which encode them in the mosquitoes Culex quinquefasciatus Say and Anopheles gambiae Giles sensu stricto have now been cloned and recombinant proteins generated (Logan and Birkett 2007). Sandflies are yet to become the focus of a similar endeavour.

The questions raised above are clearly important and from our own experience can be laborious and time consuming to answer. In the twenty plus years we have studied sandfly chemical ecology there have been some significant advances in our knowledge. However, it must be emphasised that there is a real need for wider participation and perspectives in the topic if the potential value of such work is to be fully and quickly realised in the future.

\section{References}

Alexander B and Maroli M (2003) Control of phlebotomine sandflies. Med Vet Ent 17: 1-18. 
Ashford RW (1974) Sandflies (Diptera: Phlebotomidae) from Ethiopia: taxonomic and biological notes. J Med Ent 11: 605-616.

Barth R (1961) Sobre o aparelho genital interno do macho de Phelbotomus longipalpis Lutz \& Neiva, 1912 (Diptera: Psychodidae). Mem Inst Oswaldo Cruz 59: 23-36.

Bauzer LGSR, Souza NA, Maingon RD and Peixoto AA (2007) Lutzomyia longipalpis in Brazil: a complex or a single species? A mini-review. Mem Inst Oswaldo Cruz 102: 1-12.

Beach R, Young DG and Mutinga MJ (1983) New phlebotomine sandfly colonies: rearing Phlebotomus martini, Sergentomyia schwertzi and Sergentomyia africana (Diptera: Psychodidae). J Med Ent 20: 579-584.

Boufana B (1990) The tergal gland and antennal sensilla of the sandfly Lutzomyia longipalpis (Diptera: Psychodidae). $\mathrm{PhD}$ Dissertation, University of Liverpool, Liverpool, UK.

Boufana B, Ward RD and Phillips A (1986) Development of the tergal pheromone gland in male Lutzomyia longipalpis (Diptera: Psychodidae). Trans Roy Soc Trop Med Hyg 80: 33.

Bray DP and Hamilton JGC (2007a) Courtship behaviour in the sandfly Lutzomyia longipalpis, the New World vector of visceral leishmaniasis. Med Vet Ent 21: 332-338.

Bray DP and Hamilton JGC (2007b) Host odor synergizes attraction of virgin female Lutzomyia longipalpis (Diptera: Psychodidae). J Med Ent 44: 779-787.

Cameron MM, Amerisinghe FP and Lane RP (1991) The field response of Sri Lanka sandflies and mosquitoes to synthetic cattle derived attractants. Parassitologia 33: 119-126.

Campbell-Lendrum DH, Pinto MC, Brandão-Filho SP, de Souza AA, Ready PD and Davies CR (1999) Experimental comparison of anthropophily between geographically dispersed populations of Lutzomyia whitmani (Diptera: Psychodidae). Med Vet Ent 13: 299-309.

Chaniotis BN (1967) The biology of California Phlemotomus (Diptera: Psychodidae) under laboratory conditions. J Med Ent 4: 221-233.

Chaniotis BN (1983) Improved trapping of phlebotomine sand flies (Diptera: Psychodidae) in light traps supplemented with dry ice in a neotropical rain forest. J Med Ent 20: 222-223.

Chertemps T, Duportets L, Labeur C, Ueyama M and Wicker-Thomas C (2006) A female-specific desaturase gene responsible for diene hydrocarbon biosynthesis and courtship behaviour in Drosophla melanogaster. Ins. Mol. Biol. 15: 465-473.

Christensen HA and Herrer A (1973) Attractiveness of sentinel animals to vectors of leishmaniasis in Panama. Am JTrop Med Hyg 22: 578-584.

Colvin J and Gibson G (1992) Host-seeking behaviour and management of tsetse. Ann Rev Ent 37: 21-40.

Dougherty MJ, Guerin P and Ward RD (1995) Identification of oviposition attractants for the sandfly Lutzomyia longipalpis (Diptera: Psychodidae) present in volatiles of faeces from vertebrates. Phys Ent 20: 23-32.

Dougherty MJ, Guerin PM, Ward RD and Hamilton JGC (1999) Behavioural and electrophysiological responses of the Phlebotomine sandfly Lutzomyia longipalpis (Diptera: Psychodidae) when exposed to canid host odour kairomones. Phys Ent 24: 251-262.

Dougherty MJ and Hamilton JGC (1997) Dodecanoic acid is the oviposition pheromone of Lutzomyia longipalpis. J Chem Ecol 23: 2657-2671.

Dougherty MJ, Ward RD and Hamilton JGC (1992) Evidence for the accessory glands as the site of production of the oviposition pheromone of Lutzomyia longipalpis (Diptera: Psychodidae). J Chem Ecol 18: 1165-1175.

EINaiem D and Ward RD (1991) Response of the sandfly Lutzomyia longipalpis to an oviposition pheromone associated with conspecific eggs. Med Vet Ent 5: 87-91.

EINaiem D and Ward RD (1992) Oviposition attractants and stimulants for the sandfly Lutzomyia longipalpis (Diptera: Psychodidae). J Med Ent 29: 5-12.

Ferveur JF (2005) Cuticular hydrocarbons: their evolution and roles in Drosophila pheromonal courtship. Behav Genet 35: 279-293.

Fuerborn HJ (1922) Der sexuelle Reizapparat (Shmuck-, Duft- und Beruhrungsorgane) der Psychodiden nach biologischen und physiologischen Gesichtspunkten untersucht. Arch für Natur A 88: 1-137. 
Gemetchu T (1976) The biology of a laboratory colony of Phlebotomus longipes Parrot and Martin (Diptera: Phlebotomidae). J Med Ent 12:661-671.

Gillies MT (1980) The role of carbon dioxide in host finding by mosquitoes (Diptera: Culicidae): a review. Bull Ent Res 70: 525-532.

Girling RD and Cardé RT (2006) Analysis of the courtship behavior of the navel orangeworm, Amyelois transitella (Walker) (Lepidoptera: Pyralidae), with a commentary on methods for the analysis of sequences of behavioral transitions. J Insect Behav 19: 497-520.

Hall DR, Beevor PS, Cork A and Vale GA (1984) 1-octen-3-ol, a potent olfactory stimulant and attractant for tsetse isolated from cattle odours. Insect Sci Appl 5: 335-339.

Hamilton JGC, Brazil RP, Campbell-Lendrum D et al. (2002) Distribution of putative male sex pheromones among Lutzomyia sandflies (Diptera: Psychodidae). Ann Trop Med Parasit 96: 83-92.

Hamilton JGC, Brazil RP, Morgan ED and Alexander B (1999c) Chemical analysis of oxygenated homosesquiterpenes: a putative sex pheromone from Lutzomyia lichi (Diptera: Psychodidae). Bull Ent Res 89: 139-145.

Hamilton JGC, Dawson GW and Pickett JA (1996a) 9-Methylgermacrene-B, a novel homosesquiterpene from sex pheromone glands of Lutzomyia longipalpis (Diptera: Psychodidae) from Lapinha, Brazil. J Chem Ecol 22: 1477-1491.

Hamilton JGC, Dawson GW and Pickett JA (1996b) 3-Methyl-a-himachalene; sex pheromone of Lutzomyia longipalpis (Diptera: Psychodidae) from Jacobina, Brazil. J Chem Ecol 22: 331-2340.

Hamilton JGC, Dougherty MJ and Ward RD (1994) Sex pheromone activity in a single component of tergal gland extract of Lutzomyia longipalpis (Diptera: Psychodidae) from Jacobina, Northeastern Brazil. J Chem Ecol 20: 141-151.

Hamilton JGC, Hooper AM, Mori K, Pickett JA and Sano S (1999b) 3-Methyl-a-himachalene confirmed, and the relative stereochemistry defined, by synthesis as the sex pheromone of the sandfly Lutzomyia longipalpis from Jacobina, Brazil. Chem Comms 355-356.

Hamilton JGC, Ibbotson HC, Hooper AM, Mori K, Pickett JA and Sano S (1999a) 9-Methylgermacrene-B confirmed by synthesis as the sex pheromone of the sandfly Lutzomyia longipalpis from Lapinha, Brazil, and the absolute stereochemistry defined as 95 . Chem Comms 2335-2336.

Hamilton JGC and Krishnakumari K (2004) Sex pheromone analogue for controlling Lutzomyia longipalpis. BR Patent 10500799, 20 Sept 2006.

Hamilton JGC and Ramsoondar TMC (1994) Attraction of Lutzomyia longipalpis to human skin odours. Med Vet Ent 8: 375-380.

Hamilton JGC and Ward RD (1994) Chemical analysis of a putative sex pheromone from Lutzomyia pessoai (Diptera: Psychodidae). Ann Trop Med Parasit 88: 405-412.

Howard RW and Blomquist GJ (2005) Ecological, behavioral, and biochemical aspects of insect hydrocarbons. Ann Rev Ent 50: 371-393.

Jarvis EK and Rutledge LC (1992) Laboratory observations on mating and leklike aggregations in Lutzomyia longipalpis (Diptera, Psychodidae). J Med Ent 29: 171-177.

Jones TM (1997) Sexual selection in the sandfly, Lutzomyia longipalpis. PhD Thesis, University of London.

Jones TM and Hamilton JGC (1998a) A role for pheromones in mate choice in a lekking sandfly. Anim Behav 56: 891-898.

Jones TM, Quinnell RJ and Balmford A (1998b) Fisherian flies: benefits of female choice in a lekking sandfly. Proc R Soc Lond B Biol Sci 265: 1651-1657.

Kelly DW and Dye C (1997) Pheromones, kairomones and the aggregation dynamics of the sandfly Lutzomyia longipalpis. Anim Behav 53: 721-731.

Knudsen AB, Lewis DJ, Tesh RB, Rudnick A, Jeffery J and Singh I (1979) Phlebotomine sand flies (Diptera: Psychodidae) from a primary hill forest in western Malaysia. J Med Ent 15: 286-291.

Krishnakumari B, Sarita Raj K and Hamilton JGC (2004) Synthesis of 9-methylgermacrene from germacrone, an active analogue of (S)-9-methylgermacrene-B, sex pheromone of the Phlebotomine sandfly, Lutzomyia longipalpis, from Lapinha Brazil. Paper presented at the IUPAC international conference on biodiversity and natural products: chemistry and medical applications, New Delhi, India, 26-31 January 2004. 
Lane R and Ward RD (1984) The morphology and possible function of abdominal patches in males of two forms of Lutzomyia longipalpis (Diptera: Phlebotominae). Cahiers d'Office de la Recherche Scientifique et Technique OutreMer. Ent Méd Parasit 22: 245-249.

Lane R, Phillips A, Molyneux DH, Procter G and Ward RD (1985) Chemical-analysis of the abdominal glands of two forms of Lutzomyia longipalpis - site of a possible sex-pheromone. Ann Trop Med Parasit 79: 225-229.

Lane RP, Pile MM and Amerasinghe P (1990) Anthropophagy and aggregation behaviour of the sandfly Phlebotomus argentipes in Sri Lanka. Med Vet Ent 15: 132-139.

Logan JG and Birkett MA (2007) Semiochemicals for biting fly control: their identification and exploitation. Pest Man Sci 63: 647-657.

Maingon RD, Ward RD, Hamilton JG, Bauzer LG and Peixoto AA (2008) The Lutzomyia longipalpis species complex: does population sub-structure matter to Leishmania transmission? Trends in Parasit 24: 12-17.

Mands V, Kline DL and Blackwell A (2004) Culicoides midge trap enhancement with animal odour baits in Scotland. Med Vet Ent 18: 336-342.

Mangabeira OF (1969) Sóbre a sistemática e biologia dos Phlebotomus do Ceará. Rev Bras Malariol Doencias Trop 12: 3-26.

Miles CT, Foster WA and Christensen HA (1976) Mating aggregations of male Lutzomyia sandflies at human hosts in Panama. Trans R Soc Trop Med Hyg 70: 532.

Montoya-Lerma J and Lane RP (1996) Factors affecting host preference of Lutzomyia evansi (Diptera: Psychodidae), a vector of visceral leishmaniasis in Columbia. Bull Ent Res 86: 43-50.

Morton IE and Ward RD (1989) Laboratory response of female Lutzomyia longipalpis sandflies to a host and male pheromone source over distance. Med Vet Ent 3: 219-223.

Nevatte T (2006) Changes in golden hamster behaviour and attractiveness to the sand fly Lutzomyia longipalpis as a result of Leishmania infection. PhD thesis, University of Keele, Keele, UK.

Nigam Y and Ward RD (1991) The effects of male sandfly pheromone and host factors as attractants for female Lutzomyia longipalpis. Physiol Ent 16: 305-312.

O'Shea B, Rebollar-Tellez E, Ward RD, Hamilton JG, el Naiem D and Polwart A (2002) Enhanced sandfly attraction to Leishmania infected hosts. Trans Roy Soc Trop Med Hyg 96: 117-118.

Oshaghi MA, McCall PJ and Ward RD (1994) Response of adult sandflies, Lutzomyia longipalpis (Diptera: Psychodidae), to sticky traps baited with host odour and tested in the laboratory. Ann Trop Med Parasitol 88: 439-444.

Pinto MC, Campbell-Lendrum DH, Lozovei AL, Teodoro U and Davies CR (2001) Phlebotomine sandfly responses to carbon dioxide and human odour in the field. Med Vet Ent 15: 132-139.

Quinnell RJ and Dye C (1994) Correlates of the peridomestic abundance of Lutzomyia longipalpis (Diptera, Psychodidae) in Amazonian Brazil. Med Vet Ent 8: 219-224.

Quinnell RJ, Dye C and Shaw JJ (1992) Host preferences of the phlebotomine sandfly Lutzomyia longipalpis in Amazonian Brazil. Med Vet Ent 6: 195-200.

Ready PD (1978) The feeding habits of laboratory-bred Lutzomyia longipalpis (Diptera: Psychodidae). J Med Ent 14: 545-552.

Rebollar-Tellez EA, Hamilton JGC and Ward RD (1999) Response of female Lutzomyia longipalpis to host odour kariomones from human skin. Phys Ent 24: 220-226.

Shani A (2000) Chemical communication agents (pheromones) in integrated pest management. Drug Dev Res 50: 400-405.

Souza NA, Vigoder FM, Araki AS, Ward RD, Kyriacou CP and Peixoto AA (2004) Analysis of the copulatory courtship songs of Lutzomyia longipalpis in six populations from Brazil. J Med Ent 41: 906-913.

Souza NA, Ward RD, Hamilton JG, Kyriacou CP and Peixoto AA (2002) Copulation songs in three siblings of Lutzomyia longipalpis (Diptera: Psychodidae). Trans Roy Soc Trop Med Hyg 96: 102-103.

Takken W (1991) The role of olfaction in host-seeking of mosquitos - a review. Insect Sci Appl 12: 287-295.

Talyn BC and Dowse HB (2004) The role of courtship song in sexual selection and species recognition by female Drosophila melanogaster. Anim Behav 68: 1165-1180. 
Valenta DT, Killick-Kendrick R and Killick-Kendrick M (2000) Courtship and mating by the sandfly Phlebotomus duboscqi, a vector of zoonotic cutaneous leishmaniasis in the Afrotropical region. Med Vet Ent 14: 202-212.

Ward RD and Hamilton JGC (2002) Chemical and auditory signals in Phlebotomine sandfly behaviour. In: Killick-Kendrick $\mathrm{R}$ (ed) Canine leishmaniasis: moving towards a solution. Proceedings $2^{\text {nd }}$ International Canine Leishmaniasis Forum, Seville, Spain.

Ward RD and Morton IE (1991) Pheromones in mate choice and sexual isolation between siblings of Lutzomyia longipalpis (Diptera: Psychodidae). Parassitologia 33: 527-533.

Ward RD, Morton IE, Lancaster V, Smith PA and Swift A (1989) Bioassays as an indicator of pheromone communication in Lutzomyia longipalpis (Diptera: Psychodidae). In: Hart DT (ed) Leishmaniasis, the current status and new strategies for control. NATO ASI Series A: Life Sciences, vol 163. Springer, New York, USA, pp 239-247.

Ward RD, Phillips A, Burnet B and Marucondes CB (1988) The Lutzomyia longipalpis complex: reproduction and distribution. In: Service MW (ed) Biosystematics of haematophagous insects. Oxford University Press, Oxford, UK, pp 257-269.

Ward RD, Ribeiro AL, Ready PD and Murtagh A (1983) Reproductive isolation between different forms of Lutzomyia longipalpis (Lutz and Neiva), (Diptera: Psychodidae), the vector of Leishmania dovani chagasi Cunha \& Chagas and its significance to kala-azar distribution in South America. Mem Inst Oswaldo Cruz 78: 269-280. 



\title{
10. Understanding and exploiting olfaction for the surveillance and control of Culicoides biting midges
}

\author{
James G. Logan, James I. Cook, A. Jennifer Mordue (Luntz) and Dan L. Kline
}

\begin{abstract}
Culicoides midges (Diptera: Ceratopogonidae) are found worldwide with the exception of only a few countries including New Zealand, Patagonia, the Hawaiian Isles and Antarctica. They are a nuisance pest to human beings, but transmit a number of diseases that mainly affect livestock. Like many haematophagous insects, midges have evolved highly developed and sensitive olfactory mechanisms which allow them to locate a potential mate or a suitable vertebrate host. This chapter provides a brief overview of the Culicoides midge lifecycle, its status as a vector of disease and offers an extensive review of Culicoides chemical ecology research, which has led to the identification of semiochemicals that could be exploited to control them. Host location processes can be influenced by many factors including midge physiology, host kairomones, the complex interactions of other volatile chemicals that confer differential attractiveness of hosts and also physical cues such as light levels and climatic conditions. These factors are considered in detail and future areas of work are discussed.
\end{abstract}

Keywords: biting midges, Ceratopogonidae, behaviour, olfaction

\section{Introduction}

Of the five major sensory systems (olfaction, taste, touch, vision and sound) by which organisms perceive their environment olfaction can be said to be the most important for biting flies such as midges. This sensory modality provides adult female Culicoides (Diptera: Ceratopogonidae) with essential cues for the location and identification of hosts that are required for a blood meal in order to grow their eggs. The detection of small, volatile compounds borne on the wind provides the main cues for activation and upwind flight. This, coupled with other cues such as heat, moisture and visual cues at closer range and taste on landing drive the behavioural sequence known as 'host location'. Although some of these stimuli may be involved in other aspects of midge ecology, their role in the location of a suitable host has been mostly studied and has been exploited to manipulate the behaviour of host-seeking adult females. For example, odour-baited traps can be used to lure, capture and kill adults. Although, this method has been used with some success with other biting Diptera (e.g. Glossina spp. tsetse flies), research into the olfactory processes involved in midge-host interactions is not fully understood but certainly has the potential to provide a potential means of Culicoides control.

Few insects that attack man are more annoying during their peak of activity than Culicoides midges but previous control efforts have been met with limited success, with repellents not providing full protection and with insecticides failing to provide adequate control of populations. To this end, a thorough understanding of the host-location process of each Culicoides species of medical and veterinary importance is still needed. This chapter will review current knowledge of olfaction in midge-host interactions including knowledge of experimental and commercially available traps. Protective measures are essential not only for man but also for domestic animals against midges that vector diseases such as Bluetongue of sheep and cattle and African Horse sickness. Effective 
control of targeted species is urgently required, particularly in light of the recent Bluetongue outbreak in Europe.

\section{Culicoides midge biology and ecology}

\section{Lifecycle}

Culicoides midges are members of the order Diptera, one of the largest orders within the class Insecta. They are one of four haematophagous genera within the Ceratopogonidae family and are considered to have the most medical and veterinary importance due to their biting action and ability to vector pathogens that cause disease in livestock (Kettle 1965, Linley et al. 1983). There are approximately 1,400 species of biting midge and they have a wide range of larval and adult biologies.

Midges have mostly subaquatic or subterrestrial immature stages, developing in/nearby pools, streams or bogs. However, some can also be found in a variety of terrestrial or semiterrestrial locations such as animal droppings and vegetation. The northern Palaearctic species complex $C$. obsoletus, implicated in Bluetongue virus (BTV) transmission in northern Europe, is fairly catholic in its breeding habitats occurring not only in dung but also a variety of habitats from woodland to open country and bogland to marshland (Hill 1947, Kettle and Lawson 1952). Culicoides obsoletus Meigen s.l. is strongly associated with shaded habitats and a higher green leaf density than the Old World BTV vector C. imicola Kieffer that is found in much drier, warmer habitats (e.g. straw/ dung mix at dripping water pipes) in full sun (Conte et al. 2007). Many other species troublesome to humans breed in brackish, sandy habitats along coastlines.

Culicoides are holometabolous, having four larval instars followed by a pupal stage where the adult form develops before emergence, although some species can over-winter as a diapausing fourth instar (Figure 1). Climate change, and more specifically alterations in temperature, may affect larval stages of some Culicoides species. Allingham (1991) and Bishop et al. (1996) demonstrated that Culicoides brevitarsis Kieffer emerge between $17-36^{\circ} \mathrm{C}$, with most emergence occurring in the $25-36{ }^{\circ} \mathrm{C}$ range. They also showed that average temperature in the field prior to their experiment starting, i.e. when C. brevitarsis was allowed to oviposit, played a role in determining development time. This species can survive for periods outside the optimum temperature range, but only emerge if conditions become favourable again within 50 days. Additionally, a return to favourable temperatures is not necessarily problem free. When temperatures in one experiment were raised to $25^{\circ} \mathrm{C}$ after an extended period at $12^{\circ} \mathrm{C}$ the adults that emerged appeared too small to produce viable offspring. The effect of climate change on the interaction between adult Culicoides midges and their host is an important and topical issue which is discussed in more detail later in this chapter.

The adult midge has a body length of about $1-2 \mathrm{~mm}$, a weight of approximately $0.5 \mu \mathrm{g}$ and has two compound eyes. Most adult females require blood to develop eggs, although some species, e.g. $C$. impunctatus Goetghebuer, are autogenous and therefore do not require a blood meal to develop their first batch of eggs (Blackwell et al. 1992, Boorman and Goddard 1970). All female midges must locate hosts to obtain a blood meal and this complex process can be heavily influenced and, to some extent, controlled by various environmental factors such as solar radiation, wind velocity, temperature and humidity, all of which are thought to limit flight activity (Bhasin 1996, Blackwell et al. 1997). This aspect of their ecology is discussed in more detail later in this chapter. 


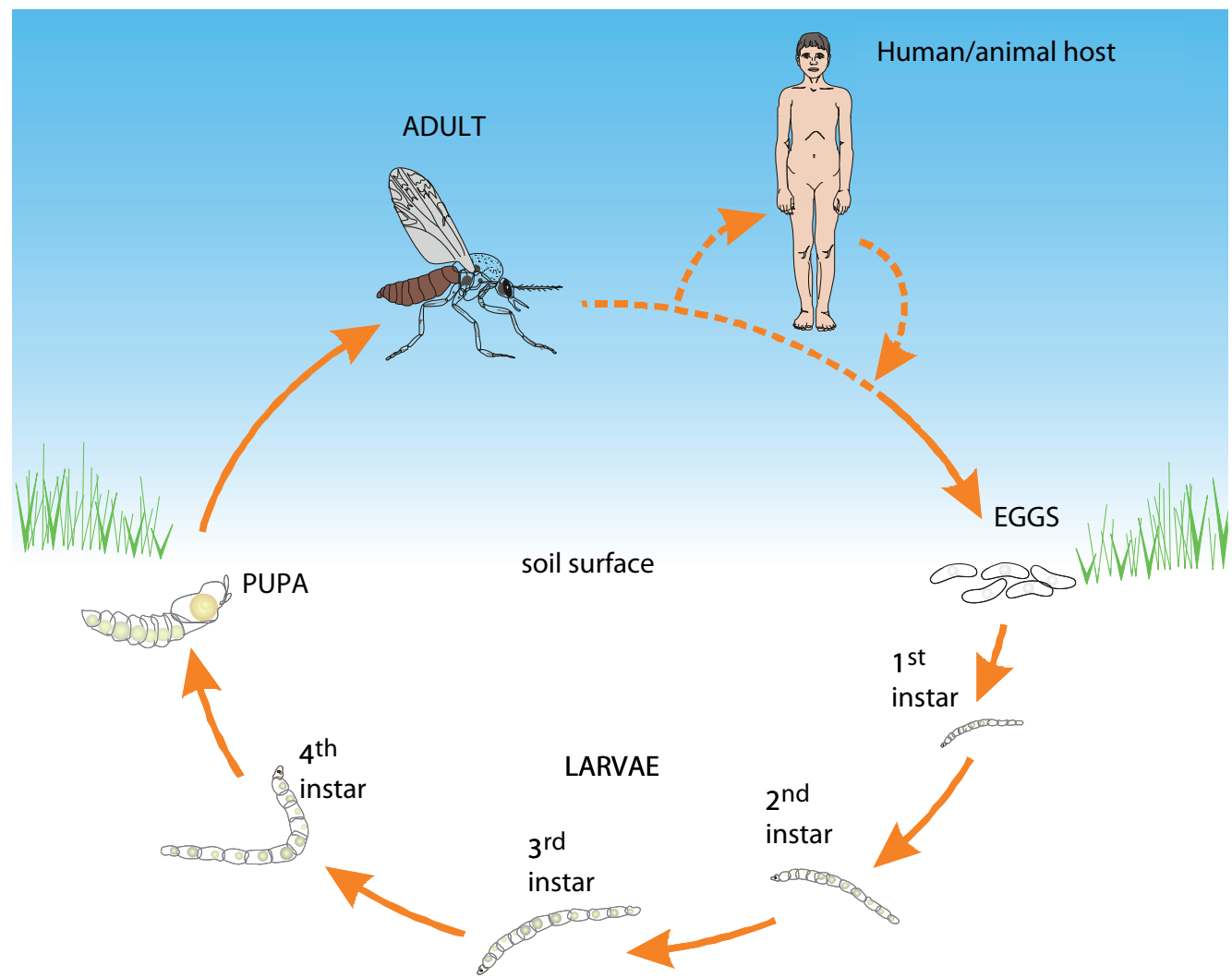

Figure 1. Illustrative representation of a female Culicoides midge lifecycle (Lynda Castle, Rothamsted Research, Harpenden, UK).

Blood meals are obtained preferentially from a variety of vertebrate hosts. For example, cattle, sheep and deer are among the predominant hosts of $C$. impunctatus although they will also readily feed on human beings (Blackwell et al. 1994b, Mands et al. 2004). ELISA tests on blood-fed C. imicola collected at farms in Ondersterpoort, RSA revealed that most midges had fed more than once on horse, cattle and sheep with horse being the most frequent (AWA Brown, AJ Mordue (Luntz) and GJ Venter, unpublished observation). The coat of cattle offers midges a greater surface area of accessible skin from which to feed than is available on sheep whose fleece they cannot penetrate so easily (Muller and Murray 1977). After blood feeding, as egg batches mature, females are thought to rest before seeking a suitable oviposition site (Boorman and Goddard 1970).

\section{Vector status/ nuisance value and geographical distribution}

Within the Ceratopogonidae, Culicoides is the most important genus regarding human and livestock comfort/welfare and health (Kettle 1965, Linley et al. 1983). Midges are found worldwide with the exception of only a few countries including New Zealand, Patagonia, the Hawaiian Isles and Antarctica. They have evolved to inhabit most temperate and tropical regions and therefore, in this chapter, we focus on those that are considered to be of high medical and veterinary importance. 
With emerging vector-borne diseases in certain countries it is vital that we understand which species spread pathogens, the hosts that are most heavily afflicted and the midge geographical distribution and habitat.

Diseases transmitted by midges mainly affect livestock. For example, Culicoides are well documented as vectors of livestock arboviruses (Kettle 1984, Purse et al. 2005), including bluetongue virus of sheep and cattle (BTV), African horse sickness virus (AHSV) and epizootic haemorrhagic disease (EHD) of deer (Mellor 1990, 1993, 1994, Sellers and Maarouf 1991). Some species also vector the filarial nematode Onchocerca cervicalis Railliet and Henry, which affects horses (Mellor 1972), or cause 'sweet itch', which is not a disease, but a distressing skin condition of horses caused by midge saliva (Mellor and McCaig 1974, Townley et al. 1984).

Bluetongue virus infects domestic and wild ruminants and can cause more than $70 \%$ mortality in sheep (Jennings and Mellor 1988). It is prevalent in Central, West and South America, Africa, Asia, the Middle East and Australia (Holbrook and Tabachnick 1995, Mellor 1990). More recently it has spread throughout Europe. Culicoides imicola is believed to be one of the main vectors of BTV. Following the introduction of $C$. imicola to Italy and the subsequent establishment of BTV (Baylis et al. 2001) several studies have been conducted to predict the likely spread of BTV (Tatem et al. 2003, Wittmann et al. 2001). In 2005-2006, Takken et al. (2008) investigated the presence and distribution of Culicoides spp. at different habitats in the Netherlands to identify where potential BTV outbreaks may occur. This information was coupled with climate data to develop a BTV risk model (De Koeijer and Elbers 2007). A mixture of habitats comprising wetland areas, peat land areas, floodplains and livestock farms was examined. In total 15 species were collected and identified with variations in abundance for each habitat type. Culicoides impunctatus was most prevalent in wetland and peat bogs/moors. Nine other species were found in the wetland areas while the diversity was much lower in the peat land environments, with only C. obsoletus and C. pulicaris species complexes being identified. Culicoides obsoletus were more prevalent in floodplains and livestock farms than C. impunctatus. Culicoides scoticus Downes and Kettle, C. dewulfi Goetghebuer and C. chiopterus Meigen were also present at the livestock farms.

In August 2006, BTV spread throughout northern Belgium, France, Germany, Luxembourg and the Netherlands. This extremely worrying outbreak occurred further north than the occurrence of $C$. imicola, the main vector of the southern Europe outbreaks. However, Culicoides dewulfi, which breeds in the dung of cattle and horses, was found RT-PCR positive to the BTV-8 serotype that had caused the northern European outbreak (Meiswinkel et al. 2007). This may present further problems as it may be possible for this species to also transmit AHSV as the virus is closely related to BTV (Meiswinkel et al. 2008). Similarly, C. obsoletus sensu stricto and C. scoticus (which comprise two of the four species in the obsoletus complex), were found to be RT-PCR positive to BTV-8. Culicoides pulicaris Linnaeus s.s. has previously been incriminated in the field transmission of BTV (Caracappa et al. 2003), and C. impunctatus (another member of the Pulicaris complex) has been implicated in the laboratory (Carpenter et al. 2006, Jennings and Mellor 1988). Despite this, the information available suggests that $C$. obsoletus played a significant role in the European outbreak (Meiswinkel et al. 2008). In June 2007 it was reported that an animal from a holding in Germany had displayed evidence of BTV-8 infection in April. This represented the first indication that the virus had successfully overwintered in the region (IAH 2008a). In September 2007 the first case of BTV was identified in the UK (IAH 2008b). There are currently 136 premises affected by BTV (DEFRA 2008). At the time of the European outbreak, temperatures soared to a record high and this appeared to promote the replication and transmission of BTV by a number of Culicoides spp. (Meiswinkel et al. 2008). It is also possible that other viruses such as AHSV, Akabane virus (AKAV), 
epizootic haemorrhagic disease virus (EHDV) and equine enchephalosis virus (EEV) may also be circulated in northern Europe under favourable climatic conditions (Meiswinkel et al. 2008). AHSV is currently prevalent in sub-Saharan Africa and causes mortality rates that exceed $90 \%$ in horses (Mellor 1994).

One of the best studied midge species is C. impunctatus, which is found throughout northern Europe, Russia and northern China but is particularly prevalent on the west coast of Scotland (Campbell and Pellham-Clinton 1960, Hill 1947). Although at least seventeen other species exist in this area, C. impunctatus outnumbers the others considerably (Blackwell et al. 1992). Culicoides impunctatus is among the most numerous of haematophagous flies in the UK where it can reach huge population densities (Hendry and Godwin 1988). Although C. impunctatus does not vector diseases in the UK presently, it causes significant losses to the economy, especially in terms of reduced tourism and outdoor activities in Scotland, where it is responsible for more than $90 \%$ of the biting attacks on humans (Blackwell et al. 1997). This is mainly due to the painful reactions caused by their bites, which discourage tourists and disrupts outdoor industries including agriculture and forestry. An estimated $20 \%$ loss in working hours has been reported in the forestry industry each year due to midge bites alone (Hendry and Godwin 1988). Their intense numbers during such attacks was recently highlighted by Mordue (Luntz) and Mordue (2003) who reported a rate of around 40,000 landings per hour on the forearms of volunteers. Similarly high numbers are found attacking people in coastal areas of Australia and the United States. In these countries several species of biting midges breed in brackish salt marshes and mangrove swamps. The encroachment of residential developments and recreational facilities into areas close to these breeding locations and the manmade extensions of breeding habitats has resulted in biting midges becoming an increasing problem.

Human pestilence by Culicoides biting midges is an acute problem along Australia's tropical and temperate shores and significant attack in the sublittoral and hinterland environs is confined to the southern parts of the country. Of the three littoral species groups involved the C.immaculatus group is of lesser importance than the other two. Both the $C$. ornatus group and the $C$. molestus group include the most seriously offending species. The former group is usually associated with mangrove habitats and the latter with the sandier reaches of open estuaries subject to tidal influence. The $C$. ornatus group is very large. It includes 50 species overall. A number of undescribed species in this group may ultimately be shown to contribute significantly towards the pestilence problem of the tropical coastline. Culicoides ornatus Taylor, C. Iongior Hagan and Reye and C. marmoratus (Skuse) are well known as serious pest species. Biting midges (e.g. Culicoides ornatus) are also serious pests around the coast of the Northern Territory. Culicoides ornatus is the dominant species in the Darwin area and numbers collected at certain locations reflect a relatively intense pest problem (Reye and Lee 1963). Prior to 1963 little was known of the biting midges on the Gold Coast of Australia as their larval habitat was isolated and they were of little consequence to man. However, this changed with the development of residential areas in close proximity to creek estuaries. Culicoides subimmaculatus Lee and Reye is the dominant pest species in these areas. With the construction of residential canal estates on the Gold Coast a new breeding habitat was created for the species C. molestus (Skuse). Here man interfered with the environment and created a vast new breeding ground for what was once a minor species. Culicoides molestus became the dominant midge species in the Gold Coast and interfered with the idyllic outdoor lifestyle for which the Gold Coast is famous. As residential development spread further to the north of the Gold Coast C. marmratus and C. henryi became the most prevalent midge species. On the Gold Coast biting midges rapidly replaced mosquitoes as the most prevalent nuisance or public health pest insect. The C. molestus group is smaller, with 11 recognised species overall. 
Perhaps the supreme pest species of the Queensland coastline is included in this group. Ironically it remains undescribed and has been regularly misidentified as C. subimmaculatus Lee and Reye from which it differs in morphological detail and most likely in its biology.

The C. immaculatus group contains a single significant human pest species in Australia, namely $C$. immaculatus Lee and Reye. Its preferred habitat is along open shores and its larval habitat remains unknown.

In the United States the tremendous numbers of C. furens (Poey), C. barbosai Wirth and Blanton, C. hollensis (Melander and Brues), C. melleus (Coquillett) and C. mississippiensis Hoffman make living in coastal areas almost unbearable at certain times of the year. Abundance of these species varies geographically along the extensive USA coastline, and spatially and temporally at different geographic locations. Tourist business is greatly affected by biting midge abundance at these locations. Kettle (1962) succinctly puts it, 'One midge is an entomological curiosity, a thousand can be hell'. However, the population level or midge density at which female midges must reach before affecting people is dependent upon personal tolerance. Established residents of a midgeinfested area will tolerate high midge densities whilst new residents a low density is too much to tolerate. The tourist business is greatly affected by midge (also called sand fly in this area) problems along many Florida beaches where there are significant populations of $C$. furens, $C$. barbosai, C. hollensis (Melander and Brues), C. melleus and C. mississippiensis. In some inland localities, particularly in the 'Highland' and 'Crystal Springs' counties, C. floridensis Beck and C. tissoti Wirth and Blanton may become a nuisance. Linley and Davies (1971) presented an excellent review of the problem of midges versus tourism, a summary of the biology of the most important species, and recommendations for control.

Culicoides furens makes life miserable in the Caribbean and on the eastern seaboard of America from New York in the north to Sao Paulo in Brazil in the south. With the aid of the wind this species may disperse 3 or 4 miles (5 to $7 \mathrm{~km}$ ) (Linley and Davies 1971). Another species, C. barbosai, had previously been confused with C. furens (Wirth and Blanton 1956). This was an important discovery because, as the local Research Unit showed later, the breeding sites of $C$. furens and $C$. barbosai were quite different. The existing measures which were directed against $C$. furens were less effective against $C$. barbosai. Culicoides furens bred in the open and in areas shaded by the white and black mangroves (Languncularia racemosa L. and Avicennia nitida Jacq., respectively) while C. barbosai bred in wetter areas in the shade of the red mangrove (Rhizophora mangle L.). Removal of the mangrove cover had no effect on the breeding of $C$. furens but a year later $C$. barbosai had almost disappeared from the cleared area (Davies 1969).

Nuisance biting and the ability to transmit diseases, as described above, along with the recent spread of Culicoides populations mean that the need for effective control is extremely important. However, the olfactory processes and behaviours associated with host location and host preference is still poorly understood and requires much research.

\section{Midge/host interactions and influencual factors}

Host location is essential for Culicoides midges to find a suitable blood meal. Midges are known to detect visual, olfactory and gustatory stimuli, which aid in their location of a host. External stimuli provided by vertebrate hosts induce a series of behaviours that ultimately lead to the female finding a host and obtaining a blood meal. Host-derived chemical odours are considered to be important as they can be detected by the insects at considerable distances and act as flight 
activators or attractants (kairomones). As midges approach their host convective heat (Brown et al. 1951), humidity, and visual cues such as shape, size, colour and contrast may also play a role (Bernier et al. 1999, Bidlingmayer 1994, Khan 1977, Muir et al. 1992, Takken 1991, Chapter 6 in this Volume). Bishop et al. (2008) used two-dimensional plastic cattle shapes and simulated stimuli to investigate host location for $C$. brevitarsis. They subsequently proposed that initial attraction was to visual stimuli, whilst host odours such as $\mathrm{CO}_{2}$ and 1-octen-3-ol were secondary and probably occurred closer to the host. Landing is suggested to be a visual response to the interface between the backline of the host and the background. Additionally, this work also revealed information on the preferential biting sites of this vector. Sticky traps placed over the replica cattle shape showed that preference is for the ridge line of the back, extending from the neck to the tail. Interestingly, attraction of the mosquito Culex annulirostris Skuse was also investigated, and the visual element was found to play no role at all. For C. impunctatus, landing responses on targets painted with black and white vertical or horizontal stripes showed that females exhibited a clear preference for black stripes irrespective of the presence of host odour or whether the target was moving (rotating) or stationary. The target was shown to be unattractive to females at a distance. Striped targets were however important at inducing a landing response with horizontal black stripes, perhaps related to edge perception, being an effective landing stimulus (A Bhasin, unpublished observation).

Most adult Culicoides are Icrepuscular, with peak activity around dawn and dusk, and to a lesser extent through the night, although a few species bite during the day (Blackwell 1997, Mellor et al. 2000). Culicoides impunctatus follows this crepuscular pattern (Blackwell et al. 1997) with the endogenous rhythm of diel flight periodicity greatly enhanced in the presence of host odour $\left(\mathrm{CO}_{2}\right)$ (A Bhasin, unpublished observation). Although for Culicoides species the onset of activity is usually triggered by falling light intensity (Mellor et al. 2000), meteorological variables other than light intensity appear to trigger activity in C. impunctatus (Blackwell 1997). Light levels are thought to accentuate an underlying rhythmically determined periodicity (Blackwell et al. 1997). This theory corresponds with that of Marsh (1986) who predicted a bimodal circadian rhythm for C. impunctatus.

Like other arthropods, Culicoides females employ semiochemicals for host-location (Mordue (Luntz) 2003). Host-related kairomones for $\mathrm{C}$. impunctatus include $\mathrm{CO}_{2}$, acetone, butanone and L-(+)-lactic acid (Bhasin et al. 2000a,b, 2001). However, the full set of olfactory stimuli has not yet been identified. To achieve this, we must examine the response of the insects to host odours and then identify the components that are physiologically relevant within those stimuli. Recently, Mands et al. (2004) investigated the response of C. impunctatus to animal extracts in a Y-tube olfactometer. They showed that water buffalo extract was the most active (threshold $0.22 \mathrm{~g} / \mathrm{ml}$ ), similar to deer extract, whereas other host extracts including pony, calf and sheep were more than ten times less active. The effects of $\mathrm{CO}_{2}$ and 1-octen-3-ol on three species of Culicoides were investigated in salt marshes, Georgia, USA (Kline et al. 1994). It was found that $\mathrm{CO}_{2}$ was an effective attractant for all three species: C. furens, C. hollensis and C. melleus. However, each species did possess a different response pattern. Contrastingly, 1-octen-3-ol was only an effective attractant for $C$. furens, and was tested both singly and in combination with $\mathrm{CO}_{2}$, where it was synergistic. Repellent effects were reported for $C$. hollensis and C. melleus. 1-Octen-3-ol has also been shown to be attractive to C. impunctatus (Blackwell et al. 1996, Mands et al. 2004) and C. vexans, C. delta C. pulicaris, C. lupicaris and C. albicans (Mands et al. 2004) in the field.

Bhasin et al. (2000b) investigated the behavioural responses of $C$. impunctatus to nine synthetic compounds that had been identified as components of mammalian host odour: 1-octen-3-ol, acetone, butanone, L-(+)-lactic acid, phenol, 3-n-propylphenol, 3-methylphenol, 4-methylphenol 
and 4-ethylphenol. The non-phenolic compounds all induced an attractive dose-dependent response over the range tested. As doses of butanone increased to supra-optimal levels arrestment of responses was noted. Furthermore, for phenol and lactic acid, supra-optimal concentrations actually appeared repellent. 1-Octen-3-ol has also been shown to arrest responses or even repel at high concentrations (Blackwell et al. 1996). Culicoides nubeculosus (Meigen) behavioural responses were tested for 1-octen-3-ol and acetone, and were shown to perform in a similar fashion to $C$. impunctatus but with lower thresholds of response (Bhasin et al. 2000b). Trapping experiments in southeastern Queensland, Australia, showed that the addition of $\mathrm{CO}_{2}$ to traps increased catches of C. histrio and C. subimmaculatus significantly. Addition of 1-octen-3-ol and $\mathrm{CO}_{2}$ increased catches of C. molestus only (Ritchie et al. 1994).

\section{Pheromones}

An interesting aspect of the host-location process is that it may also include the detection of pheromones that act synergistically with host odours. Mating in C. nubeculosus occurs either when a female flies into a swarm of males, with copulation taking place during descent to the ground, or when a female is already feeding upon a host (Pomerantzev 1932, Downes 1950, Downes 1955). In the latter case, volatiles from the blood of a vertebrate host (produced either at the wound itself, or from the excretion products resulting from concentration of the blood meal) could stimulate sex pheromone production and grooming behaviour, contributing to dispersal of the pheromone and attracting males whilst on the host. Virgin female C. nubeculosus perform a greater amount of grooming behaviour in response to blood volatiles, than to controls (Figure 2). It is hypothesised that this grooming behaviour is directly associated with the release of the sex pheromone, $\mathrm{n}$-heptadecane, and is initiated by the presence of blood volatiles (Figure 3; Mordue (Luntz) and Mordue 2003). The behaviour could be a way of transferring the pheromone from the abdomen to the wings, with wing flutter aiding pheromone dispersal. An effective trapping system for this species, therefore, may involve the combination of host-derived olfactory stimuli as well as the sex pheromone. Similarly, during feeding, C. impunctatus females are thought to produce an aggregation or 'recruitment' pheromone (Blackwell et al. 1994a). This is believed to work synergistically with host-derived kairomones such as 1-octen-3-ol and cause aggregation of female midges on a vertebrate host. This is suggested to be a means of protection from host defence by using the 'safety in numbers' strategy or a strategy that enables a midge to find a rare but non-resource-limiting host (Blackwell et al. 1994a, 1996). A contact sex pheromone, which stimulates copulation, has been identified for $C$. melleus and comprises 2-methyldocosane, 8-methyldocosane and 9-methyltricosane (Linley and Carlson 1978).

\section{Indoors/outdoors}

Culicoides midges were believed to be purely exophagic (i.e. feed on animals outdoors) and exophilic (i.e. they will not enter inside buildings). Indeed, anecdotal evidence by Muller (1991) showed that covered areas (yards, stables and sheds) significantly reduced numbers of $C$. brevitarsis reaching host cattle but had little effect on mosquitoes. However, Bishop (2002) proposed the lack of response by the midge species to be due to blocked vision, rather than an aversion to enclosed spaces. Baldet et al. (2008) recently found Culicoides indoors albeit with a lower species diversity (six species) than outdoors (nine species). Similar results have been obtained with C. impunctatus that will readily enter through a window or door of a tent or a chalet containing a human volunteer or with a light left on (JG Logan, unpublished observation). 
A

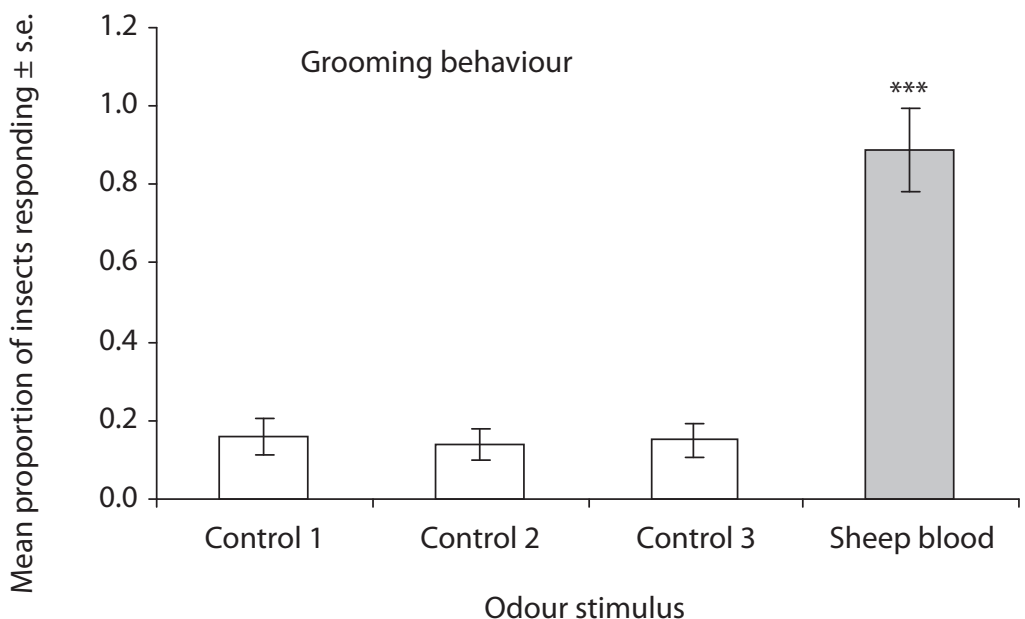

B

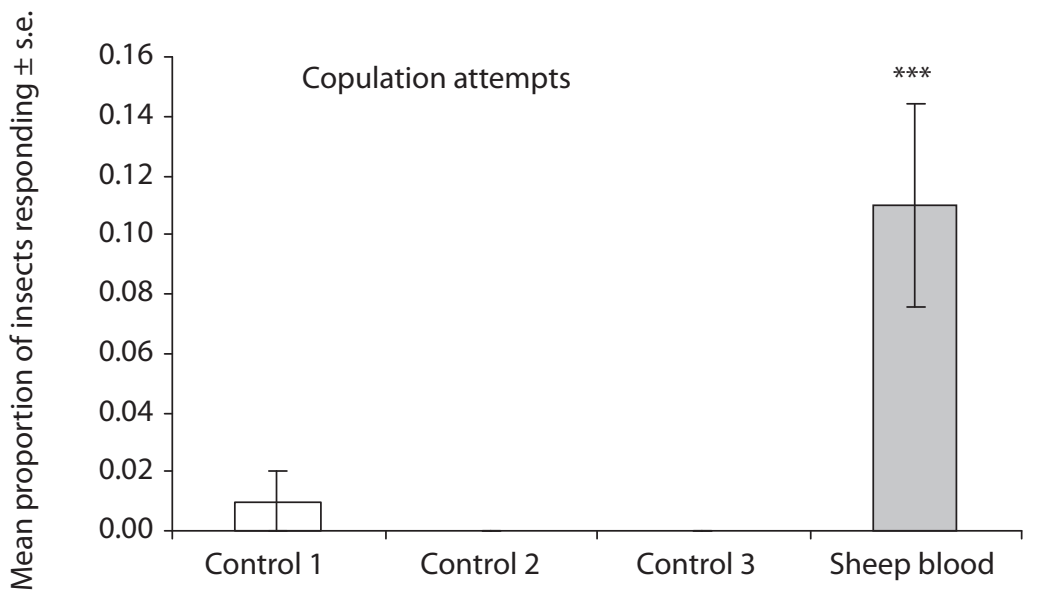

Odour stimulus

Figure 2. Behavioural responses of 24-48 hold adult Culicoides nubeculosus females to sheep blood volatiles in Alsevers solution. (A) Grooming behaviour, (B) Copulation attempts.

Control 1: empty flask; Control 2: distilled water; Control 3: Alsevers solution. Mean proportions \pm s.e. are shown. Asterisks indicate statistically significant differences from the controls (ANOVA *** $\mathrm{P}<0.001 ; n=100$ replicates and 6 insects ( 3 males and 3 female) per replicate).

In northern France, adult parous female C. obsoletus/C. scoticus activity is greater indoors than outdoors with a high proportion being freshly blood fed, but only during the autumn months. Similarly, C. bolitinos Meiswinkel, C. dewulfi and C. chiopterus are thought to be strongly endophagic whereas $C$. pulicaris and C. punctatus Meigen are more exophagic. However, it could be that the housing of all farm animals may cause exophagic/exophilic species to enter buildings to feed (Meiswinkel et al. 2000). The olfactory processes and other behaviours associated with Culicoides 


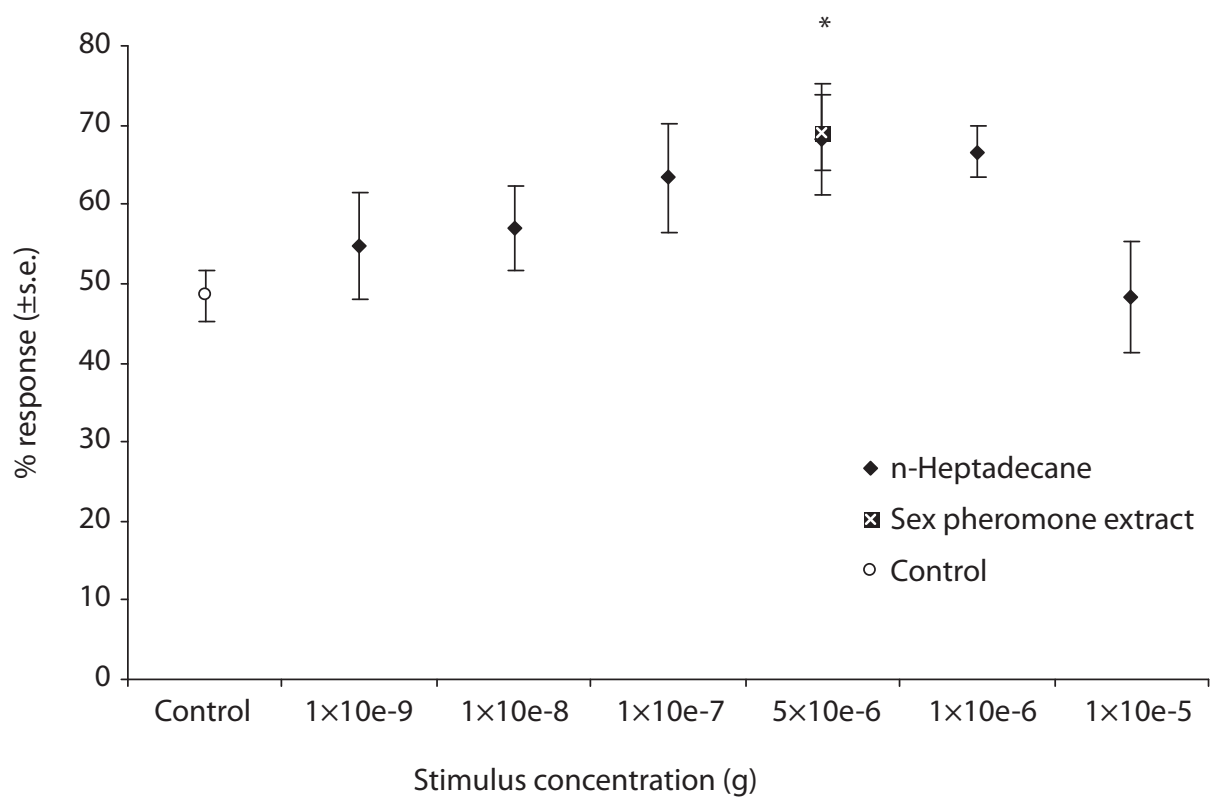

Figure 3. Behavioural responses of Culicoides nubeculosus females to a pheromone air entrainment extract and six doses of $n$-heptadecane in hexane, in a Y-tube olfactometer.

Mean percentages of midges in the treated arm of the $Y$-tube \pm s.e. are shown. Asterisks indicate statistically significant differences from the control (ANOVA, ${ }^{*} \mathrm{P}<0.05 ; n=17$, except pheromone extract where $n=6$ ).

midges entering buildings are not fully understood and may be quite different to those associated with finding a host in an open environment and requires further investigation. This is particularly important since some authorities recommend that livestock should be housed at night to reduce Culicoides attack rates.

\section{Climatic conditions}

The interaction between Culicoides midges and their host can be heavily influenced by climatic conditions. Midges display a preference for feeding in still, warm and humid conditions. Wind speeds of $1-2 \mathrm{~m} / \mathrm{s}$ are enough to inhibit flight and subsequent host-location activities (Lehane 2005). Mellor et al. (2000) reviewed information on the suppression of flight for various Culicoides species, ranging from $3 \mathrm{~m} / \mathrm{s}$ for C. imicola in Kenya (Walker 1977) to $2.2 \mathrm{~m} / \mathrm{s}$ for C. brevitarsis in Australia (Murray 1987). It has also been noted that limits are lower for mating swarm formation by certain species. In addition to the negative effects of wind on activity, it can also affect the abundance of Culicoides through dispersal of the adults (Baylis and Rawlings 1998). Similarly, lower temperature limits for flight range from $10{ }^{\circ} \mathrm{C}$ for $\mathrm{C}$. variipennis (Coquillett) (Nelson and Bellamy 1971) to $18^{\circ} \mathrm{C}$ for C. brevitarsis (Murray 1987).

In our own landing catch experiments on the west coast of Scotland, the effect of weather on host-seeking C. impunctatus females can clearly be seen (Figure 4). Although this species is active in light rain and heavy cloud cover, it is less so during heavy rain. Also, as one might expect, fewer midges land on the arms of human volunteers with increasing wind speed. In contrast to 

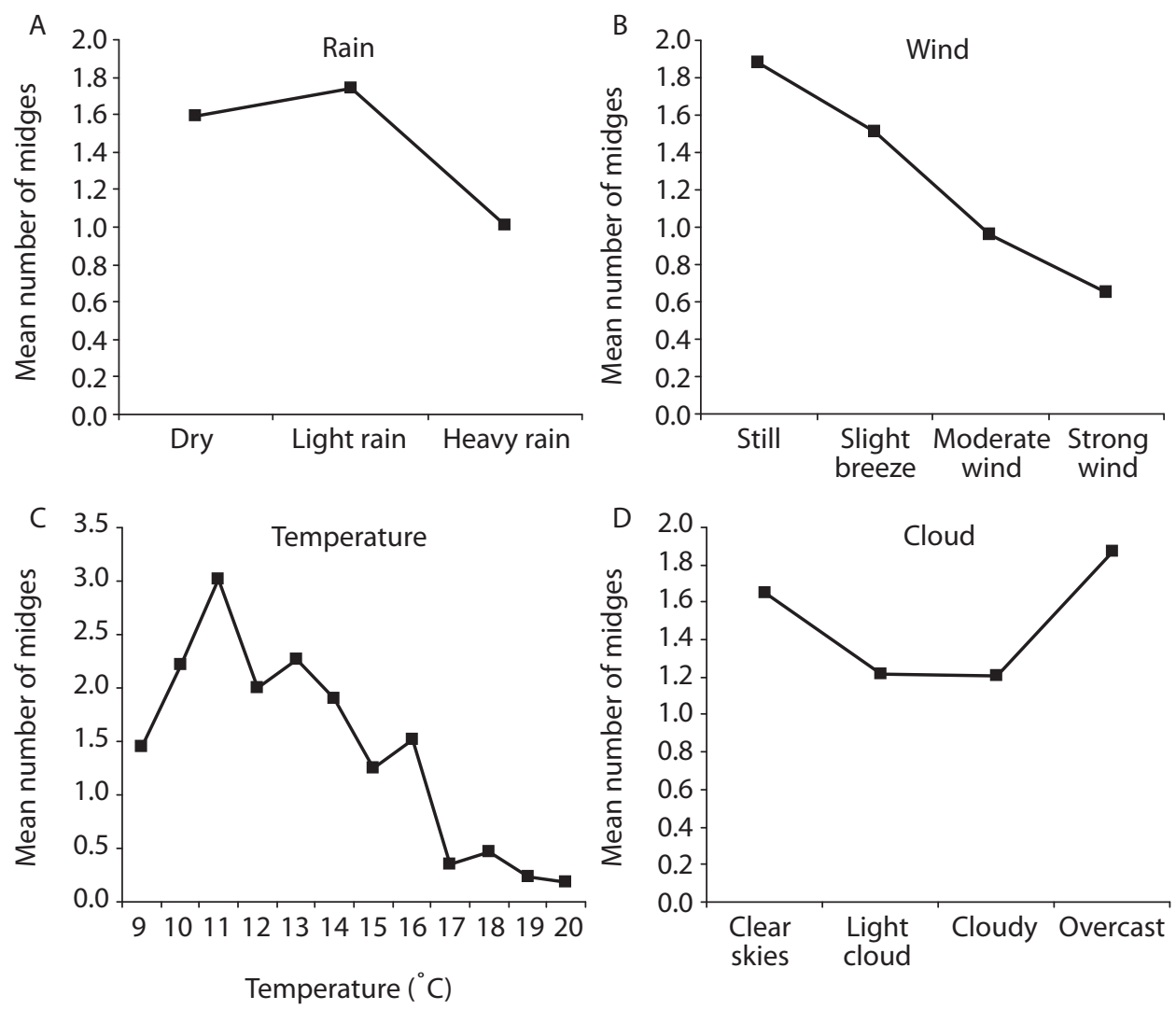

Figure 4. Mean number of Culicoides impunctatus midges landing on the forearms of volunteers under different weather conditions on the west coast of Scotland during a 3 min repellency trial.

other species, their host-seeking behaviour is high above $9{ }^{\circ} \mathrm{C}$ and optimum at around $11{ }^{\circ} \mathrm{C}$, but decreases with increasing temperature and with very little activity above temperatures of $17^{\circ} \mathrm{C}$. (JG Logan, unpublished observation)

Transmission of BTV and AHSV by C. variipennis sonorensis Wirth and Jones is favoured by high temperatures $\left(27-30^{\circ} \mathrm{C}\right)$ as the reduction in longevity is compensated for by the shorter viral extrinsic incubation period (EIP). Contrastingly, at lower temperatures where adult longevity was extended, the EIP was disproportionately prolonged, meaning few midges could survive long enough to transmit the virus (Wittmann 2000).

\section{Intra and interspecific differential responses to host}

Midges are differentially attracted to their vertebrate hosts. For example, odours collected from different host species such as waterbuck, pony, red deer, cattle and sheep result in differential trap catches of C. impunctatus females (Mands et al. 2004). Interestingly, there is anecdotal evidence that individual human hosts also show variation in their attractiveness, and it is hypothesised that more unattractive individuals produce 'masking compounds' that might indicate host unsuitability 
to midges (Mordue (Luntz) and Mordue 2003). Much evidence exists that individual human beings are differentially attractive to mosquitoes (Brady et al. 1997, Brouwer 1960, Burkot 1988, Khan et al. 1966, Lindsay et al. 1993, Mayer and James 1969, Qiu et al. 2004, Schreck et al. 1990) and there is much anecdotal evidence for biting midges. Recently, C. impunctatus has been shown to respond differentially to odours collected from individual human volunteers (Logan et al. 2009). Certain chemicals that were found in greater amounts in extracts from volunteers that caused low attractiveness, elicit a repellent effect in laboratory assays and repellency trials in the field. It is suggested that differences in the production of these natural human-derived compounds could help to explain differential 'attractiveness' between different human hosts to C. impunctatus. A mixture of two compounds in particular, 6-methyl-5-hepten-2-one and geranylacetone $((E)-6,10$ dimethylundeca-5,9-dien-2-one), showed significant repellency in the field and have the potential to be developed as novel repellents. These types of semiochemicals may interfere with olfactory mechanisms from a distance, preventing the insects from being attracted to their hosts, as has also been demonstrated for Ae. aegypti L. (Logan et al. 2008). Similar studies have shown that some individual cattle produce masking compounds such as the isoprenoid derivative, 6-methyl5-hepten-2-one, that make them less attractive to the fly, Hydrotaea irritans (Fallen), than others within a herd (Birkett et al. 2004).

Most chemicals that are described as causing positive behavioural responses in Culicoides and other haematophagous insects, such as $\mathrm{CO}_{2}$, 1-octen-3-ol, lactic acid, ammonia, acetone and fatty acids are found ubiquitously in all humans and in many other vertebrates. Logan and Birkett (2007), in their review of semiochemicals for biting fly control, proposed such chemicals may comprise a basic 'core' suite of olfactory signals that, when present, convey information to an insect that a vertebrate is nearby. Alteration of this 'core' by addition, or increase, of certain other chemicals that can 'mask' activity of these attractants, or act as repellents (Pickett et al. 1998), could be a way by which inappropriate or unsuitable vertebrate hosts are avoided by host-seeking insects.

There is some evidence that the production of repellents in some vertebrates is genetically determined (Thomas et al. 1987). If this is true for Culicoides hosts, it could be exploited to develop breeding programmes to select for unattractive individuals. However, our knowledge of Culicoides host preference to date is very poor for many Culicoides species and particularly for the vectors of BTV and therefore, this requires further investigation.

\section{Investigating host-derived semiochemicals for the control of midges}

The exploitation of host-derived semiochemicals in control technologies first requires an indepth knowledge and understanding of ecologically relevant compounds that are likely to be of practical use. It is therefore vital that we appreciate how target insects interact with such compounds at the behavioural and physiological level by investigating the ecology of the insecthost interaction. Various chemical ecology-based techniques can facilitate this. Semiochemicals can be isolated from relevant odour sources by air entrainment, solvent washings, solid phase micro extraction (SPME) and vacuum distillation. Behavioural and electrophysiological assays can then be performed to locate and identify biologically and ecologically relevant semiochemicals. Subsequent analytical chemistry techniques, such as gas chromatography (GC) and GC-mass spectrometry (GC-MS) can identify the chemicals accurately. Fractionation has been used in the past although this can be a laborious task with compounds being missed. A more efficient method is to use gas chromatography combined with electroantennography (GC-EAG) or single-sensillum recordings (GC-SSR), which is a unique and effective way of locating relevant components 
within a complex array of chemicals in natural extracts. Such techniques are used to identify semiochemicals that can be integrated into methods for the management and control of insect pests as well as enhancing our knowledge and understanding of the behaviour and evolution of mosquitoes and midges.

\section{Olfaction and electrophysiology}

Midges, like most haematophagous insects, have a well developed olfactory system and use their antenna and maxillary palps to detect semiochemicals from vertebrates, giving them information about the location and suitability of a host. A full understanding of how Culicoides detect olfactory signals from their host to date is a poorly studied area, but it is vital to the development of strategies aimed at controlling these insects (Grant and Kline 2003). Such an understanding of the olfactory process can provide important information about the way Culicoides midges detect and respond to host odour stimuli.

Insects detect odour molecules (kairomones) using olfactory receptors known as sensilla. The olfactory sensilla of different arthropods show a high degree of similarity (Hallberg and Hannson 1999). This suggests that there may consequently be a degree of similarity in odour responses of different arthropod species. Although this is the case for ubiquitous compounds such as $\mathrm{CO}_{2}$, it is often not the case for other olfactory stimuli. The sensilla are porous hairs located on the antennae and maxillary palps of the insect. Branched dendrites are found extending up into the hair and these dendrites join to a sheathed neurone, which has axons leading to the central nervous system. The sensilla are well designed for odour capture with the whole of the surface area involved in the process. An odour molecule impacting upon a sensillum diffuses laterally through the surface layers. These include an outer layer of polymerised lipid with a thin outer membrane composed of electron transport material, and an electron dense layer. After diffusing through these layers, the odour molecule reaches the receptor lymph where it is thought to be bound to an odourant binding protein (OBP) resulting in the formation of a complex. This complex is then bound by a receptor binding protein present in the membrane of the dendrite, which subsequently causes a change in membrane potential. The altered membrane potential allows an ion flux across it creating a receptor potential. The receptor potential may be large or small depending on the magnitude of the original stimulus. From the dendrite, the receptor potential spreads to the cell body and action potentials are produced. These action potentials are very rapid, lasting only a few milliseconds, and fire at different frequencies proportional to the magnitude of the receptor potential. Therefore, it can be seen how an impacting odour molecule is converted into electrical signals that can be processed by the central nervous system of the insect (Zwiebel and Takken 2004, see also Chapters 2 and 4 in this Volume). By connecting microelectrodes to an antennal preparation of an insect, the detection of a compound can be recorded as an electroantennogram (EAG), which is the electrical potential from the insect's antennae.

Culicoides antennae and maxillary palps have several olfactory sensilla types including trichodea which have multiporous walls and a number of neurones which are typical of olfactory receptors. They also possess basiconic and coeloconic sensilla which have double walls and unbranched dendrites which could be olfactory, thermo-, or hygroreceptors (Blackwell et al. 1992, Chu-Wang et al. 1975). Morphological characteristics of the olfactory apparatus, such as the size and shape of the subapical labral sensilla (also known to contain olfactory receptors), the size and depth of the palpal sensory pit, and the number and shape of heads of the palpal sensilla, can be used to classify and identify Culicoides species (Blackwell 2004, Braverman and Hulley 1979, Jamnback 1965, Lane 1984). In some cases, it is believed that differences in the number of sensilla coeloconica and 
palpal sensilla can even determine host preferences. For example, antennae having few antennal flagellomeres with pits and less coleloconic sensilla might be associated with the need to detect large amounts of odours and thus could be characteristic of mammalophilic species (Blackwell 2004, Jamnback 1965). Blackwell (2004) noted that some species found in the West Indies, such as C. darlingtonae Wirth and Blantone, C. glabellus Wirth and Blantone, C. insinuates Ortiz, C. paraensis (Goeldi), and C. pseudodiabolicus Fox are morphologically characteristic of mammalian feeders when compared to other species such as C. heliconiae Fox and Hoffman and C. flavivenula Lima whose host preferences were unknown. Although such morphological studies can be useful to identify certain Culicoides species, they may also provide some information about host preference and may even indicate the types of compounds they can detect. However, to fully understand the olfactory process, a physiological investigation is usually required.

To date, electrophysiological studies on Culicoides midges are scarce. Most work has been performed on the European species, C. nubeculosus, to look at the response of host odour extracts and individual compounds. Whilst laboratory models are useful, where possible, working with the insect of interest is preferable. It is also preferable to work with field caught insects as it has been shown for some insects, such as the tsetse fly, that responses can diminish within a few generations. The better studied (at the behavioural level) C. impunctatus has also been investigated using electrophysiology, but with more logistical difficulties due to the fact that this species is only available from field populations. Culicoides impunctatus females have a range of olfactory chemosensilla on their antennae that respond to host-derived chemicals (Bhasin et al. 2000b, Blackwell et al. 1996, Pappenberger 1996, Sutcliffe 1994) and on the maxillary palps that contain the receptors for $\mathrm{CO}_{2}$ (Bhasin et al. 2001). Culicoides impunctatus has antennal receptors that respond to a variety of other host derived kairomones including 1-octen-3-ol and other plantderived compounds including methyl salicylate (Blackwell et al. 1996, 1997). Blackwell et al. (1995, 1996) were the first authors to describe the olfactory basis of host seeking in C. impunctatus and since then, other authors have demonstrated EAG activity for other host-derived kairomones, including $\mathrm{CO}_{2}$, 1-octen-3-ol, phenols and acetone (Bhasin et al. 2000a, Blackwell et al. 1997, Grant and Kline 2003). Most recently, fifteen EAG-active compounds were found in human odour extracts and some new compounds for Culicoides midges were identified including benzaldehyde, 6-methyl-5-hepten-2-one, octanal, 2-ethylhexanol, limonene, dihydromyrcenol, nonanal, linalool, (E)-2-nonenal, menthol, naphthalene, decanal, geranylacetone and alpha-isomethylionone (Logan et al. 2009).

It is also thought that measuring the electrophysiological response of the receptors on the antennae can determine host preferences. For example host-odour extracts were assayed on parous female $C$. impunctatus response in a Y-tube olfactometer and by EAG on C. nubeculosus laboratory-reared parous females. Positive behavioural responses to host odours were dosedependent with a water buffalo extract being most active (threshold $0.22 \mathrm{~g} / \mathrm{ml}$ ), similar to deer, whereas other host extracts were less active. Correspondingly, the EAG threshold was lowest for water buffalo, 10-fold greater for deer, calf and pony, but not detected for sheep (Mands et al. 2004).

Recordings can also be made from single olfactory receptor neurones (ORNs) by inserting a microelectrode into the sensilla to detect which receptor is responding to specific compounds. In Culicoides, this has not been done for receptors on the antenna, but has for receptors on the maxillary palps. The sensilla basiconica, which are located on the maxillary palps of Culicoides, contain an ORN that responds to $\mathrm{CO}_{2}$ (Grant and Kline 2003) and shares physiological characteristics with mosquitoes (Grant et al. 1995). The $\mathrm{CO}_{2}$ receptor in Culicoides midges is thought to be extremely 
sensitive with the threshold of detection being significantly lower than that of mosquitoes. For example, stimulation with $150 \mathrm{ppm} \mathrm{CO}_{2}$ elicits a response in C. furens, whereas in Ae. aegypti, higher concentrations are required to elicit a response (Grant et al. 1995, Grant and O'Connell, this Volume). This suggests that Culicoides $\mathrm{ORNs}$ to $\mathrm{CO}_{2}$ are likely to be more active at ambient levels.

A study involving EAG is useful to screen whole extracts or single compounds that are likely to have ecological relevance to Culicoides midges. However, to fully understand how they find their hosts by way of olfaction and to identify novel semiochemicals, natural host odour, which comprises a complex mixture of relevant and irrelevant compounds, must be separated and tested. Screening all host-derived compounds (often in excess of 400 volatile chemicals) by EAG and in behavioural assays would be a time-consuming process and is highly impractical. A more efficient solution is to analyse human odour extracts by coupled gas chromatography-electroantennography (GC-EAG). This technique allows the location of EAG-active chemicals within complex extracts by taking advantage of the high-resolution of the GC while simultaneously recording the response of an antennal preparation of a female midge. This technique was first reported in 1969 (Moorhouse et al. 1969) and has been used for many insects (Logan et al. 2008, Pickett and Woodcock 1996). However, due to the difficulties with rearing Culicoides midges in the laboratory, electrophysiological techniques are complicated. Although portable EAG kits are available (see Syntech, http://www. syntech.nl/), they can be difficult to use. When searching for novel host-derived semiochemicals, it is essential to combine the electrophysiology techniques with that of gas chromatography. This poses a massive logistical problem with such high-tech equipment. However, in our laboratory, we are able to send C. impunctatus adult females (in a moist box), previously caught in Argyllshire in Scotland using a sweep net to our laboratories at Rothamsted in Hertfordshire (a distance of $\sim 400$ miles and taking around 24-48 hours). The coupled GC-EAG has been advanced to possess the capabilities of also detecting responses of single olfactory cells (GC-SCR) (Wadhams 1982), but this has never been performed on Culicoides midges.

Generally, insect olfactory systems are not only sensitive to specific molecular structures, but also to ubiquitous compounds. In the later case, sophisticated mechanisms such as coincidence detection, whereby blends of volatiles in specific ratios from a host plant are detected by insects within a complex background of volatiles from non-host plants, may be used. This might be facilitated by paired or clustered ORNs that allow fine scale resolution of such complex signals. Odours from a host are likely to occur in 'pockets' emanating from a host and therefore receptor cells that respond simultaneously may indicate the presence of a host, whereas the same receptor cells being stimulated with a delay would not (Bruce et al. 2005). Although this hypothesis has only been described for phytophagous (plant-feeding) insects thus far, coincidence detection might also occur in biting flies including Culicoides midges considering the complexity of odours within their environments (Chapter 6, this Volume).

\section{Laboratory-based experimentation}

To understand how an insect responds to natural semiochemicals, laboratory bioassays are commonly used as a first step. These behavioural experiments can be useful to determine the effect of semiochemicals on midge behaviour whilst minimising other environmental variables and provide a simple and rapid means of quantifying behavioural responses to many types of stimuli. Some of the earliest behavioural studies with haematophagous insects were conducted using host-seeking mosquitoes and used simple bioassays such as enclosed chambers or arenas to examine responses to temperature and moisture stimuli (Bar Zeeve 1960, Howlett 1910, Parker 1948, Thomson 1938). Such bioassays were only able to measure short range responses 
(Bowen 1992). More recently olfactometers that allow and encourage flight responses to odour stimuli have been developed, particularly for mosquitoes, and provide crucial information about responses of insects to olfactory stimuli in many investigations. Dual choice olfactometers, such as Y-tubes, have also been used with midges and mosquitoes and allow insects to respond to two air streams containing different odour stimuli, often comprising one test air stream containing a stimulus and one control (blank) air stream (Bhasin et al. 2000b, Blackwell et al. 1996, Brown et al. 1951, Dekker et al. 2001, Geier et al. 1999, Logan et al. 2008, 2009, Mands et al. 2004, Price et al. 1979, Roessler and Brown 1964, Smart and Brown 1957, Qiu et al. 2005).

For Culicoides midges, most laboratory-based olfactometer studies have been conducted with $C$. impunctatus. Due to the problems associated with rearing this and many other Culicoides species in the lab, these assays can be difficult. However, where possible, taking portable equipment to a field laboratory can overcome this. For example, on the West coast of Scotland, a portable Perspex wind tunnel and small glass Y-tube olfactometers (Blackwell et al. 1996, Bhasin et al. 2000b) have been used to examine the response of wild populations of Culicoides to odour stimuli. The end of the wind tunnel is placed outside a window of the field lab and the number of midges that fly upwind from outside can be counted by the number of flashes on a killing grid. For other experiments, insects can be caught by a sweep net and used in the olfactometer inside field laboratory (Logan et al. 2009). In these smaller Y-tubes, the insects walk towards a light and enter one of two arms which can contain an odour stimulus. Logan et al. (2009) showed that Culicoides midges respond to certain chemicals in the Y-tube olfactometer including benzaldehyde, 6-methyl-5-hepten-2one, octanal, 2-ethylhexanol, limonene, dihydromyrcenol, nonanal, linalool, (E)-2-nonenal, menthol, naphthalene, decanal, geranylacetone and alpha-isomethylionone.

In this Y-tube assay light is used to stimulate upwind movement towards the arms of the Y-tube, thus 'forcing' the midges to make a preferential movement to either arm. The Y-tube allows for walking and some attempts at flight but not sustained flight and as such is not entirely representative of a natural situation where $C$. impunctatus females would normally fly toward their host by way of positive odour mediated anemotaxis. Insects may not respond the same to odour stimuli when walking as opposed to flying (Kennedy 1977) and it might allow chemotactic responses to odour stimuli to occur. Additionally, there can be steep odour gradients at the interface between airstreams and this may allow chemotactic and chemokinetic responses to occur which might result in insects remaining within one air stream (Kennedy 1977). Most olfactometers may allow several behaviours to occur simultaneously such as orthokinesis, klinokinesis, chemotaxis and anemotaxis and do not allow the discrimination between close and long range responses. Therefore, caution must be taken when interpreting behavioural results. Despite such complications, olfactometric experiments are a useful tool designed to give an indication of the response of biting insects to odour stimuli under controlled conditions and are thus useful to test host odours without the host suffering the discomfort of being bitten. This is particularly important for Culicoides research where midge populations can be so immense that field trials become ethically unacceptable. Encouragingly, previous studies have demonstrated corresponding results between such laboratory trials and the field for biting midges. For example C. impunctatus show positive responses to 1-octen-3-ol in a Y-tube olfactometer in the laboratory and trap catches are enhanced by the addition of this compound to traps in the field (Bhasin et al. 2000b, Blackwell et al. 1996, Mands et al. 2004). With this in mind, it is important that studies are also conducted in the field to test semiochemicals in a more realistic and natural setting. 


\section{Control and field based experimentation}

Insecticide applications against adult biting midges have had a limited effectiveness especially when long-term relief from pestiferous populations is desired (Linley and Davies 1971). Furthermore, reduction of midge populations via larval control by the application of insecticides to the soil, or physical modification of wetland developmental sites, is not an option in many locations such as coastal wetlands and acidic bogs because immature developmental sites are extremely vast. Insecticide use in these areas is often forbidden due to environmental regulatory issues. Repellents only provide minimal and temporary relief and are not considered a long-term solution (Carpenter et al. 2005, Schreck and Kline 1981, Trigg 1996). Apart from altering one's life-style when midges are presented by staying indoors, repellents are presently the only means of self-protection. These provide an inexpensive reasonably efficient protection method against biting midges. DEET ( $N, N$-Diethyl-m-toluamide), available since 1957 , is still the most widely used repellent against insects and arthropods of medical importance such as mosquitoes and ticks and can show some efficacy against midges.

Ultimately, more effective control of Culicoides will need to rely on the development of integrated pest management programs using multiple control strategies to relieve the biting pressure of these pestiferous biting midges. New techniques are being investigated due in part to environmental concerns and desires to reduce the use of chemical insecticides. One technique that has received increased attention is the use of traps for control (Day and Sjogren 1994). The feasibility of reducing local biting midge populations through the use of removal trapping has recently been investigated by Kline and Lemire (1998; DL Kline unpublished data) in Florida, USA. They demonstrated that a single line barrier of either attractant-baited traps (CDC-type, Model 512, John Hock Company, Gainesville FL, USA) or cloth targets impregnated with an insecticide (lambdacyhalothrin) reduced mosquito and biting midge populations on a barrier island resort in Southwest Florida. Traps and targets were baited with $\mathrm{CO}_{2}$ and 1-octen-3-ol. Subsequently, Day et al. (2001) reported the reduction of $C$. furens populations in two delimited areas along the Atlantic coast in South Florida and on an island in the Bahamas, using insecticide-treated fabric targets baited with carbon dioxide and 1-octen-3-ol. This mass trapping programme against Culicoides resulted in a significant reduction in the number of Culicoides throughout the island. Cilek et al. (2003) and Cilek and Hallmon (2005) attempted to reduce Culicoides populations around individual homes in north-western Florida using either one Mosquito Magnet ${ }^{\oplus}$ Pro (American Biophysics Corporation, North Kingston RI, USA ${ }^{1}$ ) or one ABC PRO suction trap (Clarke Mosquito Products Inc., Roselle IL, USA) per backyard. The ABC PRO traps were baited with $\mathrm{CO}_{2}(500 \mathrm{ml} / \mathrm{min})$ and a 4:1:8 1-octen-3-ol/phenol mixture of 1 octen-3-ol:3-n-propylphenol:4-methylphenol, released from 16 $\mathrm{ml}$ screw capped glass vials via a single wick (Dills TM pipe cleaner, United States Tobacco Sales and Marketing, Greenwich, CT, USA). Overall release rates during the study averaged $5.39 \pm 0.54$ $\mathrm{mg} / \mathrm{h}$. Population reduction, however, was very inconsistent (ranged from $2.3 \%$ to $70.6 \%$ ) over time and these researchers concluded that 1 trap per backyard was insufficient for consistent Culicoides population reduction. They suggested that using more than one trap per backyard, or a perimeter of traps, might be the key to achieving consistent population reduction. At certain times during the study it was obvious that the attractant-baited traps were 'overwhelmed' by the sheer volume of adult blood seeking midges moving into the targeted control zone. Another possibility to achieve consistent population reduction was tested by Lloyd et al. (2008) by placing traps in all the adjacent neighbouring yards, which in effect, created a barrier along the perimeter of their backyards. Each trap was placed in the centre of a 0.30-0.40-ha area, which resulted in full

\footnotetext{
${ }^{1}$ American Biophysics Corp went out of business in 2006
} 
coverage of this residential area. In this study two commercially available attractant-baited traps were compared (two models of Mosquito Magnet ${ }^{\mathrm{TM}}$ [MM], American Biophysics Corporation, North Kingston RI, USA) on the island of Rye Key in Cedar Key, FL, USA to determine which model is more successful at capturing Culicoides spp. Functionally, the two trap models are similar with some difference in their bait-plume temperature ranges and $\mathrm{CO}_{2}$ output. The plume temperature for the MM-Freedom ${ }^{\circledast}$ (Freedom) ranges from $33.34{ }^{\circ} \mathrm{C}$ to $36.67{ }^{\circ} \mathrm{C}$ compared to $33.34{ }^{\circ} \mathrm{C}$ to $40.56{ }^{\circ} \mathrm{C}$ for the MM-Liberty Plus ${ }^{\circledR}$ (Liberty Plus) (K McKenzie, personal communication). Both traps utilised the counterflow geometry design (Kline 2002) and their suction fans were powered thermoelectrically by the combustion of propane, which also resulted in the production of the attractants $\mathrm{CO}_{2}$, moisture and heat. The Freedom produces ca. $420 \mathrm{ml} / \mathrm{min} \mathrm{CO}_{2}$, compared to ca. $550 \mathrm{ml} / \mathrm{min} \mathrm{CO}_{2^{\prime}}$ for the Liberty Plus. Both traps caught Culicoides spp. very well. Operationally, the Liberty Plus was the more reliable trap. There was an apparent species preference for the traps. The overall mean daily collection of $C$. furens in Freedom traps was significantly higher than the number collected in the Liberty Plus traps. In contrast C. mississippiensis selected the MM-Liberty Plus over the MM-Freedom. This could be due to temperature and $\mathrm{CO}_{2}$ differences between the traps. Culicoides furens is attracted to heat (Kline and Lemire 1995), $\mathrm{CO}_{2}$ (Kline et al. 1990) and 1-octen3-ol (Takken and Kline 1989). It is possible that the Freedom and Liberty Plus are presenting the attractants differently (Cooperband and Cardé 2006), which could affect a trap's ability to attract and capture host-seeking insects. Kline et al. (1994) reported that $C$. furens was the only species of three major coastal Culicoides species that were attracted to a combination of 1-octen-3-ol bait and $\mathrm{CO}_{2}$ in Sea Island, GA, USA. They reported that all Culicoides spp. were attracted to $\mathrm{CO}_{2}$. The combination of heat, 1-octen-3-ol and $\mathrm{CO}_{2}$ may be why $C$. furens was captured in such high numbers when compared with C. mississippiensis in this study. It is not certain what kairomones are used by C. mississippiensis during host location. Minimal work has been done with this species. What this means is that, for each target species, the trap type and attractant combination needs to be determined. This will be key to adult biting midge reduction using removal trap technology. For many Culicoides spp, especially those associated with livestock, this will require additional basic studies (such as those discussed earlier) to address this complex and challenging problem. The problem of protecting livestock on farms where a bite from a single viraemic midge is sufficient for transmission of BTV or AHSV remains a challenge (Mordue (Luntz) and Mordue 2006). Trap number and placement are also important points to consider. Just as important, adequate funding needs to be made available for such evaluations in order that innovative alternative control technologies can be transferred to the public in a timely manner.

\section{Push-pull control strategy}

Several ongoing research projects have evolved from these removal trapping studies to evaluating the feasibility of developing and utilising push-pull systems (DL Kline, unpublished data). Pushpull strategies involve the behavioural manipulation of pest species via the integration of stimuli that act to make the protected resource hard to locate, unattractive or unsuitable to the pests (push) while luring them toward an attractive source, such as an attractant-baited trap (pull) from where the pests are subsequently removed. Push-pull strategies maximise efficacy of behaviourmanipulating stimuli through the additive and synergistic effects of integrating their use (Cook et al. 2007). Development and use of push-pull strategies requires a thorough knowledge of a combination of behaviour-modifying stimuli that can be used to manipulate the distribution and abundance of pest insects for pest management. It requires a clear scientific understanding of the pest's biology and the behavioural/chemical ecology of the interactions with its hosts and its environment. The specific combinations of components differ in each strategy according to the pest species to be controlled (its specificity, sensory abilities, and mobility) and the resource 
targeted for protection, for example people or farm animals. The pests are repelled or deterred away from this resource (push) by using stimuli that mask host apparency or are repellents or deterrents. These stimuli may act over the long or short range and ultimately lead to the pest being repelled or deterred from the resource or not even approaching it. Long range stimuli represent the first line of defence, preventing or reducing infestation in the first place. Stimuli that act over the short range, however, can be powerful tools in preventing specific pestiferous behaviours (Cook et al. 2007). In pull components of push-pull strategies, attractive stimuli are used to divert pests from the protected resource to a trap or target. The stimuli used to achieve this act mostly over a long distance. However, short-range stimuli can be useful additions to arrest and retain the pests in a predetermined place to facilitate the concentration of their populations and to prevent them from returning to the protected resource. The stimuli can be delivered in a variety of ways. Strategies primarily include visual and chemical cues or signals. Manipulation of colour, shape, or size has been studied in a limited way in Australia. Chemical stimuli, in particular semiochemicals, have the most versatility and potential for use in pest management. The principles of the push-pull strategy are to maximise control efficacy, efficiency, sustainability, and output, while minimising negative environmental effects.

Development of effective 'push-pull' systems for biting midge population management has been hindered by a lack of resources to develop the necessary components. Limited research at several locations worldwide has been dedicated to studies necessary to reveal the details of biting midge biology, life cycles and chemical ecology that could be applied to their control by behavioural means. Studies at these locations have mainly focused on nuisance pests of people. Very little work has been conducted on the 'push' component. Some studies are underway in the U.S.A. to determine the 'masking' effect of human volatiles and compounds such as linalool and dehydrolinalool (DL Kline, unpublished data) on host attraction of Culicoides to humans and horses, and by exploiting compounds discovered during investigations on the natural differential attractiveness within a host species (U. Bernier, personal communication). In the UK, repellents such as 6-methyl-5-hepten-2-one and geranylacetone that were identified from odours of 'unattractive' volunteers are now under development as commercially available topical repellents. Other studies have focused on the effectiveness of synthetic repellent products with either DEET or Picaridin as the active ingredient. Several chemicals related to DEET have been tested against midges and shown to be similar or more effective than DEET with respect to length of and degree of protection. One such compound, Bayrepel ${ }^{\circ}$, tested by Carpenter et al. (2005) on the Scottish biting midge proved to be effective for up to 8 hours post application. Plant essential oils such as citronella and eucalyptus, PMD (p-menthane-3,8-diol), isolated from lemon eucalyptus oil of Eucalyptus citriodora Hook. have been found to be effective against C. impunctatus (Trigg 1996). Eucalyptus and other plant-derived repellents lose effectiveness more quickly with time than synthetic compounds such as DEET. Blackwell et al. (2004) demonstrated the effectiveness of neem against $C$. impunctatus and $C$. nubeculosus. Recently, spatial repellents, which repel from a distance under field conditions, have been identified for C. impunctatus (JG Logan, unpublished observation).

Most of the research efforts have been invested in determining what kinds of semiochemicals and traps/targets can be used in the 'pull' component. A limited number of the well known host kairomones that mediate behavioural responses in other haematophagous insects (Bursell et al. 1988, Hall et al. 1984, Hassanali et al. 1986) have been shown to be active in the field against biting midges. Kairomones such as those found in breath, skin emanations and urine are the main sensory cues used by haematophagous insects to find their hosts. The emphasis has been on kairomones associated with humans and livestock including mammalian-associated volatiles such 
as $\mathrm{CO}_{2}, 1$-octen-3-ol and acetone from the breath, and a mix of body odours (Bhasin et al. 2001, Gibson and Torr 1999, Logan and Birkett 2007). Carbon dioxide $\left(\mathrm{CO}_{2}\right)$ was first shown by Nelson (1965) to be an attractant for members of the C. variipennis complex and, more recently, 1-octen3-ol has been used to trap C. furens (Kline et al. 1990, Takken and Kline 1989). Carbon dioxide has a particularly important role in the attraction of haematophagous Diptera to their hosts (Gillies 1980). Stimulation by above-threshold levels of the gas, downwind of the host, activates them to respond to other kairomones and elicits upwind orientation (Bursell 1984, Healy and Copland 1995, Schofield and Brady 1997). It is released periodically from a mammalian host at an expired concentration of $4-5 \%$ above background $(0.03 \%)$, and forms a filamentous plume, the exact structure of which is determined by factors such as source size and wind speed (Brady et al. 1989, Hargrove et al. 1995, Chapter 5 in this Volume). Response thresholds for activation by $\mathrm{CO}_{2}$ are typically low: 0.006-0.01\% for Stomoxys calcitrans (L.) (Schofield et al. 1997, Warnes and Finlayson 1985 ) and $0.01 \%$ for tsetse (Bursell 1984). Combination baits used have typically utilised $\mathrm{CO}_{2}$ and 1-octen-3-ol (Ritchie et al. 1994, Takken and Kline 1989). Field studies have shown that $\mathrm{CO}_{2}$ and 1-octen-3-ol act together to exert a synergistic effect on catches of $C$. furens (Kline et al. 1994). Maximal upwind responses occur to $\mathrm{CO}_{2}$ plus 1-octen-3-ol or $\mathrm{CO}_{2}$ plus acetone but only at host emission concentrations with higher concentrations causing arrestment (Bhasin et al. 2001). A combination of 1-octen-3-ol and a pheromone produced by a host-seeking female C. impunctatus has shown activity, both in the field and in the laboratory (Blackwell et al. 1996). For the Scottish biting midge traps containing host kairomones such as $\mathrm{CO}_{2}$ with 1-octen-3-ol, acetone, a mixture of alkyl phenols, or human sweat components have been tested in the field (Bhasin et al. 2001, W Takken and AJ Mordue (Luntz), unpublished data) and promising combinations of attractant cues are being defined. Our work and that of others is beginning to reveal that certain human sweat components such as 3-methyl-2-hexanoic acid or 7-octenoic acid are either attractive to, or ignored by, midges in their repertoire of host attractants (AJ Mordue (Luntz) and J Pickett, unpublished data). Using knowledge of host specificity and preferences, the attractiveness of synthetic host odour blends can be maximised.

One difficulty with the current emphasis on host-seeking kairomones is that some species like C. impunctatus (Blackwell et al. 1992) and C. mississippiensis (Davis 1981) are autogenous species (Blackwell et al. 2004), which produce a significantly larger egg-batch in their first, host-free, period of oogenesis (Blackwell et al. 1992, Boorman and Goddard 1970). This, together with the paucity of blood meals in most areas of the highlands, would suggest that strategies acting upon the parous, biting proportion of the C. impunctatus population are unlikely to lead to more than short-term changes in the biting rates experienced, without constant, high intensity trapping. Thus research efforts should also be made to discover potential nectar sources. Both sexes utilise nectar and sugar feeding for various life cycle activities. Nectar sources are of particular importance because nectar provides energy for sustained flight in mating swarms (Downes 1969), increases adult longevity (Linley 1966a), and may play a role in egg maturation (Linley 1966b). The females of some species are not able to survive to the time of the first oviposition without nectar (Downes 1958). Volatiles from flowers may be utilised by adult biting midges to locate a nectar source. If these volatiles can be isolated and identified they can be used as attractants with traps or targets. Possible nectar sources have been determined for adult C. mississippiensis near Yankeetown, Levy County, Florida. Large numbers of adults have been found in early spring on flowering shrubs such as llex vomitoria Aiton. Other habitat related cues (apneumones) may be used by females to locate suitable oviposition or resting sites. Carpenter et al. (2001) identified the odour of Juncus spp. infusions together with the visual cue of upper layer photosynthetic sphagnum to be important in the induction of oviposition of C. impunctatus. 
Numerous types of traps are available for use in the 'pull' component. Only the Midgeater and Midg-IT were developed specifically for biting midges. Their design is very similar to the Mosquito Magnet ${ }^{\text {TM }}$ series of traps. A plethora of traps with various killing mechanisms including electrocution, drowning, sticky boards, live catching and desiccation, originally developed for mosquito control have been evaluated for their efficacy in capturing biting midges (DL Kline, unpublished data). The most widely used trap is the MM-Liberty Plus. New advances in trap technology continually need to be identified and subsequently evaluated. Various commercially available lures (several manufacturers of 1-octen-3-ol lures, Lurex [lactic acid], Lurex3 [lactic acid + ammonium bicarbonate] and BG Mesh lure [lactic acid + ammonia + propionic acid] as well as proprietary experimental lures, are currently being evaluated at Cedar Key, FL, USA. Visual aspects of trap design on biting midge attraction have not been evaluated very much, but need to be. Colour combinations, size and shape may be very important. Visual factors may enhance the effectiveness of the olfactory stimuli. For example, blue and black traps, approximating the size of a mammalian host, are used to control cattle tsetse fly (Glossina spp.). Crucial to the development of efficient traps was the finding that black stimulates landing (Gibson and Torr 1999). Traps need to be designed with visual (A Bhasin, unpublished observation) and olfactory 'pull' stimuli that help them compete effectively with the hosts that they are trying to protect and with other surrounding environmental stimuli. Trap design and positioning are important and can be maximised by adopting a systematic approach in which the behaviour of the insect is closely observed.

\section{Culcioides and climate change}

Changes in distribution and abundance of insects are likely to be amongst the most important and immediate effects of climate change (Wittmann and Baylis 2000). In addition to increases in temperature, changes in precipitation, wind patterns and climate variability are also predicted to occur (Houghton 1997). The global atmospheric concentration of $\mathrm{CO}_{2}$ is rising markedly as a result of human activity. The average level has increased from about $280 \mathrm{ppm}$ immediately before the industrial revolution to a daily average of 380 ppm in 2005 and is increasing at a rate of 2 ppm per year (Guerenstein and Hildebrand 2008). Such an increase in $\mathrm{CO}_{2}$ is expected to affect the biology of a number of living organisms including insects and therefore is stimulating research on what those effects could be. Mondor et al. (2004) quoted an Intergovernmental Panel on Climate Change (IPCC) report from 2001 which states $\mathrm{CO}_{2}$ and ozone $\left(\mathrm{O}_{3}\right)$ have risen $31 \%$ and $35 \%$ respectively since the mid-1800s. As well as contributing to effects of temperature, and the aforementioned effects on insects, these increasing levels of $\mathrm{CO}_{2}$ and $\mathrm{O}_{3}$ affect plants considerably. Elevated concentrations of these gases alter the nutritional and defensive characteristics of plants, and subsequently these effects can cascade through ecosystems and in turn impact upon higher trophic levels, such as insect herbivores and their natural enemies (Percy et al. 2002). All these changes may help create novel vector species by removing some of the barriers that render many Culicoides species refractory to infection. It is worth noting that it is not just the adult insects that may be affected. Borkent (2005) states that a variety of environmental factors have been studied for a few pest species, and the results indicate that temperature, moisture levels, chemical composition of the substrate, and population density all affect the rate of development of immatures.

Climate and weather have dramatic effects on Culicoides populations and, consequently, the epidemiologies of midge-borne viral diseases are similarly affected (Mellor et al. 2000). Due to their small size, adult Culicoides are particularly susceptible to desiccation and even brief periods at low humidity can reduce longevity (Murray 1991). However, changing meteorological factors 
are not necessarily detrimental to the insects. While there are limits to vector competence, for example BTV and AHSV are unable to develop in C. variipennis sonorensis at temperatures below $14-15^{\circ} \mathrm{C}$ (Mullens 1995, Wellby et al. 1996, Wittmann 2000), as stated previously, suitable changes in temperature may allow an increase in viable vectors.

Arndt (1995) investigated whether air pollutants react with pheromones, which would have potential ecological and economic consequences. Specifically, he studied the effects of ozone $\left(\mathrm{O}_{3}\right)$ on the pheromone-producing insect, Drosophila melanogaster Meigen. It was found that total pheromone containing extracts as well as commercially available pheromones lost their biological activity after short-term ozone fumigation at environmentally realistic levels. At the time the authors could find only one other reference relating to the subject. These findings, if true for other insects, suggest that climate change may have great impact on intraspecific and feasibly interspecific communication, particularly with the involvement of pheromones such as those for C. impunctatus and C. nubeculosus.

More recently Mondor et al. (2004) showed the existence of divergent pheromone-mediated behaviours in insects under conditions of global atmospheric change. Elevating $\mathrm{CO}_{2}$ and $\mathrm{O}_{3}$ levels, they investigated effects on a common aphid, Chaitophorus stevensis Sanborn. They showed that the aphids principle means of defence, dispersal response to alarm pheromone, decreased under elevated $\mathrm{CO}_{2}$, however, increased under elevated $\mathrm{O}_{3}$. Although the mechanism behind this differential dispersal response remains unknown, it is clear that elevated greenhouse gases may impact greatly upon intraspecific olfactory communication.

A further impact of climate change may be upon the voltinism of a species. Culicoides impunctatus, for example, is bivoltine, with two distinct emergence periods (Blackwell 1997) and has recently been shown to be orally susceptible to BTV in a laboratory study (Carpenter et al. 2006). However, alterations to temperature may create favourable conditions that allow additional generations within a given year. Tobin et al. (2008) investigated such effects using a phenology model of the grape berry moth, Paralobesia viteana (Clemens). It was designed to incorporate temperaturedependent development and diapause termination, as well as photoperiod-dependent diapause induction. They found that increases in mean surface temperature of greater than $2{ }^{\circ} \mathrm{C}$ can cause shifts in the ovipositional period resulting in dramatic effects on insect voltinism. Interestingly, they mention anecdotal evidence from grape growers that already suggests a shift, with increases in late summer populations. This will affect hosts and susceptibility and could even alter host preferences with changing farm animals and farming practices.

Such effects may have substantial implications not just from an ecological perspective, but also for how insect-derived compounds are used in pest management programmes. Different trapping methods can suggest different activity patterns and some meteorological conditions can affect the efficiency of trapping as well as the activity of the midge (Mellor et al. 2000).

Grant and Kline (2003) suggest that that a larger change in stimulus concentration is required to elicit a larger change in impulse activity than Aedes spp. mosquitoes indicating that Culicoides spp. perceive larger changes in carbon dioxide concentration than Aedes spp. It is possible that $\mathrm{CO}_{2}$ at low concentrations is similar to that produced by photosynthesising plants and therefore, in conjunction with other plant-derived volatiles, may provide information enabling location of nectar for sugar feeding. Additionally, higher concentrations may serve as a host-seeking attractant for blood feeding. Ambient levels of $\mathrm{CO}_{2}$ are higher now than ever and therefore the $\mathrm{CO}_{2}$-sensitive neurons are possibly adapted to lower, pre-industrialisation levels of carbon dioxide 
(see also Chapter 5, this Volume). This could simply reflect an earlier sensitivity, which has not genetically adapted to the dramatic $\mathrm{CO}_{2}$ increase caused by accelerated human-based emissions of carbon dioxide (Grant and Kline 2003). With a higher level of ambient $\mathrm{CO}_{2}$ this could be altering the ability of Culicoides spp. midges to respond to $\mathrm{CO}_{2}$. Because ambient concentrations are much higher, more subtle (or smaller) changes in $\mathrm{CO}_{2}$ need to be detected when host-seeking. This could hamper the ability of Culicoides spp. midges to host-seek and mosquitoes may have adapted to be able to do this. This demonstrates that specific information about Culicoides spp. midges is vital for the development of odour baited traps using $\mathrm{CO}_{2}$ and other semiochemicals as the responses at the physiological and behavioural level differs to that of other haematophagous insects. However, this sensitivity to low concentrations of $\mathrm{CO}_{2}$ may not have any effect on behaviour.

\section{Conclusions and future prospects}

For many Culicoides species, most aspects of their ecology and behaviour, and in particular the olfactory processes, remain undefined. This is in part due to the fact that Culicoides research is hindered by the difficulties associated with rearing these insects. Although $C$. sonorensis and $C$. nubeculosus have been successfully colonised, many species, including some other vectors of BTV, have not. This means that most studies are carried out with the 'laboratory models' or have to rely on field populations. For C. impunctatus, work has been carried out leading towards their successful colonisation. Suitable conditions for adult blood feeding, oogenesis, oviposition and adult survival (Carpenter et al. 2001, 2006), in the laboratory have been established but culture of the larvae remains a challenge for this species. Additional colonies of Culicoides species would allow much needed basic studies under controlled laboratory conditions to be carried out.

There are many other aspects of midge ecology that need to be elucidated. For example, climatic conditions heavily influence host location behaviour. Although there is some knowledge for species like $C$. impunctatus, other species are unstudied. This lack of knowledge, and particularly in light of climate change, is likely to affect and potentially hamper future control efforts. Additionally, oviposition site cues, those associated with the location of nectar, and pheromones involved in mate recognition and/or aggregation behaviour could provide new semiochemical tools. Investigating host preferences (at the intra and interspecific level) has already revealed potential new semiochemicals that could be exploited to develop better attractants for traps and new repellents for protection. Taking this further and understanding how unattractive odours are produced by vertebrates at the molecular/genetic level could lead to new breeding programmes whereby the trait for being unattractive is selected for. All of these areas of research warrant further investigation.

The combination of attractants and repellents could provide a push-pull control strategy, but further research is required on how this could be achieved for the many different Culicoides species. Additionally, formulation and delivery systems studies are needed to provide optimum technologies for specific species in specific geographical regions. Synthetic production of semiochemicals, and their formulation as sprays or in slow-release dispensers, will ensure standardisation and will contribute to the robustness of such strategies. GIS technologies could also be incorporated into biting midge control programs. An improved understanding of the spatial-scale effects of pest population dynamics and potential host interactions, coupled with increased capability of spatially explicit computer models, will enable us to deploy more accurate components of the various management strategies in terms of the quantities needed and their spatial distribution. Ultimately, a better understanding of the behaviour of Culicoides species midges, enabled by advances in analytical techniques, synthesis procedures, and formulation 
science, may provide us with a larger and more effective armoury of semiochemicals and other stimuli for future use.

\section{References}

Allingham PG (1991) Effect of temperature on late immature stages of Culicoides brevitarsis (Diptera: Ceratopogonidae). J Med Entom 28: 876-881.

Arndt U (1995) Air pollutants and pheromones - a problem? Chemosphere 30(6): 1023-1031.

Baldet T, Delécolle JC, Cêtre-Sossah C, Mathieu B, Meiswinkel R and Gerbier G (2008) Indoor activity of Culicoides associated with livestock in the bluetongue virus (BTV) affected region of northern France during autumn 2006. Prev Vet Med 87(1-2): 84-97.

Bar-Zeeve (1960) The reaction of mosquitoes to moisture and high humidity. Entomologica Experimentalis et Applicata 3: 198-211.

Baylis M and Rawlings P (1998) Modelling the distribution and abundance of Culicoides imicola in Morocco and Iberia using climatic data and satellite imagery. Arch Virol Suppl 14: 137-153.

Baylis M, Mellor, PS, Wittmann EJ and Rogers DJ (2001) Prediction of areas around the Mediterranean at risk of bluetongue by modelling the distribution of its vector using satellite imaging. Vet Record 149: 639-643.

Bernier UR, Booth MM and Yost RA (1999) Analysis of human skin emanations by gas chromatography mass spectrometry. 1. Thermal desorption of attractants for the yellow fever mosquito (Aedes aegypti) from handled glass beads. Analytical Chemistry 71: 1-7.

Bhasin, A (1996) Host location cues of Culicoides spp. (Diptera: Ceratopogonidae). PhD thesis, Department of Zoology. University of Aberdeen, Aberdeen, UK. 260 pp.

Bhasin A, Mordue AJ and Mordue W (2000a) Responses of the biting midge Culicoides impunctatus to acetone, $\mathrm{CO}_{2}$ and 1-octen-3-ol in a wind tunnel. Med Vet Ent 14: 300-307.

Bhasin A, Mordue AJ and Mordue W (2000b) Electrophysiological and behavioural identification of host kairomones as olfactory cues for Culicoides impunctatus and C. nubeculosus. Phys Ent 25: 6-16.

Bhasin A, Mordue AJ and Mordue W (2001) Field studies on efficacy of host odour baits for the biting midge Culicoides impunctatus in Scotland. Med Vet Ent 15: 147-156.

Bidlingmayer WL (1994) How mosquitos see traps - role of visual responses. J Am Mosq Contr Ass 10: 272-279.

Birkett MA, Agelopoulos N, Jensen V, Jespersen MB, Pickett JJA, Prijs J, Trapman HTGJ, Wadhams JLJ and Woodcock CM (2004) The role of volatile semiochemicals in mediating host location and selection by nuisance and diseasetransmitting cattle flies. Med Vet Ent 18: 313-322.

Bishop AL (2002) The response of Culicoides brevitarsis to livestock undercover. Report to Biosecurity Australia, Canberra, ACT, Australia.

Bishop AL, McKenzie HJ and Spohr LJ (2008) Attraction of Culicoides brevitarsis Kieffer (Diptera: Ceratopogonidae) and Culex annulirostris Skuse (Diptera: Culicidae) to simulated visual and chemical stimuli from cattle. Aus J Entom 47: 121-127.

Bishop AL, McKenzie HJ, Barchia IM and Harris AM (1996) Effect of temperature regimes on the development, survival and emergence of Culicoides brevitarsis Kieffer (Diptera: Ceratopogonidae) in bovine dung. Aus J Entom 35: 361368.

Blackwell A (1997) Diel flight periodicity of the biting midge Culicoides impunctatus and the effects of meteorological conditions. Med Vet Ent 11:361-367.

Blackwell A. (2004) A morphological investigation of Culicoides spp. biting midges (Diptera: Ceratopogonidae) from the Caribbean. J Vector Ecol 29: 51-61.

Blackwell A, Brown M and Mordue W (1995) The use of an enhanced Elisa method for the identification of Culicoides bloodmeals in host-preference studies. Med Vet Ent 9:214-218.

Blackwell A, Dyer C, Mordue AJ, Wadhams LJ and Mordue W (1994a) Field and laboratory evidence for a volatile pheromone produced by parous females of the Scottish biting midge, Culicoides impunctatus. Phys Entom 19: 251-257. 
Blackwell A, Dyer C, Mordue (Luntz) AJ, Wadhams LJ and Mordue W (1996) The role of 1-octen-3-ol as a host-odour attractant for the biting midge, Culicoides impuctatus Goetghebuer and the interactions of 1-octen-3-ol with a volatile pheremone produced by females. Phys Entom 21: 15-19.

Blackwell A, Evans KA, Strang RHC and Cole M (2004) Toward development of neem-based repellents against the Scottish Highland biting midge Culicoides impunctatus. Med Vet Ent 18: 449-452.

Blackwell A, Mordue AJ, Young MR and Mordue W (1992) Bivoltinism, survival rates and reproductive characteristics of the Scottish biting midge, Culicoides impunctatus (Diptera, Ceratopogonidae) in Scotland. Bull Ent Res 82: 299-306.

Blackwell A, Mordue (Luntz) AJ and Mordue W (1994b) Identification of bloodmeals of the Scottish biting midge, Culicoides impunctatus, by indirect enzyme-linked-immunosorbent-assay (Elisa). Med Vet Ent 8: 20-24.

Blackwell A, Wadhams LJ, and Mordue W (1997) Electrophysiological and behavioural studies of the biting midge, Culicoides impunctatus Goetghebuer (Diptera: Ceratopogonidae): Interactions between some plant-derived repellent compounds and a host-odour attractant, 1-octen-3-ol. Phys Entom 22: 102-108.

Boorman J and Goddard PG (1970) Observations on the biology of Culicoides impunctatus (Diptera: Ceratopogonidae) in southern England. Bull Ent Res 60: 189-198.

Borkent A (2004) The biting midges, the Ceratopogonodae (Diptera). In: Marquardt WC (ed) Biology of disease vectors. $2^{\text {nd }}$ Edition. Elsevier Academic Press, Burlington, USA, pp 113-126.

Bowen MF (1992) Terpene-sensitive receptors in female Culex pipiens mosquitoes: electrophysiology and behavious. J Insect Phys 38: 759-764.

Brady, J., Costantini, C., Sagnon, N., Gibson \& Coluzzi, M. 1997. The role of body odours in the relative attractiveness of different men to malarial vectors in Burkina Faso. Ann Trop Med Parasitol, 91, S121-S122.

Brady J, Gibson G and Packer MJ (1989) Odour movement, wind direction and the problem of host-finding by tsetse flies. Phys Entom 8: 329-333.

Braverman Y and Hulley PE (1979)The relationship between the numbers and distribution of some antennal and palpal sense organs and host preference in some Culicoides (Diptera: Ceratopogonidae) from southern Africa. J Med Entomol 15: 419-424.

Brouwer R (1960) Variations in human body odour as a cause of individual differences of attraction for malaria mosquitoes. Trop Geogr Med 12: 186-192.

Brown AWA, Sarkaria DS and Thompson RP (1951) Studies on the responses of the female Aedes mosquito. 1. The search for attractant vapours. Bull Ent Res 42: 105-106.

Bruce, TJA, Wadhams, LJ and Woodcock, CM (2005) Insect host location: a volatile situation. Trends Plant Sci 10: 269-274.

Burkot TR (1988) Non-random host selection by anopheline mosquitoes. Parasitol Today 4: 156-162.

Bursell $\mathrm{E}$ (1984) Observations on the orientation of tsetse flies (Glossina pallidipes) to wind-borne odours. Phys Entom 9: 133-137.

Bursell E, Gough AJE, Beevor PS, Cork A, Hall DR and Vale GA (1988) Identification of components of cattle urine attractive to tsetse flies, Glossina spp. (Diptera: Glossinidae). Bull Ent Res 78: 281-291.

Campbell AE and Pellham-Clinton EC (1960) A taxonomic review of the British species of Culicoides latrielle (Diptera: Ceratopogonidae). Proc R Soc Lond [B]67: 181-302.

Caracappa S, Torina A, Guercio A, Vitale F, Calabro A, Purpari G, Ferrantelli V, Vitale M and Mellor PS (2003) Identification of a novel bluetongue virus vector species of Culicoides in Sicily. Vet Rec 153: 71-74.

Carpenter S, Eyres K, McEndrick I, Smith L, Turner J, Mordue W and Mordue (Luntz) AJ (2005) Repellent efficiency of BayRepel against Culicoides impunctatus (Diptera: Ceratopogonidae). Parasitol Res 95: 427-429.

Carpenter S, Lunt HL, Arav D, Venter GJ and Mellor PS (2006) Oral susceptibility to Bluetongue virus of Culicoides (Diptera: Ceratopogonidae) from the United Kingdom. J Med Entomol 43: 73-78.

Carpenter S, Mordue (Luntz) AJ, and MordueW (2001) Oviposition in Culicoides impunctatus under laboratory conditions. Entomologia Experimentalis et Applicata 101: 123-129.

Chu-Wang I-W, Axtell RC and Kline DL (1995) Antennal and palpal sensilla of the sand fly Culicoides furens (Poey) (Diptera: Ceratopogonidae). Int J Insect Morphol Embryol 4: 131-149.

Cilek JE and Hallmon CF (2005) The effectiveness of the Mosquito Magnet trap for reducing Culicoides (Diptera: Ceratopogonidae) populations in coastal residential backyards. J Am Mosq Contr Ass 21: 218-221. 
Cilek JE, Kline DL and Hallmon CF (2003) Evaluation of a novel removal trap system to reduce Culicoides (Diptera: Ceratopogonidae) populations in Florida backyards. J Vector Ecol 28: 23-30.

Conte A, Goffredo M, Ippoliti C, Meiswinkel R (2007) Influence of biotic and abitotic factors on the distribution and abundance of Culicoides imicola and the obsoletus Complex in Italy. Vet Parasitol 150: 333-344.

Cook SM, Khan ZR and Pickett JA (2007) The use of push-pull strategies in integrated pest management. Ann Rev Entomol 52: 375-400.

Cooperband MF and Carde RT (2006) Comparison of plume structures of carbon dioxide emitted from different mosquito traps. Med Vet Ent 20: 1-10.

Davies JB (1969) Effect of felling mangroves on emergence of Culicoides spp. Jamaica Mosquito News 29: 566-571

Davis EL (1981) Laboratory studies on life cycle development and adult blood-feeding of Culicoides mississippiensis Hoffman (Diptera: Ceratopogonidae), MSc Thsesis, University of Florida, Gainesville, FL, USA.

Day JF and Sjogren RD (1994) Vector control by removal trapping. Am J Trop Med Hyg50: 126-133.

Day JF, Duxbury CV, Glasscock S and Paganessi JE (2001) Removal trapping for the control of coastal Culicoides populations. Techn Bull Florida Mosquito Contr Ass 3: 15-16.

DEFRA (2008) Bluetongue: latest situation. Department for Environment, Food and Rural Affairs (DEFRA), UK. Available at: http://www.defra.gov.uk/foodfarm/farmanimal/diseases/atoz/bluetongue/latest/index.htm. Accessed July 2008.

Dekker T, Takken W and Cardé RT (2001) Structure of host-odour plumes influences catch of Anopheles gambiae s.s. and Aedes aegypti in a dual-choice olfactometer. Phys Entom 26: 124-134.

De Koeijer AA and Elbers ARW (2007) Modelling of vector-borne diseases and transmission of bluetongue virus in Northwest Europe. In: Takken W. and Knols BGJ (eds) Emerging pests and vector-borne diseases in Europe. Ecology and control of vector-borne diseases, vol. 1. Wageningen Academic Publishers, Wageningen, the Netherlands, pp. 99-112.

Downes JA (1950) Habits and life cycle of Culicoides nubeculosus Mg. Nature 166: 510-511.

Downes JA (1955) Observations on the swarming flight and mating of Culicoides (Diptera: Ceratopogonidae). Transcr Royal Entomol Soc London 106: 213-236.

Downes JA (1958) The feeding habits of biting flies and their significance in classification. Ann Rev Entomol 3: 249-266.

Downes JA (1969) The swarming and mating flight of Diptera. Ann Rev Entomol 14: 271-98.

Geier M and Boeckh J (1999) A new Y-tube olfactometer for mosquitoes to measure the attractiveness of host odours. Entomologia Experimentalis et Applicata 92: 9-19.

Geier M, Bosch OJ and Boeckh J (1999) Ammonia as an attractive component of host odour for the yellow fever mosquito, Aedes aegypti. Chem Senses 24: 647-653.

Gibson G and Torr SJ (1999) Visual and olfactory responses of haematophagous Diptera to host stimuli. Med Vet Ent. 13: $2-23$.

Gillies MT (1980) The role of carbon dioxide in host-finding by mosquitoes (Diptera: Culicidae): a review. Bull Ent Res 70: 525-532.

Grant AJ, Wigton BE, Aghajanian JG and O'Connell RJ (1995) Electrophysiological responses of receptor neurons in mosquito maxillary palp sensilla to carbon dioxide. J Comp Physiol [A] 177: 389-396.

Grant AJ and Kline DL (2003) Electrophysiological responses from Culicoides (Diptera: Ceratopogonidae) to stimulation with carbon dioxide. J Med Entomol 40: 284-292.

Guerenstein PG and Hildebrand JG (2008) Roles and effects of environmental carbon dioxide in insect life. Ann Rev Entomol 53: 161-178.

Hall DR, Beevor PS, Cork A, Nesbitt BF and Vale GA (1984) 1-octen-3-ol, a potent olfactory stimulant and attractant for tsetse isolated from cattle odours. Insect Sci Appl 5: 335-339.

Hallberg E and Hannson BS (1999) Arthropod sensilla: morphology and phylogenetic considerations. Microsc Res Tech47: 429-440.

Hargrove JW, Holloway MTP, Vale GA, Gough AJE and Hall DR (1995) Catches of tsetse flies (Glossina spp, Diptera: Glossinidae) from traps and target baited with large doses of natural and synthetic host odor. Bull Ent Res 85: 215-227. 
Hassanali A, McDowell PG, Owaga MLA and Saini PK (1986) Identification of tsetse attractants from excretory products of a wild host animal, Syncerus caffer. Insect Sci Appl 7: 5-9.

Healy TP and Copland MJW (1995) Activation of Anopheles gambiae mosquitoes by carbon dioxide and human breath. Med Vet Ent 9: 331-336.

Hendry G and Godwin G (1988) Biting midges in Scottish forestry: a costly irritation or a trivial nuisance? Scottish Forestry 42: 113-119.

Hill MA (1947) The life-cycle and habits of Culicoides impunctatus Goetghebuer and Culicoides obsoletus Meigen, together with some observations on the life-cycle of Culicoides odibilis Austen, Culicoides pallidicornis Kieffer, Culicoides cubitalis Edwards and Culicoides chiopterus Meigen. Ann Trop Med Parasitol 41: 55-115.

Holbrook FR and Tabachnick WJ (1995) Culicoides variipennis (Diptera: Ceratopogonidae) complex in California. J Med Entomol 32: 413-419.

Houghton J (1997) Global warming: the complete briefing. $2^{\text {nd }}$ edition. Cambridge University Press, Camebridge, UK.

Jamnback H (1965) The Culicoides of New York state (Diptera: Ceratopogonidae). New York state Museum and Science Service, Albany, USA. Bulletin 399, 154 pp.

Howlett FM (1910) The influence of temperature upon the biting mosquitos. Parasitology 3: 479-484.

IAH (2008a) Bluetongue virus-infected adult midges surviving winter may have been responsible for the re-emergence of bluetongue this year in northern Europe. Institute of Animal Health, UK. Available at: http: //www.iah.ac.uk/ press_release/BT_UK_2007/BT_Statement2.html. Accessed July 2008.

IAH (2008b) Institute for Animal Health diagnoses bluetongue in Suffolk. Institute of Animal Health, UK. Available at: http: //www.iah.ac.uk/press_release/BT_UK_2007/BT_Statement1.html. Accessed July 2008.

Jennings DM and Mellor PS (1988) The vector potential of british Culicoides species for bluetongue virus. Vet Microbiol 17: 1-10.

Kennedy JS (1977) Behaviourally discriminating assays of attractants and repellents. In: Shorey HH and McKelvey JJ [eds], Chemical control of insect behaviour. John Wiley \& Sons, New york, USA, pp 91-98.

Kettle DS (1965) Biting Ceratopogonids as vectors of human and animals diseases. Acta Tropica, Basel 22(4): 356-362.

Kettle DS (1984) Medical veterinary entomology. Croom Helm, London, UK.

Kettle DS and Lawson JWH (1952) The early stages of British biting midges Culicoides Latreille (Diptera: Ceratopogonidae) and allied genera. Bull Ent Res 43: 421-467.

Khan AA (1977) Mosquito attractants and repellents. In: Shorey HH and McKelvey JJ (eds) Chemical control of insect behaviour. John Wiley \& Sons, New York. pp 305-325.

Khan AA, Maibach HI, Strauss WG and Fenley WR (1966) Quantitation of effect of several stimuli on the approach of Aedes aegypti. J Econ Entomol 59: 690-694.

Kline DL (1994) Olfactory attractants for mosquito surveillance and control - 1-octen-3-ol. J Am Mosq Contr Ass 10: 280-287.

Kline DL (2002) Evaluation of various models of propane-powered mosquito traps. J Vector Ecol 27: 1-7.

Kline DL and Lemire GF (1995) Field evaluation of heat as an added attractant to traps baited with carbon dioxide and octenol for Aedes taeniorhynchus. J Am Mosq Contr Ass 11: 454-456.

Kline DL and Lemire GF (1998) Evaluation of attractant-baited traps/targets for mosquito management on Key Island, Florida, USA. J Vector Ecol 23: 171-185.

Kline DL, Hagan DV and Wood JR (1994) Culicoides responses to 1-octen-3-ol and carbon dioxide in salt marshes near Sea Island, Georgia, U.S.A. Med Vet Ent 8: 25-30.

Kline DL, Takken W, Wood JR and Carlson DA (1990) Field studies on the potential of butanone, carbon dioxide, honey extract, 1-octen-3-ol, L-lactic acid and phenols as attractants for mosquitoes. Med Vet Ent 4: 383-391.

Lane RP (1984) Morphometric symmetry in antennae of Culicoides (Diptera: Ceratopogonidae) J Nat History 18: 651-656

Lehane M (2005) The biology of blood-sucking in insects. $2^{\text {nd }}$ Edition. Cambridge University Press, New York, USA. 336 pp.

Lindsay SW, Adiamah JH, Miller JE, Pleass RJ and Armstrong JRM (1993) Variation in attractiveness of human subjects to malaria mosquitoes (Diptera: Culicidae) in The Gambiae. J Med Entomol 30: 308-373. 
Linley JR (1966a) The ovarian cycle in Culicoides barbosai Wirth and Blanton and C. furens (Poey) (Diptera: Ceratopogonidae). Bull Ent Res 57: 1-17.

Linley JR (1966b) Effects of supplementary carbohydrate feeding on fecundity and life-length in Leptoconops becquaerti (Kieff). Bull Ent Res 57: 19-22.

Linley JR and Carlson DA (1978) A contact mating pheromone in the biting midge, Culicoides melleus. J Insect Phys 24: 423-427.

Linley JR and Davies JB (1971) Sandflies and tourism in Florida and the Bahamas and Caribbean Area. J Econ Entomol 64: 264-278.

Linley JR, Hoch AL and Pinheiro FP (1983) Biting midges (Diptera: Ceratopogonidae) and human health. J Med Entomol 20: 347-364.

Lloyd AM, Kline DL, Hogsette JA, Kaufman PE and Allan SA (2008) Evaluation of two commercial traps for the collection of Culicoides (Diptera: Ceratopogonidae). J Am Mosq Contr Ass 24(3): 253-262.

Logan JG and Birkett MA (2007) Semiochemicals for biting fly control: their identification and exploitation. Pest Manag Sci 63: 647-657.

Logan JG, Birkett MA, Clark SJ, Powers S, Seal NJ, Wadhams LJ, Mordue (Luntz) AJ, Pickett JA (2008) Identification of Human-Derived Volatile Chemicals that Interfere with Attraction of Aedes aegypti Mosquitoes. J Chem Ecol 34(3): 308-22.

Logan JG, Seal NJ, Cook JI, Stanczyk NM, Birkett MA, Clark SJ, Gezan SA, Wadhams LJ, Pickett JA and Mordue (Luntz) AJ (2009) Identification of human-derived volatile chemicals that interfere with attraction of the Scottish biting midge and their potential use as repellents. J Med Entomol 46: 208-219.

Mands V, Kline DL and Blackwell A (2004) Culicoides midge trap enhancement with animal odour baits in Scotland. Med Vet Ent 18: 336-342.

Marsh PM (1986) Ecological studies on Culicoides impunctatus (Diptera: Ceratopogonidae) with reference to its control in the highlands of Scotland. PhD thesis, University of Edinburgh, Edinburgh, UK.

Mayer MS and James JD (1969) Attraction of Aedes aegypti (L.): responses to human arms, carbon dioxide, and air currents in a new type of olfactometer. Bull. Entomol Res 58: 629-643.

Meiswinkel R, Baldet T, De Deken R, Takken W, Delecolle J-C and Mellor PS (2008) The 2006 outbreak of bluetongue in northern Europe - The entomological perspective. Prev Vet Med 87(1-2): 55-63.

Meiswinkel R, Baylis M and Labuschagne K (2000) Stabling and the protection of horses from Culicoides bolitinos (Diptera: Ceratopogonidae), a recently identified vector of African horse sickness. Bull. Entomol Res 90: 509-515.

Meiswinkel R, Van Rijn P, Leijs P and Goffredo M (2007) Potential new Culicoides vector of bluetongue virus in northern Europe. Vet Rec 161: 564-565.

Mellor, P.S. 1972. Studies on Onchocerca cervicalis (Railliet \& Henry) and its development in Culicoides (Latreille). PhD thesis, University of London, London, UK.

Mellor PS (1990) The replication of Bluetongue virus in Culicoides vectors. Curr Top Microbiol Immunol 162: 143-161.

Mellor PS (1993) African horse sickness: transmission and epidemiology. Vet Res 24: 199-212.

Mellor PS (1994) Epizootology and vectors of African horse sickness virus. Comparative Imm Microbiol Inf Dis 17: 287296.

Mellor PS and McCaig J (1974) Probable cause of sweet-itch in England. Vet Rec 95: 411-415.

Mellor PS, Boorman J and Baylis M (2000) Culicoides biting midges: Their role as arbovirus vectors. Ann Rev Entomol 45: 307-340.

Mondor EB, Trembaly ME, Awmack CS and Lindroth RL (2004) Divergent pheromone-mediated insect behaviour under global atmospheric change. Global Change Biol 10: 1820-1824.

Mordue (Luntz) AJ (2003) Arthropod semiochemicals: mosquitoes midges and sealice. Biochem Soc Trans 31: 128-133.

Mordue (Luntz) AJ and Mordue W (2003) Biting midge chemical ecology. Biologist 50: 159-162.

Moorhouse JE, Yeadon R, Beevor PS and Nesbitt BF (1969) Methods for use in studies of insect communication. Nature 232: 1174-1175.

Muir LE, Thorne MJ and Kay BH (1992) Aedes aegypti (Diptera: Culicidae) vision - spectral sensitivity and other perceptual parameters of the female eye. J Med Entomol 29: 278-281. 
Mullens BA (1995) Flight activity and response to carbon dioxide of Culicoides variipennis sonorensis (Diptera: Ceratopogonidae) in Southern California. J Med Entomol 32(3): 310-315.

Muller M (1991) Report of the Bluetongue Research Management Committee, 11-13 Septmeber, Brisbane, Australia. Muller MJ and Murray MD (1977) Blood-sucking flies feeding upon sheep in eastern Australia. Aus J Zool 25: 75-85.

Murray MD (1987) Akabane epizootics in New South Wales: evidence for long-distance dispersal of the biting midge Culicoides brevitarsis. Autralian Veterinary Journal 64: 305-308.

Murray MD (1991) The seasonal abundance of female biting-midges, Culicoides brevitarsis (Diptera: Ceratopogonidae), in coastal New South Wales. Aus J Zool 39: 333-342.

Nelson RL (1965) Carbon dioxide as an attractant for Culicoides. J Med Entomol 2: 56-57.

Nelson RL, Bellamy RE (1971) Patterns of flight activity of Culicoides variipennis (Coquillett) (Diptera: Ceratopogonidae). J Med Entomol 8: 283-291.

Pappenberger B, Geier M, and Boeckh J (1996) Responses of antennal olfactory receptors in the yellow fever mosquito Aedes aegypti to human body odours. In: Cardew G and Goode J (eds) Mosquito olfaction and olfactory mediated mosquito-host interactions. Ciba Foundation Symposium 200, pp 254-266.

Parker AH (1948) Stimuli involved in the attraction of Aedes aegypti, L., to man. Bull Ent Res 39: 387-397.

Percy KE, Awmack CS, Lindroth RL, Kubiske ME, Kopper BJ, Isebrands JG, Pregitzer KS, Hendrey GR, Dickson RE, Zak DR, Oksanen E, Sober J, Harrington R and Karnosky DF (2002) Altered performance of forest pests under atmospheres enriched by $\mathrm{CO}_{2}$ and $\mathrm{O}_{3}$. Nature 420: 403-407.

Pickett JA and Woodcock CM (1996) The role of mosquito olfaction in oviposition site location and in the avoidance of unsuitable hosts. In: Cardew G and Goode J (eds) Mosquito olfaction and olfactory mediated mosquito-host interactions. Ciba Foundation Symposium 200, pp 109-123.

Pickett J, Wadhams LJ and Woodcock CM (1998) Mate and host location by insect model systems for exploiting olfactory interactions. The Biochemist 8: 13.

Pomerantzev, BI (1932) Beitrage zur Morphologie und Anatomie der Genitalien von Culicoides (Diptera: Nematocera). Mag Parasitol 3: 183-214.

Price GD, Smith N and Carlson DA (1979) The attraction of female mosquitoes (Anopheles quadrimaculatus Say) to stored human emmanations in conjunction with adjusted levels of relative humidity, temperature and carbon dioxide. J Chem Ecol 5: 383-395.

Purse BV, Mellor PS, Rogers DJ, Samuel AR, Mertens PPC and Baylis M (2005) Climate change and the recent emergence of bluetongue in Europe. Nat Rev Microbiol 3: 171-181.

Qiu YT, Smallegange RC, Hoppe S, Van Loon JJ, Bakker EJ and Takken W (2004) Behavioural and electrophysiological responses of the malaria mosquito Anopheles gambiae Giles sensu stricto (Diptera: Culicidae) to human skin emanations. J Med Vet Entomol 18: 429-438.

Reye EJ and Lee DJ (1963) The influence of the tide cycle on certain species of Culicoides (Diptera, Ceratopogonidae). Proce Linnean Soc New South Wales 87: 377-387.

Ritchie SA, Van Essen PA, Kemme JA, Kay BH and Allaway D (1994) Response of biting midges (Diptera: Ceratopogonidae) to carbon dioxide, 1-octen-3-ol and light in southeastern Queensland. Aus J Med Entomol 31: 645-648.

Roessler P and Brown AWA (1964) Studies on the responses of the female Aedes mosquito. X. - Comparison of oestrogens and amino acids as attractants. Bull Ent Res 55: 395-403.

Schofield S and Brady J (1997) Effects of carbon dioxide, acetone and 1-octen-3-ol on the flight responses of the stable fly, Stomoxys calcitrans, in a wind tunnel. Phys Entom 22: 380-386.

Schofield S, Witty C and Brady J (1997) Effects of carbon dioxide, acetone and 1-octen-3-ol on the activity of the stable fly, Stomoxys calcitrans. Phys Entom 22(3): 256-260.

Schreck CE and Kline DL (1981) Repellency determination of four commercial products against six species of Ceratopogonid Culicoides. Mosquito News 41: 7-10.

Schreck, C.E., Kline, D.L. \& Carlson, D.A. 1990. Mosquito attraction to substances from the skin of different humans. J Am Mosq Contr Ass 6: 406-410. 
Sellers RF and Maarouf AR (1991) Possible introduction of epizootic hemorrhagic-disease of deer virus (Serotype-2) and bluetongue virus (Serotype-11) into British-Columbia in 1987 and 1988 by infected Culicoides carried on the wind. Can J Vet Res 55: 367-370.

Smart MR and Brown AWA (1957) Studies on the responses of the female Aedes mosquito. Part VII. The effect of skin temperature, hue and moisture on the attractiveness of the human hand. Bull Ent Res 47: 89-101.

Sutcliffe JF (1994) Sensory bases of attractancy - morphology of mosquito olfactory sensilla - a review. J Am Mosq Contr Ass 10: 309-315.

Takken W (1991) The role of olfaction in host-seeking of mosquitos - a review. Insect Sci Appl 12: 287-295.

Takken W and Kline DL (1989) Carbon dioxide and 1-octen-ol as mosquito attractants. J Am Mosq Contr Ass 5: 311-316.

Takken W, Verhulst N, Schotte E, Jacobs F, Jongema Y and Van Lammeran R (2008) The phenology and population dynamics of Culicoides spp. in different ecosystems in the Netherlands. Prev Vet Med 87: 41-54.

Tatem AJ, Baylis M, Mellor PS, Purse BV, Capela R, Pena I and Rogers DJ (2003) Prediction of bluetongue vector distribution in Europe and north Africa using satellite imagery. Vet Microbiol 97: 13-29.

Thomson RCM (1938) The reactions of mosquitoes to temperature and humidity. Bull Ent Res 29: 125-140.

Thomas G, Prijs HJ and Trapman JJ (1987) Factors contributing to differential risk between heifers in contracting summer mastitis. In: Thomas G, Overvecht $\mathrm{U}$ and Nansen P. Summer Mastitis. Martinus Nijhof, Dordrecht, the Netehrlenads, pp 30-35.

Tobin PC, Nagarkatti S, Loeb G and Saunders MC (2008) Historical and projected interactions between climate change and insect voltinism in a multivoltine species. Global Change Biol 14: 951-957.

Townley P, Baker KP and Quinn PJ (1984) Preferential landing and engorging sites of the Culicoides species landing on a horse in Ireland. Equine Vet J 16: 117-120.

Trigg JK (1996) Evaluation of a eucalyptus-based repellent against Culicoides impunctatus (Diptera: Ceratopogonidae) in Scotland. J Am Mosq Contr Ass 12: 329-330.

Wadhams LJ (1982) Coupled gas-chromatography - single cell recording - a new technique for use in the analysis of insect pheromones. Zeitschrift Für Naturforschung 37c: 947-952.

Walker AR (1977) Seaeonal fluctuations of Culicoides species (Diptera: Ceratopogonidae) in Kenya. Bull Ent Res 67: 217-233.

Warnes ML and Finlayson LH (1985) Responses of the stable fly, Stomoxys calcitrans (L.) (Diptera: Muscidae), to carbon dioxide and host odours. II. Orientation. Bull Ent Res 75: 717-727.

Wellby MP, Baylis M, Rawlings P and Mellor PS (1996) Effect of temperature on virogenesis of African horse sickness virus in Culicoides variipennis sonorensis (Diptera: Ceratopogonidae) and its significance in relation to the epidemiology of the disease. Bull Ent Res 86: 715-720.

Wirth WW and Blanton FS (1956) A new species of salt marsh sandfly from Florida, the Bahamas, Panama and Ecuador, its distribution and taxonomic differentiation from Culicoides furens (Poey) (Diptera, Heleidae). Florida Entomol 39: 157-162.

Wittmann EJ (2000) Temperature and the transmission of arboviruses by Culicoides biting midges. PhDThesis. University of Bristol, Bristol, UK.

Wittmann EJ and Baylis M (2000) Climate change: effects on Culicoides-transmitted viruses and implications for the UK. Vet J 160: 107-117.

Wittmann EJ, Mellor PS and Baylis M (2001) Using climate data to map the potential distribution of Culicoides imicola (Diptera: Ceratopogonidae) in Europe. OIE Sci Tech Rev 20: 731-740.

Zwiebel LJ and Takken W (2004) Olfactory regulation of mosquito-host interactions. Insect Biochem Mol Biol 34: 645652. 


\title{
11. Black fly interactions with their hosts
}

\author{
James F. Sutcliffe
}

\begin{abstract}
Haematophagous black flies (Diptera: Simuliidae) seek mammal and bird hosts as blood sources and are responsible for significant human and animal hardship and economic loss due to annoyance, effects of biting and disease transmission. Simuliids locate and choose their hosts by orienting to host-originating chemical, visual and temperature stimuli. Little is known about the nature of the stimuli involved as chemical mediators though carbon dioxide is influential and certain other breath and body odours also clearly play roles. Effects of visual stimuli are limited to the effective visual range of the host to the fly which is determined by host size and environment. Vision appears to take precedence over olfaction when the host-seeking fly makes visual contact with the host. Landing and biting site choices appear to depend on a combination of visual and skin-associated chemical and thermal stimuli. Black fly responses to potential host stimuli seem dependent on the context in which the stimuli are received. Some stimuli (e.g. warmth) induce landing or biting for flies in the host seeking mode (or context) but induce escape reactions for non-host-seeking flies. Host choice (or rejection) may be based on the ability of these host stimuli, encountered in an appropriate sequence, to sustain the host seeking mode up to its consummation (blood feeding). Research on black fly host seeking has been slowed by the relative difficulty of doing meaningful behavioural experiments in the lab with this group, by the dearth of lab colonies and the difficulties and costs of maintaining them and by the lack of adequate field tools and experimental approaches.
\end{abstract}

Keywords: attractants, blood feeding, host-seeking behaviour, sensory ecology, simuliids, visual cues

\section{Introduction}

\section{General}

Black flies (Diptera: Simuliidae) are a geographically widespread insect family found in almost all areas, including many oceanic islands, where there is running water. Crosskey (1990) includes 1,554 species $^{1}$ in his world list and notes that many more species will eventually be added as species complexes are resolved taxonomically.

Immature stages of simuliids occur in running water ranging, depending on species, from small streams such as those found in the highlands of Central America or on the Precambrian shield of Canada and Europe, to large rivers such as the Niger in West Africa or the North Saskatchewan that flows through the parklands of Alberta and Saskatchewan, Canada.

For the most part, black flies are pestiferous in numbers in rural and wilderness settings. Various factors, many of them not well-understood, appear to exclude simuliids from urban locations. In eastern Canada, black flies are a pest of humans in the spring and early summer months (late May, June, early July) while in western Canada the large river species (mainly Simulium arcticum

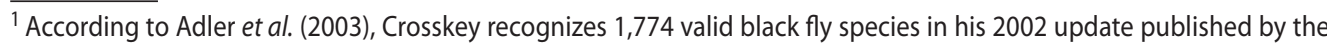
British Museum of Natural History.
} 
s.l. and S. luggeri Nicholson and Mickel) have multiple generations and are potential pests all summer long.

In their comprehensive treatment of the systematics of black flies, Adler et al. (2004) provide brief sketches of several aspects of general black fly biology (including behaviour, ecology, physiology) for North American species. Crosskey (1990) covers similar topics more extensively with a worldwide perspective.

Adler et al. (2003) provide an exhaustive revision of the taxonomic status of all North American black fly species which, because many are boreal, includes many Palearctic species as well. For the purposes of this review I use species names as reported in the original publications; however, the reader can determine the probable valid names for the species discussed by referring to the 'Taxonomic Accounts' section Adler et al.'s invaluable book.

Females of most simuliid species are blood feeders on mammals or birds though females of several species are autogenous in their first gonotrophic cycle and a few do not blood feed at any time having adapted to certain extreme conditions by adopting wholly autogenous reproductive strategies (Anderson 1986). While a few historical references have been made to black flies feeding on other insects, fish, frogs, etc., these are considered either mistaken or spurious (Crosskey 1990). The most credible report of black flies interacting with a non-mammal or non-bird vertebrate is by Smith (1969) who observed S. venustum Say swarming around a snapping turtle (Chelydra serpentina (Linnaeus)) in Algonquin Park, Ontario although none appeared to feed on it.

Black flies host seek only during the daylight hours (Crosskey 1990) suggesting that vision may be an important sensory modality in their host-seeking behaviour. While black flies are potentially active throughout the daylight hours, many species exhibit various peaks of activity in the early to mid-morning and during the late afternoon and evening. This is presumably to take advantage of the normally calmer conditions at these times and to avoid the desiccating heat of the mid-day and early afternoon hours.

\section{Medical and veterinary significance of black fly blood feeding}

Swarms of females can sometimes be massive around hosts and extremely annoying and stressful. Black fly bites can be painful and have, depending in the number of bites received and the sensitivity of the individual, consequences ranging from mild itching and irritation to severe illness and anaphylactic shock. Livestock in the parklands of western Canada have been killed by attacks of large numbers of Simulium arcticum (s.l.). Claims that this has been due to exsanguination are doubtless incorrect. Some combination airway blockage by fluid build-up around the tracheae in response to salivary antigens, allergic reactions to innumerable bites, and stress due to harassment and heat, especially in animals not previously exposed to black flies, is more credible as the cause of death (Beck 1980). Numerous examples of the pest significance of black flies are documented in many parts of the world (see Crosskey 1990 for several accounts).

Their blood feeding habit also makes black flies of significance as vectors of several important parasites and parasitic diseases of humans and animals. The most important parasite transmitted by black flies is the filarial nematode, Onchocerca volvulus (Leuckart), responsible for the condition called (among many other names) River Blindness. River Blindness is transmitted by various simuliid species in vast tracts of West and Central Africa and more focally in parts of Central and 
South America. Thylefors and Alleman (2006) provide a brief history and up-to-date summary of onchocerciasis control efforts in Africa and in the New World.

Black fly species in many parts of the world are also vectors of various other microfilarial parasites of animals including a number of Onchocerca species of livestock (Lok et al. 2000) and Dirofilaria ursi Yamaguti (Addison 1980) of black bears (Ursus americanus Pallas) in North America.

A simuliid-vectored disease of great significance in birds is an 'avian malaria' caused by geographically widespread members of the sporozoan genus Leucocytozoon. Leucocytozoon simondi Mathis and Leger is a common cause of death especially for young birds on the nesting grounds in spring and L. smithi Laveran and Lucet affects geese, turkeys and domestic fowl (Cupp 1986).

The demonstration that Vesicular Stomatitis Virus (VSV) occurs at high levels in members of some black fly species (Francy et al. 1988) and that S. vittatum Zetterstedt is capable of transmitting VSV biologically in the lab (Cupp et al. 1992) has further established the vector significance of this family. VSV does not establish high viremias in its vertebrate hosts (e.g. cattle) making its transmission by black flies (and other blood feeders) something of a mystery that appears to have been solved by Mead et al. (2000) who showed that VSV transmission can occur between black flies as they feed on the same host.

\section{Overview of black fly host preferences}

Black flies are conventionally divided into ornithophilic and mammalophilic groups in terms of broad host preferences, though the gap between these two types of hosts is spanned by some species. Black flies are generally considered to be fairly host specific within these host ranges. At one extreme, S. euryadminiculum Davies apparently restricts itself to feeding from the common loon (Adler et al. 2004) and is the poster species for the supposed high host-specificity of black flies. Mammalophilic species also appear in some cases to draw some clear distinctions between potential hosts. For instance, S. arcticum Malloch in Alberta forms massive swarms around cattle through which humans can walk and within which humans can work and rarely, if ever, get bitten (Shemanchuk 1986). Many other mammalophilic species appear to have broader host preferences (see Crosskey 1990 for a more detailed review of known black fly host preferences and Adler et al. 2004 for more recent information for North American species). It would be of great interest to know what role black fly responses to host-originating stimuli play in the evolution and expression of these host preferences.

\section{Aims of this review}

Various authors have reviewed aspects of host orientation and responses to blood hosts by biting flies. These reviews have different scopes ranging from those that look at the roles of specific types of stimuli (e.g. olfaction in mosquitoes - Takken 1991; visual stimuli in biting flies - Allan et al. 1987) to those that focus on particular parts of the process (e.g. 'distance orientation' in biting flies - Sutcliffe 1987) to others that summarise the broad scope of hosting seeking behavioural control for a number of biting fly taxa (e.g. Gibson and Torr 1999). In the first part of this review, I will deal mainly with the part of the host-black fly interaction that corresponds to active orientation of the insect to the host up to and including landing on the host and seeking out a biting site. This represents a part of the overall host location-blood feeding process in black flies that was interpreted by Sutcliffe (1986) as beginning with activation, when the blood feeder first detects 
the presence of the host, and as culminating in gorging followed by the departure of the blood fed insect out of the reach of the host's defensive reactions.

In the second part of this review I will discuss related topics including what the details of black fly responses to host stimuli may say about the evolution of host preferences, the nature of host seeking-blood feeding as a behavioural package and, finally, some of the challenges inherent in research on host seeking in simuliids.

\section{Black fly - host interactions (outside of active orientation phase)}

In the following paragraphs I provide a brief overview of the components of the black fly - host interaction outside of active orientation for purposes of setting active orientation (the main topic of this review) in its larger behaviourally meaningful context.

\section{Activation}

This larger context includes the activation step which precedes host seeking proper. In activation, the insect is stimulated out of some ground or resting state into an actively host-seeking mode by contact with host-originating stimuli (see section on 'Host seeking-blood feeding as a behavioural mode' for further discussion of this concept). In other biting flies, $\mathrm{CO}_{2}$ and other host odours are widely considered to be activating (see reviews by Gibson and Torr 1999, Takken 1991). This is likely the case for most simuliids too since many species are caught in larger numbers at traps and targets baited with $\mathrm{CO}_{2}$ only than at traps and targets without it (Sutcliffe 1986, 1987). This makes a logical case for $\mathrm{CO}_{2}$ as an activating stimulus in these instances since for flies to be caught at a trap or target they first need to have been activated. Nonetheless, there are clear exceptions to the $\mathrm{CO}_{2}$ as activator rule (if it is a rule) since certain black fly species have been caught in situations where only non- $\mathrm{CO}_{2}$ host stimuli were present. For example, in Algonquin Park, Ontario S. euryadminiculum swarmed near sources of loon uropygial odour (e.g. filter papers bearing residues from ether extracts of uropygial gland - Lowther and Wood 1964) whether $\mathrm{CO}_{2}$ was co-released or not and the inclusion of $\mathrm{CO}_{2}$ did not greatly increase numbers swarming (Fallis and Smith 1964; JF Sutcliffe personal observations). In this case, it must be concluded that it was some of the odours from uropygial gland secretions that were the activating principal(s). Examples where $\mathrm{CO}_{2}$ appears not to be necessary to catch black flies (though it may augment catches achieved with other odours) at traps or targets are also known. In field experiments in Cameroon, Thompson (1976b) found that traps baited with odours from human sweat would catch large numbers of $S$. damnosum Theobald s.l. even in the absence of $\mathrm{CO}_{2}$. By the same logic, these substances must to possess odour components that have activating properties for this species. Uropygial secretions of ducks also appear capable of activating some duck-biting species such as S. rugglesi Nicholson and Mickel and S. anatinum Wood (Fallis and Smith 1964). While odours are often cited as activating stimuli for host seeking in black flies and other biting flies, species in open savanna or parkland habitats may also rely on visual stimuli because of the longer site lines available to them. Thompson (1976a) presents evidence that suggests the savanna form of $S$. damnosum may require no more than the sight of the human form to be activated while the same appears not to be the case for the purportedly less anthropophilic (Crosskey 1990) 'forest form' of S. damnosum. 


\section{Biting site selection}

Post active orientation (i.e. post landing) components of the host-black fly interaction include several complex activities such as biting site selection, biting, gorging and disengagement from the host followed by escape.

Biting site selection in many black flies is characterised by rapid movement over the skin, fur or feathers accompanied by a patting or scanning action of the prothoracic legs. The prothoracic tarsi bear larger numbers of gustatory sensilla than the mesothoracic or metathoracic tarsi (Sutcliffe and Mclver 1976, 1987). This suggests that chemical cues are involved in mediating the biting response though we still know very little about the nature of these cues or whether others (e.g. thermal cues) also play a role in biting site selection. See the discussion under 'Heat and moisture cues' and 'Non-breath odours (including natural repellents)' below for further consideration of this topic.

Biting proper is accomplished by a complex interaction of the mouthparts with the skin. This process is quite well understood from a mechanical - anatomical perspective. Various authors have examined simuliid mouthparts in detail (Sutcliffe 1985, Sutcliffe and Mclver 1982, Wenk 1962) and Sutcliffe and Mclver (1984) provided a detailed analysis of the mouthpart sensilla of S. venustum s.l. and their various possible roles during biting. The make-up and properties of black fly saliva has been investigated as well. Like other blood feeders' saliva, black fly saliva contains a range of substances many of which are suited to interrupting the haemostatic process (Cupp and Cupp 1997) thus allowing feeding to proceed as quickly and efficiently as possible. Studies by (Stallings et al. 2002) on S. vittatum further reveal that black fly saliva has properties that may help microfilariae in nearby sub-cutaneous tissues to move toward the black fly's mouthparts during biting.

\section{Active feeding}

The onset of active feeding (gorging) is known to be stimulated by certain substances (gorging stimulants, phagostimulants) in the blood. Cessation of feeding is probably due to inputs from gut stretch receptors though no specific information about this exists for black flies. Phagostimulants for S. venustum s.l. were studied using artificial feeding methods (Smith and Friend 1982, Sutcliffe and Mclver 1979) and include various nucleotides (in particular ADP) which can stimulate full gorging at concentrations as low as $10^{-5} \mathrm{M}$ in saline.

Of particular interest in further understanding how biting and feeding are controlled in black flies are electrophysiological studies of the tarsal, antennal, and mouthpart and food canal sensilla. Putative functions for these receptors are reviewed by Mclver and Sutcliffe (1986) based on sensillar morphology and placement but until tested electrophysiologically, these proposed functions must be considered unconfirmed.

\section{Active orientation to the host}

\section{Carbon dioxide}

Carbon dioxide is a powerful mediator of host seeking for many biting flies including many black fly species. For this reason, $\mathrm{CO}_{2}$ from a block of dry ice or a tank has been a standard method used to increase the black fly catch at many types of traps and targets. In such applications, $\mathrm{CO}_{2}$ 
is often referred to as an 'attractant'. This is a term that has been used loosely by many authors (including this one, e.g. Sutcliffe 1986) over the years. For a chemical mediator of host seeking to qualify as a true attractant it must induce upwind anemotaxis. It is not possible to know simply by observing the accumulated swarm of flies around the trap, or by the increased catch of a sticky target or entry trap, whether the greater numbers are due to an attractant effect of $\mathrm{CO}_{2}$ or whether they are, instead, due to elevated $\mathrm{CO}_{2}$ levels simply stimulating increasing unoriented black fly flight activity that, in turn, causes more flies to encounter the arresting stimulus of the visually conspicuous trap or get caught on the sticky target.

To address this and similar questions, a method of intercepting black flies in their host-seeking flight is needed. If interception traps can be deployed some distance away around a $\mathrm{CO}_{2}$ source, it should be possible to differentiate between attraction and increased unoriented flight activity since the former will be characterised by high catches on the downwind sides of traps while the latter will be characterised by more or less equal catches on downwind and upwind sides. Working in Alberta on populations made up mostly of S. arcticum (IIS-10.11), Sutcliffe et al. (1995) deployed six Tangletrap-coated clear Lexan panels (upright sticky traps - USTs) as interception traps (see section entitled 'Challenges in the study of black fly host seeking behaviour' for further discussion of these traps) in a $12 \mathrm{~m}$ diameter ring around a $\mathrm{CO}_{2}$-baited cow silhouette trap (CST). Approximately $82 \%$ of the black flies caught were on the downwind sides of the panels supporting the interpretation that they had flown upwind in the $\mathrm{CO}_{2}$ plume. These results are similar to those of Golini and Davies (1971) for S. venustum Say in Algonquin Park, Ontario. Such results justify the use of the term 'attractant' with respect to $\mathrm{CO}_{2}$ for these particular black fly species and, they may also be taken as some indication of $\mathrm{CO}_{2}$ acting as an attractant for other black fly species. Nonetheless, this is a term that should always be used with due consideration.

In general in simuliids there appears to be good evidence for an increased response (in the form of increased trap catches) with increased release of $\mathrm{CO}_{2}$, i.e. a dose-effect. However, the shape of the dose-response curve, whether it is different for different species and what affects the curve's shape is poorly known. Part of this is due to the fact that much of the field work that reports on the effects of $\mathrm{CO}_{2}$ dose on numbers of black flies caught has been based on very rough methods of controlling and measuring $\mathrm{CO}_{2}$ release rate. To be most worthwhile, such work should employ precision release valves and accurate rotameter type flow gauges to regulate and measure release rates from $\mathrm{CO}_{2}$ tanks fitted with two stage regulators.

Fallis et al. (1967) measured the $\mathrm{CO}_{2}$ dose-response for black flies in Algonquin Park, Ontario, Canada and found that catch rate at $\mathrm{CO}_{2}$-baited fan traps increased in decreasing proportion to $\mathrm{CO}_{2}$ release rates from 0 to $800 \mathrm{ml} / \mathrm{min}$. Sutcliffe and Schofield (unpublished results) have also found a clear dose-response of $\mathrm{CO}_{2}$ for UST catches for several black fly species in Alberta and Ontario. As in Fallis et al. (1967), these dose-effects 'top out' at higher, though still physiologically relevant for cattle, $\mathrm{CO}_{2}$ release rates. It is not clear whether this is due to the plume simply recruiting more and more black flies in its active space, until there are no more flies left to recruit, or if it is due to an ever-increasing recruitment by the plume coupled with a fall off in numbers that succeed in navigating the greater distances to the USTs. Torr (1990) found that $\mathrm{CO}_{2}$ plumes were not readily navigable for Glossina pallidipes Austen but became much more so when acetone was co-released. It would be interesting to see the effects of other chemical mediators of black fly host-seeking on the shape of the $\mathrm{CO}_{2}$ dose-response curve.

Livestock under black fly attack can often be observed to bunch up apparently for the same reasons other herd animals bunch up when under threat of attack from a predator - there is 
safety in numbers. What this means in the context of biting fly attack is not clear. The 'topping out' of the $\mathrm{CO}_{2}$ dose-response in black flies may provide the explanation for this behaviour in animals under black fly attack since it suggests that though a large group of animals will produce a great deal of $\mathrm{CO}_{2}$, the per unit return in terms of black flies attacking will not be linear, i.e. the ratio of flies to individual cattle should drop and so, therefore, should individual host biting rate. Ratti et al. (2006) present quantitative data showing a dilution effect on black fly biting on black grouse proportional to group size. They do not discuss why this occurs but the shape of $\mathrm{CO}_{2}$ doseresponse curve may be part of the explanation.

Schofield and Sutcliffe (1996) show that the amount of $\mathrm{CO}_{2}$ produced by human individuals also appears to account for individual host 'attractiveness' for human biting black flies in Algonquin Park. Black fly catch on sticky Lexan panels mounted above the heads of seated, similarly dressed human subjects proved to be significantly and consistently different over two years of experiments. Removal of breath from the vicinity of the subjects (by having them breathe out through long tubes with their openings many meters away at right angles to the wind) eliminated individual differences and reduced catches on the panels by about $85 \%$. This was shown to have been due to the removal of the $\mathrm{CO}_{2}$ component, and not the non- $\mathrm{CO}_{2}$ breath odours, by the fact that release of tanked $\mathrm{CO}_{2}$ from a tube at the subjects' head levels at subject-specific rates restored individual panel catches to about $90 \%$ of their previous levels and completely restored individual differences.

\section{Non- $\mathrm{CO}_{2}$ breath odours}

To date there is very little known about the chemical identities of non- $\mathrm{CO}_{2}$ breath odours that mediate black fly host seeking though there is a variety of evidence that such substances exist for at least some species (Sutcliffe 1986, 1987). It may be significant, for instance, that Schofield and Sutcliffe (1996) were only able to restore about $90 \%$ of the whole breath catch on sticky panels mounted above the heads human subjects in field experiments in Algonquin Park (see experiment description above) when subject-specific amounts of tanked $\mathrm{CO}_{2}$ were released. This could mean that non- $\mathrm{CO}_{2}$ components of breath are responsible for larger numbers of black flies responding to the plume or that non- $\mathrm{CO}_{2}$ breath components play a role in 'focussing' the responders so that more of them were intercepted by the sticky panels. The latter explanation is supported by results from field work in Algonquin Park by Dean (2004). In these experiments, a yellow square $(46 \times 46 \mathrm{~cm})$ or cylinder $\left(20 \mathrm{~cm}\right.$ high $\times 15 \mathrm{~cm}$ diameter) target was baited either with nothing, $\mathrm{CO}_{2}$ at $300 \mathrm{ml} / \mathrm{min}$ or with whole breath (adjusted to contain $300 \mathrm{ml} / \mathrm{min}$ of $\mathrm{CO}_{2}$ ) of a single adult male subject. At the same time, a black cloth target or cylinder of the same configuration as its yellow counterpart, was placed across the wind from the yellow target at separations of $0 \mathrm{~m}, 1 \mathrm{~m}$ or 3 $\mathrm{m}$. Total number of flies caught on sticky interception screens placed immediately in front of the yellow and black targets were not significantly different for $\mathrm{CO}_{2}$ and breath-baited trials but, for any given separation, yellow targets baited with whole breath captured a higher proportion of the total than did $\mathrm{CO}_{2}$ baited yellow targets. This suggests that whole breath contained substances that 'focussed' the responding flies more tightly in the odour plume (perhaps by making the plume more navigable) but that these substances did not recruit more flies to host seeking. Similar properties have been found in tsetse flies for acetone and octenol which Torr (1990) found improve the navigability of a $\mathrm{CO}_{2}$ plume for Glossina pallidipes.

Sutcliffe et al. (1994) tested several substances found in cattle breath for their abilities to enhance catches of black flies in Athabasca, Alberta. These included acetone and 1-octen-3-ol (hereafter termed octenol), 1- and 2-octanol and octanoic (caprylic) acid each at 'high' and 'low' release rates and each accompanied by $\mathrm{CO}_{2}$ released from a pressurised tank. Only acetone at the high rate 
produced significantly higher catches than the $\mathrm{CO}_{2}$-only control. Unfortunately, this work was plagued by inadequate $\mathrm{CO}_{2}$ flow control problems and the release rates of the chemicals was not quantified. This, and the fact that the octenol effect in those host seekers it is known to occur in is dose dependent (Takken and Knols 1999, Vale et al. 1985) means the lack of an octenol effect in these experiments should not be taken for the lack of an octenol effect. Subsequent work done in Alberta incorporating more precise $\mathrm{CO}_{2}$ flow control and using UST catching methods (JF Sutcliffe unpublished results) failed to reproduce an acetone effect and further failed to show any effect of octenol.

Atwood and Meisch (1993) also investigated the effects of octenol odours (presented with and without $\mathrm{CO}_{2}$ ) on black fly catches in tent traps in Arkansas. Results were mixed though octenol (released from pipe cleaner wicks inserted into vials) plus $\mathrm{CO}_{2}$ resulted in significantly larger catches of Cnephia pecuarum Riley than $\mathrm{CO}_{2}$ alone in one trial of three. In the single trial in which S. meridionale Riley catches are reported, octenol plus $\mathrm{CO}_{2}$ produced much smaller catches than $\mathrm{CO}_{2}$ alone.

Results from experiments such as those reported in this section reveal some of the problems that must be addressed for field experiments on odour mediation of black fly host seeking to be maximally valuable. For instance, neither study could report precise or even estimated dose rates for chemicals tested in the field because release from wicks (Atwood and Meisch 1993) and from vials with different sized holes in the caps (Sutcliffe et al. 1994) would have varied through the day depending on environmental conditions. The importance of such information in the interpretation of results like these is difficult to over-state. Other features of these studies, such as inadequate control of $\mathrm{CO}_{2}$ release rate and collecting devices (tent trap and CST, respectively) that require landing and or trap entry for the fly to become part of the sample are also problematic. The former is easily done with high precision valves and flow meters attached to a $\mathrm{CO}_{2}$ tank with a two-stage regulator. The latter can best be addressed by using simple interception traps such as USTs (Sutcliffe et al. 1995, see 'Challenges in the study of black fly host seeking behaviour' section for further discussion of this).

\section{Non-breath odours}

One of the most dramatic examples of non-breath chemical mediation of black fly host seeking is the effect of secretions of the uropygial glands of certain waterfowl on some ornithophilic species. Lowther and Wood (1964) first reported that S. euryadminiculum is strongly and apparently exclusively 'attracted' to loon uropygial gland extracts with or without a visual stimulus and with or without $\mathrm{CO}_{2}$ (Fallis and Smith 1964). In this same study, catches of other waterfowl biters (e.g. S. rugglesi, S. anatinum) in sweep net collections over filter papers impregnated with various extracts of duck uropygial glands were also greater than control sweeps though, in this case, the addition of $\mathrm{CO}_{2}$ significantly increased catch of these species. The active specifics in loon and duck uropygial secretions have not been isolated and identified though we have unpublished data (SW Schofield and JF Sutcliffe) that indicates that long chain alcohols in loon uropygial gland extracts may play a role.

Sutcliffe et al. (1994) examined the ability of crude and fractionated 'whole cow odour' (WCO) and cow urine to enhance CST catches of black flies (largely S. arcticum IIS-10.11) in Alberta. While crude WCO plus $\mathrm{CO}_{2}$ resulted in catches significantly higher than the control $\left(\mathrm{CO}_{2}\right.$ only), none of the fractions did so suggesting a possible synergism between two or more components of the WCO. Cow urine in combination with $\mathrm{CO}_{2}$, while it resulted in higher-than-control catches in CSTs 
than $\mathrm{CO}_{2}$ alone, did not have a significant effect though it was close to significance $(P<0.12)$. In work done subsequently in Alberta (JF Sutcliffe, unpublished results) with better $\mathrm{CO}_{2}$ flow control and using USTs instead of CSTs, cow urine (stored for no more than 3 weeks in a $4{ }^{\circ} \mathrm{C}$ refrigerator) plus $\mathrm{CO}_{2}$ was found to result in larger catches than $\mathrm{CO}_{2}$ alone.

Information on human odours other than breath as mediators of black fly host orientation is scant. The first evidence that such substances influence human biters was provided by Thompson $(1976 a, b)$ who found that clothing that had been worn for some time enhanced trap catches of the forest form of S. damnosum s.l. in Cameroon even in the absence of $\mathrm{CO}_{2}$. Thompson (1976b) makes a circumstantial case for the source of this substance being apocrine sweat though this has not been investigated further.

Schofield and Sutcliffe (1997) provide the only other evidence to date for an effect of human skin secretions on biting responses of black flies. In field-lab experiments with human biting species (S. venustum s.l.) in Algonquin Park, they first established that human test subjects had different 'bitabilities' (defined as each subject's likelihood of being bitten by a given black fly) by placing black flies caught individually in vials off a $\mathrm{CO}_{2}$-baited cloth target, directly onto a subject's skin and observing for a biting response for three minutes. Different subjects had significantly different bitabilities that were maintained over several weeks. To isolate a possible skin substance effect from other subject-specific characteristics that may have been influencing biting, similarly caught black flies were given the opportunity to bite artificial latex rubber membranes that had been treated with subject skin secretions and then stretched over a warmed (approx $32-37^{\circ} \mathrm{C}$ ) aluminum block. The hierarchy of individual bitability and subject-membrane bitability corresponded closely although in an unexpected manner. Treated membranes were bitten less frequently than control membranes and membranes of more bitable individuals were so because they were less 'repellent' than membranes corresponding to less bitable individuals. Nothing is known of the chemical properties of these apparent biting inhibitors though the observation that flies in vials on treated membranes stayed on the sides and top of the vials could suggest that the effect was, at least in part, due to volatiles. This work raises the possibility of what Schofield and Sutcliffe (1997) refer to as 'antibitants' in human skin. Many a black fly field worker has experienced severe black fly attack immediately after bathing and hair washing. The temporary removal of antibitants from the skin and hair brought about by bathing and shampooing may help explain this phenomenon. While the notion of antibitants or natural 'repellents' has not been pursued further for simuliids, there is mounting evidence for host-derived compounds that variously interfere with aspects of host seeking and blood feeding in other haematophagous arthropods (e.g. Logan et al. 2008).

While on the face of it the treatment effect described above looks like repellence, interpretations of such experiments must be done carefully. Schofield and Sutcliffe (1997) recognise this and propose alternatively that what is an apparent repellence could actually be the effect of skin substances that stimulate walking activity that normally occurs when the insect lands on the skin (see description of 'patting' behaviour in 'Biting site selection' section). Confinement of the black fly in the vial might make this look like an attempt to escape a repellent substance.

A final, indirect, mode of chemical interaction between the host and the blood feeding black fly may be what McCall and Lemoh (1997) refer to as the 'invitation effect' observed in certain members of the $S$. damnosum complex. This is suggested to be a pheromone effect between black flies in the act of biting the host and black flies that have not yet selected a biting site. The effect is to increase the number of flies biting on a given area of the host's skin. The sensory basis of this effect has not been elucidated nor has it been determined who (the sender or the receiver) 
benefits from the message though the term 'invitation effect' implies the sender. It is arguable, however, that by biting where saliva from previous bites has already increased blood flow to the area, the receiving individual may benefit by being able to feed rapidly at that site. If this is the case, it might be better to re-name this the 'party crasher effect'.

\section{Visual interactions with hosts}

In Sutcliffe's (1986) framework for host seeking in simuliids, chemically-mediated orientation to the host eventually brings the fly into visual contact with the host. This contact would first be with the host animal as a whole but with closer approach, the fly would be able to see and respond to particular physical and textural features of the host.

All other things being equal, the range at which the host first becomes visible for different species would be determined by the physical setting. Visual obstructions and the complexity in woodland surroundings may make it more difficult for the host seeker to see a host animal of a given size, shape and colouration than in relatively less complex, more open settings such as savanna and parkland. However, the relative ambiguity of odour information about the location of an odour source (such as a host animal - Brady et al. 1990) should mean that the host-seeking fly will give priority to much less ambiguous visual information when it becomes available. This, in turn, suggests that woodland species may rely on olfactory cues for longer than open landscape species which may rely more on visual cues. Relatively little information on this question is available though some support for this in the savanna form versus the forest form of $S$. damnosum is presented in the section titled 'Activation'.

Based on field experiments, Sutcliffe et al. (1995) estimated that host seeking S. arcticum (IIS-10.11) responded visually to a host-mimicking CST (110 cm long by $46 \mathrm{~cm}$ wide) from about $8 \mathrm{~m}$. At this distance, they calculated that the image of the CST would subtend an angle of about $8^{\circ}$ (in the horizontal plane) and would cover all or parts of up 15 ommatidia. If other species of black flies have a similar visual resolution capability for their hosts, it would mean that something the size of a human would be resolved at a distance of approximately $20 \mathrm{~m}$ while a host the size of a loon would not become apparent until it was within about $100 \mathrm{~cm}$. Black fly species that bite smaller birds in leafed out, relatively dimly lit wooded areas might not make effective visual contact until within a few centimetres.

When visual and chemical stimuli are both 'in play', which take precedence? The reasoning presented at the beginning of this section indicates that the less ambiguous visual signal should. In field experiments in Algonquin Park, Ontario, Canada, Dean (2004) undertook to examine this question for host-seeking mammalophilic black flies. In these experiments a yellow square $(46 \times 46$ $\mathrm{cm}$ ) or cylindrical $\left(20 \mathrm{~cm}\right.$ high $\times 15 \mathrm{~cm}$ diameter) target was baited either with nothing, $\mathrm{CO}_{2}$ at $300 \mathrm{ml} / \mathrm{min}$ or with whole breath of a single adult male adjusted to $300 \mathrm{ml}$ per minute $\mathrm{CO}_{2}$. At the same time, a black cloth target or cylinder of the same configuration as its yellow counterpart, was placed either beside the yellow target ( $0 \mathrm{~m}$ separation) or at separations of 1 or $3 \mathrm{~m}$. When at $0 \mathrm{~m}$ separation, the sticky screens in front of black target always caught significantly more than half of the flies irrespective of the bait. However, when the targets were separated by 1 or $3 \mathrm{~m}$, the sticky screens in front of the baited (yellow) targets collected relatively more flies than those in front of the dark targets. Clearly, our understanding of how responses to different modalities of stimulus are ordered during host seeking in simuliids requires further examination and probably needs to integrate elements such host/target movement. 
Crosskey (1990) provides a detailed discussion of the preferred feeding sites for different groups of mammal-biting black flies. While details vary from host to host, he notes that most species biting large mammals do so on either on the muzzle and ears or on the belly, chest and front legs. The adaptive reasons for black flies having preferred biting areas are poorly researched but presumably relate to some degree to avoidance of host defensive behaviours. Other reasons for certain areas of the body being preferred for biting may also relate to avoiding competition with other black fly or biting fly species. In ornithophilic species such as S. euryadminiculum and S. rugglesi, preferred biting sites are on the head and neck Bennett et al. (1972) again presumably because the animal is less able to defend itself from flies in such places.

Visual cues play an important role in determining where on the host the black fly will land, i.e. in leading the fly to its preferred biting area on the host. Extensive study of colour and shape preferences of a number of black fly species (reviewed by Sutcliffe 1986, 1987) have established that black flies generally prefer to land on darker colours and matte surfaces. While black flies do not seem to assess overall shape prior to landing, some species do exhibit a preference for landing near edges and on prominences. Choice of landing site appears to be largely independent of odour stimuli. Yee and Anderson (1995) showed that females of several black fly species they studied in California would land on the head ends of deer-mimicking three dimensional models irrespective of whether $\mathrm{CO}_{2}$ used to bait the models was released from the head end of the model or from beneath it. Similarly, the black fly Wilhelmia equina L. will orient to the head end of a host model even of the odour bait is at the tail end (Wenk and Schlorer 1963). When not given a head or ears to indicate which end of a CST is which, S. arcticum landed equally on both ends (Sutcliffe et al. 1995). These and similar findings suggest that though there is a range over which the host seeking black fly is guided by host odours, at very close range, visual stimuli take over and guide landing/biting site selection.

\section{Heat and moisture cues}

The importance of heat/thermal stimuli for host-seeking black flies is implied by the fact that simuliids bite only warm-blooded hosts (birds and mammals). The role of thermal stimuli in mediating landing and biting in human biting black flies in Algonquin Park Canada was examined by Sutcliffe and Mclver (1979), who showed that female flies collected individually in small vials after landing on a human host and subsequently transferred onto a warmed latex membrane, would bite the membrane more readily when the temperature differential between the membrane and the air just above it was greater. They argued that this means that skin (or membrane) temperature alone is not the key stimulus and suggested that the thermal differential may have served as a short range landing cue. Schofield and Sutcliffe (1997) in their experiments on the bitability of latex membranes treated with human skin secretions also measured the effect of the temperature differential between the air and the artificial biting surface. Again, black flies that experienced a greater temperature differential $\left(14-17^{\circ} \mathrm{C}\right)$ between the air and the membrane were much more likely to bite the membrane than flies that experienced a lesser differential $\left(11-14^{\circ} \mathrm{C}\right)$. The question of whether the temperature differential is a short range orientation/landing stimulus (Sutcliffe and Mclver 1979) or a biting stimulus (Smith and Friend 1982) remains an open one.

Virtually no information exists for the effects of water vapour either in breath or from the skin on black fly host seeking. 


\section{Discussion}

\section{Host seeking-blood feeding as a behavioural mode}

The concept of 'activation' is used extensively in the discussion of host seeking in biting Diptera though what is meant by the term is not always clear. Sutcliffe $(1986,1987)$ suggested that activation is the event that causes a 'switching over' from some ground state of behaviour into a host seeking 'behavioural package' or mode. While in the host seeking mode the host seeker would be biased to respond to host originating stimuli in a manner appropriate to the host seeking circumstances but, under different circumstances (e.g. in an oviposition behavioural mode), the insect might respond to the same stimulus differently or not at all. This suggests that the activation and continuation of black fly host seeking may fit the 'rolling fulcrum' model (Miller and Strickler 1984) developed as a theoretical framework to explain host-seeking behaviour in plant feeding insects.

During artificial feeding experiments in Algonquin Park, Sutcliffe and Mclver (1979) observed that black flies captured individually in vials after landing on a host would only bite the skinsimulating artificially-warmed membrane if given the opportunity to do so within a few minutes of collection. Flies held for too long would often not bite when placed on the membrane and many appeared agitated and to be trying to get away from the warmth of the membrane. They interpreted this as the result of the flies having switched out of the host-seeking mode during the holding period. In the framework of the rolling fulcrum model, the host-related stimuli that served to establish (activate) and sustain host seeking-appropriate responses had been interrupted long enough for some of the flies (those that would not bite) to revert to some more basic behavioural mode in which sources of heat might be interpreted as representing and increased desiccation risk. When black flies were held in vials for different lengths of time after being collected from a host, we found that there was a gradual decrease in the proportion of the sample that would bite the artificial membrane such that the 'half life' of biting persistence was only a few minutes (JF Sutcliffe, unpublished results). Dean (2004) investigated this phenomenon in human biting black fly species in Algonquin Park and tried to determine factors that account for some flies switching out of the host seeking mode before others and to see if biting persistence could be extended in captive female black flies by providing flies with continued contact with simulated host stimuli (in this case, slightly elevated levels of $\mathrm{CO}_{2}$ in an air stream) during the holding period. The treatment had no effect and has not been followed up though it would be interesting to do so with more complex and complete mixtures of host-odour stimuli.

The existence of a behavioural mode that appears to establish a behavioural context for the host seeking stimulus-responses has implications for the interpretation of results from experiments designed to study the host seeker-host interaction and for the circumstances under which such work can be done meaningfully. In my experience, it is simply not possible to get female black flies that have been held after being collected in the field to respond 'appropriately' to host stimuli in the lab. This makes behavioural work on these problems 'out of season' and anywhere but in the field problematic. Thus, lab-based olfactometer and wind tunnel experiments, odour choice tests, artificial feeding experiments using previously collected flies or using flies reared from pupae and so-on are exceedingly difficult to perform for this group. Even if it were possible to get black flies to 'behave' in the lab, the fidelity with which lab responses reflect what would happen in a natural setting would be open to question. Indeed, research on host-seeking responses in biting flies in general should be carefully interpreted since the concept of host seeking as a context-sensitive behavioural mode is not unique to black flies. 


\section{Host preferences}

While the range of potential black fly host preferences is determined at one level by ecological and temporal factors (i.e. the fly and the prospective host species must occur in the same habitat at the same time), whether a given black fly species actually prefers a particular host species is presumably determined by subtleties of sensory cues given off by hosts and by the responses of the host seeker to those stimuli.

The precise dimensions of the host preferences for most black fly species are not well known partly because many black fly species are very difficult to tell apart in the field and can often only be differentiated at the species level with cytogenetic or molecular methods. Another reason for our poor understanding of black fly host preferences is that is very difficult to field-collect black flies with appreciable amounts leftover blood meals in the field. Thus, it has been difficult to apply ELISA and other immunological methods to the analysis of black fly blood meal sources. However, the current widespread availability of very sensitive molecular methods may make it more feasible than ever to determine the origins of blood meal remnants in flies even several days after feeding. Though this opportunity has not been exploited widely yet, some researchers have taken advantage (e.g. Malmqvist et al. 2004) and the approach has already yielded information that has implications for black fly interactions with their hosts. Hellgren et al. (2008) found that three of four specimens of S. annulus (Lundstrom) collected with a car top trap at points 200 km apart in northern Sweden had all fed from Common Cranes (Grus grus (Linnaeus)) despite the fact this bird is not numerous in the study area. This suggests that $S$. annulus may orient preferentially to G. grus because of specific odour or visual stimuli the bird possesses. Results like these when investigated further may lead to many more examples of specific, host stimulus-based preferences in black flies like those that exist between, for instance, S. euryadminiculum and loons and between S. rugglesi and waterfowl.

A better understanding of the stimuli that the host seeking black fly encounters and how the fly reacts to these stimuli may explain much about the sensory and behavioural mechanisms that result in host preferences. For instance, where in the host-seeking process does host the preference exert itself (i.e. where does host discrimination occur)? Is it from the start of active orientation or does the insect begin host seeking based on initial minimal information about the prospective host and make decisions about whether to continue further host seeking when subsequent cues fail to, or succeed in, satisfying certain 'expected' criteria (e.g. host-specific body or breath odours, visual host qualities that the insect will respond to in the host seeking behavioural context) that confirm that the prospective host is indeed suitable. That the insect could have enough information about the prospective host from initial contact seems highly unlikely unless the stimulus is highly specialised such as in the case of loon uropygial odours for S. euryadminiculum. For many more generalist species, first contact may be with more generalised stimuli such as elevated levels of $\mathrm{CO}_{2}$. In the latter situation, host discrimination must be ongoing as the insect continues to move closer to the host coming into contact with more and more hostrelated stimuli that ultimately allow the insect to determine whether it should continue to invest effort and invoke risk both of which host seeking and the act of blood feeding entail.

The range of host preferences exhibited in black flies, and in biting flies in general, must reflect evolutionary 'choices' made by different species or lineages. Different host-seeking strategies would come with their own sets of advantages and disadvantages. A high degree of host species specialisation could be combined with a narrow range of specific host-related stimuli that the insect would require to initiate and engage in sustained host seeking. This strategy would narrow 
the number of host animals available for the specialist to feed on but would help ensure that its investment of energy in host seeking would be most likely to result in a blood meal with minimum risk. Alternatively, for biting flies with broader host ranges, a better strategy might be to respond initially to more general host-related stimuli (e.g. $\mathrm{CO}_{2}$ ) and discriminate as more information, in the form of host-related stimuli, becomes available. This greatly enlarges the number of potential host animals available but it also increases the probability of investing energy and undertaking additional risk for no return.

Members of the Anopheles gambiae Giles mosquito species complex are host specialists and appear to conform to this pattern. In behavioural experiments in Burkina Faso, Costantini et al. (2001) showed that An. gambiae s.l. females relied less on $\mathrm{CO}_{2}$ cues and more on human-specific odours to mediate host seeking. Perhaps An. gambiae made the evolutionary 'choice' to specialise on humans long ago. This is plausible for an African species since it could have co-evolved with humans over several hundred thousand years. The human biting black fly counterparts to An. gambiae could be the savannah and forest forms of African species $S$. damnosum s.l. Thompson $(1976 a, b)$ working in Cameroon showed that substances in human skin and sweat (with or without $\mathrm{CO}_{2}$ ) result in large swarms of the forest form around traps while the human profile without any skin or breath odours, is swarmed heavily by savannah form. Certain black flies that bite nonhumans are also highly host specific and also fit the pattern. Simulium euryadminiculum appears to specialise on loons and swarms en masse to odours of extracts of loon uropygial gland. The addition of $\mathrm{CO}_{2}$ to uropygial gland odour appears not to increase numbers. Species such as $\mathrm{S}$. rugglesi and $S$. anatinum with reportedly somewhat broader host preferences (various ducks and to a limited extent other birds) only respond to duck uropygial gland secretions if accompanied by $\mathrm{CO}_{2}$ and to a lesser extent to $\mathrm{CO}_{2}$ alone (Fallis and Smith 1964). Other ornithophilic species such as S. aureum s.l. and Prosimulium decemarticulatum Twinn which have even broader host preferences among waterfowl and passerines, respond in large numbers to $\mathrm{CO}_{2}$ alone but not to uropygial gland secretions.

Given the broad occurrence of $\mathrm{CO}_{2}$ as a sensory cue for insects of many types including for many non-biting Diptera (Guerenstein and Hildebrand 2008), perhaps $\mathrm{CO}_{2}$ represents the ancestral host odour for black flies and sensitivity to other host odours evolved subsequently. This process could have resulted in the various degrees of host specificity that are represented in the family today and to a gradual de-emphasis of the $\mathrm{CO}_{2}$ cue for those with the greatest degree of host-specificity.

\section{Challenges in the study of black fly host-seeking behaviour}

Notwithstanding the concerns about the interpretation of lab-evoked 'host seeking' responses (above), work with many mosquito species and with certain other biting flies (e.g. stable flies, tsetse) from lab colonies has helped elucidate various features of host seeking in these groups. However, to date, virtually all work that sheds light on aspects of host seeking in black flies has been field-based because black fly colonies are few and far between because they are difficult to establish and labour-intensive and costly to maintain. Edman and Simmons (1986) summarise the challenges, which include the infrastructure requirements associated with maintaining an insect that oviposits and whose larval stages occur in flowing water as well as the particular solutions that must be found to inducing mating, blood feeding, and oviposition by adults. According to Adler et al. (2004), only four North American black fly species have been successfully colonised on a continuing basis and only a handful of colonies currently exist. 
The usefulness of colony reared black flies for host seeking work, if they were available, would probably also be limited for reasons already discussed. Indeed, many attempts to colonise black flies have involved autogenous species since they do not have to blood feed (or to be induced to perform some lab equivalent of host seeking) to produce eggs. Though inducing meaningful hostseeking responses with colonised black flies is problematic, there are other related constructive uses that black flies from a lab colony could be put to. For instance, colonised black flies could be used for electroantennogram-screening of candidate host-mediating chemicals thus helping focus efforts in the field.

Tsetse fly trap and target responses are relatively well worked out (Gibson and Torr 1999; Chapter 12 , this volume) making it possible to design field experiments appropriately for the purposes intended. Black fly host-seeking research over the years has relied on a variety of host mimicking traps and targets of various shapes and sizes but, unlike in the tsetse world, how black fly traps and targets work is not well understood nor do we know in detail the responses they evoke from the host-seeking black fly. Electric nets in various configurations and applications have been one of the key tools in the analysis of tsetse host seeking behaviour because they can be used to intercept host orienting flies in a non-interactive way. Black fly work has not had an equivalent of the electric net. In an attempt to address this, we have used both sticky Lexan squares and sticky neutral coloured fibreglass window screening in such applications (e.g. Schofield and Sutcliffe 1996, Sutcliffe et al. 1996). In experiments where the desire is to assess the effects of candidate attractants without the introduction of other variables such colour preferences, trap entry behavior, and so-on, these methods are superior to more complex systems such as CSTs. However, sticky Lexan sheets and screens still have not been assessed for their ability to truly intercept host seeking black flies. This would be a worthwhile undertaking as would an assessment of the applicability of electric net technology to black fly host seeking research.

\section{Conclusions}

As we attempt to come to grips with the most basic of questions relating to host-seeking behaviour in black flies and biting flies in general, the landscape itself is changing. The most recent ingredient added to the host-insect interaction picture is the growing appreciation that the parasites that blood feeding Diptera transmit manipulate many aspects of their vertebrate and insect hosts' physiologies including those aspects that mediate host-seeking. The ability of certain malaria parasites to manipulate their mosquito vectors during blood feeding has been known for many years (e.g. Rossignol et al. 1984, 1986) but evidence is now mounting that parasite-infected host seekers may respond differently than non-infected host seekers during host seeking. There is also considerable evidence that parasite-infected vertebrate hosts may be more attractive for vector species (see reviews by Hurd 2003, Lefevre and Thomas 2008; Chapter 16, this volume). Kruppa and Burchard (1999) compared the ability of Onchocerca volvulus-infected individuals to 'attract' filarial-carrying and non-carrying black flies but found no differences. Nonetheless, there is no doubt that there are parasite manipulations of hosts that influence the host-biting fly interaction (indeed, that make this a three-way interaction) and that they will also have to be accounted for before a full understanding of biting fly host seeking behaviours can be achieved.

\section{References}

Addison EM (1980) Transmission of Dirofilaria ursi Yamaguti, 1941 (Nematoda: Onchocercidae) of black bears (Ursus americanus) by blackflies (Simuliidae). Can J Zool 58: 1913-1922. 
Adler PH, Currie DC and Wood DM (2003) The black flies (Simuliidae) of North America. Comstock Publishing, Cornell, USA.

Allan SA, Day JF and Edman JD (1987) Visual ecology of biting flies. Annu Rev Entomol 32: 297-316.

Anderson, JR (1986) Reproductive strategies and gonotrophic cycles of black flies. In: Kim KC and Merritt RW (eds) Black flies. Ecology, population management and annotated world list. Pennsylvania State University, University Park, PA, USA, pp 63-76.

Atwood DW and Meisch MV (1993) Evaluation of 1-octen-3-ol and carbon dioxide as black fly (Diptera, Simuliidae) attractants in Arkansas. J Amer Mosq Contr Assoc 9: 143-146.

Beck BE (1980) Clinical studies of the effect of the black fly Simulium arcticum on cattle. In: Haufe WO and Croome GCR (eds) Control of black flies in the Athabasca River. Alberta Environment, Lethbridge, Alberta, Canada, pp 233-237.

Bennett GF, Fallis AM and Campbell AG (1972) The response of Simulium (Eusimulium) euryadminiculum Davies (Diptera: Simuliidae) to some olfactory and visual stimuli. Can J Zool 50:793-800.

Brady J, Packer MJ and Gibson G (1990) Odor plume shape and host finding by tsetse. Ins Sci Applic 11: 377-384.

Costantini C, Birkett MA, Gibson G, Ziesmann J, Sagnon N'F, Mohammed HA, Coluzzi M and Pickett JA (2001) Electroantennogram and behavioural responses of the malaria vector Anopheles gambiae to human-specific sweat components. Med Vet Entomol 15: 259-266.

Crosskey RW (1990) The natural history of blackflies. Wiley, Chichester, UK.

Cupp EW (1986) The epizootiology of livestock and poultry diseases associated with black flies. In: Kim KC and Merritt RW (eds) Black flies. Ecology, population management and annotated world list. Pennsylvania State University, University Park, PA, USA, pp 387-395.

Cupp EW and Cupp MS (1997) Black fly (Diptera: Simuliidae) salivary secretions: Importance in vector competence and disease. J Med Entomol 34: 87-94.

Cupp EW, Mare CJ, Cupp MS and Rowley FB (1992) Biological transmission of Vesicular Stomatitis Virus (New Jersey) by Simulium vittatum (Diptera, Simuliidae). J Med Entomol 29: 137-140.

Dean BL (2004) Factors affecting host location behaviour in a field population of the black fly Simulium venustum (sensu lato) (Diptera: Simuliidae). MSc Dissertation, Trent University, Peterborough, Canada.

Edman JD and Simmons KR (1986) Maintaining black flies in the laboratory. In: Kim KC, Merritt RW (eds) Black flies. Ecology, population management and annotated world list. Pennsylvania State University, University Park, PA, USA, pp 305-312.

Fallis AM and Smith SM (1964) Ether extracts from birds and $\mathrm{CO}_{2}$ as attractants for some ornithophilic simuliids. Can J Zool 42: 723-730.

Fallis AM, Bennett GF, Griggs $G$ and Allen T (1967) Collecting Simulium venustum females in fan traps and on silhouettes with the aid of carbon dioxide. Can J Zool 45: 1011-1017.

Francy DB, Moore CG, Smith GC, Jacob WL, Taylor SH and Calisher CH (1988) Epizootic vesicular stomatitis in Colorado, 1982 - isolation of virus from insects collected along the northern Colorado Rocky Mountain front range. J Med Entomol 25: 343-347.

Gibson G and Torr SJ (1999) Visual and olfactory responses of haematophagous Diptera to host stimuli. Med Vet Entomol 13: 2-23.

Golini VI and Davies DM (1971) Upwind orientation of female Simulium venustum Say (Diptera) in Algonquin Park, Ontario. Proc Entomol Soc Ont 101: 49-54.

Guerenstein PG and Hildebrand JG (2008) Roles and effects of environmental carbon dioxide on insect life. Annu Rev Entomol 53: 161-178.

Hellgren O, Bensch S and Malmqvist B (2008) Bird hosts, blood parasites and their vectors - association uncovered by molecular analyses of blackfly blood meals. Molecular Ecol 17: 1605-1613.

Hurd H (2003) Manipulation of medically important insect vectors by their parasites. Annu Rev Entomol 48: 141-61.

Kruppa TF and Burchard GD (1999) Similar attraction by onchocerciasis patients and individuals putatively immune to Onchocerca volvulus. Trans R Soc Trop Med Hyg 93: 365-367.

Lefèvre $T$ and Thomas $F$ (2008) Behind the scene, something else is pulling the strings: Emphasizing parasitic manipulation in vector-borne diseases. Infection, Genetics and Evolution 8: 504-519. 
Logan LG, Birkett MA, Clark SJ, Powers S, Seal NJ, Wadhams LJ, Mordue Luntz AJ and Pickett JA (2008) Identification of human-derived volatile chemicals that interfere with attraction of Aedes aegypti mosquitoes. J Chem Ecol 34: 308-322.

Lok JB, Walker ED and Scoles GA (2000) Filariasis. In: Eldridge BF, Edman JD (eds) Medical entomology. A textbook on public health and veterinary problems caused by arthropods. Kluwer, Dordrecht, the Netherlands, pp 299-375.

Lowther JK and Wood. DM (1964) Specificity of a black fly, Simulium euryadminiculum Davies, towards its host, the common loon. Can Entomol 96: 911-913.

Malmqvist B, Strasevicius D, Hellgren O, Adler PH, Bensch S (2004) Vertebrate host specificity of wild-caught blackflies revealed by mitochondrial DNA in blood. Proc R Soc Lond B 271: S152-S155.

McCall PJ and Lemoh PA (1997) Evidence for the "invitation effect" during bloodfeeding by blackflies of the Simulium damnosum complex (Diptera: Simuliidae). J Insect Behav 10: 299-303.

Mclver SB and Sutcliffe JF (1986) Sensory basis of behavior and structural adaptations for feeding in black flies. In: Kim KC, Merritt RW (eds) Black flies. Ecology, population management and annotated world list. Pennsylvania State University, University Park, PA, USA.

Mead DG, Ramberg, FB, Besselsen DG and Mare CJ (2000) Transmission of vesicular stomatitis virus from infected to noninfected black flies co-feeding on nonviremic deer mice. Science 287: 485-487.

Miller JR and Strickler KS (1984) Finding and accepting host plants. In: Bell WJ, Cardé TR (eds) Chemical ecology of insects. Chapman Hall, London, UK, pp 127-157.

Ratti O, Ojanen U and Helle P (2006) Increasing group size dilutes black fly attack rate in Black Grouse. Ornis Fennica 83: 86-90.

Rossignol PA, Ribeiro JMC and Spielman A (1984) Increased intradermal probing time in sporozoite-infected mosquitoes. Am J Trop Med Hyg 33: 17-20.

Rossignol PA, Ribeiro JMC and Spielman A (1986) Increased biting rate and reduced fertility in sporozoite-infected mosquitoes. Am J Trop Med Hyg 35: 277-279.

Schofield SW and Sutcliffe JF (1996) Human individuals vary in attractiveness for host-seeking black flies (Diptera: Simuliidae) based on exhaled carbon dioxide. J Med Entomol 33: 102-108.

Schofield SW and Sutcliffe JF (1997) Humans vary in their ability to elicit biting responses from Simulium venustum (Diptera: Simuliidae). J Med Entomol 34: 64-67.

Shemanchuk JA (1986) Host-seeking behavior and host preference of Simulium arcticum. In: Kim KC and Merritt RW (eds) Black flies. Ecology, Population Management and Annotated World List. Pennsylvania State University, University Park, PA, USA, pp 250-260.

Smith SM (1969) The black fly, Simulium venustum, attracted to the turtle, Chelydra serpentina. Entomol News 80: 107108.

Smith JJB and Friend WG (1982) Feeding behaviour in response to blood fractions and chemical phagostimulants in the black-fly, Simulium venustum. Physiol Entomol 7: 219-226.

Stallings T, Cupp MS and Cupp EW (2002) Orientation of Onchocerca linealis Stiles (Filaroidea: Onchocercidae) microfilariae to black fly saliva. J Med Entomol 39: 908-914.

Sutcliffe JF (1985) Anatomy of membranous mouthpart cuticles and their roles in feeding in black flies. J Morphol 186: 53-68.

Sutcliffe JF (1986) Black fly host location: a review. Can J Zool 64: 1041-1053.

Sutcliffe JF (1987). Distance orientation of biting flies to their hosts. Insect Sci Appl 8: 611-616.

Sutcliffe JF and Mclver SB (1976) External morphology of the sensilla on the legs of selected black fly species (Diptera: Simuliidae). Can J Zool 54: 1779-1787.

Sutcliffe JF and Mclver SB (1979) Experiments on biting and gorging behaviour in the black fly, Simulium venustum. Physiol Entomol 4: 393-400.

Sutcliffe JF and Mclver SB (1982) Innervation and structure of mouthpart sensilla in females of the black fly, Simulium venustum (Diptera: Simuliidae). J Morphol 171: 245-258.

Sutcliffe JF and Mclver SB (1984) Mechanics of blood feeding in black flies (Diptera: Simuliidae). J Morphol 180: 124-144. 
Sutcliffe JF and Mclver SB (1987) Fine structure of tarsal sensilla of male and female Simulium vittatum (Diptera: Simuliidae). J Morphol 192: 13-26.

Sutcliffe JF, Shemanchuk JA and McKeown DB (1994) Preliminary survey of odours that attract the black fly, Simulium arcticum (IIS-10.11)(Diptera: Simuliidae) to its cattle hosts in the Athabasca region of Alberta. Insect Sci and Its Application 15: 487-494.

Sutcliffe JF, Steer DJ and Beardsall D (1995) Studies of host location behaviour in the black fly, Simulium arcticum Malloch (IIS-10.11)(Diptera: Simuliidae): Aspects of close-range trap orientation. Bull Entomol Res 85: 415-424.

Takken W (1991) The role of olfaction in host-seeking of mosquitoes: a review. Insect Sci Applic 12: 297-295.

Takken W and Knols BG (1999) Odor-mediated behavior of Afrotropical malaria mosquitoes. Annu Rev Entomol 44: 131-157.

Thompson BH (1976a) Studies on the attraction of Simulium damnosum s.l. (Diptera: Simuliidae) to its hosts. I. The relative importance of sight, exhaled breath, and smell. Tropenmed. Parasitol 27: 355-473.

Thompson BH (1976b) Studies on the attraction of Simulium damnosum s.l. (Diptera: Simuliidae) to its hosts. II. The nature of substances of the human skin responsible for attractant olfactory stimuli. Tropenmed Parasitol 27: 83-90.

Thylefors B and Alleman M (2006) Towards the elimination of onchocerciasis. Ann Trop Med Parasitol 100: 733-746.

Torr SJ (1990) Dose response of tsetse flies (Glossina) to carbon dioxide, acetone and octenol in the field. Physiol Entomol 15: 93-103.

Vale GA and Hall DR (1985) The role of 1-octen-3-ol, acetone and carbon dioxide in the attraction of tsetse flies, Glossina spp. Bull Entomol Res 75: 209-217.

Wenk P (1962) Anatomie des Kopfes von Wilhelmia equina L. Females (Simuliidae syn. Melusinidae, Diptera). Zool Jahrb Abt Anat Ontog Tiere 80: 81-134.

Wenk $P$ and Schlorer G (1963) Wirstorientierung und Kopulation bei blutsaugenden Simuliiden (Diptera). Z. Tropenmed Parasitol 14: 177-191.

Yee WL and Anderson JR (1995) Trapping black flies (Diptera-Simuliidae) in northern California. 2. Testing visual cues used in attraction to $\mathrm{CO}_{2}$-baited animal head models. J Vector Ecol 20: 26-39. 


\title{
12. Olfaction in Glossina - host interactions: a tale of two tsetse
}

\author{
Steve J. Torr and Philippe Solano
}

\begin{abstract}
The role of olfaction in the host-oriented behaviour of tsetse is reviewed. For all tsetse, odours play a role in host location but the relative importance of olfactory stimuli varies between species. For Morsitans-group tsetse such as Glossina pallidipes and G. m. morsitans, host odours elicit longrange $(\sim 100 \mathrm{~m})$ orientation whereas Palpalis group species such as $G$. palpalis spp. and G. fuscipes spp., while showing significant olfactory responses, are much less responsive. Hence baiting traps with natural host odours can increase the catches of some Morsitans tsetse ten-fold compared to only a doubling with Palpalis tsetse. Species that are less sensitive to odours seem to be more responsive to visual stimuli. The semiochemicals present in host odour include carbon dioxide, 1-octen-3-ol, acetone, butanone, 4-methylphenol and 3-n-propylphenol. For Morsitans tsetse, odours from hosts are not, in general, significantly different from those of non-hosts and thus olfaction does not seem to play an important role in host selection. The notable exception to this is human odour which is repellent to some species of Morsitans tsetse. Traps and insecticide-treated targets baited with blends of 1-octen-3-ol, ketones and phenols are, arguably, the most successful application of artificial baits to vector control worldwide. The current use and future prospects of using natural and artificial baits to control tsetse are discussed.
\end{abstract}

Keywords: Glossina, biting flies, host stimuli, vector behaviour, semiochemicals, allomones, kairomones, attractants, repellents

\section{Introduction}

Tsetse (Glossina spp.) are unlike most other haematophagous Diptera in that both sexes of tsetse rely exclusively on blood for their development and maintenance and hence, every $~ 3$ days they visit their hosts to feed. Tsetse also have an unusual form of reproduction: adenotrophic viviparity. From the age of $\sim 6$ days, females produce a single egg which develops within the uterus over a period of 7-12 days, dependent on temperature. The mature larvae, consisting of equal numbers of males and females, are then deposited on the soil where they burrow to pupariate, emerging $\sim 30$ days later as adults. If there were no deaths of pupae or adults, and larvae were produced with perfect efficiency, then the minimum period that a female must live to ensure population replacement is $\sim 25$ days, but in reality the average female must live nearly twice as long. This combination of regular blood feeding and longevity makes tsetse efficient cyclical vectors of Trypanosoma spp., the causative agents of trypanosomiasis in humans (sleeping sickness) and livestock (nagana); the trypanosomes have sufficient time to develop in the fly and a high probability of subsequent transmission to other hosts.

The unusual bionomics of tsetse have several important impacts on efforts to control tsetseborne diseases. First, classical interventions directed against the immature stages are impossible - the larva is contained within the mother and the pupa is buried in the ground. Second, the slow reproductive rate means that applying a relatively low, but persistent, mortality against a tsetse population can drive it to extinction; a control measure that kills 3\% of the adult females/ day will eliminate a population of tsetse (Hargrove 1988). Third, the absolute reliance of tsetse on feeding regularly from their hosts makes them vulnerable to interventions that disrupt - or exploit - this behaviour. Indeed, this vulnerability was apparent from virtually the moment that 
the role of tsetse in the transmission of trypanosomes was recognised. In the 1890s, an epidemic of rinderpest devastated ungulate populations across East and southern Africa and thus had a huge impact on the distribution and abundance of tsetse hosts. Consequently, large areas of southern Africa became tsetse free. The realisation that tsetse could be controlled by eliminating their hosts led to the widespread use of game elimination to control tsetse-borne diseases.

Some of the earliest interventions against tsetse attempted to kill flies as they approached natural or artificial hosts. On the island of Principe, sticky panels were attached to the backs of humans working in tsetse-infested area; the density of tsetse was reduced greatly although the relative contribution of the sticky-backed humans to this success is unclear. In South Africa, Harris developed traps that simulated some of the visual properties of natural hosts and used these in an attempt to control tsetse in present-day KwaZulu-Natal. Huge numbers of tsetse were captured, and the population numbers declined, but local elimination of the tsetse population was never achieved. In Zululand too, however, the contribution of traps is also in doubt, since hunting operations to eliminate the hosts of tsetse were performed at the same time (Buxton 1955).

The early interest in these approaches to tsetse control did not persist, however: concerns about the gross environmental impact of game elimination, the failure of traps as a control tool, and the development of organochlorine insecticides led to a decline in host-based interventions and, in their place, there emerged control methods which relied on the widespread application of insecticides to the resting sites of tsetse. In Francophone West Africa, the wide-spread use of drugs rather than vector control formed the mainstay of efforts against Gambian sleeping sickness. In present-day Cameroon and Burkina Faso, Eugene Jamot developed the use of highly mobile medical teams to detect and treat cases of sleeping sickness and between 1925 and 1935 hundreds of thousands of people were treated (see reviews by Courtin et al. 2008 and Maudlin 2006). This emphasis on case detection and treatment to combat Gambian sleeping sickness has persisted to the present day as evidenced by the strategy currently promoted by WHO (Simarro et al. 2008). It was not until the 1970s that interest in exploiting the host-orientated behaviour of tsetse was revived. The research that underpinned this revival, and its subsequent impact on tsetse control, is the subject of this review.

\section{The geopolitics of tsetse and humans}

Tsetse infest some 10 million $\mathrm{km}^{2}$ of sub-Saharan Africa but within this area the various groups of tsetse are associated with particular ecological zones (Table 1). The Fusca group of species is generally associated with densely-forested regions of Central Africa; the Palpalis group occurs mainly in riverine areas of Central and West Africa, and the Morsitans group is associated with savannah regions across the continent. These differences in the geographical distribution of tsetse species have important epidemiological implications. The savannah areas are the main areas for cattle production and hence species from the Morsitans group are important in the epidemiology of animal trypanosomiasis. On the other hand, the Palpalis group is associated with mangrove swamps and densely-settled riverine systems of West Africa where $T$. brucei gambiense occurs (Solano et al. 2008); this species of Trypanosoma accounts for $>90 \%$ of sleeping sickness cases and hence the Palpalis group is particularly important in the transmission of this disease. The forest regions infested by Fusca group are sparsely populated and hence species from this group play little part in the transmission of trypanosomiasis in most places.

Not surprisingly, most research has been focussed on species of epidemiological importance. As with many aspects of tsetse biology and control, an account of the olfactory interactions of tsetse 
Table 1. Species and subspecies of tsetse.

Subgenus Nemorhina (Palpalis group)

1a. G. palpalis palpalis Robineau-Desvoidy, 1830

1b. G. palpalis gambiensis Vanderplank, 1949

2. G. tachinoides Westwood, 1850

3a. G. pallicera pallicera Bigot, 1891

3b. G. pallicera newsteadi Austen, 1929

4a. G. fuscipes fuscipes Newstead, 1910

4b. G. fuscipes martinii Zumpt, 1935

4c. G. fuscipes quanzensis Pires, 1948

5. G. caliginea Austen, 1911

Subgenus Glossina s. str. (Morsitans group)

1. G. longipalpis Wiedemann, 1830

2a. G. morsitans morsitans Westwood, 1850

2b. G. morsitans centralis Machado, 1970

2c. G. morsitans submorsitans Newstead, 1910

3. G. pallidipes Austen, 1903

4. G. austeni Newstead, 1912

5. G. swynnertoni Austen, 1923

Subgenus Austenina (Fusca group)

1a. G. fusca fusca Walker, 1849

1b. G. fusca congolensis Newstead and Evans, 1921

2a. G. nigrofusca nigrofusca Newstead, 1910

2b. G. nigrofusca hopkinsi Van Emden, 1944

3. G. fuscipleuris Austen, 1911

4. G. severini Newstead, 1913

5. G. vanhoofi Henrard, 1952

6. G. nashi Potts, 1955

7. G. tabaniformis Westwood, 1850

8. G. longipennis Corti, 1895

9. G. brevipalpis Newstead, 1910

10. G. medicorum Austen, 1911

11. G. schewtzi Newstead and Evans, 1921

12. G. haningtoni Newstead and Evans, 1922

13. G. frezili Gouteux, 1987

and their hosts has two parts: one concerned with the savannah species of East and southern Africa and another concerned with the riverine flies of West Africa.

\section{Host-orientated behaviour of savannah flies}

Species within the Morsitans-group have associations with particular types of savannah woodland. For instance, G. m. morsitans Westwood and G. m. centralis Machado in East and southern Africa are associated with 'miombo' woodland, dominated by species of Isoberlinia, Brachystegia and Julbernadia, and the West African G. m. submorsitans Newstead is found in similar 'doka' woodland dominated by Isoberlinia doka. Glossina Pallidipes Austen is regarded generally as 
being predominant in dense thicket. However, G. pallidipes and G. m. morsitans may also be found together in, for example, the extensive mopane (Colophospermum mopane) woodlands of Zambia and Zimbabwe. Several species have relatively confined geographical distributions: G. swynnertoni Austen is found only in the savannah woodlands of the Maasai steppe and G. austeni Newstead is associated with evergreen thicket lying within $200 \mathrm{~km}$ of the east coast of Africa, but extending in a strip from Somalia to South Africa. The Morsitans fly can also display distributions akin to riverine flies, especially in the dry season when suitable resting sites are confined to riparian woodland (Hargrove and Vale 1980, Torr and Hargrove 1999; Vale 1971). The diet of Morsitans group tsetse is less variable than that of Palpalis group species. The diet of all Morsitans-group species comprises blood from suid and bovid hosts (Clausen et al. 1998). The particular host species with these families can vary according to availability; meals from cattle, for instance, may be more important in settled areas whereas buffalo and kudu may be important in game reserves. Nonetheless this variability is less than that of Palpalis group flies which can persist feeding on largely on reptiles or mammals. Hence, the removal of just six key host species can eliminate Morsitans-group tsetse (Vale and Cumming 1976) but not Palpalis group flies.

\section{Are host odours important?}

Until the 1970s, it was generally assumed that savannah species of tsetse located their hosts largely by vision, an opinion based primarily on the observation that tsetse were attracted to moving objects such as cars and walking men. It was not until the development of techniques for separating the responses to olfactory and visual stimuli that this assumption could be tested objectively. Two technical developments were particularly important for this. First, electric nets (Vale 1974a) provided an objective method for assessing the numbers of tsetse in the vicinity of moving or stationary hosts. Second, the use of ventilated pits (Vale 1974b) provided a means of producing natural host odour with or without any associated visual stimulus. Hence, Vale (1974b) was able to compare the numbers of tsetse in the vicinity of a moving or stationary warthog, or the numbers approaching a visual model of a warthog with or without natural host odour. In a series of ground-breaking experiments, Vale (1974b) demonstrated unequivocally that tsetse were indeed attracted to mobile hosts largely in response to the animal's movement rather than its odours. On the other hand, most ( 90\%) tsetse arriving at a stationary ox or warthog did so in response to the host's odours. Increasing the dose of odour, by placing more hosts in the ventilated pits, increased the numbers of tsetse attracted; each ten-fold increase in dose producing a $\sim 2.5$-fold increase in the catch (Hargrove and Vale 1978, Hargrove et al. 1995).

Following earlier reviewers (e.g. Gibson and Torr 1999, Sutcliffe 1987), it is useful to consider three main phases in the odour-orientated responses of tsetse: (1) activation, which marks the initiation of host-orientated responses; (2) long-range responses, which bring the activated fly to the vicinity of the host and (3) short-range responses, which culminate in it landing and feeding on a host. The host-finding sequence ceases with the insect alighting on the host, and consequently we do not consider the probing and feeding responses.

\section{Activation}

Tsetse are faced with the problem of locating highly mobile hosts that are frequently hidden in dense vegetation. To overcome this challenge, there are two possible search strategies: either 'sit-and-wait' for an animal to pass by or 'range' so as to increase the probability of encountering a host. Tsetse are diurnally active (Brady and Crump 1978, et ante) but their total daily flight time is limited to $<30$ minutes (Bursell and Taylor 1980). For most of the day, they rest on branches 
and boles (Hadaway 1977) or, when temperatures are $>32{ }^{\circ} \mathrm{C}$, in 'refuges' such as holes in trees. Torr (1988a) studied the responses of wild tsetse resting in refuges to natural ox odour or a visual stimulus similar in its size, colour and speed of movement to a passing warthog. These stimuli were presented at 15 min intervals over a 2 hour period in the late afternoon, the period when savannah flies are most active (Hargrove and Brady 1992). Only $15 \%$ of tsetse were activated by natural ox odour compared to $\sim 35 \%$ in response to the visual stimulus or $5 \%$ in the absence of any stimuli. Thus most flies left the refuge in the apparent absence of any host stimuli, presumably in response to their endogenous rhythm of spontaneous activity modulated by nutritional condition, environmental temperature and falling light intensity (Brady 1972, Brady and Crump 1978, Torr and Hargrove 1999). Vale (1980b) also used electric nets to estimate, amongst other things, whether tsetse arriving at a stationary host had been resting or ranging when they first encountered host odour. His results also indicated that most (>80\%) tsetse were ranging when they first responded to host stimuli.

Video studies of tsetse flying in the apparent absence of any host stimuli, and thus presumed to be 'ranging' for a host, showed that they flew with a downwind bias (Gibson et al. 1991). This accords with theoretical considerations of strategies for maximising the probability of encountering host odours. In typical tsetse habitat, variations in wind direction (Griffiths and Brady 1995, Zollner et al. 2004) are likely to create wide swathes of odour which are more likely to be intercepted by flying up- or downwind, with the latter being more energetically efficient (Sabelis and Schippers 1984).

\section{Long-range responses}

Vale (1977b) showed that tsetse respond to the odours produced by a single ox at $\sim 90 \mathrm{~m}$ downwind and hence we regard 'long-range responses' as being those occurring at $>10 \mathrm{~m}$ downwind of a host.

Field studies, using video and various arrangements of electric nets, have shown that in response to host odour, tsetse fly upwind (Gibson and Brady 1988, Torr 1988b, Vale 1974b) at $\sim 6 \mathrm{~m} / \mathrm{s}$ (Gibson and Brady 1985), <0.5 m above ground (Torr 1988b). On losing contact with odour, they execute a reverse turn to bring them back into the odour plume where they turn upwind (Gibson and Brady 1988, Torr 1988b). Wind tunnel studies (Colvin et al. 1989) showed that tsetse use optomotor anemotaxis (Kennedy 1977) to orientate upwind, and video observation of tsetse in odour plumes also indicated that they detected wind direction in flight (Gibson and Brady 1988) and altered their course accordingly.

Griffiths et al. (1995) measured the speed with which marked flies navigated up an odour plume. Half of those that navigated successfully up the plume did so with a mean speed of $4 \mathrm{~m} / \mathrm{s}$, which accords with the notion that they flew up the entire plume in virtually straight and continuous flight. The remainder however arrived at the odour source with constant probability for at least 20 minutes after take-off. The proline-based metabolism of tsetse prevents them from flying for extended periods (Hargrove 1975) and it seems likely that these late arrivals must have landed at some point en route to the odour source. Direct observation of tsetse in the field showed that, after losing contact with odour, tsetse land, wait for variations in wind direction to bring the plume back to them and then take-off upwind when contact is re-established (Bursell 1984a). Bursell $(1984 a, b)$ suggested that tsetse assessed wind direction while on the ground and moved up the plume in a series of straight flights upwind. 
Which way is up?

In the absence of vegetation, packets of odour-laden air are carried downwind in virtually straight lines (David et al. 1982). Hence, a strategy of flying directly upwind whenever odour is detected should lead a fly to its host. However, a series of studies in tsetse-infested woodland in Zimbabwe suggested that this simple view of plume dispersal is not applicable. To visualise the dispersal of an odour plume, Brady et al. (1989) observed the movement of parcels of smoke. They found that at the low $(<1 \mathrm{~m} / \mathrm{s})$ wind speeds typically prevailing when tsetse are active, the parcels did not travel in straight lines through vegetation. Instead, they changed direction such that even 5 $\mathrm{m}$ from the source, $25 \%$ of flies travelling upwind would be heading $>90^{\circ}$ away from host. This error was even greater at lower wind speeds, greater distances from the source and in areas with dense vegetation.

However such navigational errors may not be as serious as Brady et al. (1990) suggested. First, theoretical analysis of host-location strategies suggests that for tsetse starting $100 \mathrm{~m}$ downwind of a host, even a 20-40\% upwind bias in flight direction would still enable virtually all flies to arrive at the source within $300 \mathrm{~s}$ (Williams 1994). Second, in nature, the vegetation and local topography constrains the direction of flight and hence flight directly towards the source may be frequently impossible. Indeed, there is evidence from Kenya and Zimbabwe that tsetse fly along game paths and through gaps in bushes (Paynter and Brady 1993, Vale 1998). In such situations, tsetse may need only to gauge whether to fly up or down a path rather than the precise direction of the host.

The general impression of the long-range orientation of tsetse is therefore not of a precisely orientated navigation up an odour plume but, rather, a 'quick-and-dirty' strategy of fast, mainly upwind, flight that rarely leads directly to the host (Griffiths et al. 1995). For fast-flying insects such as tsetse, this so-called 'biased random walk' strategy for locating odour sources may be optimal (Brady et al. 1990, Griffiths et al. 1995).

\section{Short-range responses}

Odour-orientated responses bring the fly to the vicinity of the host, but the final location is largely a response to visual cues. Indeed, tsetse are unable to locate an odour source precisely without a visual target (Vale 1974b) and flies approaching an odour source can be diverted towards an odourless visual target (Torr 1989). The colour, shape and size of the target govern the strength of the response. For example, blue or black objects are more attractive than green-yellow; larger objects attract more flies than small ones, and horizontal oblongs or squares are more attractive than vertical oblongs. In general, these same cues also increase landing responses, with the exception that black elicits a stronger landing response than blue. Host odours appear to have no effect on close-range orientation but they do increase landing responses (Hargrove 1980, Vale 1974b, Warnes 1995).

\section{Components of host odour}

A combination of random screening and chemical analysis of host odours using gas chromatography linked with electroantennography (GC-EAG) (e.g. Bursell et al. 1988, Hall et al. 1984) has identified ten chemicals as being important host kairomones in ox odour. The most active of these are: carbon dioxide, acetone (Vale 1980a), 1-octen-3-ol (henceforth termed octenol; Vale and Hall 1985a), 4-methylphenol and 3-n-propylphenol (Bursell et al. 1988, Hassanali et al. 1986) and butanone (Torr et al. 1995). When dispensed at the known natural doses, a synthetic blend of 
these is about half as effective as natural ox odour, indicating that other kairomones must also exist (Hargrove et al. 1995, Torr et al. 1995, 2006).

\section{Behavioural roles of host kairomones}

Various field and laboratory studies have assessed whether the components of host odour have specific behavioural effects.

\section{Activation}

Laboratory and field studies both suggest that carbon dioxide has an important activating effect whereas other components such as acetone or octenol do not (Bursell 1984a, Torr 1988a).

\section{Long-range responses}

Wind-tunnel studies have shown that tsetse exhibit optomotor anemotaxis (Kennedy 1977) in response to carbon dioxide (Colvin et al. 1989), acetone and octenol (Bursell 1984a; Paynter and Brady 1993). The field evidence for this is however equivocal: video studies suggest that upwind anemotaxis occurs in response to all the known attractants (Brady and Griffiths 1993, Paynter and Brady 1993) whereas studies using electric nets (Torr 1988c, 1990, Torr and Mangwiro 1996) or visual observations (Bursell 1987, Torr 1988c) suggest that upwind flight occurs only in the presence of carbon dioxide. Field studies have also shown strong synergistic effects between carbon dioxide and both octenol and acetone (Torr 1990, Vale and Hall 1985a). For instance, Torr (1990) showed that carbon dioxide and acetone dispensed individually at doses of 1,200 I/h and $50 \mathrm{mg} / \mathrm{h}$, respectively, doubled the catch compared to a 16-fold increase when both odours were dispensed together. He suggested that the synergy arises because strong upwind flight is elicited only when acetone and carbon dioxide are both present. Various laboratory studies have indicated that carbon dioxide, acetone and octenol also elicit orthokinetic and klinokinetic responses (Paynter and Brady 1993, Warnes 1990) but field evidence for this is poor (Gibson and Brady 1988).

\section{Short-range responses}

Torr (1989) showed that the close-range orientation of tsetse towards a host was unaffected by odour composition, but the final alighting response is affected by the presence of carbon dioxide (Vale and Hall 1985b) and unidentified chemicals present on the skin (Warnes 1995). However, visual factors such as colour type and pattern (Brady and Shereni 1988, Doku and Brady 1989, Gibson 1992, Green 1986), shape (Vale 1974b, Torr 1989) and size (Hargrove 1980) appear to be more important.

All the above studies of the responses of tsetse to specific host kairomones highlight the importance of carbon dioxide. Earlier studies (e.g. Brady and Griffiths 1993, Torr 1988b) suggested that at any great distance downwind of a host, the background noise of carbon dioxide in the atmosphere would obscure that produced by the host. Thus, it was argued that host carbon dioxide could not play a role in long range responses and activation - behavioural responses which are likely to occur up to $90 \mathrm{~m}$ downwind of the host. However, detection of carbon dioxide is not necessarily limited by its presence in the atmosphere but rather, its variability (Zollner et al. 2004). Using high performance (10 Hz to an accuracy of $\pm 0.1 \mathrm{ppm}$ ) infra-red carbon dioxide analysers, Zollner et al. (2004) showed that carbon dioxide released 4-20 I/min can be detected 
up to $64 \mathrm{~m}$ downwind. The resolution and sensitivity of the instruments are comparable to those of an insect, suggesting that, in principle, carbon dioxide is detectable by insects at long range.

\section{The role of olfaction in host selection}

Savannah species of tsetse feed on a wide range of animals but some species are fed on more than others. For instance, meals from pigs (warthog and bushpig) and ungulates (e.g., buffalo, kudu) are repeatedly identified as being important hosts whereas blood meals from primates, including humans, are relatively rare (Clausen et al. 1998). Moreover, while ruminants consistently form a large part of the diet, some relatively common species such as impala are notable by their virtual absence in the diet.

Various studies have assessed whether host selection is governed by olfactory responses. Vale (1974b) showed that the numbers of tsetse attracted to different hosts was roughly correlated with their respective masses. For example, odour from an ox $(\sim 450 \mathrm{~kg})$ attracted $5 \times$ more than a goat $(\sim 32 \mathrm{~kg})$ whereas the number attracted to an impala and a bushpig (both $\sim 73 \mathrm{~kg}$ ) were roughly equal. The only exception to the general correlation between mass and attractiveness was for humans: the numbers of tsetse attracted to odour from a single human $(74 \mathrm{~kg})$ was a fifth of that attracted to goat odour, and adding human odour to ox odour virtually annulled the attractiveness of the latter. Human odour depressed not only the numbers of tsetse attracted to an odour source but also the alighting (Hargrove 1976) and feeding (Vale 1977a) responses. In further studies, Vale (1979) showed that the repellent effects of humans were due to unidentified chemicals present in body odour rather than the breath.

The preponderance of Suidae in the diet of tsetse has prompted several detailed studies of the responses of tsetse to odours from bushpig and warthog. Vale (1974b) reported that many tsetse landed near the eyes of warthogs and he suggested that this response may be related to secretions from the pre-orbital gland. Torr (1994) confirmed Vale's observations but suggested that the landing response was a visual response to the dark patch produced by eye secretions rather than an olfactory response. Moreover, adding natural warthog odour to a blend of artificial ox odour (i.e. carbon dioxide, acetone, octenol and phenols) did not increase the catch significantly, suggesting that there are no kairomones unique to warthogs (Torr 1994). Studies of the responses of tsetse to odours from bushpig on the other hand do suggest that this host does may produce unidentified attractants; placing hessian sacks soiled with residues from a live bushpig increased the catch of tsetse (Vale et al. 1986a). Vale et al. (1986a) suggested that the unidentified kairomones present in bushpig residues were not identical to those in ox urine (i.e. various phenols) but no direct comparisons of bushpig residues and phenols have been undertaken to test this suggestion.

Several papers by Gikonyo and colleagues have assessed the responses of tsetse to waterbuck (Kobus ellipsiprymnus (Ogilby)), an ungulate which, they argue, is fed on less than might be expected from its relative abundance. Gikonyo et al. (2000) compared the responses of tsetse to waterbuck and oxen. Single flies were placed in a cage, which was then applied to a live host, or a feeding membrane treated with secretions from the host's coat. There was no significant difference in initial landing rates - but landing duration, probability of probing and feeding rates were all lower with waterbuck. They suggested that these differences were due to allomones unique to waterbuck. Further investigations showed that the chemical profiles of waterbuck and buffalo differ (Gikonyo et al. 2002), that tsetse show electrophysiological responses to these chemicals (Gikonyo et al. 2003) and that some of these chemicals elicit upwind flight in olfactometers (Gikonyo et al. 2003, Mwangi et al. 2008). Curiously, the bioassays of putative allomones did 
not repeat Gikonyo's (2003) initial bioassay based on landing responses, and hence there is no evidence that the putative allomones are indeed those responsible for differences in landing responses. Moreover, the behavioural implications of results from olfactometers with small $(<2$ $\mathrm{m}$ long $\times 0.24 \mathrm{~m}$ wide, Gikonyo et al. 2003) flight chambers are unclear. In the only field study of waterbuck odours, Madubunyi et al. (1996) found that traps baited with urines from buffalo, cattle and waterbuck caught similar numbers of tsetse, and chemical analyses showed that these urines contained 4-methylphenol and 3-n-propylphenol in similar ratios. Thus there are no field data to confirm the hypothesis that waterbuck odours are repellent to tsetse.

On the other hand, there are examples of host choice being influenced by responses to cues other than host odours. For example, the low feeding rates of tsetse on impala and goat seems to be explained by their inherently high rate of grooming, which deters tsetse from landing and engorging; an impala attracted fewer flies than an ox, consistent with its smaller size, but no flies fed on it whereas $35 \%$ of tsetse approaching an ox fed to completion (Vale 1977a). Visual stimuli may also play a role in host selection: the low number of feeds from zebra seems to be due, in part at least, to the cryptic effect of their stripes (Gibson 1992).

The advent of DNA-based tools for identifying the individual specific sources of blood meals allows us to determinate whether some individuals within a herd are bitten more than others (Torr et al. 2001). The results show that for tsetse feeding on cattle there is a marked preponderance of meals from larger/older individuals within a herd (Torr et al. 2007b). This bias seems to be due to younger cattle having higher rates of defensive behaviour (Torr and Mangwiro 2000) and producing low doses of kairomones, particularly carbon dioxide and phenols (Torr et al. 2006).

\section{Repellents}

The search for attractants and analyses of odours from hosts has led, almost incidentally, to the identification of a number of chemicals naturally present in host odours that reduce the catch of tsetse from traps (Vale 1979, 1980a, Vale et al. 1988a). These findings might suggest that these chemicals may play a role in host selection (Torr et al. 1996). However, results with traps do not necessarily indicate that these chemicals reduce the numbers of tsetse attracted to and/or feeding on hosts in nature. Thus, while 2-methoxyphenol reduces the numbers of G. pallidipes caught by odour-baited traps by $\sim 85 \%$, it has no significant effect on the numbers attracted to and feeding on an ox (Torr et al. 1996). More recently, Saini and Hassanali (2007) showed that 4-methyl-2methoxyphenol reduced the numbers of tsetse feeding on cattle by $95 \%$. However, this chemical has not been detected in natural host odours.

\section{Intraspecific variation in olfactory responses}

Much of the foregoing description of olfactory responses relates primarily to G. pallidipes and G. $m$. morsitans populations in Zimbabwe and Kenya. Other species and populations of Morsitans-group flies have been less intensively studied; nonetheless, there is evidence that there are similarities and differences between species and populations. G. longipalpis Wiedemann seems to be the species most similar to $G$. pallidipes being responsive to phenols, acetone and octenol (Späth 1995). The G. morsitans subspecies and G. swynnertoni are responsive to acetone and octenol but show little if any response to the phenols (G. m. morsitans: Vale 1980a, Vale et al. 1988a; G. m. submorsitans: Mérot et al. 1988b, Politzar and Merot 1984; G. m. centralis Machado: Modo 1999; G. swynnertoni: Brightwell and Dransfield 1997, Ndegwa and Mihok 1999). Glossina austeni seems to be the least responsive species, with only carbon dioxide being an effective kairomone 
(Kappmeier-Green 2001). There is evidence of intra-specific variations in the olfactory responses of Morsitans-group species. Studies of G. pallidipes in Somalia showed that acetone or octenol were only effective in the presence of 4-methylphenol and 3-n-propylphenol, and baiting a target with the odour from an ox only doubled the catch. By contrast, acetone, octenol and phenols are effective on their own for G. pallidipes in Zimbabwe (Vale 1980a, Vale and Hall 1985a) and Kenya (Baylis and Nambiro 1993), and odour from a single ox increases the catch from a target $\sim 10 x$ (Vale 1974b).

\section{Fusca group species}

Fusca group species play a less important role in the transmission of trypanosomes and hence their olfactory responses have been studied less than those of the Morsitans group. Nonetheless a few species are locally important as vectors of disease and/or are sympatric with Morsitans group species and hence we do have some knowledge of their responses. The impression from these few studies is that the group displays somewhat similar responses to Morsitans group species. For instance, baiting traps with acetone, octenol or 4-methylphenol all increase the catches of $G$. longipennis Corti (Baylis and Nambiro 1993, Kyorku et al. 1990), G. brevipalpis Newstead (Kappmeier and Nevill 1999). Studies of G. medicorum Austen in west Africa suggest that 3-methylphenol, 4-methylphenol and octenol are effective, although acetone is not (Späth 1995).

\section{West side story: olfactory responses of riverine flies}

\section{Ecology of riverine flies}

Riverine species of tsetse show marked associations with particular ecotypes, which, in turn, have important implications for their interactions with hosts. It is therefore pertinent to summarise briefly the main features of the important Palpalis group species (reviewed in Solano et al. 2009).

\section{Distribution}

\section{Glossina tachinoides Westwood}

This species generally occurs in narrow thickets along river courses in thickets consisting of Mimosa pigra and Morelia senegalensis (Sudanese ecotype), swampy forests of Mitragyna inermis and in the 'open' gallery forests consisting predominantly of Syzygium guineense, Cola laurifolia and Pterocarpus santalinoides (Sudano-Guinean ecotype). It is often found with G. p. gambiensis Vanderplank in riverine habitats, and occasionally with G. p. palpalis Robineau-Desvoidy in forested areas. The distribution of $G$. tachinoides is largely between $12^{\circ}-8^{\circ} \mathrm{N}$ and $2^{\circ} \mathrm{W}-21^{\circ} \mathrm{E}$, but there are also a few isolated populations found as far east as Ethiopia.

\section{Glossina palpalis}

The distribution of this species extends along the Atlantic coast from Senegal to Angola. Glossina p. palpalis is found closer to the coast within degraded forest areas and wet savannahs, whereas G. p. gambiensis is found in the riparian networks within drier woodland savannahs and 'niayes lowland areas associated with oil palm plantations in north-west Senegal. Thus G. p. gambiensis is associated with the Guinean and Sudano-Guinean ecotypes extending from Senegal to Benin, while G. p. palpalis occurs from Liberia to Angola. Both subspecies can be found in mangrove areas along the coast, and in areas ranging from peridomestic areas, such as 'sacred woodlands' 
or mango plantations in savannah areas and coffee/cocoa plantations in forested areas, to sites within major urban centres such as Abidjan and Dakar. This close association with humans makes this species a particularly important vector of human and animal trypanosomiases.

\section{Glossina fuscipes Newstead}

This species is associated with the riverine and lacustrine systems of the Congo basin and Lakes Victoria and Tanganyika, particularly vegetated areas associated with rivers, lakes and the forestsavannah mosaics. G. fuscipes is an important vector of human trypanosomiasis in Central Africa (DRC, Congo Brazzaville) and some countries of East Africa (Uganda, Kenya), as well as animal trypanosomiasis in, for example, the Central African Republic.

\section{Seasonal effects on local distribution}

The local distribution of Palpalis group species varies markedly between seasons, with flies being closer to water bodies during the drier months. Within the riverine system, flies disperse linearly along the river, oscillating up- and down stream over relatively short distances (200-300 m). In the dry season, displacements are largely within the river whereas in the wet season they are more likely to disperse away from the river, crossing adjacent savannah woodlands and moving from one hydrographic network to another. In the hot season, some individuals can fly long distances (17 km in three days for G. tachinoides; $22 \mathrm{~km}$ in five days for G. p. gambiensis) along rivers.

\section{Host range}

The diet of Palpalis group species seems more variable than that of Morsitans group flies, being considered more 'opportunistic' in their diet (Sané et al. 2000; Simo et al. 2008 ). For instance, in Côte d'Ivoire, G. palpalis feeds mainly on humans in settlements in the absence of pigs, but takes $75 \%$ of its blood meals on pigs when they are abundant and in plantations, it feeds at equal rates on bushbuck and humans. In the hot season, when humans and small ungulates are concentrated around water bodies, the fly takes $40 \%$ of its blood meals from bushbuck and 35$55 \%$ from humans. In the cold season on the other hand, it feeds mainly on reptiles (54-67\%).

Tsetse fly populations (G. palpalis, G. tachinoides) that inhabit atypical habitats around villages of wet areas can feed exclusively on pigs bred by villagers. There is some evidence that individual $G$. p. gambiensis may learn to feed on a particular species in some circumstance (Bouyer et al. 2007a).

\section{Olfactory responses of Palpalis group flies}

In general, Palpalis group species show markedly weaker responses to host odours. This has meant that researchers have seldom published their findings and, hence, previous reviewers (e.g. Gibson and Torr 1999, Green 1994) have had to rely on unpublished data and personal communications. At the time of writing, published data on the responses of Palpalis group flies is still confined largely to just two species: G. tachinoides and G. fuscipes.

\section{Glossina tachinoides}

Merot et al. (1986), in Burkina Faso, compared the numbers attracted to sources of natural odour from a man $(60 \mathrm{~kg})$, a pig $(60 \mathrm{~kg})$ and a cow $(150 \mathrm{~kg})$ following Vale's (1974b) protocol of placing the hosts in a ventilated pit. Tsetse were captured using an electrocuting target placed at the odour 
source. The results showed that these odours increased the catch only $1.2 \times$. Even placing four cattle in the pit to increase the dose of odour produced only a $1.8 \times$ increase in catch. In accordance with these relatively modest increases in catch, carbon dioxide dispensed at $3 \mathrm{l} / \mathrm{min}$ increased the catch only $1.2 \times$. In a subsequent study, Galey et al. (1986) showed that baiting traps with carbon dioxide (0.5-20 I/min) increased the catch 1.2-3.3x. Filledier and Mérot (1989b) showed that urine from a Baoule taurine tripled the catch of $G$. tachinoides.

Merot et al. (1988a) undertook a series of experiments to determine whether the kairomones known to attract G. pallidipes were also effective for G. tachinoides. Their results (Mérot et al. 1988a) showed that a blend of carbon dioxide $(1.5 \mathrm{I} / \mathrm{min})$, acetone, octenol and phenols doubled the catch compared to a $1.3 \times$ increase produced by the odour from three cattle. Studies with traps suggested that (1) a combination of phenol(s) and octenol doubled the catch, but (2) these odours were ineffective on their own, and (3) their effect was antagonised by acetone. Filledier and Mérot (1989a) showed that baiting traps with a blend of 3-methylphenol and octenol doubled (1.3$2.5 \times$ ) the catch of tsetse. Späth (1995) suggested a blend of 3-methylphenol, 4-methylphenol and acetone were more effective but his results do not show that the addition of 4-methylphenol was significantly more effective than the combination of octenol and 3-methylphenol alone.

Blood meal analyses of $G$. tachinoides show a preponderance of meals from hippopotamus, ruminants (mainly bushbuck) and monitor lizards (Clausen et al. 1998). Accordingly, Späth (1997) assessed the effect of baiting traps with odours from these hosts. Placing a caged monitor lizard adjacent to a trap had no effect but hexane skin washings from the lizard increased the catch $1.3 \times$. Skin washings from a warthog and the phenolic fraction of bushbuck urine also increased the catch significantly $1.4 \times$ and $1.8 \times$, respectively. No studies have been undertaken to confirm these findings or to test whether these products contain novel attractants.

\section{G. fuscipes fuscipes Newstead}

Like other Palpalis group species, G. f. fuscipes is associated with riverine and lacustrine habitats. Studies of their diet consistently show a high proportion of meals from reptiles (Clausen et al. 1998), particularly monitor lizards (Varanus niloticus niloticus Linnaeus), and this has prompted several studies of the responses of $G$. f. fuscipes to lizards.

In the Central African Republic, Gouteux et al. (1995) showed that placing a caged monitor lizard adjacent to a trap increased the catch of G. f. fuscipes significantly. The cages were hidden by covering them with leaves and hence Gouteux et al. argued that the catch increases were due to olfactory rather than visual stimuli. In Kenya, Mohamed-Ahmed (1998), used various arrangements of traps and electric nets to study the responses of G. f. fuscipes to monitor lizards. Placing a cage containing three lizards adjacent to an electric net doubled the catch of tsetse, although the increase was significant only for females. The lizards within the cage were visible and hence the increase could be due to either visual or olfactory responses. Accordingly, Mohamed-Ahmed compared the numbers of tsetse landing on a black cylinder $(1.5 \times 0.1 \mathrm{~m})$ with or without a single lizard ( $~ 5 \mathrm{~kg}$ ) inside. The cylinder was perforated with small ( $13 \mathrm{~mm}$ dia) holes along its length to allow odours from the lizard to escape. An electric grid that covered the surface of the cylinder caught flies as they landed. The results showed that when the lizard was inside, twice as many tsetse landed, but the increases were not significant for either sex. Baiting cylinders with lizard urine increased the catch of tsetse attracted to the vicinity of the cylinder but the increase $(2 x)$ was only significant for females. By contrast, baiting traps with lizard urine increased significantly the catch of males $(\sim 1.4 \times)$ but not females. 
Glossina f. fuscipes is an important vector of Trypanosoma spp. (Waiswa et al. 2006, Welburn et al. 2006) and hence, not surprisingly, meals from primates - including presumably humans - and ungulates are also relatively common (Clausen et al. 1998). However, despite feeding on cattle, G. $f$. fuscipes does not appear responsive to the tsetse attractants present naturally in ox odour; baiting traps with acetone, octenol or phenols, dispensed either on their own or in blends known to be effective for other species of tsetse, did not have any significant effects (Mwangelwa et al. 1995). Mohamed-Ahmed and Mihok (1999) studied the numbers of G. f. fuscipes attracted to traps baited with carbon dioxide. In an experiment conducted in a 'linear forest', baiting a trap with carbon dioxide (5 I/min) had no significant effect on the catch of tsetse. However, when the experiment was repeated in a 'dense forest', carbon dioxide $(2.5 \mathrm{l} / \mathrm{min})$ doubled the catch of females but had no significant effect for males. In a third experiment, conducted in 'dense forest', they used electric nets to gauge the numbers of tsetse in the vicinity of a trap with or without carbon dioxide (2.5 I/ $\mathrm{min})$. Their results show that carbon dioxide doubled the total (i.e. catch from trap+electric nets) catch of females but had, yet again, no significant effect for males. The similar effects obtained with either (1) traps alone or (2) traps+electric nets suggest that the main effect of carbon dioxide was to increase the numbers of tsetse attracted to the odour source rather than increasing trap efficiency (Vale and Hargrove 1979). Mohamed-Ahmed and Mihok (1999) argued that the absence of any effect for carbon dioxide in the linear forest was due to local topography and vegetation; for example, if tsetse were confined to the linear forest, which was only 5-10 m wide, then the area from which they could be recruited by host odours would be necessarily restricted. Nonetheless, even in habitats where carbon dioxide or lizard odours were effective, it is striking that the overall effects are relatively small $(\sim 2 x)$ and confined to females only.

\section{Other Palpalis group species}

Published data on other Palpalis group species are extremely scant. In Liberia, Cheke and Garms (1988) showed that acetone and octenol doubled the catch of G. p. palpalis from traps but these odours had no significant effect when dispensed in combination with a blend of phenols. Finally, in Congo-Brazzaville, Frezil and Carnevale (1976) showed that baiting a trap with carbon dioxide increased the catch of G. f. quanzensis Pires $\sim 40 \mathrm{x}$.

\section{Current research on Palpalis group species}

Currently, a major research programme supported by the Bill and Melinda Gates Foundation and the EC, amongst others, is assessing the olfactory responses of five Palpalis group species: $G$. $p$. gambiensis, G. p. palpalis, G. f. fuscipes, G. f. quanzensis and G. tachinoides. Preliminary findings (Omolo et al. 2009, Rayaisse et al. 2010) suggest that these species show moderate but significant responses to host odours. For instance, studies in Burkina Faso have shown that there is a significant $\sim 1.5 \times$ increase in the numbers of G. p. gambiensis attracted to a source of ox odour, and that a blend of acetone, 4-methylphenol, 3-n-propylphenol and octenol can double the catch from traps (IAEA 2003, Rayaisse et al. 2010). Studies of G. fuscipes species (Omolo et al. 2009) confirm that G. f. fuscipes responds to odours from lizard but not to those from cattle, humans or pigs whereas G. f. quanzensis appears to show significant responses to pig odours. The absence of any significant response by G. f. fuscipes to cattle odour is particularly intriguing since some experiments showed that even the odour from four cattle, which would have contained $\sim 8 \mathrm{l} / \mathrm{min}$ of carbon dioxide, was ineffective. Previous studies (Mohamed-Ahmed and Mihok 1999) have also reported a variable response to carbon dioxide (for details see above). For the other Palpalis group species, carbon dioxide does appear to be effective but not all of the olfactory response is accounted for by this kairomone. Overall, Palpalis group flies show a significant response to host 
odours, but the effect is small in comparison to, say, G. pallidipes. Research is currently in progress to identify the kairomones present in lizard, pig and cattle odours.

\section{Attacking tsetse with attractants}

Not surprisingly, the practical use of attractants for controlling and monitoring tsetse has been largely confined to operations directed against the Morsitans group. Ironically, however the revival of bait methods to control tsetse was led by Claude Laveissière and co-workers in West Africa who used unbaited traps to control Palpalis group species in sleeping sickness foci of Ivory Coast (see Table 2 in Green 1994). These operations were initiated in the late 1970s, some four years before the first large-scale use of odour-baited traps and targets (see Table 1 in Green 1994). Nonetheless, for the purposes of this review we focus mainly on the use of odour baits against Morsitans group species.

\section{Use of odour baits to control Morsitans group species}

The first successful application of odour-baited traps and targets to control tsetse was undertaken in Zimbabwe where traps and insecticide-treated targets baited with synthetic host odours were used to eliminate G. m. morsitans and G. pallidipes from an island $\left(5 \mathrm{~km}^{2}\right)$ in Lake Kariba (Vale et al. 1986b). Following on from this success, large-scale use of the technology began in 1984, with the deployment of insecticide-treated targets over an area of $\sim 1000 \mathrm{~km}^{2}$ in the Zambezi valley of Zimbabwe. At the commencement of the operation in 1984, these targets were baited with acetone and octenol and these devices, deployed at a density of $4 / \mathrm{km}^{2}$ eliminated G. m. morsitans and reduced the population of G. pallidipes by $>99.9 \%$ (Vale et al. 1988b). These early successes led to a rapid adoption of bait methods for tsetse control in Zimbabwe (Figure 1) and elsewhere (e.g. Zambia: Willemse 1991; Kenya: Dransfield et al. 1990). By the late 1980s, phenols were identified as attractants for G. pallidipes and since then a blend of acetone $(\sim 100 \mathrm{mg} / \mathrm{h})$, octenol $(\sim 0.5 \mathrm{mg} / \mathrm{h})$, 4-methylphenol $(\sim 1 \mathrm{mg} / \mathrm{h})$ and 3-n-propylphenol $(\sim 0.1 \mathrm{mg} / \mathrm{h})$ has become the standard blend for traps and targets used to monitor and/or control G. pallidipes (Torr et al. 1997), while blends of acetone and octenol have been used in operations against G. morsitans spp. (Kgori et al. 2006, Willemse 1991). For further details of control operations based on the use of odour-baited traps and targets, see reviews by Green (1994), Hargrove (2003) and Torr et al. (2005).

The relatively low cost of odour-baited traps and targets, and the possibility that local people could make and deploy them, made the use of such devices appealing to local communities and, more especially, the government institutions, donor agencies and NGOs that advised them. Accordingly, the 1990s saw a proliferation of community-based interventions against tsetse. Over the last decade, artificial baits have been somewhat eclipsed by the increasing use of insecticidetreated cattle, as illustrated by the case of Zimbabwe (Figure 1). Insecticide-treated cattle are identical to insecticide-treated targets in their effect on a tsetse population but they have several practical advantages including being: cheaper, less susceptible to theft and vandalism and effective against tick-borne diseases (Eisler et al. 2003). A further advantage of insecticide-treated cattle over artificial baits is that livestock keepers are more likely to treat their cattle than use traps and targets which are regarded as 'public goods' (Kamuanga et al. 2001).

Recent studies of the behaviour of tsetse feeding on cattle (Bouyer et al. 2007b; Torr et al. 2007a,b) showed that by applying insecticide to the legs and belly of the larger cattle within a herd, the cost of trypanosomiasis control can be reduced still further to $\sim 2$ per head per year (Shaw et al. 


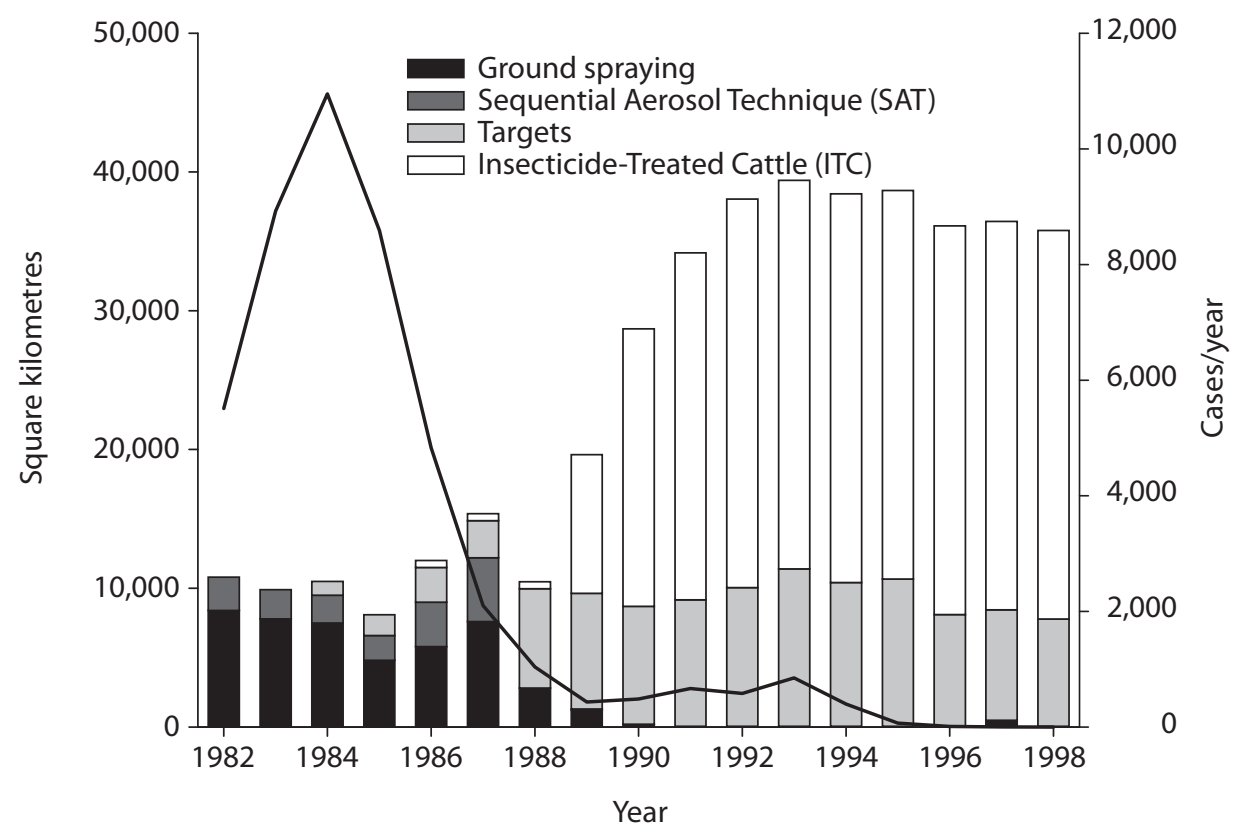

Figure 1. Areas of Zimbabwe treated annually by various methods of tsetse control (vertical bars) and annual number of trypanosomiasis cases (solid line) (redrawn from data presented in Vale and Torr 2004 and Torr et al. 2005).

2006). Hence in areas where cattle are already present it seems that, in principle, the use of natural rather than artificial baits will be the method of choice.

\section{Discussion}

\section{Variation in the olfactory responses of tsetse}

East vs. West

Intraspecific comparisons of the olfactory responses of different species in different zoogeographical zones are hampered by the range of experimental methods used by various researchers. To overcome this, let us consider only experiments where an electrocuting target was baited with odour from a single adult ox or cow, thereby ignoring the potentially confounding effects of using different trap designs, and the effects of host odour on trap efficiency. The results (Figure 2) show that the large responses to host odours are neither exclusive to Morsitans group species (cf. G. longipennis, a Fusca group species) nor do all Morsitans group species show strong responses to host odour (cf. G. austeni, G. m. submorsitans, G. pallidipes in Somalia). On the other hand, published data, and studies currently being undertaken by us in West and Central Africa, do indicate that no Palpalis group species show the strong olfactory responses exhibited by, say, G. pallidipes in Zimbabwe. 


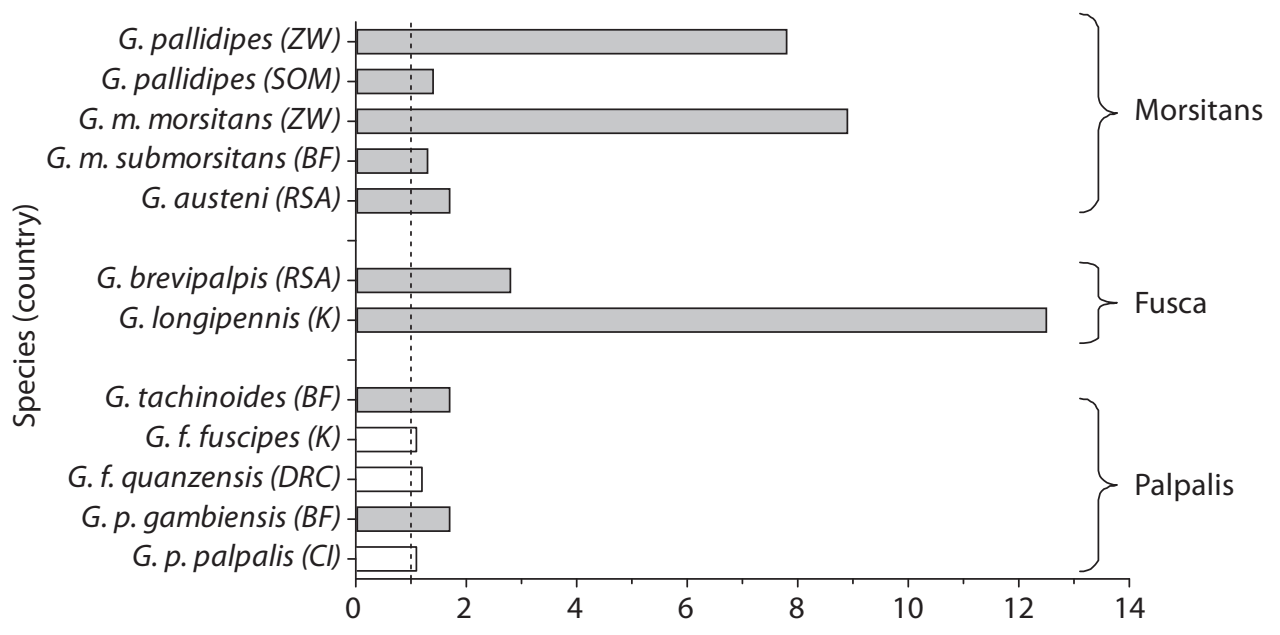

Catch index

Figure 2. Catch of tsetse from an electrocuting target baited with odour from 1-2 cattle. Catches are expressed as a proportion ('Catch index') of that from an unbaited target. An index of 1 (dotted vertical line) indicates that the odour has no significant effect; solid bars indicate indices that are significantly $(\mathrm{P}<0.05)$ different from unity. Indices for each species are derived from: Vale 1974b (G. m. morsitans \& G. pallidipes in Zimbabwe (ZW)); Mérot et al. 1986 (G. m. submorsitans \& G. tachinoides in Burkina Faso (BF)); Torr et al. 1989 (G. pallidipes in Somalia (SOM)); Baylis and Nambiro 1993 (G. longipennis in Kenya (K); Kappmeier-Green 2001 (G. brevipalpis and G. austeni in South Africa (RSA)); Omolo et al. 2009 (G. fuscipes spp and G. f. quanzensis in Kenya (K) and the Democratic Republic of Congo (DRC)); Rayaisse et al. 2009 (G. p. gambiensis and G. tachinoides in Burkina Faso (BF)); G. p. palpalis in Côte d'Ivoire (CI)).

The current model of the odour-orientated behaviour of tsetse, based largely on the responses of G. pallidipes in Zimbabwe and Kenya, may not be as widely applicable as is assumed. We suggest that there is an urgent need to analyse the basic host-orientated responses of these 'less responsive' species. For instance, the current paradigm for the long-range orientation of tsetse is based on upwind anemotactic responses to host odours. However, might klinokinetic or orthokinetic responses (Warnes 1990) rather than anemotactic responses be more important for Palpalis group species? Might host kairomones modulate the visual responses of tsetse (Torr 1989)? Having established what species do not do, we now need to find out what they do do. A better understanding of the behaviour of these species could provide improved means of controlling tsetse in much the same way that Vale's discovery of the olfactory responses of tsetse in Zimbabwe led to a revolution in tsetse control methods there (Vale 1993).

\section{Intraspecific genetic variation}

The differences in the olfactory responses of G. pallidipes from Zimbabwe and Somalia may be due to genetic differences between these populations. Virtually no information is currently available on relationships between genetic structure and the olfactory responses of tsetse populations. The few studies dealing with tsetse population genetics in East Africa have focused on genetic differentiation measures between geographically distant populations (see Gooding and Krafsur 
2005 for review). In West Africa, several studies have focused on within-population structure (e.g. Ravel et al. 2007, Solano et al. 2000). These studies suggest that, on a microgeographic scale, $G$. palpalis populations are composed of several 'clusters' or subgroups, genetically differentiated from each other with separate geographical and host ranges (as illustrated in Figure 3). Such a population structure might explain, in part, why Palpalis group flies have variable responses to host odours. For example, a trap baited with reptile kairomones might only be effective for the sub-population that feeds on lizards and not those that feed on, say, humans or pigs. Further research is needed to assess this possibility.

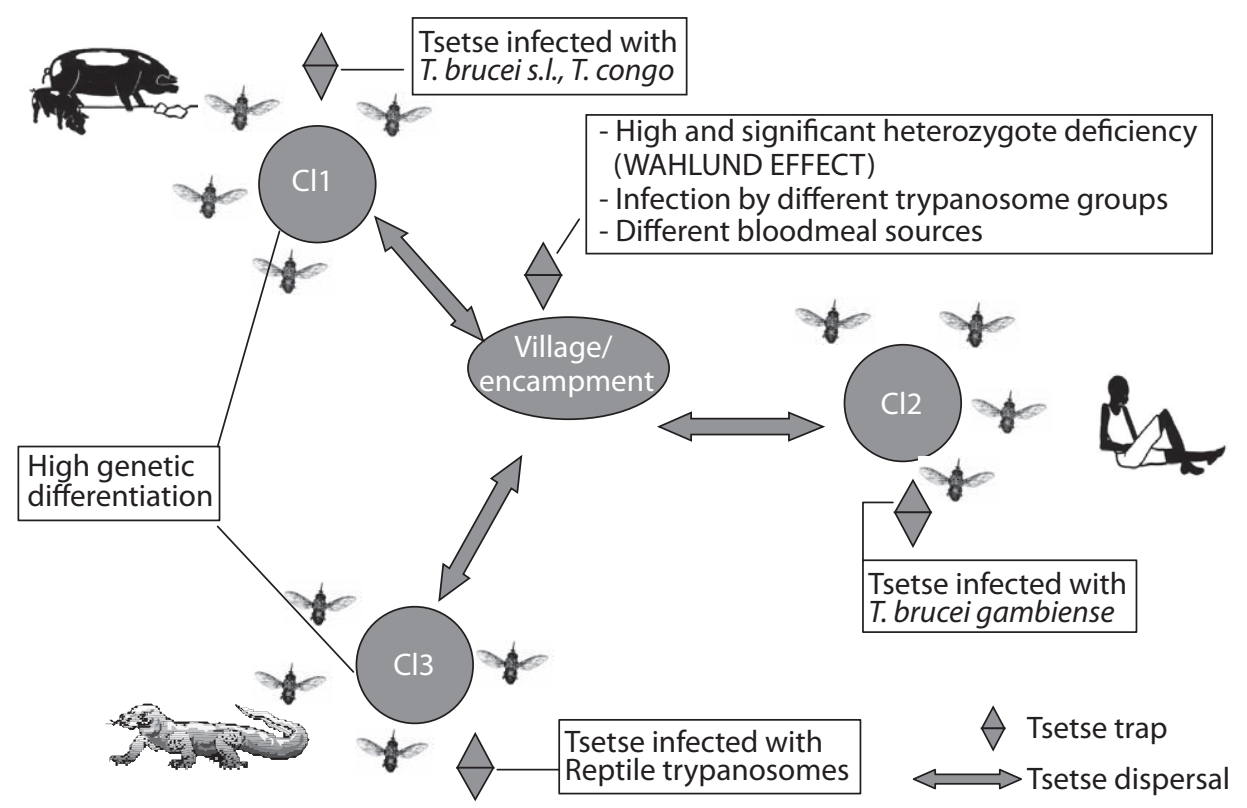

Figure 3. Representation of the Wahlund effect with a population of G. palpalis, as suggested by genetic results obtained using microsatellite DNA markers in West Africa (Solano et al. 2000, Ravel et al. 2007). Tsetse caught in different traps in the environs of a single 'village' comprise flies from different clusters $(\mathrm{Cl} 1$ to $\mathrm{Cl} 3)$ that are spatially and genetically distinct. In this example, $\mathrm{Cl} 1$ is associated with pigs infected by $\mathrm{T}$. brucei and T. congolense, $\mathrm{Cl}$ 2 is the vector of pathogenic trypanosomes causing sleeping sickness and $\mathrm{Cl} 3$ feeds mostly on monitor lizards infected with non-pathogenic trypanosomes.

\section{Future directions}

\section{Attractants}

The past decade has seen a decline in research aimed at developing improved baits for tsetse, perhaps because there was a general perception that all the important attractants had been identified for savannah species and there was none to find for riverine flies. However, it is clear that neither view is correct: unidentified attractants for savannah species remain to be identified (Torr et al. 1995, 2006) and, more excitingly, Palpalis group flies do respond to host kairomones other than carbon dioxide (Filledier and Mérot 1989a; Mohamed-Ahmed 1998; Rayaisse et al. 2010). 
Would the identification of novel attractants have an impact on tsetse control? The basic studies of the responses of some species to host odours might suggest that host odours will be of little practical importance. For instance, the modest $(\sim 1.3 \times)$ increases in catch for G. pallidipes in Somalia or both G. m. submorsitans and G. tachinoides in Burkina Faso produced by baiting a target with ox odour (Figure 2) might have suggested that the effect is due largely to carbon dioxide only and hence there are no useful attractants to identify. In practice however, traps baited with blends of acetone, octenol and phenols have been shown to produce 2-4x increases in catch (Filledier and Mérot 1989a, Politzar and Merot 1984, Torr et al. 1989). We should be wary therefore of assuming that the modest catch increases obtained with natural odours means that we cannot do better with synthetic blends.

The identification of odours that improve the performance of traps and targets, say, four-fold could have important implications for the control of tsetse, especially those riverine species, which play an important role in the transmission of T. brucei spp.. For the control of Palpalis group species, baits must currently be deployed at densities of 30 targets $/ \mathrm{km}^{2}$ or more, compared to 4 targets/ $\mathrm{km}^{2}$ for savannah species. These higher densities make the method prohibitively expensive for community-based schemes; Shaw et al. (2006) estimated that the field costs of using artifical baits deployed at a conservative density of just 10 targets $/ \mathrm{km}^{2}$ to control G. f. fuscipes in Uganda was $\$ 706 / \mathrm{km}^{2}$ compared to the $\$ 283 / \mathrm{km}^{2}$ needed for 4 targets $/ \mathrm{km}^{2}$ to control G. pallidipes. The identification of cheap attractants that improved the performance of baits $2-4 \times$ might reasonably be expected to make the costs of controlling riverine and savannah species comparable.

Moreover, while targets must be deployed over large $\left(>500 \mathrm{~km}^{2}\right)$ areas to control savannah species, experience with the use of baits against riverine species shows that relatively small- scale $(<100$ $\mathrm{km}^{2}$ ) interventions can have a major impact on tsetse and sleeping sickness (see examples in Green 1994, and Laveissière and Grébaut 2003). In general, their more restricted distribution and lower densities make populations of Palpalis group species more amenable to control. In addition, the lower rates of infection with $T$. brucei s.l., compared to $T$. vivax or $T$. congolense, mean that a given level of tsetse control has a greater impact on human than animal trypanosomiasis (Rogers 1988). Thus if better baits for riverine flies can be developed, the prospects for their wider use, especially to control sleeping sickness, seem good.

While the prospects of halving the costs of baits to control riverine flies seem reasonable, the cost $\left(\sim 300 / \mathrm{km}^{-2}\right)$ will still be much greater than that $\left(\$ 12 / \mathrm{km}^{-2}\right)$ of using insecticide-treated cattle (Shaw et al. 2006). There are no examples of large-scale uptake of traps and targets by communities to control tsetse, whereas livestock keepers are prepared to pay the cost ( 2 cents/animal/month) of treating their cattle with insecticide to control ticks and tsetse, as evidenced by the sale of $\sim 37,000$ doses to Ugandan farmers (I. Maudlin, personal communication) over two months in 2008. Given the decline in government and donor support for tsetse control, and the greater role assumed by livestock keepers and communities living in tsetse-infested areas, it seems likely that the insecticide-treated cattle rather than artificial baits will be the mainstay of tsetse control. However, while insecticide-treated cattle offer a particularly cheap and sustainable method of tsetse, we suggest that odour-baited targets and traps will continue to play an important role. First, cattle are not evenly distributed and thus to achieve success it is frequently necessary to deploy artificial baits in areas where cattle are absent or where other hosts are abundant (Hargrove et al. 2003, Warnes et al. 1999). Second, while insecticide-treated cattle may be effective for controlling vectors of $T$. $b$. rhodesiense, this approach may be less applicable for vectors of $T$. b. gambiense, where cattle play a less important role in both disease epidemiology and tsetse diet. Third, all tsetse control operations are bedevilled by tsetse re-invading from either neighbouring areas of 
infestation, or pockets of survivors, and the only effective method of preventing reinvasion from these is to deploy a barrier of insecticide-treated targets (Cuisance and Politzar 1983, Hargrove 1993, Kgori et al. 2006, Muzari and Hargrove 1996). Finally, all control operations rely on surveys to assess the extent of the tsetse infestation and the impact of control operations; odour-baited traps play a key role in the success of these surveys, including current control operations in West Africa (I. Sidibe, personal communication).

\section{Repellents}

In some respects it is surprising that more research has not been conducted on identifying repellents for tsetse. We know that standard repellents such as DEET are not effective against savannah species of tsetse (Vale 1979; Torr et al. 1996) but potent, unidentified, repellents are present in human odours (Vale 1974b). Presumably, the methods that were used to identify attractants for tsetse could also be used for repellents.

Torr et al. (1996) suggested that repellents would be most effective in areas where there are low densities of tsetse and low infection rates in the vector populations, conditions that are typical of sleeping sickness foci in West Africa. It would therefore be pertinent to assess the responses of Palpalis group species to chemicals (e.g. 4-methyl-2-methoxyphenol) and natural odours (e.g. human body odour) which appear to be repellent for savannah flies.

\section{The application of science}

The last 40 years has seen a tumultuous body of research on the olfactory responses of tsetse to their hosts, resulting in new methods of vector control and surveillance. However, measured in terms of impact on vector populations and disease incidence, the impact of this research has been disappointing: tsetse are, roughly, as widespread as ever, sleeping sickness still kills $\sim 40,000$ people a year and the alleviation of animal trypanosomiasis is more dependent on drugs than vector control.

Baits - artificial and natural - offer the prospect of playing a greater role in the future, but it is clear that we need to understand and promote the correct use of these technologies; too often they have been used inappropriately and hence had little or no sustained impact (Torr et al. 2005). The development of web-based systems (e.g. www.tsetse.org; www.sleeping-sickness.idr.fr) to promote the correct use of baits goes some way to addressing this need.

More significantly, the formation of the Pan-African Tsetse and Trypanosomiasis Eradication Campaign (PATTEC) signals a new determination by African governments and leaders to support greater efforts against tsetse. The emergence of cheap and effective methods of vector control and surveillance, based largely on host-orientated behaviour, seem set to play an important role in this initiative.

\section{Acknowledgements}

We thank Professors Glyn Vale and John Hargrove and Dr Gay Gibson for helpful comments on earlier drafts of this chapter. We also thank our colleagues Drs. Johan Esterhuizen and Maurice Omolo and messrs. Jean-Baptiste Rayaisse and Inaki Tirados for helpful discussions. 


\section{References}

Baylis M and Nambiro CO (1993) The responses of Glossina pallidipes and G. Iongipennis (Diptera: Glossinidae) to odourbaited traps and targets at Galana Ranch, south-eastern Kenya. Bull Entomol Res 83: 145-151.

Bouyer J, Pruvot M, Bengaly Z, Guerin PM and Lancelot R (2007a) Learning influences host choice in tsetse. Biol Letters 3: 113-U111.

Bouyer J, Stachurski F, Kabore I, Bauer B and Lancelot R (2007b) Tsetse control in cattle from pyrethroid footbaths. Prev Vet Med 78: 223-238.

Brady J (1972) Spontaneous, circadian components of tsetse fly activity. J Insect Physiol 18: 471-484.

Brady J and Crump AJ (1978) Control of circadian activity rhythems in tsetse flies - environment of physiological clock? Physiol Entomol 3: 177-190.

Brady J and Griffiths N (1993) Upwind flight responses of tsetse flies (Glossina spp) (Diptera: Glossinidae) to acetone, octenol and phenols in nature - a video study. Bull Entomol Res 83: 329-333.

Brady J and Shereni W (1988) Landing responses of the tsetse fly Glossina morsitans morsitans Westwood and the stable fly Stomoxys calcitrans (L) (Diptera, Glossinidae and Muscidae) to black-and-white patterns - a laboratory study. Bull Entomol Res 78: 301-311.

Brady J, Gibson G and Packer MJ (1989) Odour movement, wind direction, and the problem of host-finding by tsetse flies. Physiol Entomol 14:369-380.

Brady J, Packer MJ and Gibson B (1990) Odour plume shape and host finding by tsetse. Insect Sci Appl 11: 377-384.

Brightwell R and Dransfield R (1997) Odour attractants for tsetse: Glossina austeni, G. brevipalpis and G. swynnertoni. Med Vet Entomol 11:297-299.

Bursell E (1987) The effect of wind-borne odours on the direction of flight in tsetse flies. Physiol Entomol 12: 149-156.

Bursell E (1984a) Effects of host odour on the behaviour of tsetse. Insect Sci Appl 5: 345-349.

Bursell E (1984b) Observations on the orientation of tsetse flies (Glossina pallidipes) to wind-borne odours. Physiol Entomol 9: 133-137.

Bursell E and Taylor P (1980) An energy budget for Glossina (Diptera: Glossinidae). Bull Entomol Res 70: 187-196.

Bursell E, Gough AJE, Beevor PS, Cork A, Hall DR and Vale GA (1988) Identification of components of cattle urine attractive to tsetse flies, Glossina spp (Diptera: Glossinidae) Bull Entomol Res 78: 281-291.

Buxton PA (1955) The natural history of tsetse flies - an account of the biology of the genus Glossina (Diptera). H.K. Lewis, London, UK. 816 pp.

Cheke RA and Garms R (1988) Trials of compounds to enhance trap catches of Glossina palpalis palpalis in Liberia. Med Vet Entomol 2: 199-200.

Clausen PH, Adeyemi I, Bauer B, Breloeer M, Salchow F and Staak C (1998) Host preferences of tsetse (Diptera: Glossinidae) based on bloodmeal identifications. Med Vet Entomol 12: 169-180.

Colvin J, Brady J and Gibson G (1989) Visually-guided, upwind turning behaviour of free-flying tsetse flies in odourladen wind: a wind-tunnel study. Physiol Entomol 14:31-39.

Courtin F, Jamonneau V, Duvallet G, Garcia A, Coulibaly B, Doumenge JP, Cuny G and Solano P (2008) Sleeping sickness in West Africa (1906-2006): changes in spatial repartition and lessons from the past. Trop Med Int Health 13: 334344.

Cuisance D and Politzar H (1983) Etude sur l'efficacité contre Glossina palpalis gambiensis et G. tachinoides de barrières constituées d'écrans ou de pièges biconiques imprégnés de DDT, de deltaméthrine, ou de dieldrine. Rev Elev Med Vet Pays Trop 36: 159-168.

David CT, Kennedy JS, Ludlow AR, Perry J.N. and Wall C (1982) A re-appraisal of insect flight towards a point source of wind-borne odour. J Chem Ecol 8: 1207-1215.

Doku C and Brady J (1989) Landing site preferences of Glossina morsitans morsitans Westwood (Diptera: Glossinidae) in the laboratory: avoidance of horizontal features. Bull Entomol Res 79: 521-528.

Dransfield RD, Brightwell R, Kyorku C and Williams B (1990) Control of tsetse fly (Diptera: Glossinidae) populations using traps at Nguruman, south-west Kenya. Bull Entomol Res 80: 265-276. 
Eisler MC, Torr SJ, Coleman PG, Machila N and Morton JF (2003) Integrated control of vector-borne diseases of livestock - pyrethroids: panacea or poison? Trends Parasitol 19:341-345.

Filledier J and Mérot P (1989a) Pouvoir attractif de l'association m-crésol, 1-octen-3-ol dans un type de diffuseur pratique pour Glossina tachinoides au Burkina Faso. Rev Elev Med Vet Pays Trop 42: 541-544.

Filledier J and Mérot PR (1989b) Etude de l'attractivité de solutions isolées par fractionnement de l'urine de bovin Baoulé pour Glossina tachinoides Westwood, 1850 au Burkina Faso. Rev Elev Med Vet Pays Trop 42 453-455.

Frezil J-L and Carnevale P (1976) Utilisation de la caroglace pour la capture de Glossin fuscipes quanzensis Pires, 1948, avec le piège Challier-Laveissière. Consequences épidémiologiques. Cahiers ORSTOM séries entomologie médicale et parasitologie 14: 225-233.

Galey JB, Mérot P, Mitteault A, Filledier J and Politzar H (1986) Efficacité du dioxyde de carbone comme attractif pour Glossina tachinoides en savane humide d'Afrique de I'Ouest. Rev Elev Med Vet Pays Trop 39: 351-354.

Gibson G (1992) Do tsetse 'see' zebras? A field study of the visual response of tsetse to striped targets. Physiol Entomol 17: 141-147.

Gibson G and Brady J (1985) Anemotactic'flight paths of tsetse flies in relation to host odour: a preliminary video study in nature of the response to loss of odour. Physiol Entomol 10:375-406.

Gibson G and Brady J (1988) Flight behaviour of tsetse flies in host odour plumes - the initial response to leaving or entering odour. Physiol Entomol 13: 29-42.

Gibson G, Packer MJ, Steullet P and Brady J (1991) Orientation of tsetse flies to wind, within and outside host odour plumes in the field. Physiol Entomol 16: 47-56.

Gibson G and Torr SJ (1999) Visual and olfactory responses of haematophagous Diptera to host stimuli. Med Vet Entomol 13: 2-23.

Gikonyo NK, Hassanali A, Njagi PGN, Gitu PM and Midiwo JO (2002) Odour composition of preferred (buffalo and ox) and nonpreferred (waterbuck) hosts of some savannah tsetse flies. J Chem Ecol 28: 969-981.

Gikonyo NK, Hassanali A, Njagi PGN and Saini RK (2000) Behaviour of Glossina morsitans morsitans Westwood (Diptera: Glossinidae) on waterbuck Kobus defassa Ruppel and feeding membranes smeared with waterbuck sebum indicates the presence of allomones. Acta Tropica 77: 295-303.

Gikonyo NK, Hassanali A, Njagi PGN and Saini RK (2003) Responses of Glossina morsitans morsitans to blends of electroantennographically active compounds in the odors of its preferred (buffalo and ox) and nonpreferred (waterbuck) hosts. J Chem Ecol 29: 2331-2345.

Gooding RH and Krafsur ES (2005) Tsetse genetics: contributions to biology, systematics, and control of tsetse flies. Ann Rev Entomol 50: 101-123.

Gouteux JP, Blank F, Cuisance D, D'Amico F and Kota Guinza A (1995) Trials of olfactory attractants to enhance trap catches of Glossina fuscipes fuscipes (Diptera: Glossinidae) in the Central African Republic. Vet Res26: 33-340.

Green CH (1986) Effects of colours and synthetic odours on the attraction of Glossina pallidipes and Glossina morsitans morsitans to traps and screens. Physiol Entomol 11:411-421.

Green CH (1994) Bait methods for tsetse fly control. In: Baker JR and Muller R (eds) Advances in Parasitology, Vol 34, Academic Publishers, pp. 229-291.

Griffiths N and Brady J (1995) Wind structure in relation to odour plumes in tsetse fly habitats. Physiol Entomol 20: 286-292.

Griffiths N, Paynter Q. and Brady J (1995) Rates of progress up odour plumes by tsetse flies: a mark-release video study of the timing of odour source location by Glossina pallidipes. Physiol Entomol 20: 100-108.

Hadaway AB (1977) Resting behaviour of tsetse flies, and its relevance to their control with residual insecticides. Miscellaneous Report No. 36. Centre for Overseas Pest Research, London, UK, 11 pp.

Hall DR, Beevor PS, Cork A, Nesbitt BF and Vale GA (1984) 1-octen-3-ol - potent olfactory stimulant and attractants for tsetse isolated from cattle odours. Insect Sci Appl 5: 335-339.

Hargrove JW (1975) Some changes in the flight apparatus of tsetse flies, Glossina morsitans and G. pallidipes during maturation. J Insect Physiol 21: 1485-1489.

Hargrove JW (1976) Effect of human presence on behaviour of tsetse (Glossina spp) (Diptera: Glossinidae) near a stationary ox. Bull Entomol Res 66: 173-178. 
Hargrove JW (1980) The effect of model size and ox odour on the alighting response of Glossina morsitans Westwood and Glossina pallidipes Austen (Diptera: Glossinidae). Bull Entomol Res 70: 229-234.

Hargrove JW (1988) Tsetse - the limits to population growth. Med Vet Entomol 2: 203-217.

Hargrove JW (1993) Target barriers for tsetse flies (Glossina spp) (Diptera: Glossinidae): quick estimates of target densities and barrier widths. Bull Entomol Res 83: 197-200.

Hargrove JW (2003) Tsetse eradication: sufficiency, necessity and desirability. DFID Animal Health Programme, Centre for Tropical Veterinary Medicine, University of Edinburgh, Edinburgh, UK. 134 pp.

Hargrove JW and Brady J (1992) Activity rhythms of tsetse flies (Glossina spp) (Diptera: Glossinidae) at low and high temperatures in nature. Bull Entomol Res 82: 321-326.

Hargrove JW, Holloway MTP, Vale GA, Gough AJE and Hall DR (1995) Catches of tsetse (Glossina spp.) (Diptera: Glossinidae) from traps and targets baited with large doses of natural and synthetic host odour. Bull Entomol Res 85: 215-227.

Hargrove JW, Torr SJ and Kindness HM (2003) Insecticide-treated cattle against tsetse (Diptera: Glossinidae): what governs success? Bull Entomol Res 93: 203-217.

Hargrove JW and Vale GA (1978) Effect of host odour concentration on catches of tsetse flies (Glossinidae) and other Diptera in the field. Bull Entomol Res 68: 607-612.

Hargrove JW and Vale GA (1980) Catches of Glossina morsitans morsitans Westwood and Glossina pallidipes Austen (Diptera: Glossinidae) in odour-baited traps in riverine and deciduous woodlands in the Zambezi valley of Zimbabwe. Bull Entomol Res 70: 571-578.

Hassanali A, McDowell PG, Owaga MLA and Saini RK (1986) Identification of tsetse attractants from excretory products of a wild host animal, Syncerus caffer. Insect Sci Appl 7: 5-9.

IAEA (2003) Improved attractants for enhancing tsetse fly suppression. Final report of a co-ordinated research project 1006-2002, International Atomic Energy Agency, Vienna, Austria.

Kamuanga M, Swallow BM, Sigue' H and Bauer B (2001) Evaluating contingent and actual contributions to a local public good: tsetse control in the Yale agro-pastoral zone, Burkina Faso. Ecol Econ 39: 115-130.

Kappmeier-Green K (2001) Strategy for monitoring and sustainable integrated control or eradication of Glossina brevipalpis and G. austeni (Diptera: Glossinidae) in South Africa. PhD thesis, University of Pretoria: 245.

Kappmeier K and Nevill EM (1999) Evaluation of conventional odour attractants for Glossina brevipalpis and Glossina austeni (Diptera: Glossinidae) in South Africa. Ond J Vet Res 66: 307-316.

Kennedy J S (1977) Olfactory responses to distant plants and odor sources. In: Shorey HH and McKelvey JJ (eds) Chemical control of insect behavior: theory and application. John Wiley \& Sons, New York, USA, pp 67-91.

Kgori PM, Modo S and Torr SJ (2006) The use of aerial spraying to eliminate tsetse from the Okavango Delta of Botswana. Acta Tropica 99: 184-199.

Kyorku C, Brightwell R and Dransfield RD (1990) Traps and odour baits for the tsetse fly, Glossina longipennis (Diptera: Glossinidae). Bull Entomol Res 80: 405-415.

Laveissière $C$ and Grébaut $P$ (2003) The risk of sleeping sickness transmission: simplifying the calculation of the index. Insect Sci Appl 23: 99-102.

Madubunyi LC, Hassanali A, Ouma W, Nyarango D and Kabii J (1996) Chemoecological role of mammalian urine in host location by tsetse, Glossina spp (Diptera: Glossinidae). J Chem Ecol 22: 1187-1199.

Maudlin I (2006) African trypanosomiasis. Ann Trop Med Parasitol 100: 679-701.

Mérot P, Filledier J and Mulato C (1988) Pouvoir attractif, pour Glossina tachinoides, de produits chimiques isolés des odeurs animales. Rev Elev Med Vet Pays Trop 41: 79-85.

Mérot P, Galey JB, Politzar H, Filledier J and Mitteault A (1986) Pouvoir attractif de l'odeur des hôtes nourriciers pour Glossina tachinoides en zone soudano-guinéenne (Burkina Faso). Rev Elev Med Vet Pays Trop 39: 345-350.

Modo S (1999) Odour attractants for the tsetse fly Glossina morsitans centralis Machado in Botswana. MSc thesis, University of Zimbabwe, Harare, Zimbabwe.

Mohamed-Ahmed MM (1998) Olfactory responses of Glossina fuscipes fuscipes (Diptera: Glossinidae) to the monitor lizard Varanus niloticus niloticus. Bull Entomol Res 88: 311-317.

Mohamed-Ahmed MM and Mihok S (1999) Responses of Glossina fuscipes fuscipes (Diptera: Glossinidae) and other Diptera to carbon dioxide in linear and dense forests. Bull Entomol Res 89: 177-184. 
Muzari MO and Hargrove JW (1996) The design of target barriers for tsetse flies, Glossina spp (Diptera: Glossinidae). Bull Entomol Res 86: 579-583.

Mwangelwa MI, Dransfield RD, Otieno LH and Mbata KJ (1995) The responses of Glossina fuscipes fuscipes Newstead to odour attractants and traps. J Afr Zool 109: 23-30.

Mwangi MT, Gikonyo NK and Ndiege IO (2008) Repellent properties of delta-octalactone against the tsetse fly, Glossina morsitans morsitans. J Insect Sci 8: paper 43.

Ndegwa PN and Mihok S (1999) Development of odour-baited traps for Glossina swynnertoni (Diptera: Glossinidae). Bull Entomol Res 89: 255-261.

Omolo MO, Hassanali A, Mpiana S, Esterhuizen J, Lehane MJ, Solano P, Rayaisse J-B, Vale GA, Torr SJ and Tirados I (2009) Prospects for developing odour baits to control G. fuscipes spp., the major vector of human African trypanosomiasis. PloS Negl Trop Dis 3(5): e435. doi:10.1371/journal.pntd.0000435.

Paynter Q and Brady J (1993) Flight responses of tsetse flies (Glossina) to octenol and acetone vapour in a wind tunnel. Physiol Entomol 18: 102-108.

Politzar H and Merot P (1984) Attraction of the tsetse fly Glossina morsitans submorsitans to acetone, 1-octen-3-ol, and the combination of these compounds in West Africa. Rev Elev Med Vet Pays Trop 37: 468-473.

Ravel S, de Meeus T, Dujardin JP, Zeze DG, Gooding RH, Dusfour I, Sane B, Cuny G and Solano P (2007) The tsetse fly Glossina palpalis palpalis is composed of several genetically differentiated small populations in the sleeping sickness focus of Bonon, Cote d'Ivoire. Infect Genet Evol 7: 116-125.

Rayaisse J-B, Solano P, Dramane K, Torr SJ, Tirados I, Lehane MJ and Esterhuizen J (2010) Responses of G. tachinoides and G. palpalis spp. to natural host odours. PLoS Negl Trop Dis 4(3): e632. doi:10.1371/journal.pntd.0000632.

Rogers DJ (1988) A general model for the African trypanosomiases. Parasitology 97: 193-212.

Sabelis MW and Schippers P (1984) Variable wind directions and anemotactic strategies of searching for an odour plume. Oecologia 63: 225-228.

Saini RK and Hassanali A (2007) A 4-alkyl-substituted analogue of guaiacol shows greater repellency to Savannah tsetse (Glossina spp.). J Chem Ecol 33: 985-995.

Sané B, Laveissière C and Meda HA (2000) Diversité du régime alimentaire de Glossina palpalis palpalis en zone forestière de Côte d'Ivoire: relation avec la prévalence de la trypanosomiase humaine africaine. Trop Med Int Health 5: 73-78.

Shaw A, Torr S, Waiswa C and Robinson T (2006) Comparable costings of alternatives for dealing with tsetse: estimates for Uganda. Proceedings 11th International Congress of Parasitology ICOPA XI, Medimond International Proceedings, Bologna, Italy, Glasgow, UK.

Simarro PP, Jannin J and Cattand P (2008) Eliminating human African trypanosomiasis: Where do we stand and what comes next? Plos Med 5: 174-180.

Simo G, Njiokou F, Mbida Mbida JA, Njitchouang GR, Herder S, Asonganyi T and Cuny G (2008) Tsetse fly host preference from sleeping sickness foci in Cameroon: epidemiological implications. Infect Genet Evol 8: 34-39.

Solano P, de La Rocque S, de Meeus T, Cuny G, Duvallet G and Cuisance D (2000) Microsatellite DNA markers reveal genetic differentiation among populations of Glossina palpalis gambiensis collected in the agro-pastoral zone of Sideradougou, Burkina Faso. Insect Mol Biol 9: 433-439.

Späth J (1995) Olfactory attractants for West African tsetse flies, Glossina spp (Diptera: Glossinidae). Trop Med Parasitol 46: $253-257$.

Späth J (1997) Natural host odours as possible attractants for Glossina tachinoides and G. longipalpis (Diptera: Glossinidae). Acta Tropica 68: 149-158.

Sutcliffe JF (1987) Distance orientation of biting flies to their hosts. Insect Sci Appl 8: 611-616.

Torr SJ (1988a) The activation of resting tsetse flies (Glossina) in response to visual and olfactory stimuli in the field. Physiol Entomol 13: 315-325.

Torr SJ (1988b) The flight and landing of tsetse (Glossina) in response to components of host odour in the field. Physiol Entomol 13: 453-465.

Torr SJ (1988c) Behaviour of tsetse flies (Glossina) in host odour plumes in the field. Physiol Entomol 13: 467-478.

Torr SJ (1989) The host-orientated behaviour of tsetse flies (Glossina): the interaction of visual and olfactory stimuli. Physiol Entomol 14: 325-340. 
Torr SJ (1990) Dose responses of tsetse flies (Glossina) to carbon dioxide, acetone and octenol in the field. Physiol Entomol 15: 93-103.

Torr SJ (1994) Responses of tsetse flies (Diptera: Glossinidae) to warthog (Phacochoerus aethiopicus Pallas). Bull Entomol Res 84: 411-419.

Torr SJ, Hall DR, Phelps RJ and Vale GA (1997) Methods for dispensing odour attractants for tsetse flies (Diptera:Glossinidae). Bull Entomol Res 87: 299-311.

Torr SJ, Hall DR and Smith JL (1995) Responses of tsetse flies (Diptera: Glossinidae) to natural and synthetic ox odours. Bull Entomol Res 85: 157-166.

Torr SJ and Hargrove JW (1999) Behaviour of tsetse (Diptera: Glossinidae) during the hot season in Zimbabwe: the interaction of micro-climate and reproductive status. Bull Entomol Res 89: 365-379.

Torr SJ, Hargrove JW and Vale GA (2005) Towards a rational policy for dealing with tsetse. Trends Parasitol 21: 537-541.

Torr SJ and Mangwiro TNC (1996) Upwind flight of tsetse (Glossina spp) in response to natural and synthetic host odour in the field. Physiol Entomol 21: 143-150.

Torr SJ and Mangwiro TNC (2000) Interactions between cattle and biting flies: effects on the feeding rate of tsetse. Med Vet Entomol 14: 400-409.

Torr SJ, Mangwiro TNC and Hall DR (1996) Responses of Glossina pallidipes (Diptera: Glossinidae) to synthetic repellents in the field. Bull Entomol Res 86: 609-616.

Torr SJ, Mangwiro TNC and Hall DR (2006) The effects of host physiology on the attraction of tsetse (Diptera: Glossinidae) and Stomoxys (Diptera: Muscidae) to cattle. Bull Entomol Res 96: 71-84.

Torr SJ, Maudlin I and Vale GA (2007a) Less is more: restricted application of insecticide to cattle to improve the cost and efficacy of tsetse control. Med Vet Entomol 21: 53-64.

Torr SJ, Parker AG and Leigh-Browne G (1989) The responses of Glossina pallidipes Austen (Diptera: Glossinidae) to odour-baited traps and targets in Somalia. Bull Entomol Res 79: 99-108.

Torr SJ, Prior A, Wilson PJ and Schofield S (2007b) Is there safety in numbers? The effect of cattle herding on biting risk from tsetse flies. Med Vet Entomol 21:301-311.

Torr SJ, Wilson PJ, Schofield S, Mangwiro TNC, Akber S and White BN (2001) Application of DNA markers to identify the individual-specific hosts of tsetse feeding on cattle. Med Vet Entomol 15: 78-86.

Vale GA (1971) Artificial refuges for tsetse flies (Glossina spp). Bull Entomol Res 61: 331-350.

Vale GA (1974a) New field methods for studying responses of tsetse flies (Diptera; Glossinidae) to hosts. Bull Entomol Res 64: 199-208.

Vale GA (1974b) Responses of tsetse flies (Diptera, Glossinidae) to mobile and stationary baits. Bull Entomol Res 64: 545-588.

Vale GA (1977a) Feeding responses of tsetse flies (Diptera-Glossinidae) to stationary hosts. Bull Entomol Res 67: 635-649.

Vale GA (1977b) Flight of tsetse flies (Diptera: Glossinidae) to and from a stationary ox. Bull Entomol Res 67: 297-303.

Vale GA (1979) Field responses of tsetse flies (Diptera: Glossinidae) to odours of men, lactic acid and carbon dioxide. Bull Entomol Res 69: 459-467.

Vale GA (1980a) Field studies of the responses of tsetse flies (Glossinidae) and other Diptera to carbon dioxide, acetone and other chemicals. Bull Entomol Res 70: 563-570.

Vale GA (1980b) Flight as a factor in the host-finding behaviour of tsetse flies (Diptera: Glossinidae). Bull Entomol Res 70: 299-307.

Vale GA (1993) Development of baits for tsetse flies (Diptera: Glossinidae) in Zimbabwe. J Med Entomol 30: 831-842.

Vale GA (1998) Responses of tsetse flies (Diptera: Glossinidae) to vegetation in Zimbabwe: implications for population distribution and bait siting. Bul Entomol Res 80 (Suppl 1): 1-59.

Vale GA and Cumming DHM (1976) Effects of selective elimination of hosts on a population of tsetse flies (Glossina morsitans morsitans (Diptera: Glossinidae)). Bull Entomol Res 66: 713-729.

Vale GA, Flint S and Hall DR (1986a) The field responses of tsetse flies, Glossina spp. (Diptera: Glossinidae), to odours of host residues. Bull Entomol Res 76: 685-693.

Vale GA and Hall DR (1985a) The role of 1-octen-3-ol, acetone and carbon dioxide in the attraction of tsetse flies, Glossina spp. (Diptera: Glossinidae), to ox odour. Bull Entomol Res 75: 209-217. 
Vale GA and Hall DR (1985b) The use of 1-octen-3-ol, acetone and carbon dioxide to improve baits for tsetse flies, Glossina spp. (Diptera: Glossinidae). Bull Entomol Res 75: 219-231.

Vale GA and Torr SJ (2004) Development of bait technology to control tsetse. In: Maudlin I, Holmes PH and Miles MA (eds) The trypanosomiases. CABI, Wallingford, UK, pp 509-523.

Vale GA, Hall DR and Gough AJE (1988a) The olfactory responses of tsetse flies, Glossina spp (Diptera: Glossinidae) to phenols and urine in the field. Bull Entomol Res 78: 293-300.

Vale GA and Hargrove JW (1979) Methods of studying the efficiency of traps for tsetse flies (Diptera: Glossinidae) and other insects. Bull Entomol Res 69: 183-193.

Vale GA, Hargrove JW, Cockbill GF and Phelps RJ (1986b) Field trials of baits to control populations of Glossina morsitans morsitans Westwood and Glossina pallidipes Austen (Diptera: Glossinidae). Bull Entomol Res 76: 179-193.

Vale GA, Lovemore DF, Flint S and Cockbill GF (1988b) Odour-baited targets to control tsetse flies, Glossina spp. (Diptera, Glossinidae), in Zimbabwe. Bull Entomol Res 78: 31-49.

Waiswa C, Picozzi K, Katunguka-Rwakishaya E, Olaho-Mukani W, Musoke RA and Welburn SC (2006) Glossina fuscipes fuscipes in the trypanosomiasis endemic areas of south eastern Uganda: Apparent density, trypanosome infection rates and host feeding preferences. Acta Tropica 99: 23-29.

Warnes ML (1990) The effect of host odour and carbon dioxide on the flight of tsetse flies (Glossina spp) in the laboratory. Journal of Insect Physiology 36: 607-611.

Warnes ML (1995) Field studies on the effect of cattle skin secretion on the behaviour of tsetse. Med Vet Entomol 9: 284-288.

Warnes ML, van den Bossche P, Chihiya J, Mudenge D, Robinson TP, Shereni W and Chadenga V (1999) Evaluation of insecticide-treated cattle as a barrier to re-invasion of tsetse to cleared areas in northeastern Zimbabwe. Med Vet Entomol 13: 177-184.

Welburn SC, Coleman PG, Maudlin I, Fevre EM, Odiit M and Eisler MC (2006) Crisis, what crisis? Control of Rhodesian sleeping sickness. Trends Parasitol 22: 123-128.

Willemse L (1991) A trial of odour baited targets to control the tsetse fly, Glossina morsitans centralis (Diptera: Glossinidae) in west Zambia. Bull Entomol Res 81: 351-357.

Williams BG (1994) Models of trap seeking by tsetse flies: anemotaxis, kinesis and edge detection. J Theor Biol 168: 105-115.

Zollner GE, Torr SJ, Ammann C and Meixner FX (2004) Dispersion of carbon dioxide plumes in African woodland: implications for host-finding by tsetse flies. Physiol Entomol 29: 381-394. 



\title{
13. Behavioural modalities of 'non-vector' biting Diptera: from olfaction to feeding
}

Steve Schofield and Steve J. Torr

\begin{abstract}
Though they are not considered to be important vectors, stable flies and related species can have direct economic impacts, and as well might influence pathogen transmission by affecting the feeding behaviour of flies such as tsetse. We discuss here the responses of Stomoxyinae to hostbased kairomones. Further, we consider the interplay between these Diptera, other vectors and their common hosts as it relates to disease epidemiology and control strategies. In general, we find that the odour-evoked responses of stable flies in the laboratory are similar to other groups of fast moving Diptera that blood feed (e.g. tsetse), i.e. activity increases and flights are steered upwind. In field studies, several compounds have been associated with increased catch of Stomoxyinae from traps, but only $\mathrm{CO}_{2}$ consistently achieved this effect. On the whole, results suggest that non$\mathrm{CO}_{2}$ kairomones are not that important to host finding by Stomoxys and, further, that synthetic host odours in general might not have a place in sampling or control schemes for this pest. We also describe the interplay between Stomoxyinae and their hosts, highlighting the role that their feeding behaviour might have as regards the epidemiology of animal trypanosomiasis. Finally, we discuss briefly some of the potential practical applications that might arise from a better understanding of the interrelationship between Stomoxys species and their hosts.
\end{abstract}

Keywords: behaviour, host odour, olfaction, Stomoxys, Glossina, stable fly, tsetse fly

\section{Introduction}

The blood feeding habit has arisen independently in several orders of insects (Lehane 2005) and, with this, has come a means of spreading a variety of human and veterinary pathogens. These range from those with a modest or focal impact on human population health (e.g. Murray Valley encephalitis, La Crosse encephalitis), to others that play a significant role more-or-less globally (e.g. malaria, dengue). It is our desire to manage the threats presented by vector-borne pathogens that, to a large extent, has driven research to understand the behavioural ecology underpinning haematophagy. Such efforts have aimed to provide novel means of controlling disease by, for example, luring vectors to lethal baits or developing means of preventing them from finding and biting a host. Unfortunately, and outside some notable exceptions, efforts in this area often have not contributed meaningfully to control efforts. There are many reasons for this, but it no doubt at least partly reflects a systematic failure on the part of investigators to appreciate and/or evaluate behaviour sensu stricto. Instead, the tendency has been to measure the final outcome of a complex repertoire of behaviours. To paraphrase, we have spent entirely too much time catching biting insects in traps (or huts, or on hosts, or in olfactometer ports) and not nearly enough time analysing, quantitatively, the behavioural processes that got them there in the first place. Why this should be the case is difficult to fathom, especially given that some of the earliest research (Kennedy 1939) in the field seemed to avoid this pitfall. Moreover, early workers did warn of the potential problems with taking a 'black-box' approach to behavioural research. One of the most vocal in this regard was John Kennedy, who often discussed the potential for bias in behavioural work, especially where experimental endpoints are framed through an anthropomorphic lens (Kennedy 1992). In any event, one result of not heeding these cautions is that our understanding 
of the behavioural ecology of vectors is poorer than it ought to be. Whether or not this failing has impacted on the effectiveness of interventions that target vectors remains unclear.

On the upside, in recent years the need to analyse vector behaviour objectively and quantitatively seems to have been re-emphasised, and there has been technological improvement and new funding opportunities to support this research. The result has been a greater volume and quality of work on vector behaviour. Examples include other chapters in this book, as well as recent studies in the literature (Cooperband and Cardé 2006, Dekker et al. 2005, Torr et al. 2008).

\section{Chapter scope}

This book focuses on disease vectors, whether veterinary or medical. So the inclusion of a chapter on 'non-vector' biting flies seems odd. However, it is probably more precise to say that the main subjects of this chapter are not especially good or important vectors. By no means are we suggesting that they do not have direct or indirect impacts on health or productivity, because they might and do (Hansens 1951, Mullens et al. 2006, Pickard 1968, Schofield and Torr 2002, Schwinghammer et al. 1986, 1987, Zumpt 1973). We also feel that the information consolidated herein might have some relevance to vectors more generally, for the emphasis of this book is on behaviour rather than diseases or pathogens. In particular, it can be revealing to contrast the behaviours of non-vector biting flies with better-studied vectors such as the Glossinidae, not so much to make note of similarities, which abound, but rather to highlight intriguing differences.

For simplicity, and to allow a more in-depth treatment of the subject material, we decided to limit our coverage to taxa of non-vector biting flies that occur globally, and for which a reasonable body of laboratory and field-based behavioural data exists. The resulting list is very short indeed, i.e. it is arguably comprised of species that fall within a single group, i.e. the Stomoxyinae. Within this subfamily, our emphasis will be on the synanthropic and cosmopolitan species Stomoxys calcitrans (L.) (the stable fly).

In terms of chapter structure, our thoughts are presented along a continuum that starts with the insect as a sensory receptor, and then moves through events that might culminate in feeding on a vertebrate host. Hence, though much of the discussion focuses on the role of olfaction in distance orientation, visual cues are touched upon and near- or on-host feeding behaviours are discussed. We also consider laboratory data related to distance orientation in tsetse in this chapter. Finally, while we acknowledge that interesting olfactory work has been done in several areas of stable fly biology (e.g. see Carlson et al. 2000, Romero et al. 2006), we focus on behaviour related to haematophagy.

\section{A framework for host orientation}

Despite our above caution regarding the need to be aware of bias and anthropomorphisms, we begin our discussion with a model for host-seeking behaviour that is somewhat dated, oversimplified, perhaps biased and almost certainly anthropomorphic. Nevertheless, it provides a useful framework around which thoughts - and manuscripts - can be organised.

Many species of haematophagous Diptera, including stable flies, must find their hosts from a distance. Elements of this process can be divided along spatial and temporal lines and, though it is impossible to attribute the logical framework to a single author, it is well summarised by Sutcliffe $(1986,1987)$. In this model, the receptivity of a given insect to host-based stimuli is 
modulated by factors such as hunger (Klowden and Lea 1979, Klowden et al. 1987), competing behavioural priorities (e.g. oviposition) and endogenous activity cycles (Jones and Gubbins 1978, Nayar and Sauerman 1971). Presuming an insect that is receptive, host orientation sensu stricto is thought to be 'activated' by some type(s) of host cue. What follows are behavioural manoeuvres, often mediated by odour, that bias movement towards a host. For flying insects, environmental landmarks are critically important during this period, e.g. to steer flights. As the orienting insect approaches the blood source, host-based visual and/or other cues are thought to become increasingly relevant as behavioural modulators. Ultimately the searching insect might find and land upon the host and then feed to repletion.

\section{Blood lust: the role of blood feeding in the biology of Stomoxys}

Much, though not all (Kunz and Monty 1976, Mihok et al. 1995, 1996, 2007, Zumpt 1973) of our knowledge concerning Stomoxys biology comes from work done on stable flies. Like tsetse, stable flies are day-active and both sexes blood feed, which provides the materials necessary for males to develop reproductive competence (Anderson 1978, Venkatesh and Morrison 1980, 1982), and females to develop ovaries (Chia et al. 1982, Kuzina 1942, Spates et al. 1988, Venkatesh and Morrison 1980). In contrast to tsetse, stable flies also feed on plant-related sugar sources (Jones et al. 1992, Parr 1962) and blood feeding is often daily (Venkatesh and Morrison 1980) or even more frequent (S Schofield, unpublished data). In terms of endogenous activity, laboratory studies suggest a single daily peak, with a free running period of approximately 26 hours (Schofield and Brady 1996). Diel field activity is often bimodal (Beresford and Sutcliffe 2006, Charlwood and Lopes 1980, Kunz and Monty 1976, Schofield 1998), for example with morning and evening peaks, though unimodal patterns have also been described (Berry and Campbell 1985, Harley 1964). Regardless of the specific feeding periodicity or activity cycle, the reality for stable flies, especially females, is that they feed often. From a host location perspective, this presumably means that unless they have recently fed, they are likely to be receptive to host-based stimuli.

\section{Stable flies as olfactory receptors}

The molecular and physiological bases for insect olfaction are well covered elsewhere, including Chapters 2, 3 and 4 in this book. We therefore limit our discussion to a brief overview of electrophysiological studies that have evaluated stable fly responses to odour.

In this fly, olfactory receptors are concentrated on the third antennal segment (Lewis 1971, 1972), where single cell recordings by Lewis (1972) and later Bidgood (1980) demonstrated responses to the ubiquitous kairomone $\mathrm{CO}_{2}$. Warnes and Finlayson (1986) similarly demonstrated responses to $\mathrm{CO}_{2}$, but in this case it was through use of electroantennogram (EAG). With the same technique, they also recorded responses to acetone, 1-octen-3-ol, cattle odour, cattle odour filtered through sodalime, human breath and the odour of cattle faeces. Schofield et al. (1995) were the first to screen a modest number of compounds using stable flies as electrophysiological receptors. Like Warnes and Finalyson, they used EAG and found that 1-octen-3-ol evoked substantial activity, whereas acetone did not. For saturated primary aliphatic alcohols, responses increased with carbon length to octan-1-ol; and for 1-octen-3-ol with dose over several orders of magnitude. Responses to a $\mathrm{C}_{8}$ aldehyde, carboxylic acid and ketone were less that to $\mathrm{C}_{8}$ alcohols. Several recent studies have expanded upon the number of compounds screened for electrophysiological activity. Birkett et al. (2004) worked with a number of biting and nuisance flies and discerned stable fly EAG responses to several types of compound including phenols ( $m$ - and $p$-cresol), alcohols (including 1-octen3-ol), ketones, linalool and citronellol. These authors also tested the horn fly, Haematobia irritans 
(L.), with responses being similar to those reported for stable flies, though with a few exceptions, notably, for example, an absence of response to phenols. Jeanbourquin and Guerin $(2007 a, b)$ focused on rumen and dung odours, and reported that 1-octen-3-ol and dimethyl trisulphide evoked the strongest EAG responses, but carboxylic acids, short chain alcohols and aldehydes, ketones, indoles, phenols and terpenes also elicited significant EAG responses. So what do the electrophysiological results tell us? For one, they indicate that some compounds consistently evoke responses (e.g. 1-octen-3-ol), whereas others do not (e.g. ketones, carboxylic acids). There are many possible explanations for such differences, some of which are explored by Schofield et al. (1995). Second, that stables flies act as receptors for a variety of host-derived compounds, and that these same compounds would be reasonable targets for further investigation. What electrophysiological outcomes do not address is whether or not active compounds have any behavioural (or biological) relevance - it is this question that comprises the subject matter of the next several chapter sections.

\section{Starting the search engine: initiation of host-oriented behaviour}

Activation is thought to represent a transition in behavioural state whereby the insect becomes (more) receptive to host-based cues. Hence, measurement of activation itself is not possible. Rather, early and observable events in the host-seeking process are used as indicators that activation has occurred. While one can debate as to whether activation is a useful concept, i.e. it is not testable, the behaviours that have been used as surrogate markers do provide insight into host-seeking processes. In this context, measurement of activation has traditionally employed frequency-based measures (e.g. flight periodicity) as behavioural indicators. As well, at least for stable flies, studies have been informed by results for other groups of blood feeding Diptera, in particular as regards the positive association between exposure to $\mathrm{CO}_{2}$ and mosquito (Daykin et al. 1965) or tsetse (Bursell 1984, Turner 1971) activity.

To our knowledge, the earliest work on behaviour initiation by stable flies was done by Gatehouse and Lewis (1973), who recorded the number of stable fly landings on targets situated within a wind tunnel. They observed more landings in the presence of $\mathrm{CO}_{2}$, and suggested that this kairomone evoked flights and/or increased the probability that a flight would terminate on a target. Warnes and Finlayson (1985a) followed this up with a study using stable flies that were placed singly into a test chamber and exposed to various concentrations of $\mathrm{CO}_{2}$, or to human breath. They found that flight activity generally increased with $\mathrm{CO}_{2}$ concentration, was positively associated with hunger status, and that breath was associated with higher levels of activity than could be accounted for by $\mathrm{CO}_{2}$ alone. Alzogaray and Carlson (2000) also suggested an activationassociated effect for $\mathrm{CO}_{2}$, in this case based on net movement of stable flies away from one end of an olfactometer. In a more comprehensive study, Schofield et al. (1997) evaluated responses of groups of four stable flies that were placed into a test chamber situated in a wind tunnel and that were then exposed to $\mathrm{CO}_{2}$. In line with the results of earlier studies, $\mathrm{CO}_{2}$ was associated with increased flight frequency, decreased latency to take-off, and increased likelihood that flights would terminate on a visual target placed in the test chamber. Moreover, the authors pointed out that the response threshold to $\mathrm{CO}_{2}$ was similar in their and Warnes and Finalyson's work, e.g. $0.006 \%$ compared to $0.01 \%$. However, they did caution against reading too much into this similarity. First, because inter-study differences in plume structure could affect responses. Second, because these thresholds, if expressed as odour flux, were about an order of magnitude different. Finally, because the activity increase was manifest as a step function, whereas in Warnes and Finlayson's work there was a dose-response relationship. 
Schofield et al. (1997) also evaluated responses to acetone and 1-octen-3-ol. For acetone, outcomes were more muted but followed a similar pattern to those for $\mathrm{CO}_{2}$, i.e. flight activity increased with a response threshold of about $0.01 \mathrm{ug} / \mathrm{l}$, and the proportion of flights terminating on a target increased in the presence of this compound. By contrast, the principle impact of 1-octen-3-ol was a decrease in flight activity at the highest concentrations. This latter finding matches well with at least some work on tsetse where high release rates of 1-octen-3-ol have been associated with reduced: trap catch (Vale and Hall 1985a), upwind turning response (Paynter and Brady 1993), and activity (Bursell 1984).

More recently, Jeanbourquin and Guerin $(2007 a, b)$ examined the behavioural responses of stable flies to rumen and dung-based volatiles. In one experiment, groups of five flies were placed into a release chamber situated in a wind tunnel, with 'activation' defined by a fly leaving the chamber. Increased rates of leaving were evident for raw rumen volatiles, as well as several individual chemicals (dimethyl trisulphide, $\mathrm{p}$-cresol and butanoic acid). As with previous studies, 1-octen3-ol was not associated with an increase in activity. Nor, as was documented in the Schofield et al. (1997) study, was it associated with a decrease in activity. In another experiment, single flies placed into a wind tunnel were exposed to raw dung volatiles, with activation being defined as three or more take-offs within the flight area. Both cow and horse dung were associated with increased 'activation' compared against the control group, e.g. about $70 \%$ of flies were activated compared against 30\%. Unfortunately, the authors did not characterise responses over a range of compound exposures, nor did they use survivorship analyses to explore the data. As a result, potentially informative comparisons that might have been made with earlier studies regarding, for example, latency of response or nature of the dose-response curve, are not possible.

\section{In-flight responses to odour: wind tunnel results}

If the intent of behavioural work is to inform vector control, then research should ultimately be done in, or at least translate to, the field. As pointed out earlier in this chapter, much of the existing field research on haematophagous Diptera has not looked at behaviour per se, but rather has recorded behavioural endpoints, e.g. trap catch. Clearly, this partly reflects the difficulty of executing and interpreting behavioural assays in the field. It is for this reason that laboratory assays of behaviour can be especially useful, i.e. they can document behaviour under carefully controlled and relatively easy to set up conditions. Despite this, most such work has, just as for field studies, measured behavioural endpoints, e.g. position in an olfactometer, net displacement in a wind tunnel. For example, using the set-up described earlier, Gatehouse and Lewis (1973) suggested that while $\mathrm{CO}_{2}$ increased flight activity, it was not associated with a landing bias on a baited versus unbaited target, and hence they concluded that it did not elicit directional responses. On the other hand, they found that emanations from a hand resulted in relatively more landings on a baited target and therefore inferred direction orientation. Of course, in the absence of actual measurements of behaviour per se, it is impossible to validate these inferences. Indeed, subsequent work on stable flies, as we shall see, contradicts their conclusion concerning $\mathrm{CO}_{2}$. Warnes and Finlayson (1985b) evaluated responses of stable flies to $\mathrm{CO}_{2}$ and acetone in a low speed wind tunnel, using net movement over a three-minute period to infer (or not) directed orientation. They found that various concentrations of $\mathrm{CO}_{2}$ resulted in net upwind movement, as did acetone $(0.8 \mathrm{~g} / \mathrm{min})$, expired breath and odour from guinea pigs. Certainly, a parsimonious explanation for these results is upwind anemotaxis, but by no means was this behavioural strategy specifically demonstrated. Indeed, it has been argued that the results for acetone might also reflect an arrestant artefact because cessation of upwind flight responses to acetone have been documented at a concentration/flux some 20 times less than was tested in this study (Schofield 
and Brady 1997). In the last couple of years, several additional studies have evaluated orientation behaviours of stable flies in the laboratory. Jeanbourquin and Guerin $(2007 a, b)$ were interested in stable fly responses to rumen and dung volatiles. 'Attraction' in these experiments was determined on the basis of whether flies showed a minimum upwind displacement in a wind tunnel. As above, while these positive outcomes are suggestive of directional upwind movement, they do not establish causation. In any event, these authors reported that a number of compounds, including horse and cow dung, raw rumen volatiles, dimethyl trisulphide, p-cresol and butanoic acid were associated with attraction in a wind tunnel. Though flights were also classified as being oriented (or not) on the basis of 'sinuous flights in the direction of the odour source', the authors did not provide the detail necessary to validate this classification scheme, nor the related interpretations. Most recently, Beresford and Sutcliffe (2008), looked at the influence of male age on the responsiveness of stable flies to $\mathrm{CO}_{2}$ in a wind tunnel. Interestingly, net upwind displacement at the end of a two-hour observation period was inversely correlated with age, which the authors suggest might reflect an age-based bias in the propensity of males to seek blood meals versus female conspecifics. However, inferring orientation on the basis of fly position after an extended period cannot be considered a reliable indicator of directional orientation, especially because a negative control was not employed, e.g. these results also could reflect age-based variability in activity levels or dispersal tendencies.

To our knowledge, the only laboratory experiment that has recorded and critically evaluated inflight manoeuvres of stable flies was done by Schofield and Brady (1997). This work has much in common previous work on tsetse flies where upwind anemotaxis (Colvin et al. 1989, Paynter 1991, Paynter and Brady 1993, 1996) and ortho- and klinokinetic responses (Warnes 1990, Paynter and Brady 1993, 1996) to host-based odour were observed. All of these studies analysed, quantitatively, the actual flight tracks of test insects. In the interest of efficiency, we will discuss the tsetse work here (instead of in Chapter 12, this volume), with particular attention being paid to studies (Colvin et al. 1989, Paynter and Brady 1993, 1996) that used a set-up very similar to that employed for the stable fly experiments. Bursell (1984) was one of the first to evaluate carefully tsetse behaviour in the laboratory, and suggested that resting flies took off and flew in an upwind direction when exposed to $\mathrm{CO}_{2}$. Following this, Colvin et al. (1989) released tsetse flies (Glossina morsitans morsitans Westwood) from a crosswind position into a wind tunnel and analysed their responses to: (1) the olfactory stimulus of a $\mathrm{CO}_{2}$ odour plume; (2) the visual stimulus of a moving floor with a strong visual pattern and (3) the physical stimulus generated by wind moving at $0.25 \mathrm{~m} / \mathrm{s}$. By using clever combinations of these three stimuli, the authors demonstrated that flights were indeed steered into the wind in the presence of this compound, and that this was guided by visual flow, i.e. that upwind anemotaxis was occurring. This reinforced results from field work where upwind turning had been observed (see Chapter 13), but more importantly provided strong evidence for the mechanism of in-flight steering. Paynter and Brady (1996) expanded upon this work by examining tsetse responses to $\mathrm{CO}_{2}$ at various wind speeds. In addition to upwind anemotaxis, they described kinetic responses to $\mathrm{CO}_{2}$, specifically that tsetse slowed down and turned more in its presence. However, these effects and upwind anemotaxis were not evident at a low wind speed (e.g. $0.1 \mathrm{~m} / \mathrm{s})$. The authors suggested that this might reflect a limit on the part of tsetse to detect wind-induced drift. Not surprisingly, stable flies tested using a similar apparatus (Schofield et al. 1997) also moved upwind when exposed to $\mathrm{CO}_{2}$. In this case, responses were dosedependent with flies showing a substantial propensity to move and exit and turn upwind, and to execute a major turn over three orders of magnitude of $\mathrm{CO}_{2}$ concentration (Table 1). Moreover, flight kinetics differed between the negative control and the odour treatments, and between different concentrations of $\mathrm{CO}_{2}$ (Table 2). In other words, and as with tsetse, stable flies slowed down, turned more, and headed upwind in the presence of $\mathrm{CO}_{2}$. However, they differed from 
Table 1. Percentage of flights exiting upwind, exhibiting major turns and turning upwind in $\mathrm{CO}_{2}$, acetone, and 1-octen-3-ol. Controls consisted of clean air. $\mathrm{CO}_{2}$ concentration is given as percent above ambient (from Schofield and Brady 1997).

\begin{tabular}{|c|c|c|c|}
\hline Concentration & Exit up & Major turn & Turn up \\
\hline \multicolumn{4}{|l|}{$\mathrm{CO}_{2}(\%)$} \\
\hline Control & 25 & 38 & 54 \\
\hline 0.0012 & 47 & 42 & 63 \\
\hline 0.006 & 58 & 65 & 74 \\
\hline 0.012 & 69 & 68 & 73 \\
\hline 0.06 & 70 & 80 & 84 \\
\hline 0.12 & 84 & 80 & 88 \\
\hline \multicolumn{4}{|l|}{ Acetone $(\mu \mathrm{g} / \mathrm{l})$} \\
\hline Control & 30 & 33 & 43 \\
\hline 0.001 & 34 & 32 & 50 \\
\hline 0.01 & 59 & 61 & 79 \\
\hline 0.1 & 50 & 57 & 73 \\
\hline 1.0 & 43 & 79 & 40 \\
\hline 10.0 & 20 & 72 & 34 \\
\hline \multicolumn{4}{|l|}{ 1-octen-3-ol $(\mu \mathrm{g} / \mathrm{l})$} \\
\hline Control & 30 & 39 & 48 \\
\hline 0.0002 & 58 & 61 & 64 \\
\hline 0.002 & 58 & 60 & 62 \\
\hline 0.02 & 57 & 60 & 76 \\
\hline 0.2 & 26 & 66 & 36 \\
\hline 2.0 & 19 & 61 & 21 \\
\hline
\end{tabular}

$n$ for each row: $\mathrm{CO}_{2}=73-92$, acetone $=43-74,1$-octen-3-ol $=$ 57-79.

tsetse by displaying substantially slower flight speeds and higher turning rates in clean air, and in plumes containing $\mathrm{CO}_{2}$.

In-flight responses of tsetse and stable flies to acetone and 1-octen-3-ol have also been evaluated in the laboratory. For tsetse and over a range of doses, both of these compounds were associated with an increased probability of upwind flight (Paynter and Brady 1993). As regards kineses, tsetse exposed to 1-octen-3-ol slowed down and turned more at the highest doses tested, whereas acetone did not produce consistent concentration-dependent kinetic effects. For stable flies, exposure to acetone or 1-octen-3-ol yielded modest upwind responses with more flies flying upwind and/or executing a major turn over several concentration increments. However, the highest 1-octen-3-ol exposures were associated with a reduction in upwind flight compared against the negative control (Table 1).

Overall, the results of laboratory studies implicate a number of kairomones as being of potential importance to host-orientation for stable flies (and tsetse flies). These include $\mathrm{CO}_{2}$, acetone and 1-octen-3-ol. Perhaps more importantly, they also highlight specific behavioural strategies (taxes and kineses) that occur in the presence of odour that reasonably can be expected to increase the probability of host finding. Indeed, it has been postulated that taxes and kinesis, depending on 
Table 2. Mean ( $\pm S E)$ values for angular velocity, sinuosity and velocity of video-recorded stable fly flights in $\mathrm{CO}_{2^{\prime}}$ acetone, and 1-octen-3-ol. Controls consisted of clean air (from Schofield and Brady 1997).

\begin{tabular}{llll}
\hline Concentration & Angular velocity $(\% / \mathrm{s})$ & Sinuosity $(\% / \mathrm{cm})$ & Velocity $(\mathrm{cm} / \mathrm{s})$ \\
$\mathrm{CO}_{2}(\%)$ & & & \\
Control & $114.6 \pm 10.8$ & $0.7 \pm 0.11$ & $170.9 \pm 5.3$ \\
0.0012 & $129.1 \pm 10.5$ & $0.8 \pm 0.09$ & $188.4 \pm 5.1$ \\
0.006 & $162.8 \pm 10.1$ & $1.1 \pm 0.09$ & $172.2 \pm 4.9$ \\
0.012 & $192.2 \pm 10.9$ & $1.5 \pm 0.12$ & $151.3 \pm 5.3$ \\
0.06 & $191.5 \pm 9.4$ & $1.5 \pm 0.08$ & $139.4 \pm 4.6$ \\
0.12 & $212.9 \pm 9.9$ & $1.9 \pm 0.09$ & $133.3 \pm 4.8$ \\
Acetone $(\mu \mathrm{g} / \mathrm{l})$ & & & \\
Control & $102.2 \pm 12.4$ & $0.6 \pm 0.11$ & $169.8 \pm 5.6$ \\
0.001 & $124.7 \pm 11.5$ & $0.8 \pm 0.10$ & $160.3 \pm 5.2$ \\
0.01 & $122.9 \pm 9.7$ & $0.9 \pm 0.08$ & $149.8 \pm 4.4$ \\
0.1 & $151.9 \pm 9.5$ & $1.1 \pm 0.08$ & $157.1 \pm 4.3$ \\
1.0 & $167.0 \pm 9.4$ & $1.3 \pm 0.12$ & $145.5 \pm 4.3$ \\
10.0 & $156.3 \pm 8.4$ & $1.2 \pm 0.07$ & $140.3 \pm 3.8$ \\
$1-0$ - & & & \\
Control & $114.2 \pm 9.3$ & $0.7 \pm 0.08$ & $170.2 \pm 3.7$ \\
0.0002 & $151.4 \pm 10.9$ & $1.1 \pm 0.10$ & $142.0 \pm 4.3$ \\
0.002 & $148.4 \pm 11.2$ & $1.3 \pm 0.10$ & $136.9 \pm 4.4$ \\
0.02 & $152.7 \pm 10.4$ & $1.1 \pm 0.08$ & $148.6 \pm 4.1$ \\
0.2 & $151.5 \pm 9.4$ & $1.2 \pm 0.08$ & $139.5 \pm 3.7$ \\
2.0 & $137.7 \pm 11.4$ & $1.2 \pm 0.10$ & $123.5 \pm 4.8$ \\
\hline
\end{tabular}

$n$ for each row: $\mathrm{CO}_{2}=73-92$, acetone $=43-74,1$-octen-3-ol $=$ 57-79.

the environment, might play a more or less important role in the orientation response. This idea was explored by Williams (1994), who suggested that the consistency of wind direction could be an important determinant of the specific strategy deployed by foraging tsetse. In this model, if wind-generated directional information was reliable at the time of odour detection, then upwind anemotaxis with various odour sampling frequencies should optimise the probability of host encounter. By contrast, if directional information was very poor then kinetic responses to odour detection should be preferred, i.e. the fly should slow down and turn more. Supporting this model, there is field evidence to suggest that tsetse can move upwind very quickly to an odour source (Griffiths et al. 1995). While the model was developed for tsetse, the general approach and its conclusions are applicable to any fast-flying insect responding to an odour source.

Of course, as with all laboratory studies, the important uncertainty is their relevance to the field situation. Aside from the usual litany of issues such as the use of laboratory-reared insects and artificial test conditions, we make note here of several additional points for consideration. First, stable flies and tsetse move quickly relative to some other groups of blood feeding Diptera (e.g. mosquitoes), or other insects for which orientation behaviours have been well documented (e.g. moths). The upshot is that laboratory observations of Stomoxys and tsetse are, by default, usually limited to relatively few behavioural events, at least compared to the types of records that can be obtained for moths and mosquitoes. The consequence is that we have not been 
able to observe directly how sequences of behaviours are put together for these groups, the comparator being the elegant work that has been done on moths as they fly into and out of odour plumes (Murlis et al. 1992, Chapter 6 in this volume). This limitation does come with some analytic benefit. In particular, 'less' behaviour allows for easier analyses, and the rapid horizontal movement displayed by these groups makes two-dimensional analyses more tractable. Second, analyses have focused on concentration as a measure of exposure, with limited consideration of odour flux (i.e. amount of material moving through area per unit time, often expressed as: moles $/ \mathrm{s} / \mathrm{cm}^{2}$ ). It remains uncertain which, if either, of these measures is more appropriate. Is this a critical issue? Perhaps not, but certainly it can affect inter-study validation, e.g. as pointed out above in our discussion of the threshold at which $\mathrm{CO}_{2}$ evokes stable fly flight activity. However, because flux loses its distinction from concentration where flights are substantially faster (e.g. stable flies, tsetse) or slower than wind speed (Paynter and Brady 1996, Schofield 1996), and because it is more challenging computationally, it seems prudent to express exposure intensity based on concentration. However, this comes with the caveat that impacts of test conditions on odour presentation should be considered when interpreting results.

\section{Field work on the responses of Stomoxyinae to host odour}

Before discussing the specifics of field studies evaluating responses of Stomoxyinae to host kairomones, we wish to present a conclusion from our review. As far as we can tell, there is little evidence to indicate that trap-out strategies have broad-based utility as a control intervention for stable flies, and even less data to suggest that host-odour might contribute significantly to any such approach.

While we cannot say whether this conclusion will hold into the future, the current lack of evidence does beg a question - what is the practical aim of carrying out such work? For one, it might allow for heightened sensitivity of surveillance systems. Of course this needs to be weighed against the need for a surveillance system itself (i.e. for a non-vector), and the net benefit accrued by adding host odour into the surveillance equation. Regarding the former, the nuisance and consequent economic costs associated with Stomoxyinae seem to validate the need for at least targeted surveillance, though the need to employ host odour as part of a surveillance system has not been convincingly demonstrated. Another area where this work has proved to be useful, albeit tangentially, is the investigation of host-stable fly interactions. Work in this area, which partly originated due to interest in responses of flies to host odour, has yielded useful insights into intraand interspecific host-parasite relationships (see below)

In any event, visual cues, as well as traps and targets based on various combinations of these have long been used as surveillance tools for stable flies (Schofield 1996). It has been around these technologies that most odour-based research has been carried out. With the exception of a few studies where trap efficiency was also considered (Mihok et al. 2007, Schofield 1998, Vale and Hall 1985b), the bulk of the field work on Stomoxyinae has used a behavioural endpoint, i.e. trap catch, as the outcome measure. As regards specific kairomones, the only compound that has yielded consistent and positive results from the field is $\mathrm{CO}_{2}$. Hoy (1970) reported that Malaise traps releasing $\mathrm{CO}_{2}$ at $3 \mathrm{l} / \mathrm{min}$ caught about three times more stable flies than unbaited traps, and Roberts (1972) reported that catches from a Malaise trap baited with $3.5 \mathrm{I} / \mathrm{min} \mathrm{CO}_{2}$ were similar to those from a trap baited with odour from a single steer. More recently, Mihok et al. (1996) found that Vavoua traps baited with $\mathrm{CO}_{2}$ at $2 \mathrm{I} /$ min caught about twice as many Stomoxyinae flies as unbaited traps. Some of the early work on tsetse responses to odour also yielded useful information for Stomoxyinae. Vale (1980), while investigating the responses of tsetse to host 
odour, also collected Stomoxyinae; later work (Schofield 1998) suggests that the Stomoxys spp. collected by Vale comprised a mixture of three species: S. niger niger Macquart, S. sitiens Rhodani and $S$. calcitrans. Flies were intercepted using electric nets that were positioned downwind of a stationary target. When $\mathrm{CO}_{2}$ was released at 2.5 or $15 \mathrm{l} / \mathrm{min}$ from near to the target, catch was increased by more than an order of magnitude. For the variety of other compounds tested in this study, which included ketones, aldehydes, alcohols and acids, the only one that was observed to affect the behaviour of Stomoxyinae was acetic acid (at $0.7 \mathrm{~g} / \mathrm{hr}$ ), which reduced the catch. Vale and Hall (1985a) found that catches of Stomoxyinae at beta traps or electrified targets increased with $\mathrm{CO}_{2}$ release rate and was some 6 or 16 times higher, respectively, at the maximum tested (20 $\mathrm{I} / \mathrm{min}$ ) when compared against unbaited controls. The potency of $\mathrm{CO}_{2}$ was again demonstrated by Vale and Hall (1985b) with catches at baited targets $(2 \mathrm{l} / \mathrm{min}$ ) being $>10 \times$ greater than at an unbaited trap. Several de facto dose responses to $\mathrm{CO}_{2}$ also have been obtained from Zimbabwe. In these experiments, Stomoxyinae were collected from electric targets baited with the odour from various sized groups of cattle (Hargrove et al. 1995, Torr et al. 2007). For both studies, catch rose with bait mass in a logarithmic fashion (Figure 1). Schofield (1998) also deployed electric targets in Zimbabwe to evaluate the influence of $\mathrm{CO}_{2}\left(2 \mathrm{I} / \mathrm{min} \mathrm{CO}_{2}\right)$ and found that it more than doubled catches when compared against an unbaited target. Target efficiency, estimated by positioning an electric screen next to the target, was $>50 \%$ in this study and was not affected by $\mathrm{CO}_{2}$.

Can non- $\mathrm{CO}_{2}$ odours derived from hosts increase the catch of stable flies in the field? Some of the early studies in Zimbabwe suggested not (Vale 1980, Vale and Hall 1985a,b). However, since this work, evidence has emerged to indicate that other kairomones, especially 1-octen-3-ol, can sometimes affect trap catch. Holloway and Phelps (1991) found that F3 traps baited with 1-octen3-ol ( $0.7 \mathrm{mg} / \mathrm{hr}$ ) caught about twice as many Stomoxys spp. as unbaited traps, though subsequent work carried out in the same area (Schofield 1996) with the same type of trap was not able to

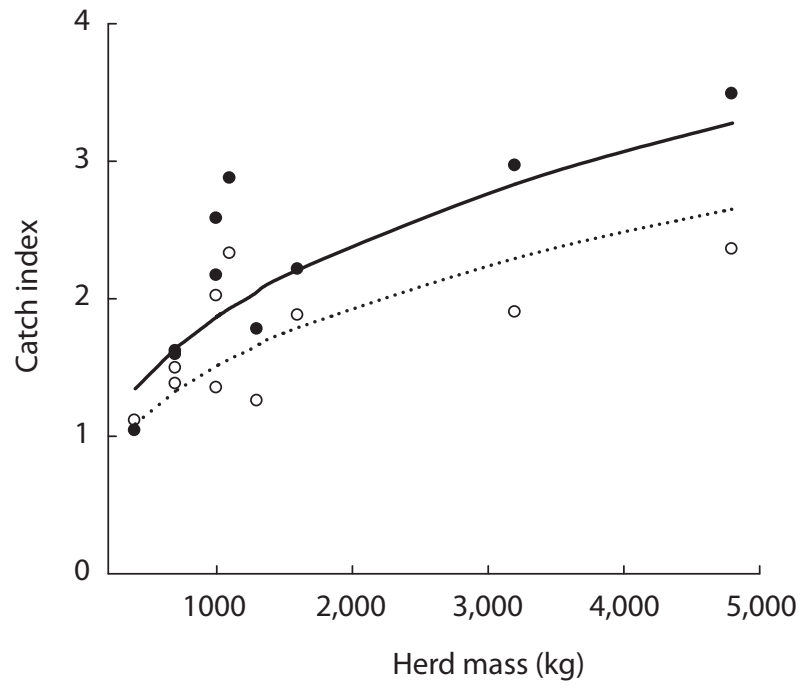

Figure 1. Catch indices of tsetse (solid circles) and Stomoxys (open circles) attracted to herds of cattle of various size. Indices are calculated as the detransformed mean catch from a herd divided by that from a single ox weighing $400 \mathrm{~kg}$. Lines fitted by regression of $\log _{10}$ (herd mass) against $\log _{10}$ (catch index); regression co-efficients for tsetse and Stomoxys were $0.41( \pm 0.070, S E)$ and 0.25 ( \pm 0.093$)$, respectively (adapted from Torr et al. 2007). 
duplicate this result, and earlier studies, also in this area (Vale and Hall 1985a,b), were only able to show a 'repellent' effect for 1-octen-3-ol (5-500 mg/hr) when it was released with ox odour. Of importance, most of the above studies only classified catch to the genus level, and hence interspecies variability might have affected outcomes.

In Kenya, Mihok et al. (1995) evaluated the influence of acetone, 1-octen-3-ol and various urines and dungs on the catch of Stomoxys spp. and other Stomoxyinae (e.g. Haematobosca, Prostomoxys, Rhinomusca, Stygeromyia) using Vavoua traps. Most of the test compounds did not affect trap catch, though 1-octen-3-ol yielded mixed results and sometimes was associated with increased (about 3-fold) catch, especially at high doses (2 mg/hr). In a similar study, Mihok et al. (1996) did not find a consistent effect for 1-octen-3-ol. In two experiments it did not enhance catch of Stomoxys compared to $\mathrm{CO}_{2}$ alone, and when released by itself caught less than half the number of flies as a $\mathrm{CO}_{2}$-baited trap, which is similar to the outcome (Mihok et al. 1995) for a $\mathrm{CO}_{2}$-baited Vavoua trap against an unbaited trap. However, catch of Haematobosca spp. were about 2-3 times higher for one of the experiments in which $\mathrm{CO}_{2}$ was compared against $\mathrm{CO}_{2}$ and 1-octen-3-ol. In work carried out in North America (Cilek 1999), release of 1-octen-3-ol from alsynite cylinders was not associated with increased stable fly catch, though acetone $(50 \mathrm{mg} / \mathrm{hr}$ ) or a mixture of phenols were. Finally, a series of studies carried out in North America and Africa (Mihok et al. 2007) using Nzi traps yielded similarly mixed results, i.e. 1-octenol sometimes was associated with modest increases in catch, and sometimes not. These same authors also reported that traps baited with acetone $(890 \mathrm{mg} / \mathrm{hr}$ ) in Ethiopia caught about twice as many flies as unbaited traps, but increases were not evident when acetone was combined with 1-octen-3-ol and/or cattle urine.

Mihok et al. (2007) put forward an interesting hypothesis to explain the variable results for 1-octen3-ol. Specifically that it is not efficacious when presented in combination baits or in areas where livestock are near. We refer the reader to the paper for a fuller explanation, but do point out that in addition to the exception noted by the authors (Cilek 1999), Vale and Hall (1985b) and Schofield (1996) tested 1-octen-3-ol without cattle in the immediate vicinity, and did not observe increased trap catch. Albeit, substantial numbers of wild ungulates were present around the test sites. Moreover, even though they might not have been immediately adjacent to the test area, there was a large $(>100)$ herd of cattle at the research station in Zimbabwe, as well as wild ungulates, when Holloway and Phelps (1991) carried out their studies. Several alternative, though not necessarily mutually exclusive, explanations for this inconsistent response are possible. For one, even where 1-octen-3-ol has had demonstrable effects, catch increases have been rather modest (e.g. about 2-3 fold). Assuming a substantial random error term and a small expected difference between treatment and control, experiments might have been underpowered for detecting differences. Second, 1-octen-3-ol has been shown to have both positive (e.g. upwind anemotaxis) and negative (activation, reduction in upwind movement, reduced trap catches) effects in the laboratory and field, and exposure to high levels of 1-octen-3-ol have been associated with physiological refractoriness in stable flies (Schofield et al. 1995). Given this variability, it would seem that there is a fairly narrow window to capture 'positive' responses to 1-octen-3-ol, which might be manifest as highly variable results for field work. Finally, we suggest that non- $\mathrm{CO}_{2}$ kairomones simply might not be that important to host orientation in stable flies. Yes, they are active in the laboratory and sometimes in the field, but their relative importance seems small compared to $\mathrm{CO}_{2}$. This last perspective is supported by the work of Torr et al. (2006) who found that differences between cattle in terms of 'attractiveness' to Stomoxys were reduced by standardising $\mathrm{CO}_{2}$ release.

Hence, the story for Stomoxys, though heavily influenced by tsetse work, turns out to be somewhat different as regards the diversity of host-based kairomones that consistently evoke field responses. 
For tsetse, there are several kairomones (Chapter 12, this volume), for Stomoxys there is just one. Of course, additional compounds might be identified in the future, and novel uses for these compounds could be developed. However, as it stands, and likely for at least the near future, there seems to be limited operational advantage to deploying non- $\mathrm{CO}_{2}$ odours, or arguably even $\mathrm{CO}_{2}$, for the purpose of stable fly surveillance or control.

\section{Feeding by Stomoxyinae: strategies and host selection}

The work of Torr et al. (2006) described above highlights that conspecific hosts can vary in their ability to draw Stomoxys to their vicinity, something that also has been demonstrated for other blood feeding insects (Mukubana et al. 2002, Schofield and Sutcliffe 1996). Of course, once an insect gets near to a host a variety of cues, perhaps including host odours, influence whether or not feeding occurs. As it turns out, several groups of Stomoxyinae have received attention in this regard. For example, the load of Haematobia irritans (L.) on cattle has been shown to be affected by a variety of factors (e.g. breed, hair density, sebum, host size, host colour) (Jensen et al. 2004). Similarly and as pointed out by Mullens et al. (2006), it has long been known that cattle show substantial variation in the stable fly load that they support (see Cheng 1958, Mullens and Meyer 1987). Most of the work that critically examines this relationship has been published in the last decade or so. The exception is Warnes and Finlayson (1987). These authors carried out experiments in a laboratory arena and found that calves that displayed higher rates of defensive behaviour had lower fly roads. Subsequent to this, and often in concert with studies examining tsetse, several authors have considered the interrelationship between stable flies, their hosts and other insects. Torr and Mangwiro (2000) evaluated interactions between tsetse feeding success, host, and populations of other flies by observing individual cattle placed within an incomplete ring of electric nets. Though not intended to focus on Stomoxys behaviour, the study was relevant because in addition to documenting that tsetse feeding 'success' varied between hosts, related largely to the host's age, it linked this effect to the intensity of host defensive behaviour. Further, rather than defensive behaviour primarily being regulated by tsetse load, the strongest association was with the numbers of Stomoxys attacking the host. Hence, their suggestion that interspecific interaction (driven by Stomoxys populations) might have important effects on feeding outcomes for tsetse, and by extension could affect trypanosomiasis epidemiology. Of note, while this study considered stable fly populations, it did not collect detailed information on their feeding behaviour. The interplay suggested by Torr and Mangwiro (2002) found substantial support in a follow-up study (Torr et al. 2007). In this case, per capita load of Stomoxys was associated with a significant and negative reduction in tsetse feeding success, from about $80 \%$ at a per capita load of 20 flies to about $40 \%$ at a per capita load of 1000 flies (Figure 2). Though not assessed for Stomoxys specifically, results from this study are also intriguing because they demonstrate the substantial heterogeneity that is possible with respect to host selection, something that can have interesting and potentially important implications for disease epidemiology. A more specific evaluation of Stomoxys feeding behaviour was made by Schofield and Torr (2002) who carried out two experiments in which hostdefensive behaviour and feeding success was directly observed. Interestingly, most Stomoxys were disturbed while feeding (73\%), whether by other flies (65\%) or by host-defensive behaviour (35\%). Moreover, feeding success varied with host (Figure 3 ) and, somewhat paradoxically, there was a positive (though not significant) trend between Stomoxys density and increased feeding success. This last observation runs contrary to the conventional wisdom that this interaction, if anything, should be in the negative direction. The plausible explanation for this, which is somewhat unique as regards the extant literature, is that the majority of Stomoxys were dislodged by non-biting Diptera versus host-based defensive behaviour. Hence, increasing the number of Stomoxys on the host would presumably reduce their per capita exposure to non-biting Diptera, and in turn might 

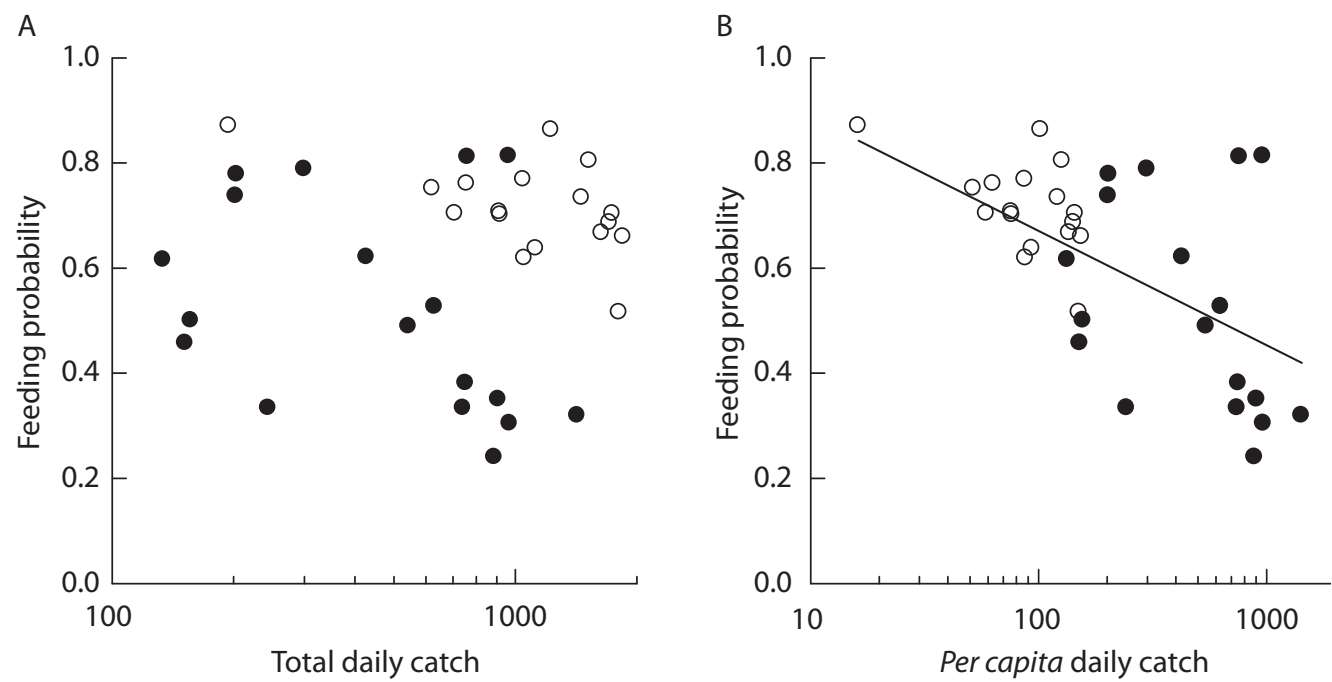

Figure 2. Feeding probability of Glossina plotted against (A) total daily catch of Stomoxys or (B) per capita catch of Stomoxys for a single ox (solid dots) or a herd of 12 cattle (open circles). Line fitted by logistic regression (adapted from Torr et al. 2007).

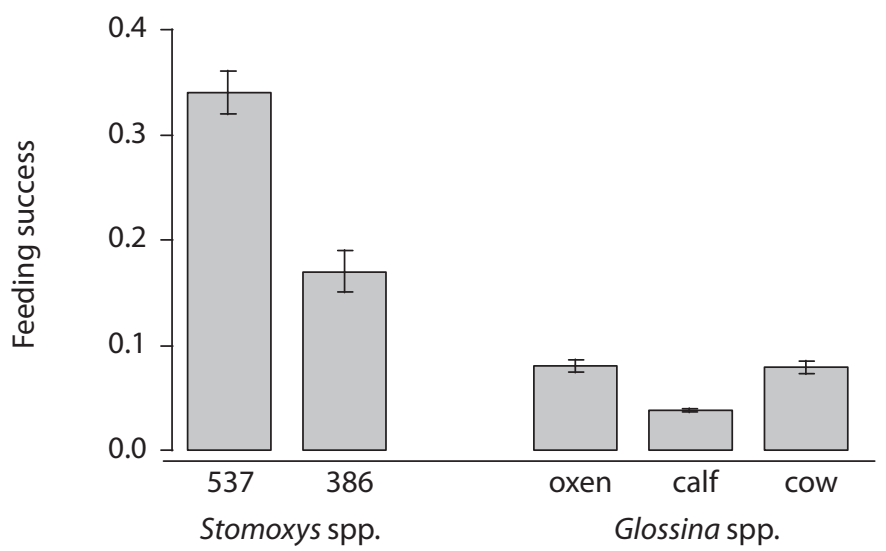

Figure 3. Feeding success ( \pm SE) of Stomoxys on two different adult oxen, designated by ear tags 537 and 386, and feeding success of tsetse on different types of cattle in Zimbabwe (adapted from Schofield and Torr 2002).

increase the probability of a complete feed. In terms of the comparison of Stomoxys and tsetse, the principal differences observed were that the former showed a higher rate of feeding success, a lower relative rate of host-induced disturbance, a longer average period on the host (whether or not disturbed) and a non-constant rate of leaving the host. The authors suggest that these differences are compatible with the life attributes of these groups. On the one hand, Stomoxys are rather short lived (daily survival has been estimated to be about 0.75-0.9 (Berry et al. 1981, Scholl 1986)), and feed daily or even more often. Hence, expectation is for a more persistent, 'riskier' 
feeding strategy. On the other hand, tsetse are longer lived (daily survival >0.95; Hargrove 1988) and likely feed only every $\sim 3$ days. Thus, at least compared to stable flies, they should be 'choosier' feeders. So, just as for kairomones, while some of the gross patterns of behaviour for these groups are similar (e.g. host-selection heterogeneities), they show marked differences at a finer scale.

One of the most ambitious studies of interactions between stable flies and their hosts was undertaken in California by Mullens et al. (2006). In this work, individuals in four herds of 25 cows were observed 8-10 times per week for 12 weeks. For each observation period, the numbers of flies and defensive behaviours were recorded. In addition to a very strong correlation $\left(r^{2}>0.8\right)$ between the weekly mean estimates for defensive behaviour and fly populations, the authors were able to demonstrate that ranked fly load per animal was consistent over the experiment, as was ranked intensity of defensive behaviour. Consistent with work for tsetse, older cows tended to maintain higher fly loads and demonstrated a lower rate of effective defensive manoeuvres (i.e. leg stamps) than younger animals. Finally, the per capita rate of defensive behaviour against stable flies decreased with time, suggesting that animals became habituated to stable fly attack. Though the authors were unable to characterise economically relevant impacts of fly attack (e.g. decreased milk yield), they make the valid point that more work is required to relate economic impact - to host behaviour - to biting fly pressure. Finally, they suggest that monitoring defensive behaviour (instead of fly population) might provide a simpler, more efficient and sensitive indictor for decision-making around pest management.

In contrast to the situation for distance-orientation kairomones, results from studies evaluating close-range or on-host behaviours/interactions would seem to have direct operational relevance to stable fly management. This might simply be in terms of developing efficient surveillance indicators (Mullens et al. 2006), or more ambitiously could be articulated in the context of developing more sustainable and/or cost-effective control strategies (see Mullens et al. 2006, Prior 2003, Torr et al. 2001, 2007).

\section{Conclusion}

While odour-based orientation to host animals is necessary to stable flies, it might not be readily exploited from a control perspective. This is substantially different to the situation for at least some species of tsetse, where research on and eventual use of host odour has powered successful control programs (see Chapter 12 in this volume). The example of tsetse research also shows how a better appreciation of the host-orientation behaviour of flies can enhance our understanding of host-parasite interactions, which in turn might lead to improved methods of control (e.g. insecticide-treated cattle to control tsetse, see Torr et al. 2007). Whether or not this also will be the case for stable flies remains to be seen.

Aside from these practical considerations, the odour-oriented behaviour of Stomoxys and tsetse provide many examples of where host selection is governed more by its defensive behaviour than its kairomone profile. By contrast, much research on identifying 'attractants' and 'repellents' for mosquitoes and midges has focussed on comparing the odour profiles of individuals that are bitten more or less (see other chapters in this book). The relative importance of odours in host selection by different species of biting fly may be related, in part, to their respective activity periods: day-active species can make better use of visual cues such as host-colour,-movement and -shape whereas odour is, perhaps, the only cue that can be relied on to distinguish one sleeping host from another. However, we also suggest that this might reflect an underlying assumption that odours alone are responsible for inter-host variability in attractiveness. The cases of Stomoxys 
and tsetse provide numerous examples where this is not the case. Perhaps we should be checking that the same does not apply to other vectors?

\section{References}

Alzogaray RA and Carlson DA (2000) Evaluation of Stomoxys calcitrans (Diptera: Muscidae) behavioral response to human and related odors in a triple cage olfactometer with insect traps. J Med Entomol 37: 308-315.

Anderson JR (1978) Effects of a blood meal on the mating drive of Stomoxys calcitrans males and its necessity as a prerequisite for proper insemination of females. J Econ Entom 71: 379-386.

Beresford DV and Sutcliffe JF (2006) Studies on the effectiveness of coroplast sticky traps for sampling stable flies (Diptera: Muscidae), including a comparison to Alsynite. J Econ Entomol 99: 1025-1035.

Beresford DV and Sutcliffe JF (2008) Male stable fly (Stomoxys calcitrans) response to $\mathrm{CO}_{2}$ changes with age: evidence from wind tunnel experiments and field collections. J Vector Ecol 33: 247-254.

Berry IL and Campbell JB (1985) Time and weather effects on daily feeding patterns of stable flies (Diptera: Muscidae). Environ Entomol 14: 336-342.

Berry IL, Scholl PJ and Shugart JI (1981) A mark and recapture procedure for estimating population sizes of adult stable flies. Environ Entomol 10: 88-93.

Bidgood HM (1980) Host location in Stomoxys calcitrans (L.). Dissertation, University of Birmingham, Birmingham, UK.

Birkett MA, Agelopoulos N, Jensen KM, Jespersen JB, Pickett JA, Prijs HJ, Thomas G, Trapman JJ, Wadhams LJ and Woodcock CM (2004) The role of volatile semiochemicals in mediating host location and selection by nuisance and disease-transmitting cattle flies. Med Vet Entomol 18: 313-322.

Bursell E (1984) Effects of host odour on the behaviour of tsetse. Insect Sci Appl 5: 343-349.

Carlson DA, Alzogaray RA and Hogsette JA (2000) Behavioral response of Stomoxys calcitrans (Diptera: Muscidae) to conspecific feces and feces extracts. J Med Entomol 37: 957-961.

Charlwood JD and Lopes, J. (1980) The age structure and biting behavior of Stomoxys calcitrans (Diptera: Muscidae) from Manuas, Brazil. Bull Ent Res 70: 549-556.

Cheng T (1958) The effect of biting fly control on weight gain in beef cattle. J Econ Entomol 51: 275-278.

Chia LS, Baxter JA and Morrison PE (1982) Quantitative relationship between ingested blood and follicular growth in the stable fly, Stomoxys calcitrans. Can J Zool 60: 1917-1921.

Cilek JE (1999) Evaluation of various substances to increase adult Stomoxys calcitrans (Diptera: Muscidae) collections on alsynite cylinder traps in north Florida. J Med Entomol 36: 605-609.

Colvin J, Brady J and Gibson G. (1989) Visually-guided, upwind turning behaviour of free-flying tsetse flies in odourladen wind: a video study. Physiol Entomol 14: 31-39.

Cooperband MF and Cardé RT (2006) Orientation of Culex mosquitoes to carbon dioxide-baited traps: flight manoeuvres and trapping efficiency. Med Vet Entomol 20: 11-26.

Daykin PN, Kellogg FE and Wright RH (1965) Host-finding and repulsion of Aedes aegypti. Can Entomol 97: 239-263.

Dekker T, Geier M and Cardé RT (2005) Carbon dioxide instantly sensitizes female yellow fever mosquitoes to human skin odours. J Exp Biol 208: 2963-2972.

Gatehouse AG and Lewis CT (1973) Host location behaviour of Stomoxys calcitrans. Entomol Exp Appl 16: 275-290.

Griffiths N, Paynter Q and Brady J (1995) Rates of progress up odour plumes by tsetse flies: a mark-release video study of the timing of odour source location by Glossina pallidipes. Physiol Entomol 20: 100-108.

Hansens EJ (1951) The stable fly and its effect on seashore recreational areas in New Jersey. J Econ Entomol 44: 482-487.

Hargrove JW (1988) Tsetse - the limits to population growth. Med Vet Ent 2: 203-217.

Hargrove JW, Holloway MTP, Vale GA, Gough AJE and Hall DJ (1995) Catches of tsetse flies (Glossina spp.) (Diptera: Glossinidae) from traps baited with large doses of natural and synthetic host odour. Bull Ent Res 85: 215-227.

Harley JMB (1965) Seasonal abundance and diurnal variations in activity of Stomoxys and Tabanidae in Uganda. Bull Ent Res 56: 319-332.

Holloway MTP and Phelps RJ (1991) The responses of Stomoxys spp. (Diptera: Muscidae) to traps and artificial host odours in the field. Bull of Ent Res 81: 51-55. 
Hoy JB (1970) A recent test of Gambusia for mosquito control in a rice field. Proc Pap Annu Conf Calif Mosq Control Assoc.38: 73-74.

Jeanbourquin P and Guerin PM (2007a) Sensory and behavioural responses of the stable fly Stomoxys calcitrans to rumen volatiles. Med Vet Entomol 21:217-224.

Jeanbourquin P and Guerin PM (2007b) Chemostimuli implicated in selection of oviposition substrates by the stable fly Stomoxys calcitrans. Med Vet Entomol 21:209-216.

Jensen KM, Jespersen JB, Birkett MA, Pickett JA, Thomas G, Wadhams LJ and Woodcock CM (2004) Variation in the load of the horn fly, Haematobia irritans, in cattle herds is determined by the presence or absence of individual heifers. Med Vet Entomol 18: 275-280.

Jones MDR and Gubbins SJ (1978) Changes in the circadian flight activity of the mosquito Anopheles gambiae in relation to insemination, feeding and oviposition. Physiol Entomol 3: 213-220.

Jones CJ, Milne DE, Patterson RS, Schreiber ET and Milio JA (1992) Nectar feeding by Stomoxys calcitrans (Diptera: Muscidae): effects on reproduction and survival. Environ Entomol 21: 141-147.

Kennedy JS (1939) The visual responses of flying mosquitoes. Proc Zool Soc Lond 109: 221a-242a.

Kennedy JS (1992) The new anthropomorphism. Cambridge University Press, Cambridge, UK.

Klowden MJ and Lea AO (1979) Effect of defensive host behaviour on the bloodmeal size and feeding success of natural populations of mosquitoes (Diptera: Culicidae). J Med Entomol 15: 514-517.

Klowden MJ, Davis EE and Bowen MF (1987) Role of the fat body in the regulation of host-seeking behaviour in the mosquito Aedes aegypti. J Insect Physiol 33: 643-646.

Kunz SE and Monty J (1976) Biology and ecology of Stomoxys nigra Marquart and Stomoxys calcitrans (L.) (Diptera: Muscidae) in Mauritius. Bull Ent Res 66: 745-755.

Kuzina OS (1942) Gonotrophic relationships in Stomoxys calcitrans L. and Haematobia stimulans L. Med Parisitol 11: 70-78.

Lehane MF (2005) The biology of blood sucking insects. Cambridge University Press, New York, USA.

Lewis CT (1971) Superficial sense organs on the antennae of the fly, Stomoxys calcitrans. J Insect Physiol 17: 449-461.

Lewis CT (1972) Chemoreceptors in haematophagous insects. Behavioural aspects of parasite transmission. Zoot. J. Linn. Soc. 51 Suppl. I: 201-213.

Mihok S, Carlson DA and Ndegwa PN (2007) Tsetse and other biting fly responses to Nzi traps baited with octenol, phenols and acetone. Med Vet Entomol 21:70-84.

Mihok S, Kang'ethe EK and Githaiga, KK (1995) Trials of traps and attractants for Stomoxys spp. (Diptera: Muscidae). J Med Entomol 32: 283-289.

Mihok S, Maramba O, Munyoki E and Saleh K (1996) Phenology of Stomoxyinae in a Kenyan forest. Med Vet Entomol 10: $305-316$.

Mukabana WR, Takken W, Coe R and Knols BG (2002) Host-specific cues cause differential attractiveness of Kenyan men to the African malaria vector Anopheles gambiae. Malar J 1: 17.

Mullens BA and Meyer JA (1987) Seasonal abundance of stable flies (Diptera: Muscidae) on California dairies. J Econ Entomol 5: 1039-1043.

Mullens BA, Lii KS, Mao Y, Meyer JA, Peterson NG and Szijj CE (2006) Behavioural responses of dairy cattle to the stable fly, Stomoxys calcitrans, in an open field environment. Med Vet Entomol 20: 122-137.

Murlis J, Elkinton JS and Cardé RT (1992) Odour plumes and how insects use them. Ann Rev Entomol 37: 505-532.

Nayar JK and Sauerman DM (1971) The effect of light regimes on the circadian rhythm of flight activity in the mosquito Aedes taeniorhynchus. J Exp Biol 54: 745-756.

Parr HCM (1962) Studies on Stomoxys calcitrans L. in Uganda, East Africa. II Notes on life history and behaviour. Bull Ent Res 53: 437-443.

Paynter QE (1991) The effects of host odours on the flight behaviour of tsetse flies. Dissertation, Imperial College, University of London, London, UK.

Paynter Q and Brady J (1993). Flight responses of tsetse flies (Glossina) to octenol and acetone vapour in a wind-tunnel. Physiol Entomol 18: 102-108. 


\section{Behavioural modalities of 'non-vector' biting Diptera: from olfaction to feeding}

Paynter Q and Brady J (1996) The effect of wind speed on the flight responses of tsetse flies to $\mathrm{CO}_{2}$ : a wind-tunnel study. Physiol Entomol 21: 309-312.

Pickard E (1968) Stomoxys calcitrans (L.) breeding along TVA reservoir shorelines. Mosquito News 28: 644-646.

Prior, A.R. (2003) Written in blood: the use of microsatellite markers to study the feeding behaviour of haematophagous Diptera. PhD thesis, University of Greenwich, Greenwich, UK. 240 p.

Roberts RH (1972) The effectiveness of several types of malaise traps for the collection of Tabanidae and Culicidae. Mosquito News 32: 542-547.

Romero A, Broce A and Zurek L (2006). Role of bacteria in the oviposition behaviour and larval development of stable flies. Med Vet Entomol 20: 115-121.

Schofield S (1996) Visual and olfactory responses of the stable fly. Dissertation, Imperial College, University of London, London, UK.

Schofield S (1998) Field activity and seasonal changes in Stomoxys populations in Zimbabwe. Bull Ent Res 88: 627-632.

Schofield S and Brady J (1996) Circadian activity pattern in the stable fly, Stomoxys calcitrans. Physiol Entomol 21: 159163.

Schofield S and Brady J (1997) Effects of carbon dioxide, acetone and 1-octen-3-ol on the flight responses of the stable fly, Stomoxys calcitrans, in a wind tunnel. Physiol Entomol 22: 380-386.

Schofield S and Torr SJ (2002) A comparison of the feeding behaviour of tsetse and stable flies. Med Vet Entomol 16: 177-185.

Schofield S, Cork A and Brady J (1995). Electroantennogram responses of the stable fly, Stomoxys calcitrans, to components of host odour. Physiol Entomol 20: 273-280.

Schofield SW and Sutcliffe JF (1996) Human individuals vary in attractiveness for host-seeking black flies (Diptera: Simuliidae) based on exhaled carbon dioxide. J Med Entomol 33: 102-108.

Schofield S, Witty C and Brady J (1997) Effects of carbon dioxide, acetone and 1-octen-3-ol flux on the landing responses of the stable fly, Stomoxys calcitrans. Physiol Entomol 22: 256-260.

Scholl PJ (1986) Field population studies of Stomoxys calcitrans (L.) in Eastern Nebraska. Southwest Entomol 11: 155-160.

Schwinghammer KA, Knapp FW and Boling JA (1987) Physiological and nutritional response of beef steers to combined infestations of horn fly and stable fly (Diptera: Muscidae). J Econ Entomol 80: 120-125.

Schwinghammer KA, Knapp FW, Boling JA and Schillo KK (1986) Physiological and nutritional response of beef steers to infestations of the horn fly (Diptera: Muscidae). J Econ Entomol 79: 1010-1015.

Spates GE, DeLoach JR and Chen AC (1988) Ingestion, utilization and excretion of blood meal sterols by the stable fly, Stomoxys calcitrans. J Insect Physiol 34: 1055-1061.

Sutcliffe JF (1986) Black fly host location: a review. Can J Zool 64: 1041-1053.

Sutcliffe JF (1987) Distance orientation of biting flies to their hosts. Insect Sci Appl 8: 611-616.

Torr SJ and Mangwiro TN (2000) Interactions between cattle and biting flies: effects on the feeding rate of tsetse. Med Vet Entomol 14: 400-409.

Torr SJ, Della Torre A, Calzetta M, Costantini C and Vale GA (2008) Towards a fuller understanding of mosquito behaviour: use of electrocuting grids to compare the odour-orientated responses of Anopheles arabiensis and An. quadriannulatus in the field. Med Vet Entomol 22: 93-108.

Torr SJ, Mangwiro TN and Hall DR (2006) The effects of host physiology on the attraction of tsetse (Diptera: Glossinidae) and Stomoxys (Diptera: Muscidae) to cattle. Bull Ent Res 96: 71-84.

Torr SJ, Prior A, Wilson PJ and Schofield S (2007) Is there safety in numbers? The effect of cattle herding on biting risk from tsetse flies. Med Vet Entomol 21: 301-311.

Torr SJ, Wilson PJ, Schofield S, Mangwiro TN, Akber S and White BN (2001) Application of DNA markers to identify the individual-specific hosts of tsetse feeding on cattle. Med Vet Entomol 15: 78-86.

Turner DA (1971) Olfactory perception of live hosts and carbon dioxide by the tsetse fly Glossina morsitans orientalis Vanderplank. Bull Ent Res 6: 75-96.

Vale GA (1980) Field studies of the responses of tsetse flies (Glossinidae) and other Diptera to carbon dioxide, acetone and other chemicals. Bull Ent Res 70: 563-570. 
Vale G and Hall D (1985a). The role of 1-octen-3-ol, acetone and carbon dioxide in the attraction of tsetse flies, Glossina spp. (Diptera: Glossinidae), to ox odour. Bull Ent Res 75: 209-217.

Vale $G$ and Hall D (1985b). The use of 1-octen-3-ol, acetone and carbon dioxide to improve baits for tsetse flies, Glossina spp. (Diptera: Glossinidae). Bull Ent Res 75: 219-231.

Venkatesh K and Morrison PE (1980) Some aspects of oogenesis in the stable fly, Stomoxys calcitrans (Diptera: Muscidae). J Insect Physiol 26: 711-715.

Venkatesh Kand Morrison PE (1982) Bloodmeal as a regulator of triacylglyceral synthesis in the haematophagous stable fly, Stomoxys calcitrans. J Comp Physiol 147: 49-52.

Warnes ML and Finlayson LH (1985a) Responses of the stable fly, Stomoxys calcitrans (L.) (Diptera: Muscidae), to carbon dioxide and host odours. I. Activation. Bull Ent Res 75: 519-527.

Warnes ML and Finlayson LH (1985b) Responses of the stable fly, Stomoxys calcitrans (L.)(Diptera: Muscidae), to carbon dioxide and host odours. II. Orientation. Bull Ent Res 75: 717-727.

Warnes ML and Finlayson LH (1986) Electroantennogram responses of the stable fly, Stomoxys calcitrans, to carbon dioxide and other odours. Physiol Entomol 11: 469-473.

Warnes ML and Finlayson LH (1987) Effect of host behaviour on host preference in Stomoxys calcitrans. Med Vet Entomol 1:53-57.

Warnes ML (1990) The effects of host odour and carbon dioxide on the flight of tsetse flies (Glossina spp.) in the laboratory. J Insect Physiol 36: 607-611.

Williams B (1994) Models of trap seeking by tsetse flies: anemotaxis, klinotaxis and edge detection. J Theor Biol 168: 105-115.

Zumpt F (1973) The stomoxyine biting flies of the world. Gustav Fischer Verlag, Stuttgart, Germany. 


\title{
14. The role of olfaction in host seeking of triatomine bugs
}

\author{
Pablo G. Guerenstein and Claudio R. Lazzari
}

\begin{abstract}
Triatomine bugs (Hemiptera: Reduviidae) are hemimetabolous insects that depend on a blood meal along their entire life cycle. They live in close association with their vertebrate hosts, including humans. Triatomines are the vectors of Chagas disease in the Americas. They transmit the protozoan parasite Trypanosoma cruzi during or immediately after feeding by depositing their faeces on a host. Olfactory cues play a major role in host seeking in triatomines. Some of the odorants to which the odour receptor cells respond to are already known. Most of those odorants (known to also be detected by other haematophagous insects) are of vertebrate origin, and activate odour receptor cells in antennal grooved pegs and basiconic sensilla. Behavioural responses (activation and/or attraction) to single odorants often occur mainly at concentrations close to those that represent the response threshold for the related olfactory receptor cell. Information about relevant single odorants (e.g. $\mathrm{CO}_{2}$ ) is used to build synthetic odour mixtures capable of attracting the bugs efficiently. Thus, a few odour mixtures that evoke higher attraction than that evoked by its odorant constituents when presented singly (i.e. showing additive or synergistic effects) have been developed. $L$ (+) lactic acid plays a fundamental role in enhancing the attraction to those mixtures. Triatomine bugs are nocturnal, and the behavioural response to host odours is temporally modulated. Thus, behavioural responsiveness to $\mathrm{CO}_{2}$ is controlled by a circadian clock, and is limited to the early night, the moment when the bugs leave their refuges to search a host. A prototype of odour baited trap that exploits a particular behaviour of triatomines has been developed. However, more research is needed in order to achieve a practical, cheap and efficient odour lure for a trap able to accomplish the necessary task of surveillance of the bugs in the field.
\end{abstract}

Keywords: attraction, chemoreception, modulation, odour bait, olfactory sensilla, orientation, Rhodnius, Triatoma

\section{Introduction}

Triatomine bugs, also named kissing bugs, cone-noses or assassin bugs, are Heteropterans belonging to the family Reduviidae, subfamily Triatominae. They are hemimetabolous insects with a life cycle that lasts from a few months to a year depending on the species, and that includes five wingless nymphal (larval) stages followed by a winged adult stage. Triatomines are obligate haematophagous along their entire life cycle, as nymphs, males and females feed on the blood of vertebrates. Most of their hosts are warm-blooded vertebrates (birds and mammals), but some species live in association and feed on ectothermic animals or even are able to suck haemolymph from other insects (e.g. Almeida et al. 2000).

Around 120 species of triatomines have been described in the New World, half of them capable of transmitting the flagellate protozoan Trypanosoma cruzi Chagas, the causative agent of Chagas disease, which menace about 28 million people living in Latin America and accounts for 670,000 disability-adjusted life years per annum (DALY; Tarleton et al. 2007). The epidemiologically most important species are Triatoma infestans Klug (Figure 1), T. dimidiata Latreille, and Rhodnius prolixus Stål. About 13 species are known in the Old World although they are not vectors of Trypanosoma 


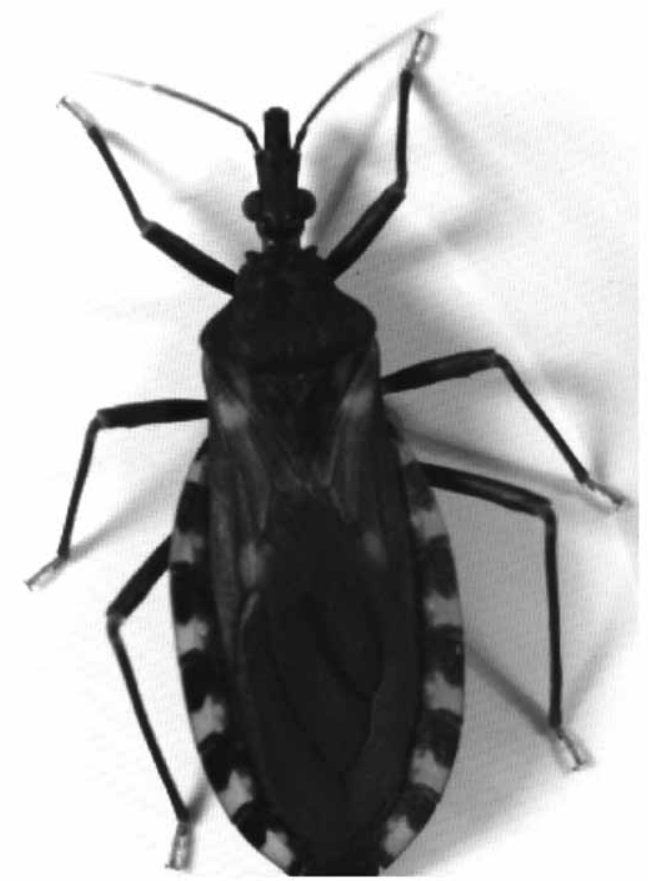

Figure 1. An adult female of Triatoma infestans, an epidemiologically important species.

cruzi. They are endemic in southern China, in Malaysia, Indonesia, New Guinea, northeastern Australia and India (Lent and Wygodzinsky 1979).

Taking a blood meal could take up to 30 min, according to the species, and such a meal most often represents several times their initial body weight. The bite is often painless and, if the host is infected with Trypanosoma cruzi, the bug may acquire the infection with its meal. The parasite then colonises the hindgut of the insects. The bugs can transmit the parasite to a vertebrate host when infected excrements (faeces) are left over the host's skin. The parasite can then enter the host's bloodstream through the skin or mucous membrane, such as that of the mouth if the insect or its dejections are ingested. An important factor affecting the vectorial capacity of a given triatomine species is the time elapsed between feeding and defecation. Those species releasing excrements during or just after feeding increase the probability of parasite transmission.

The importance of triatomines as disease vectors was first established by the Brazilian scientist Carlos Chagas in 1909, who discovered the first human cases of a hitherto unknown trypanosomiasis. Chagas proved that the vector of the causative agent of the disease, Trypanosoma cruzi, was an insect of the order Hemiptera, Panstrongylus megistus Burmeister (Lent and Wygodzinky 1979). Further research on the vectors revealed that the parasite is actually present in several species of the subfamily Triatominae found in and around human dwellings as well as in those living in sylvatic habitats. The finding that armadillos, opossums and other mammals are naturally infected with the parasite made clear that Chagas' disease is a zoonosis, and that the secondary adaptation of some vector species to human habitations resulted in the domestic cycle of the parasite (Lent and Wydgodzinsky 1979). No vaccine against the parasite responsible for Chagas' disease is at 
present available. Control of this disease depends on the surveillance and chemical elimination of the triatomine vectors (by applying insecticides), screening of blood banks and detection of parasites in pregnant women, followed by treatment of the newborns from infected mothers. Being the presence of the vectors strongly associated to the precarious structure of houses in less favoured rural areas of endemic countries, house improvement to reduce the number of potential refuges (e.g. wall crevices) for bugs constitutes is another way to reduce transmission.

Regional coordinate actions against Chagas disease started in 1992 by the Southern Cone Initiative, signed by Brazil, Argentina, Bolivia, Chile, Paraguay, Uruguay, and Peru. It was followed by two similar multinational initiatives against Chagas disease in Central America and the Andean Pact region, both launched in 1997, and a large-scale Chagas disease surveillance initiative for the countries of the Amazon basin launched in 2002 (Schofield and Kabayo 2008).

The surveillance of the arrival to houses and the detection of bugs present in low-density are important components of control strategies. So, the possibility to exploit olfactory responses to actively attract bugs into traps or detection devices becomes relevant (e.g. Guerenstein et al. 1995), as well as the characterisation of their chemosensory system and olfactory responses.

Most triatomine bugs live in close association with their vertebrate hosts, usually inside human housings, in the case of the most antropophilic species, or in the nests or burrows of marsupials, edentates, rodents, carnivores, bats, and birds. Triatomines are also found among rocks and in stone walls, where they feed on small rodents and eventually on iguanas and other lizards that share their habitat. In addition, the insects are found under fallen logs and in hollow trees, among exposed roots and under loose bark, in palm fronds and in epiphytic bromeliads (Lent and Wygodzinsky 1979). Many triatomines have become peridomestic, occurring in stables and corrals, in rabbit and guinea pig houses, and in chicken and pigeon coops. From an epidemiological point of view, the most important habitat of triatomine bugs is human dwellings, in particular in rural areas of Latin America. Thus, triatomines can be classified into sylvatic and domestic species, with an intermediate category of peridomestic species. Inside houses, during daytime, triatomines hide in dark and sheltered places such as wall cracks, furniture, trunks, suitcases, and boxes filled with old clothes or papers. They are found among curtains and clothes hanging from the walls, behind pictures or calendars, in various types of roofing materials, such as thatch, palm fronds, or even loose shingles, among mattresses and in cots and beds of all kinds, in hammocks and cradles, and in woodpiles (Lent and Wygodzinsky 1979).

Bugs spend daytime in akinesis, inside their 'refuges' in the neighbourhood of their hosts, presumably to avoid detection by predators, including their active hosts. The akinesis behaviour is controlled and maintained by external cues, such as aggregation arresting pheromones and tactile stimulation by physical contact with the substrate and with conspecifics (thigmotaxis), in combination with an internal motivational state of inactivity, controlled by a circadian clock. During the early night, the internal circadian clock determines that one to two hours after dusk, when their hosts are resting, the bugs become active (Lazzari 1992), and leave their refuges searching for cues revealing the presence of a potential host to feed on.

Once a blood meal has been obtained, bugs walk away from the host, but do not return to their refuge immediately (Lorenzo and Lazzari 1998). The time spent outside just after feeding would allow bugs to complete most of their copious diuresis that follows a blood meal. At dawn, they return to their shelters guided by chemical cues present in their faeces, which are actively deposited around the refuge accesses (Lorenzo and Lazzari 1996). Chemicals present in the 
faeces constitute one of the aggregation pheromones of triatomines (Lorenzo Figueiras et al. 1994, Schofield and Patterson 1977). A second one, associated with the cuticle, is left behind on surfaces over which the bugs walked (Lorenzo Figueiras and Lazzari 1998). The two pheromones, together with thigmotaxis, cause the insects to remain inactive inside their refuge during daytime.

\section{Olfactory physiology: detection of host odours}

The antenna of triatomines consists on four segments, a proximal scapus, a pedicelus and two flagellar articles. All segments bear sensilla, hair-like cuticular structures that house sensory cells of different modalities, including chemoreception, mechanoreception, thermoreception and hygroreception (Barth 1952, 1953, Catalá 1994, Catalá and Schofield 1994, Diehl et al. 2003, Guerenstein and Guerin 2001, Mayer 1968, Bernard 1974, Insausti et al. 1999, Justo and Tramezzani 1977, Mclver and Siemicki 1984, 1985, Lazzari and Wicklein 1994, Taneja and Guerin 1997, Wigglesworth and Gillet 1934).

Triatomine chemoreceptor cells specialised in odour detection (odour receptor cells, ORCs), and contained in olfactory sensilla, are the best studied. Thus, the odour 'tuning' of many of them is known. To date, the structure of three morphologically distinguishable types of olfactory sensilla has been described on the antennae of triatomines. Those sensilla are the grooved pegs (GP; Diehl et al. 2003, Guerenstein and Guerin 2001, Taneja and Guerin 1997, named basiconica in Catalá 1997), the basiconic sensilla (BS, Guerenstein and Guerin 2001, named trichoid thin-walled in Catalá 1997) and the trichoid sensilla (Guerenstein 1999, named trichoid thick-walled in Catalá 1997). The latter are $35 \mu \mathrm{m}$ long single (thick)-walled wall-pore sensilla (Bernard 1974). They are the most numerous olfactory sensilla on the adult antenna and yet the odour tuning of its ORCs (1-2 in T. infestans nymphs, Bernard 1974; 5-6 in R. prolixus adults, Wigglesworth and Gillet 1934) is unknown because no olfactory stimulus tested up to date evoked a response from them (Bernard 1974, PG Guerenstein and P Guerin, unpublished results). An olfactory function is attributed to this sensillum type because of its morphological characteristics. On the contrary, the odour tuning and physiology of some ORCs in the other two types of olfactory sensilla have been studied in detail (see below).

To study the odour tuning of identified ORCs, single sensillum recordings (SSRs) are performed on different, individual sensilla. A number of monomolecular odours (odorants), individually placed in odour cartridges, can then be screened and the responses to them recorded for analysis. Alternatively, a headspace extract of a natural odour source (e.g. a host odour) can be prepared. This extract can then be injected into a gas chromatograph (GC) that is coupled to the antennal preparation. As the constituents of the extract elute from the GC column, the bioactive odorants are detected by the insect ORCs as recorded by means of a SSR, a technique named gas chromatography coupled to single sensillum recording (GC-SSR; e.g. Guerenstein and Guerin 2001, see also Chapter 3 in this volume).

The morphology and ultrastructure of the GPs of $5^{\text {th }}$ instar nymphs of $T$. infestans have been studied in detail (Diehl et al. 2003). They are non-articulated, double-walled wall-pore sensilla, 8-18 $\mu \mathrm{m}$ long, with a diameter of about $2.5 \mu \mathrm{m}$ at their base. The wall pores in the 13-18 longitudinal grooves communicate to the inner sensillum lymph cavity through radial spoke channels. These sensilla house four (ca. $54 \%$ of GPs) or five receptor cells with an unbranched outer dendritic segment that can reach the tip of the sensillum. Areas of direct contact between cell membranes of the soma of the receptor cells are found, and may represent gap junctions. This suggests possible interaction between the responses of the sensory cells. Some pegs have a terminal pore whereas 
others do not. To date, the proportion of GPs with a terminal pore has not been established and, moreover, it is unknown if this pore has any functional role. Fifth instar nymphs posses $80-110$ of such sensilla per antenna (Diehl et al. 2003).

As in other insects (e.g. Altner et al. 1977), the ORCs of this type of sensillum respond to polar odorants. According to the odour tuning of their ORCs, different functional types of GPs are found (Table 1). In $5^{\text {th }}$ instar nymphs GP1 represents ca. $36 \%$ of the total GP population, whereas GP2 and GP3 represent 10 and $43 \%$ respectively. The receptor cells of $11 \%$ of the GPs did not respond to any of the olfactory stimuli tested so far (Diehl et al. 2003). At least one of the unresponsive receptor cell types is a hygroreceptor cell (Bernard 1974).

All odorants that evoke responses in ORCs of GPs are known to be emitted by vertebrate hosts. Thus, short-chain carboxylic acids are constituents of vertebrate odour (see Guerenstein and Guerin 2001), including human sweat (e.g. Cork and Park 1996). GC-SSR experiments using the antenna of $T$. infestans nymphs allowed the identification of one of them, isobutyric acid, as an active constituent of the headspace extract of rabbit odour (Guerenstein and Guerin 2001). In addition, ammonia and short-chain aliphatic amines have been identified in vertebrate effluvia, including sweat and urine odour (see Diehl et al. 2003, Taneja and Guerin 1997).

Our knowledge about the morphology and physiology of BS is more limited. As for GPs, data were collected mainly from $5^{\text {th }}$ instar nymphs of T. infestans. BS are non-articulated, single (thin)-walled wall-pore sensilla, about $26 \mu \mathrm{m}$ long, with a diameter of about $2 \mu \mathrm{m}$ at their base (Guerenstein and Guerin 2001). The numerous wall-pores are linked to pore tubules (Bernard 1974). These sensilla house a highly variable number of receptor cells, from 21 to 41, with extensive dendritic branching in the sensillar lumen (Guerenstein and Guerin 2001).

Table1. Odorants that evoke a response in grooved peg sensilla of $5^{\text {th }}$ instar T. infestans. Best stimuli are indicated whereas less effective stimuli are in parentheses. Responses consist on an increase in spike rate unless otherwise indicated. Data based on Bernard (1974), Diehl et al. (2003), Guerenstein and Guerin (2001) and Taneja and Guerin (1997).

\begin{tabular}{llll}
\hline & GP1 & GP2 & GP3 \\
$\begin{array}{l}\text { ORC1 } \\
\text { (high amplitude spike) }\end{array}$ & $\begin{array}{l}\text { ammonia (methyl-, } \\
\text { dimethyl-, ethyl-amine) }\end{array}$ & $\begin{array}{l}\text { isobutyric acid (butyric, } \\
\text { isovaleric, 2-methylbutyric } \\
\text { acids) }\end{array}$ & C $_{4-5}$ aliphatic amines $^{\text {b }}$ \\
\hline $\begin{array}{l}\text { ORC2 } \\
\text { (intermediate amplitude spike) }\end{array}$ & $-^{\text {a }}$ & $\begin{array}{l}\text { ammonia (methyl-, } \\
\text { dimethyl-, Ethyl-amine) }\end{array}$ & $-^{\text {a }}$ \\
\hline $\begin{array}{l}\text { ORC3 } \\
\text { (low amplitude spike) }\end{array}$ & $\begin{array}{l}\text { isobutylamine } \\
\text { (isopentylamine) }\end{array}$ & $\begin{array}{l}\text { isobutylamine } \\
\text { (2-butylamine, butylamine, } \\
\text { 3-methyl 2-butylamine, } \\
\text { isopentylamine) }\end{array}$ & $\begin{array}{l}\text { isobutylamine } \\
\text { (2-butylamine, } \\
\text { butylamine, 3-methyl } \\
\text { 2-butylamine, } \\
\text { isopentylamine) }\end{array}$ \\
\hline
\end{tabular}

a No odorant tested up to date evoked a response.

${ }^{b}$ Response consists on a decrease in spike frequency. 
Experiments with GC-SSR using sheep odour indicated that BS posses an ORC highly sensitive to aliphatic aldehydes, responding maximally to heptanal, octanal and nonanal (Guerenstein and Guerin 2001). Such an ORC is also present in BS of $1^{\text {st }}$ and $5^{\text {th }}$ instar nymphs and adults of $T$. infestans and in $5^{\text {th }}$ instar nymphs and adults of R. prolixus and Dipetalogaster maxima (Uhler) (Guerenstein 1999). In addition, screenings of odorants in BS ORCs of $1^{\text {st }}$ and $5^{\text {th }}$ instar nymphs of T. infestans indicated cells selectively sensitive to terpenes (Guerenstein 1999). One of these ORCs responds maximally to $\mathrm{a}(+)$ pinene whereas another responds to both $\mathrm{a}(+)$ pinene and $(-)$ limonene. Finally, an ORC that responds to terpinen-4-ol and a(+) terpineol is also present. Sensilla basiconica of adult $T$. infestans possess an ORC sensitive to the heterocyclic-aromatics pyridine and furan (Mayer 1968).

As in the case of GPs, most odorants that evoke responses in ORCs of BS are of vertebrate origin. Thus, $C_{7}-C_{9}$ saturated aliphatic aldehydes occur in different vertebrate odours including chicken feather odour (Guerenstein and Guerin 2001), and human effluvia (e.g. Preti et al. 1977). In addition, $\mathrm{a}(+)$ pinene, $(-)$ limonene, and $\mathrm{a}(+)$ terpineol have been identified, for example, in chicken feather and human urine odour (see Guerenstein 1999). On the other hand, terpinen-4-ol appears not to be emitted by vertebrates nor by triatomines, and is known as a constituent of the male sex pheromone of some heteropterans (Aldrich 1995).

It should be noted that in adults, the spatial pattern of chemosensory sensilla in the antennal pedicelus shows differences between species or even populations of the same species. Thus, for example, in the tribe Triatomini, the density and number of types of chemosensory sensilla in this antennal segment is positively correlated with the habitat range of the species (Carbajal de la Fuente and Catalá 2002, Catalá 1997).

\section{Olfactory behaviour: host-seeking}

Olfactory cues play a major role in host seeking in triatomines although they are not the only relevant sensory cues. The heat emitted by the host body constitutes a main orienting cue for triatomines, whose thermal sense and their use of thermal cues have been the object of diverse studies (e.g. Ferreira et al. 2007, Flores and Lazzari 1996, Guerenstein and Lazzari 2009, Lazzari and Núñez 1989a,b, Wigglesworth and Gillett 1934). The behavioural sensitivity of triatomines to heat is extremely high and they remain the only group of blood-sucking insects where the ability to perceive the infrared radiation emitted by the host body has been demonstrated (Lazzari and Núñez 1989a, Schmitz et al. 2000).

As mentioned in the introduction, triatomines are nocturnal and stay inactive, hidden in their refuges, during daytime. Shortly after dusk the bugs become active. Around that time their hosts are asleep, emitting volatile odorant blends released by their skin and breath that are dispersed by air currents. Some of these chemicals activate nearby bugs, which increase their locomotion activity (e.g. Núñez 1982), thus, increasing the probability of encountering other host-associated cues after leaving their refuge. The bimodal stimulation of antennal receptors by mechano(air movement) and chemical (odours) stimuli triggers long-range, positive odour-modulated anemotaxis, which here we also refer to as upwind orientation or attraction (e.g. Barrozo and Lazzari 2004a, Taneja and Guerin 1995, see also Chapter 6 in this volume). Provided that airstreams pass the host's body before reaching the bugs, they carry the host odours. Then, just walking upwind (positive anemotaxis) represents a simple solution to reach the host. As the bug approaches the host, cues of additional modalities (heat, moisture) are detected and integrated at the CNS level, assigning an appetitive value to the complex, multimodal signal. 
The behavioural response of triatomines to a number of odorants that are constituents of natural odour blends, has been recorded using different experimental devices including an olfactometer (e.g. Núñez 1982) and servospheres (e.g. Barrozo and Lazzari 2004a, Taneja and Guerin 1995). A servosphere (also called locomotion compensator) is an experimental device in which a walking insect is constantly kept at the apex of a sphere. The insect's displacements are indirectly tracked by sensors that detect the displacements of the sphere, which moves as a result of the walking activity of the insect. Thus, parameters related to activation, such as walking speed and distance walked, can be measured. In addition, attraction can be assessed by recording the walking angles with respect to an airstream carrying the odour stimulus, and the percent displacement in the upwind direction. In a servosphere the insect cannot change its position with respect to the stimulus (i.e. it is an 'open loop' device). Two models of servosphere were used in triatomine research, the 'Kramer sphere' (Kramer 1976), and the 'Dahmen sphere' (Dahmen 1980, modified by Lazzari). The two models differ technically but measure virtually the same parameters. In the case of triatomines, two different odour delivery systems were used in servosphere experiments. Thus, one (e.g. Taneja and Guerin 1995) or two (e.g. Barrozo and Lazzari 2004a) airstreams reached the walking insect. In the former case (used with the Kramer sphere), the control consisted of the response of the bugs towards clean air delivered before and after odour delivery. In the latter (used with the Dahmen sphere), one of the streams carried the test stimulus whereas the other (at $180^{\circ}$ from the first) represented an odourless, simultaneous control.

Different odorants evoke activation and/or attraction on triatomines even when presented singly. One such odorant is carbon dioxide $\left(\mathrm{CO}_{2}\right.$ (Guerenstein and Hildebrand 2008). It is not completely understood if $\mathrm{CO}_{2}$ activates triatomines per se because in different studies, using the different experimental set-ups mentioned above, $\mathrm{CO}_{2}$ stimuli evoked little (Guerenstein and Guerin 2001, Taneja and Guerin 1995) or no (Barrozo and Lazzari 2004a, Núñez 1982) activation of the bugs. In any case, a strong activating effect has never been reported. On the contrary, a clear-cut oriented response of the bugs (nymphs and adults) towards a $\mathrm{CO}_{2}$ source has been shown (Barrozo and Lazzari 2004a, Guerenstein and Guerin 2001, Núñez 1982, Taneja and Guerin 1995, see also Trapping of triatomines using host-odour cues). Thus, the mean walking angle was statistically not different from the direction of the $\mathrm{CO}_{2}$ stimulus. On the Dahmen servosphere the response threshold falls between 300 and 400 ppm above ambient $\mathrm{CO}_{2}$ background (see also below for more information on threshold), whereas the response increases with concentration up to 2,300 $\mathrm{ppm}$, the highest level tested on that device. For these tests the $\mathrm{CO}_{2}$ level in the control airstream was ambient $\mathrm{CO}_{2}$ (Barrozo and Lazzari 2004a).

Nymphs of $T$. infestans are able to orientate (walk) towards a $\mathrm{CO}_{2}$ source under continuous stimulation (Barrozo and Lazzari 2006). This is in contrast to $\mathrm{CO}_{2}$-tracking in other, flying, haematophagous insects like mosquitoes, which are able to follow a $\mathrm{CO}_{2}$ plume only if this odorant is presented in an intermittent (pulsed) manner (e.g. Geier et al. 1999). Thus, mosquitoes make use of the high-frequency intermittent pattern of $\mathrm{CO}_{2}$ presentation within a natural host odour plume to orientate towards a $\mathrm{CO}_{2}$ source. In triatomines, however, pulsed stimulation of $\mathrm{CO}_{2}$ evokes repellency when delivered at a frequency of $1 \mathrm{~Hz}$ (Barrozo and Lazzari 2006). The reason why $T$. infestans bugs evolved to orientate within a continuous plume may be related to the fact that in their usually domestic habitat, $\mathrm{CO}_{2}$ may disperse (and be encountered by walking bugs) in a more homogeneous way (Lehane 2005). To attempt to clarify this, it would be interesting to test the orientation towards $\mathrm{CO}_{2}$ in the sylvatic triatomine species. 
$\mathrm{CO}_{2}$ is sufficient but not necessary (Barrozo and Lazzari 2004a,b, Guerenstein and Guerin 2001, Núñ̃ez 1982, Taneja and Guerin 1997) to evoke an attractive behavioural response in triatomines, similarly to some mosquitoes (e.g. Bernier et al. 2003, Bosch et al. 2000, Gibson and Torr 1999).

Another host odorant that evokes a behavioural response per se is nonanal (see Olfactory physiology: detection of host odours). Nonanal, at $8 \mathrm{ppb}$ in ambient air, activates (increases walking speed) but does not attract triatomine nymphs on the servosphere (Guerenstein and Guerin 2001). Lower and higher doses do not evoke any response. The activating concentration of $8 \mathrm{ppb}$ is just a few ppb under the estimated response threshold of the aldehyde ORC in BS (Guerenstein and Guerin 2001).

In addition, ammonia (see Olfactory physiology: detection of host odours) both activates (increases walking speed) and attracts triatomines at doses of 3-17 ppb but not at a lower dose (Taneja and Guerin 1997). The behavioural threshold of $3 \mathrm{ppb}$ is just one ppb above the estimated response threshold of the ammonia ORC in GP1 (Taneja and Guerin 1997), which is similar to that of the ammonia ORC in GP2 (Diehl et al. 2003).

Other odorants, isobutyric acid and 1-octen-3-ol, both constituents of human sweat odour (Cork and Park 1996), also evoke a response, attraction, when presented singly to nymphs on a servosphere (Guerenstein and Guerin 2001, Barrozo and Lazzari 2004a). In the case of 1-octen-3-ol, five concentrations in log-steps were tested and yet attraction is observed only at an intermediate dose (Barrozo and Lazzari 2004a). Attraction to isobutyric acid is obtained at doses (0.02-10 ppb in air) that evoke low to moderate $(<40 \mathrm{~Hz})$ increases in the firing rate of the ORC excited by carboxylic acids in GP2 sensilla, whereas at a dose (100 ppb) that evokes higher firing rates $(>40 \mathrm{~Hz}$ ) the bugs are not attracted (Guerenstein and Guerin 2001). As in the case of nonanal, the behavioural threshold of $0.02 \mathrm{ppb}$ for isobutyric acid is just under the estimated response threshold of the carboxylic acid ORC in GP2 (Guerenstein and Guerin 2001). It should be mentioned that isobutyric acid has also been identified as a main constituent of the alarm pheromone of triatomines (e.g. Cruz López et al. 1995, Guerenstein and Guerin 2004, Manrique et al. 2006, Rojas et al. 2002, Schofield 1979a,b). Thus, a parsimonious use of this odorant by these insects has been proposed (Guerenstein and Guerin 2001, 2004, Guerenstein and Lazzari 2009) because it is used by triatomines as, at least, host attractant and alarm signal depending on its concentration, sensory (in particular odour blend) context and the physiological state of the perceiving bug. It should be noted that odorants tested singly are often effective only within a narrow range of concentrations (Barrozo and Lazzari 2004a, Guerenstein and Guerin 2001).

Testing odorants singly is important to assess the significance of a blend constituent per se, and as a control for any enhanced (additive, positive-synergistic) or diminished response to any odour mixture that includes that constituent. However, in nature, the insects are confronted to blends of odours consisting of several constituents presented simultaneously at particular proportions. Identifying those blends correctly should help increase the certainty that the odour source is the expected host (Lehane 2005). In fact, natural odour blends and even some synthetic mixtures developed attract triatomines more efficiently than single odorants (e.g. Barrozo and Lazzari 2004b, Taneja and Guerin 1995). A list of synthetic mixtures that evoke higher attraction than that evoked by its odorant constituents per se is provided in Table 2.

A number of other mixtures tested elicited no attraction, evoked attraction at levels not higher than those evoked by the odorants when presented singly or lacked the controls of its odorant constituents tested singly to assess if the response to the mixture was different to that to the 
Table 2. Synthetic odour mixtures that evoke higher attraction than that evoked by its odorant constituents when presented singly (i.e. additive or synergistic effects observed). Tests carried out with T. infestans nymphs (unless otherwise indicated).

\begin{tabular}{|c|c|c|c|}
\hline Mixture & Mixture constituents ${ }^{\mathrm{a}}$ & Increased attraction & References \\
\hline 1 & $\mathrm{~L}-\mathrm{LA}+\mathrm{CO}_{2}$ & yes $^{c}$ & Núñez $1987^{b}$, Barrozo and Lazzari 2004a \\
\hline 2 & $\begin{array}{l}\mathrm{L}-\mathrm{LA}+\mathrm{C}_{3} \\
\mathrm{~L}-\mathrm{LA}+\mathrm{C}_{4} \\
\mathrm{~L}-\mathrm{LA}+\mathrm{C}_{5}\end{array}$ & yes $^{c}$ & Barrozo and Lazzari 2004b \\
\hline 3 & $\mathrm{~L}-\mathrm{LA}+\mathrm{C}_{3}+\mathrm{C}_{4}+\mathrm{C}_{5}$ & yes ${ }^{c}$ & Barrozo and Lazzari 2004b \\
\hline 4 & $\mathrm{~L}-\mathrm{LA}+\mathrm{C}_{3}+\mathrm{C}_{4}+\mathrm{C}_{5}+\mathrm{CO}_{2}$ & yes ${ }^{c, d}$ & Barrozo and Lazzari 2004b \\
\hline
\end{tabular}

a L-LA: L (+) lactic acid; $C_{3-6}:$ n-aliphatic carboxylic acids.

${ }^{\mathrm{b}}$ Tested on nymphs of $R$. prolixus.

c Synergistic effect.

${ }^{d}$ Attraction similar to that evoked by mouse odour.

constituents (Barrozo and Lazzari 2004a,b, Guerenstein and Guerin 2001, Otálora-Luna et al. 2004). The mixtures that do not evoke attraction higher than that elicited by their odorant constituents when presented singly include $L(+)$ lactic acid $(L-L A)+C_{6}$ n-aliphatic carboxylic acid, $C_{3}+C_{4}+$ $\mathrm{C}_{5}$ n-aliphatic carboxylic acids, $\mathrm{C}_{3}+\mathrm{C}_{4}+\mathrm{C}_{5}$ n-aliphatic carboxylic acids $+\mathrm{CO}_{2}$, isobutyric acid + nonanal, isobutyric acid + ammonia, isobutyric acid $+\mathrm{CO}_{2}$ and 1-octen-3-ol $+\mathrm{CO}_{2}$. At present, results from mixtures that evoked no additive or synergistic response are of limited value because only one or a limited number of proportions and concentrations have been tested.

The synergistic effect between L-LA (a human skin odorant; e.g. Cork and Park 1996) and $\mathrm{CO}_{2}$ in mixture 1 of Table 2 is evinced when measuring the threshold of the oriented response towards $\mathrm{CO}_{2}$. Thus, this threshold diminishes from $300-400 \mathrm{ppm}$ when $\mathrm{CO}_{2}$ is tested singly (see above) to 75-150 ppm when presented together with L-LA, which does not evoke a response per se (Barrozo and Lazzari 2004a). On the other hand, when testing different doses of L-LA with a fixed level of $\mathrm{CO}_{2}(300 \mathrm{ppm})$, only the intermediate dose out of five tested evokes attraction (Barrozo and Lazzari 2004a). In mosquitoes, a mixture of L-LA and $\mathrm{CO}_{2}$ also acts in a synergistic way (e.g. Geier and Boeckh 1999).

Mixtures of L-LA plus $\mathrm{C}_{3}, \mathrm{C}_{4}$ or $\mathrm{C}_{5}$ n-aliphatic carboxylic acids (mixtures 2 in Table 2) also evince a synergistic effect as none of those odorants evoke attraction when presented singly, even when those carboxylic acids (present in human sweat and skin odour; Bernier et al. 2000, Cork and Park 1996) have been tested at five different doses, including the same and higher concentrations than that in the mixture (Barrozo and Lazzari 2004b). In addition, a mixture of L-LA plus $C_{3}, C_{4}$ and $C_{5}$ $\mathrm{n}$-aliphatic carboxylic acids (mixture 3 in Table 2), at a particular odorant ratio and concentration, is attractive even when that $\mathrm{C}_{3-5}$ mixture is not, again showing synergistic attraction (Barrozo and Lazzari 2004b). Moreover, when the latter mixture is combined with $\mathrm{CO}_{2}$ (mixture 4 in Table 2), an additional synergistic effect is revealed, making that mixture as attractive as a mouse, when tested on the servosphere (Barrozo and Lazzari 2004b). This is remarkable considering that the positive control (a mouse) is a source of a plethora of sensory cues (including heat, water vapour and a complete, natural, odour blend) and triatomines are able to detect many of them (Guerenstein and Lazzari 2009). 
It should be noted that all mixtures in Table 2 include L-LA but not all of them have $\mathrm{CO}_{2}$ as constituent. This emphasizes the role of L-LA in synergistic interactions between odorant constituents (Barrozo and Lazzari 2004a,b). Moreover, as L-LA is not attractive by itself, its role in triatomine host seeking seems to be limited to enhance the response to odour mixtures, a role similar to that found in Anopheles gambiae Giles mosquitoes (Smallegange et al. 2005).

Table 3 includes the full list of host odorants known to be detected by triatomines to date, according to neurophysiological and behavioural data. Most of those odorants are known to be also detected by other haematophagous insects (Guerenstein and Lazzari 2009).

Trypanosoma cruzi, the parasite that triatomines transmit, is considered subpathogenic for the vectors. Thus, the parasite affects the capacity to resist starvation but neither the developmental nor the mortality rate (Vallejo et al. 2009). However, changes in the behaviour of infected bugs also occur. Thus, it has been suggested that bugs infected with $T$. cruzi detect a host more rapidly than uninfected insects (Botto-Mahan et al. 2006). To date, it is unclear if the olfactory system is involved in this behavioural change. Other changes reported as induced by parasite infection include a higher biting rate (Botto-Mahan et al. 2006).

Table 3. Host odorants known to be detected by triatomines. Both behavioural and neurophysiological data were considered to develop this list.

\begin{tabular}{|c|c|}
\hline Odorant & Reference \\
\hline $\mathrm{CO}_{2}$ & $\begin{array}{l}\text { Guerenstein and Hildebrand 2008, Guerenstein and } \\
\text { Lazzari } 2009 \text { (reviews) }\end{array}$ \\
\hline $\mathrm{C}_{7-9} \mathrm{n}$-aliphatic aldehydes & Guerenstein and Guerin 2001 \\
\hline 1-octen-3-ol & Barrozo and Lazzari 2004a \\
\hline $\mathrm{L}(+)$ lactic acid & Núñez 1987, Barrozo and Lazzari 2004a \\
\hline $\begin{array}{l}\text { isobutyric acid and } \mathrm{C}_{3-5} \text { n-carboxylic acids (and } \mathrm{C}_{6} \\
\text { n-carboxylic acid) }\end{array}$ & $\begin{array}{l}\text { Guerenstein and Guerin 2001, Diehl et al. 2003, Barrozo } \\
\text { 2003, Barrozo and Lazzari 2004b }\end{array}$ \\
\hline ammonia, (ethylamine, dimethylamine) $)^{1}$ & Taneja and Guerin 1997, Diehl et al. 2003 \\
\hline$C_{4-5}$ aliphatic amines as isobutylamine ${ }^{1}$ & Diehl et al. 2003 \\
\hline terpenes as $a(+)$ pinene and terpinen- $4-\left.0\right|^{1}$ & Guerenstein 1999 \\
\hline pyridine, furan 1 & Mayer 1968 \\
\hline amyl acetate ${ }^{1}$ & Bernard 1974 \\
\hline
\end{tabular}

${ }^{1}$ No behavioural response known (only neurophysiological evidence).

\section{The modulation of olfactory responses}

The behaviour of an animal, for instance its response to environmental cues, does not only depend on exogenous factors, such as the presence of a stimulus, but it is also affected by endogenous physiological factors. Haematophagous insects are not an exception, and such dependence on the physiological state has been analysed in detail in mosquitoes, where feeding and reproduction alternate, giving place to a gonotrophic cycle (Klowden 1981, 1990, 1995, Klowden and Briegel 1994, Takken et al. 2001). 
In the case of triatomines, the physiological state-dependency of their response to host-associated cues has been analysed recently (Bodin et al. 2008, 2009a,b, Barrozo and Lazzari 2004a). The responses to chemical and thermal cues of insects of different physiological state have been characterised as a function of the time of the day, the moulting cycle, and the nutritional and reproductive states (Bodin et al. 2008, 2009a,b).

Triatomine insects exhibit a marked circadian organisation of many activities (e.g. locomotor activity, egg hatching, ecdysis, oviposition, thermopreference, visual sensitivity (Barrozo et al. 2004b)). These different physiological and behavioural processes take place at either one or two temporal windows, one at the beginning and the other at the end of the scotophase. Particularly, they exhibit a maximal motivation to feed at dusk (Lorenzo and Lazzari 1998), and during this time they leave their shelters and seek for a blood meal guided by $\mathrm{CO}_{2}$ and other olfactory cues relevant for host searching (see Olfactory behaviour: host-seeking). At dawn, the bugs return to their shelters using the aggregation pheromone present in their excrements as chemical landmarks to guide them back to refuges (Lorenzo Figueiras et al. 1994). For this, they continuously deposit faecal drops at the entrance of their shelters (Lorenzo and Lazzari 1996). As a consequence, they must discriminate and react to distinct olfactory stimuli always present in their habitat, at the right moment.

It has been shown that some insect species, such as fruit flies and cockroaches, vary their olfactory sensitivity along the day, under the influence of a circadian clock, which modifies the sensitivity of the antenna to chemical stimuli. In those species, the sensitivity of all ORCs is modulated simultaneously, making the insects more or less sensitive to all odours at the same time (Krishnan et al. 1999, Page and Koelling 2003). This is not the case of modulation of olfactory sensitivity in triatomines. Recent work in two species of triatomines, T. infestans and R. prolixus, has revealed that the responsiveness to $\mathrm{CO}_{2}$ is limited to a particular temporal window corresponding to the early night, the moment when they leave their refuges to search for a host (Barrozo and Lazzari 2004a, Barrozo et al. 2004a, Bodin et al. 2008). Furthermore, their responsiveness to aggregation pheromones is also limited to a particular moment of the day, but in this case, the end of the night, when they use them as chemical landmarks for returning to their refuges (Bodin et al. 2008). Interestingly enough, the mechanism controlling both responses (to $\mathrm{CO}_{2}$ and to aggregation pheromones) are not the same. Whereas the behavioural responsiveness to $\mathrm{CO}_{2}$ is submitted to the control of a circadian clock, responsiveness to aggregation pheromone is not, and it follows exogenous cues, such as the light-dark cycle (Bodin et al. 2008).

The particularities of the circadian organisation of activities in triatomines can be summarised as follows. First, the temporal modulation of their chemical sensitivity is not general, but their responsiveness to each odour is specifically modulated. Second, this control is executed by a combination of endogenous and exogenous mechanisms acting together. Finally, the sensitivity to each odour is maximal at the moment when that odour becomes biologically relevant and thus, when the insect displays a particular related behaviour. Thus, the sensitivity is specifically modulated according to the behavioural (temporal) context, each odour is associated with.

Concerning the moulting cycle, and the nutritional and reproductive states, triatomines offer the possibility of studying their effect independently. Because of their haemimetabolous development and obligate haematophagy of nymphs and adults of both sexes, those factors can be analysed avoiding any interference from the others. This is not the case of mosquitoes, were only adult females feed on blood and nutrition and reproduction are closely related. 
First studies on the effect of the nutritional state suggest no effect of the starvation time on the responsiveness of $T$. infestans nymphs to host-related cues (Barrozo and Lazzari 2004a), but this was recently shown not to be always the case. Bodin et al. $(2009 a, b)$ characterised the influence of all three factors, nutrition, reproduction and moulting on the response of $R$. prolixus to hostassociated odours and heat, as well as the motivation to feed on blood, in nymphs, males and females. Thus, insects recently fed are not attracted by host odours, and the proximity of the ecdysis, both before and after, reduces the responsiveness of the insects to host signals, as well as their motivation to feed. The same depression in feeding behaviour was observed in females a few days after feeding, when they are ready for egg-laying (Bodin et al. 2009b). These results emphasise the necessity of precisely controlling the physiological state and the temporal synchronisation of experimental animals in the study of the behavioural responses of triatomine bugs, as well as to consider the temporal context (daytime) associated to the response and cues under analysis (Lazzari et al. 2004, Takken 2005).

\section{Trapping of triatomines using host odour cues}

When seeking a host, many triatomines let themselves fall from ceilings upon detection of host odours from below. In fact, one of the most popular common names for triatomines in Spanish, 'vinchuca', is a word derived from Quechua, and meaning 'bug that falls'. Taking advantage of this behaviour, an olfactometer was designed to study the attractiveness of different odour stimuli and, furthermore, to test the ability of those odours to capture bugs (Guerenstein et al. 1995).

This dual-choice 'trapping olfactometer' consists on a closed rectangular arena with three plastic tubes connected to it from below. In one side of the arena, one of the tubes (the 'release tube') contains the bugs at the beginning of the experiment. Bugs could reach the arena surface by climbing onto a piece of cardboard inside this tube. On the opposite side of the arena, two 'capture-tubes' (control and test) are placed. The insects can drop into the capture-tubes but cannot escape from them. All tubes have cloth mesh at the bottom whereas below the capturetubes an odour source and its control are placed. Air is pulled out from the release tube and, therefore, an airstream from the odour source (and its control) constantly reaches the release tube via the capture-tubes and the arena (Guerenstein et al. 1995). This device has already been useful not only to provide proof of concept for the experimental design of the olfactometers but also to test the odour emission from a culture of yeast (Saccharomyces cerevisiae Meynen ex Hansen, cultured with water and sugar) as activator and attractant for triatomines (Guerenstein et al. 1995).

Interestingly, triatomines are significantly activated by yeast odours, that is, more bugs fall into the capture tubes when yeast odour is present below the test tube (a test experiment) than in control experiments in which both capture tubes are 'controls' (water with sugar). Moreover, the odour from the yeast culture also attracts triatomines in test experiments, as most of the bugs captured fell in the test (yeast) tube while very few were found in the control tube. Under these experimental conditions, the levels of activation and attraction evoked by yeast do not differ significantly from those evoked by a living mouse (Guerenstein et al. 1995). Carbon dioxide is likely the main responsible for the responses to yeast because its removal using potassium hydroxide resulted in a loss of response by the bugs (Guerenstein et al. 1995). The high levels of activated $(68-85 \%)$ and attracted (76-87\%) bugs throughout the experiments indicate that the rationale behind the design of the trapping olfactometer is compelling.

Inspired by the trapping olfactometer, a baited trap for triatomines has been designed (Guerenstein et al. 1995; Figure 2). The trap consists of a circular ramp that surrounds a plastic container. At the 


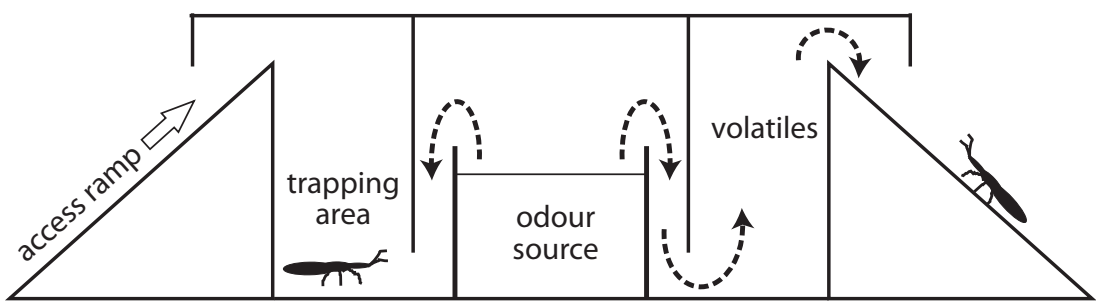

$2 \mathrm{~cm}$

Figure 2. Trap for triatomines. A source of volatile odorants (e.g. a culture of baker yeast, Guerenstein et al. 1995) is placed inside the trap. When bugs climb the circular ramp, they perceive the odours from below. This induces the bugs to jump inside the trapping area, from where they cannot escape.

centre of the container, a vial with an odour source is placed. The odour is forced to descend to the floor of the trap where it reaches a series of exit slits in the rim of an inverted cup. The odour then diffuses up to the dark lid of the trap device. When host seeking bugs reach the upper edge of the ramp they detect the odour as coming from below and drop into the plastic container from which they cannot escape. For laboratory assays, a test and a control trap are placed in opposite sides of an experimental arena; bugs are released in the evening and collected the following morning. Experiments with such a trap confirmed that a yeast culture activates (52-66\%) and attracts (86$89 \%)$ of the bugs. Moreover, the results showed that a yeast culture constitutes an suitable odour bait, and that the trap is efficient in laboratory tests (Guerenstein et al. 1995).

The trap developed is effective for different, relevant, species of triatomines. Thus, in the laboratory, the yeast-baited trap can be used to catch T. infestans (e.g. Guerenstein et al. 1995), T. dimidiata, T. pallidipennis (Stål) (Pimenta et al. 2007), T. sordida (Stål), Panstrongylus megistus (Burmeister) (Pires et al. 2000) and R. prolixus (Lorenzo et al. 1999). However, some species of triatomines (T. brasiliensis (Neiva 1911) and T. pseudomaculata (Correa and Espínola 1964)) do not fall into the traps (Pires et al. 2000), possibly because of particularities of their host-seeking behaviour.

The triatomine trap is also capable to catch bugs under semi-natural and field conditions. Thus, the yeast-baited trap is able to catch significant numbers of bugs in chicken-coops located in open fields exposed to natural climatic conditions at an endemic area for Chagas Disease (Lorenzo et al. 1998). It should be mentioned, however, that no host was present during those tests and, therefore, the traps did not have to compete with the multimodal cues from such a host, including its natural odour blend. In the field, a trap model using the same principle as the one described above, caught wild, sylvatic, triatomines when a source of pure $\mathrm{CO}_{2}$ (dry ice) was used as bait (Botto-Mahan et al. 2002).

In large-scale field tests, a trap that uses a live bait (a mouse) successfully caught triatomines (e.g. Noireau et al. 2002). However, to date, no trap baited with synthetic odorants has been tested in such field tests. Further understanding of the olfactory behaviour of triatomines should lead to the development of practical, cheap and efficient baited trap devices, able to monitor the bugs in the field and thus help implement a rational control strategy that includes selective intervention procedures (Schofield and Kabayo 2008, Schofield et al. 2006, Tarleton et al. 2007). 


\section{Acknowledgments}

The National Research Council (CONICET) and National University of Entre Rios, Argentina, and ANR, CNRS and University of Tours, France, are acknowledged for contributing financial support.

\section{References:}

Aldrich J (1995) Chemical communication in the true bugs and parasitoid exploitation. In: Cardé RT and Bell WJ (eds) Chemical ecology of insects 2. Chapman and Hall, New York, USA. pp 318-363.

Almeida C, Costa Vinhaes M, Silveira A, Silveira AC and Costa J (2000) Monitoring the domiciliary and peridomiciliary invasion process of Triatoma rubrovaria in the state of Rio Grande do Sul, Brazil. Mem Inst O Cruz 95: 761-768.

Altner H, Sass H and Altner I (1977) Relationship between structure and function of antennal chemo-, hygro-, and thermoreceptive sensilla in Periplaneta americana. Cell Tissue Res 176: 389-405.

Barrozo RB (2003) Orientación al hospedador en la vinchuca Triatoma infestans (Heteroptera: Reduviidae): Claves sensoriales responsables. PhD Thesis, University of Buenos Aires, Argentina.

Barrozo RB and Lazzari C (2004a) The response of the blood-sucking bug Triatoma infestans to carbon dioxide and other host odours. Chem Senses 29:319-329.

Barrozo RB and Lazzari CR (2004b) Orientation behaviour of the blood-sucking bug Triatoma infestans to short-chain fatty acids: synergistic effect of L-lactic acid and carbon dioxide. Chem Senses 29: 833-841.

Barrozo RB and Lazzari CR (2006) Orientation response of haematophagous bugs to $\mathrm{CO}_{2}$ : the effect of the temporal structure of the stimulus. J Comp Physiol A 192: 827-831.

Barrozo RB, Minoli SA and Lazzari CR (2004a) Circadian rhythm of behavioural responsiveness to carbon dioxide in the blood-sucking bug Triatoma infestans (Heteroptera: Reduviidae). J Insect Physiol 50: 249-254.

Barrozo RB, Schilman PE, Minoli SA and Lazzari CR (2004b) Daily rhythms in disease-vector insects. Biol Rhythm Res 35: 79-92.

Barth R (1952) Estudos anatômicos e histológicos sôbre a subfamília Triatominae (Hemiptera-Reduviidae). II parte: Um novo orgao sensíbel das Triatominae. Bol Inst Oswaldo Cruz 5: 1-4.

Barth R (1953) Estudos anatômicos e histolôgicos sôbre a subfamília Triatominae (Hemiptera-Reduviidae). III parte: Pesquisas sôbre o mecanismo de picada das Triatominae. Mem Inst O Cruz 51: 11-33.

Bernard J (1974) Etude électrophysiologique de récepteurs impliqués dans l'orientation vers l'hôte et dans l'acte hématophage chez un Hémiptère: Triatoma infestans. PhD Thesis, University of Rennes, Rennes, France.

Bernier UR, Kline DL, Barnard DR, Schreck CE and Yost RA (2000) Analysis of human skin emanations by gas chromatography/mass spectrometry. 2. Identification of volatile compounds that are candidate attractants for the yellow fever mosquito (Aedes aegypti). Anal Chem 72: 747-756.

Bernier UR, Kline DL, Posey KH, Booth MM, Yost RA and Barnard DR (2003) Synergistic attraction of Aedes aegypti (L.) to binary blends of L-lactic acid and acetone, dichloromethane, or dimethyl disulfide. J Med Entomol 40: 653-656.

Bodin A, Barrozo RB, Couton L and Lazzari CR (2008) Temporal modulation and adaptive control of the behavioural response to odours in Rhodnius prolixus. J Insect Physiol 54: 1343-1348.

Bodin A, Vinauger C and Lazzari CR (2009a) State-dependency of host seeking in Rhodnius prolixus: The post-ecdysis time. J Insect Physiol 55: 574-579.

Bodin A, Vinauger C and Lazzari CR (2009b) State-dependency of host seeking in blood-sucking insects: Behaviour and physiology. J Exp Biol 212: 2386-2393.

Bosch O, Geier M and Boeckh J (2000) Contribution of fatty acids to olfactory host finding of female Aedes aegypti. Chem Senses 25: 323-330.

Botto-Mahan C, Cattan P and Canals M (2002) Field tests of carbon dioxide and conspecifics as baits for Mepraia spinolai, wild vector of Chagas disease. Acta Trop 82: 377-380.

Botto-Mahan C, Cattan P and Medel R (2006) Chagas disease parasite induces behavioural changes in the kissing bug Mepraia spinolai. Acta Trop 98: 219-223. 
Carbajal de la Fuente A and Catalá S (2002) Relationship between antennal sensilla pattern and habitat in six species of Triatominae. Mem Inst O Cruz 97: 1121-1125.

Catalá S (1994) The cave organ of Triatominae (Hemiptera, Reduviidae) under scanning electron-microscopy. Mem Inst O Cruz 89: 275-277.

Catalá S (1997) Antennal sensilla of Triatominae (Hemiptera, Reduviidae): A comparative study of five genera. Int J Insect Morphol Embryol 26: 67-73.

Catalá S and Schofield C (1994) Antennal sensilla of Rhodnius. J Morphol 219: 193-203.

Cork A and Park K (1996) Identification of electrophysiologically-active compounds for the malaria mosquito, Anopheles gambiae, in human sweat extracts. Med Vet Entomol 10:269-276.

Cruz López L, Morgan E and Ondarza R (1995) Brindley's gland exocrine products of Triatoma infestans. Med Vet Entomol 9: 403-406.

Dahmen H (1980) A simple apparatus to investigate the orientation of walking insects. Experientia 36: 685-687.

Diehl PA, Vlimant M, Guerenstein P and Guerin PM (2003) Ultrastructure and receptor cell responses of the antennal grooved peg sensilla of Triatoma infestans (Hemiptera: Reduviidae). Arthropod Struct Dev 31: 271-285.

Ferreira RA, Lazzari CR, Lorenzo MG and Pereira MH (2007) Do haematophagous bugs assess skin surface temperature to detect blood vessels? PLoS One 2: e932. doi: 10.1371/ journal.pone.0000932.

Flores GB and Lazzari CR (1996) The role of the antennae in Triatoma infestans: orientation towards thermal sources. J Insect Physiol 42: 433-440.

Geier M and Boeckh J (1999) A new Y-tube olfactometer for mosquitoes to measure the attractiveness of host odours. Entomol Exp Appl 92: 9-19.

Geier M, Bosch O and Boeckh J (1999) Influence of odour plume structure on upwind flight of mosquitoes towards hosts. J Exp Biol 202: 1639-1648.

Gibson G and Torr S (1999) Visual and olfactory responses of haematophagous Diptera to host stimuli. Med Vet Entomol 13:2-23.

Guerenstein PG (1999) Sensory and behavioural responses of Triatoma infestans to host and conspecific odours. PhD Thesis, University of Neuchâtel, Neuchâtel, Switzerland.

Guerenstein PG and Guerin P (2001) Olfactory and behavioural responses of the blood-sucking bug Triatoma infestans to odours of vertebrate hosts. J Exp Biol 204: 585-597.

Guerenstein PG and Guerin P (2004) A comparison of volatiles emitted by adults of three triatomine species. Entomol Exp Appl 111: 151-155.

Guerenstein PG and Hildebrand J (2008) Roles and effects of environmental carbon dioxide in insect life. Ann Rev Entomol 53: 161-178.

Guerenstein PG and Lazzari CR (2009) Host-seeking: how triatomines acquire and make use of information to find blood. Acta Trop 110: 148-158.

Guerenstein PG, Lorenzo MG, Núñez JA and Lazzari CR (1995) Bakers-yeast, an attractant for baiting traps for Chagasdisease vectors. Experientia 51: 834-837.

Insausti TC, Lazzari CR and Campanucci VA (1999) Neurobiology of behaviour. A: Morphology of the nervous system and sense organs. In: Carcavallo RU, Girón Galíndez I, Jurberg J and Lent H (eds) Atlas of Chagas' disease vectors in America, Vol 3, Editora Fiocruz, Rio de Janeiro, Brazil, pp. 1017-1051.

Justo S and Tramezzani J (1977) Estructuras cuticulares de la cabeza del Triatoma infestans (Klug, 1834). Physis Sección C 37: 343-367.

Klowden M (1981) Initiation and termination of host-seeking inhibition in Aedes aegypti during oocyte maturation. J Insect Physiol 27: 799-803.

Klowden M (1990) The endogenous regulation of mosquito reproductive behavior. Experientia 46: 660-670.

Klowden M (1995) Blood, sex, and the mosquito, the mechanisms that control mosquito blood-feeding behavior. Bioscience 45: 326-331.

Klowden M and Briegel H (1994) Mosquito gonotrophic cycle and multiple feeding potential: contrasts between Anopheles and Aedes (Diptera: Culicidea). J Med Entomol 31: 618-622. 
Kramer E (1976) The orientation of walking honeybees in odour fields with small concentration gradients. Physiol Entomol 1:27-37.

Krishnan B, Dryer S and Hardin P (1999) Circadian rhythms in olfactory responses of Drosophila melanogaster. Nature 400: 375-378.

Lazzari CR (1992) Circadian organization of locomotion activity in the haematophagous bug Triatoma infestans. J Insect Physiol 38: 895-903.

Lazzari CR and Núñez JA (1989a) The response to radiant heat and the estimation of the temperature of distant sources in Triatoma infestans. J Insect Physiol 35: 525-529.

Lazzari CR and Núñez JA (1989b) Blood temperature and feeding behavior in Triatoma infestans (Heteroptera: Reduviidae). Entomol Gener 14: 183-188.

Lazzari CR and Wicklein M (1994) The cave-like sense organ in the antennae of blood-sucking bugs. Mem Inst O Cruz 89: 643-648.

Lazzari CR, Minoli SA and Barrozo RB (2004) Chemical ecology of insect vectors: The neglected temporal dimension. Trends Parasitol 20: 506-507.

Lehane M (2005) The biology of blood-sucking in insects. Cambridge University Press, New York, USA.

Lent $\mathrm{H}$ and Wygodzinsky P (1979) Revision of the Triatominae (Hemiptera, Reduviidae), and their significance as vectors of Chagas disease. Bull Am Mus Nat His 163: 123-520.

Lorenzo MG and Lazzari CR (1996) The spatial pattern of defecation in Triatoma infestans and the role of faeces as a chemical mark of the refuge. J Insect Physiol 42: 903-907.

Lorenzo MG and Lazzari CR (1998) Activity pattern in relation to refuge exploitation and feeding in Triatoma infestans (Hemiptera: Reduviidae). Acta Trop 70: 163-170.

Lorenzo MG, Reisenman CE and Lazzari CR (1998) Triatoma infestans can be captured under natural climatic conditions using yeast-baited traps. Acta Trop. 70: 277-284.

Lorenzo MG, Manrique G, Pires HH, de Brito Sánchez MG, Diotaiuti L and Lazzari CR (1999) Yeast culture volatiles as attractants for Rhodnius prolixus: electroantennogram responses and captures in yeast-baited traps. Acta Trop 72: 119-124.

Lorenzo Figueiras AN and Lazzari CR (1998) Aggregation in the haematophagous bug Triatoma infestans: a novel assembling factor. Physiol Entomol 23: 33-37.

Lorenzo Figueras AN, Kenigsten A and Lazzari CR (1994) Aggregation in the haematophagous bug Triatoma infestans: Chemical signals and temporal pattern. J Insect Physiol 40:311-316.

Manrique G, Vitta AC, Ferreira RA, Zani CL, Unelius CR, Lazzari CR, Diotaiuti L and Lorenzo MG (2006) Chemical communication in Chagas disease vectors. Source, identity, and potential function of volatiles released by the metasternal and Brindley's glands of Triatoma infestans adults. J Chem Ecol 32: 2035-2052.

Mayer M (1968) Response of single olfactory cell of Triatoma infestans to human breath. Nature 220: 924-925.

Mclver S and Siemicki R (1984) Fine structure of antennal mechanosensilla of adult Rhodnius prolixus Stäl (Hemiptera: Reduviidae). J Morphol 180: 19-28.

Mclver S and Siemicki R (1985) Fine structure of antennal putative thermo-/hygrosensilla of adult Rhodnius prolixus Stal (Hemiptera: Reduviidae). J Morphol 183: 15-23.

Noireau F, Abad-Franch F, Valente SAS, Dias-Lima A, Lopes CM, Cunha V, Valente VC, Palomeque FS, Carvalho-Pinto CJ, Sherlock I, Aguilar M, Steindel M, Grisard EC and Jurberg J (2002) Trapping Triatominae in sylvatic habitats. Mem Inst O Cruz 97: 61-63.

Núñez JA (1982) Food source orientation and activity in Rhodnius prolixus Stal (Hemiptera, Reduviidae). Bull Entomol Res 72: 253-262.

Núñez JA (1987) Behaviour of Triatominae bugs. In: Brenner R and Stoka A (eds) Chagas' disease vectors, Vol II, CRC Press, Boca Raton, USA, pp 1-28.

Otálora-Luna F, Perret J and Guerin P (2004) Appetence behaviours of the triatomine bug Rhodnius prolixus on a servosphere in response to the host metabolites carbon dioxide and ammonia. J Comp Physiol A 190: 847-854.

Page T and Koelling E (2003) Circadian rhythm in olfactory response in the antennae controlled by the optic lobe in the cockroach. J Insect Physiol 49: 697-707. 
Pimenta F, Diotaiuti L, Lustosa Lima A and Lorenzo MG (2007) Evaluation of cultures of Saccharomyces cerevisae as baits for Triatoma dimidiata and Triatoma pallidipennis. Mem Inst O Cruz 102: 229-231.

Pires HH, Lazzari CR, Diotaiuti L and Lorenzo MG (2000) Performance of yeast-baited traps with Triatoma sordida, Triatoma brasiliensis, Triatoma pseudomaculata and Panstrongylus megistus in laboratory assays. Pan Am J Public Health 7: 384-388.

Preti G, Smith A and Beauchamp G (1977) Chemical and behavioral complexity in mammalian chemical communication systems: guinea pigs (Cavia porcellus), marmosets (Saguinus fuscicollis) and humans (Homo sapiens). In: MullerSchwarze D and Mozell M (eds) Chemical signals in vertebrates. Plenum Press, New York, USA, pp 95-114.

Rojas JC, Rios-Candelaria E, Cruz-López L, Santiesteban A, Bond-Compean JG, Brindis Y and Malo EA (2002) A reinvestigation of Brindley's gland exocrine compounds of Rhodnius prolixus (Hemiptera: Reduviidae). J Med Entomol 39: 256-265.

Schmitz H, Trenner S, Hofmann MH and Bleckmann H (2000) The ability of Rhodnius prolixus (Hemiptera; Reduviidae) to approach a thermal source solely by its infrared radiation. J Insect Physiol 46: 745-751.

Schofield C (1979a). Behavior of Triatominae (Hemiptera, Reduviidae) - review. Bull Ent Res 69: 363-379.

Schofield C (1979b) Demonstration of isobutyric acid in some Triatomine bugs. Acta Trop 36: 103-105.

Schofield C and Patterson J (1977) Assembly pheromone of Triatoma infestans and Rhodnius prolixus nymphs (Hemiptera: Reduviidae). J Med Entomol 13: 727-734.

Schofield C and Kabayo J (2008) Trypanosomiasis vector control in Africa and Latin America. Paras. Vectors 1: 24. doi: 10.1186/1756-3305-1-24.

Schofield C, Jannin J and Salvatella R (2006) The future of Chagas disease control. Trends Parasitol 22: 583-588.

Smallegange RC, Qiu YT, Van Loon JJ and Takken W (2005) Synergism between ammonia, lactic acid and carboxylic acids as kairomones in the host-seeking behaviour of the malaria mosquito Anopheles gambiae sensu stricto (Diptera: Culicidae). Chem Senses 30: 145-152.

Takken W (2005) Chemical ecology of insect vectors: temporal, environmental and physiological aspects. Trends Parasitol 21:57.

Takken W, Van Loon J and Adam W (2001) Inhibition of host-seeking response and olfactory responsiveness in Anopheles gambiae following blood feeding. J Insect Physiol 47: 303-310.

Taneja J and Guerin P (1995) Oriented responses of the triatomine bugs Rhodnius prolixus and Triatoma infestans to vertebrate odors on a servosphere. J Comp Physiol A 176: 455-464.

Taneja J and Guerin P (1997) Ammonia attracts the haematophagous bug Triatoma infestans: Behavioural and neurophysiological data on nymphs. J Comp Physiol A 181:21-34.

Tarleton RL, Reithinger R, Urbina JA, Kitron U and Gürtler RE (2007) The challenges of Chagas disease - Grim outlook or glimmer of hope? PLoS Med 4: e332. doi: 10.1371/journal.pmed.0040332.

Vallejo G, Guhl F and Schaub G (2009) Triatominae-Trypanosoma cruzi/T. rangeli:Vector-parasite interactions. Acta Trop. 110: 137-147.

Wigglesworth V and Gillet (1934) The function of the antennae in Rhodnius prolixus (Hemiptera) and the mechanism of orientation to the host. J Exp Biol 11: 120-138. 



\title{
15. Chemical ecology of tick-host interactions
}

Sandra A. Allan

\begin{abstract}
Ticks impact the health of livestock and humans world-wide through their roles as pests and vectors of diseases such as heartwater, theileriosis, anaplasmosis, babesiosis, Lyme disease, erhlichiosis, tick-borne encephalitis and Crimean Congo Haemorrhagic fever. Intrinsic to their capacity to serve in these roles is the ability to locate vertebrate hosts. Olfactory cues are the primary method used for location of potential hosts and these include both emanations from hosts and pheromones produced by ticks feeding on the host. Through behavioural, chemical and electrophysiological methods, volatile compounds from host breath, ruminant digestion, skin and glandular substances, and other blood-feeding ticks have been identified and their role in the host-finding process elucidated. These volatile compounds can provide the basis for development of surveillance methods or be used in the development of push-pull strategies.
\end{abstract}

Keywords: host, tick, vector, control, surveillance, $\mathrm{CO}_{2}$, pheromone, ruminant, Amblyomma, Ixodes

\section{Introduction}

Ticks are highly specialised ectoparasites challenged by the requirement of finding suitable hosts for blood feeding several times through their life cycle. While strategies vary greatly between species with some species feeding on a single host during a life cycle, and others requiring multiple hosts to complete the life cycle, the location of potential hosts is a problem faced by all individuals. Their environment is complex and filled with a multitude of chemical and physical cues most of which are irrelevant to host-finding. Olfactory information relating to the location of potential hosts identified with specialised receptors guides their host-seeking behaviours that enhance the likelihood of host contact. In addition to host emanations, tick-produced odours also enhance contact between ticks and a potential host, and these are reviewed in this chapter. For species such as Amblyomma variegatum (Fabricius), the roles of host-produced and tick-produced odours in host location are heavily intertwined. Essential to a discussion of tick-vector interactions are definitions of several behavioural terms (Box 1).

\section{Strategies for host-finding}

Strategies for host-finding by ticks can be described as either nidicolous or non-nidicolous (Sonenshine 1991). Nidicolous ticks generally live in nests, burrows or roosts in close association with hosts and include most of the soft ticks (Argasidae) and some hard ticks (Ixodidae)(e.g. Ixodes angustus Neumann). While these ticks may live many years, most of that time is spent off the host, with feeding occurring for short periods of time ( $\leq 1 \mathrm{hr}$ ) and nymphs and adults feeding repeatedly (Klompen et al. 1996). Nidicolous ticks respond to host cues (i.e. carbon dioxide $\left(\mathrm{CO}_{2}\right)$ ), heat and moisture with these stimuli generally detected over relatively short distances (Sonenshine 1993). Host-finding by these ticks occurs in a relatively protected environment and in close proximity to potential hosts. Observations of host-finding by four Australian ticks feeding on reptiles revealed that while ticks responded to host odour by adapting a questing position, the responding ticks were always close to the host $(<10 \mathrm{~cm})$ (Belan and Bull 1991). 


\section{Box 1. Definitions of behavioural terms used in tick-vector interactions.}

Activation: Initiation of movement from a resting behaviour to locomotory behaviour similar to the activity caused by stimulants described by (Dethier et al. 1960).

Attraction: Oriented movement towards a stimulus source (Dethier et al. 1960).

Allomone: A chemical that favours the emitter but is detrimental to the perceiving individual.

Kairomone: A chemical released from one organism that induces a favourable response by the receiving individual (Shorey 1977). These are compounds produced by prospective hosts (e.g. from breath, glandular secretions, urine) that elicit responses by host-seeking ticks are kairomones.

Pheromone: A chemical that is released from one organism and induces a response by another individual of the same species (Shorey 1977).

Questing: An off-host behaviour of ticks consisting of a resting position with the forelegs held rigidly in front (Lees 1948). After contact with host stimuli, forelegs are waved in the air presumably for detection of volatile compounds by Haller's organs on the tarsi.

Repellent: Initially described as a chemical that causes an insect to make oriented movements away from the source (Dethier et al. 1960), this term is used loosely here to include actions such as attraction-agonists, spatial repellents and attraction inhibitors (Bernier et al. 2007).

In contrast, non-nidicolous ticks occur in open and exposed habitats such as forests, savannahs, scrub, moorlands, pastures, meadows, brush and deserts, although some may have an association with nests and burrows (Sonenshine 1993). Most of the hard ticks (Ixodidae) are non-nidicolous and for these ticks, blood-feeding is slow (i.e. 3-14 days), occurs only once per life stage and 94$97 \%$ of the life is spent off of the host. Hosts of different stages may be the same individual but usually are different individuals and often different species. In many cases, hosts for immatures are smaller vertebrates (e.g. birds, rodents) and hosts for adults are larger (e.g. cattle, deer, dogs). Hosts may be present over a large area in the environment and host stimuli maybe effective over longer distances than for nidicolous ticks.

Strategies used by hard ticks for location of vertebrate hosts are often classified into two groups (Waladde and Rice 1982). The first group utilises a passive ambush tactic. Ambush strategists climb vegetation and rest on the tops of grass blades or twigs with legs folded. Once in contact with stimuli such as host-associated odours, heat, vibrations or shadows, the forelegs are extended and waved presumably to enhance contact of odours with sensory structures on the tarsus of the foreleg. If a host is contacted as it brushes past, the ticks cling to the host, locate a feeding site and attach. Species that are examples of this type of strategy include Ixodes ricinus (Linnaeus) (Lees 1948) and Rhipicephalus (Boophilus) microplus (Canestrini) (Wilkinson 1953).

Other ticks such as Hyalomma dromedarii Koch, $A$. variegatum and $A$. hebraeum Koch use an active host-seeking or hunter strategy and actively crawl or run towards the host. Increased activity occurs in the response to a stimulus such as $\mathrm{CO}_{2}$ or attraction-aggregation-attachment (AAA) pheromones in the case of large mammal-feeding Amblyomma. Species may vary between strategies between life stage or may exhibit both types of strategies (i.e. A. americanum (L.)) (Sonenshine 1993). 
Host specificity varies considerably between ticks and the several species considered to be onehost ticks are restricted almost exclusively to a single host (e.g. $R$. microplus on cattle, I. texanus Banks on raccoons). Larvae of $R$. microplus are the only life stage that seeks hosts as all of the remaining active life stages occur on the bovine hosts. While there are some 2-hosts ticks, most species are 3-host ticks and may feed on different host species during each life stage. With a broad host range, a large number of host species may be used by a species (i.e. I. persulcatus Schulze has 200 species of recorded hosts) (Anderson and Magnarelli 2008).

Clearly there are different challenges in location of potential hosts according to the type of habitats that the ticks are in and the type of strategy used for host location. For all of these species, olfactory cues contribute to the determination of a potential host.

\section{Odour detection}

The primary chemosensory structures used by ticks to detect volatile host chemosensory stimuli are located on the dorsal surface of the tarsi of the first pair of legs (forelegs) in a unique organ. This structure, Haller's organ, consists of a posterior capsule (Pc) (Figure 1), which is covered by a

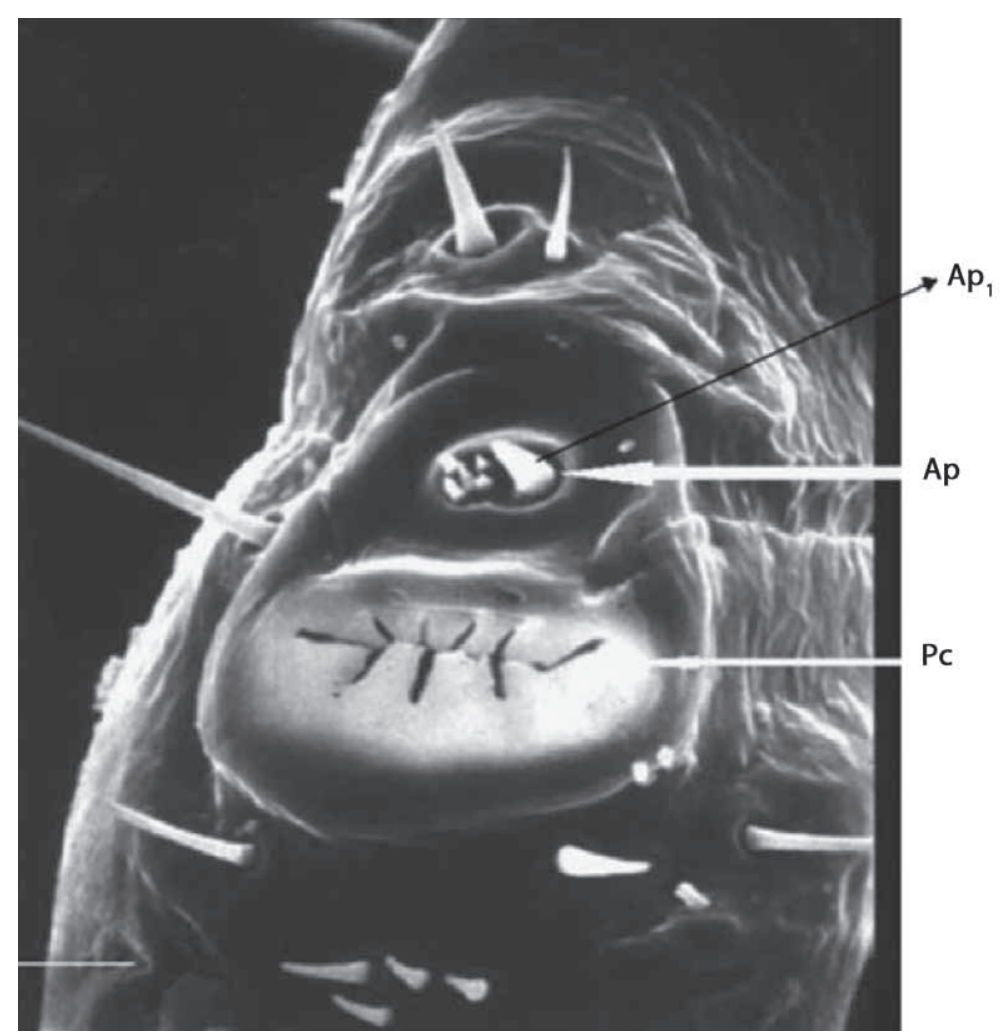

Figure 1. Scanning electron micrograph of the tarsus of the foreleg of Dermacentor variabilis showing Haller's organ. Arrows show the location of olfactory sensilla. $A p=$ Anterior pit; $A p_{1}=$ multiporose olfactory sensilla; $P C=$ Posterior capsule. Bar $=50 \mu \mathrm{m}$ (from Sonenshine 2004). 
membrane with a slit in most species, and an anterior pit (Ap) that contains several small sensilla (Figure 2). Amblyomma variegatum, which is one of the best studied ticks, has 19 tarsal olfactory sensilla, three of which are in the anterior pit and seven are in the capsule of Haller's organ. These sensilla consist of 68-94 olfactory receptors per tarsus for adults while larvae with 14 sensilla have 57-77 olfactory receptors (Hess and Vlimant 1986). The number of sensilla and receptors in each location varies slightly between species.

Receptors present in the capsule and anterior pit of Haller's organ that have been characterised by single sensillum electrophysiology consist of receptors for $\mathrm{CO}_{2}$ (Steullet and Guerin 1992a), hydrogen sulphide $\left(\mathrm{H}_{2} \mathrm{~S}\right.$ ) (Steullet and Guerin 1992b), aromatic aldehydes, lactones and phenols as well as short-chain fatty acids and aldehydes (Steullet and Guerin 1994a,b) (Table 1 and 2).

The presence of two $\mathrm{CO}_{2}$ receptors in $\mathrm{A}$. variegatum that differ in sensitivity optimises the ability to detect very small changes in host emission of this kairomone (Steullet and Guerin 1992a). The $\mathrm{CO}_{2}$-inhibited receptor is sensitive to very small concentration changes just above ambient $\mathrm{CO}_{2}$ levels. The $\mathrm{CO}_{2}$-excited receptor monitors changes in concentration above $0.1 \%$ which would include concentrations encountered near a potential host (Steullet and Guerin 1992a). Previously, Holscher et al. (1980) recorded responses to $\mathrm{CO}_{2}$ from single sensillum recordings from A. americanum, A. maculatum Koch and Dermacentor variabilis (Say).

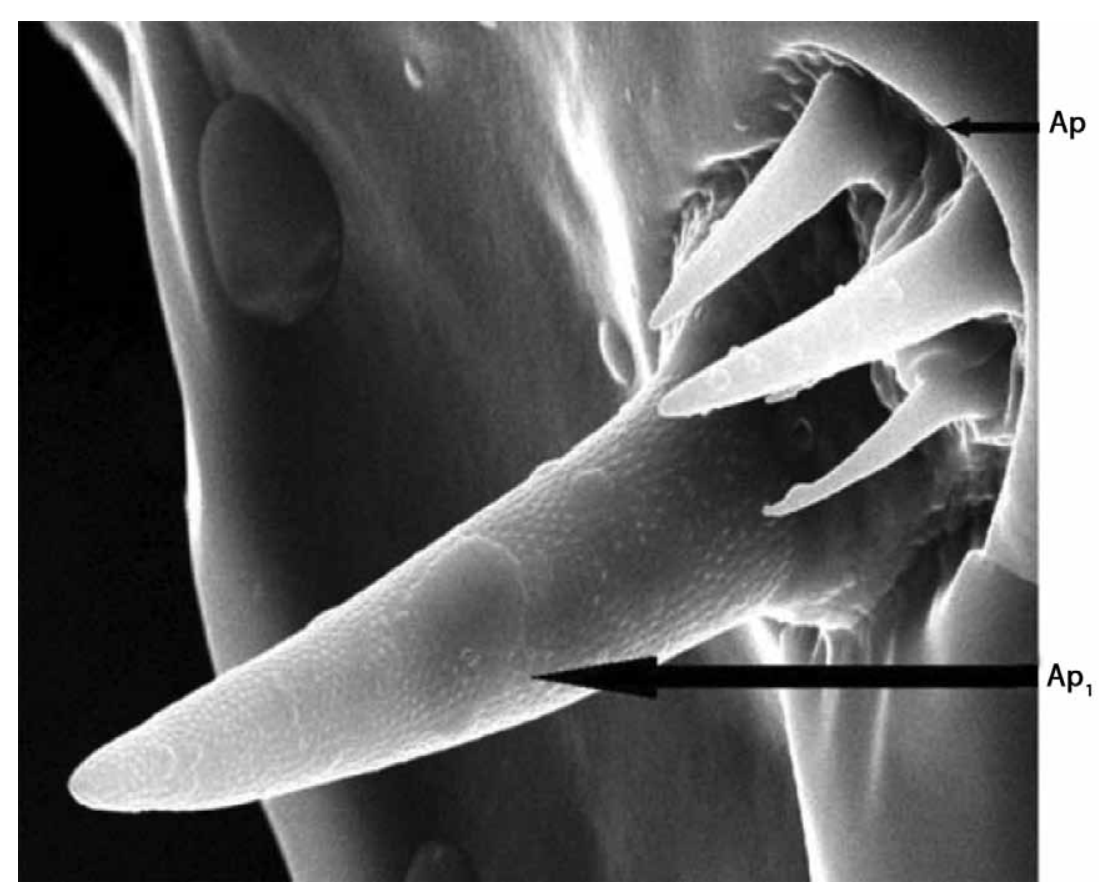

Figure 2. Scanning electron micrograph illustrating the large olfactory sensillum, Ap 1 in the anterior pit of Haller's organ surrounded by other sensillae. Bar $=5 \mu \mathrm{m}$. Ap = anterior pit; $A p_{1}=$ olfactory sensillum (from Sonenshine 2004). 
Table 1. Olfactory receptor types related to vertebrate host location in the capsule of Haller's organ on the forelegs of ticks as characterised by single sensillum electrophysiology (adapted from Steullett and Guerin 1994a).

\begin{tabular}{|c|c|c|c|}
\hline Receptor types & Tick species & Characteristics & Reference \\
\hline Aldehyde - type 1 & A. variegatum & $\begin{array}{l}\text { Saturated and unsaturated } \mathrm{C}_{6} \text { aliphatic } \\
\text { aldehydes; most sensitive to hexanal }\end{array}$ & Steullet and Guerin 1994a \\
\hline Aldehyde - type 2 & A. variegatum & $\begin{array}{l}\text { Saturated aldehydes such as heptanal } \\
\text { and hexanal }\end{array}$ & Steullet and Guerin 1994a \\
\hline Aldehyde - type 3 & A. variegatum & $\begin{array}{l}\text { Unsaturated aliphatic aldehydes such as } \\
\text { E-2-heptenal }\end{array}$ & Steullet and Guerin 1994a \\
\hline Benzaldehyde & A. variegatum & $\begin{array}{l}\text { Most sensitive to benzaldehyde, also to } \\
\text { furfural }\end{array}$ & Steullet and Guerin 1994a \\
\hline $\mathrm{CO}_{2}$-excited & A. variegatum & Sensitive to concentrations above $0.1 \%$ & Steullet and Guerin 1992a \\
\hline $\mathrm{CO}_{2}$-inhibited & A. variegatum & $\begin{array}{l}\text { Sensitive to small concentration changes } \\
\text { between } 0 \text { and } 0.2 \% \text {; Increase of } 0.001 \text { - } \\
0.002 \% \text { inhibits }\end{array}$ & Steullet and Guerin 1992a \\
\hline $\mathrm{H}_{2} \mathrm{~S}-$ type 1 & A. variegatum & $\begin{array}{l}\text { More firing with } \mathrm{H}_{2} \mathrm{~S} \text {; no response to } \\
\text { dimethyl sulfide }\end{array}$ & Steullet and Guerin 1992b \\
\hline $\mathrm{H}_{2} \mathrm{~S}-$ type 2 & A. variegatum & $\begin{array}{l}\text { Ethyl mercaptan elicits stronger response; } \\
\text { dimethyl sulfide responses }\end{array}$ & Steullet and Guerin 1992b \\
\hline 2-hydroxybenzaldehyde & A. variegatum & Most sensitive to 3-hydroxybenzaldehyde & Steullet and Guerin 1994a \\
\hline Lactone & A. variegatum & $\begin{array}{l}\text { Most sensitive to } \gamma \text {-valerolactone, also } \\
\text { 8-butyrolactone, } 6 \text {-caprolactone }\end{array}$ & Steullet and Guerin 1994a \\
\hline Methyl salicylate & $\begin{array}{l}\text { A. variegatum } \\
\text { I. ricinus } \\
\text { R. microplus }\end{array}$ & $\begin{array}{l}\text { AAA pheromone component } \\
\text { AAA pheromone component } \\
\text { AAA pheromone component }\end{array}$ & $\begin{array}{l}\text { Hess and Vlimant } 1986 \\
\text { Steullet } 1993 \\
\text { Steullet } 1993\end{array}$ \\
\hline
\end{tabular}

Two types of hydrogen sulfide $\left(\mathrm{H}_{2} \mathrm{~S}\right)$ receptors have been identified in separate wall-pore sensilla in the capsule of the Haller's organ of A. variegatum (Steullet and Guerin 1992b) (Table 1). Both receptor types were highly sensitive to $\mathrm{H}_{2} \mathrm{~S}$ with a response threshold of $0.1 \mathrm{ppb}$ and sensitive up to $10 \mathrm{ppm}$; the responses to sulfides and mercaptans were dissimilar. The type 1 receptor fired more with stimulation from $\mathrm{H}_{2} \mathrm{~S}$, while the type 2 receptor responded more to eythlmercaptan whereas dimethyl sulfide only stimulated the type 2 receptor. In a behavioural assay $\mathrm{H}_{2} \mathrm{~S}$ at 0.02 and $1 \mathrm{ppm}$ aroused $60 \%$ of resting ticks with two-thirds questing and the remainder searching actively. In mixtures with $\mathrm{CO}_{2}, \mathrm{H}_{2} \mathrm{~S}$ appeared to act antagonistically with $\mathrm{H}_{2} \mathrm{~S}$ at $1 \mathrm{ppm}$ mixed with $\mathrm{CO}_{2}$ decreased questing behaviour. It is likely that these sulfide receptors activate with other sulfide compounds and that their presence in other body odours such as those from axillary secretions may play a role in attraction to potential hosts or predilection sites (Steullet and Guerin 1992b). Response to these sulfides may explain the reported attraction of $D$. variabilis to carrion odour (Kneidel 1984, McNemee et al. 2003).

Volatiles collected from human breath, axillary secretions and rabbit odour collected on adsorbent material (Porapak) did not stimulate any receptors on the surface of the foreleg tarsus of $A$. variegatum (Steullet and Guerin 1994b). Bovine odour, however, did stimulate receptors in the DII.1 sensilla, and 2-nitrophenol and 4-methyl-2-nitrophenol also stimulated these sensilla. Using other known vertebrate-associated volatiles, numerous receptor types were characterised 
Table 2. Olfactory receptor types related to vertebrate host location in the anterior pit near Haller's organ on the forelegs of ticks as characterised by single sensillum electrophysiology (adapted from Steullet and Guerin 1994b).

\begin{tabular}{|c|c|c|c|c|}
\hline Receptor type & Tick species & $\begin{array}{l}\text { Sensilla in } \\
\text { anterior } \\
\text { pit }\end{array}$ & Characteristics & Reference \\
\hline Ammonia - type 1 & A. variegatum & DII.6 & Responsive to $\mathrm{NH}_{3}$ & Steullet and Guerin 1994b \\
\hline Ammonia - type 2 & A. variegatum & DII.6 & $\begin{array}{l}\text { Responsive to } \mathrm{NH}_{3} \text { with different } \\
\text { dose-response curve }\end{array}$ & Steullet and Guerin 1994b \\
\hline \multirow[t]{2}{*}{ Ammonia - type 3} & A. variegatum & DIV & $\begin{array}{l}\text { Weakly stimulated by high } \\
\text { concentrations }\end{array}$ & Steullet and Guerin 1994b \\
\hline & R. sanguineus & & & Haggart and Davis 1980 \\
\hline Fatty acid - type 1 & A. variegatum & DII.1 & $\begin{array}{l}\text { Most sensitive to pentanoic acid, } \\
\text { also to 3-methylbutanoic acid and } \\
\text { butanoic acid }\end{array}$ & Steullet and Guerin 1994b \\
\hline Fatty acid - type 2 & A. variegatum & DII.5 & $\begin{array}{l}\mathrm{C}_{4} \text { and } \mathrm{C}_{5} \text { fatty acids, weak } \\
\text { response to hexanoic, heptanoic } \\
\text { and nonanoic acids }\end{array}$ & Steullet and Guerin 1994b \\
\hline Fatty acid - type 3 & A. variegatum & DII.5 & $\begin{array}{l}\text { Most sensitive to } \\
\text { 2-methylpropanoic acid }\end{array}$ & Steullet and Guerin 1994b \\
\hline \multirow[t]{4}{*}{ Lactone } & A. variegatum & DI.1 & $\begin{array}{l}\text { Most sensitive to } \gamma \text {-valerolactone, } \\
\text { also 6-caprolactone }\end{array}$ & Steullet and Guerin 1994b \\
\hline & A. hebraeum & & & Steullet 1993 \\
\hline & R. microplus & & & Steullet 1993 \\
\hline & I. ricinus & & & Leonovich 2004 \\
\hline \multirow[t]{4}{*}{$\begin{array}{l}\text { Nitrophenol } \\
\text { (phenol) }\end{array}$} & A. variegatum & DII.1 & $\begin{array}{l}\text { Sensitive to 2-nitrophenol and } \\
\text { 4-methyl-nitrophenol }\end{array}$ & Steullet and Guerin 1994b \\
\hline & A. variegatum & & & Schöni et al. 1984 \\
\hline & R. microplus & & & De Bruyne and Guerin 1994 \\
\hline & I. ricinus & & & Leonovich 2004 \\
\hline 3-pentanone & A. variegatum & DIV & Responded to 3-pentanone & Steullet and Guerin 1994b \\
\hline
\end{tabular}

(Steullet and Guerin 1994a). These included a benzaldehyde receptor, a hydroxybenzaldehyde receptor, three types of receptors responding differently to aliphatic aldehydes, three receptors responding to ammonia and a lactone receptor (Table 2). Ammonia receptors were previously reported by Haggart and Davis (1980). Leonovich (2004) also reported that a distal knoll sensillum of Haller's organ in I. ricinus was sensitive to steer hair extract as well as o-cresol (2-methyl phenol) and $\mathrm{p}$-valerolactone.

Gustatory receptors detect components of the arrestment pheromones. Grenacher et al. (2001) demonstrated that the arrestment behaviour in the sheep tick, I. ricinus to its own faeces was mediated by gustatory receptor cells housed in the terminal wall-pore sensilla on the foreleg tarsus. Waladde (1987) speculated that olfactory receptors on the palpal organ of ticks may serve to perceive olfactory stimuli at very close range. 


\section{Behavioural responses to host-produced compounds}

\section{Breath}

Breath from potential vertebrate hosts strongly stimulates ticks with an increase in host-seeking behaviour, generally including questing behaviour or movement toward the source (Guerin et al. 2000). Breath contains various cues such as moisture and heat in addition to volatile compounds such as $\mathrm{CO}_{2}$, acetone, ammonia, and $\mathrm{H}_{2} \mathrm{~S}$ and behavioural responses to these compounds are presented in Table 3. In ruminants, numerous compounds that are by-products of rumen digestion, such as 1-octen-3-ol, also induce behavioural responses (Donzé et al. 2004).

Table 3. Compounds other than $\mathrm{CO}_{2}$ eliciting responses associated with host seeking in ticks.

\begin{tabular}{|c|c|c|c|c|}
\hline Chemical & Tick species & Component of & Behaviour & Reference \\
\hline Acetone & A. variegatum & Breath (bovine) & Attraction (lab) & McMahon and Guerin 2002 \\
\hline \multirow[t]{2}{*}{ Acetic acid } & A. variegatum & Breath/rumen (bovine) & $\begin{array}{l}\text { Upwind movement, } \\
\text { increased speed }\end{array}$ & Donzé et al. 2004 \\
\hline & O. erraticus & Breath & Walking in a dish & El-Ziady 1958 \\
\hline \multirow[t]{2}{*}{ Ammonia } & O. erraticus & Breath & Walking in dish & El-Ziady 1958 \\
\hline & R. sanguineus & Breath & Questing & Haggart and Davis 1980 \\
\hline \multirow[t]{2}{*}{ Benzaldehyde } & A. hebraeum & AAA pheromone & Attraction (lab) & Apps et al. 1988 \\
\hline & $\begin{array}{l}\text { A. hebraeum, } A \text {. } \\
\text { variegatum }\end{array}$ & AAA pheromone & Attraction (lab) & Yunker et al. 1992 \\
\hline Benzoic acid & R. microplus & Skin/hair (bovine) & Questing & Osterkamp et al. 1999 \\
\hline \multirow[t]{2}{*}{ Butanoic acid } & A. variegatum & Breath/rumen (bovine) & $\begin{array}{l}\text { Upwind movement, } \\
\text { increased speed }\end{array}$ & Donzé et al. 2004, \\
\hline & R. microplus & Skin/hair (bovine) & Questing & Osterkamp et al. 1999 \\
\hline $\begin{array}{l}\text { 2-ethylhexanoic } \\
\text { acid }\end{array}$ & R. microplus & Skin/hair (bovine) & Questing & Osterkamp et al. 1999 \\
\hline \multirow[t]{4}{*}{ Guanine } & A. walkerae & Arrestment pheromone & Akinesis & Neitz and Gothe 1984 \\
\hline & $\begin{array}{l}\text { A. cohaerens, } R \text {. } \\
\text { appendiculatus, } \\
\text { A. persicus }\end{array}$ & Arrestment pheromone & Akinesis & Otieno et al. 1985 \\
\hline & I. scapularis & Arrestment pheromone & Akinesis & Allan and Sonenshine 2002 \\
\hline & A. americanum & Arrestment pheromone & Akinesis & Yoder et al. 2008 \\
\hline Hematin & I. scapularis & Arrestment pheromone & Akinesis & Allan and Sonenshine 2002 \\
\hline Heptadecane & $\begin{array}{l}\text { A. hebraeum, } A . \\
\text { variegatum }\end{array}$ & AAA pheromone & Attraction & Yunker et al. 1992 \\
\hline Heptanoic acid & R. microplus & Skin/hair (bovine) & Questing & Osterkamp et al. 1999 \\
\hline Hexanal & R. microplus & Skin/hair (bovine) & Questing & Osterkamp et al. 1999 \\
\hline Hexanoic acid & R. microplus & Skin/hair (bovine) & Questing & Osterkamp et al. 1999 \\
\hline Hydrogen sulfide & A. variegatum & Breath & Questing & Steullet and Guerin 1992b \\
\hline \multirow[t]{2}{*}{ Hypoxanthine } & A. persicus & Arrestment pheromone & Akinesis & Otieno et al. 1985 \\
\hline & I. scapularis & Arrestment pheromone & Akinesis & Allan and Sonenshine 2002 \\
\hline Inosine & I. scapularis & Arrestment pheromone & Akinesis & Allan and Sonenshine 2002 \\
\hline Isobutanoic acid & A. variegatum & Breath/rumen (bovine) & $\begin{array}{l}\text { Upwind movement, } \\
\text { increased speed }\end{array}$ & Donzé et al. 2004 \\
\hline
\end{tabular}


Table 3. Continued.

\begin{tabular}{|c|c|c|c|c|}
\hline Chemical & Tick species & Component of & Behaviour & Reference \\
\hline Isobutyric acid & A. variegatum & AAA pheromone & Attraction & Apps et al. 1988 \\
\hline 3-methylindole & A. variegatum & Breath/rumen (bovine) & $\begin{array}{l}\text { Upwind movement, } \\
\text { increased speed }\end{array}$ & Donzé et al. 2004 \\
\hline 4-methylphenol & A. variegatum & Breath/rumen (bovine) & $\begin{array}{l}\text { Upwind movement, } \\
\text { increased speed }\end{array}$ & Donzé et al. 2004 \\
\hline \multirow[t]{2}{*}{$\begin{array}{l}\text { 2-methyl } \\
\text { propanoic acid }\end{array}$} & $\begin{array}{c}\text { A. hebraeum, } A . \\
\text { variegatum }\end{array}$ & AAA pheromone & Attraction (lab) & Yunker et al. 1992 \\
\hline & R. microplus & Skin/hair (bovine) & Questing & Osterkamp et al. 1999 \\
\hline \multirow[t]{2}{*}{ Methyl salicylate } & A. variegatum & AAA pheromone & $\begin{array}{l}\text { Questing, increased } \\
\text { angular velocity } \\
\text { and walking } \\
\text { speed }\end{array}$ & McMahon and Guerin 2000 \\
\hline & $\begin{array}{c}\text { A. hebraeum, } A . \\
\text { variegatum }\end{array}$ & AAA pheromone & Attraction (lab) & Yunker et al. 1992 \\
\hline Nonanoic acid & $\begin{array}{l}\text { A. hebraeum, } A . \\
\text { variegatum }\end{array}$ & AAA pheromone & Attraction (lab) & Yunker et al. 1992 \\
\hline Nitric oxide & A. variegatum & Breath (bovine) & Attraction (lab) & McMahon and Guerin 2002 \\
\hline \multirow[t]{6}{*}{ 2-nitrophenol } & R. microplus & Skin/hair (bovine) & Questing & Osterkamp et al. 1999 \\
\hline & A. variegatum & AAA pheromone & $\begin{array}{l}\text { Questing, increased } \\
\text { angular velocity } \\
\text { and walking } \\
\text { speed }\end{array}$ & McMahon and Guerin 2000 \\
\hline & A. variegatum & AAA pheromone & $\begin{array}{l}\text { Long-range } \\
\text { attraction }\end{array}$ & Norval et al. 1991, 1993 \\
\hline & A. variegatum & AAA pheromone & $\begin{array}{l}\text { Aggregation, } \\
\text { attachment }\end{array}$ & Schöni et al. 1984 \\
\hline & A. hebraeum & AAA pheromone & Attraction & Norval et al. 1991 \\
\hline & A. hebraeum & AAA pheromone & Attachment & Yunker et al. 1992 \\
\hline \multirow[t]{2}{*}{ 1-octen-3-ol } & A. variegatum & $\begin{array}{l}\text { Breath (bovine), } \\
\text { disturbed tick }\end{array}$ & $\begin{array}{l}\text { Attraction (low } \\
\text { levels) }\end{array}$ & McMahon et al. 2001 \\
\hline & R. microplus & Skin/hair (bovine) & Questing & Osterkamp et al. 1999 \\
\hline Pentanoic acid & R. microplus & Skin/hair (bovine) & Questing & Osterkamp et al. 1999 \\
\hline Propanoic acid & A. variegatum & Breath/rumen (bovine) & $\begin{array}{l}\text { Upwind movement, } \\
\text { increased speed }\end{array}$ & Donzé et al. 2004 \\
\hline Pyruvate & R. microplus & Skin/hair (bovine) & Questing & Osterkamp et al. 1999 \\
\hline Salicylaldehyde & $\begin{array}{l}\text { A. hebraeum, } A \text {. } \\
\text { variegatum }\end{array}$ & AAA pheromone & Attraction (lab) & Yunker et al. 1992 \\
\hline Squalene & $\begin{array}{l}\text { A. americanum, } \\
\text { D. variabilis }\end{array}$ & Skin & Attraction & Yoder et al. 1998 \\
\hline \multirow[t]{4}{*}{ Uric acid } & A. persicus & Arrestment pheromone & Akinesis & Otieno et al. 1985 \\
\hline & I. scapularis & Arrestment pheromone & Akinesis & Allan et al. 2002 \\
\hline & I. uriae & Arrestment pheromone & Akinesis & Benoit et al. 2008 \\
\hline & A. americanum & Arrestment pheromone & Akinesis & Yoder et al. 2008 \\
\hline \multirow[t]{2}{*}{ Xanthine } & A. persicus & Arrestment pheromone & Akinesis & Otieno et al. 1985 \\
\hline & I. scapularis & Arrestment pheromone & Akinesis & Allan and Sonenshine 2002 \\
\hline
\end{tabular}


$\mathrm{CO}_{2}$ is a strong activating stimulus for many tick species with ticks moving from a quiescent state with legs curled under the body to questing behaviour or walking. In addition to being an activator, $\mathrm{CO}_{2}$ has also been reported as an attractant source in both laboratory (Anderson et al. 1998, Sauer et al. 1974) and field studies (Barré et al. 1997, Gray 1985, Wilson et al. 1972). In a wind tunnel, $\mathrm{CO}_{2}$ acted as both a locomotor stimulant and an attractant for $A$. variegatum (Steullet and Guerin 1992a). The presence of $\mathrm{CO}_{2}$ often enhances or synergises responses to host odours and tick pheromones (Barré et al. 1997, Beelitz and Gothe 1991, Maranga et al. 2003, Norval et al. 1989a). In a laboratory study with the soft tick, Argas walkerae Kaiser and Hoogstraal, over $90 \%$ of unfed females became active within 17 seconds and ran quickly to the drum containing the chicken when exposed to a gradient of $\mathrm{CO}_{2}$ that increased close to the bird (Beelitz and Gothe 1991). When the same treatment was tested, but with $\mathrm{CO}_{2}$ removed with an adsorbent (sodium hydroxide), only $67.5 \%$ of ticks migrated to the chicken. If $\mathrm{CO}_{2}$ was present in concentrations different than the gradient towards a chicken, tick movement was altered. If only a heat source was presented in the absence of chicken odour or $\mathrm{CO}_{2}, 55 \%$ of the ticks migrated to the source. Beelitz and Gothe (1991) concluded that odours from the chicken represented an essential stimulus modality with heat and $\mathrm{CO}_{2}$ as secondary, non-specific stimulants. In an olfactometer with pulsed $\mathrm{CO}_{2}$ delivery, Perritt et al. (1993) reported that adult $A$. americanum and $D$. variabilis perceived and responded to a $\mathrm{CO}_{2}$ level of $9 \mathrm{ppm}$ above the mean ambient level. While, attraction to a $\mathrm{CO}_{2}$ source in the field has been reported as far as $21 \mathrm{~m}$ for $A$. americanum (Wilson et al. 1972), it is generally effective over a shorter distance. Ixodes spp. appear to respond less to $\mathrm{CO}_{2}$ than do species of other tick genera (Anderson et al. 1998, Crooks and Randolph 2006, Fourie et al. 1993, Ginsberg and Ewing 1989, Schulze et al. 1997) and it has been postulated that $\mathrm{CO}_{2}$ might be more effective as an excitant or attractant for 'hunter' ticks then 'ambush' ticks.

Human breath, which contains $\mathrm{H}_{2} \mathrm{~S}$, stimulates the sulfide-sensitive cells in Haller's organ of $A$. variegatum. Ruminants produce large amounts of $\mathrm{H}_{2} \mathrm{~S}$ during eructation and can likely be detected by sulfide receptors at considerable distances from the host (Steullet and Guerin 1992b). Ticks, even in the absence of $\mathrm{CO}_{2}$, may be stimulated into activation or questing by the presence of sulfides (Steullet and Guerin 1992b).

In a comparison of walking responses of $A$. variegatum on a servosphere to five components of breath, none of the compounds were as attractive as natural breath. Acetone, however, was the most attractive compound after natural breath and induced the greatest reduction in speed and highest angular velocity (McMahon and Guerin 2002). However, field evaluation of acetone has not been sucessful and Norval et al. (1987) reported no attraction of $A$. hebraeum. Nitric oxide and isoprene were also breath constituents evaluated by McMahon and Guerin (2002) with no activity for isoprene and weak attraction reported for nitric oxide.

Ammonia is commonly associated with vertebrates and present in breath as well as skin odours and urine (Taneja and Guerin 1997). Ammonia has been reported to elicit leg waving in $R$. sanguineus (Latreille) in laboratory assays (Haggart and Davis 1980). The soft tick Ornithodoros erraticus (Lucas) walked more on the sides of petri dishes containing $0.5 \%$ ammonia than sides without odour (ElZiady 1958). McMahon and Guerin (2002) did not detect any effects of ammonia on the walking behaviour of $A$. variegatum in servosphere assays and speculated that this compound may need to be presented in combination with other compounds to elicit a behavioural response.

The physiological state of ticks impacts host-seeking behaviour. Questing, movement and latency (time after exposure to questing) to $\mathrm{CO}_{2}$ were evaluated for different stages of $A$. hebraeum in a laboratory study (Anderson et al. 1998). Responsiveness to $\mathrm{CO}_{2}$ as indicated by behaviour was 
greatest for ticks six weeks after molting compared to newly molted ticks, and by partially-fed females compared to replete females. Males removed from a host at any stage of feeding remained highly responsive to $\mathrm{CO}_{2}$.

\section{Ruminant by-products}

Wild and domestic ruminants are critical in the maintenance of populations of many of the hard tick species associated with debilitating human and livestock diseases such as Lyme disease, erhlichiosis, babesiosis, heartwater, and theileriosis (Sonenshine 1993). Ruminants regularly eruct gases from the foregut to relieve pressure and Donzé et al. (2004) reported attraction in the laboratory of adult A. variegatum, A. hebraeum, I. scapularis Say, I. persulcatus and I. ricinus to the odour of rumen fluid from freshly slaughtered cattle. Three species (A. variegatum, I. persulcatus and I. ricinus) significantly increased speed, and removal of the odour from the air stream resulted in intense local search behaviour by $A$. variegatum. Using gas chromatography (GC) coupled with electrophysiological recordings from the wall-pore olfactory sensilla on the first leg tarsus of $A$. variegatum and I. ricinus, butanoic, isopentanoic, pentanoic and hexanoic acids, 2-nitrophenol, 4-methyl-2-nitrophenol, 4-methylphenol, indole and 3-methylphenol were determined to be biologically active. Receptor cells from both tick species also responded to acetic acid, propionic acid, phenol and 2-methylphenol. Attraction of $A$. variegatum was observed with butanoic and isobutanoic acid, 4-methylphenol or 3-methylindole individually. The dose and proportions of these compounds in a mixture were critical in eliciting oriented walking responses from $A$. variegatum and $I$. ricinus. Of the components identified from cattle, 1-octen-3-ol is an effective attractant for tsetse (Hall et al. 1984) and other biting flies but it did not attract A. hebraeum in the field (Norval et al. 1987). Location of large hosts such as ruminants appears to be facilitated by the attraction of ticks to volatile rumen end-products of digestion.

\section{Urine}

Phenolic components of urine are known to attract haematophagous insects (Bursell et al. 1988, Hassanali et al. 1986). There is, however, little evidence to support the role of host urine in tick attraction. Carroll et al. (1995) observed arrestant responses by l. scapularis females to urine from a white-tailed deer (Odocoileus virginianus (Zimmerman)) doe in a laboratory assay. Urine from bucks resulted in avoidance responses on similar assays (Carroll 1999) and responses to urine appeared to be enhanced at 50\% RH compared to 95\% RH (Carroll 2000).

\section{Skin and feathers}

Questing ticks contact the skin and associated hair or feathers of a host and presumably, based on the suitability of the stimuli perceived, continue contact on the host until attachment occurs or detach and continue searching until a suitable host is found. Host-seeking D. variabilis are often present along trails (Carroll et al. 1991, Newhouse 1983) and this may reflect the presence of compounds from hosts (e.g. dogs) present along the trails. Lees (1969) reported large aggregations of unfed I. canisuga Johnson on walls of dog kennels at dog height and speculated that the ticks were attracted by odours left by dogs as they rubbed against the wall. In laboratory assays, rubbings from dog ears resulted in arrestment responses by female I. scapularis, A. americanum and both sexes of D. variabilis (Carroll 2002) and walking responses by I. ricinus (Crooks and Randolph 2006). Extracts of canine skin and hair were attractive to Ixodes spp. (Dobrotvorsky et al. 2000) and D. variabilis, A. americanum and R. sanguineus nymphs (Dukes and Rodriguez 1976) in laboratory assays. 
The questing behaviour of $R$. microplus (one-host tick), and I. ricinus (with a broad host range) larvae were examined to compounds extracted from either the hair or skin surface from bovines and several other animals was examined and divided into lipid and volatile components (Osterkamp et al. 1999). Bovine skin lipid extracts and volatile extracts elicited the strongest questing responses from $R$. microplus larvae. Larvae of $I$. ricinus responded strongly to cattle, pig and human lipid extracts and equally to volatile extracts of cattle, humans, mice, and deer. Based on GC analysis of extracts, a blend of 37 compounds was formulated that elicited high questing responses. Seven key components of the blend essential for questing responses were identified and consisted of benzoic acid, 2-ethylhexanoic acid, hexanoic acid, 2-nitrophenol, 1-octen-3-ol, pentanoic acid and pyruvate. Comparisons of questing responses of the two species to bovine odour and different blends of compounds indicated inter-specific species differences in responses to compound combinations. For R. microplus, 2-nitrophenol and 1-octen-3-ol accounted for $80 \%$ of the response to the entire blend of seven compounds. Osterkamp et al. (1999) concluded that responses to individual compounds were obtained at high concentrations. However, when tested mixtures, lower doses were effective in eliciting responses thereby indicating additive or synergistic effects in the mixtures. Squalene, a component of mammalian skin, and also a glandular secretion of $D$. variabilis, was found to be attractive to A. americanum and D. variabilis adults (Yoder et al. 1998) and may play a role in host selection.

Compounds present on skin or feathers also may serve as natural repellents against ectoparasites. The crested auklet (Aethia cristatella (Pallas)) emits a seasonally elevated citrus scent and GC analysis of whole-bird headspace collections and of neck feather extracts revealed several aldehydes (Douglas et al. 2004). Of these, octenal and hexanal as well as a synthetic blend representing the crested auklets natural odorants were repellent against nymphs of $A$. americanum and $I$. uriae (White).

\section{Glandular substances}

Glandular secretions from integumentary glands are an important form of chemical communication for many vertebrates. These secretions from deer and antelope have been reported as kairomones for host-seeking Ixodes ticks. Substances from the ante-orbital glands of the klipspringer antelope (Oreotragus oreotragus (Zimmerman)) rubbed on twigs elicited attraction and aggregation of adults of I. neitzi (Clifford, Walker and Keirans) (Rechav et al. 1978). These aggregations increase the likelihood of future encounters with potential hosts as ticks often quest from these vantage points. Carroll et al. (1996) reported arrestment responses of male and female I. scapularis to rubbings from tarsal, interdigital and preorbital glands of white-tailed deer (Odocoileus virginianus (Zimmerman)). Only weak arrestment responses were reported from rubbings of forehead, back and non-glandular areas of the feet. Tarsal gland substances from female deer (does) but not male deer (bucks) elicited arrestment responses of male and female I. scapularis (Carroll et al. 1995). In the same study, interdigital gland substances influenced climbing behaviour associated with arrestment. These arrestment responses were verified in the field using field-collected ticks and wooden skewers rubbed with gland substances (Carroll et al. 1996). Both foreleg and hind leg interdigital glands produce substances that elicit arrestment responses by female 1 . scapularis (Carroll 2001). Doe urine, under certain circumstances, elicited arrestment responses from female I. scapularis. Avoidance, however, appeared to occur in response to urine from dominant reproductive bucks (Carroll 2000). White-tailed deer urinate on tarsal glands as part of their scentmarking behaviours and it is difficult to differentiate possible attractant responses to urine or tarsal gland secretions as in general urine was repellent (Carroll 2000). 
Glandular secretions of the tick, Haemaphysalis leachi (Audouin), a tick commonly found on dogs, contain a dog-repelling allomone that inhibits removal of ticks by grooming (Burger et al. 2006). Based on responses in bioassays, the allomones may consist of six aliphatic aldehydes from hexanal to undecanal.

\section{Feeding-site preference}

Some species of tick exhibit a strong predilection for feeding on certain sites on the hosts body. Two such species are $R$. appendiculatus Neumann and R. evertsi Neumann, which exhibit strong preferences to feed inside the ears and around the anal regions of catlle, respectively. In a study by Wanzala et al. (2004), ticks of each species were released on six different body locations on cattle with most ticks of each species migrating over the body and reaching the preferred feeding site. Given the apparent avoidance of the preferred feeding site by the other species, the authors proposed that a push-pull effect, with some compounds attracting and some compounds repelling, influenced the orientation behaviour. Using dichloromethane extracts of volatiles from ears or the anal region adsorbed onto charcoal or silica, tick responses were assayed in a tick climbing bioassay apparatus. Odours trapped from cattle ears attracted $R$. appendiculatus but repelled $R$. evertsii. Odour from the anal region had the opposite effect. It seems likely that other species with strong predilection sites for feeding are similarly guided by chemical cues.

One impact of feeding aggregations of ticks, particularly those containing immatures, is the potential for pathogen transmission through nonsystemic means by co-feeding of infected and uninfected ticks (Randolph et al. 1996). This is best supported from field data with Lyme disease spirochetes and lxodes ticks (Kimura et al. 1995, Stafford et al. 1995).

\section{Behavioural response to tick pheromones that enhance host contact}

For some tick species, vector-host interactions are facilitated by the presence of tick pheromones. The pheromones that facilitate host contact fall into two categories: attraction-aggregationattachment (AAA) pheromones or arrestment (assembly) pheromones.

\section{Attraction-aggregation-attachment pheromones}

In many of the Amblyomma species that feed on large hosts such as cattle, the location of suitable hosts and the development of feeding aggregations of ticks on hosts are facilitated by the emission of volatile compounds by blood-feeding conspecific males on the hosts. These attraction-aggregation-attachment (AAA) pheromones are a multi-component blend that can elicit a series of behavioural steps that ultimately result in the feeding aggregations observed on hosts.

This strategy has been well documented for the bont ticks, A. variegatum and A. hebraeum (Apps et al. 1988, Diehl et al. 1991, Norval and Rechav 1979). Pioneer male ticks locate a suitable host and migrate on the host to attach at a preferred feeding site. After blood-feeding for five or more days, males emit a mixture of volatile compounds that elicit attraction of unfed conspecific nymphs and adults (secondary colonisers) to the infested host individual (Hess and deCastro 1986; Norval et al. 1989a 1989b; Steullet 1993). Once on the host, ticks migrate to form aggregations in the preferred attachment sites around the feeding males emitting the volatiles. At this point, they will attach to the host and feed. While males may attach to potential hosts in the absence of females, females will only attach and feed near a source of pheromone (blood-feeding males) (Norval and 
Rechav 1979). The principal components of the AAA pheromone for A. variegatum and A. hebraeum include 2-nitrophenol (o-nitrophenol), methyl salicylate,

, 2,6-dichlorophenol, with some differences in presence and abundance between species (Diehl et al.1991, Lusby et al. 1991, Schöni et al. 1984). In general, 2-nitrophenol and methyl salicylate stimulate long range, interspecific attraction and aggregation; whereas, methyl salicylate and nonanoic acid induce attachment (Lusby et al. 1991).

The behavioural effects of these pheromones are enhanced in combination with $\mathrm{CO}_{2}$ (Hess and deCastro 1986, Norval et al. 1989a) and host odours (which are not attractive on their own) (McMahon and Guerin 2000) with attraction in the field from over $10 \mathrm{~m}$ distance. Hosts with attached feeding males attract more ticks than uninfected hosts (Norval et al. 1989b). A similar pheromone is produced by blood-feeding males of $A$. maculatum. A hexane extract of blood-fed males that was placed on one ear (preferred feeding site) of cattle resulted in 4-fold increase of ticks attached to the treated ear over the control ear (Gladney et al. 1974). A similar pheromone appears to be present in A. cajennense (F.) but may only induce attachment, not aggregation (Rechav et al. 1997). Maranga et al. (2003) examined responses of released $A$. variegatum to different doses of the synthetic pheromone blend (o-nitrophenol, methyl salicylate and nonanoic acid in paraffin oil) in the presence or absence of $\mathrm{CO}_{2}$. Up to $90 \%$ of the released ticks were attracted to the pheromone source in the presence of $\mathrm{CO}_{2}$ within three hours. $\mathrm{CO}_{2}$ alone was unattractive and the pheromone blend alone was only moderately attractive.

An additional tick-produced compound, 1-octen-3-ol, was identified from various tick materials (disturbed adults, exuviae, faeces, carcasses) of A. variegatum (McMahon et al. 2001). In servosphere trials, 1-octen-3-ol elicited attraction in unfed A. variegatum, however, it is unclear how this compound mediates tick responses and if it would play any role in host attraction. Previous studies investigated 1-octen-3-ol as a possible attractant for $A$. variegatum as it is a component of ruminant digestion, however, no significant attraction was detected (Norval et al. 1987).

\section{Arrestment pheromones}

Arrestment or assembly pheromones cause locomotion to stop and result in clusters of individuals in the environment. Upon contacting these pheromones, ticks cease crawling, curl their legs under the body and become akinetic (Sonenshine 2004, 2006). These pheromones act within and between sexes, may affect immatures and tend to be present in caves, under ledges, rookeries, in cracks or crevices or other sheltered locations (Sonenshine 1991). They are particularly widespread in nidicolous soft ticks (at least 14 species in the Argasidae family) but are also reported from at least eight species of hard ticks (e.g. I. scapularis, I. ricinus, R. appendiculatus (Sonenshine 1991)). These assemblies may enhance contact with potential hosts, enhance survival by keeping ticks in favourable microhabitats and ensuring mating for those species that mate off hosts. Guanine and other related purines such as uric acid, xanthine, hypoxanthine and inosine which are constituents of tick faeces and present in exuvia comprise the active components of this pheromone (Allan and Sonenshine 2002, Dusbábek et al.1991, Grenacher et al. 2001, Hassanali et al. 1989, Otieno et al. 1985, Sonenshine et al. 2003). Haematin and 8-azaguanine also contributed to assembly responses (Grenacher et al. 2001, Sonenshine et al. 2003). Excretion of nitrogenous waste products by ticks generally occurs after blood-feeding and immediately after moulting. Locations with accumulations of these materials are likely indicative of an environment favourable for survival and the historic presence of a host (Otieno et al. 1985). Benoit et al. (2008) reported that guanine and other components of tick excreta function as an assembly pheromone for the seabird tick, $I$. 
uriae, in promoting the formation of off-host aggregations, while uric acid and other components of penguin guano functioned as kairomones used by the tick to locate its penguin host. Similarly, unfed $A$. americanum responded to an assembly pheromone, which contains uric acid and suggests a kairomonal role of uric acid from bird and reptile faeces. The latter animals are the preferred hosts for immatures of this species (Yoder et al. 2008). Such clustering of unfed ticks in the environment greatly enhances successful host contact if clusters are located optimally.

\section{Factors that enhance chemical attractants}

In nature, chemical attractants are rarely present without other factors that often enhance or synergise responses of tick responses. Although eyes and light receptors are simple in ticks, visual contrast against a background or detection of movement are known to enhance responses (Philis and Cromroy 1977, Waladde and Rice 1982). Radiant heat has been reported to be synergistic when combined with host odours (Lees 1948, Sonenshine 1993) and is detected by thermoreceptors on the tarsi of the first pair of legs, which are waved in the air during active searching.

\section{Practical applications}

\section{Surveillance}

Host cues have been used successfully for the development of effective surveillance tools for arthropods. As $\mathrm{CO}_{2}$ is known to activate and possibly attract ticks at a considerable distance from the host, it has been examined for tick surveillance. A trap using $\mathrm{CO}_{2}$ as an attractant used for collection of I. ricinus (Gray 1985) attracted ticks from distances of up to $3.5 \mathrm{~m}$ and subsequently attraction for numerous species of ticks has been reported. Attraction of $A$. americanum to $\mathrm{CO}_{2}$ was reported to be from several meters (Sonenshine 1993) and Wilson et al. (1972) collected $A$. americanum from as far away as $21 \mathrm{~m}$. $\mathrm{CO}_{2}$-baited traps have been used to sample a wide range of species including O. turicata Duges (Adeyeye and Butler 1991). Solberg et al. (1992) reported that dry ice-baited traps collected larger number of I. scapularis and A. americanum than did drag or human-host collections.

Host odours or tick-produced odours have also been combined with $\mathrm{CO}_{2}$ for surveillance. Attraction of $A$. hebraeum to traps baited with $\mathrm{CO}_{2}$ in conjunction with an AAA pheromone was effective from distances as far as $11 \mathrm{~m}$ in 90 minutes (Norval et al. 1989a). Subsequently these traps were used for surveillance of Ehrlichia (Cowdria) ruminantium in field populations (Norval et al. 1990). Use of synthetic AAA pheromone (methyl salicylate, o-nitrophenol, nonanoic acid) greatly increased the efficacy of $\mathrm{CO}_{2}$ traps for $A$. variegatum in Guadeloupe (Barré et al. 1997). Attraction of $A$. variegatum to different doses of AAA pheromone and $\mathrm{CO}_{2}(500 \mathrm{ml} / \mathrm{min})$ resulted in collection of up to $90 \%$ of the released ticks (Maranga et al. 2003) and these traps were used to evaluate efficacy of a subsequent control study (Maranga et al. 2006). Because attractants are considered particularly useful for ticks using the hunter strategy for host-finding (Sonenshine 1993), they have promise for development of effective surveillance strategies for these ticks.

Another standard approach for tick surveillance is the use of the tick drag or flag (Sonenshine 1993). The addition of host odour (from sheep wool) onto the cloth increased efficacy from 2.4-2.8 fold (for flagging and dragging, respectively) for I. rubicundus Neumann. Additionally, duration of tick contact on the cloth increased from 9.1 seconds to 32.9 seconds (Fourie et al. 1995). 


\section{Targeted on-host control}

Chemical attractants have been evaluated for targeted on-host control of ticks by combining attractant compounds with acaricides. In an initial study by Gladney et al. (1974), extracts of blood-fed males of $A$. maculatum, which contained an attractant pheromone, combined with insecticide were used to kill female ticks that attached to the treated site. A tick decoy concept was developed using the AAA pheromones for targeted control of the bont ticks, A. variegatum and $A$. hebraeum, on cattle (Norval et al. 1996). Components of the AAA pheromone (methyl salicylate, o-nitrophenol) combined with a tick sex pheromone (2,6-dichlorophenol) and another attractant (phenylacetaldehyde), along with insecticides, were attached to tails of cattle. The pheromones served as attractants for unfed nymphs, males and females to the site on the body where pesticide was present. Once at the treated site, prolonged contact during the aggregation, attachment and feeding behaviour resulted in mortality. When these tags were evaluated in Zimbabwe against $A$. hebraeum, tick numbers decreased on cattle over a 12 week period (Norval et al. 1996). In the two trials, efficacy ranged from 87.5 to $99.3 \%$ for cyfluthrin and flumethrin-impregnated tags. In an additional study in Guadeloupe, acaricide and AAA-impregnated tags were attached to the necks and tails of cattle for control of $A$. variegatum (Allan et al. 1998). In this trial, tick numbers were reduced similarly between pheromone and acaricide tags and acaricide tags only. Cattle supplied with pheromone tags had higher proportions of ticks on the neck (18.2\%) and hind ( $81 \%)$ regions of the body than did cattle with no pheromone tags (necks $8.2 \%$, hind $62.5 \%$ ), indicating that pheromone delivery did affect tick aggregations. While this approach appears promising, efficacy is difficult to evaluate as dead ticks may not remain on the host.

There is potential for a push-pull approach for tick control to be developed with the discovery of semiochemicals that mediate attraction to different attachment sites by the vectors of East Coast fever, $R$. appendiculatus and $R$. evertsi on cattle. Such an approach could combine the use of a synthetic or botanical tick repellent at the ear and an attractant-baited trap treated with a fungal pathogen or acaricide located on the back of the animal (Hassanali et al. 2008).

\section{Targeted off-host control}

Several approaches have been taken for targeted off-host control of ticks that incorporate olfactory cues that facilitate host location. The combination of $\mathrm{CO}_{2}(500 \mathrm{ml} / \mathrm{min})$ with AAA pheromones as attractants to self-treatment traps baited with spores of fungal conidia (Beauveria bassiana (Bals.) and Metarhizium anisopliae (Sorok.)) appeared effective in treating ticks released onto a field plot (Maranga et al. 2006). Three weeks after the treatment only $34 \%$ of the released ticks were recovered on plots with fungi whereas 76 to $85 \%$ of ticks were recovered on control plots.

The combination of arrestment pheromone components with acaricides was effective in laboratory studies with Argas persicus (Oken) (Dusbábek et al. 1997). For I. scapularis, the combination of pheromone components into a slow-release matrix along with permethrin provided promising results when evaluated in a petri dish assay (Sonenshine et al. 2003). Application of this material on vegetation in a field trial resulted in significant reduction of I. scapularis nymphs near Armonk, New York (T. Daniels and R. Falco, personal communication).

\section{Repellents}

One of the most effective methods for prevention of tick-borne diseases of humans is the use of repellents. Towards this end, considerable effort has been made to evaluate compounds that 
interfere with host-finding by ticks or deter ticks from treated sites. Different systems for testing tick repellents have been reviewed by Dautel (2004) who differentiated between assays that evaluate repellency in the absence or presence of host stimuli. In the absence of host stimuli, such in petri dishes or tick climbing assays, repellency is measured by the absence of ticks in a location containing the potential repellent. These assays do not correspond directly to sensory perception of potential hosts but may impact behavioural steps that contribute to successful host-finding (i.e. climbing vegetation, arrestment in locations suitable for the host). In assays that include host stimuli, repellency is measured by avoidance in the presence of host stimuli. Studies by Carroll et al. (2005) indicated that I. scapularis and A. americanum responded to DEET ( $\mathrm{N}, \mathrm{N}$-diethyl-3methylbenzamide) and SS220 (1S,2S'-2-methylpiperidinyl-3-cyclohexene-1-carboxamide) in the vapour phase to avoid host contact. Similarly Dautel et al. (1999) reported that I. ricinus nymphs would not contact DEET-treated filter paper. McMahon et al. (2003) reported that indalone in an airstream caused $A$. variegatum to walk downwind, however airborne DEET did not repel ticks attracted to the AAA pheromone. Permethrin, while a toxicant, also repels a range of tick species and is used for treatment of military uniforms (Mehlhorn et al. 2003, McCain and Leach 2007, Schreck et al. 1995). In contrast, a less common effect has been reported in which fabric impregnated with permethrin as a repellent enhanced attachment behaviour of $H$. dromedarii with more rapid attachment with greater numbers attached than the untreated controls (Fryauff et al.1994). These results were presumably related to the increased neurosecretory production which activated salivary secretion in $\mathrm{H}$. dromedarii ticks exposed to permethrin (Mohamed et al. 2000). Questions remain as to the mode of action of many repellents (i.e. deterrency, inhibition of attraction, masking of detection)(Bernier et al. 2007) and as to whether repellent compounds act by olfaction, contact with gustatory receptors or both.

Host-derived volatiles that interfere with attraction are potential sources for new repellent compounds for several insect species (Bernier et al. 2007, Logan et al. 2009, Weldon and Carroll 2007) and may also hold potential for ticks. Research on volatile compounds emitted from unsuitable predilection sites on hosts such as cattle (Wanzala et al. 2004) may provide new approaches for management of livestock from tick infestation and tick-borne diseases using a push-pull approach (Hassanali et al. 2008). The basis for naturally 'tick-resistant' breeds or species of hosts appears to be related to host immune response (Martinez et al. 2006), however, differences in volatile emissions (Weldon and Carroll 2007), if present, provide potential compounds useful for livestock protection. Plant-based compounds evaluated against ticks may provide new active ingredients for protection of human and even livestock (Bissinger et al. 2009, Carroll et al. 2007, Dietrich et al. 2006, Feaster et al. 2009, Palsson et al. 2008).

The use of host odours for development of in vitro feeding systems for hard ticks can address ethical concerns, high costs of maintaining suitable hosts and provides opportunities for development of products against ticks (Kröber and Guerin 2007). Feeding on membranes has been facilitated by the addition of the AAA pheromones, host hair, tick faeces and bovine pelage (Kröber and Guerin 2007, Kuhnert et al. 1995). Development of these feeding systems could accelerate development of methods for high throughput screening of candidate repellents.

\section{Conclusions}

The challenges for ticks to find potential hosts in a complex environment are met, at least partially, by an array of olfactory cues. These cues are primarily host-produced but for some species, tickproduced odours also contribute to host location. One of the most well-studied tick species is A. variegatum, which feeds primarily on large ungulate mammals. This tick, highly responsive 
to rumen odours and AAA pheromones, may only provide some insight into the olfactory cues used by other tick species. Interactions between ticks and potential hosts are complex and our understanding of them has been advanced significantly through the use of electrophysiological, sophisticated behavioural and highly sensitive analytical chemical methods. Certainly, additional research into the chemical ecology of additional tick-host interactions will provide compounds that can be used to develop new surveillance methods or tools for development of push-pull strategies against ticks that are both pests of vectors of human and livestock diseases.

The field of insect olfactory ecology is advancing quickly with the convergence of genomic, physiological, and ecological data and detailed examination of the processes between odour molecules and odour receptor proteins (De Bruyne and Baker 2008). Relatively little, however, is known for ticks about the three major groups of proteins (odorant receptors, odorant-binding proteins and odour-degrading enzymes) that are of critical importance to insect olfactory systems for detection of many of the same chemicals. These may provide feasible molecular targets for the development of attractants and novel repellent strategies. For ticks, progress in our understanding of the molecular basis for olfaction has been slower and important differences between insect and acarines may be discovered. Robertson and Kent (2009) examined the evolution of three protein genes related to the carbon dioxide receptor in three species of mosquitoes, the silk moth and red flour beetles. That gene lineage is absent from the I. scapularis genome indicating that ticks utilise another pathway to detect carbon dioxide. Unique gene evolutionary processes in ticks have also been reported by Wang et al. (2007), and with completion of sequencing of different tick genome, a better understanding of these processes will be obtained. The degree of conservation within insect odorant receptor genes and their relation to acarine genes is yet to be determined and this information will provide insight into approaches for rational design of novel control strategies targeted against gene orthologs.

\section{References}

Adeyeye OA and Butler JF (1991) Field evaluation of carbon dioxide baits for sampling Ornithodoros turicata (Acari: Argasidae) in gopher tortoise burrows. J Med Entomol 28: 45-48.

Allan SA, Barré N, Sonenshine DE and Burridge MJ (1998) Efficacy of tags impregnated with pheromone and acaricide for control of Amblyomma variegatum. Med Vet Entomol 12: 141-150.

Allan SA and Sonenshine DE (2002) Evidence of an assembly pheromone in the black-legged tick, Ixodes scapularis. J Chem Ecol 28: 15-27.

Anderson JF and Magnarelli LA (2008) Biology of ticks. In: Edlow JA (ed) Infectious disease clinics of North America: tick borne diseases, Lyme disease. WB Saunders, Philadelphia, USA, pp. 195-215.

Anderson RB, Scrimgeour GJ and Kaufman WR (1998) Responses of the tick, Amblyomma hebraeum (Acari: Ixodidae), to carbon dioxide. Exp Appl Acarol 22: 667-681.

Apps PJ, Viljoen HW and Pretorius V (1988) Aggregation pheromone of the bont tick Amblyomma hebraeum: identification of candidates for bioassay. Onderstepoort J Vet Res 55: 135-137.

Barré N, Garris Gl and Lorvelec O (1997) Field sampling of the tick Amblyomma variegatum (Acari: Ixodidae) on pastures in Guadeloupe; attraction of $\mathrm{CO}_{2}$ and/or tick pheromones and conditions of use. Exp Appl Acarol 21: 95-108.

Belan I and Bull CM (1991) Host detection by four Australian tick species. J Parasitol 77: 337-340.

Beelitz P and Gothe R (1991) Investigations on the host-seeking and finding of Argas (Persicargas) walkerae (Ixodoidea: Argasidae). Parasitol Res 77: 622-628.

Benoit JB, Lopez-Martinez G, Phillips SA, Elnitsky MA, Yoder JA, Lee RE Jr and Denlinger DL (2008) The seabird tick, Ixodes uriae, uses uric acid in penguin guano as a kairomone and guanine in tick feces as an assembly pheromone on the Antarctic Peninsula. Polar Biol 31: 1445-1451. 
Bernier UB, Kline DL and Posey KH (2007) Human emanations and related natural compounds that inhibit mosquito host-finding abilities. In: Debboun M, Frances SP and Strickman D (eds) Insect repellents principles, methods, and uses. CRC Press, New York, USA, pp 77-100.

Bissinger BW, Apperson CS, Sonenshine DE, Watson DW and Roe RM 2009. Efficacy of the new repellent BioUD ${ }^{\circledR}$ against three species of ixodid ticks. Exp Appl Acarol 48: 239-250.

Burger BV, Marx A, Roux ML and Oelofsen BW (2006) Characterization of dog repellent factor from cuticular secretion of female yellow dog tick, Haemaphysalis leachi. J Chem Ecol 32: 125-136.

Bursell E, Gough AJE, Beevor PS, Cork A, Hall DR and Vale GA (1988) Identification of components of cattle urine attractive to tsetse flies, Glossina spp. (Diptera: Glossinidae). Bull Entomol Res 78: 281-291.

Carroll JF (1999) Responses of three species of adult ticks (Acari: Ixodidae) to chemicals in the coats of principal and minor hosts. J Med Entomol 36: 238-242.

Carroll JF (2000) Responses of adult Ixodes scapularis (Acari: Ixodidae) to urine produced by white-tailed deer of various reproductive conditions. J Med Entomol 37: 472-475.

Carroll JF (2001) Interdigital gland substances of white-tailed deer and the response of host-seeking ticks (Acari: Ixodidae). J Med Entomol 38: 114-117.

Carroll JF (2002) How specific are host-produced kairomones to host-seeking ixodid ticks? Exp Appl Acarol 28: 155-161.

Carroll, JF, Russek-Cohen E, Nichols JD and Hines JE (1991) Populations dynamics of American dog ticks (Acari: Ixodidae) along park trails. Environ Entomol 20: 922-929.

Carroll JF, Klun JA and Schmidtmann ET (1995) Evidence for kairomonal influence on selection of host-ambushing sites by adult Ixodes scapularis (Acari: Ixodidae). J Med Entomol 32: 119-125.

Carroll JF, Mills GD and Schmidtmann ET (1996) Field and laboratory responses of adult /xodes scapularis (Acari: Ixodidae) to kairomones produced by white-tailed deer. J Med Entomol 33: 640-644.

Carroll JF, Klun JA and Debboun M (2005) Repellency of Deet and SS220 applied to skin involves olfactory sensing by two species of ticks. Med Vet Entomol 19: 101-106.

Carroll, JF, Cantrell CL, Klun JA and Kramer M (2007) Repellency of two terpenoid compounds isolated from Callicarpa americana (Lamiaceae) against Ixodes scapularis and Amblyomma americanum ticks. Exp Appl Acarol 41:215-224.

Crooks E and Randolf SE (2006) Walking by Ixodes ricinus ticks: intrinsic and extrinsic factors determine the attraction of moisture or host odour. J Exp Biol 209: 2183-2142.

Dautel H (2004) Test systems for tick repellents. Int J Med Microbiol 293 Suppl 37: 182-188.

Dautel H, Kahl O, Siema K, Oppenrieder M, Muller-Kuhrt L and Hilker M (1999) A novel test system for detection of tick repellents. Ent Exp Appl 91: 431-441.

De Bruyne M and Baker TC (2008) Odor detection in insects: volatile codes. J Chem Ecol 34: 882-897.

De Bruyne M and Guerin PM (1994) Isolation of 2,6-dichlorophenol from the cattle tick Boophilus microplus: receptor cell responses but no evidence for a behavioural response. J Insect Physiol 40: 143-154.

Dethier VG, Baron Browne L and Smith CN (1960) The designation of chemicals in terms of the responses they elicit from insects. J Econ Entomol 53: 134-136.

Diehl, PA, Guerin PM, Vlimant M and Steullet P (1991) Biosynthesis, production site, and emission rates of aggregationattachment pheromone in male of two Amblyomma ticks. J Chem Ecol 17: 833-847.

Dietrich G, Dolan MC, Peralta-Cruz J, Schmidt J, Piesman J, Eisen RJ and Karchesy JJ (2006) Repellent activity of fractioned compounds from Chamaecyparis nootkatensis essential oil against nymphal Ixodes scapularis (Acari: Ixodidae). J Med Entomol 43: 957-961.

Dobrotvorsky AK, Tkachev AV, Maumov RL and Carroll JF (2000) Study of host odour determinants of questing behavior in Ixodes ticks. In: Proc $3^{\text {rd }}$ International conference on Tick and Tick-borne Pathogens, High Tatra Mountains, Slovakia, pp 235-240.

Donzé G, McMahon and Guerin PM (2004) Rumen metabolites serve ticks to exploit large mammals. J Exp Biology 207: 4283-4289.

Dukes JC and Rodriguez JC (1976) A bioassay for host-seeking response of tick nymphs (Ixodidae). J Kansas Entomol Soc 49: 562-566. 
Dusbábek F, Simek P, Jegorov A and Troska J (1991) Identification of xanthine and hypoxanthine as components of assembly pheromone in excreta of argasid ticks. Exp Appl Acarol 11:307-316.

Dusbábek F, Rupeš V, Šimek P and Zahradínčková H (1997) Enhancement of permethrin efficacy in acaricide-attractant mixtures for control of the fowl tick Argas persicus (Acari: Argasicae). Exp Appl Acarology 21: 293-305.

Douglas HD III, Co JE, Jones TH and Conner WE (2004) Interspecific differences in Anthia spp. auklet odorants and evidence for chemical defense against ectoparasites. J Chem Ecol 30: 1921-1934.

El-Ziady S (1958) The behavior of Ornightodoros erraticus (Lucas, 1849), small form (Ixodoidea, Argasidae), towards certain environmental factors. Ann Entomol Soc Amer 51:317-336.

Feaster JE, Scialdone MA, Todd RG, Gonzalez YI, Foster JP and Hallahan DL (2009) Dihydronepetalactones deter feeding activity by mosquitoes, stable flies, and deer ticks. J Med Entomol 46: 832-840.

Fryauff DJ, Shoukry MA and Schreck CE (1994) Stimulation of attachment in a camel tick, Hyalomma dromedarii (Acari: Ixodidae): the unintended result of sublethal exposure to permethrin-impregnated fabric. J Med Entomol 31:23-29.

Fourie LJ, Snyman A, Kok DJ, Horak IG and van Zyl JM (1993) The appetence behavior of two south African paralysisinducing ixodid ticks. Exp Appl Acarol 17: 921-930.

Fourie LJ, Van der Lingen F and Kok DJ (1995) Improvement of field sampling methods for adult Karoo paralysis ticks, Ixodes rubicundus (Acari: Ixodidae), through addition of host odour. Exp Appl Acarol 19: 93-101.

Ginsberg HS and Ewing CP (1989) Comparison of flagging, walking, trapping and collecting from hosts as sampling methods for northern deer ticks, Ixodes dammini,and lone star ticks, Amblyomma americanum (Acari: Ixodidae). Exp Appl Acarol 7: 313-322.

Gladney WJ, Grabbe RR, Ernst SE and Oehler DD (1974) The Gulf Coast tick: evidence of a pheromone produced by males. J Med Entomol 11: 303-306.

Gray JS (1985) A carbon dioxide trap for prolonged sampling of Ixodes ricinus L. populations. Exp Appl Acarol 1: 35-44.

Grenacher S, Kröber T, Guerin PM and Vlimant M. (2001) Behavioral and chemoreceptor cell responses of the tick, Ixodes ricinus, to its own faeces and faecal consitituents. Exp Appl Acarol 25: 641-660.

Guerin PM, Kröber T, McMahon C, Guerenstein P, Genacher S, Vlimant M, Diehl P, Steullet P and Syed Z (2000) Chemosensory and behavioural adaptations of ectoparasitic arthropods. Noza Acta Leopoldina NF $83 \mathrm{nr} 316: 213-$ 229.

Haggart DA and Davis EE (1980) Ammonia-sensitive neurons on the first tarsi of the tick, Rhipicephalus sanguineus. J Insect Physiol 26: 517-523.

Hall DR, Beevor PS, Cork A, Nesbitt BF and Vale GA (1984) 1-octen-3-ol. A potent olfactory stimulant and attractant for tsetse isolated from cattle odours. Insect Sci Appl 5: 335-339.

Hassanali A, McDowell PC, Owaga MLA and Saini RK (1986) Identification of tsetse attractants from excretory products of a wild host animal, Syncerus caffer. Insect Sc Appl 7: 5-9.

Hassanali A, Nyandat E, Obenchain FD, Otieno DA and Galun R (1989) Humidity effect on response of Argas persicus (Oken) to guanine, an assembly pheromone of ticks. J. Chem Ecol 15: 791-798.

Hassanali A, Herren H, Khan ZR, Pickett JA and Woodcock CM (2008) Integrated pest management: the push-pull approach for controlling insect pests and weeds of cereals, and its potential for other agricultural systems including animal husbandry. Phil Trans R Soc B 363: 611-621.

Hess E and De Castro JJ (1986) Field tests of the response of female Amblyomma variegatum (Acari: Ixodidae) to the synthetic aggregation-attachment pheromone and its components. Exp Appl Acarol 2: 249-255.

Hess E and Vlimant M (1986) Leg sense organs of ticks. In: Sauer JR and Hair JA (eds) Morphology, physiology and behavioural biology of ticks. Ellis Horwood, Chicester, UK, pp 361-390.

Holscher KH, Gearhart HL and Barker RW (1980) Electrophysiological response of three tick species to carbon dioxide in the laboratory and field. Ann Entomol Soc Am 73: 288-292.

Kimura K, Isogai E, Isogai H, Kamewaka Y, Nishikawa T, Ishii N and Fujii N (1995) Detection of Lyme disease spirochetes in the skin of naturally infected wild Sika deer (Cervus nippon yesoensis) by PCR. Appl Environ Micro 61: 1641-1642.

Klompen JSH, Black WC IV, Keirans JE and Oliver JH Jr (1996) Evolution of ticks. Ann Rev Entomol 41: 141-161.

Kneidel KA (1984) Carrion as an attractant to the American dog tick, Dermacentor variabilis (Say). J NY Entomol Soc 92: 405-406. 
Kröber T and Guerin PM (2007) An in vitro feeding assay to test acaricides for control of hard ticks. Pest Manag Sci 62: $17-22$.

Kuhnert F, Diehl PA and Guerin PM (1995) The life-cycle of the bont tick Amblyomma hebraeum in vitro. Int J Parasitol 25: 887-896.

Lees AD (1948) The sensory physiology of the sheep tick, Ixodes ricinus (L.) J Exp Biol 25: 145-207.

Lees AD (1969) The behaviour and physiology of ticks. Acarologia 11: 397-406.

Leonovich SA (2004) Phenol and lactone receptors in the distal sensilla of the Haller's organ in Ixodes ricinus ticks and their possible role in host perception. Exp Appl Acarol 32: 89-102.

Logan, JG, Birkett MA, Clark SJ, Powers S, Seal NJ, Wadhams LJ, Mordue (Luntz) JA and Pickett JA (2009) Identification of human-derived volatile chemicals that interfere with attraction of Aedes aegypti mosquitoes J Chem Ecol 34: 308-322.

Lusby WR, Sonenshine DE, Yunker CE, Norval RA and Burridge MJ (1991) Comparison of known and suspected pheromonal constituents in males of the African ticks, Amblyomma hebraeum Koch and Amblyomma variegatum (Fabricius). Exp Appl Acarol 13: 143-152.

Maranga RO, Hassanali A, Kaaya GP and Mueke JM (2003) Attraction of Amblyomma variegatum (ticks) to the attractionaggregation-attachment pheromone with or without carbon dioxide. Exp Appl Acarol 29: 121-130.

Maranga RO, Hassanali A, Kaaya GP and Mueke JM (2006) Performance of a prototype baited-trap in attracting and infecting the tick Amblyomma variegatum (Acari: Ixodidae) in field experiments. Exp Appl Acarol 38: 211-218.

Martinez ML, Machado MA, Nascimento CS, Silva MVGB, Teodoro RL, Furlong J, Prata MCA, Campos AL, Guimaraes MFM, Azevedo ALS, Pires MFA and Verneque RS (2006) Association of BoLA-DRB3.2 alleles with tick (Boophilus microplus) resistance in cattle. Genetics Mol Res 5: 513-524.

McCain WC and Leach GJ (2007) Repellents used in fabric: The experience of the U.S. military. In: Debboun M, Frances SP and Strickman D (eds) Insect repellents principles, methods, and uses. CRC Press, New York, USA, pp. 261-274.

McNemee RB Jr, James WJ IV and Maloney FA Jr (2003) Occurrence of Dermacentor variabilis (Acari: Ixodidae) around a porcupine (Rodentia: Erthethizontidae) carcass at Camp Ripley, Minnesota. J Med Entomol 40: 108-111.

McMahon C and Guerin PM (2000) Responses of the tropical bont tick, Amblyomma variegatum (Fabricius), to its aggregation-attachment pheromone presented in an air stream on a servosphere. J Comp Physiol A 186: 95-103.

McMahon C and Guerin PM (2002) Attraction of the tropical bont tick, Amblyomma variegatum, to human breath and to the breath components acetone, $\mathrm{NO}$ and $\mathrm{CO}_{2}$. Naturwissen 89: 311-315.

McMahon C, Guerin PM and Syed Z (2001) 1-octen-3-ol isolated from bont ticks attracts Amblyomma variegatum. J Chem Ecol 27: 471-486.

McMahon C, Kröber K and Guerin PM (2003) In vitro assays for repellents and deterrents for ticks: differing effects of products when tested with attractant or arrestment stimuli. Med Vet Entomol 17: 370-378.

Mehlhorn H, Schmahl G, Mencke N and Bach T (2003) In vitro and in vivo studies on the effect of a combination containing 10\% imidacloprid and 50\% permethrin against /xodes ricinus ticks. Parasitol Res 89: 323-325.

Mohamed FSA, Abbassy MM, Darwish ZEA, Tetreault, Matzouk AS, Shoukry MA, Fryauff DJ and Beavers GM (2000) Effects of permethrin on the salivary glands and neuroendocrine organs of unfed female Hyalomma (Hyalomma) dromedarii (Ixodoidea: Ixodidae). J Med Entomol 37: 393-400.

Neitz AWH and Gothe R (1984) Investigations into the volatility of female pheromones and the aggregation-inducing property of guanine in Argas (Persicargas) walkerae. Onderstepoort J Vet Res 51: 197-201.

Newhouse V (1983) Variations in population density, movement and rickettsial infection rates in a local population of Dermacentor variabilis (Acarina: Ixodidae) ticks in the Piedmont of Georgia. Environ Entomol 2: 1737-1746.

Norval RAI and Rechav YH (1979) An assembly pheromone and its perception in the tick Amblyomma variegatum (Acarina, Ixodidae). J Med Entomol 16: 507-511.

Norval RAI, Yunker CE and Butler JF (1987) Field sampling of unfed adults of Amblyomma hebraeum Koch. Exp Appl Acarol 3: 213-217.

Norval RAI, Butler JF and Yunker CE. (1989a) Use of carbon dioxide and natural or synthetic aggregation-attachment pheromone of the bont tick, Amblyomma hebraeum, to attract and trap unfed adults in the field. Exp Appl Acarol 7: 171-180. 
Norval, RAI, Andrew HR and Yunker CE (1989b) Pheromone-mediation of host-selection in bont ticks (Amblyomma hebraeum Koch). Science 243: 364-365.

Norval, RAl, Andrew HR and Yunker CE (1990) Infection rates with Cowdria ruminantium of nymphs and adults of the bont tick (Amblyomma hebraeum) collected in the field in Zimbabwe. Vet Parasitol 36: 277-283.

Norval RAI, Peter T, Yunker CE, Sonenshine DE and Burridge MJ. (1991) Responses to the ticks Amblyomma hebraeum and $A$. variegatum to known or potential components of the aggregation-attachment pheromone. II. Attachment stimulation. Exp Appl Acarol 13: 19-26.

Norval RAl, Peter T, Yunker CE, Sonenshine DE and Burridge MJ (1993) Response of the ticks Amblyomma hebraeum and $A$. variegatum to known or potential components of the aggregation-attachment pheromone. III. Short-range attraction and aggregation. Exp Appl Acarol 16: 237-245.

Norval RAI, Sonenshine DE, Allan SA and Burridge MJ (1996) Efficacy of pheromone-acaricide-impregnated tail-tag decoys for controlling the bont tick, Amblyomma hebraeum (Acari: Ixodidae) on cattle in Zimbabwe. Exp Appl Acarol 20: 31-46.

Osterkamp J, Wahl U, Schmulfuss G and Haas W (1999) Host-odour recognition in two tick species is coded in a blend of vertebrate volatiles J Comp Physiol A 185: 59-67.

Otieno DA, Hassanali A, Obenchain FD, Sternberg A and Galun R (1985) Identification of guanine as an assembly pheromone of ticks. Insect Sci Appl 6: 667-670.

Palsson K, Jaenson TGT, Baeckstrom and Borg-Karlson A (2008) Tick repellent substances in the essential oil of Tanacetum vulgare. J Med Entomol 45: 88-93.

Perritt, DW, Couger G and Barker RW (1993) Computer-controlled olfactometer system for studying responses of ticks to carbon dioxide. J Med Entomol 30: 571-578.

Philis WA and Cromroy HL (1977) The microanatomy of the eye of Amblyomma americanum (Acari: Ixodidae) and resultant implications of its structure. J Med Entomol 13: 685-698.

Randolph SE, Gern L and Nuttall PA (1996) Co-feeding ticks: Epidemiological significance for tick-borne pathogen transmission. Parasitol Today 12: 472-479.

Rechav Y, Goldberg M and Fielden L (1997) Evidence for attachment pheromones in the Cayenne tick (Acari: Ixodidae). J Med Entomol 34: 234-237.

Rechav Y, Norval RAI, Tannock J and Colborne J (1978) Attraction of the tick Ixodes neitzi to twigs marked by the klipspringer antelope. Nature 275: 310-311.

Robertson HM and Kent LB (2009) Evolution of the gene lineage encoding the carbon dioxide receptor in insects. J Insect Sci 9: 19, available online: insectscience.org/9.19. Accessed 5 Oct 2009.

Sauer JR, Hair JA and Houts MS (1974) Chemo-attraction in the lone star tick (Acarina: Ixodidae). 2. Responses to various concentrations of $\mathrm{CO}_{2}$. Ann Entomol Soc Amer 67: 150-152.

Schöni, R, Hess E, Blum W and Ramstein K. (1984) The aggregation-attachment pheromone of the tropical bont tick Amblyomma variegatum, Fabricius (Acari: Ixodoidea): isolation, identification and action of its components. J Insect Physiol 30: 613-618.

Schreck CE, Fish D and McGovern TP (1995) Activity of repellents applied to skin for protection against Amblyomma americanum and Ixodes scapularis ticks (Acari: Ixodidae). J Amer Mosq Control Assoc 11: 136-140.

Schulze TL, Jordan RA and Hung RW (1997) Biases associated with several sampling methods used to estimated abundance of Ixodes scapularis and Amblyomma americanum (Acari: Ixodidae). J Med Entomol 34: 615-623.

Shorey HH (1977) Interaction of insects with their chemical environment. In: Shorey HH and McKelvey JJ Jr (eds) Chemical control of insect behavior theory and application. John Wiley \& Sons. New York, USA, pp. 1-6.

Solberg VB, Neidhardt K, Sardelis MR, Hildbrandt C, Hoffmann FJ and Boobar LR (1992) Quantitative evaluation of sampling methods for Ixodes dammini and Amblyomma americanum (Acari: Ixodidae). J Med Entomol 29: 451-456.

Sonenshine DE (1991) Biology of ticks, Volume 1. Oxford University Press, New York, USA.

Sonenshine, DE (1993) Biology of ticks, Volume 2. Oxford University Press, New York, USA.

Sonenshine DE (2004) Pheromones and other semiochemicals of ticks and their use in tick control. Pararsitol 129: S405-S425.

Sonenshine DE (2006) Tick pheromones and their use in tick control. Ann Rev Entomol 51: 557-580. 
Sonenshine DE, Adams T, Allan SA, McLaughlin JR and Webster FX (2003) Chemical composition of some components of the arrestment pheromone of the black-legged tick, Ixodes scapularis (Acari: Ixodidae), and their use in tick control. J Med Entomol 40: 849-859.

Stafford KC III, Bladen VC and Magnarelli LA (1995) Ticks (Acari: Ixodidae) infesting wild birds (Aves) and white-footed mice in Lyme, CT. J Med Entomol 32: 453-466.

Steullet P (1993) Perception of vertebrate volatiles in the tropical bont tick, Amblyomma variegatum Fabricius. Dissertation, Université de Neuchâtel, Neuchâtel, Switzerland.

Steullet P and Guerin PM (1992a) Perception of breath components by the topical bont tick, Amblyomma variegatum Fabricius (Ixodidae) I. $\mathrm{CO}_{2}$-excited and $\mathrm{CO}_{2}$-inhibited receptors. J Comp Physiol A 170: 665-676.

Steullet P and Guerin PM (1992b) Perception of breath components by the tropical bont tick Amblyomma variegatum Fabricius (Ixodidae) II. Sulfide receptors. J Comp Physiol A 170: 677-685.

Steullet P and Guerin PM (1994a) Identification of vertebrate volatiles stimulating olfactory receptors on tarsus 1 of the tick Amblyomma variegatum Fabricius (Ixodidae) I. Receptors within the Haller's organ capsule. J. Comp Physiol A 174: 27-38.

Steullet P and Guerin PM (1994b) Identification of vertebrate volatiles stimulating olfactory receptors on tarsus 1 of the tick Amblyomma variegatum Fabricius (Ixodidae) II. Receptors outside the Haller's organ capsule. J. Comp Physiol A 174: 39-47.

Taneja J and Guerin PM (1997) Ammonia attracts the hemaematophagous bug Triatoma infestans: behavioural and neurophysiological data on nymphs. J Comp Physio A 181: 21-34.

Waladde SM (1987) Receptors involved in host location and feeding in ticks. Insect Sci Appl 8: 643-647.

Waladde SM and Rice MJ (1982) The sensory basis of tick feeding behaviour. In: Obenchain FD and Galun R (eds) The physiology of ticks. Pergamon Press, Oxford, UK, pp 71-118.

Wanzala W, Sika NFK, Gule S and Hassanali A (2004) Attractive and repellent host odours guide ticks to their respective feeding sites. Chemoecology 14: 229-232.

Wang M, Guerrero FD, Pertea G and Nene VM (2007) Global comparative analysis of ESTs from the southern cattle tick, Rhipicephalus (Boophilus) microplus. BMC Genomics 8: 368.

Weldon PJ and Carroll JF (2007) Vertebrate chemical defense: secreted and topically acquired deterrents of arthropods. In: Debboun M, Frances SP and Strickman D (eds) Insect epellents principles, methods, and uses. CRC Press, New York, USA, pp. 47-75.

Wilkinson PR (1953) Observations on the sensory physiology and behaviour of larvae of the cattle tick, Boophilus microplus (Can.)(Ixodidae). Austr J Zool 1:345-516.

Wilson JG, Kinzer DR, Sauer JR and Hair JA (1972) Chemo-attraction in the lone star tick (Acarina: Ixodidae). I. Response of different developmental stages to carbon dioxide administered via traps. J Med Entomol 9:245-252.

Yoder JA, Atwood AD and Stevens BW (1998) Attraction to squalene by ticks (Acari: Ixodidae): first demonstration of a host-derived attractant. Internat J Acarol 24: 143-147.

Yoder JA, Ark JT and Farrell AC (2008) Failure by engorged stages of the lone star tick, Amblyomma americanum, to react to assembly pheromone, guanine and uric acid. Med Vet Entomol 22: 135-139.

Yunker CD, Peter T, Norval RAI, Sonenshine DE, Buridge MJ and Butler JF (1992) Olfactory responses of adult Amblyomma hebraeum and A. variegatum (Acari: Ixodidae) to attractant chemicals in laboratory tests. Exp. Appl. Acarol 13: 295301. 


\title{
16. Parasite-mediated enhancement of transmission by haematophagous insects
}

Hilary Hurd

\begin{abstract}
Blood-sucking arthropods are vectors for a variety of parasites and pathogens, including many that infect man and his domestic animals. Infective stages are imbibed during blood feeding and their life cycle is completed when they are transferred back to their vertebrate host during another blood-feeding episode. Transmission of vector-borne parasites is therefore dependent upon contact between hosts and haematophagous arthropods. In order for blood feeding to occur, a vector performs a series of behaviour patterns that are initiated in response to both endogenous and exogenous triggers; the latter including odour signals from the host. If the host is infected these signals may change in ways that makes them more attractive to the vector of the parasite. In addition, infection-induced lethargy in the host may make blood feeding less risky and blood that has fewer erythrocytes is easier to imbibe. When vectors are infected several aspects of blood-feeding behaviour may be changed in ways that increase host contact or facilitate feeding. Mosquitoes infected with rodent malarias have been shown to exhibit increased response to host odours, to have a lower blood volume threshold at which they cease feeding and to show increased feeding persistence in the face of host-defensive behaviour. Increased feeding persistence is also observed in Leishmania-infected sand flies, as is increased biting behaviour. The latter is due to blockage of the gut with promastigote secretory gel. Vector infections also alter mechanisms that combat host haemostasis, such as the mosquito anti-platelet aggregation factor, apyrase. This causes additional probing attempts when sporozoite infections are present. The evidence that some vector-transmitted parasites and pathogens increase transmission prospects by altering the blood-feeding process suggests that they are manipulating the host or the vector. Host contact determines the spread of infection thus information concerning parasite-induced changes in haematophagy needs to be incorporated into epidemiological models.
\end{abstract}

Keywords: vectors, parasites, transmission, haematophagy, blood feeding, behaviour manipulation

\section{Introduction}

The particular interest in the olfactory response of haematophagous insects witnessed in this volume is undoubtedly prompted by their capacity to transmit the causative agents of infectious diseases to humans and their domestic animals. The majority of these vector-borne infectious agents have complex lifecycles that require two or more hosts and one of the major problems associated with these parasitic lifestyles is the safe transmission from host to host. Parasites have evolved different strategies to solve the various problems associated with transmission, one of which is a reliance on insect vectors to ferry them between hosts. Insects that are used as vectors invariably visit vertebrates to feed upon their blood or other body fluids, thus a prerequisite for this form of transmission is that the parasite is in easy reach of an insect vector when it is feeding. The transmission stages of these parasites thus inhabit the blood or superficial skin layers (reviewed in Lehane 2005) and their vectors are usually females requiring a protein rich meal for egg production (but see Otranto et al. 2008). Not only does vertebrate blood provide a nutritious food source for haematophagous insects, it is also an ideal environment for the development of a 
multitude of parasitic organisms ranging in size from arboviruses to helminths (for lists see Lehane 2005 and Otranto et al. 2008).

Vector insects were once regarded as flying syringes, doing little more than sucking up parasites in one host and injecting them into another. It is now recognised that complex molecular, biochemical and behavioural interactions occur between parasites and vectors. These interactions affect the fitness of the insect and the outcome of parasite transmission (Hurd 2003). The exception to this is when mechanical transmission occurs, during which pathogens are rapidly moved from insect to insect on contaminated mouth parts. One example is Stomoxys nigra Macquart, which transmit Trypanosoma evansi Steel in this way (Mihok et al. 1995, Sumba et al. 1998). The more general pattern is biological transmission, where the parasite may multiply in the insect vector, change into another developmental stage or both change and multiply (Hamilton and Hurd 2002, Lehane 2005). All of the developmental stages may occur within the midgut lumen, as is the case for Leishmania spp. infecting sand flies or Trypanosoma cruzi Chagas that multiply in the crop and midgut of the triatomine bug, Triatoma infectans Klug, and are deposited on the skin when the bug defecates during blood feeding. In many cases, however, the gut wall is traversed and parasites invade tissues such as the thoracic muscle in the case of the filarial worms Wuchereria bancrofti (Cobbold) and Onchocerca volvulus Bickel or the Malpighian tubules, as does Dirofilaria immitis (Leidy). Once these worms have moulted into L3 larvae they make their way to the mouthparts, ready for transmission back to a mammalian host during the vector's next blood meal. Although the malaria parasite, Plasmodium, passes through the midgut cells, it remains on the gut wall beneath the basal lamina undergoing sporogony to produce the infective sporozoites which migrate to the salivary gland and are transferred into the vertebrate host in the saliva.

Pathogens transmitted by haematophagous insects interact with their vertebrate hosts at many different levels, often causing severe morbidity or mortality. Many of the diseases that disproportionately affect the poor and marginalised populations of the world are caused by insect-transmitted parasites. These diseases include malaria, caused by protozoan parasites of the genus Plasmodium; sleeping sickness, caused by the flagellates Trypansoma brucei rhodesiense and T. b. gambiense; Chagas' disease, caused by Trypanosoma cruzi; leishmaniasis cause by Leishmania spp.; lymphatic filariasis caused by Wuchereria bancrofti or Brugia malayi Brug; river blindness caused by Onchocerca volvulus and dengue fever, caused by DEN viruses.

Once established in haematophagous insects, many vector transmitted parasites interact with their hosts in ways that result in a decrease in vector longevity and /or reproductive fitness (see reviews by Ferguson and Read 2002, Hurd 1993, Hurd 2003 and Moore 1993). In keeping with the theme of this volume, this chapter will focus on the point of contact between vector and vertebrate host and how this contact is affected by pathogens and parasites that may be present in host or vector.

For transmission to occur, a minimum of two contacts must be made, one to transmit the infectious organism to the vector and one to return it to a vertebrate host. Thus I will consider transmission from an infected host to an uninfected insect and from an infected insect back to a vertebrate; thereby considering transmission at both stages of the parasite's life cycle. The blood feeding that causes both of these contacts to occur can be broken down into a series of vector behaviour patterns namely; appetitive search, attraction to the host, landing, probing and feeding (Hamilton and Hurd 2002). There is sparse, but growing, evidence that several aspects of this sequence of events can be altered by infectious agents and that, although the mechanism underlying these changes may differ, the resulting consequences are similar. This chapter will present an overview 
of parasite-induced changes in blood-feeding behaviour that affect each point of contact between vector and host. As much of this was reviewed in Hamilton and Hurd (2002) and Hurd (2003), and as space is limiting, the focus of this article will be upon detailing more recent studies.

\section{The attractiveness of the odour of infected-hosts}

Parasitic infection is known to modify the odour profile of host urine, exhaled breath or skin microbial flora (Braks et al. 1999, Hamilton and Hurd 2002, Kavaliers and Colwell 1995, Penn and Potts 1998). These odours can be detected by conspecifics and may act as chemical signals during social interactions (Kavaliers and Colwell 1995). In mice they play a role in avoidance behaviours and mate choice (Barthelemy et al. 2005, Kavaliers et al. 1998). It is conceivable that over evolutionary time selection pressures will have favoured parasites that exploit or manipulate host odour changes that influence the host-seeking behaviour of the respective vector, and thereby enhance their transmission.

The odour profile of individual hosts differ considerably (Qiu et al. 2006, Rebollar-Tellez et al. 1999) and haematophagous insects clearly distinguish between them, as reviewed by Clements (1999). Surprisingly, there are very few published studies that compare the response of vectors to parasite-infected hosts and most of these have examined the response of mosquitoes to malaria or arboviral-infected hosts (reviewed by Clements (1999)). Sand flies have been shown to be more attracted to Leishmania-infected dogs (Coleman et al. 1988) and, in a bioassay of the entrained odours of hamsters infected with L. infantum Nicolle, they were shown to be more attractive to the sand fly, Lutzomyia longipalpis (Lutz and Neiva), than uninfected hamster odour (O'Shea et al. 2002). Likewise, pilot studies with rodent malaria-infected mice showed a similar preferential attraction of uninfected anopheline mosquitoes to the odours of infected hosts, attractiveness increasing with parasitaemia. (reviewed by Hamilton and Hurd (2002)). The latter studies corroborated the earlier findings of Day and colleagues (Day et al. 1983) studying mice infected with rodent malarias. Increased attractiveness of a host will only benefit parasite transmission if stages infective to the vector are present. In the case of malaria parasites these are the gametocytes. Rodent malarias have been shown to cause the mouse host to be most attractive when gametocytes are circulating, suggesting that these hosts may receive more bites and thus infect more mosquitoes (Day and Edman 1983). A positive association was found between both asexual parasitaemia and gametocyte density and the proportion of mosquitoes feeding on $P$. chabaudi-infected mice at 14 days post-infection (when gametocytes are present in this species) (Ferguson et al. 2003). Mosquitoes four days post-infection with P. chabaudi I. Landau \& A. Chabaud were also found to be more attracted to infected mice than uninfected ones when offered a second blood meal (Ferguson and Read 2004). However, chickens infected with P. gallinaceum Brumpt were found to be less attractive to Aedes aegypti (L.) than uninfected ones (Freier and Friedman 1976). Although this latter study did not state whether gametocytes were present, other experiments presented in the paper suggest their chickens would have had a parasitaemia of at least $28 \%$. Plasmodium gallinaceum gametocytes are present in chickens with rising parasitaemias of $10+\%$ (Warburg et al. 1992), thus in this species gametocyte presence does not seem to make the host more attractive and the authors ascribed the decrease in attractiveness of infected hosts to infection associated metabolic changes that lead to odour changes (Day and Edman 1983).

The majority of studies of rodent malarias are made in the laboratory, using host / parasite / vector models that do not occur in nature and would not have co-evolved. If increased host attractiveness at a time of peak infectivity also occurs in malaria-infected humans then the increase in biting rate and hence increased parasite transmission that this may cause would have important implications 
for disease epidemiology. This is predicted for example in the simulations made by Kingsolver (1987) using increasing-preference and switching behaviour models that took account of nonrandom feeding behaviours. For example, increasing consistent host preference for infected hosts was predicted to make it easier to maintain a stable infection, relative to the random choice model (Kingsolver 1987). A very elegantly designed study undertaken in the field in western Kenya was able to confirm that non-random feeding on malaria-infected humans does occur (Lacroix et al. 2005). Twelve groups of three children between 3 and 5 years old were initially exposed to Anopheles gambiae Giles s.s. mosquitoes. One child in each group was uninfected, one was naturally infected with the asexual stages of $P$. falciparum Welch (that are not transmissible to mosquitoes) and the third child harboured gametocytes. None of these children had symptomatic malaria. The same children were then given anti-malaria treatment and again exposed to mosquitoes when parasites had been cleared from the blood. In the first assay, gametocytaemic children were twice as attractive as other children, but this increased attractiveness disappeared once the infection had been cleared. Thus increased attractiveness was a function of the presence of transmission stages of the parasite alone, and not due to an intrinsic attractiveness of this group of children. In contrast, a field study undertaken in Papua New Guinea failed to detect any increase in the attractiveness of Anopheles punctulatus Dönitz to malaria or filarial-infected individuals (Burkot et al. 1989).

It is likely that other vector-transmitted parasites also increase host attractiveness as has been shown in a field studiy that detected greater attraction of Glossina pallidipes Austen towards oxen infected with T. congolense Broden than uninfected or T. vivax Grassi and Feletti-infected oxen (Baylis and Mbwabi 1995b). However there are surprisingly few studies that have examined this issue, even though it is of such importance for disease epidemiology. Other practical aspects that may follow from studies of host odour changes are the potential for diagnosis of infectious diseases using odour profiles and the value of using infection-specific odours as baits to lure haematophagous insects away from their targets.

\section{Host temperature and attractiveness}

Alteration in long range attraction to infected hosts is likely to be the result of changes in odour profile, however, at close range host temperature may also come into play. Are vectors attracted to hosts with elevated temperatures? Increased body temperature is associated with many parasitic infections making this an attractive proposition, but this hypothesis is not supported by empirical evidence, in fact quite the contrary. Studies of arbovirus-infected chickens, Rift Valley Fever virus-infected lambs and rodents infected with malaria all failed to make an association between increased feeding and elevated host-temperatures. Indeed, mice infected with rodent malaria experience depressed rather than elevated temperatures, yet are more attractive to Ae. aegypti and Culex quinquefasciatus Say (reviewed by Clements 1999). Although these studies did not demonstrate a role for infection-induced temperature elevation in altering the attractiveness of infected hosts, the relationship between host temperature and parasite transmission may not be obvious. For instance, the increased attractiveness of pregnant women to mosquitoes has been ascribed to elevation of body temperature and an increased release of volatile substances (Lindsay et al. 2000). This may, in part, explain the increased vulnerability to malaria experienced by pregnant women (Ansell et al. 2002). 


\section{Defensive behaviour of infected hosts}

Animals react to insect blood feeding with defensive behavioural responses that are likely to interrupt the vector feeding process and lead to the taking of multiple meals (Klowden and Lea 1979). This increases the chance that a vector will feed on several different hosts, including some infected ones. However, the pathology associated with some vector transmitted diseases induces host lethargy thus decreasing defensive behaviour, making it easier for the vector to fully engorge. For example, Plasmodium infection causes lethargy in infected mice, which limits their defensive behaviour; the timing of the onset of lethargy varying depending upon the species of rodent malaria. The vulnerability of infected mice to mosquito biting was shown to increase in synchrony with increased lethargy (Day et al. 1983). The advantage of feeding on a defenceless host seems obvious, yet few examples of increased blood feeding on lethargic hosts exist, as discussed by Clements (1999) and Moore (1993). This could be a consequence of the lack of investigations that would demonstrate it. Alternatively, it may not be as advantageous for the vector as it first seems. If a parasite decreases the fitness of its vector then an easy to come by, but infective, meal will have its own costs. Selection pressured that mitigate against favouring infected hosts when foraging may be operating. Additionally, in terms of transmission success, the times at which transmission stages of the parasite are present may not coincide with disease symptoms that induce pathology, as seen for instance in P. falciparum infection, where gametocytes are largely present in asymptomatic people.

Even when defensive behaviour fails, vertebrates are not the passive victims of the blood feeding attempts of other organisms. They have a battery of internal defence mechanisms to combat this theft of blood. These are reviewed by Lehane (2005) and include haemostasis mechanism such as coagulation and platelet aggregation that control bleeding at the wound site. As is to be expected, an arms race has ensued to produce counter measures to these defences. Thus the saliva of haematophagous arthropods in a complex mix of biologically active proteins that assist the feeding mechanism by resisting host haemostasis, including anticoagulants, immunomodulators, vasodilators and anti-platelet compounds. It is becoming evident that many of these molecules have been usurped by vector transmitted parasites to enhance their transmission prospects.

One such example is seen in ticks. Unlike the brief encounter that blood-sucking insects have with their hosts, ticks engorge on vertebrates for several days, engendering the recruitment of inflammatory cells such as neutrophils to the bite site in an attempt to disrupt tick feeding. These efforts are countered by an array of salivary gland proteins (Nuttall and Labuda 2003). One such is the antioxidant Salp25D, expressed in the midgut and salivary glands of the tick Ixodes scapularis Say (Das et al. 2001). This tick is a vector for several pathogens including the spirochete Borrelia burgdorferi (Johnson), causative agent of Lyme disease. By silencing salivary gland salp25D using dsRNA injection, Narasimhan and colleagues (Narasimhan et al. 2007) showed that, although engorgement was not affected, acquisition of a spirochete burden in the midgut was significantly reduced by the first $24 \mathrm{~h}$ of feeding, compared to control ticks. In contrast, transmission of Borrelia from infected ticks to mice was not impaired. This study further demonstrated that Salp25D is likely to protect the incoming spirochete from the damaging hydroxylradicles generated at the bite site. Thus transfer of this pathogen from the vertebrate host to the tick is enhanced by the activity of a salivary gland protein used by the vector to produce an anti-inflammatory response. The spirochete is, in effect, piggy backing on a mechanism involved in normal feeding behaviour. 


\section{Blood feeding on an infected host}

As we have seen, blood feeding is a dangerous strategy for insects to pursue, as the act of piercing or biting the host engenders defensive actions on the part of the host that are likely to be lethal to the vector. The majority of haematophagous arthropods thus visit their feeding site only briefly and the morphology and mechanics of their highly specialised feeding apparatuses have evolved to efficiently penetrate the host tissue and obtain a meal in as short a time as possible. Blood is a viscous fluid and flows up the food canal as a result of the negative pressure applied by the pharangeal pump and/or the cibarial pump, both located in the head capsule. Models used to describe the flow of blood during vector feeding suggested that the radius of the food canal has the largest influence over feeding time (Daniel and Kingsolver 1983), however, the Daniel and Kingsolver model has been challenged, as discussed by Clements (1999). Terminal diameters of feeding canals are not much greater than the diameter of single red blood cells and it would appear that the size may be limited by the need to pierce the skin efficiently and without detection. This is discussed in detail by Lehane (2005).

\section{Host blood quality}

In addition to the mechanics of the insect's feeding apparatus, the nature of the blood will influence feeding success. The proportion of erythrocytes present (haematocrit) will affect the viscosity of the blood and hence its rate of flow, its nutritious value (as much of blood protein is contained in the red blood cells) and, in the case of parasites that infect erythrocyte, the level of parasitaemia. Chronic parasitic infections such as malaria can induce long term reductions in haematocrit which may affect vector feeding success. This was demonstrated in a study of mice infected with the rodent malaria, P. yoelii nigeriensis (Killick-Kendrick). Mice exhibited a drop in haematocrit (measured as packed cell volume (PCV)) as days-post-infection and parasitaemia rose. Early in infection, when PCV has only dropped 1-3\%, mosquitoes allowed to feed on anaesthetised infected mice were able to imbibe significantly larger amounts of haemoglobin (a proxy measurement for red blood cells) than mosquitoes feeding on control mice. However, as parasitaemia increased and PCV fell below $6 \%$ of the control mice the haemoglobin taken by mosquitoes feeding on infected mice was significantly reduced (Taylor and Hurd 2001). Thus, initially, the predicted decrease in viscosity as PCV falls appears to enhance erythrocyte uptake. The infective, gametocyte stages of $P$. y. nigeriensis are present early in infection when haematocrit has fallen only slightly. It is thus likely that their transmission to mosquitoes will be enhanced by a small drop in PCV.

In addition to increasing erythrocyte uptake, the speed at which blood was initially imbibed was also demonstrated to increase when the yellow fever mosquito, Ae. aegypti, fed on P. chabaudi infected mice or hamsters infected with the Rift Valley Fever virus compared with their uninfected counterparts (Shieh and Rossignol 1992). This was due to a reduction in the time taken to locate blood during probing which, the authors proposed, may be due to an infection-induced reduction in the number of host platelets.

Both the reduction in probing time and the increased erythrocyte uptake seen when mosquitoes feed on slightly anaemic blood may be advantageous. It will enhance the chances that mosquitoes take enough protein for egg production before host-defensive behaviour is initiated. However, mosquitoes feeding on very anaemic blood will need to seek further feeding opportunities, thereby increase risky host contact. If the advantage of obtaining a quick meal outweighs the disadvantage of incurring an infection, then mosquitoes may favour feeding on slightly anaemic 
hosts. Nevertheless, it must be noted that associations used in these study do not occur in the field and it would be necessary to repeat these findings in a naturally occurring host/parasite/ vector system before the value of enhanced feeding on an anaemic host could be hypothesised to enhance transmission. Whether these trade-offs operate in favour of human parasite transmission in a malaria endemic area, where slight anaemia is likely to be commonplace, has yet to be tested.

The affect of host haematocrit on blood feeding has also been investigated in tsetse flies. The consumption of ox blood by G. pallidipes decreases as PCV exceeded 30\% (Baylis and Mbwabi 1995a). However, although tsetse fly feeding was greater on cattle infected with $T$. congolense and $T$. vivax, this was not attributed to host anaemia but to vasodilation caused by $T$. congolense attaching to the wall of the host microvasculature (Moloo et al. 2000).

\section{The importance of correct parasite location in the host}

Many vectors exhibit peak biting activity at specific times of the day. For example, An. gambiae are nocturnal feeders with host landing building up from 22:00 $\mathrm{h}$ to $04: 00 \mathrm{~h}$ and continuing until just before day break. In contrast, An. funestus Giles show a peak feeding activity around 6:00 h (reviewed by Clements (1999). Some vector- transmitted parasites have a circadian rhythm that matches that of their vector. They are present in the peripheral blood vessels during the period that their vector is most likely to bite. This phenomenon is particularly evident in species of filarial nematodes. It is known as 'microfilarial periodicity' and has been discussed by Lehane (2005). Of particular interest is the difference in periodicity shown by two strains of $W$. bancrofti. In one strain microfilariae congregate in the lungs during the day and are only found in the peripheral blood vessels at night; coinciding with the biting pattern of their vector $C x$. quinquefasciatus. In contrast, the strain transmitted by the day-biting vector, Ae. polynesiensis (Marks), are subperiodic and can also be found circulating during the day (Hawking 1962). Parasite periodicity, known as the 'Hawking phenomenon', has also been reported to occur in several species of malaria (Gautret and Motard 1999), but could not be demonstrated following a field study of biting behaviour of $A n$. gambiae feeding on P. falciparum-infected children in Kenya (Githeko et al. 1993). The significance of parasite periodicity as a means of enhancing transmission has not been fully determined but, especially in situations where parasitaemia is low, it could enhance the chances that transmission stages will be imbibed by a suitable vector and, in the case of Plasmodium, in numbers that maximise the chances of fertilisation.

Parasites are also known to congregate in locations that vectors have access to. Lutzomyia longipalpis (Lutz \& Neiva) preferentially probes on skin lesions rich in Leishmania mexicana (Biagi) amastigotes, the infective stage to the vector (Coleman and Edman 1988). Developing gametocytes of $P$. falciparum are sequestered in the microcirculature whilst developing, thus they are only exposed to potential transmission when they are mature. There is also evidence that suggests that circulating gametocytes are aggregated, rather than having a homeogeneous distribution. This aggregation is reflected in infection patterns in mosquitoes and may help to facilitate the chance of macrogametocyte fertilisation in the midgut (Pichon et al. 2000).

\section{The blood-feeding behaviour of infected vectors}

Arthropod haematophagy consists of a series of physiological and behavioural steps beginning with an appetitive search, and proceeding through activation and orientation, attraction, landing, probing or biting and finally imbibing blood (Clements 1999, Hamilton and Hurd 2002, Lehane 2005). The initiation and completion of all of these stages is influenced by exogenous 
and endogenous factors including, in some cases, the presence of parasites. Although parasite transmission will only occur during the latter two behavioural phases, all of the preliminary events are crucial. We can surmise that changes to any of them that result in more host contact will increase the prospects of parasite transmission, once the parasite has developed into the life cycle stage that is transmitted back to the vertebrate host (Hurd 2003, Lefevre et al. 2006). Various vector-transmitted parasite, including bacteria, protozoans and nematodes do indeed alter one or more stages of blood-feeding behaviour and, in a few cases, this has been shown to increase transmission chances. A few such examples have been well documented in the reviews quoted in this chapter and here the focus will be upon vector/parasite associations that have recently been explored in greater depth.

\section{Appetitive search and attraction}

Once parasites have reached the infective stage of their development, any host contact on the part of their vector will provide an opportunity for transmission. We can therefore argue that changes in vector behaviour that enhance appetitive searches and make the vector more responsive to hosts odours will favour parasite transmission back to the vertebrate, thereby completing its life cycle. Unfortunately, there are few studies that specifically measure these response in vectors infected with transmissible stages of parasites. Comparisons need to be made between vectors with immature and mature parasite stages. Increased attraction at an inappropriate time would not benefit the parasites and may just be a response, for instance, to food robbery caused by the parasite increasing a hunger response rather than manipulated behaviour (as seen in T. cruziinfected triatomines (Schaub 2006)).

Those studies that have specifically investigated the effect of infection on host attractiveness have focused on mosquitoes. For example, an increase in the olfactory response of $P$. gallinaceum sporozoites-infected Ae. aegypti to host odours has been reported (Rossignol et al. 1986). Additionally, the proportion of An. stephensi infected with P. chabaudi that took a second blood meal from an anaesthetised mouse rose from 0.39 to 0.56 if they were infected with oocysts and this change in behaviour was not influenced by the burden of infection (Ferguson and Read 2004). The significance of the latter finding is, however, unclear as increased host contact at this stage of infection would not benefit parasite transmission and the experimental design did not make it possible to clarify whether the behaviour change was actually due to a change in response to host odours.

\section{Feeding persistence and the role of host-defensive reactions}

As has already been discussed, in a natural situation haematophagy is often interrupted by hostdefensive responses that can result in the death of the insect. Feeding persistence is the repeated attempt to feed, despite these interruptions.

In the face of host-defensive responses, mosquitoes infected with sporozoites of the rodent malaria P. y. nigeriensis are significantly more persistent than uninfected mosquitoes in their feeding attempts when interrupted before any blood is imbibed. This is a complete reversal of the reduction in feeding persistence that occurs when the parasites is developing on the midgut wall, before it reaches the infective stage (Anderson et al. 1999). Stage specific alteration of biting behaviour was also seen by Koella and colleagues, investigating the size of blood meal that would inhibit further blood feeding following interruption. They showed that Ae. aegypti infected with oocysts of $P$. gallinaceum had a lower threshold value for blood meal size and were thus less likely 
to re-feed than uninfected mosquitoes. In contrast, the presence of sporozoites increased the threshold volume required to inhibit host-seeking behaviour success (Koella et al. 2002).

In addition to these laboratory investigations, several studies have examined the feeding behaviour of malaria-infected mosquitoes in field situations. Not only are peak feeding times of anopheline mosquitoes changed (reviewed in Hamilton and Hurd (2002) and Hurd (2003)), infected mosquitoes have been shown to engorge more fully (Koella and Packer 1996, Koella et al. 1998, Maxwell et al. 1998) suggesting that more tenacious feeding behaviour also occurs in naturally infected mosquitoes.

A similar increase in blood-feeding persistence has also been observed to occur in sand flies infected with the metacyclic promastigote forms (infective to mammalian hosts) of $L$. infantum or L. mexicana. Sand flies were allowed to feed on an anaesthetised mouse but feeding was artificially interrupted by brushing the antennae of the flies. Rogers and Bates found a positive correlation between feeding persistence and the number of metacyclic promastigotes per fly (Rogers and Bates 2007). In both cases only the infective-sand fly stage caused this behavioural change.

\section{Changes in biting and probing behaviour}

In addition to an increase in feeding persistence, vector biting or probing behaviour has been shown to be affected by several vector/parasite associations including malaria/mosquitoes, Leishmania/sand flies, Trypanosoma/tsetse flies and Yersinia pestis/fleas. Of these, recent studies of the changes in biting behaviour induced by Leishmania infections are beginning to elucidate mechanisms underlying behavioural changes that have been recognised for over a century and have been reviewed by Hurd (2003), Ready (2008) and Rogers and Bates (2007).

Sand flies become infected with amastigote forms of Leishmania following an infected blood meal and the parasites complete their entire developmental process within the sand fly gut. The final phase takes place in the stomodeal valve where they transform into motile metacyclic promastigote forms that are infective to mammals. Transmission occurs when these promastigotes are regurgitated within a gel-like material that fills the cardia and stomodeal valve, causing a blockage. This promastigote secretory gel (PSG) is formed by the parasites and has been shown to enhance cutaneous infections when deposited onto the skin with sand fly saliva (Rogers et al. 2004, Titus 1998). A major component of PGS is a filamentous glycoprotein, proteophosphoglycan (fPPG) (Ilg et al. 1996), which forms the 3D matrix of the plug that blocks the gut (Stierhof et al. 1999) and keeps the valve open. The production of PSG has been shown to occur is several Leishmania-sand fly combinations (Stierhof et al. 1999). It has been suggested that the plug facilitates regurgitation and, by restricting blood flow into the fly, prolongs feeding time and causes flies to bite more, a concept known as 'the blocked fly hypothesis'.

Sand flies harbouring infective stages of Leishmania take longer to feed on an anaesthetised mouse than uninfected flies, but do not probe more often (Rogers and Bates 2007). These authors concluded that the interruption of feeding as a result of host-defensive behaviour (which is not occurring when the host is anaesthetised) and the subsequent persistence in attempts to feed is crucial to engendering multiple feeding attempts, and may be more important than the 'blocked fly hypothesis'. They suggest that the presence of fPPG may inhibit the functioning of the mechanoreceptors in the foregut that detect blood flow, thereby altering the hunger state or increasing the threshold blood volume at which feeding is deceased. 
If this behavioural change is to be advantageous to the parasite, it must increase the basic reproductive number $\left(R_{0}\right)$ of the infection by increasing the chance that an infectious vector will feed on more than one host. Rogers and Bates (2007) showed that infected sand flies were more likely to feed on multiple hosts in a laboratory setting.

Promastigote secretory gel is one of the few examples of a product produced by parasites that, via its action in the vector, appears to manipulate blood-feeding behaviour in a way that increases parasite fitness by enhancing transmission. Rogers and Bates (2007) argue that this is an example of true manipulation as it fulfils the criteria set out by (Poulin 1995) and discussed further by Thomas and colleagues (Thomas et al. 2005). Briefly, it is an example of a complex change in behaviour that achieves the purpose of increasing transmission chances, it fits its purpose as increased feeding behaviour only occurs once the infective stages are present and it occurs in several sand fly/Leishmania combinations. PGS could thus be regarded as a manipulator molecule.

The saliva of haematophagous insects contains a cornucopia of molecules that, in one way or another, assist the blood-feeding process including anaesthetics, anti-coagulation factors, antiplatelet factors and vasodilators than combat the haemostatic defences of the host. Our current knowledge of the nature and wide range of these molecules is reviewed in detail by Lehane (2005). Many species contain an enzyme, apyrase, which inhibits the action of the platelet-aggregation factor ADP by converting it to AMP and orthophosphate. The presence of apyrase at the site of a blood vessel wound made by a probing insect will thus inhibit the sealing of a lanced blood vessel. This would allow a haematoma to form and the insect to locate blood more quickly and feed more efficiently, and for longer. This has been aptly demonstrated in mosquitoes where feeding time has been related to the quantity of apyrase in the glands of different anopheline mosquitoes (Ribeiro et al. 1985). More recently, Boisson and colleagues (Boisson et al. 2006) confirmed the role of apyrase in efficient mosquito feeding using gene silencing to reduced the expression of the apyrase gene AgApy. Saliva from mosquitoes injected with ds-AgAyp exhibited reduced enzyme activity and was less efficient at inhibiting platelet activity. $d s-A g A y p$-injected females took twice as long probing before obtaining a blood meal. The authors suggested that their results indicate that there may be redundant apyrase activity and also that products of the AgApyLike1 and Ag9 genes may also be involved in inhibiting platelet aggregation.

Malaria sporozoites are transmitted to the vertebrate host during the probing phase of feeding (Rodriguez and Hernandez-Hernandez 2004). Salivary gland infections are associated with an increase in the time vectors spend probing. Median blood-location time for Ae. aegypti infected with the avian malaria, P. gallinaceum, thus increases three-fold compared to uninfected mosquitoes. This is probably as a result of a four fold decrease in the activity of salivary apyrase, even though the volume of saliva produced is constant (Rossignol et al. 1984). A similar increase in probing behaviour has been detected in malaria-infected anopheline mosquitoes, as reviewed recently (Hamilton and Hurd 2002; Hurd 2003). One study provided evidence that sporozoite infections cause multiple feeding episodes that lead to enhanced transmission. Using microsatellite markers to identify the source of blood meals in the midguts of mosquitoes feeding in a field situation, Koella and colleagues (Koella et al. 1998) were able to demonstrate that $22 \%$ of P. falciparuminfected An. gambiae had fed on more than one person during the night, compared with only $10 \%$ of uninfected females. Multiple feeding on different hosts had previously been detected in Ae. aegypti infected with P. gallinaceum (Kelly and Edman 1992). However, an alternative interpretation of these observations is that mosquitoes that become infected may have a propensity to multiple feeding. 
Malaria sporozoites inhabit the distal lobes of mosquito salivary glands, the same location in which apyrase is produced (Sterling et al. 1973). Surprisingly we still do not know the mechanism causing the parasite-associated reduction in apyrase. Is it caused by damage incurred as the sporozoites migrate through the glands or do the sporozoites secrete an inhibitor that suppresses transcription or inhibits translation? The former would indicate that transmission is enhanced by an accidental by-product of pathology the latter that this may be another example of vector manipulation.

A final example comes not from mosquitoes but from fleas infected with plague bacilli. In nature, Y. pestis, the causative agent of plague, circulates amongst rodent species generally causing little overt disease symptoms. Violent outbreaks resulting in high mortality periodically occur amongst susceptible rodent populations and can increase the risk of human infection. Plague transmission occurs via flea bites. Within the flea gut, ingested bacteria are found in a mass of coagulated blood that blocks the proventriculus. The blockage results in the regurgitation of plague bacilli into the mammalian host during feeding. A coagulase- and fibrinolysin associated (pla) gene encoded on the bacilli plasmid, pKYP1, has been associated with coagulase activity that facilitates the formation of the proventricular mass (Cavanaugh 1971). Mcdonough and co-workers (Mcdonough et al. 1993) have shown that fleas infected with pla bacteria had higher mortality than that caused by pla- bacteria and that mortality was not linked to bacterial load. They suggest that the pla gene product may disrupt the flea's digestive process leading to blockage, starvation, and early death. Increased feeding attempts early in this process will enhance plague transmission. Although further studies are needed to substantiate these suggestions, the pla gene can be regarded as a promising manipulator molecule candidate with which $Y$. pestis may modify its vector's feeding behaviour to suit its own ends.

\section{Are parasites manipulating vectors?}

Much has been written about host manipulation by parasites. There are many incredible examples that are favourites amongst teachers and students alike (Moore 2002). In comparison with the fate of crickets infected with hair worms, or snails with trematode sporocysts in their tentacles, changes in vector feeding persistence or probing behaviour are far from dramatic. Despite this, the co-evolutionary implications of host and vector behavioural changes are similar. Many of the changes we see can be expected, and in a few cases have been shown, to lead to an increase in vector transmission that may be accompanied by a decrease in vector fitness due to the greater danger associated with increased host contact. Do these unequal consequences for the symbiotic partners imply that the parasite is manipulating its host? Alternatively, is the behavioural change a by-product of pathology caused by the infection or are we seeing attempts by the vector to compensate for the presence of the parasite? Here is not the place to detail the pros and cons of these theories, which have been much discussed (most recently by Lefevre et al. (2006) and Lefevre and Thomas (2008), and until we fully understand the mechanisms that govern them, it is difficult to do much more than speculate.

Parasite fitness can be expressed as $R_{0}$ which, for microparasites, can be defined as the number of secondary infections that arise from a primary infection. Following the seminal work of Ross and Macdonald (Macdonald 1957), models developed to take into account factors that affect $R_{0}$ were developed by Anderson and May $(1979,1992)$ and have since been modified and refined by many authors interested in the epidemiology of infectious disease. 
Host contact is probably the most important factor limiting the spread of vector transmitted diseases and this concept, known as vectorial capacity, is incorporated into many of these models. Saul (2003) developed two deterministic models, one for stable transmission and one for epidemic situations. They were based on the cyclic feeding model he and his colleagues had previously developed (Saul et al. 1990) rather than the a continuous feeding model refined from Ross' model by Macdonald (1957). Saul's model assumed that mosquitoes will not feed for a few days after becoming engorged; whilst the respective gonotrophic cycle is completed (cyclical feeding). His model showed that search-related mortality of mosquitoes will have an important impact on malaria transmission. The sensitivity of malaria transmission to mosquito mortality has been also recognised by others including Smith and McKenzie (2004). One parameter of Saul's model (Saul 2003) is the 'constant attraction rate constant'. Saul recognised that this will, in fact, be dependent upon factors such as host emission of attractants and host availability to vectors (e.g. are they sleeping under bednets?). Saul's model was used to explore the effectiveness of using 'bait' animals to lure vectors away from biting humans and predicted that insecticide treatment of livestock will have an appreciable effect on the human inoculation rate (Saul 2003).

The classical models did not recognise that the parasite itself may have a role to play in affecting host contact. We have seen that there is evidence that parasitic infections of both mammalian hosts and arthropod vectors alter feeding behaviour in ways that increase host contact; that infected hosts are more attractive than uninfected ones and that mosquito biting is heterogeneous. The assumptions that mosquitoes, for instance, genuinely feed at random and that infected mosquitoes make the same host contacts as uninfected ones is probably erroneous. Smith et al. (2007) discuss classical and neoclassical models that estimate $R_{0}$ for malaria and reconsider $R_{0}$ in finite population with heterogeneous biting. They recognise the importance of $R_{0}$ as an important metric with which to plan malaria control programmes and that, amongst other measures, it may be more productive to target interventions towards those individuals who are bitten most, than those who are bitten least. Clearly it is germane to incorporate many more specific features of the parasite's life cycle and its effect on vector feeding behaviour into mathematic models that are to be of use for planning control strategies.

\section{Conclusions}

Parasite-induced changes in vector haematophagy have been recognised for over a century. However the overriding impression that I have gained whilst preparing this chapter is that, relative to the number of parasites transmitted by vectors, the documented cases of parasites changing vector feeding behaviour is very small and, in particular, the epidemiological impacts of these changes have been neglected. Investigations have mostly focused on malaria and Leishmania infections and we are certainly in no position yet to assume that this is a common strategy that parasites employ to enhance transmission. Hopefully, the burgeoning of studies of olfaction in vector-host interactions will provide further opportunities to incorporate more parasites into these investigations.

Although observations of biting behaviour in a laboratory setting are useful, they are severely limited as to the information they can give us about the consequences for parasite transmission in the field. Studies performed in field settings with natural hosts that can display normal defensive behaviours must be performed to evaluate laboratory findings, although ethical issues associated with investigating human infections must be considered. The few investigations of this kind that have been performed to date are exemplars to be followed. 
Parasitic infection can alter several host behaviour patterns simultaneously and these changes can themselves alter as parasites mature. Experiments need to be planned to isolate individual aspects of feeding behaviour and determine which are affected, and when. Only then can we can decide whether, for instance, changes in feeding persistence have more influence on parasite transmission than changes in biting or probing.

In addition, to gaining an insight into the degree to which vector feeding behaviour can affect disease transmission, a knowledge of the mechanisms underlying these changes will help us to evaluate the evolutionary implications of behavioural change (Lefevre et al. 2006). As the genome sequences of more and more vectors and parasites are published and we move further into the post genomic era, investigation of protein profiles and gene transcription of nervous and other tissues that may govern changes in odour perception, flight behaviour, landing, probing and imbibing blood is becoming feasible. Some promising headway has recently been made by Lefevre and colleagues who have examined changes in the head proteome of tsetse flies infected with trypanosomes and of malaria-infected mosquitoes (Lefevre et al. 2007a,b).

The recent expansion of studies on olfactory behaviour into a wide range of disease vectors and the advanced technologies for more precise understanding of these behaviours, as described in the preceding chapters, is likely to stimulate incorporation of pathogens and parasites in these studies. If this happens we will be able to use knowledge such as this to determine who is manipulating whom, to understand the implication for parasite transmission and to apply our understanding to the planning of novel disease control strategies such as the deployment of transgenic technology to alter feeding behaviour and divert vectors away from anthropophagy.

\section{Acknowledgements}

The helpful comments of an anonymous referee helped to improve this chapter and they are gratefully acknowledged.

\section{References}

Anderson RA, Koella JC and Hurd H (1999) The effect of Plasmodium yoelii nigeriensis infection on the feeding persistence of Anopheles stephensi Liston throughout the sporogonic cycle. Proc R Soc Lond B Biol Sci 266: 1729-1733.

Anderson RM and May RM (1979) Population biology of infectious diseases:Part 1. Nature 280: 361-367.

Anderson RM and May RM (1992) Infectious diseases of humans. Oxford University Press, Oxford, UK.

Ansell J, Hamilton KA, Pinder M, Walraven GE and Lindsay SW (2002) Short-range attractiveness of pregnant women to Anopheles gambiae mosquitoes. Trans R Soc Trop Med Hyg 96: 113-116.

Barthelemy M, Gabrion C and Petit G (2005) Does chronic malaria modify the odours of its male mouse host? Can J Zool 83: 1079-1086.

Baylis M and Mbwabi AL (1995a) Effect of host packed cell volume on the bloodmeal size of male tsetse flies, Glossina pallidipes. Med Vet Entomol 9: 399-402.

Baylis M and Mbwabi AL (1995b) Feeding behaviour of tsetse flies (Glossina pallidipes Austen) on Trypanosoma-infected oxen in Kenya. Parasitology 110 (Pt 3): 297-305.

Boisson B, Jacques JC, Choumet V, Martin E, Xu JN, Vernick K and Bourgouin C (2006) Gene silencing in mosquito salivary glands by RNAi. Febs Letters 580: 1988-1992.

Braks MAH, Anderson RA and Knols BGJ (1999) Infochemicals in mosquito host selection: Human skin microflora and Plasmodium parasites. Parasitology Today 15: 409-413.

Burkot T, Paru A, Graves P and Garner P (1989) Human host selection by Anophelines: no evidence for preferential selection of malaria or microfilariae-infected individuals in a hyperendemic area. Parasitology 98: 337-342. 
Cavanaugh DC (1971) Specific effect of temperature upon transmission of the plague bacillus by the oriental rat flea, Xenopsylla cheopis. Am J Trop Med Hyg 20: 264-273.

Clements AN (1999) The biology of mosquitoes, volume 2 sensory reception and behaviour. CABI Publishing, Oxford, UK, $740 \mathrm{pp}$.

Coleman RE and Edman JD (1988) Feeding-site selection of Lutzomyia longipalpis (Diptera: Psychodidae) on mice infected with Leishmania mexicana amazonensis. J Med Entomol 25: 229-233.

Coleman RE, Edman JD and Semprevivo LH (1988) Interactions between malaria (Plasmodium yoelii) and leishmaniasis (Leishmania mexicana amazonensis): effect of concomitant infection on host activity, host body temperature, and vector engorgement success. J Med Entomol 25: 467-471.

Daniel TL and Kingsolver JG (1983) Feeding strategy and the mechanics of blood sucking in insects. J Theor Biol 105: 661-677.

Das S, Banerjee G, DePonte K, Marcantonio N, Kantor FS and Fikrig E (2001) Salp25D, an Ixodes scapularis antioxidant, is 1 of 14 immunodominant antigens in engorged tick salivary glands. J Infect Dis 184: 1056-1064.

Day JF, Ebert KM and Edman JD (1983) Feeding patterns of mosquitoes (Diptera: Culicidae) simultaneously exposed to malarious and healthy mice, including a method for separating blood meals from conspecific hosts. J Med Entomol 20: $120-127$.

Day JF and Edman JD (1983) Malaria renders mice susceptible to mosquito feeding when gametocytes are most infective. J Parasitol 69: 163-170.

Ferguson HM and Read AF (2002) Why is the effect of malaria parasites on mosquito survival still unresolved? Trends Parasitol 18: 256-261.

Ferguson HM and Read AF (2004) Mosquito appetite for blood is stimulated by Plasmodium chabaudi infections in themselves and their vertebrate hosts. Malar J 3: 12.

Ferguson HM, Rivero A and Read AF (2003) The influence of malaria parasite genetic diversity and anaemia on mosquito feeding and fecundity. Parasitology 127: 9-19.

Freier JE and Friedman S (1976) Effect of host infection with Plasmodium gallinaceum on the reproductive capacity of Aedes aegypti. J Invert Pathol 28: 161-166.

Gautret P and Motard A (1999) Periodic infectivity of Plasmodium gametocytes to the vector. A review. Parasite 6: 103111.

Githeko AK, Brandlingbennett AD, Beier M, Mbogo CM, Atieli FK, Owaga ML, Juma F and Collins FH (1993) Confirmation that Plasmodium falciparum has aperiodic infectivity to Anopheles gambiae. Med Vet Ent 7: 373-376.

Hamilton JCG and Hurd H (2002) Parasite manipulation of vector behaviour. In: Lewis EE, Cambell JF and Sukhdeo MVK (eds) The behavioural ecology of parasites. CABI, Wallingford, UK, pp 259-281.

Hawking $F$ (1962) Microfilaria infection as an instance of periodic phenomena seen in host-parasite relationships. Ann N Y Acad Sci 98: 940-953.

Hurd H (1993) Reproductive disturbances induced by parasites and pathogens of insects. In: Beckage NE, Thompson SN and Federici BA (eds) Parasites and pathogens of insects. Academic Press Inc, San Diego, USA, pp 87-105.

Hurd H (2003) Manipulation of medically important insect vectors by their parasites. Annu Rev Entomol 48: 141-161.

Ilg T, Stierhof YD, Craik D, Simpson R, Handman E and Bacic A (1996) Purification and structural characterization of a filamentous, mucin-like proteophosphoglycan secreted by Leishmania parasites. J Biol Chem 271: 21583-21596.

Kavaliers M and Colwell DD (1995) Discrimination by female mice between the odours of parasitized and non-parasitized males. Proc R Soc Lond B Biol Sci 261: 31-35.

Kavaliers M, Colwell DD and Choleris E (1998) Parasitized female mice display reduced aversive responses to the odours of infected males. Proc R Soc Lond B Biol Sci 265: 1111-1118.

Kelly R and Edman JD (1992) Multiple transmission of Plasmodium gallinaceum (Eucoccida, Plasmodiidae) during serial probing by Aedes aegypti (Diptera, Culicidae) on several hosts. J Med Ent 29: 329-331.

Kingsolver JG (1987) Mosquito host choice and the epidemiology of malaria. Am Nat 130: 811-827.

Klowden MJ and Lea AO (1979) Effect of defensive host behavior on the blood meal size and feeding success of natural populations of mosquitoes (Diptera: Culicidae). J Med Entomol 15: 514-517. 
Koella JC and Packer MJ (1996) Malaria parasites enhance blood-feeding of their naturally infected vector Anopheles punctulatus. Parasitology 113 (Pt 2): 105-109.

Koella JC, Rieu L and Paul REL (2002) Stage-specific manipulation of a mosquito's host-seeking behavior by the malaria parasite Plasmodium gallinaceum. Behav Ecol 13: 816-820.

Koella JC, Sorensen FL and Anderson RA (1998) The malaria parasite, Plasmodium falciparum, increases the frequency of multiple feeding of its mosquito vector, Anopheles gambiae. Proc R Soc Lond B Biol Sci 265: 763-768.

Lacroix R, Mukabana WR, Gouagna LC and Koella JC (2005) Malaria infection increases attractiveness of humans to mosquitoes. Plos Biol 3: 1590-1593.

Lefevre T, Koella JC, Renaud F, Hurd H, Biron DG and Thomas F (2006) New prospects for research on manipulation of insect vectors by pathogens. Plos Pathogens 2: 633-635.

Lefevre T and Thomas F (2008) Behind the scene, something else is pulling the strings: Emphasizing parasitic manipulation in vector-borne diseases. Infect Genet Evol 8: 504-519.

Lefevre T, Thomas F, Ravel S, Patrel D, Renault L, Le Bourligu L, Cuny G and Biron DG (2007a) Trypanosoma brucei brucei induces alteration in the head proteome of the tsetse fly vector Glossina palpalis gambiensis. Insect Mol Biol 16: 651-660.

Lefevre T, Thomas F, Schwartz A, Levashina E, Blandin S, Brizard JP, Le Bourligu L, Demettre E, Renaud F and Biron DG (2007b) Malaria Plasmodium agent induces alteration in the head proteome of their Anopheles mosquito host. Proteomics 7: 1908-1915.

Lehane MJ (2005) The biology of blood-sucking insects. Cambridge University Press, Cambridge, UK. 321 pp.

Lindsay S, Ansell J, Selman C, Cox V, Hamilton K and Walraven G (2000) Effect of pregnancy on exposure to malaria mosquitoes. Lancet 355: 1972-1975.

Macdonald G (1957) The epidemiology and control of malaria. Oxford University Press, London, UK.

Maxwell CA, Wakibara J, Tho S and Curtis CF (1998) Malaria-infective biting at different hours of the night. Med Vet Entomol 12: 325-327.

Mcdonough KA, Barnes AM, Quan TJ, Montenieri J and Falkow S (1993) Mutation in the Pla gene of Yersinia pestis alters the course of the plague bacillus-flea (Siphonaptera, Ceratophyllidae) interaction. J Med Ent 30: 772-780.

Mihok S, Maramba O, Munyoki E and Kagoiya J (1995) Mechanical transmission of Trypanosoma spp by African Stomoxyinae (Diptera, Muscidae). Trop Med Parasitol 46: 103-105.

Moloo SK, Sabwa CL and Baylis M (2000) Feeding behaviour of Glossina pallidipes and G. morsitans centralis on Boran cattle infected with Trypanosoma congolense or T. vivax under laboratory conditions. Medical and Veterinary Entomology 14: 290-299.

Moore J (1993) Parasites and the behavior of biting flies. J Parasitol 79: 1-16.

Moore J (2002) Parasites and the behaviour of animals. Oxford University Press Inc, New York, USA.

Narasimhan S, Sukumaran B, Bozdogan U, Thomas V, Liang X, DePonte K, Marcantonio N, Koski RA, Anderson JF, Kantor F and Fikrig $E$ (2007) A tick antioxidant facilitates the Lyme disease agent's successful migration from the mammalian host to the arthropod vector. Cell Host Microbe 2: 7-18.

Nuttall PA and Labuda M (2003) Dynamics of infection in tick vectors and at the tick-host interface. Adv Virus Res 60: 233-272.

O'Shea B, Rebollar-Tellez E, Ward RD, Hamilton JGC, El Naiem D and Polwart A (2002) Enhanced sandfly attraction to Leishmania-infected hosts. T Roy Soc Trop Med H 96: 117-118.

Otranto D, Stevens JR, Cantacessi C and Gasser RB (2008) Parasite transmission by insects: a female affair? Trends Parasitol 24: 116-120.

Penn D and Potts WK (1998) Chemical signals and parasite-mediated sexual selection. Trends Ecol Evol 13: 391-396.

Pichon G, Awono-Ambene HP and Robert V (2000) High heterogeneity in the number of Plasmodium falciparum gametocytes in the bloodmeal of mosquitoes fed on the same host. Parasitology 121: 115-120.

Poulin R (1995) 'Adaptive' changes in the behaviour of parasitized animals: A critical review. Int J Parasitol 25: 1371-1383.

Qiu YT, Smallegange RC, Van Loon JJA, Ter Braak CJF and Takken W (2006) Interindividual variation in the attractiveness of human odours to the malaria mosquito Anopheles gambiae s. s. Med Vet Entomol 20: 280-287.

Ready PD (2008) Leishmania manipulates sandfly feeding to enhance its transmission. Trends Parasitol 24: 151-153. 
Rebollar-Tellez EA, Hamilton JGC and Ward RD (1999) Response of female Lutzomyia longipalpis to host odour kairomones from human skin. Physiol Entomol 24: 220-226.

Ribeiro JM, Rossignol PA and Speilman A (1985) Salivary gland apyrase determines probing time in anopheline mosquitoes. J Insect Physiol 31: 689-692.

Rodriguez MH and Hernandez-Hernandez FD (2004) Insect-malaria parasites interactions: the salivary gland. Insect Biochem Molec 34: 615-624.

Rogers ME and Bates PA (2007) Leishmania manipulation of sand fly feeding behavior results in enhanced transmission. Plos Pathogens 3: 818-825.

Rogers ME, Ilg T, Nikolaev AV, Ferguson MAJ and Bates PA (2004) Transmission of cutaneous leishmaniasis by sand flies is enhanced by regurgitation of fPPG. Nature 430: 463-467.

Rossignol PA, Ribeiro JM and Spielman A (1984) Increased intradermal probing time in sporozoite-infected mosquitoes. Am J Trop Med Hyg 33: 17-20.

Rossignol PA, Ribeiro JM and Spielman A (1986) Increased biting rate and reduced fertility in sporozoite-infected mosquitoes. Am J Trop Med Hyg 35: 277-279.

Saul A (2003) Zooprophylaxis or zoopotentiation: the outcome of introducing animals on vector transmission is highly dependent on the mosquito mortality while searching. Malaria J 2: 32.

Saul AJ, Graves PM and Kay BH (1990) A cyclical feeding model for pathogen transmission and its application to determine vectorial capacity from vector infection-rates. J Appl Ecol 27: 123-133.

Schaub GA (2006) Parasitogenic alterations of vector behaviour. Int J Med Microbiol 296 37-40.

Shieh JN and Rossignol PA (1992) Opposite influences of host anemia on blood feeding rate and fecundity of mosquitoes. Parasitology 105: 159-163.

Smith DL and McKenzie FE (2004) Statics and dynamics of malaria infection in Anopheles mosquitoes. Malar J 3: 13.

Smith DL, McKenzie FE, Snow RW and Hay SI (2007) Revisiting the basic reproductive number for malaria and its implications for malaria control. PLoS Biol 5: e42.

Sterling CR, Aikawa M and Vanderberg JP (1973) The passage of Plasmodium berghei sporozoites through the salivary glands of Anopheles stephensi: an electron microscope study. J Parasitol 59: 593-605.

Stierhof YD, Bates PA, Jacobson RL, Rogers ME, Schlein Y, Handman E and Ilg T (1999) Filamentous proteophosphoglycan secreted by Leishmania promastigotes forms gel-like three-dimensional networks that obstruct the digestive tract of infected sandfly vectors. Eur J Cell Biol 78: 675-689.

Sumba AL, Mihok S and Oyieke FA (1998) Mechanical transmission of Trypanosoma evansi and T-congolense by Stomoxys niger and S-taeniatus in a laboratory mouse model. Med Vet Entomol 12: 417-422.

Taylor PJ and Hurd H (2001) The influence of host haematocrit on the blood feeding success of Anopheles stephensi: implications for enhanced malaria transmission. Parasitology 122: 491-496.

Thomas F, Adamo S and Moore J (2005) Parasitic manipulation: where are we and where should we go? Behav Processes 68: 185-199.

Titus RG (1998) Salivary gland lysate from the sand fly Lutzomyia longipalpis suppresses the immune response of mice to sheep red blood cells in vivo and concanavalin A in vitro. Exp Parasitol 89: 133-136.

Warburg A, Touray M, Krettli AU and Miller LH (1992) Plasmodium gallinaceum: antibodies to circumsporozoite proetein prevent sporozoites from invading the salivary glands of Aedes aegypti. Expt Parasitol 75: 303-307. 


\title{
17. Practical application of olfactory cues for monitoring and control of Aedes aegypti in Brazil: a case study
}

\author{
Alvaro E. Eiras, Martin Geier, Andreas Rose and Owen Jones
}

\begin{abstract}
The increasing importance of managing the mosquito vectors of dengue, mainly Aedes aegypti, is recognised world-wide as the disease becomes more prevalent in many countries. The complexity of the urban environment where the vectors breed makes it especially difficult to organise control efforts to reduce their numbers and their impact on disease transmission. Existing control programmes depend on larval surveys for population assessment but these are often unreliable and become available too late for the application of control measures to have a significant impact on disease transmission. The need for better and more timely information about the mosquito vectors of dengue has been recognised in Brazil and several new technologies for more reliable information on vector distribution and population densities have emerged that are making control efforts more effective. Olfactory cues from oviposition sites are now being used to lure gravid Ae. aegypti females into sticky traps for population assessments and indices of dengue risk. The development of the so-called MosquiTRAP which incorporates an oviposition attractant is described and its efficiency relative to more traditional monitoring methods is discussed. Volatile chemicals that attract host-seeking female Ae. aegypti have also been identified. These have been formulated for controlled release ('BG-Lure') and incorporated into novel trap designs ('BG-Sentinel') with much improved capture of Ae. aegypti compared to the standard traps that have been used previously. Both trapping systems are now being used to monitor and survey these important disease vectors. The MosquiTRAP is also an important cornerstone of an online GIS-based surveillance system called MI Dengue (Dengue Intelligent Monitoring System) which is being implemented in Brazil. Results to date have demonstrated that the accuracy of its predictions relating to dengue risk is much greater than that obtained using more traditional methods. Real-time decision-making related to vector management is now possible using the new surveillance systems, and this should greatly impact the management of the disease.
\end{abstract}

Keywords: Aedes aegypti, oviposition attractant, host odours, MosquiTRAP, BG-Sentinel, MIDengue, disease risk assessment, Brazil

\section{Introduction}

Dengue is ranked as the most important mosquito-borne viral disease in the world, where in the last 50 years incidence has increased 30-fold. The World Health Organization (WHO) has estimated that 2.5 billion people live in over 100 countries of Africa, South-East Asia, the eastern Mediterranean, the western Pacific, and the Americas where dengue viruses can be transmitted. Up to 50 million infections occur annually, with 500,000 cases of dengue haemorrhagic fever and 22,000 deaths, mainly among children (WHO 2009).

The virus responsible for dengue fever (DF) and dengue haemorrhagic fever (DHF) belongs to the family Flaviviridae (the same family as the yellow fever virus), and four different viral serotypes exist: DEN-1, DEN-2, DEN-3, and DEN-4 (Gubler 2004). Infection with one dengue serotype provides immunity for years, but it does not protect against infection with the other serotypes. Dengue haemorrhagic fever is more common in children and it can lead to shock from blood loss, and even 
death (Gubler 1998). Dengue virus is transmitted to humans during blood feeding by infected mosquitoes, which have usually acquired the virus by ingesting blood of infected and viremic humans and possibly also by transovarial transmission. The mosquito Aedes aegypti (L.) (Diptera: Culicidae) is considered the main vector, although other Aedes species, including Aedes albopictus (Skuse), have been implicated in rural epidemics as well as some urban ones (Effler at al. 2005). The mosquito Ae. aegypti lives in close association with humans in urban and suburban environments. The mosquito feeds predominantly on human blood and breeds in artificial containers such as drums, buckets, tyres, flowerpots, and vases (Forattini et al. 1995). Therefore, the epidemiology of dengue is highly related to the biology of the mosquito vector and human behaviour, as well as to the environment and the virus itself.

As there is no effective vaccine for dengue, vector control is the main approach for control and prevention. Although insecticide spraying has been used extensively, larval source reduction (eliminating or cleaning water-filled containers that can harbour Ae. aegypti larvae) is considered the most effective way of reducing and controlling the mosquito populations (Gubler 1998).

Scientific interest in research on dengue has grown in many countries, and opportunities for investigators to obtain funds for that research have considerably improved. Funding agencies, such as governments and international organisations, have increased the amount of money available for research in response to dengue's growing global incidence and impact on public health. In Brazil, special programme funds have been allocated by the Federal and State governments directed towards the achievement of new knowledge, control tools and new technologies for dengue and other neglected diseases (CNPq 2009).

In subtropical countries such as Brazil, mosquito population density, dengue transmission, and the number of DF cases start to increase at the beginning of the rainy season (October), with dengue cases peaking more than three months later (Coutinho et al. 2006, Forattini et al. 1995). In Brazil, dengue fever re-emerged as a major urban epidemic in 1986 (Marques et al. 1993) and currently is considered the most important arthropod-borne viral disease (MS 2007a,b). In 2000, all 26 states had reported DF cases and during 1986-2007 a total of 4,559,818 cases were officially reported (MS 2007a) with 493 deaths (MS 2007b).

Transmission of dengue depends on a variety of variables in the relation between virus, mosquito vector and human host (Focks et al. 1995, Kuno 1997). In the assessment of dengue risk, important factors are the level of herd immunity in the human population and the ratio of mosquito to human density, which influences the probability of vector-host contacts. To measure mosquito density, Ae. aegypti control programmes usually still mainly rely on the surveillance of immature mosquito stages (eggs, larvae and pupae) to determine the dengue risk. These surveillance methods rely on tedious and repetitive house-to-house surveys. Data from these surveys are used to calculate indices like the Premise Index (PI) or the Breteau Index (BI). The Premise Index was described in the early 1920s for yellow fever outbreaks and is based on the percentage of premises infected with Aedes larvae or pupae (Connor and Monroe 1923), whereas the BI indicates the number of containers with larvae and/or pupae of Ae. aegypti (Breteau 1954). Both indices are used by WHO as indicators of dengue transmission risk (Focks 2003).

In general, these traditional indices are poorly related to the risk of dengue transmission (Coelho et al. 2008, Focks 2003, Méndez et al. 2006, Reiter et al. 1991, Scott and Morrison 2002). More advanced assessments of immature stages therefore focus only on pupae, as their abundance is more closely linked to adult population size (Focks 2003). A direct surveillance of the adult 
mosquito population was previously possible only with much time and effort (using aspirators or human landing collections) or were inefficient due to low catching rates of nonspecific adult traps (Schoeler et al. 2004). However, studies in Brazil, combining newly-developed specific traps for adult Ae. aegypti with geographic information systems, are now showing that an efficient and real-time surveillance of adult vectors is possible, resulting in a quick determination of dengue risk and effective vector control.

\section{Overview of standard collections methods for dengue vectors}

The collection of host-seeking dengue mosquitoes directly from human volunteers (humanlanding collection or catch) can provide a real-time surveillance of the mosquito population that is actually involved in producing a dengue risk. During a dengue outbreak, however, when it is important to assess the efficacy of the control measures, human-landing collections expose the field workers to an intolerable risk of infection. In addition, the human-landing collection is time consuming, labour intensive and yields variable results due to differences in human attractiveness and in the skill of the field workers. Therefore other methods to collect adult mosquitoes are usually preferred for the collection of dengue vectors. These methods focus either on the detection of dengue mosquito eggs in oviposition traps (ovitraps), on finding their larvae or pupae, or on collecting adult mosquitoes (Service 1993, Silver 2008).

Resting adults can be collected using aspirators, which is labour intensive and costly, but collects large numbers of mosquitoes. Conventional adult traps use only visual cues to attract mosquitoes (Fay-Prince trap, Wilton trap). Catch rates are usually too low when set in relation to their price and the fact that these traps need electricity adds to their inconvenience. The addition of carbon dioxide raises the attractiveness of visual traps, but its use on a larger scale will also be too costly and complicated in most urban dengue-risk areas.

Recent research has led to the development of new traps and attractants that are highly specific for dengue mosquitoes. Sticky traps with oviposition attractants capture mosquitoes that have already ingested a quantity of blood sufficient to produce eggs (e.g. MosquiTRAP ${ }^{\mathrm{TM}}$ ). A new ventilator trap (BG-Sentinel ${ }^{\mathrm{TM}}$ ) used with a combination of odorant substances found on human skin focuses on host-seeking dengue mosquitoes. The BG-Sentinel trap has been shown to have much better catching rates than conventional traps (Kröckel et al. 2006, Meeraus et al. 2008). Both trap types allow for more specific and refined vector control campaigns in situations where the traditional tools are often marginally effective.

\section{Dengue mosquito monitoring through the detection of eggs: ovitraps}

Eggs are collected with an oviposition trap (ovitrap), which is commonly used to detect the presence of Aedes mosquitoes in low density areas. The ovitrap was first described by Fay and Eliason (1966) and consists of a black container filled partially with water and a wooden paddle placed vertically as an oviposition substrate. This trap requires laboratory infra-structure and human resources for counting the eggs deposited on the oviposition substrate and identifying the larval species of Aedes.

Historically, ovitraps have provided useful information in the early detection of new infestations, spatial (presence or absence) and temporal (seasonal) distribution and in monitoring the impact of control measures on Ae. aegypti, including those using pesticides (Focks 2003, Reiter and Nathan 2001, Reiter et al. 1991). However, many comparative studies between the larval survey 
and ovitrap use have shown that the ovitrap, besides being more sensitive in the detection of Ae. aegypti, also has a lower cost but demonstrates limited operational viability in entomological surveillance (Braga et al. 2000, Rawlins et al. 1998). When ovitraps are used in urban areas where several species of the Stegomyia subgenus occur sympatrically, the eggs are morphologically indistinguishable and, thus there is a need for identification of larvae.

Further studies are necessary in order to use ovitraps as a methodological approach to indicate risk of dengue fever and yellow fever, because of the difficulties involved in calculating the population density of adult vectors (Focks 2003).

\section{Dengue mosquito monitoring through the detection of larvae and/or pupae}

\section{Active search for immature stages}

The search of premises for containers that harbour larvae or pupae is still a widespread surveillance method for dengue vectors. The results are usually used to calculate surveillance indices such as the House Index (percentage of houses with larvae and/or pupae, also: Premise Index), the Container Index (percentage of water-containers with larvae and/or pupae) or the Breteau Index (number of positive containers per 100 houses) (Silver 2008). However, these indices do not provide for a significant assessment of the number of adults that are actually produced and the associated dengue transmission risk (Coelho et al. 2008, Focks 2003, Méndez et al. 2006, Reiter et al. 1991, Scott and Morrison 2002).

Because the time from pupa to adult is short and pupal mortality is low, the number of pupae found at a given premise provides the best estimation of adult density (Focks 2003). Linking the number of pupae to human density can thus give an improved estimate of an important factor in dengue risk assessment, the relation of mosquito density to human density. Focks (2003) therefore promotes a 'pupal/ demographic survey method'. This method combines interviews to determine the number of people living or sleeping at the examined premises with the examination of the water-holding containers for pupae. Pupae are collected and, in the case of the presence of various container-breeding species, kept in the laboratory until adult emergence for identification. Using standardised classification methods to identify and focus on the most productive containers should both make surveillance and the control through source reduction more efficient (Barrera et al. 2006, Chadee et al. 2007, Focks and Alexander 2006, Koenraadt et al. 2007). However, pupal habitats may vary greatly in different settings, making a comparison and standardisation difficult.

\section{Larval traps}

Larval traps of various designs made of automobile tyres (tyre-section traps) have been used for monitoring Aedes oviposition activity (WHO 1999). A standard larval trap ('larvitrap') consists of a water-filled section of a tyre that facilitates visual inspection of the water in situ and allows for the ready transfer of larvae to another container for examination. However, such a trap has many disadvantages such as low sensitivity and the eggs laid in the trap have to be in contact with water for hatching. In Brazil, the tyre-section larval trap was evaluated in the field and the results showed that ovitraps are much more sensitive than larval traps (Marques et al. 1993). In spite of the difference in sensitivity, larval traps are still widely used in Ae. aegypti surveillance programmes and studies in Brazil (Dibo et al. 2005) and other countries, such as Argentina (Micieli and Campos 2003), Peru (Morrison et al. 2004), Nicaragua (Lugo et al. 2005), Thailand (Tsuda et al. 
2006), Columbia (Cuéllar-Jiménez et al. 2007, Maestre-Serrano et al. 2008) or Cuba (Suárez Ramírez and Colás Bonne 2008).

\section{Floating larval trap}

An alternative larval trap, the 'floating larval trap', was developed and evaluated for eliminating biases commonly associated with human collection (larval survey) (Harrison et al. 1982). The trap collects diving mosquito larvae and pupae when they float back to the water surface. Both field and laboratory data revealed that the trap was highly efficient and sensitive for detecting immature stages of Ae. aegypti and Culex quinquefasciatus Say. The trap was claimed to function as a random sampling device, providing an equal chance for the capture of immatures. However, fewer $1^{\text {st }}$ and $2^{\text {nd }}$ instars larvae are collected because they often remain closer to the water surface and do not dive as deeply when disturbed. Specimens captured in the trap remained alive for at least $48 \mathrm{~h}$. Although the size of this instrument $(>13 \mathrm{~cm}$ diam.) could probably be reduced, its use excludes important Ae. aegypti larval habitats such as vases, tyres, small plastic containers and most footbaths.

\section{Detection of adult dengue mosquitoes}

\section{Human landing collection}

Although laborious, collecting mosquitoes directly from a human host obviously provides the best indication of the number of mosquitoes that are currently involved in the transmission cycle and can therefore give key information in the assessment of the dengue risk. The method has been used to determine daily biting periodicity in different areas of the world (Chadee and Martinez 2000). However, catch rates depend on the time of day and the skill, motivation and attractiveness of the individual field worker. Results are therefore variable and may lack the accuracy needed for a reproducible and meaningful comparison of data, especially in the study of seasonal variations. Because the technique exposes field workers to infected mosquitoes, it is currently undergoing ethical restrictions (Focks 2003, PNCD 2002). The human landing collection is therefore not used as a standard surveillance method for dengue mosquitoes, but remains an important reference method in the assessment and comparison of other collection methods (Jones et al. 2003, Kröckel et al. 2006, Schoeler et al. 2004).

\section{Sampling resting adults with aspirators}

Aspirators are used to capture resting adult mosquitoes, especially indoors (Nasci 1981) in dark places and this method provides an estimate of adult density in the sampled houses (Morrison et al. 2004; Schoeler et al. 2004). Aspirators are either carried on the back of the technician (e.g. CDC backpack aspirator, John W. Hock Co., Gainesville, FL, USA) or hand held as in the Nasci aspirator. As the field worker needs to enter private homes, this method is invasive and requires high tolerance of the inhabitants. It is also labour intensive and results may be affected by the efficiency of field technicians (Clark et al. 1994, Favaro et al. 2006). Using backpack aspirators is therefore usually considered more for research than for routine activities of dengue control programmes (Favaro et al. 2008). 


\section{Traps for gravid Aedes aegypti}

Artificial oviposition sites can be used to attract and catch gravid mosquito females. Their attractiveness for dengue mosquitoes can be augmented by the addition of decomposing organic matter in fermenting infusions made from grass, hay, leaves or other plant material (Chadee 1993, Du and Millar 1999, Reiter et al. 1991, Sant'Ana et al. 2006, Takken and Mboera 2000). The CDC gravid trap (John W. Hock Co., Gainesville, FL, USA) was originally designed by Reiter (1983). A battery-powered fan draws mosquitoes attracted by grass or hay infusions into a catch bag. In contrast, sticky oviposition traps use glue boards to capture landing mosquitoes and do not need electricity. Different models have been developed to capture gravid Ae. aegypti (Fávaro et al. 2006, Gama et al. 2007, Kay et al. 2000, Muir and Kay 1998, Ordóñez-Gonzalez et al. 2001, Ritchie et al. 2003, 2004) and Ae. albopictus (Facchinelli et al. 2007). Like the CDC gravid trap, they use decomposing organic material in water to attract mosquito females searching for an oviposition site. Although these infusions are relatively easy to produce, they need approximately one to three weeks time to ferment. Their composition also changes with age and time in the traps, potentially altering their attractiveness and making a comparison of trapping results more difficult.

\section{Traps using visual cues}

The use of light traps constitutes a method for capturing a large variety of adult insects and is often used to collect nocturnal mosquito species. However, light traps are not effective at capturing the diurnal Ae. aegypti (Silver 2008). The Fay-Prince trap (John W. Hock Co., Gainesville, FL, USA) is a daytime trap with black and white contrasts and uses a fan to draw approaching mosquitoes into a catch bag (Fay and Prince 1970). Another trap designed to capture day-active mosquitoes such as Ae. aegypti is the Wilton trap (Wilton 1985) (John W. Hock Co., Gainesville, FL, USA). The Wilton trap consists of a glossy black cylinder with a fan and collection cup inside. Both traps catch mosquitoes of both sexes and all physiological stages. The catch rates for visual traps are, however, only a fraction of the human landing collection (Jones et al. 2003, Kröckel et al. 2006, Schoeler et al. 2004).

\section{Traps using carbon dioxide}

Carbon dioxide is a general attractant to a large majority of mosquito species. Liberated either from dry ice or pressurised gas bottles, $\mathrm{CO}_{2}$ enhances the catch rates of the Fay-Prince and the Wilton traps, but results for Ae. aegypti are poor compared to human landing collections (Canyon and Hii 1997, Schoeler et al. 2004). Some trap types that are commercially available for the end consumer ${ }^{1}$ produce $\mathrm{CO}_{2}$ from the combustion of propane gas. The reaction is usually supported by a catalyst and yields warm, humid $\mathrm{CO}_{2}$ of a concentration attractive to mosquitoes (Kline 2002). The attractiveness of these traps for dengue mosquitoes has not been investigated. As these traps are relatively expensive, they are not practical for use in routine monitoring programmes. Other traps with carbon dioxide that are routinely used to monitor mosquitoes, such as the EVS-trap or the CDC-light-traps with $\mathrm{CO}_{2}$, also catch Ae. aegypti and Ae. albopictus (Russel 2004), but the costs, efforts and logistics that come along with the use of $\mathrm{CO}_{2}$ from any of these sources currently do not make theirr application feasible in most field situations.

\footnotetext{
${ }^{1}$ E.g. Woodstream Mosquito Magnet, Blue Rhino Skeeter Vac, Flowtron Mosquito Power Trap, Lentek Mosquito Trap Guardian (list does not claim to be exhaustive).
} 


\section{The need for simple, efficient, and specific collection tools}

A suitable tool for collecting adult dengue vectors in routine monitoring operations should be sensitive enough to detect adults also when they occur in low densities. The tool should be robust and workable for routine uses under various conditions and the caught mosquitoes should be easily removable from the collection device to be identified and/or processed for further virus detection. The collection rate should correlate very closely with human landing collections in order to establish meaningful risk indicators.

\section{MosquiTRAP for collection of gravid Aedes aegypti}

\section{Olfactory cues}

The deposition of eggs by mosquitoes is mediated by olfactory cues, which originate from the breeding site as a result of microbial activity (Bentley and Day 1989; Clements 1999). Apparently, Metha (1934) was the first to report the existence of olfactory cues in recognition and selection of oviposition sites by mosquitoes. Water containing decomposing organic matter was found attractive for gravid mosquitoes and has been widely used for mosquito surveillance and possibly, mosquito control (Chadee et al. 1993, Eiras 2001, Millar et al. 1992, Reiter et al. 1991, Takken and Mboera 2000). Hay and grass infusions have been reported since the 1980's to be attractive for ovipositing females of a wide range of mosquito species, including Ae. albopictus (Holck et al. 1988), Ae. aegypti (Chadee et al. 1993), Ae. triseriatus (Say) (Holck et al. 1988), Cx. nigripalpus Theobald (Ritchie 1984), Cx. pipiens Linnaeus (Reiter 1986), Cx. quinquefasciatus (Mboera et al. 2000). The infusions are usually made of many different organic materials, such as leaves, grass and sod (Allan and Kline 1995, Chadee et al. 1993, Rawlins et al. 1998, Reiter et al. 1991).

There are differences in the selection of breeding sites between Ae. albopictus and Ae. aegypti. The former species breeds in natural and manmade containers and is often most abundant in rural and sylvan areas, but is able to rapidly colonise suburban an urban areas with sylvan characteristics (Hornby et al. 1994). The second species is a container-inhabiting mosquito that occurs in urban areas and oviposits in many breeding sites such as tyres, plant vases, and other water-holding containers associated with human activities. Many reports have shown that gravid Ae. aegypti and Ae. albopictus lay more eggs in ovitraps when baited with grass infusions compared to clean water (Chadee et al. 1993, Reiter et al. 1991, Sant'Ana et al. 2006).

Many organic materials have been used for increase the efficacy of ovitraps for sampling Aedes eggs in the field, such as oak leaves (Quercus spp.) (Allan and Kline 1995), hay infusions of Axonopus compressus (Sw.) Beauv. (Rawlins et al. 1998), Bermuda hay infusions (Reiter et al. 1991), infusions of dried Sclerica bractaeda (L.) (Chadee et al. 1993), palletised plant-based animal feeds (Ritchie et al. 2003) and leaves of Panicum maximum Jacq. (Sant'Ana et al. 2006). Sant'Ana et al. (2006) have shown that the oviposition responses of Aedes (Stegomyia) mosquitoes changed with respect to grass species, stage of plant development and type of fermentation, thus suggesting that grass leaves have different chemical profiles and bacteria and other microorganisms present in the infusions and that they may produce several chemical lures for gravid mosquitoes. Neglecting to take into account the age of infusions for monitoring seasonal abundance of mosquitoes by ovitraps baited with grass infusions may constitute a significant source of variability in the results of these studies. The period of fermentation of grass infusions has a significant effect on the response of gravid mosquitoes and infusions of known and standardised fermentation periods should be used only. For instance, studying various ages of fermentations (e.g. 1, 3, 5, 7, 15, 20 and 
30 days), Sant'Ana et al. (2006) demonstrated that the best fermentation ages for grass infusions of P. maximum were 15 and 20 days. Most likely, the period of fermentation may change the chemical profile of volatiles produced by grass infusions and, consequently, the oviposition response of $A e$. aegypti and Ae. albopictus may be affected.

Identification of oviposition attractants for Aedes aegypti from Panicum maximum grass infusion

The use of specific synthetic oviposition attractant chemicals in monitoring programmes for Aedes (Stegomyia) mosquitoes would eliminate the need to create infusions and would allow for the preparation of precise formulations of an oviposition medium. Therefore, synthetic oviposition attractants can eliminate variation in infusion quality and attractiveness.

One important aspect of the use of oviposition attractants in trapping devices is to understand the role of the individual active chemicals. Several studies used dual-choice bioassays at close-range, where the oviposition behaviour comes at the end of a behavioural sequence and thus, they did not discriminate between long-range and short-range responses. As it is likely that oviposition stimulants act mainly at close-range, such studies may underestimate the role of these stimulants at long range.

There are several publications on the evaluation of synthetic oviposition attractants for mosquitoes in the field (Allan and Kline 1995, Beehler et al. 1994, Mboera et al. 2000). However, only few chemical identifications of oviposition attractants have been conducted for Culex (Du and Millar 1999, Hwang et al. 1980, Leal et al. 2008) and Aedes mosquitoes (Bentley et al. 1979, Ponnusamy et al. 2008, Sant'Ana 2003). Some compounds such as nonanal, p-cresol, indole and 3-methylindole have been identified as oviposition attractants for $C_{x}$. quinquefasciatus and $C_{x}$. tarsalis mosquitoes (Du and Millar 1999, Leal et al. 2008) and Aedes mosquitoes (Bentley et al. 1979). Some specific bacteria associated with carboxylic acids and methyl esters have been described in binary choice assays as potent oviposition stimulants for gravid Ae. aegypti, but the elucidation of these compounds has not yet been completed (Ponnusamy et al. 2008)

Oviposition attractants for gravid Ae. aegypti were isolated and identified from $P$. maximum grass infusions (Sant'Ana et al. 2006) and these are considered an important tool for increasing the catch of gravid Ae. aegypti in the sticky trap MosquiTRAP (Eiras and Resende 2009, Eiras and Sant'Ana 2002, Favaro et al. 2006, 2008, Lourenço-de-Oliveira et al. 2008). The chemical identification of volatiles produced by grass infusion of $P$. maximum that enhanced oviposition responses of gravid female Ae. aegypti was possible only by means of a combination of studies using electrophysiology (electroantennography), gas-chromatography coupled with mass-spectrometry (GC-MS), gaschromatography coupled with electroantennography (GC-EAD) and behaviour using ovitraps (Eiras et al. 2001, Eiras and Sant'Ana 2002; Sant'Ana 2003; Eiras et al. 2004). Firstly, the grass infusions of different ages (Sant'Ana et al. 2006) were extracted individually by solvent and by solid phase micro extraction (SPME) and submitted individually to GC-MS, GC-EAD and EAG and compared with results from oviposition responses. It was interesting that the responses of gravid female Ae. aegypti antennae to EAG extract of grass infusions of different ages followed a similar pattern to the oviposition responses to grass infusion extracts in the field (Figure 1), showing that either the quantity of active compounds increased with ageing of the grass infusion or that new compounds were produced as the grass infusions fermented. Later, the active compounds present in infusion extracts of high EAG and oviposition responses were submitted to GC-MS for identification. Seven compounds were identified as candidates for an oviposition stimulant or attractant of gravid Ae. aegypti: nonanal, benzothiazol, decanal, p-cresol, indole, 3-methyl indole and limonene) (Eiras and 


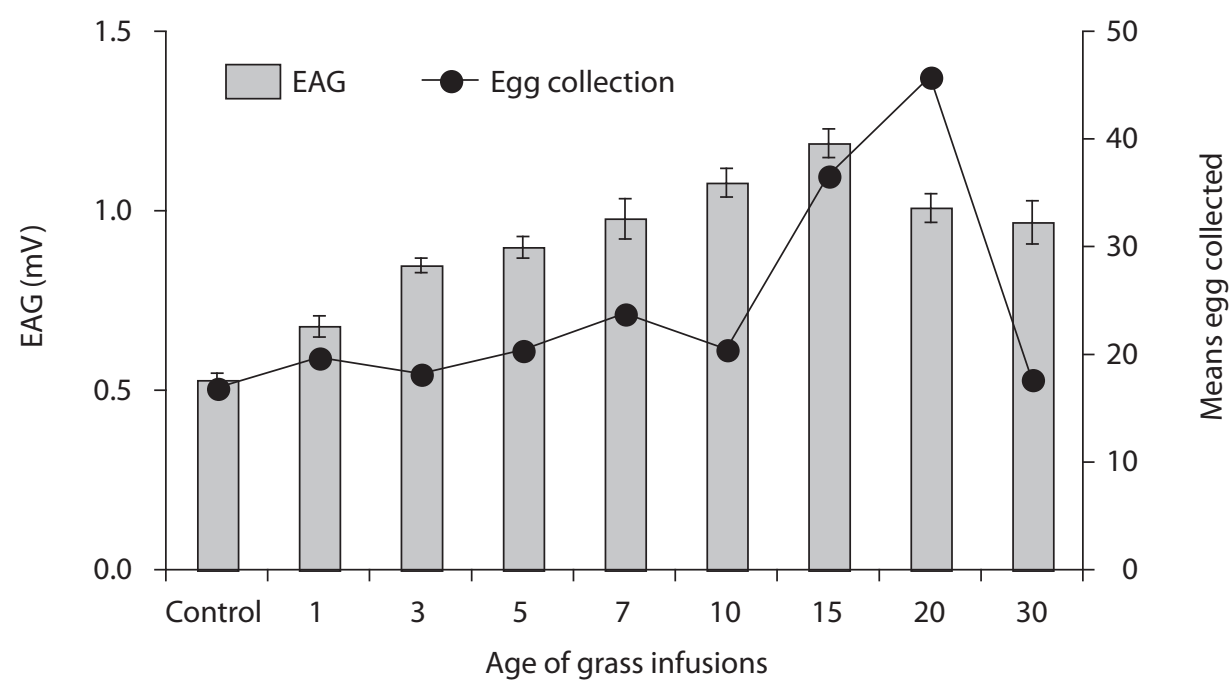

Figure 1. Behavioural (ovitrap) and electrophysiological (EAG) responses of gravid Aedes aegypti to Panicum maximum grass infusions of different ages (Sant'Ana 2004).

Sant'Ana 2002 - patent pending). The EAG studies of the seven individually identified compounds showed that the aldehydes (nonanal) elicited the highest antennal responses of gravid female Ae. aegypti. The GC analysis showed that the grass infusion produced a large number of volatiles. The compound 3-methylindole has a very unpleasant and strong smell even in small concentrations, and has been shown to modify the response of gravid female Ae. aegypti (Allan and Kline 1995, Sant'Ana 2003). However, 3-methylindole was revealed to have a repellent effect on Ae. albopictus in laboratory assays and in the field using ovitraps (Trexler et al. 2003). By contrast, 3-methylindole is an oviposition stimulant for Cx. quinquefasciatus at very low concentrations (Mboera et al. 2000).

Based on the fact that many substances are produced by grass infusions and that only a few key chemical compounds are responsible for attracting gravid mosquitoes to breeding sites, many of the volatiles present in infusions of organic material are likely to be produced by other sources, such as plants or decaying materials. The responses of gravid Ae. aegypti mosquitoes to a single compound could result in attraction to an inadequate place for oviposition, but the complexity of chemical signals may be crucial as an indicator of a potential breeding site.

\section{Formulation of lures for Aedes aegypti from synthetic oviposition attractants}

The majority of semiochemicals that have been described as oviposition attractants for Aedes species would volatilise very quickly if simply added to the water surface of an oviposition trap. Some form of controlled-release technology is required to release the active substances at a rate that is optimal for continued attraction of the target species to the oviposition trap. In the case of Aedes species, two types of controlled-release devices have been used. One was a solid matrix polymer, within which the active ingredient nonanal was evenly distributed while the other was released from a reservoir system that took the form of sealed tube. The reservoir system tend to release more chemicals at the beginning of their field life in contrast with that released from solid matrix polymer, which was more towards the end of their field life (Figure 2). There is a high 


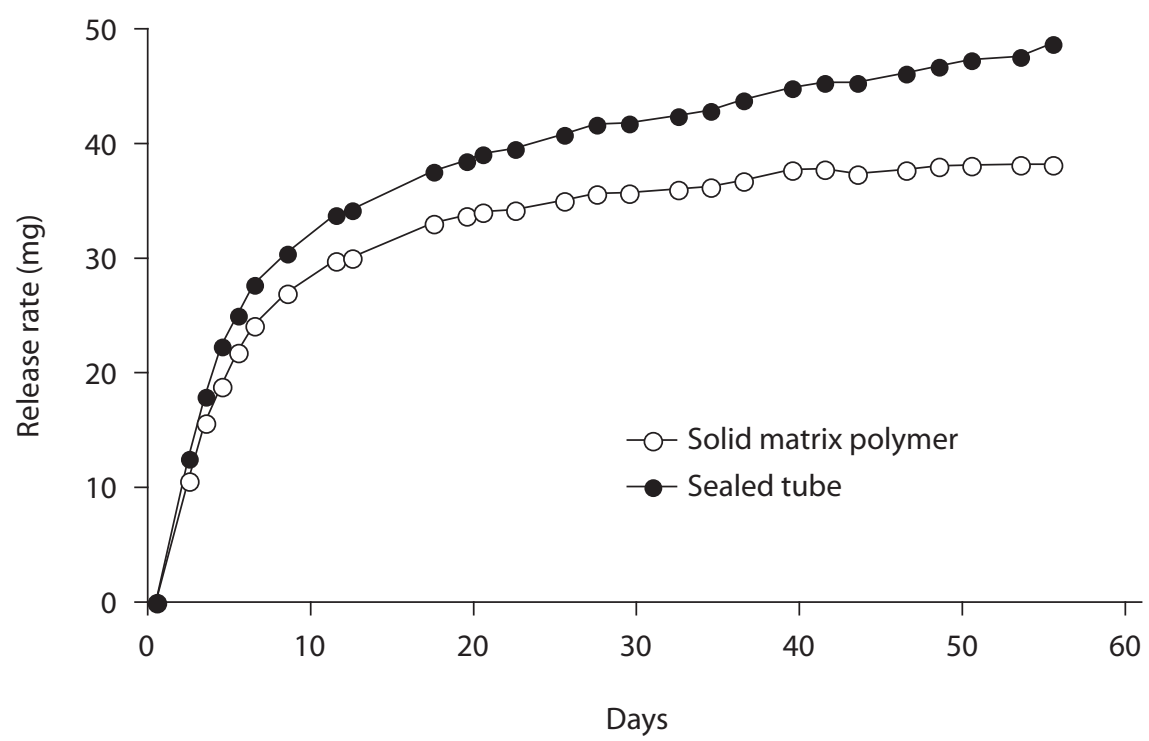

Figure 2. Release rate of nonanal (AtrAedes) at solid matrix tube and sealed tube dispensers at $25.1 \pm 1.87^{\circ} \mathrm{C}$ (Data not published).

evaporation rate during the first 10 days before both devices get a steady release rate. This is often referred to as a 'first order' release profile, while the preferred profile would be one where the amount of active substance released remains constant during the whole field life of the dispenser and referred to as a 'zero order' release profile. Dispensers with a reservoir system tend to show a zero order release profile and therefore are preferred as dispensers, as the attraction rate remains more consistent during the whole length of the life of the dispenser in the field.

\section{Laboratory bioassays}

Behavioural studies of gravid female Ae. aegypti exposed to synthetic lures were carried out in a dual-port olfactometer (Geier and Boeckh 1999) in response to log-doses of nonanal. It is interesting that the response of host-seeking Ae. aegypti to a human hand in the olfactometer bioassay is usually about $85-100 \%$, whereas the response of gravid females to grass infusions is about $40-50 \%$ (Mota 2003). The response of gravid Ae. aegypti to oviposition attractants was consistently within the range of 31 to $45 \%$ responding whereas the response of host-seeking mosquitoes to human odour was about $80 \%$ (Table 1). The synthetic oviposition attractant (nonanal) and the AtrAedes lure elicited similar responses in gravid Ae. aegypti.

\section{Semi field test}

Semi-field systems have been used for a variety of studies on mosquitoes, especially on the behavioural ecology of malaria vectors (Knols et al. 2002, Schmied et al. 2008) and for evaluating trapping devices for Ae. aegypti (Kline 2002). Although there are some limitations associated with these studies, for instance that long-range dispersal cannot be studied. Semi-field cages are considered a controlled environment that offers intermediate conditions between the laboratory and the field for behavioural studies, where both laboratory and wild-caught insects can be tested. 
Table 1. Behavioural responses of host seeking and gravid female Aedes aegypti to different stimuli in a dual port olfactometer (Data not published).

\begin{tabular}{llrlr}
\hline & Stimulus & N & Control & Test \\
Host-seeking female & Human hand & 30 & $81.9 \% \pm 10.8$ & $1.1 \% \pm 2.6^{*}$ \\
Gravid female & Grass infusion & 33 & $35.0 \% \pm 4.0$ & $21.1 \% \pm 3.8^{\text {ns }}$ \\
& Nonanal $(10 \mu \mathrm{g})$ & 8 & $31.8 \% \pm 13.1$ & $22.6 \% \pm 6.4^{\text {ns }}$ \\
& AtrAedes ${ }^{\text {TM }}$ Lure & 8 & $43.4 \% \pm 13.6$ & $25.2 \% \pm 6.0^{\text {ns }}$ \\
\hline
\end{tabular}

* Means $P<0.05$ and ns $=$ means $P>0.05$.

In Brazil, a semi-field experimental area with eight test cages was developed for studying behavioural responses of gravid Ae. aegypti mosquito to evaluate prototype traps, controlled release devices and the development of new formulations of synthetic oviposition attractants (Roque and Eiras 2008). As laboratory-reared mosquitoes are used in these field cages, the age and physiological status of test mosquitoes, which are free of viruses and bacterial contamination, are well established. Field cage results showed that gravid Ae. aegypti mosquitoes had similar responses in a field cage as in field tests in urban areas, confirming that the semi-field set up is reliable, safe and gives reproducible results.

Behavioural studies in semi-field cages (Roque and Eiras 2008) showed that the MosquiTRAP baited with a formulation of synthetic oviposition cues caught similar proportions of gravid Ae. aegypti to traps baited with infusions of 10-45 days of fermentation (Figure 3). However, the oviposition cues caught a significantly higher proportion of mosquitoes than infusions aged 5 or 60 days $(P<0.01)$ (Roque 2007). Therefore, the synthetic oviposition cue can replace the natural grass infusions. The behavioural response of gravid Ae. aegypti is likely to be governed by a complex mixture of

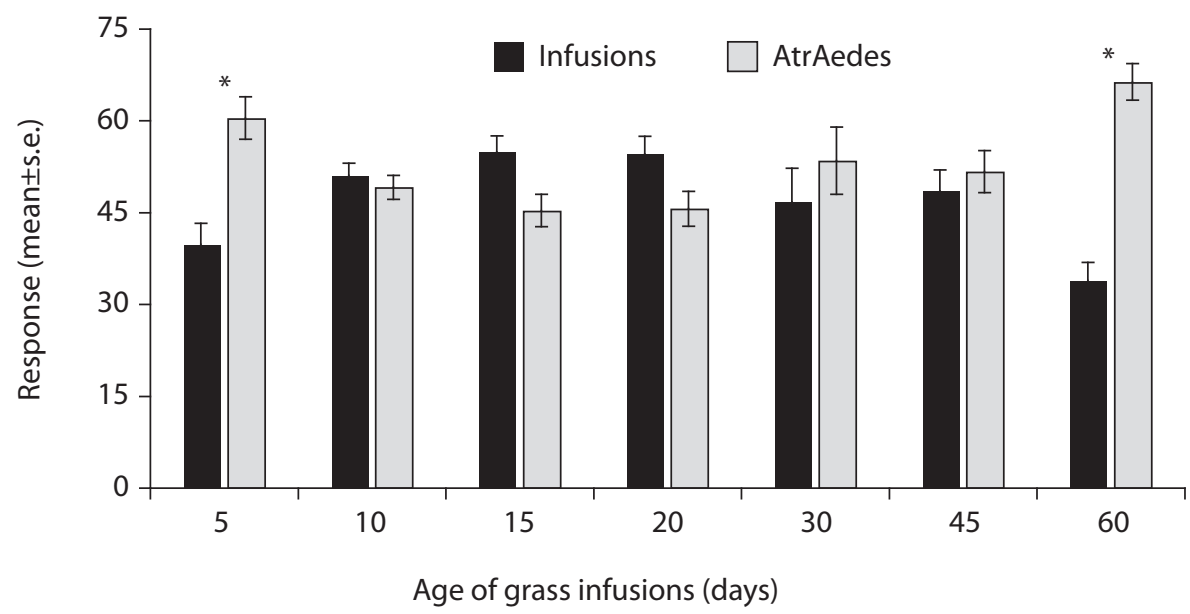

Figure 3. Behavioural responses of gravid females Aedes aegypti to the sticky trap (MosquiTRAP) within field cages to grass infusion (Panicum maximum) and synthetic oviposition attractants (AtrAedes) (Roque 2007).

${ }^{*}$ Means $\mathrm{P}<0.05$. 
several compounds having specific release rates. A single compound at a very precise release rate can replace the grass infusion. Again, oviposition behaviour is probably mediated by a complex combination of many compounds rather than by one single compound (Eiras 2001). Moreover, the concentration of the compound in the odour plume may play an import role as chemical signal to gravid mosquitoes.

\section{Field evaluation of oviposition lures for Aedes aegypti}

Field studies showed that the AtrAedes lures are still attractive after 30 days of field use (Eiras et al. 2004) and their performance as bait for the Brazilian sticky trap (MosquiTRAP) has been evaluated with adult collection methods, using an aspirator. In Brazil, the sensitivity of the sticky trap was compared with manual aspirations (Nasci 1981) to detect the presence of A. aegypti females during the rainy season for 23 weeks and it was concluded that the sensitivities of both methods were similar (Favaro et al. 2008). The authors pointed out that the relative efficiency of use of the MosquiTRAP was 30 traps per person per day whereas that of manual aspirators was five collections per person per day. Collection with aspirators requires the collector to enter indoor sites, thus causing potential problems with the residents. In a dengue endemic area in Rio de Janeiro city during the rainy season, the sticky trap baited with AtrAedes lures was also compared with CDC backpack aspirators in a mark-release-recapture experiment. From a total of 1,240 gravid dust-marked Ae. aegypti released in the field, 127 (10.2\%) were recaptured by baited MosquiTRAPs whereas only $47(3.8 \%)$ were recaptured with the backpack aspirator at 10, respectively 8 , days after release(Maciel de Freitas et al. 2008).

\section{Traps for collection of gravid adult Aedes aegypti}

Only a few traps are specifically designed for catching previously blood fed, i.e. gravid female Ae. aegypti. The CDC gravid trap (John W. Hock Co., Gainesville, FL, USA) was originally designed by Reiter (Reiter 1993) to attract and catch gravid mosquitoes. It has a battery-powered fan mounted inside that draws mosquitoes attracted by grass or hay infusions into a catch bag. Later, the CDCgravid trap was modified by Dennet et al. (2007) and visual aspects were incorporated to increase the catch of gravid Ae. albopictus. The modified gravid trap did not turn out to be effective at catching gravid Ae. albopictus, probably because the standard oviposition attractant used had been developed for Culex spp. and may not have been attractive to Ae. albopictus.

Several sticky trap models have been developed to capture gravid Ae. aegypti (Favaro et al. 2006, Gama et al. 2007, Kay et al. 2000, Muir and Kay 1998; Ordonez et al. 1997, Ordonez-Gonzalez et al. 2001, Ritchie et al. 2003, 2004) and Ae. albopictus (Facchinelli et al. 2007). The efficacy of a sticky ovitrap lined with an adhesive paper strip was tested in a dengue area in Guadelupe, Mexico using mark-release-recapture (MRR) methods (Ordonez-Gonzalez et al. 2001). A similar type of sticky ovitrap with natural oviposition attractants was developed and evaluated in an Ae. aegypti infested area in Cairns, Australia, where an association between the number of adult females captured and the risk of dengue transmission was found (Ritchie et al. 2004). The Australian sticky ovitraps have also been used for surveillance and behavioural investigations of Ae. aegypti and Ae. polynesiensis (Marks) in Moorea, French Polynesia (Russell and Ritchie 2004) and in the Torres Straits, Australia (Ritchie et al. 2006). In Italy, Facchichelli et al. (2007) also developed a new sticky trap design, which was shown to be specific for Ae. albopictus in field trials in Rome. The MosquiTRAP has been used for studies of mark-release-recapture in a suburban residential area of Rio de Janeiro (Brazil) (Maciel de Freitas and Lourenço de Oliveira 2009, Maciel de Freitas et al. 2008) and in Cairns, Australia (Russell et al. 2005). 
In Brazil the MosquitoTRAP is used for monitoring populations of Ae. aegypti in urban areas (Eiras and Resende 2009). Such sticky traps are a low-cost device without the need for electricity, laboratory facilities or human resources, providing faster results than the ovitrap, larval survey and other adult traps for dengue control. Mosquito behaviour in sticky traps appears to be similar in different areas (Favaro et al. 2006, Gama et al. 2007, Ritchie et al. 2004); once stuck, the mosquito remains in resting position. Those that escape, usually loose one or more legs that remain adhered to the sticky card. Identification of collected mosquitoes is still possible with their thoraces, which usually remain somewhat visible (Gama et al. 2007, Ritchie et al. 2003).

The MosquiTRAP Version 1.0 (patent pending) was developed in 2001 at the Federal University of Minas Gerais and consisted of a 1 litre black plastic cylinder filled with $300 \mathrm{ml}$ of grass infusion (as a natural oviposition attractant) and a removable sticky card on which the mosquitoes are captured (Eiras 2002). During the dry season of 2003, field studies showed that the MosquiTRAP Version 1.0 was more sensitive to detect the presence of Ae. aegypti in urban areas than the traditional larval survey used in Brazil (Gama et al. 2007), especially during the dry season when the larval method cannot detect this mosquito. Therefore, the Brazilian National Programme of Dengue Control (NPDC) stimulated and provided funds for further studies. In 2004-2005, the Brazilian NPDC sponsored field evaluations of the MosquiTRAP version 2.0 and the MI-Dengue technology in 10 Brazilian cities, aiming to supplement new tools and develop new entomological indices for dengue vector control (Eiras et al. 2005, 2007). For such studies, it was necessary to develop a new sticky trap prototype that could be produced on a large scale. The MosquiTRAP Version 2.0 was developed at the University of Minas Gerais and prototyped by professional designers, looking for easy and rapid functionality for field workers. This trap version was funded by several Brazilian public grants that encouraged the creation of a 'spin-off' company, namely Ecovec Ltda., in order to commercialise it.

The MosquiTRAP Version 2.0 was evaluated and compared with larval surveys and ovitraps in 10 Brazilian cities. The results demonstrated that field workers were able to identify 95 to $100 \%$ of Ae. aegypti captured in MosquiTRAPs and that the larval survey was less sensitive than the sticky trap, whereas the ovitrap was more sensitive than both methods (Eiras et al. 2005).

The position in which the sticky trap is placed in houses is very important in order to avoid rejection by the residents. Studies in Brazil demonstrated that the MosquiTRAPs placed outdoors captured five times more females than traps placed indoors (Favaro et al. 2006). This is probably because Ae. aegypti feeds on human blood indoors and lays eggs outdoors, where more breeding places are available. Outdoor traps have also a great advantage of being a non-invasive method of mosquito sampling because trap inspection does not require house entry for mosquito inspections, which is obviously inconvenient for homeowners as well as for vector control workers. Beside the sticky trap being easy to manipulate, it was readily accepted by the residents and local community. A study in Brazil has shown that the MosquiTRAP had a high level of acceptance by the local community (95.6 to $99.3 \%)$, whereas the larval survey had a low acceptance $(60-90 \%)$. The time spent for sticky trap inspection is about 3-5 min whereas the larval survey is about 12-25 min (Eiras et al. 2005). The selectivity of the MosquiTRAP in catching gravid Ae. aegypti was shown by Favaro et al. (2006): of 488 dissected females, 426 were gravid (87.3\%).

Several studies have been conducted to compare the sensitivity of the sticky trap with that of the ovitrap (Facchinelli et al. 2007, Fávaro et al. 2006, 2008, Gama et al. 2007, Honorio et al. 2009, Lourenço-de-Oliveira et al. 2008, Ritchie et al. 2003, 2004) and the aspirator (Favaro et al. 2008, Maciel de Freitas et al. 2008). Generally, gravid Ae. aegypti lay eggs in both natural and artificial 
containers, indoors or outdoors and exhibit an oviposition pattern known as 'skip-oviposition', which means that a single gravid female lays her eggs in several containers (Cobert and Chadee 1993). Consequently, one recipient, such as an ovitrap, may contain eggs from several Ae. aegypti females (Reiter et al. 1991), which precludes the possibility of knowing the number of females that laid eggs in the ovitrap. The sensitivity of the ovitrap (presence of at least one egg) and the sticky trap (presence of at least one adult female Ae. aegypti) was measured in field trials. Gama et al. (2007) reported that during the dry season, when the population of Ae. aegypti is low, the ovitrap is more sensitive (16-67\%) than the sticky trap (0-32\%) whereas Favaro et al. (2006) showed a similar sensitivity of the MosquiTRAP (82.1\%) and the ovitrap (89.7\%) during the rainy season. In Australia, Ritchie et al. (2003) reported that the sensitivity of sticky ovitraps (67.5\%) was slightly higher than that of oviposition traps (64\%). The sensitivity of a trap to detect the presence of disease vectors and to measure population fluctuations with time is a key aspect in effective monitoring of dengue risk and dengue control programmes.

A new MosquiTRAP design (Version 3.0) (Figure 4) consists of a polyethylene mat black container (33 cm high by $15 \mathrm{~cm}$ wide), divided into two parts: a base, filled with approximately $300 \mathrm{ml}$ of tap water, and an upper part with a funnel-shaped opening, facilitating the mosquito's entry and hindering its exit (Eiras and Resende 2009). The sticky card is attached from the water line in the base to the upper part of the trap and the synthetic oviposition attractant (AtrAedes) is attached to the sticky card. This new MosquiTRAP model \#3.0 was compared with the previous model \#2.0 in semi-field and urban areas of Brazil. In the semi-field, the new version caught a significantly higher number of gravid Ae. aegypti (total of 244; mean 5.5 \pm 0.46 ) than the previous version (total of 91; mean 2.1 \pm 0.29 ) (Eiras et al. 2005). In urban areas (Boa Vista, Roraima State, Brazil) 40 sticky traps of each version were set in 40 blocks and the evaluation ran for 12 weeks. The previous MosquiTRAP version 2.0 caught 47 gravid female Ae. aegypti whereas the new version 3.0 caught 127.

This new MosquiTRAP version has been evaluated for dispersion of Ae. aegypti (Maciel de Freitas and Lourenço-de-Oliveira 2009, Maciel de Freitas et al. 2007, 2008) and temporal patterns of Ae. aegypti population dynamics (Honorio et al. 2009) and in a novel field data acquisition of adult female Ae. aegypti and processing-generating GIS real-time web-site surveillance system (Eiras and Resende 2009).

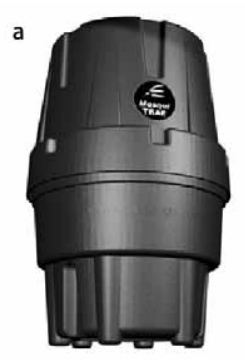

b
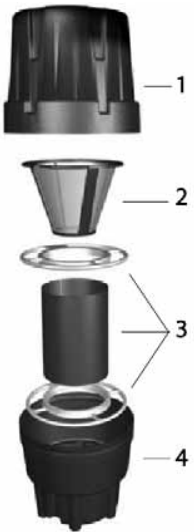

Figure 4. The Brazilian sticky trap MosquiTRAP'TM: (A) Sticky trap assembled and (B) its inner parts: (1) upper opening; (2) funnel shape entering; (3) sticky card set on rings and (4) lower pot with water (Eiras et al. 2007). 


\section{Traps for collection of host-seeking adult Aedes aegypti}

\section{Host odours attractive for Aedes aegypti}

\section{Composition of host odours attractive for Aedes aegypti}

In the collection of mosquitoes in general, the most effective attractant is carbon dioxide (Chapter 5 , this volume). For the highly anthropophilic Ae. aegypti, carbon dioxide is, however, not very effective if used as the sole attractant for gravid females. As the gas is either liberated from dry ice, from pressurised bottles or from the combustion of propane gas, its use is costly, complicated and not feasible in typical dengue endemic urban environments. The search for Ae. aegypti attractants other than carbon dioxide first focused on lactic acid. This substance, present on human skin as well as in breath, was repeatedly shown to attract Ae. aegypti, but until the beginning of the 1990's only in combination with carbon dioxide (Acree et al. 1968, Eiras and Jepson 1991, 1994, Smith et al. 1970).

Attraction to lactic acid without the addition of carbon dioxide was first shown in a Y-tube olfactometer that allowed for an especially fine distinction of the effect of olfactory attractants (Geier and Boeckh 1999). The apparatus also demonstrated the attraction of female Ae. aegypti to a human skin extract. This extract had been produced by rubbing the skin of a human test subject with ethanol-soaked cotton pads and a subsequent extraction of these pads. Although lactic acid was also a component of the skin extract, the extract was more attractive than lactic acid alone and was the same as that of a human finger introduced into the olfactometer.

Ammonia has also been found to be an attractive component. It had already been shown to be attractive for other haematopageous insects (Hribar et al. 1992, Taneja and Guerin 1997), but earlier authors could not demonstrate the attraction of Ae. aegypti to ammonia (Brown et al. 1951, Müller 1968, Rössler 1961;). It had never before been tested in combination with lactic acid. Reinvestigation of this combination showed a synergistic effect of ammonia with lactic acid (Geier et al. 1999a). However, the combination of lactic acid and ammonia was again not as attractive as the complete skin extract, indicating that yet other components play an additional role.

Because fatty acids are widely present on human skin, their possible role as attractants for mosquitoes had already been proposed by earlier authors (Carlson et al. 1973, Knols et al. 1997, Rössler 1961). This was confirmed for Ae. aegypti by Bosch et al. (2000), whose experiments suggested two groups of carboxylic acids with different chain lengths: $\mathrm{C} 1$ to $\mathrm{C} 3$ and $\mathrm{C} 5$ to $\mathrm{C} 8$ (C9 and $\mathrm{C} 11$ carboxylic acids reduced the attractiveness of lactic acid in the olfactometer).

Later investigations showed that hexanoic acid (caproic acid) was one substance with which the attractiveness of the lactic acid and ammonia combination for Ae. aegypti could be augmented further, but only in a closely defined proportion to each other. A dispenser liberating the different substances in the right quantities for a prolonged period of time would have to account for the different physical and chemical properties of the individual components. Due to their relatively large volume and vapour pressure, reservoir devices have been used mainly for lactic acid controlled release. Tube structures such as those described earlier for oviposition attractants have been used with the factor determining the release rate being the material from which the tube is made. For ammonia and hexanoic acid, solid polymer release matrices have been used. In this way, each substance in the three-component lure has its own release mechanism so that the ratios between the three components remain the same during the whole of the field life of 
the lure. The same principle of separated release devices for each compound in one dispenser unit is also used in an advanced version of a commercially-available dispenser, the Sweetscent ${ }^{\mathrm{TM}}$ dispenser, in which thin polymer coatings determine the release rate of each compound out of its reservoir pouch. Figure 5 shows the BG-Lure ${ }^{\mathrm{TM}}$ and the Sweetscent ${ }^{\mathrm{TM}}$, which are made up of the three controlled release formulations of the active substances, all contained in a mesh bag for ease of manipulation and placement in the BG Sentinel trap.

A

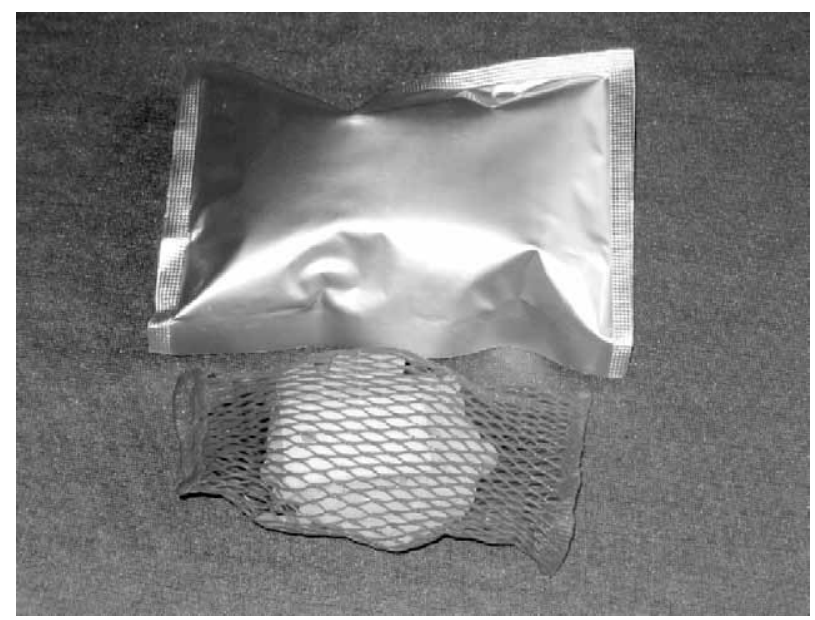

B

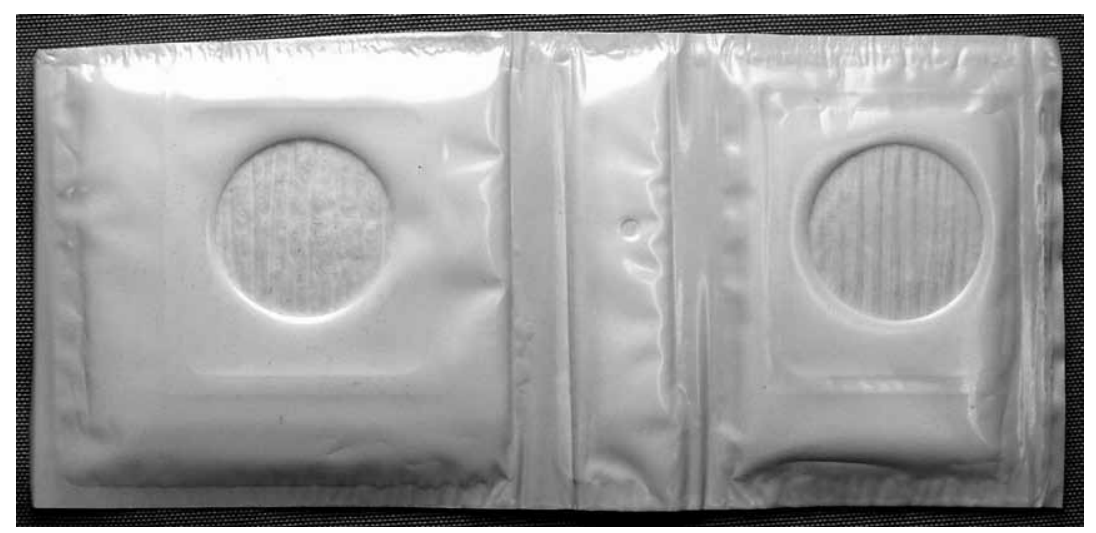

Figure 5. (A) Bg-Lure ${ }^{\mathrm{TM}}$ and (B) the Sweetscent ${ }^{\mathrm{TM}}$ dispensers containing lactic acid, ammonia and hexanoic acid (Source: Biogents AG).

Plume structure of host odours attractive for Aedes aegypti

Not only the composition, but also the structure of attractant odour plumes carries important information for host-seeking Ae. aegypti. This was indicated for the first time by a closer analysis of its flight behaviour in a Y-tube olfactometer (Geier et al. 1999b). The attractiveness of carbon dioxide, of human skin odours rubbed on a glass support and of L-(+)-lactic acid were tested in homogeneous, turbulent and filamentous odour plumes. A marked difference between carbon 
dioxide on the one side and the skin odours on the other side was observed. A turbulent and filamentous carbon dioxide plume with large fluctuations in the concentration lured more mosquitoes upwind than a homogeneous $\mathrm{CO}_{2}$ plume. The opposite was found with plumes of human skin odour: the highest number of mosquitoes flew upwind in the homogeneous plume, whereas in filamentous plumes, their numbers were significantly lower. 3-D video analyses of the flight behaviour of individual Ae. aegypti females in a larger wind tunnel confirmed these findings (Dekker et al. 2005). Heterogeneous odour plumes, both from skin odour and from carbon dioxide, elicited a meandering flight pattern with many turns and transversal parts. Homogeneous skin odour resulted in a quick and direct upwind flight, while mosquitoes presented with a homogeneous carbon dioxide plume flew mostly sidewards, much as in the control experiments (see also Chapter 6, this volume).

\section{The Biogents-Sentinel trap design}

The patented design of the BG-Sentinel incorporates attractive host-odour plumes for Ae. aegyti (Figure 6) (Geier et al. 2004 - patent pending). A faint upward air stream, charged with artificial skin odours, is produced through a gauze making up the top of the trap. The individual components are lactic acid, ammonia and caproic acid, all liberated in a fixed ratio from a specially designed dispenser, the BG-Lure, which is placed inside the trap. The artificial host-odour plume mimics the upward convection currents produced by a human body. Approaching mosquitoes are furthermore attracted over short range by the black and white contrast between the top of the trap and the centre tube, where the mosquitoes are drawn into a catch bag. One fan produces the attractive plume and sucks the mosquitoes into a catch bag.
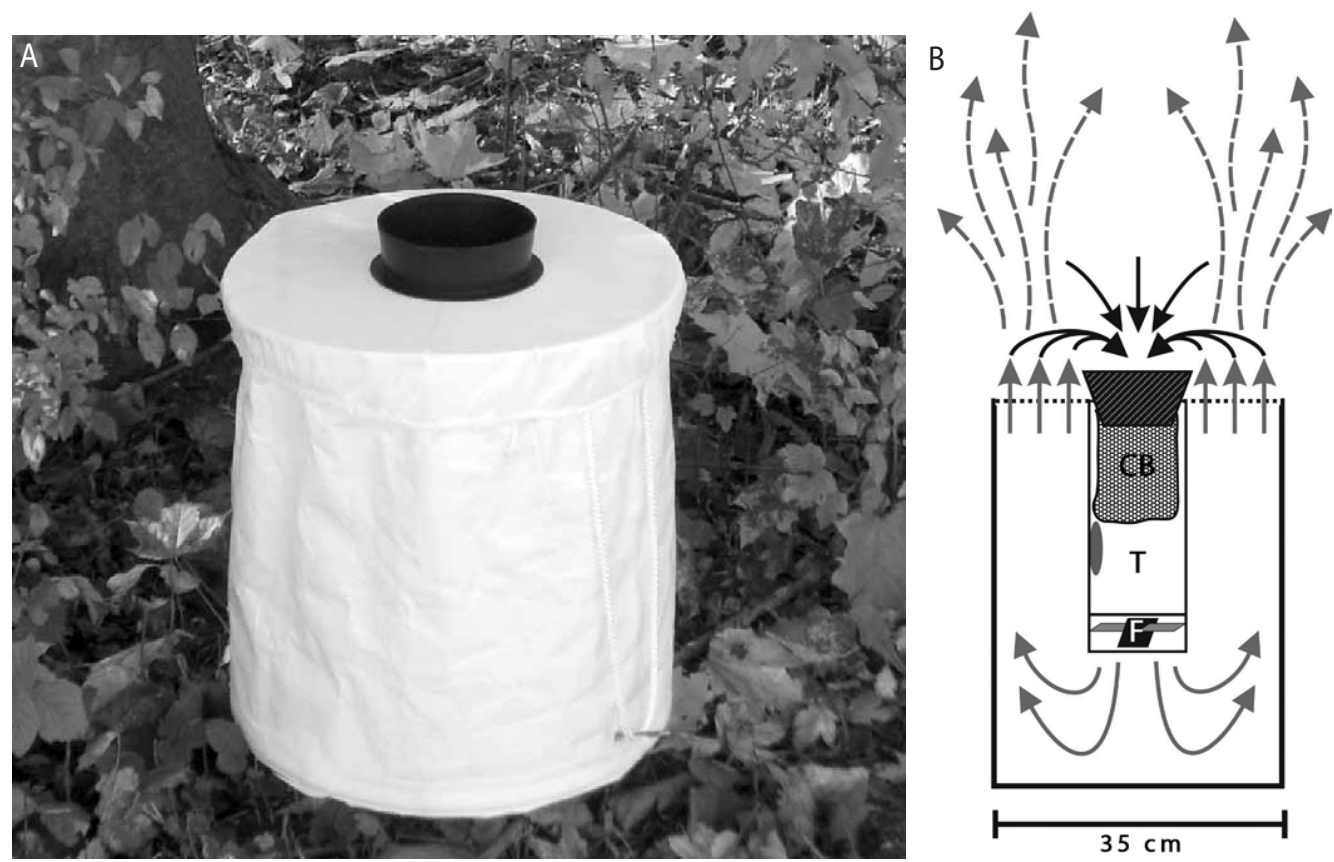

Figure 6. (A) BG-Sentinel trap ${ }^{\mathrm{TM}}$ assembled and (B) its functional diagram: longitudinal section of the trap. $C B$ : catch bag; T: black tube; F: fan; arrows indicate the direction of the airflow. 
A number of field studies have demonstrated that the BG-Sentinel trap is an excellent tool for capturing Ae. aegypti (Feltner and Ferrao 2008, Krueger and Hagen 2007, Maciel de Freitas et al. 2006, 2007, Meeraus et al. 2007, 2008, Morrison et al. 2008, Williams et al. 2007, Solberg et al. 2007). Kröckel et al. (2006) compared the BG-Sentinel to the Fay-Prince trap and a trap that produces carbon dioxide through the combustion of propane gas (the Mosquito Magnet Liberty ${ }^{\mathrm{TM}}$ ), and human landing collections in Belo Horizonte, Brazil. They reported significantly better catching rates for female Ae. aegypti with the BG-Sentinel (between 3 and 15 times better catching rates than the MML trap and between 8 and 15 times better rates than the Fay-Prince traps (Figure 7). Compared to human landing collections, the BG-Sentinel trap (without the addition of carbon dioxide) catches about $40 \%$ fewer female Ae. aegypti than a human collector. The trap also captures male Ae. aegypti.

In comparison to a backpack aspirator (used in a standard protocol of 20 minutes per premise) in Rio de Janeiro, Brazil, the BG-Sentinel (placed at each premise for 24 hours) captured significantly more female and male Ae. aegypti (Maciel de Freitas et al. 2006). A similar field study in Cairns, Australia, confirmed significantly higher catching rates of the BG-Sentinel than a back pack aspirator and EVS-trap baited with carbon dioxide (Williams et al. 2006). Additional experiments demonstrated the BG-Sentinel's potential as an Ae. aegypti-population sampling tool both in dengue risk assessment as well as the assessment of the success of mosquito control programmes (Williams et al. 2007). As with a conventional CDC light trap, the BG-Sentinel requires electrical power to operate the ventilator. Although the ventilator requires only about three Watts, it has some disadvantages in routine monitoring operations. Passive trapping systems that use sticky surfaces to catch mosquitoes do not require electricity and are low cost tools, but the catching rates of these systems are much lower (between 10 to 50 times, Kröckel et al. 2006). For a sensitive and high resolution spatial analysis of dengue vectors both trapping systems are useful, the

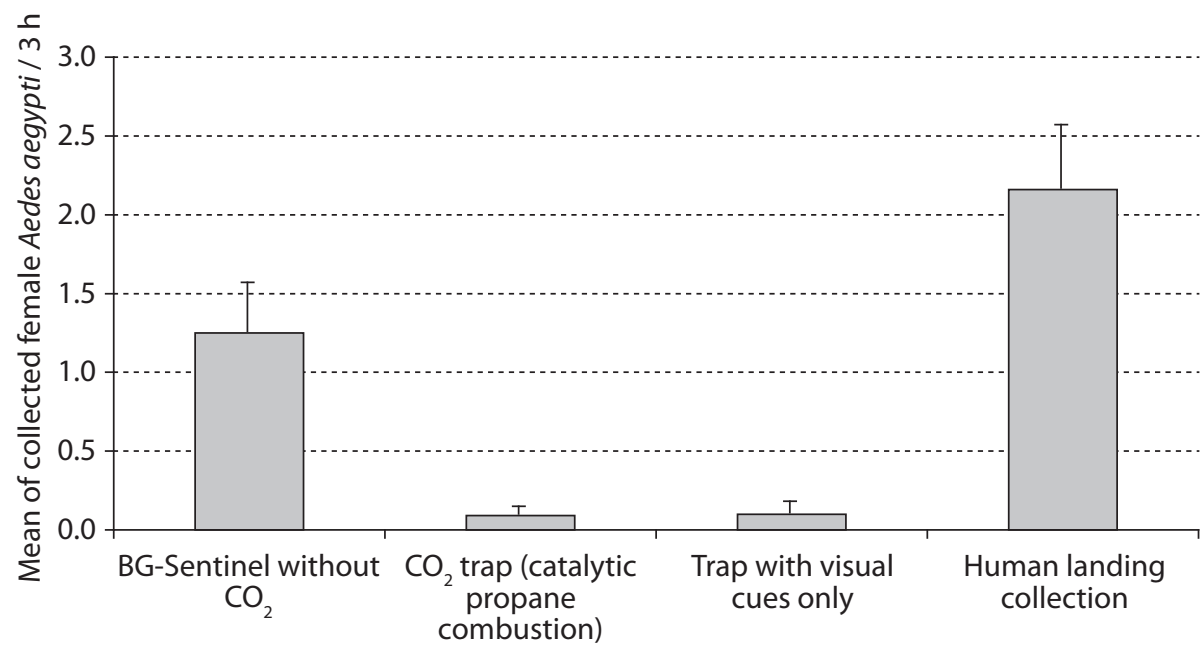

Figure 7. Mean values ( \pm standard error) for caught Aedes aegypti (syn. Stegomyia aegypti) from three-hour catching periods over an eight week period. BG-Sentinel trap with lure dispenser $B G$ Lure $(n=32) ; \mathrm{CO}_{2}$ trap: Mosquito Magnet Liberty generates moist, warm $\mathrm{CO}_{2}(n=32)$; Trap with visual cues only: Fay Prince trap with black and white contrast ( $n=30)$; Human landing collection: Voluntary test person as a mosquito catcher $(n=32)$ (Kröckel et al. 2006). 
sticky traps to provide a high spatial resolution and the BG-Sentinels to provide a fast and highly sensitive measure of the density of adult vectors.

The high collection rates of the BG-Sentinel allow an accurate determination of vector population densities and provide sufficient sample material to establish virus infection rates by pooling mosquitoes. In the transmission of vector-borne diseases, two factors play a role: first, the density of vectors and, second, the incidence of disease agents in their population (infection rate). With efficient sampling tools for the vectors involved and with quick tests for the presence of viruses in these vectors, it is thus possible to assess transmission risk by calculating the Vector Index. This gives the most meaningful risk index which can be used in the efficient planning and rapid implementation of the control of vector-borne diseases (J Arias, personal communication).

Although originally developed for catching host-finding females, it has been demonstrated that the BG-Sentinel trap also catches gravid females, parous and nulliparous females, males, and even blood-fed females in larger numbers than expected (Maciel de Freitas et al. 2006, Williams et al. 2006). Catching a broad spectrum of mosquitoes in different physiological stages allows a more precise analysis of the mosquito population in a certain area.

A semi-field study showed that an established population of Ae. aegyti in a greenhouse could be completely eradicated by the placement of one BG-Sentinel trap. Already two days after the trap's placement, the human biting rate in the greenhouse was reduced from 15 bites per minute to less than one bite per minute (Obermayr 2006). Within six weeks of continuous operation of the trap, the mosquito population was completely extinguished with no eggs, larvae or adult mosquitoes being found. A large scale field study with about 600 households is currently ongoing in Manaus, Brazil to demonstrate that the traps can be also used for control of local mosquito populations and for lowering the risk of dengue transmission in private homes.

\section{Traps for sampling host-seeking Aedes albopictus}

The BG-Sentinel with the BG-Lure has also proven to be a useful trap for another species of the subgenus Stegomyia, Aedes albopictus. This was reported for the first time in 2005, when Ae. albopictus were detected in two locations in Italy where ovitraps failed to collect this species (Bitzhenner et al. 2005). In the same year, Ae. albopictus was detected in BG-Sentinel traps placed on islands in the Torres strait near Australia (Ritchie et al. 2006). In 2006, Ae. albopictus was found in BG-Sentinels placed in Libreville, Gabon (Krüger and Hagen 2007). A study comparing the CDC Light trap baited with carbon dioxide to the BG-Sentinel and another new trap, the CMT-20TM was published by Meeraus et al. (2007). The BG-Sentinel baited only with the BG-Lure captured six times more Ae. albopictus that the $\mathrm{CDC}$ trap with carbon dioxide. Adding $\mathrm{CO}_{2}$ to the $\mathrm{BG}$-Sentinel and the BG-Lure improved the catching rate to 33 times that of the carbon dioxide-baited CDC trap. The CMT-20 (with SkinLure ${ }^{T M}$, an attractant containing lactic acid) collected significantly more Ae. albopictus when used with $\mathrm{CO}_{2}$ than without $\mathrm{CO}_{2}$, but did not collect significantly more Ae. albopictus than the $\mathrm{CO}_{2}$-baited $\mathrm{CDC}$ light trap.

The high catching rates of the BG-Sentinel traps for Ae. albopictus have led to the idea of using the traps for mosquito control. A long-term field study in Cesena, Italy, was conducted from May to October 2008 in order to investigate the effect of the traps on local mosquito populations. Human biting rates as well as egg collections with ovitraps were significantly reduced in areas with trap placement compared to areas without traps (Englbrecht et al. 2009). 


\section{Practical application of population data of Aedes aegypti}

\section{The role of GIS in public health surveillance}

Geographic Information Systems (GIS) have been widely used by many countries for health surveillance. In Canada, the Public Health Agency of Canada developed a Disease Surveillance OnLine web site that provides mapping and other services for cancer, cardiovascular diseases, major chronic diseases, notifiable diseases, and injury surveillance (PHAC 2009). Mosquito surveillance systems are also currently using GIS as an important tool to monitor the distribution and spread of West Nile virus (BCCDC 2009), dengue (Sithprasasna et al. 1997) and malaria (WHO 2009).

Geographic information systems consist of an automated computer-based system that has the ability to capture, retrieve, manage, display and analyse large quantities of spatial and temporal data in a geographical context. The system comprises hardware (computer and printer), software (GIS software), digitised base maps, information and a whole set of procedures such as data collection, management and updating (WHO 2009). Vector-borne diseases such as that carried by Ae. aegypti can be mapped temporally and spatially. Such information, when mapped together creates a powerful tool for monitoring and controlling the dengue vector. Operations for dengue control based on GIS were established in Singapore in 1998. Streets, residential buildings and other relevant databases were obtained and mapped to form the base map layer, such as Aedes breeding sites, dengue case incidences, complainants' addresses, sensitive areas, weather data (rainfall, temperature and relative humidity) were mapped into the GIS (Ai-leen and Song 2000).

In Recife, northeastern Brazil, a new approach to dengue vector surveillance based on the use of spatial analysis techniques, such as the Kernel density estimator, for the identification of hotspots of vector populations using data from oviptraps, can be very useful for guiding vector control operations. The long-term use of modified ovitraps provided with Bacillus thuringiensis var. israelensis (Bti) as a larvicide was evaluated in various intra-urban landscapes. Results from the first year of egg collections indicated that this could be a promising strategy for detecting Ae. aegypti population outbreaks (Regis et al. 2008). It is also possible to integrate remote sensing satellite imagery, trap placement, meteorological data and census data. Similarly, environmental data such as temperature, relative humidity, and rainfall can be recorded daily at meteorological point-stations and also integrated into the system. The main disadvantage of the spatial analysis technique is that it still relies on the use of oviposition traps, requiring much personnel and laboratory facilities. Although their use is recommended for the detection of low populations of $A e$. aegypti, their value in the assessment of vector abundance, also between blocks or neighborhoods is questionable (Focks 2003).

\section{Intelligent Dengue Monitoring System}

Because of the difficulties of field data collection of larval surveys by the Brazilian National Programme for Dengue Control, a new field data acquisition of adult female Ae. aegypti and processing-generating GIS real-time web-site surveillance system was developed and evaluated in Brazil. The technology is known as 'Intelligent Dengue Monitoring system' (MI-Dengue) and was developed by a 'spin-off' company of the Federal University of Minas Gerais, Belo Horizonte, Brazil. The MI-Dengue consists of (a) a MosquiTRAP baited with synthetic oviposition attractant that captures adult female Ae. aegypti, which allows for the identification of the adult vectors during trap inspection; (b) recording and sending entomological data on electronic spreadsheets or by mobile phone during trap inspection and (c) an internet site that is an integrated real- 
time adult mosquito surveillance system providing entomological indices and GIS technology (Eiras and Resende 2009). The MI-Dengue permits researchers to send and make data available on the internet for municipalities' health managers to access information on the density of gravid female Ae. aegypti on georeferenced maps and in analytical tables of the sites monitored with MosquiTRAP.

The MI-Dengue web-data system consists of three software programmes developed to simplify information: (a) 'geo-dengue collection' installed in portable devices (e.g. palmtops or cell phones) to record Ae. aegypti field capture data; (b) 'geo-dengue monitoring' to process the field data and produce tables with entomological indices and graphs with trends for analysis; and (c) 'geodengue', which produces georeferenced maps of mosquitoes captured with MosquiTRAP and makes them available to users on the Internet on a weekly basis.

The MI-Dengue technology provides a wide range of GIS tools for public health surveillance and can be adapted for surveillance of other diseases, such as AIDS, tuberculosis, malaria, leishmaniosis, etc.

\section{Data acquisition}

Field data collected by ovitrap, larval surveys, adult traps and aspirator are traditionally recorded on printed spreadsheets, attached to a clipboard by field workers. Later, the spreadsheets are sent to be typed and consolidated on a personal computer. Not only is this method prone to typing errors, it is also time consuming and labour intensive if a large amount of field data is collected.

An alternative and faster method of acquisition and transport of entomological data is by means of an electronic spreadsheet installed in palmtop computers or mobile phone (Eiras and Resende 2009). These data are transferred automatically to the municipality's database, and the internet site automatically generates the GIS maps and entomological tables for the city council's dengue control service. The electronic spreadsheet allows for recording household information (e.g. resident's name, address, postal code, and emplacement of the trap within the residence) as well as number of adult mosquitoes caught in each trap/premise.

There are several advantages of using electronic spreadsheet or mobile phone over conventional field data acquisition systems. The field data can be accessed immediately (premises visited and scheduled for visits, trap locations, residents' names, and so on.) and the entomological indices can be produced automatically. The operation cost is low because these applications and systems are maintained and operated automatically and there is no delay between data reported to the database and data available for web mapping and public health access.

\section{GIS for identifying infestations of female adult Aedes aegypti}

Infestations of gravid female Ae. aegypti are measured by the MI-Dengue system in five different ways for focused vector control: (1) weekly monitoring of Ae. aegypti infestation in the urban blocks; (2) monitoring re-infestation of blocks; (3) hot spot areas; (4) entomological index and (5) monitoring infection of adult mosquitoes with dengue virus (Eiras and Resende 2009).

All blocks of urban areas are weekly monitored for Ae. aegypti infestation and the number of female Ae. aegypti captured with the sticky trap is used to establish colour categories for classifying blocks as green (zero captures), yellow (one female Ae. aegypti/MosquiTRAP/week = low infestation), 
orange (two females/trap/week = medium infestation), and red ( $>$ three females/trap/week = high infestation) (Eiras and Resende 2009). These parameters were based on studies by Ritchie et al. (2004) showing that in Australia, more than two gravid females A. aegypti/trap/week was associated with a risk of dengue cases.

These weekly data on infested sites and vector infestation levels provided important information to the municipal dengue control workers who assisted municipal health managers in targeting and optimising their Ae. aegypti control activities. The georeferenced maps produced by MI-Dengue technology and provided weekly on the internet, allowed municipal health managers to identify urban blocks by colour according to the number of female Ae. aegypti specimens captured. Thus, dengue control activities could be focused on infested blocks within a 200 -meter radius. This focused vector control strategy, supported by a weekly infestation monitoring system, allowed spatial localisation of vector infestation and the evaluation of control measures within the radius represented by the trap.

\section{Entomological index}

Two indices are used to calculate the El: (1) the Mean Female Aedes Index (MFAl), which is the mean number of female Ae. aegypti specimens caught by traps installed per week and is used for monitoring vector infestations in neighborhoods and municipalities (Figure 8) (Eiras and Resende 2009); (2) the Temporal Mean Female Aedes Index (MFAlt), which is the average of MFAI values for the three previous, consecutive weeks and is calculated on a monthly basis for Ae. aegypti. The MFAlt in neighbourhoods are colour-coded green (MFAlt $<0.2)$, yellow $(0.2<$ MFAlt $<0.4)$, and red (MFAIt>0.4). The MFAlt was developed based on correlations between the number of dengue cases and the MFAl entomological index during an outbreak of dengue in Vitoria city, Espírito Santo State, Brazil, where clusters of dengue cases and mosquitoes were observed. The entomological index $t$ is classified as 'risk of dengue' if MFAlt $\geq 0.4$, while 'dengue alerts' are issued when MFAlt ranges between 0.20 and 0.39. The situation is considered 'risk-free' in terms of dengue when MFAlt $\leq 0.2$. Using this trategy, the municipality of Presidente Epitacio, Sao Paulo, Brazil, experienced a shift from 'dengue alert' to 'risk free' was observed). A reduction in entomological indices after several weeks of monitoring indicated a strong change in the Ae. aegypti population density that was influenced either by environmental conditions or by vector control in the targeted and priority areas, or both (Table 2) (Eiras and Resende 2009).

\section{Monitoring re-infestation of blocks}

Three variables are used as follows: (a) recurrence of positive traps (at least one female Ae. aegypti caught); (b) time (chronological order of the week in which the capture occurred), and (c) number of female Ae. aegypti in the traps during the previous four weeks in the monitored blocks (Eiras and Resende 2009).

\section{Monitoring block infestations with adult female Aedes aegypti using GIS}

The city council's mosquito control unit can access GIS-maps on the website for the weekly infestations of the blocks by female Ae. aegypti, and can thus obtain a spatial and temporal understanding of the infestations (Eiras and Resende 2009). The black dots on the maps represent the exact trap location by GPS. The users (city council) can have access to the georeferenced information (latitude and longitude), household address, block and neighbourhood numbers, and number of mosquitoes captured during each epidemiological week by clicking on these 

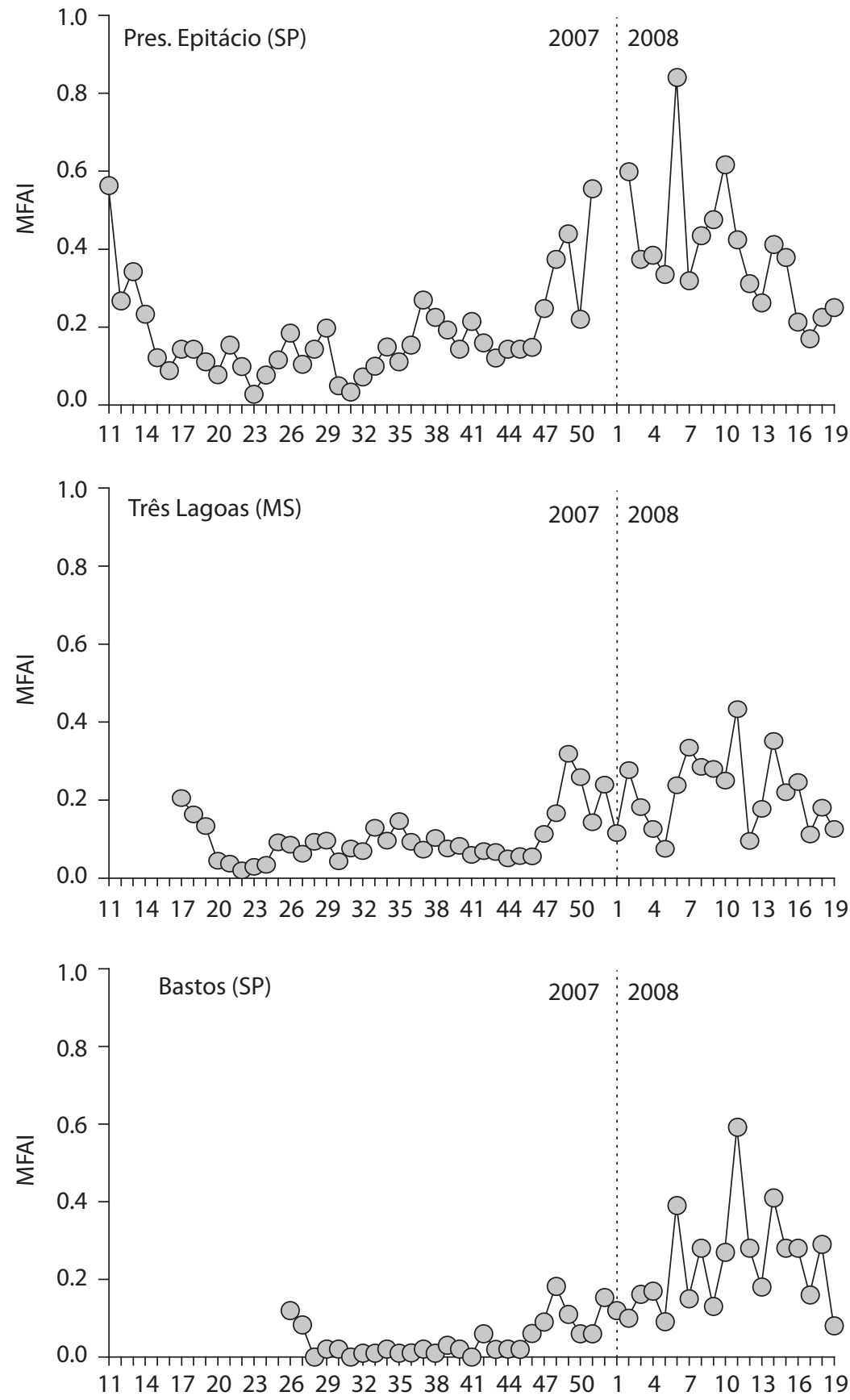

Weeks

Figure 8. Weekly monitoring of Mean Female Aedes Index (MFAI) furnished by MI-Dengue in three municipalities in 2007 and 2008 (Eiras and Resende 2009). 
Table 2. Example of evolution in entomological indices in neighbourhoods in the Municipality of Presidente Epitacio (São Paulo State, Brazil), classified according to temporal Mean Female Aedes Index (MFAlt) in the previous four epidemiological weeks (Eiras and Resende 2009).

\begin{tabular}{|c|c|c|c|}
\hline \multirow[t]{2}{*}{ Entomological weeks } & \multicolumn{3}{|c|}{$\%$ neighbourhood classified } \\
\hline & Non-critical (MFAlt<0.2) & $\begin{array}{l}\text { Dengue alert } \\
(0.2<\text { MFAlt }<0.4)\end{array}$ & Critical (MFAlt $>0.4)$ \\
\hline $7-10$ & 0.0 & 44.4 & 55.6 \\
\hline $8-11$ & 22.2 & 33.3 & 44.4 \\
\hline $9-12$ & 22.2 & 44.4 & 33.3 \\
\hline $10-13$ & 22.2 & 66.7 & 11.1 \\
\hline $11-14$ & 33.3 & 55.6 & 11.1 \\
\hline $12-15$ & 66.7 & 33.3 & 0.0 \\
\hline $13-16$ & 88.9 & 0.0 & 11.1 \\
\hline $14-17$ & 88.9 & 11.1 & 0.0 \\
\hline
\end{tabular}

dots (traps). The weekly infestation levels are provided by colour-coded information on the blocks, based on the number of female Ae. aegypti captured in each block. The dengue vector control measures can be based on the weekly observations of infestation levels, where effort is concentrated in given areas or neighbourhoods of red blocks, thus facilitating implementation of targeted control measures.

Monthly monitoring of Ae. aegypti reinfestation in the neighbourhoods

Eiras and Resende (2009) showed in preliminary studies that using the MI-Dengue technology in three cities in Brazil reduced entomological indices after seven weeks of monitoring adult female Ae. aegypti followed by directional control measures. The MI-Dengue technology was used in three districts (30,000-75,000 inhabitants) during one year and vector control was based on the website information of 'dengue alert' or 'hot spots' of adult female Ae. aegypti. Results showed that there was a trend of shifting from categories 'dengue alert' and 'critical' to 'risk free', indicating a strong seasonal variation in the Ae. aegypti population density that was probably influenced by the climatic conditions and/or targeted control measures. The incidence of dengue cases was also significantly reduced when compared with the neighbourhood districts (Figure 9). Although, this was a pilot study, further data are currently being collected in 45 Brazilian cities during 2009 with the populations of the cities ranging from 30,000 to 700,000 inhabitants.

\section{Hot spot areas}

Cluster analysis of the capture of female mosquitoes by MosquiTRAP is based on the weekly mean number of female Aedes index per block. Space-time Permutation Scan Statistical Model (STPSSM) (Kulldorff et al. 2005) and Poisson Scan Statistic Model (PSSM) (Kulldorff and Nagarwalla 1995, Kulldorff et al. 1998) were used to identify whether Ae. aegypti clusters in space are randomly distributed or not (Figure 10). 
A - - Andradina-SP $\quad-\mathrm{O}$ - Presidente Venceslau-SP $\_-$Rancharia-SP

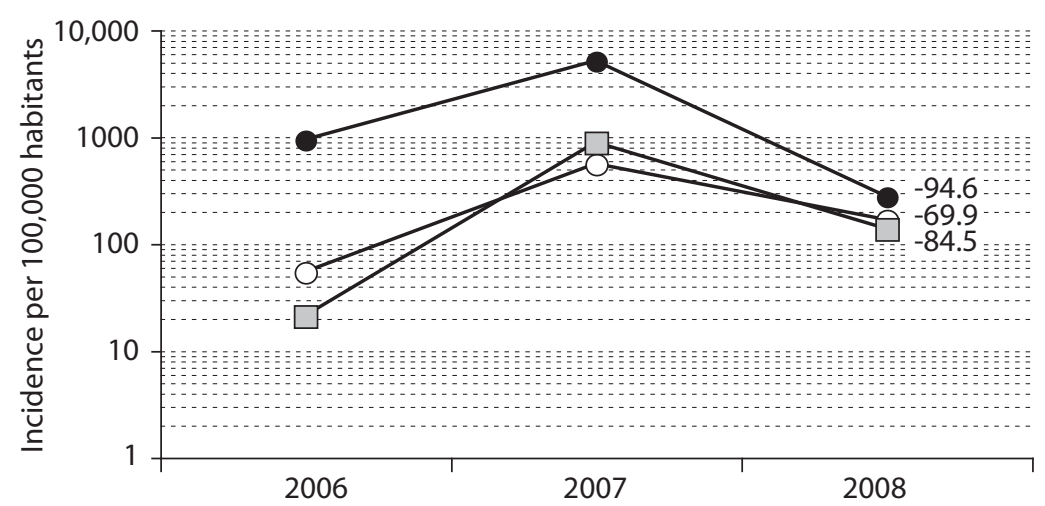

B -0 Divinópolis-MG -O- Pará de Minas-MG $\quad \square-$ Santa Luzia-MG

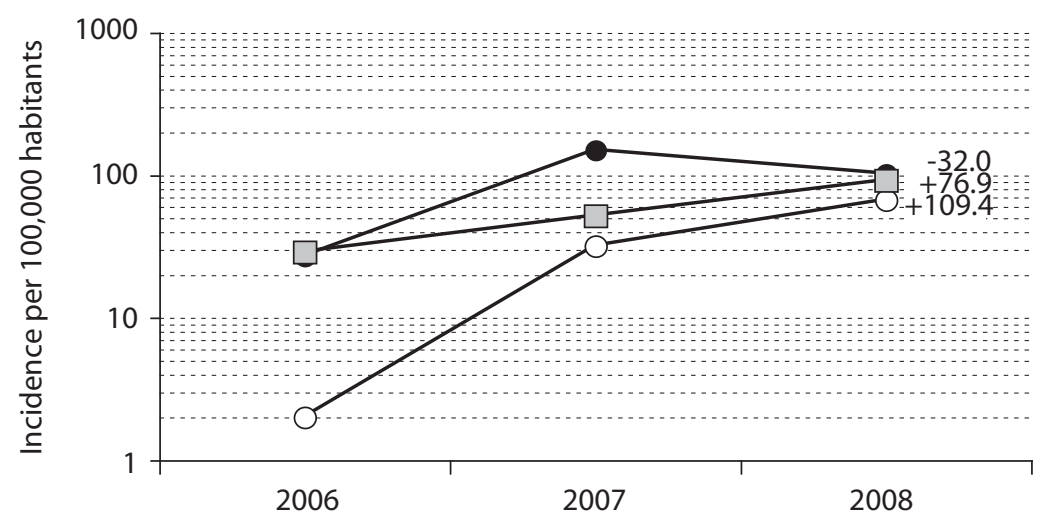

C - Três Lagoas-MS -O-Presidente Epitácio-SP $\quad \square-$ Bastos-MG

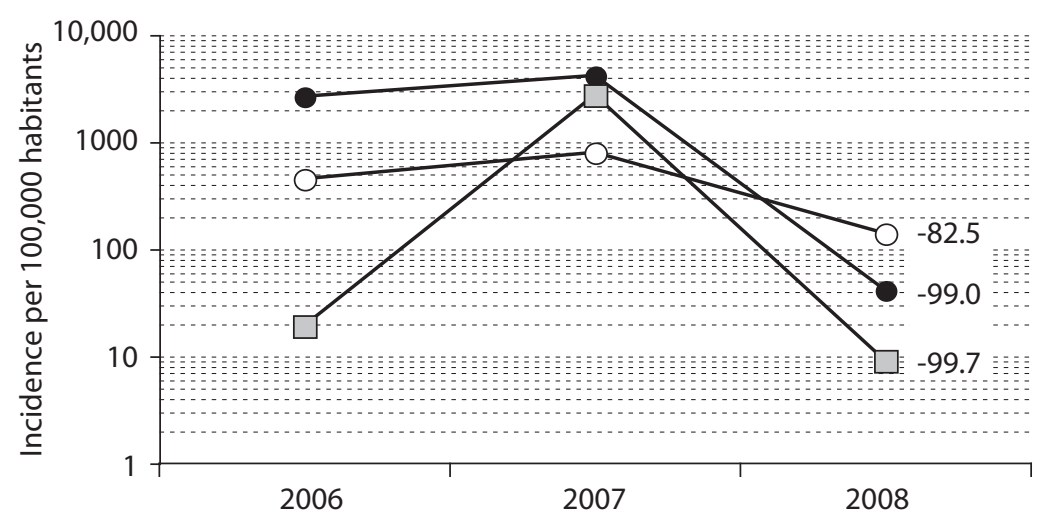

Figure 9. Total autochthonous dengue cases and incidence in municipalities that (A) did not adopt MI-Dengue, (B) partially MI-Dengue and (C) fully adopted MI-Dengue in early 2008. Numbers in parenthesis means percentage of (-) reduction or (+) increasing in incidence of autochthonous dengue in 2008 (adapted from Eiras and Resende 2009). 
A

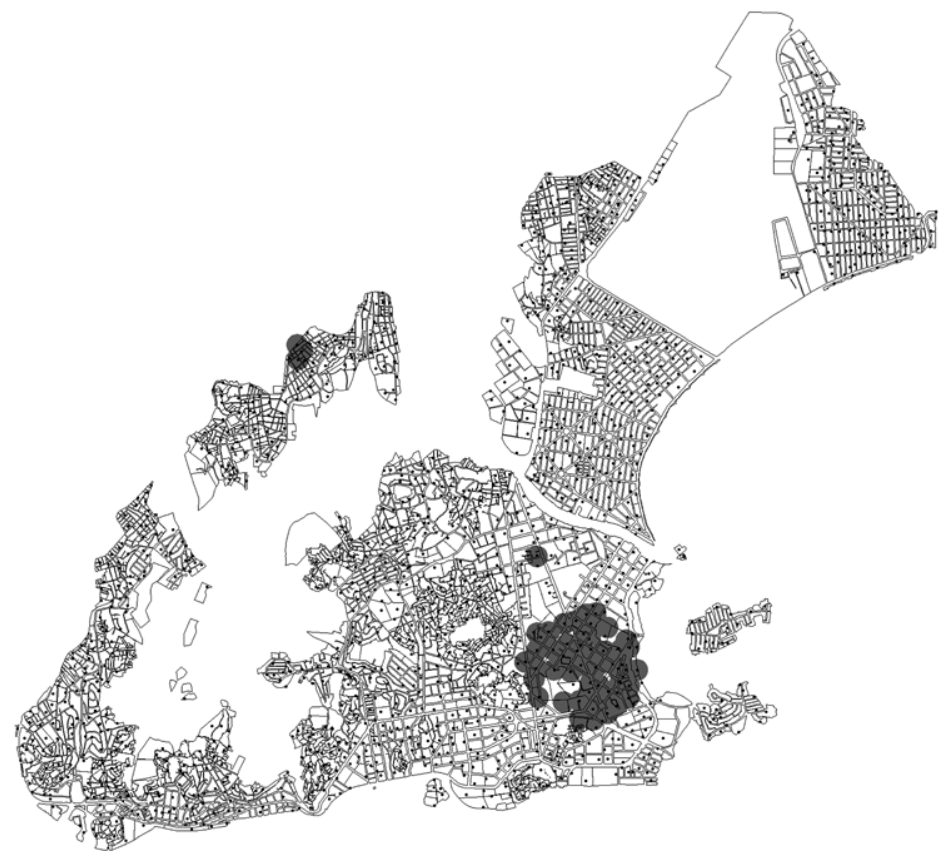

B

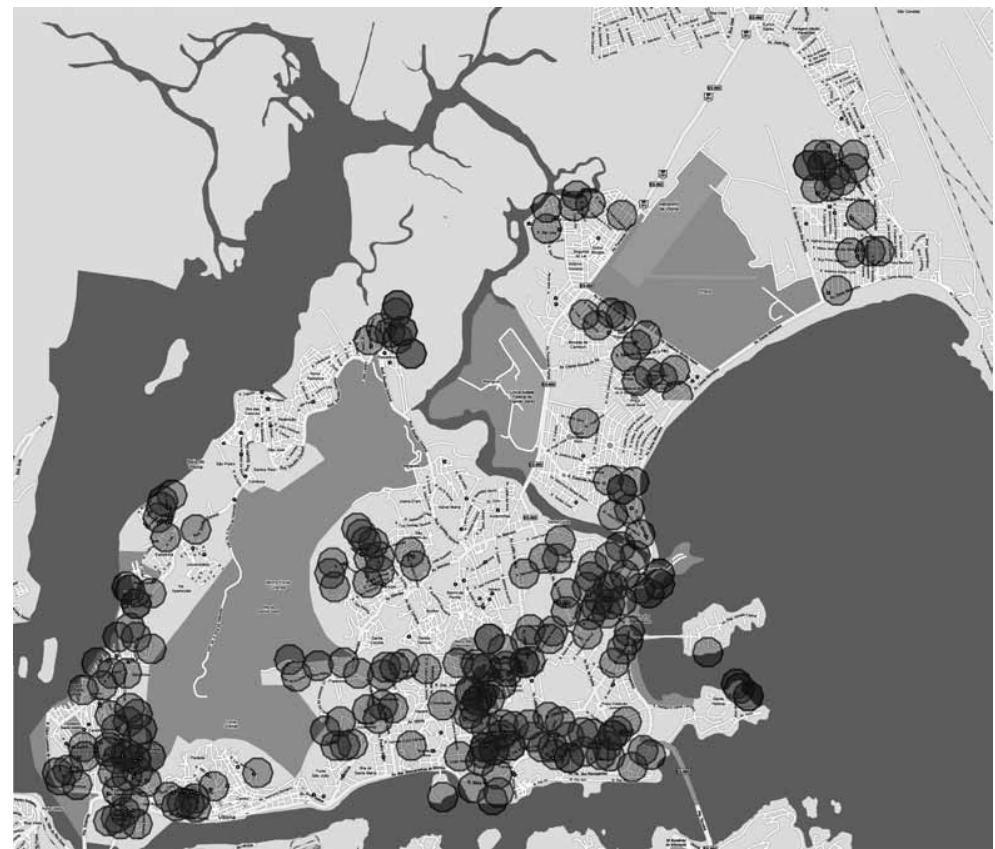

Figure 10. (A) Presence of hot spot areas of gravid female Aedes aegypti in Vitoria (Espirito Santo State, Brazil) detected by MI-Dengue weekly monitoring at week 12 (2009). The window shown on the screen allowed to follow weekly maps. (B) GIS of infected mosquitoes with dengue virus (small circles are dengue cases and bigger circles are infected Aedes aegypti mosquitoes with dengue virus). 
Measurement of dengue- infected Aedes aegypti mosquitoes using GIS

In order to control and prevent dengue transmission, it is also important to rapidly detect and type the virus in adult mosquitoes. For instance, in Brazil, the control of adult Ae. aegypti is trigged only when a person gets dengue fever (PNCD 2002). Thus, information indicating the number of $A e$. aegypti infected by dengue virus may replace the current indicator for adult Ae. aegypti control.

Recently in Brazil, a rapid and stable method for detection and typing of dengue virus (DEN) in Ae. aegypti was established and associated with the MI-Dengue technology. Vitoria city, Espirito Santo State, Brazil, has been using the MI-Dengue technology since January 2007 and approximately 1,450 sticky traps were placed all over the urban area. The entomological index and GIS maps are produced on a weekly basis. The adult female Ae. aegypti mosquitoes that are trapped in the MosquiTRAP, are pooled in groups of about 20 mosquitoes and submitted for virus detection and type identification by reverse transcriptase RT-PCR. Data on infected mosquitoes is plotted in GIS web-online for the city council. At the moment of writing, it takes 10 days to analyse mosquitoes for virus infection because samples need to be transported and the analysis is done manually. In future, it is expected that the detection of virus in mosquitoes can be automated. It was found that Ae. aegypti was infected with virus DENV-1, DENV-2 and DENV-3 in urban areas during many months (Figure 10B). The hot spot areas with infected mosquitoes were considered priorities for vector control. In a preliminary study of an outbreak of dengue in Vitoria city, it was observed that $85 \%$ of dengue cases occurred within a $200 \mathrm{~m}$ radius of sticky traps having caught infected female Ae. aegypti (AE Eiras, unpublished data) This method is very helpful and it has been used as baseline to develop a new early surveillance system to provide early warning for dengue fever epidemics, in furnishing information for epidemiologic studies, and for effective vector control measures.

Monitoring gravid female Ae. aegypti with MosquiTRAP and MI-Dengue allows the Municipal Health Secretariats to conduct weekly follow-up of infestation trends in neighbourhoods and municipalities. The practicality of MosquiTRAP and MI-Dengue to monitor female adult Ae. aegypti in real time is substantial when compared with other georeferenced studies that use sampling of immature forms of the mosquito (eggs and larvae) (Ai-leen and Song 2000; Chansang and Kittayapong 2007), which require time and infrastructure for identification, quantification, and data processing. Our experience has shown that these innovative technological tools are not difficult to learn nor are they difficult to integrate into existing dengue control programmes.

\section{Potential use of traps for dengue suppression}

Efficient tools for the monitoring of adult Ae. aegypti, such as the MosquiTRAP and the BG-Sentinel trap, have a wide range of applications in both dengue control operations and research. Both trap types allow the detection of virus-positive female Ae. aegypti during standard weekly monitoring (even if the incidence of these females is very low) and at the peak of acute epidemics.

A novel strategy has been proposed to effectively suppress dengue transmission in urbanised areas by the use of innovative mosquito traps. This has not been tried before, probably because of the low performance, high costs, and complexity of existing trapping technologies. The BGSentinel and MosquiTRAP have the potential to be produced economically at a large scale due to their simple technology. Although the efficacy of the trap has been demonstrated already in scientific studies and monitoring programmes, the trap has not been used before as a control tool to suppress dengue transmission. In 2009, two pilot projects were designed to prove that 
a lure and kill control strategy encompassing environmentally-friendly traps for adult vectors can suppress dengue transmission. Both projects are currently being implemented in Manaus city, Amazon State, Brazil, and the Salvador, Bahia State, Brazil). Using an integrated communitybased approach for proper education and training of the trap users, the traps are installed in the household or premises, public buildings, and working places. They can be placed indoors as well as outdoors, depending on the situation and prevalence of the vectors. The traps should run continuously throughout the whole season or year. The theoretical benefits of both traps are increased vector mortality, reduction of human biting rates, early indication of breeding sites through monitoring, enhancement of individual responsibility in community based programmes, environmentally friendly (no toxic substances are used) and sustainable. These are important preconditions for a successful implementation of the traps and the acceptance of the users. Education and training of the trap user is very important to achieve all the benefits of the traps. Therefore the implementation of the proposed trapping strategy has to be integrated in already existing or newly implemented community-based dengue control programs. The use of the traps is expected to have a positive effect on the continued motivation of individuals to take action against the vectors, by source reduction, using additional traps, or applying insecticide treatments.

\section{Acknowledgments}

Alvaro Eiras thanks to CNPq, FAPEMIG, SEBRAE, FINEP and the Programa Nacional de Controle da Dengue (PNCD), the Brazilian Health Ministry and the 'spinoff' Ecovec for supporting the development and evaluation of the MI-Dengue technology.

\section{References}

Acree F Jr., Turner, RB, Gouck HK, Beroza M and Smith N (1968) L-Lactic acid: a mosquito attractant isolated from humans. Science 161: 1346-1347.

Ai-leen GT and Song RJ (2000) The use of GIS in ovitrap monitoringfor dengue control in Singapore Dengue Bull 24: 110-116.

Allan SA and Kline DL (1995) Evaluation of organic infusions and synthetic compounds mediating oviposition in Aedes albopictus and Aedes aegypti (Diptera: Culicidae). J Chem Ecol 21: 1847-1860.

Barrera R, Amador M and Clark GG (2006) Use of the pupal survey technique for measuring Aedes aegypti (Diptera: Culicidae) productivity in Puerto Rico. Am J Trop Med Hyg 74: 290-302.

Beehler JW, Millar JG and Mulla MS (1994) Field evaluation of synthetic compounds mediating oviposition in Culex mosquitoes (Diptera: Culicidae). J Chem Ecol 20: 281-291.

Bentley MD and Day JF (1989) Chemical ecology and behavior aspects of mosquito oviposition. Annu Rev Entomol 34: 401-421.

Bentley MD, McDaniel IN, Yatagai M, Lee H-P and Maynard R (1979) p-Cresol: an oviposition attractant of Aedes triseriatus. Environ Entomol 8: 206-209.

Bitzhenner M, Guaraglia Ch, Geier M, Rose A and Talbalaghi A (2005) Evaluation of the BG-Sentinel, a new monitoring trap for mosquitoes, in northern Italy. Poster presentation at the $4^{\text {th }}$ International Congress of Vector Ecology, Reno, Nevada, USA, 2-7 October 2005.

Bosch JO, Geier M and Boeckh J (2000) Contribution of fatty acids to olfactory host finding of female Aedes aegypti. Chem Senses 25: 323-330.

Braga IA, Gomes AC, Nelson M, Mello RCG, Bergamaschi DP and Souza JMP (2000) Comparação entre pesquisa larvária e armadilha de oviposição, para detecção de Aedes aegypti. Rev Bras Med Trop 33: 347-353.

Breteau H (1954) La fiève jaune en Afrique occidentale française. Un aspect de la médecine préventive massive. Bulletin WHO 11: 453-481. 
British Columbia Centre for Disease Control (BCCDC) (2009) Interactive GIS mapping for West Nile virus. Available at http: //maps.bccdc.org.

Brown AWA, Sarkaria DS and Thompson RP (1951) Studies on the responses of the female Aedes mosquito. Part I. - The search for attractant vapours. Bull Ent Res 42: 105-114.

Canyon DV and Hii JLK (1997) Efficacy of carbon dioxide, 1-octen-3-ol, and lactic acid in modified Fay-Prince traps as compared to man-landing catch of Aedes aegypti. J Am Mosq Control Assoc 13: 66-70.

Carlson DA, Smith N, Gouck HK and Goodwin DR (1973) Yellow fever mosquitoes: compounds related to lactic acid that attract females. J Econ Entomol 66: 329-331.

Chadee DD (1993) Oviposition response of Aedes aegypti (L.) to the presence of conspecific eggs in the field in Trinidad, WI. J Florida Mosq Contr Ass 64: 63-66.

Chadee DD, Lakkan A, Ramdath WR and Persad RC (1993) Oviposition response of Aedes aegypti mosquitoes to different concentrations of hay infusion in Trinidad, West Indies. J Am Mosq Control Assoc 9: 346-348.

Chadee DD and Martinez R (2000) Landing periodicity of Aedes aegypti with implications for dengue transmission in Trinidad, West Indies. J Vector Ecol 25(2): 158-163.

Chadee DD, Doon R and Severson DW (2007) Surveillance of dengue fever cases using a novel Aedes aegypti population sampling method in Trinidad, West Indies: the cardinal points approach. Acta Trop 104: 1-7

Chansang C and Kittayapong P (2007) Application of mosquito sampling count and geospatial methods to improve dengue vector surveillance. Am J. Trop Med Hyg 77: 897-902.

Clark GG, Seda H and Gubler DJ (1994) Use of the 'CDC backpack aspirator' for surveillance of Aedes aegypti in San Juan, Puerto Rico. J Am Mosq Control Assoc 10: 119-124.

Clements AN (1999) The biology mosquitoes. Volume 2: sensory reception and behaviour. CABI, Wallingford, UK.

Cobert PS and Chadee DD (1993) An improved method for detecting substrate preferences shown by mosquitoes that exhibit skip oviposition. Physiol Entomol 18: 114-118.

Coelho GE, Burattini MN, Teixeira MG, Coutinho FAB and Massad E (2008) Dynamics of the 2006/2007 dengue outbreak in Brazil. Mem Inst Oswaldo Cruz 103: 535-539.

Connor ME and Monroe WM (1923) Stegomyia índices and their value in the yellow fever control. Am JTrop Med 3: 9-19.

Conselho Nacional de Desenvolvimento Científico e Tecnológico (CNPq) (2009) Edital doenças neglegenciadas - MCT/ CNPq/CT-Saúde/MS/SCTIE/DECIT No. 034/2008. Available at: http: //www.cnpq.br/editais/ct/encerrados.htm.

Coutinho FAB, Burattini MN, Lopez LF and Massad E (2006) Threshold conditions for a non-autonomous epidemic system describing the population dynamics of dengue. Bull Math Biol 68: 2263-2282.

Cuéllar-Jiménez ME, Velásquez-Escobar OL, González-Obando R and Morales-Reichmann CA (2007) Detección de Aedes albopictus (Skuse) (Diptera: Culicidae) en la ciudad de Cali, Valle del Cauca, Colombia. Biomédica 27: 273-279.

Dekker T, Geier M and Cardé RT (2005) Carbon dioxide instantly sensitizes female yellow fever mosquitoes to human skin odours. J Exp Biol 208: 2963-2972

Dennett JA, Wuithiranyagool T, Reyna-Nava M, Bala A, Tesh RB, Parsons RE and Bueno RJr (2007) Description and use of the Harris County Gravid trap for West Nile virus surveillance 2003-06. J Am Mosq Control Assoc. 23: 359-362.

Dibo MR, Chiaravalloti-Neto F, Battigaglia M, Mondini A, Favaro EA, Barbosa AC and Glasser CM (2005) Identification of the best ovitrap installation sites for gravid Aedes (Stegomyia) aegypti in residences in Mirassol, state of São Paulo, Brazil. Mem Inst Oswaldo Cruz,100: 339-343

Du YJ and Millar JG (1999) Electroantennogram and oviposition bioassay responses of Culex quinquefasciatus and Culex tarsalis (Diptera: Culicidae) to chemicals in odors from bermuda grass infusions. J Med Entomol 36: 158-166.

Effler PV, Pang L, Kitsutani PI, Vorndam V, Nakata M, Ayers T, Elm J, Tom T, Reiter P, Rigau-Perez JG, Hayes JM, Mills K, Napier M, Clark GG and Gubler (2005) Dengue fever, Hawaii, 2001-2002. Emerg Infect Dis 11: 742-749.

Eiras AE (2001) Mediadores químicos entre hospedeiros e insetos vetores de doenças médico-veterinárias. In: Vilela EF and Lúcia MTD (eds) Feromônios de insetos: biologia, química e emprego no manejo de pragas. Editora Holos, Ribeirão Preto, SP, Brasil.

Eiras AE (2002) Armadilha para captura de mosquitos. Brazilian Patent PI0203907, 9 Sept 2002.

Eiras AE and Jepson PC (1991) Host location by Aedes aegypti (Diptera: Culicidae): A wind tunnel study of chemical cues. Bull Entomol Res 81: 151-160. 
Eiras AE and Jepson PC (1994) Responses of female Aedes aegypti (Diptera: Culicidae) to host odours and convection currents using an olfactometer bioassay. Bull Entomol Res 84: 207-211.

Eiras AE and Resende MC (2009) Preliminary evaluation of the 'Dengue-MI' technology for Aedes aegypti monitoring and control. Cad Saude Publica 25 Suppl 1: S45-58.

Eiras AE and Sant'Ana AL (2002) Atraentes de oviposição para mosquitos. Brazilian Patent PI 0106701-0, Dec 2001.

Eiras AE, Resende MC and Silva IM (2007) Proposta de validação da MosquiTRAP e do sistema de monitoramento (MI-Dengue): uma nova tecnologia para a geração de novos índices de vigilância entomológica para o Programa Nacional de Controle da Dengue (PNCD). Research Report, Fundação Nacional de Saúde (FUNASA), Brasilia, Brasil.

Eiras AE, Sant'Ana AL and Stein K (2001) Identification of volatiles from grass infusions that attract gravid Aedes aegypti mosquito. Presentation at the $3^{\text {rd }}$ International Congress of Vetor Ecology, Barcelona, Spain, 16-21 September 2001.

Eiras AE, Silva IM and Resende MC (2005) Proposta de novo método de monitoramento e de novos índices de vigilância entomológica usando MosquiTRAP, uma nova armadilha para a captura de adultos do mosquito Aedes aegypti. Research Report, Fundação Nacional de Saúde (FUNASA), Brasilia, Brasil.

Eiras AE, Silva IM, Roque RA, Matosinhos IM and Geier M (2004) Behavioural responses of gravid Aedes aegypti (Diptera: Culicidae) to synthetic oviposition attractants identified from grass infusions volatiles. Presentation at the XXII International Congress of Entomology, Brisbane, Queensland, Australia, 15-21 August 2004.

Englbrecht Ch, Venturelli C, Rose A and Geier M (2009) Continuous trapping of adult Asian tiger mosquitoes (Aedes albopictus) with BG-Sentinel traps reduced the human landing rate and density indices in an urban environment in Cesena, Italy. Oral presentation at the $5^{\text {th }}$ European Mosquito Control Association Workshop, Turin, Italy, 9-13 March 2009.

Facchinelli L, Valerio L, Pombi M, Reiter P, Constantini C and Della Torre A (2007) Development of a novel sticky trap for container-breeding mosquitoes and evaluation of its sampling properties to monitor urban populations of Aedes albopictus. Med Vet Entomol 21: 183-195.

Favaro AE, Dibo MR, Mondini A, Ferreira AC, Barbosa AAC, Eiras AE, Barata EAMF and Chiaravalloti-Neto F (2006) Physiological state of Aedes (Stegomyia) aegypti mosquitoes captured with MosquiTRAPs in Mirassol, São Paulo, Brazil. J Vector Ecol 31: 285-291.

Favaro EA, Mondini A, Dibo MR, Barbosa AAC, Eiras AE, Barata EAMF and Chiaravalloti-Neto F (2008) Assessment of entomological indicators of Aedes aegypti (L.) from adult and egg collections in São Paulo, Brazil. J Vector Ecol 33: 8-16.

Fay RW and Eliason DA (1966) A preferred oviposition site as a surveillance method for Aedes aegypti. Mosq News 26: 531-535.

Fay RW and Prince WH (1970) A modified visual trap for Aedes aegypti. Mosq News 30: 20-23.

Feltner $\mathrm{H}$ and Ferrao $\mathrm{P}$ (2008) Evaluating efficacy of the BG-Lure attractant using three mosquito trap designs in the city of Alexandria, Virginia. Presenation at the $33^{\text {rd }}$ Annual Conference of the Mid-Atlantic Mosquito Control Association, Baltimore, Maryland, USA, 27-29 Feb 2008. Available at: http://www.bg-sentinel.com/bilder/Feltner_Ferrao-2008Evaluating_efficacy_of_BG-Lure.pdf.

Focks DA (2003) A review of entomological sampling methods and indicators for dengue vectors. TDR/ IDE/Den/03.1 WHO, Geneva, Switzerland.

Focks DA and Alexander N (2006) Multicountry study of Aedes aegypti pupal productivity survey methodology: findings and recommendations. TDR/IRM/Den /06.1 WHO, Geneva.

Focks DA, Daniels E, Haile DG and Keesling JE (1995) A simulation model of the epidemiology of urban dengue fever: literature analysis, model development, preliminary validation, and samples of simulation results. Am J Trop Med Hyg 53: 489-506.

Forattini OP, Kakitani I, Massad E and Marucci D (1995) Studies mosquitoes (Diptera: Culicidae) and anthropic environment. 9. Synanthropic and epidemiological vector role of Aedes scapularis in South-Eastern Brazil. Rev Saúde Pública 29: 199-207.

Gama RA, Silva EM, Silva IM, Resende MC and Eiras AE (2007) Evaluation of the sticky MosquiTRAP'M for detecting Aedes (Stegomyia) aegypti (L.) (Diptera: Culicidae) during the dry season in Belo Horizonte, Minas Gerais, Brazil. Neotrop Entomol 36: 294-302. 
Geier M and Boeckh JA (1999) A new Y-tube olfactometer for mosquitoes to measure the attractiveness of host odours. Entomol Exp Appl 92: 9-19.

Geier M, Bosch JO and Boeckh J (1999) Ammonia as an attractive component of host odour for the yellow fever mosquito, Aedes aegypti. Chem Senses 24: 647-653.

Geier M, Bosch JO and Boeckh J (1999) Influence of odour plume structure on upwind flight of mosquitoes towards hosts. J Exp Biology 202: 1639-1648.

Geier M, Rose A and Eiras AE (2004) Insect trap. International Patent WO2004/054358A2, 1 Jul 2004

Gubler DJ (1998) Dengue and dengue hemorrhagic fever. Clin Microbiol Rev 11: 480-496.

Gubler DJ (2004) The changing epidemiology of yellow fever and dengue, 1900 to 2003: full circle? Comp Immun Microbiol Infect Dis 27: 319-330.

Harrison BA, Callahan MC, Watts DM and Panthusiri L (1982) An efficient floating larval trap for sampling Aedes aegypti populations (Diptera: Culicidae). J Med Entomol 19: 722-727.

Holck AR, Meek CL and Holck JC (1988) Attractant enhanced ovitraps for the surveillance of container breeding mosquitoes. J Am Mosq Control Assoc 4: 97-98.

Honorio NA, Codeço CT, Alves FC, Magalhães MAFM and Lourenço-de-Oliveira R (2009) Temporal distribution of Aedes aegypti in different districts of Rio de Janeiro, Brazil, measured by two types of traps. J Med Entomol 46: 1001-1014

Hornby JA, Moore DE and Miller TW (1994) Aedes albopictus, distribution, abundance, and colonization in Lee County, Florida, and its effect on Aedes aegypti. J Am Mosq Control Assoc 10: 397-402.

Hribar LJ, Leprince DJ and Foil LD (1992). Ammonia as an attractant for adult Hybomitra lasiophthalma (Diptera: Tabanidae). J Med Entomol 29: 346-348.

Hwang YS, Kramer WL and Mulla M (1980) Isolation and identification of oviposition repellents for Culex mosquitoes. J Chem Ecol 6: 71-80.

Jones JW, Sithiprasasna R, Schleich S and Coleman RE (2003) Evaluation of selected traps as tools for conducting surveillance for adult Aedes aegypti in Thailand. J Am Mosq Control Assoc 19: 148-150.

Kay BH, Sutton KA and Russell BM (2000) A sticky entry-exit trap for sampling mosquitoes in subterranean habitats. J Am Mosq Contr Assoc 16: 262-265.

Kline DL (2002) Evaluation of various models of propane-powered mosquito traps. J Vector Ecol 27: 1-7.

Knols BGJ, Van Loon JJA, Cork A, Robinson RD, Adam W, Meijerink J, De Jong R, Takken W (1997) Behavioural and electrophysiological responses of the female malaria mosquito Anopheles gambiae s.s. Giles (Diptera: Culicidae) towards Limburger cheese volatiles. Bull Entomol Res. 87: 151-159.

Knols BGJ, Njiru BNN, Mathenge EM, Mukabana WR, Beier JC and Killeen GF (2002) MalariaSphere: A greenhouseenclosed simulation of a natural Anopheles gambiae (Diptera: Culicidae) ecosystem in western Kenya. Malaria J 1: 19.

Koenraadt CJM, Jones JW, Sithiprasasna R and Scott TW (2007) Standardizing container classification for immature Aedes aegypti surveillance in Kamphaeng Phet, Thailand. J Med Entomol 44: 938-944.

Kröckel U, Rose A, Eiras AE and Geier M (2006) New tools for surveillance of adult yellow fever mosquitoes: Comparison of trap catches with human landing rates in an urban environment. J Am Mosq Control Assoc 22: 229-238.

Krueger A and Hagen RM (2007) Short communication: first record of Aedes albopictus in Gabon, Central Africa. Trop Med Int Health 12: 1105-1107.

Kulldorff M, Athas WF, Feurer EJ, Miller BA and Key CR (1998) Evaluating cluster alarms: a space-time scan statistic and brain cancer in Los Alamos, New Mexico. Am J Pub Health 88: 1377-1380.

Kulldorff M, Heffernan R, Hartman J, Assunção R and Mostashari F (2005) A space-time permutation scan statistic for disease outbreak detection. PLoS Med 2: e59.

Kuno G (1997) Factors influencing the transmission of dengue viruses. In: Gubler DJ and Kuno G (eds) Dengue and dengue hemorrhagic fever. CABI, Wallingford, UK, pp 61-88.

Leal WS, Barbosa RMR, Xu W, Ishida Y and Syed Z (2008) Reverse and conventional chemical ecology approaches for the development of oviposition attractants for Culex mosquitoes Plos One 3: e3045.

Lourenço-de-Oliveira R, Lima JB, Peres R, Alves FC, Eiras AE and Codeço CT (2008) Comparison of different uses of adult traps and ovitraps for assessing dengue vector infestation in endemic areas. J Am Mosq Control Assoc 24: 387-392. 
Lugo E del C, Moreno G, Zachariah MA, López MM, López JD, Delgado MA, Valle SI, Espinoza PM, Salgado MJ, Pérez R, Hammond SN and Harris E (2005) Identification of Aedes albopictus in urban Nicaragua. J Am Mosq Control Assoc 21: 325-327.

Maciel de Freitas R, Codeco CT and Lourenço de Oliveira R (2007) Daily survival rates and dispersal of Aedes aegypti females in Rio de Janeiro, Brazil. Am J Trop Med Hyg 76: 659-665.

Maciel de Freitas R, Eiras AE and Lourenço de Oliveira R (2006) Field evaluation of effectiveness of the BG-Sentinel, a new trap for capturing adult Aedes aegypti (Diptera: Culicidae). Mem Inst Oswaldo Cruz 101: $321-325$.

Maciel de Freitas R, Eiras AE and Lourenco de Oliveira R (2008) Calculating the survival rate and estimated population density of gravid Aedes aegypti (Diptera, Culicidae) in Rio de Janeiro, Brazil. Cad Saúde Pública 24: 2747-2754.

Maciel de Freitas R and Lourenco de Oliveira R (2009) Presumed unconstrained dispersal of Aedes aegypti in the city of Rio de Janeiro, Brazil. Rev Saúde Pública 43: 8-12.

Maestre-Serrano R, Vergara-Sanchez C, Berrueco-Rodriguez G, Bello-Novoa B and Brochero H (2008) Presencia de Haemagogus equinus Theobald, 1903 (Diptera: Culicidae) en los municipios de Soledad y Malambo en el departamento del Atlántico, Colombia, 1998-2005. Biomédica 28: 99-107.

Marques CCA, Marques GRAM, Brito M, Santos Neto LG, Ishibashi VC and Gomes FA (1993) Estudo comparativo de eficácia de larvitrampas e ovitrampas para vigilância de vetores de dengue e febre amarela. Rev Saúde Pública 27: 237-241.

Mboera LEG, Takken W, Mdira KY and Pickett JA (2000) Sampling gravid Culex quinquefasciatus (Diptera: Culicidae) in Tanzania with traps baited with synthetic oviposition pheromone and grass infusions. J Med Entomol 37: 172-176.

Meeraus W, Johnson J and Arias JR (2007) Field comparison of novel and industrial standard traps for collecting Aedes albopictus in northern Virginia. Poster presentation at the $73^{\text {rd }}$ Annual Meeting of American Mosquito Control Association, Orlando, Florida, USA, 1-5 April 2007.

Meeraus WH, Armistead JS and Arias JR (2008) Field comparison of novel and gold standard traps for collecting Aedes albopictus in northern Virginia. J Am Mosq Control Assoc 24: 244-248.

Méndez F, Barreto M, Arias JF, Rengifo G, Muñoz J, Burbano ME and Parra B. (2006) Human and mosquito infections by dengue viruses during and after epidemics in a dengue-endemic region of Colombia. Am J Trop Med Hyg. 74(4): 678-83.

Metha DR (1934) Effect of saline and free ammonia on the oviposition of Anopheles culicifacies and Anopheles subpiscus (Rossi). Rev Mal Surv India 4: 411-420.

Micieli MV and Campos RE (2003) Oviposition activity and seasonal pattern of a population of Aedes (Stegomyia) aegypti (L.) (Diptera: Culicidae) in subtropical Argentina. Mem Inst Oswaldo Cruz 98: 659-663.

Millar JG, Chaney JD and Mulla MS (1992) Identification of oviposition attractants for Culex quinquefasciatus from fermented Bermuda grass infusions. J Am Mosq Control Assoc 8: 11-17.

Morrison AC, Gray K, Getis A, Astete A, Sihuincha M, Focks D, Watts D, Stancil JD, Olson JG, Blair P and Scott TW (2004) Temporal and geographic patterns of Aedes aegypti (Diptera: Culicidae) production in Iquitos, Peru. J Med Entomol 41: 1123-1142.

Morrison AC, Zielinski-Gutierrez E, Scott TW and Rosenberg R (2008) Defining challenges and proposing solutions for control of the virus vector Aedes aegypti. PLoS Med 5: 0362-0366.

Mota RN (2003) Construção de um olfatômetro de dupla escolha para estudos de orientação de fêmeas grávidas de Aedes aegypti (Linnaeus, 1792) (Diptera: Culicidae) à atraentes de oviposição. MSc Dissertation, Universidade Federal de Minas Gerais, Brazil.

Ministério da Saúde (MS) (2007a) Graphic showing the historical series of reported dengue cases between 1980 and 2005. Available at: http://portal.saude.gov.br/portal/arquivos/pdf/Graficos_DNC_dengue_verde.pdf.

Ministério da Saúde (MS) (2007b) Graphic showing the historical series of mortality due to dengue cases between 1980 and 2005. Available at: http://portal.saude.gov.br/portal/arquivos/pdf/Graficos_DNC_dengue_vermelho.pdf.

Muir LE and Kay BH (1998) Aedes aegypti survival and dispersal estimated by mark-release-recapture in Northern Australia. Am J Trop Med Hyg 58: 277-282.

Müller W.(1968). Die Distanz- und Kontaktorientierung der Stechmücken Aedes aegypti (Wirtsfindung, Stechverhalten und Blutmahlzeit). Z vergl Physiol 58: 241-303. 
Nasci RS (1981) A light weight battery-powered aspirator for collecting mosquitoes in the field. Mosq News 41: 808-811. Obermayr R (2006) Are new trapping technologies useful for mosquito control interventions? Vector Ecol Newsl 37 (3): 11-12.

Ordonez JG, Fernandez Salas I and Flores-Leal A (1997) Monitoring dispersal of marked Aedes aegypti females under field conditions using sticky ovitraps in Monterrey, northeastern Mexico. J Am Mosq Assoc 13: 121.

Ordóñez-Gonzalez JG, Mercado-Hernandez R, Flores-Suarez AE and Fernández-Salas I (2001) The use of sticky ovitraps to estimate dispersal of Aedes aegypti in Northeastern Mexico. J Am Mosq Contr Assoc 17: 93-97.

PNCD (2002) Programa Nacional de Controle da Dengue. Ministério da Saúde Fundação Nacional de Saúde, Brasília, Brasil. Available at: http://bvsms.saude.gov.br/bvs/publicacoes/pncd_2002.pdf.

Public Health Agency of Canada (PHAC) (2009) Notifiable diseases on-line. Available at: http://dsol-smed.phac-aspc. gc.ca/dsol-smed/ndis/index-eng.php.

Ponnusamy L, Xu N, Nojima S, Wesson DM, Schal C and Apperson CA (2008) Identification of bacteria and bacteriaassociated chemical cues that mediate oviposition site preferences by Aedes aegypti. PNAS 105: 9262-9267.

Rawlins SC, Martinez R, Wiltshire S and Legall G (1998) A comparison of surveillance systems for the dengue vector Aedes aegypti in port of Spain, Trinidad. J Am Mosq Control Assoc 14: 131-136.

Regis L, Monteiro AM, Varjal de Melo-Santo MA, Silveira Jr JC, Freire Furtado A, Veiga Aciolis R, Santo GM, Nkazawa M, Sa Carvalho M, Ribeiro Jr PJ, Viera de Souza W (2008) Developing new approaches for detecting and preventing Aedes aegypti population outbreaks: basis for surveillance, alert and control system. Mem Inst Oswaldo Cruz 103: 50-59.

Reiter P (1983) A portable, battery-powered trap for collecting gravid Culex mosquitoes. Mosq News 4: 496-498.

Reiter P (1986) A standardized procedure for the quantitative surveillance of certain Culex mosquitoes by egg raft collection. J Am Mosq Control Assoc 3: 494-501.

Reiter P, Amador MA and Colon N (1991) Enhancement of the CDC ovitrap with hay infusion for daily monitoring of $A$. aegypti populations. J Am Mosq Control Assoc 7: 52-55.

Reiter P, Nathan MB (2001) Guidelines for assessing the efficacy of insecticide space sprays for the control of the Dengue vector Aedes aegypti. WHO/CDS/CPE/PVC/2001.1, WHO, Geneva, Switzerland.

Ritchie SA, Long S, Hart A, Webb CE and Russell RC (2003) An adulticidal sticky ovitrap for sampling container-breeding mosquitoes. J Am Mosq Control Assoc 19: 235-242.

Ritchie SA (1984) Hay infusion and isopropyl alcohol-baited CDC light trap; a simple effective trap for gravid Culex mosquitoes. Mosq News 44: 404-407.

Ritchie SA, Moore P, Carruthers M (2006) Discovery of a widespread infestation of Aedes albopictus in the Torres Strait, Australia. J Am Mosq Control Assoc 22: 358-365.

Ritchie SA, Long S, Smith G, Pike A and Knox TB (2004) Entomological investigations in a focus of dengue transmission in Cairns, Queesland, Australia, by using the sticky ovitraps. J Med Entomol 41: 1-4.

Roque RA (2007) Formulação e avaliação de atraentes sintéticos de oviposição, identificados em infusões do capim colonião (Panicum maximum) para fêmeas grávidas do mosquito Aedes (Stegomyia) aegypti (Linnaeus, 1762) (Diptera: Culicidae) em condições de campo e semi-campo. Dissertation, Universidade Federal de Minas Gerais, Brazil.

Roque RA and Eiras AE (2008) Calibration and evaluation of field cage for oviposition study with Aedes (Stegomyia) aegypti female (L.) (Diptera: Culicidae). Neotrop Entomol 37: 478-485.

Rössler HP (1961) Versuche zur geruchlichen Anlockung weiblicher Stechmücken (Aedes aegypti L. (Culicidae). Z Vgl Physiol 44: 184-231.

Russell RC (2004) The relative attractiveness of carbon dioxide and octenol in CDC- and EVS-type light traps for sampling the mosquitoes Aedes aegypti (L.), Aedes polynesiensis Marks, and Culex quinquefasciatus Say in Moorea, French Polynesia. J Vector Ecol 29: 309-314.

Russel RC, Webb CE, Williams CR and Ritchie SA (2005) Mark-release-recapture study to measure dispersal of the mosquito Aedes aegypti in Cairns, Queensland, Australia. Med Vet Entomol 19: 1-7.

Russell RC and Ritchie SA (2004) Surveillance and behavioral investigations of Aedes aegypti and Aedes polynesisensis in Moorea, French Polynesia, using a sticky ovitrap. J Am Mosq Control Assoc 20: 370-375. 
Sant'Ana AL (2003) Avaliação, extração, identificação e estudos eletrofisiológicos dos voláteis presentes em infusões de Panicum maximum que estimulam e/ou atraem fêmeas de Aedes (Stegomyia) aegypti Linnaeus, 1762 (Diptera: Culicidae) para oviposição. Dissertation, Universidade Federal de Minas Gerais, Minas Gerais, Brazil.

Sant'Ana AL, Roque RA and Eiras AE (2006) Characteristics of grass infusions as oviposition attractants to Aedes (Stegomyia) (Diptera: Culicidae). J Med Entomol 43: 214-20.

Service MW (1993) Mosquito ecology: field sampling methods. Second edition. Chapman \& Hall, London, UK.

Schoeler GB, Schleich SS, Manweiler SA and Sifuentes VA (2004) Evaluation of surveillance devices for monitoring Aedes aegypti in an urban area of northeastern Peru. J Am Mosq Control Assoc 20: 6-11.

Schmied WH, Takken W, Killen GF, Knols BGJ and Smallegange (2008) Evaluation of two counterflow traps for testing behaviour-mediating compounds for the malaria vector Anopheles gambiae s.s. under semi-field conditions in Tanzania. Malaria J 7: 230.

Scott TW and Morrison AC (2002) Aedes aegypti density and the risk of dengue-virus transmission. In: Takken W and Scott TW (eds) Ecological aspects for application of genetically modified mosquitoes. Proceedings of the Frontis workshop on ecological challenges concerning the use of genetically modified mosquitoes for disease control. Wageningen, the Netherlands 26-29 June 2002. Wageningen UR Frontis Series, pp 187-206.

Silver JB 2008. Mosquito ecology: field sampling methods. $3^{\text {rd }}$ edition. Springer Co., New York, USA.

Sithprasasna R, Linthicum KJ, Lerdthusnee K and Brewer TG (1997) Use of Geographical information system to study the epidemiology of dengue haemorrhagic fever in Thailand. Dengue Bull 21:68-72.

Smith CN, Smith N, Gouck HK, Weidhaas DE, Gilbert IH, Mayer MS, Smittle BJ and Hofbauer A (1970) L-Lactic acid as a factor in the attraction of Aedes aegypti (Diptera: Culicidae) to human hosts. Ann Entomol Soc Am 7: 99-117.

Solberg VB, Sithiprasasna R and Fansiri T (2007) Efficacy testing of eight unique or standard mosquito surveillance traps/ methods in Thailand mouses. Presentation at the ESA Annual Meeting, San Diego, California, USA, 9-12 Dec 2007.

Suárez Ramírez N and Colás Bonne M (2008) Afectación de la sensibilidad del sistema de vigilancia entomológica mediante larvitrampas en un área de salud. Medisan 12(4). available at: http://bvs.sld.cu/revistas/san/vol12_4_08/ san11408.htm.

Takken W and Mboera LEG (2000) Effects of chemical stimuli on oviposition of Culex quinquefasciatus (Diptera: Culicidae) in Tanzania. Proc Exp Appl Entomol 11: 182-187.

Taneja J. and Guerin PM (1997) Ammonia attracts the haematophagus bug Triatoma infestans: behavioural and neurophysiological data on nymphs. J Comp Physiol 181:21-34.

Trexler JD, Apperson CS, Gemeno C, Perich MJ, Carlson D and Schal C (2003) Field and laboratory evaluations of potential oviposition attractants for Aedes albopictus (Diptera: Culicidae). J Am Mosq Control Assoc 19: 228-34.

Tsuda Y, Suwonkerd W, Chawprom S, Prajakwong S and Takagi M. (2006) Different spatial distribution of Aedes aegypti and Aedes albopictus along an urban-rural gradient and the relating environmental factors examined in three villages in northern Thailand. J Am Mosq Control Assoc 22: 222-228.

WHO (1999) Strengthening implementation of the global strategy for dengue fever/ dengue haemorrhagic fever prevention and control. Report of the Informal Consultation. WHO, Geneva, Switzerland.

WHO (2009) Dengue and dengue hemorrhagic fever. Available at: http://www.who.int/csr/disease/dengue/impact/en/.

Williams CR, Long SA, Russel RC and Ritchie SA (2006) Field efficacy of the BG-Sentinel compared with the CDC Backpack Aspirator and CO2-baited EVS trap for collection of adult Aedes aegypti in Cairns, Queensland, Australia. J Am Mosq Control Assoc 22: 296- 300.

Williams CR, Long SA, Webb CE, Bitzhenner M, Geier M, Russel RC and Ritchie SA (2007) Aedes aegypti population sampling using BG-Sentinel traps in north Queensland Australia: statistical considerations for trap deployment and sampling strategy. J Med Entomol 44: 345-350.

Wilton DP (1985) Preliminary evaluation of a black cylinder suction trap for Aedes aegypti and Culex quinquefasciatus (Diptera: Culicidae). J Med Entomol 22: 113-114. 


\title{
18. Exploitation of olfactory-mediated behaviour
}

\author{
Willem Takken and Bart G.J. Knols
}

\begin{abstract}
Intra- and interspecific communication between arthropods and their blood hosts is to a large extent mediated by chemical cues. The information provided in this book shows the many and significant advancements in our knowledge of these interactions, from DNA regulation to natural interactions of wild arthropod populations and their hosts. Recent information from molecular genetics, neurobiology, behavioural ecology and chemistry have resulted in understanding how these organisms are affected by small molecules, mostly produced by the vertebrate host, and that the insects and ticks respond to blends of odorants rather than single cues. Whereas $\mathrm{CO}_{2}$ is a universal kairomone for these blood-feeding arthropods, other host-derived odorants are often required to attain behavioural responses similar to those observed with natural hosts. The collective data described in this book provide opportunities and challenges for integration of olfactory tools as novel interventions for the control of these arthropods, many of which are vectors of infectious diseases or nuisance insects. Examples of such a strategy are provided, notably with mosquitoes, as these are among the group of arthropods for which alternative control strategies are most urgently required. Finally, the gaps in research that need to be addressed are discussed, focusing on identification and formulation of semiochemicals, sampling technologies and the integration of this in existing control programmes.
\end{abstract}

Keywords: semiochemicals, blood-feeding arthropods, chemical communication, challenge, vector control, attractant, repellent

\section{Introduction}

Chemical communication is an important mechanism of intra- and interspecific communication of arthropods. This book, which reviews the role of olfaction in vector-host interactions, shows progress made in our understanding of this process in blood feeding insects and ticks, from cellular level to population. Whereas the role of olfactory cues in host seeking of disease vectors was known for many decades, the regulatory mechanisms of this behaviour remained largely hidden until recent advancements in the fields of molecular genetics, analytical chemistry and visual technology. These technologies allowed for the discovery of olfactory genes and examination of the genetic regulation of gene expression in several disease vectors. In sensory physiology advances in technology and chemistry have made detailed studies possible on the activation of olfactory processing from the periphery to the insect brain. Advanced knowledge on insect behaviour led to the development of tools with which flight behaviour and responses to olfactory cues can be accurately assessed in laboratory and field. This book provides numerous examples of these advances over a wide range of insects and ticks. The collected information should be used for the development of tools that can be exploited for the control of vector-borne disease transmission and reduction of biting nuisance by blood-feeding arthropods, and several examples of these will be discussed in this concluding chapter.

\section{Molecular regulation of olfactory behaviour}

The recent publication of the genomes of several disease vectors (2002: Anopheles gambiae Giles sensu stricto; 2006: Culex pipiens quinquefasciatus Say; 2007: Aedes aegypti (L.); 2007: Pediculus 
humanus Linnaeus; 2008: Ixodes scapularis Say (see www.vectorbase.org for all genomes) creates unprecedented opportunities for studying the genetic and cellular regulation of insect behaviour. For example, mechanisms that affect the expression of olfactory genes in mosquitoes can now be studied in detail, allowing for a comprehensive understanding of the insect response to selected odorants (Touhara and Vosshall 2009, Chapter 2 in this volume). In addition, recent data on odorant binding proteins (OBPs) demonstrate the significance of these molecules in transporting volatile chemicals through the sensillum fluid to the receptor membrane, but also how gene silencing, using RNAi technologies, can fundamentally alter these processes, suggesting behavioural changes that went hitherto unexplained (Pelletier and Leal 2009, Sengul and Tu 2008, Zhou et al. 2008). The challenge is how this information can be maximally exploited for vector-borne disease control. For example, it has been suggested that silencing of selected olfactory genes might lead to mosquitoes that no longer recognise human odours, or not any vertebrate indeed. Exploitation of this knowledge would require the stable transformation of genetic traits to produce lines of mosquitoes with altered host-seeking behaviour and/or host-seeking strategies, so that fewer bites would occur on humans. We can then envisage the release of such mosquitoes in natural ecosystems to replace wild sibling populations as a contribution to disease control. In recent years, the use of such genetically modified vectors has been debated intensively as the socio-ethical and legal aspects of this technology remain unresolved (Benedict et al. 2008, Knols and Louis 2004, Scott et al. 2002, 2008). The World Health Organization, through its Tropical Disease Research (TDR) programme, discussed this topic at length during a meeting in May 2009, and it is expected that the organisation will provide guidance on the use of this technology in disease-endemic countries (Mumford et al. 2009). As the focus of genetic modification for vector-borne disease control appears to be more on the alteration of vector competence then on other biological traits (Catteruccia et al. 2009), the development of mosquitoes with altered biting behaviour is at present remote.

\section{Neurophysiology}

Recent advances in the neurophysiology of olfaction of disease vectors focused mostly on mosquitoes (Chapters 3 and 4) although studies on sandflies (Chapter 9) and kissing bugs (Chapter 14) also provided new insights in chemoreception of these insects. Drosophila melanogaster Meigen serves as a universal model insect for our understanding of processing of olfactory information in insects. Studies on Ae. aegypti, An. gambiae sensu stricto and Cx. quinquefasciatus demonstrate many similarities with $D$. melanogaster in olfactory processing, confirming the validity of using this species as a model for our understanding of insect olfaction. Whereas olfactory receptor cells can be broadly tuned to a wide range of chemical cues (Chapter 3, this volume and Carey et al. 2010), internal processing of these signals, perhaps governed by olfactory binding proteins (OBPs) and interaction at the neural membrane cause a first selective barrier for the action of semiochemicals, which is further regulated at the primary olfactory centre in the brain (Chapter 4). Functional dimorphism has been described for insects from different orders, but for mosquitoes this still has to be described. If so, this might explain the observed differences in olfactory responses of mosquitoes with respect to mating, host seeking and oviposition. It is interesting that odours, and specifically odour quality, ultimately are encoded by spatial patterns of activation in defined sets of glomeruli (Chapter 4). Such information needs to be linked to behavioural studies in which the behavioural response of insects to qualitatively different blends of odorants are being examined (Bernier et al. 2007, Jawara et al. 2009, Okumu et al. 2010a, Smallegange et al. 2009, Syed and Leal 2009, Williams et al. 2006). Whereas in recent years significant advances have been made in our understanding of the olfactory processing of odour cues of vectors, there is a lack of congruence between these results and those of the behavioural essays reported elsewhere in this volume 
(Chapters 7 and 8). For rapid assessment of the quality of candidate odorant cues as behaviourally relevant, we propose the development of a model that would predict the behavioural outcome of the signal-transduction cascade of semiochemicals.

\section{Flight behaviour}

It is now generally accepted that blood-feeding insects respond to host odours, which as kairomones serve to guide the insects to the host. In this volume mosquitoes were selected as an example for reviewing upwind flight behaviour (Chapter 6). During the flight, mosquitoes use both chemical and visual cues, while temperature is detected only within a short range from the host. The effect of carbon dioxide during upwind flight has become better understood in recent years but the overall effect of blends of kairomones on in-flight behaviour, however, remains to be clarified. For example, $\mathrm{CO}_{2}$ is sensed from a long distance, but we do not know over which distance other chemical cues are being detected and cause a behavioural effect. This is puzzling, as the principles of odour dispersal predict that packages of odour move downwind in a concentration similar to that at the source (Murlis et al. 2000). In a wind tunnel, Dekker et al. (2005) demonstrated that the yellow fever mosquito Ae. aegypti responded to homogeneous plumes of host odour and to turbulent plumes of $\mathrm{CO}_{2}$. A similar phenomenon was shown to occur in the malaria mosquito An. gambiae s.s. (Spitzen et al. 2008). Recent semi-field and field studies showed that host odours alone attracted few mosquitoes to odour-baited traps, and that addition of $\mathrm{CO}_{2}$ increased the catch significantly (Qiu et al. 2007, Schmied et al. 2008), providing support for these laboratory findings. These results suggest that mosquitoes sense a host from a distance with $\mathrm{CO}_{2}$ as a principle component and that close-range host orientation is guided by other host odours, principally derived from the skin. It remains a challenge to elucidate whether such host odours induce upwind flight behaviour from a distance (Chapter 6). Tools such as windtunnels provided with imaging recorders, capable to record flights at 6,000 images per second (Anonymous, 2010) and equipped with on-line analysis software, as well as the use of electric nets for recording host-seeking flights in a natural environment (Chapters 6 and 12) suggest that details or odourmodulated flight behaviour of insects will rapidly advance. This, in turn, provides opportunities to better understand the role of specific host cues in the host-seeking strategy of disease vectors. Indoor host-seeking behaviour has remained largely unexplored, yet given the tendency of many vectors to bite indoors (endophagy) this deserves more focus. Exactly how host-seeking insects behave in a 'no-wind' environment could serve as a means to interfere with odour-modulated behaviour indoors and optimise methods to divert, trap, or repel insects close to hosts.

\section{Behavioural assays}

The study of odour-modulated behaviour of flying insects is, by nature, complex as the target organism moves fast through the natural environment such that direct observations are highly unreliable. This complexity is even enhanced as we consider that odour plumes are highly motile and affected by wind (Chapter 6 , this volume). A consensus seems to have been reached among scientists that this behaviour should be studied at different levels: (a) under controlled conditions in the laboratory, (b) in semi-field enclosures, where the insects can be observed in a simulated near-natural environment and (c) in the field. Examples of these studies are reported in this volume, with mosquitoes, sandflies, stable flies, blackflies, tsetse flies, kissing bugs and ticks as target arthropods. The latter two groups require different observation tools as they move by walking and, hence, are more easily observable by the researcher. In the cascade of behavioural observations, the order of laboratory - semi field - field is desirable, but as the use of semi field systems is of recent origin (Ferguson et al. 2008, Knols et al. 2002), many studies move directly from 
the laboratory to the field. Video recording can be used for detailed studies of in-flight behaviour (Beeuwkes et al. 2008, Cooperband and Cardé 2006, Dekker et al. 2005, Gibson and Brady 1985, 1988), and may reveal behavioural manoeuvres associated with particular olfactory cues (Dekker et al. 2005, Spitzen et al. 2008) and advanced software is available that enables detailed analysis of flight paths according to essential behavioural parameters (e.g. see www.noldus.com).

Several standard assays for laboratory studies have emerged, which provide robust tools for examining the behavioural response of blood-feeding insects and ticks to sensory cues such as odour, heat, humidity and visual objects. For the study of mosquito behaviour, a wind tunnel or olfactometer is widely used, and using these devices, semiochemicals that affect the behaviour of mosquitoes have been identified. The most recent studies report odour blends of chemicals identified from the natural volatiles of blood hosts (Bernier et al. 2007, Okumu et al. 2010a, Chapter 7 in this volume). Although this work initially focused on tsetse flies, it is encouraging to note that these studies are rapidly expanding to other blood-feeding species, leading to several promising odour blends with which these insects can be manipulated. Laboratory studies now produce blends of odorants to which the insects are attracted or by which they are repelled, depending on the odour composition. The challenge now is to provide proof that these blends are also effective in the field, as was done for tsetse flies. Only then can these studies be justified and perhaps improved to provide a large range of odorant cues with which nuisance insects and disease vectors can be effectively controlled. The recent study by Okumu et al. (2010a) in Tanzania and field studies in Brazil (Chapter 17, this volume) as well as a field study in Scotland (Logan et al. 2009) demonstrate that such cues are effective against different mosquito and biting midge species on several continents. Ongoing studies are expected to lead to a rapid expansion of the available odour cues affecting these insects.

\section{Semiochemicals}

For many years, odorant baits for mosquitoes consisted of carbon dioxide $\left(\mathrm{CO}_{2}\right)$ or live hosts (Takken and Knols 1999). Detailed research on tsetse flies and screwworm flies, however, resulted in the identification of chemical cues to which these insects respond by attraction or aversion (Cork and Hall 2007, Vale 1993, Chapter 12 in this volume). Inspired by these developments, studies on odour baits for other vectors were actively initiated, and in the preceding chapters of this volume the state-of-the art of these studies are reviewed. Carbon dioxide acts as a universal kairomone (activator and attractant) for blood-feeding arthropods (Chapter 5, this volume), which suggests an evolutionary-safe adaptation to vector-host interactions, as all vertebrates exhale this compound. Any response to $\mathrm{CO}_{2}$ is expected to lead the arthropod to a live host. However, $\mathrm{CO}_{2}$ is a not a kairomone that arthropods can exploit to satisfy their intrinsic preference for biting specific host species. For several mosquito species, which express a strong anthropophilic behaviour, studies focused on the chemical identification of human odorants and on selection of those compounds that cause a behavioural response in the mosquito (Bernier et al. 2000, 2002, Logan et al. 2009). This has led to the publication of blends of odorant chemicals that cause attraction of Ae. aegypti (Bernier et al. 2003) and An. gambiae (Okumu et al. 2010a, Smallegange et al. 2009). Both species are important vectors of arboviruses such as dengue, yellow fever, and Chikungunya (Ae. aegypti) and malaria (An. gambiae), and the availability of attractive blends opens the way for improved surveillance of these vectors, as well as for the development of removal trapping systems. In Chapter 17 (this volume) a practical application for the surveillance of Ae. aegypti in Brazil is described, which is the immediate result of the detailed chemical, physiological and behavioural studies reviewed in other chapters of this book. Compared to mosquitoes, the identification of kairomones for other vectors has been less intense, but recent data on biting 
midges (Chapter 10, this volume), kissing bugs (Chapter 14, this volume) and sandflies (Chapter 9 , this volume) suggest that these insects can soon also be sampled using attractive odour baits. It has been suggested that only baits that are as attractive as natural host odours can be applied for the successful manipulation of vectors, such as for example the control of tsetse populations in southern Africa (Hargrove 2005), and that baits that are less attractive than natural hosts are not relevant for vector control. We argue that a constant removal of fractions of vector populations over time will cause a significant reduction in density irrespective of the degree of attractiveness, although the speed of population reduction will be much higher when the baits are powerful. This of course on condition that the target vector population does not develop a change in behaviour by selection. We are not aware of publications of behavioural resistance as a result of olfactory manipulation, and suggest that the probability of this to occur is small as the insects can usually feed on alternative hosts (Killeen et al. 2001), which express a different odour profile.

\section{Pathogen-induced effects on olfactory behaviour}

The few reports on manipulation of vector behaviour by pathogens remains intriguing, as it is evolutionary advantageous for these microorganisms to control their transmission from host to vector and vice versa. Although there is much evidence for the manipulation of feeding behaviour by pathogens, the olfactory evidence of these interactions remains limited to studies on Plasmodium and Leishmania (Chapter 16, this volume). Recent work on vector manipulation by viruses in plants demonstrated convincingly that the pathogens affect the composition of semiochemicals that guide their insect vectors to the plant (Mauck et al. 2010) and match earlier work on malaria vectors (Lacroix et al. 2005). It is, however, not known whether these effects apply to a broad range of vector-borne microorganisms and of those where such behavioural manipulation was found, the nature of the semiochemicals involved is unknown to date. Yet, detailed knowledge of these interactions would provide insight in specific cues that attract vectors to their hosts, and can be used for vector manipulation.

\section{Future challenges and prospects}

Our knowledge on the role of olfactory cues in host-seeking behaviour of blood-feeding arthropods has made significant advances in the last decade (this volume). Tools for the detailed observation and analysis of this behaviour are now being used by a large group of scientists, which is expected to answer questions that have eluded us this far. The field of research has broadened, to include molecular genetics, cellular regulation of signal transduction, analytical chemistry, behavioural ecology and parasite-vector interactions. While these studies answer fundamental questions about the role of odorants in arthropod behaviour, several challenges need to be resolved. For example, the sampling of blood-feeding insects relies currently on crude tools such as traps, knock-down sprays, resting collections or the human biting catch (Chapters 8, 11 and 12 , this volume). The rationale for trap design is often unknown, and studies as described by Cooperband et al. (2006) show the importance of understanding the way insects approach a trap while responding to host volatiles. All mosquito traps currently marketed operate a strong suction airflow to draw insects into a collection bag. The disadvantage of such traps is that they require a power source to operate the fan, which is often lacking in rural areas where such traps need to be operated. Research into passive trapping systems or even targets should receive more attention. Recently, for the first time, odour-baited stations for mosquitoes have been field tested (Okumu et al. 2010b). Although still not practical and in need of a $\mathrm{CO}_{2}$ source provided from a pressurised gas cylinder, this study marks the first step towards effective use of attractive kairomone blends for operational use in developing countries. Such systems can be used as a 'lure and kill' tactic, 
whereby insects are attracted to an insecticide-treated resting site. Alternatively, insects can be contaminated with biological control agents such as entomopathogenic fungi, through a'lure and contaminate' strategy (Lwetoijera et al., in press).

An important goal of research on olfactory behaviour of arthropods is to understand how odour cues can be used operationally. Many blood-feeding arthropods are disease vectors, and hence are targeted for control. The work on tsetse flies has convincingly shown that odour cues can be used to control disease vectors (Torr et al. 2005), but currently there are no examples of other disease vectors that can be controlled in this way.

Much work reviewed in this book concerns the role of kairomones in vector-host interactions, and for some vector species effective strategies are available that are used for their control (Chapter 12 , this volume). An older, and more widely applied strategy of vector manipulation is the use of repellents such as $\mathrm{N}, \mathrm{N}$-diethyl-3-methylbenzamide (DEET) (Katz et al. 2008). DEET has been in use for more than 50 years, and is effective against mosquitoes, sand flies and ticks. Because of its relatively long persistence and high efficacy, it is not only used as protection against nuisance mosquitoes but also as personal protection against malaria and dengue vectors. Other compounds like IR3535((R)) and Picaridin have been shown to provide a similar degree of protection as DEET. Recently, olfactory receptors for DEET were reported, providing exciting prospects for behavioural manipulation for other candidate chemicals with repellent action (Ditzen et al. 2008, Syed and Leal 2008). For instance, it is known that at low concentrations 1-octen-3-ol is attractive to some mosquito species and tsetse flies, but that higher concentrations cause a repellent effect. A different, but repellent effect was observed with carboxylic acids in anopheline mosquitoes, which was overcome by the addition of ammonia + lactic acid to the acid mixture (Smallegange et al. 2005). From these studies it is suggested that semiochemicals can be strategically applied for behavioural manipulation of disease vectors, opening the way for push-pull strategies as have been developed for herbivorous insects (Agelopoulos et al. 1999, Khan et al. 2008).

The emergence and spread of infectious diseases is studied using models and for vector-borne diseases these include the interaction between vectors, parasites and hosts (Anderson and May 1992). The vector-component in these models is, however, surprisingly inaccurate as vector behaviour is often not taken into account. For example, vectors exhibit different biting behaviour depending on physiological stage, age and infectious stage. The preceding chapters provide evidence that behavioural responses to blood hosts can be much affected by these factors, and therefore should be included in these models. Models can also be used to assess the impact of behavioural manipulation, for instance by removal trapping or the impact of a push-pull system. Agent-based models have recently been developed to assess the efficacy of bed net interventions on a micro scale, and such models can similarly be developed for removal trapping (Gu and Novak 2009). We suggest that field studies on the risk of vector-borne disease incorporate a behavioural component, so that better use can be made of behavioural manipulation through vector control or through personal protection.

This book focuses on advances in our knowledge on olfactory mediation of arthropod-host interactions. To study these interactions in natural situations in the field, sampling systems are needed to assess the behavioural response to semiochemicals. Although the efficacy of the many tools used for the collection of arthropods is not discussed here, it is obvious that such tools are essential in behavioural studies. A growing number of semiochemicals is becoming available for behavioural manipulation of nuisance insects and disease vectors and proof of principle of their operational use is becoming available (Okumu et al. 2010b). We suggest that the private pest 
control sector becomes more closely involved in this work, so that the concept of arthropod manipulation with semiochemicals can be further developed. One successful example of such a development is that of the insecticide-treated bed nets, where the private sector became closely involved in disease control (Lengeler et al. 2007, Noor et al. 2009), allowing for a massive surge in technological advances using a private/public partnership. The control of tsetse flies using semiochemicals has received commercial interest, but to date only on a limited scale. On a larger scale the control of nuisance mosquitoes using semiochemical technology was undertaken by American Biophysics Corp. The latter company, however, dissolved after only a few years of successful operation. Although the reasons for the breakup of this company remain elusive, the results of their activities show that there was a broad private and public interest in this method of pest control. Now that better and more advanced methods of semiochemical production have become available, it seems worthwhile to examine the possible integration of this technology into the control of nuisance insects and disease vectors.

The reviews in this book demonstrate the importance of chemical ecology in vector-host interactions. The rapid advances in this field made in recent years suggest that we can now begin to consider applying the collective knowledge gained for manipulation of these vectors, introducing a novel, sustainable tool for disease control. The example of the successful control of tsetse flies using semiochemicals remains the best proof that this strategy can be highly effective. Semiochemicals also provide a solid base for studies of population densities and regulation of disease vectors, so that more accurate and objective measurements of transmission risk as well as the impact of vector control can be obtained.

\section{References}

Agelopoulos N, Birkett MA, Hick AJ, Hooper AM, Pickett JA, Pow EM, Smart LE, Smiley DWM, Wadhams LJ and Woodcock CM (1999) Exploiting semiochemicals in insect control. Pest Sci 55: 225-235.

Anderson RM and May RM (1992) Infectious diseases of humans - Dynamics and control. Oxford University Press, Oxford, UK. 757 pp.

Anonymous (2010) Malaria. Available at: http://intellectualventureslab.com/?page_id=563. Accessed 23 Feb 2010.

Beeuwkes J, Spitzen J, Spoor CW, Van Leeuwen JL and Takken W (2008) 3-D flight behaviour of the malaria mosquito Anopheles gambiae s.s. inside an odour plume. Proc Exp Appl Entomol 19: 137-146.

Benedict M, D'Abbs P, Dobson S, Gottlieb M, Harrington L, Higgs S, James A, James S, Knols B, Lavery J, O'Neill S, Scott T, Takken W and Toure $Y$ (2008) Guidance for contained field trials of vector mosquitoes engineered to contain a gene drive system: recommendations of a scientific working group. Vector Borne Zoonotic Dis 8: 127-166.

Bernier UR, Kline DL, Allan SA and Barnard DR (2007) Laboratory comparison of Aedes aegypti attraction to human odors and to synthetic human odor compounds and blends. J Am Mosq Control Assoc 23: 288-293.

Bernier UR, Kline DL, Barnard DR, Schreck CE and Yost RA (2000) Analysis of human skin emanations by gas chromatography/mass spectrometry. 2. Identification of volatile compounds that are candidate attractants for yellow fever mosquito (Aedes aegypti). Anal Chem A 72: 747-756.

Bernier UR, Kline DL, Posey KH, Booth MM, Yost RA and Barnard DR (2003) Synergistic attraction of Aedes aegypti (L.) to binary blends of L-lactic acid and acetone, dichloromethane, or dimethyl Disulfide. J Med Entomol 40: 653-656.

Bernier UR, Kline DL, Schreck CE, Yost RA and Barnard DR (2002) Chemical analysis of human skin emanations: comparison of volatiles from humans that differ in attraction of Aedes aegypti (Diptera: Culicidae). J Am Mosq Contr Ass 18: 186-195.

Carey AF, Wang G, Su CY, Zwiebel LJ and Carlson JR (2010) Odorant reception in the malaria mosquito Anopheles gambiae. Nature (in press). Doi:10.1038/nature08834.

Catteruccia F, Crisanti A and Wimmer EA (2009) Transgenic technologies to induce sterility. Malaria J 8 (Suppl 2): S7. 
Cooperband MF and Cardé RT (2006) Orientation of Culex mosquitoes to carbon dioxide-baited traps: flight manoeuvres and trapping efficiency. Med Vet Entomol 20: 11-26.

Cork A and Hall MJR (2007) Development of an odour-baited target for female New World screwworm, Cochliomyia hominivorax: studies with host baits and synthetic wound fluids. Med Vet Entomol 21: 85-92.

Dekker T, Geier M and Cardé RT (2005) Carbon dioxide instantly sensitizes female yellow fever mosquitoes to human skin odours. J Exp Biol 208: 2963-2972.

Ditzen M, Pellegrino M and Vosshall LB (2008) Insect odorant receptors are molecular targets of the insect repellent DEET. Science 319: 1838-1842.

Ferguson HM, Ng'habi KR, Walder T, Kadungula D, Moore SJ, Lyimo I, Russell TL, Urassa H, Mshinda H, Killeen GF and Knols BG (2008) Establishment of a large semi-field system for experimental study of African malaria vector ecology and control in Tanzania. Malar J 7: 158.

Gibson G and Brady J (1985) 'Anemotactic' flight paths of tsetse flies in relation to host odour: a preliminary video study in nature of the response to loss of odour. Physiol Entomol 10:395-406.

Gibson G and Brady J (1988) Flight behaviour of tsetse flies in host odour plumes: the initial response to leaving or entering odour. Physiol Entomol 13: 29-42.

Gu W and Novak RJ (2009) Predicting the impact of insecticide-treated bed nets on malaria transmission: The devil is in the detail. Malaria J 8: 256.

Hargrove JW (2005) Extinction probabilities and times to extinction for populations of tsetse flies Glossina spp. (Diptera: Glossinidae) subjected to various control measures. Bull Entomol Res 95: 13-21.

Jawara M, Smallegange RC, Jeffries D, Nwakanma DC, Awolola TS, Knols BGJ, Takken W and Conway DJ (2009) Optimizing odor-baited trap methods for collecting mosquitoes during the malaria season in The Gambia. PLOS ONE 4: e8167.

Katz TM, Miller JH and Hebert AA (2008) Insect repellents: historical perspectives and new developments. J Am Acad Dermatol 58: 865-871.

Khan ZR, Midega CAO, Amudavi DM, Hassanali A and Pickett JA (2008) On-farm evaluation of the'push-pull'technology for the control of stemborers and striga weed on maize in western Kenya. Field Crops Res 106: 224-233.

Killeen GF, McKenzie FE, Foy BD, Bogh C and Beier JC (2001) The availability of potential hosts as a determinant of feeding behaviours and malaria transmission by African mosquito populations. Trans Roy Soc Trop Med Hygiene 95: 469-476.

Knols BG, Njiru BN, Mathenge EM, Mukabana WR, Beier JC and Killeen GF (2002) MalariaSphere: A greenhouse-enclosed simulation of a natural Anopheles gambiae (Diptera: Culicidae) ecosystem in western Kenya. Malar J 1: 19.

Knols BGJ and Louis C (2004) Bridging laboratory and field research for genetic control of disease vectors. In: Knols BGJ and Louis C (eds) Proceedings of the joint WHO/TDR, NIAID, IAEA and Frontis Workshop, Nairobi, Kenya 14-16 July 2004. Wageningen UR Frontis series Vol. 11. Springer Science + Business Media, Berlin, pp 225.

Lacroix R, Mukabana WR, Gouagna LC and Koella JC (2005) Malaria infection increases attractiveness of humans to mosquitoes. PLoS Biol 3: e298.

Lengeler C, Grabowsky M, McGuire D and Desavigny D (2007) Quick wins versus sustainability: options for the upscaling of insecticide-treated nets. Am J Trop Med Hygiene 77 Suppl S: 222-226.

Logan JG, Seal NJ, Cook JI, Stanczyk NM, Birkett MA, Clark SJ, Gezan SA, Wadhams LJ, Pickett JA and Mordue J (2009) Identification of human-derived volatile chemicals that interfere with attraction of the Scottish biting midge and their potential use as repellents. J Med Entomol 46: 208-219.

Lwetoijera DW, Sumaye RD, Madumla EP, Kavishe DR, Mnyone LL, Russell TL and Okumu FO (2010) An extra-domiciliary method for delivering entomopathogenic fungi, Metharizium anisopliae IP 46 against malaria vectors, Anopheles arabiensis. Parasites and Vectors (in press).

Mauck KE, De Moraes CM and Mescher MC (2010) Deceptive chemical signals induced by a plant virus attract insect vectors to inferior hosts. PNAS 107: 3600-3605.

Mumford J, Quinlan MM, Beech C, Alphey L, Bayard V, Capurro ML, Kittayapong P, Knight JD, Marrelli MT, Ombongi K, Ramsey JM and Reuben R (2009) MosqGuide: A project to develop best practice guidance for the deployment of innovative genetic vector control strategies for malaria and dengue. Asia-Pacific J Mol Biol Biotech 17: 93-95. 
Murlis J, Willis MA and Cardé RT (2000) Spatial and temporal structures of pheromone plumes in fields and forests. Physiol Entomol 25: 211-222.

Noor AM, Mutheu JJ, Tatem AJ, Hay SI and Snow RW (2009) Insecticide-treated net coverage in Africa: mapping progress in 2000-07. Lancet 373: 58-67.

Okumu FO, Killeen GF, Ogoma S, Biswaro L, Smallegange RC, Mbeyela E, Titus E, Munk C, Ngonyani H, Takken W, Mshinda H, Mukabana WR and Moore SJ (2010a) Development and field evaluation of a synthetic mosquito lure that is more attractive than humans. PLoS ONE 5: e8951.

Okumu FO, Madumla EP, John AN, Dickson W, Lwetoijera DW and Sumaye RD (2010b) Attracting, trapping and killing disease-transmitting mosquitoes using odor-baited stations -The Ifakara odor-baited stations. Parasites and Vectors 3: 12.

Pelletier J and Leal WS (2009) Genome analysis and expression patterns of odorant-binding proteins from the southern house mosquito Culex pipiens quinquefasciatus. PLoS One 4: e6237.

Qiu YT, Smallegange RC, Ter Braak CJF, Spitzen J, Van Loon JJA, Jawara M, Milligan P, Galimard AM, Van Beek TA, Knols BGJ and Takken W (2007) Attractiveness of MM-X traps baited with human or synthetic odor to mosquitoes (Diptera: Culicidae) in The Gambia. J Med Entomol 44: 970-983.

Schmied WH, Takken W, Killeen GF, Knols BG and Smallegange RC (2008) Evaluation of two counterflow traps for testing behaviour-mediating compounds for the malaria vector Anopheles gambiae s.s. under semi-field conditions in Tanzania. Malar J 7: 230.

Scott TW, Harrington LC, Knols BGJ and Takken W (2008) Applications of mosquito ecology for successful insect transgenesis-based disease prevention programs. In: Aksoy S (ed) Transgenesis and the management of vectorborne disease. Advances in experimental medicine and biology. Springer-Verlag, Berlin, Germany, pp 151-168.

Scott TW, Takken W, Knols BGJ and Boëte C (2002) The ecology of genetically modified mosquitoes. Science 298: 117119.

Sengul MS and Tu Z (2008) Characterization and expression of the odorant-binding protein 7 gene in Anopheles stephensi and comparative analysis among five mosquito species. Insect Mol Biol.

Smallegange RC, Qiu YT, Bukovinszkine-Kiss G, Van Loon JJA and Takken W (2009) The effect of aliphatic carboxylic acids on olfaction-based host-seeking of the malaria mosquito Anopheles gambiae sensu stricto.J Chem Ecol 35: 933-943.

Smallegange RC, Qiu YT, Van Loon JJ and Takken W (2005) Synergism between ammonia, lactic acid and carboxylic acids as kairomones in the host-seeking behaviour of the malaria mosquito Anopheles gambiae sensu stricto (Diptera: Culicidae). Chem Senses 30: 145-152.

Spitzen J, Smallegange RC and Takken W (2008) Effect of human odours and positioning of CO2 release point on trap catches of the malaria mosquito Anopheles gambiae sensu stricto in an olfactometer. Physiol Entomol 33: 116-122.

Syed $Z$ and Leal WS (2008) Mosquitoes smell and avoid the insect repellent DEET. PNAS 105: 13598-13603.

Syed Z and Leal WS (2009) Acute olfactory response of Culex mosquitoes to a human- and bird-derived attractant. PNAS 106: 18803-18808.

Takken W and Knols BGJ (1999) Odor-mediated behavior of afrotropical malaria mosquitoes. Ann Rev Entomol 44: 131-157.

Torr SJ, Hargrove JW and Vale GA (2005) Towards a rational policy for dealing with tsetse. Trends Parasitol 21: 537-541.

Touhara K and Vosshall LB (2009) Sensing odorants and pheromones with chemosensory receptors. Ann Rev Physiol 71: 307-332.

Vale GA (1993) Development of baits for tsetse flies (Diptera: Glossinidae) in Zimbabwe. J Med Entomol 30: 831-842.

Williams CR, Bergbauer R, Geier M, Kline DL, Bernier UR, Russell RC and Ritchie SA (2006) Laboratory and field assessment of some kairomone blends for host-seeking Aedes aegypti. J Am Mosq Control Assoc 22: 641-647.

Zhou JJ, He XL, Pickett JA and Field LM (2008) Identification of odorant-binding proteins of the yellow fever mosquito Aedes aegypti: genome annotation and comparative analyses. Insect Mol Biol 17: 147-163. 



\section{About the editors}

Willem Takken (1951) is Professor in Medical and Veterinary Entomology at Wageningen University, the Netherlands. He studied in Wageningen and obtained his PhD degree in 1980 based on research on the biology and feeding behaviour of tsetse flies. He worked in several African countries on the control of animal trypanosomiasis. Upon his return to Europe, he was appointed as lecturer at the Laboratory of Entomology in Wageningen, where he introduced Medical and Veterinary Entomology to the Wageningen academic society. His work involved mosquito-host interactions, in particular the host-seeking behaviour of malaria mosquitoes. He later expanded this work to include field research in Tanzania, Kenya and other tropical countries. Today, his work involves the

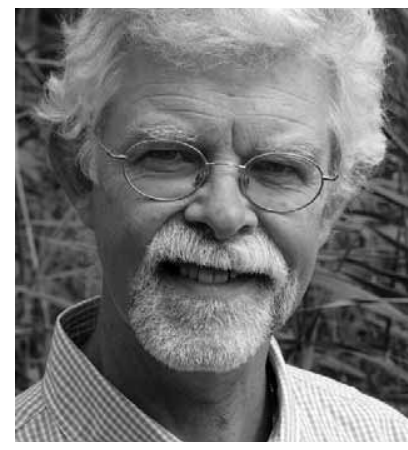
ecology of mosquitoes, biological control of mosquitoes and the impact of environmental change on malaria vectors. In Europe, Willem studies the ecology of Lyme disease vectors and vectors of other, emerging infectious diseases. He emphasises collaboration with other institutions, and has an extensive network of national and international collaborators. Willem is a member of the Council of the International Congress of Entomology and serves on several editorial boards and advisory committees. He has (co)authored more than 160 scientific publications and book chapters.

Bart G.J. Knols (1965) is Visiting Scientist at the Division of Infectious Diseases, Tropical Medicine \& AIDS of the Academic Medical Centre in Amsterdam, the Netherlands and holds an Honorary Professorship in medical entomology at the University of Witwatersrand, South Africa. He studied biology in Wageningen where he also obtained his PhD degree in Medical Entomology in 1996 based on studies on odour-mediated host seeking behaviour of the African malaria vector Anopheles gambiae. He has lived and worked in East and Southern Africa for eleven years. In Zambia he was involved in large-scale tsetse fly control campaigns using odour-baited targets. In Tanzania he studied the behavioural ecology of malaria mosquitoes for several years, before working

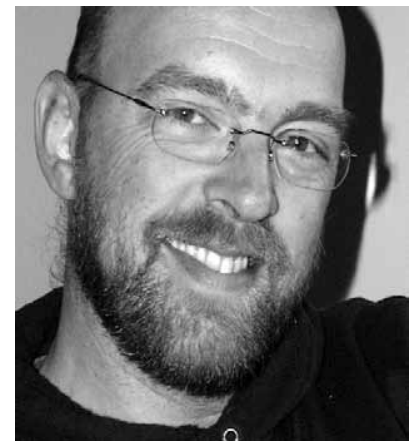
at the International Centre of Insect Physiology and Ecology (ICIPE) in Kenya from 1997-2002. There he led a research team of thirty students and scientists on the shores of Lake Victoria (at Mbita Point). Bart worked for the United Nations from 2003-2006 (IAEA in Vienna) to develop genetic control strategies for mosquitoes, and was Assistant Professor at Wageningen University until 2009. His career is marked by the discovery of Limburger cheese as a surrogate attractant for An. gambiae (Ig Nobel Prize 2006), besides his pioneering work in semi-field systems in Africa and the discovery of a fungal entomopathogen for adult malaria vector control. Bart obtained his MBA degree (Open University, UK) in 2006 and is a member of the Royal Dutch Academy of Arts and Sciences (KNAW-DJA). He has (co)authored more than 130 scientific publications and book chapters. He currently directs K\&S Consulting, a company engaged in the global dissemination of medical information (www.malariaworld.org), organisation building, and consultancies in infectious disease control.

Willem and Bart were the joint recipients of the Eijkman medal in 2007, which is the highest award in the field of tropical medicine and international health in the Netherlands. 



\title{
Contributors
}

\section{Sandra A. Allan (USA)}

Center for Medical, Agricultural and Veterinary Entomology, ARS/USDA, Gainesville, FL 32608, USA. Email: sandy.allan@ars.usda.gov

\section{Jonathan D. Bohbot (USA)}

Plant Sciences, USDA-ARS, Room 024, 10300 Baltimore avenue, Bldg 007 Barc-West, Beltsville, MD 20705, USA. Email: jonathan.bohbot@ars.usda.gov

\section{Daniel P. Bray (United Kingdom)}

Centre for Applied Entomology and Parasitology, Institute for Science and Technology in Medicine, Keele University, Keele, Staffordshire, ST5 5BG, UK. Email: d.p.bray@biol.keele.ac.uk

\section{Ring T. Cardé (USA)}

Department of Entomology, University of California, Riverside, CA 92521, USA. Email:

ring.carde@ucr.edu

\section{James I. Cook (United Kingdom)}

Rothamsted Research, Harpenden, Hertfordshire, AL5 2JQ, UK. Email: james-i.cook@bbsrc.ac.uk

\author{
Alvaro E. Eiras (Brazil) \\ Horizonte, MG, Brazil. Email: alvaro@icb.ufmg.br

\section{Martin Geier (Germany)} \\ Institut für Zoologie, Universität Regensburg, 93053 Regensburg, Germany. \\ Biogents AG, Bruderwöhrdstr 15b, 93053 Regensburg, Germany. Email: \\ martin.geier@biologie.uni-regensburg.de
}

Lab. Ecologia Química de Insetos Vetores, Depto. Parasitologia,Instituto de Ciências Biológicas. Univ. Federal de Minas Gerais, Av. Presidente Antônio Carlos 6627, Pampulha, P.O. Box 486, 31270-901 Belo

\section{Gabriella Gibson (United Kingdom)}

Natural Resources Institute, University of Greenwich at Medway, Chatham, Kent ME4 4TB, UK.

School of Life Sciences, University of Sussex, Falmer, Brighton, BN1 9 QG, UK. Email: g.gibson@gre.ac.uk

\section{Alan J. Grant (USA)}

Harvard School of Public Health, 665 Huntington Avenue, Boston, MA 02115, USA. Email: laaj@rcn.com

\section{Pablo G. Guerenstein (Argentina)}

Centro de Investigaciones Científicas y Transferencia de Tecnología a la Producción, CONICET, Matteri 49 (esq. España), 3105 Diamante, Entre Ríos, Argentina. Email: pabloguerenstein@cicyttp.org.ar

\section{James G.C. Hamilton (United Kingdom)}

Centre for Applied Entomology and Parasitology, Institute for Science and Technology in Medicine, Keele University, Keele, Staffordshire, ST5 5BG, UK. Email: j.g.c.hamilton@keele.ac.uk

\section{Bill S. Hansson (Germany)}

Max Planck Institute for Chemical Ecology, Department of Evolutionary Neuroethology, Hans-KnoellStrasse 8, D-07745 Jena, Germany. Email: hansson@ice.mpg.de 


\section{Sharon R. Hill (Sweden)}

SLU, Division of Chemical Ecology, Department of Plant Protection Biology, P.O. Box 102, Sundsvägen 14, 23053 Alnarp, Sweden. Email: sharon.hill@ltj.slu.se

\section{Hilary Hurd (United Kingdom)}

Centre for Applied Entomology and Parasitology, School of Life Sciences, Keele University, Keele, Staffordshire, ST5 5BG, UK. Email: h.hurd@keele.ac.uk

\section{Rickard Ignell (Sweden)}

SLU, Division of Chemical Ecology, Department of Plant Protection Biology, P.O. Box 102, Sundsvägen 14, 23053 Alnarp, Sweden. Email: rickard.ignell@vv.slu.se

\section{Owen Jones (United Kingdom)}

AgriSense BCS Ltd, Treforest Industrial Estate Pontypridd, South Wales CF37 5SU, UK. Email: owenj@agrisense.demon.co.uk

\section{Dan L. Kline (USA)}

USDA/ARS, Center for Medical, Agricultural and Veterinary Entomology, P.O. Box 14565, 1600/1700 SW 23rd Dr., Gainesville, FL 32608, USA. Email: dan.kline@ars.usda.gov

\section{Bart G.J. Knols (the Netherlands)}

Division of Infectious Diseases, Tropical Medicine \& AIDS, Academic Medical Centre, Amsterdam, the Netherlands.

K\&S Consulting, Kalkestraat 20, 6669 CP Dodewaard, the Netherlands. Email: bart@malariaworld.org

\section{Claudio R. Lazzari (France)}

Institut de Recherche sur la Biologie de l'Insecte, UMR 6035 CNRS, Université François Rabelais, Institut de Recherche sur la Biologie de I'Insecte, UMR 6035 CNRS, Université François Rabelais, Av. Monge, Parc Grandmont, 37200 Tours, France. Email: claudio.lazzari@univ-tours.fr

\section{James G. Logan (United Kingdom)}

Rothamsted Research, Harpenden, Hertfordshire, AL5 2JQ, UK. Email: james.logan@bbsrc.ac.uk

\section{Tan Lu (USA)}

Vanderbilt University/Vanderbilt University Medical Center, Institutes for Chemical Biology \& Global Health, Center for Molecular Neuroscience, VU Station B 351634, Nashville, TN 37235-3582, USA. Email: tan.lu@vanderbilt.edu

\section{A. Jennifer Mordue (Luntz) (United Kingdom)}

Aberdeen University, School of Biological Sciences, Zoology Building, Tillydrone Avenue, Aberdeen AB24 2TZ, UK. Email: a.j.mordue@abdn.ac.uk

\section{Wolfgang R. Mukabana (Kenya)}

School of Biological Sciences, University of Nairobi, P.O. Box 30197, 00100 GPO, Nairobi, Kenya and International Centre of Insect Physiology and Ecology, P.O. Box 30772, 00100, GPO, Nairobi, Kenya. Email: rmukabana@yahoo.co.uk 
Robert J. O'Connell (USA)

Brudnick Neuropsychiatric Research Institute, University of Massachusetts Medical School, 303 Belmont Street, Worcester, MA 01604, USA. Email: robert.oconnell@umassmed.edu

\section{Evelyn A. Olanga (Kenya)}

International Centre of Insect Physiology and Ecology, P.O. Box 30772, 00100, GPO, Nairobi, Kenya and Department of Zoological Sciences, Kenyatta University, P.O. Box 43844, 00100 GPO, Nairobi, Kenya. Email: eveolanga@gmail.com

\section{Yu Tong Qiu (the Netherlands)}

Laboratory of Entomology, Wageningen University and Research Centre, PO Box 8031, $6700 \mathrm{EH}$ Wageningen, the Netherlands. Email: yu.tongqiu@wur.nl

\section{Andreas Rose (Germany)}

Institut für Zoologie, Universität Regensburg, 93053 Regensburg, Germany.

Biogents AG, Bruderwöhrdstr 15b, 93053 Regensburg, Germany. Email: andreas.rose@biogents.com

\section{Steve Schofield (Canada)}

Force Health Protection, Communicable Disease Control Program, CF H Svcs Gp HQ Ottawa, Department of National Defence, 1745 Alta Vista Drive, Ottawa, Ontario K1A OK6, Canada. Email:

schofield.sw@forces.gc.ca

\section{Meryem S. Sengul (Sweden)}

SLU, Division of Chemical Ecology, Department of Plant Protection Biology, P.O. Box 102, Sundsvägen 14, 23053 Alnarp, Sweden. Email: msengul@vt.edu

\section{Renate C. Smallegange (the Netherlands)}

Laboratory of Entomology, Wageningen University and Research Centre, P.O. Box 8031, $6700 \mathrm{EH}$ Wageningen, the Netherlands. Email: renate.smallegange@wur.nl

\section{Philippe Solano (Burkina Faso)}

Institut de Recherche pour le Développement (IRD), UMR 177 IRD-CIRAD/ Centre International de Recherche-Développement sur I'Elevage en zone Subhumide(CIRDES), Bobo Dioulasso, Burkina Faso. Email: Philippe.Solano@ird.bf

\section{James F. Sutcliffe (Canada)}

Department of Biology, Trent University, 1600 West Bank Drive, Peterborough, Ontario, K9J 2B6, Canada. Email: jsutcliffe@trentu.ca

\section{Willem Takken (the Netherlands)}

Laboratory of Entomology, Wageningen University and Research Centre, P.O. Box 8031, 6700 EH Wageningen, the Netherlands. Email: willem.takken@wur.nl

\section{Steve J.Torr (United Kingdom)}

Natural Resources Institute (NRI), University of Greenwich, Natural Resources Institute, University of Greenwich at Medway, Central Avenue, Chatham Maritime, Chatham, Kent ME4, 4TB, UK. Email: s.torr@greenwich.ac.uk 
Joop J.A. van Loon (the Netherlands)

Laboratory of Entomology, Wageningen University and Research Centre, PO Box 8031, $6700 \mathrm{EH}$ Wageningen, the Netherlands. Email: joop.vanloon@wur.nl

\section{Richard D. Ward (United Kingdom)}

Centre for Applied Entomology and Parasitology, Institute for Science and Technology in Medicine, Keele University, Keele, Staffordshire, ST5 5BG, UK. Email: bia40@keele.ac.uk

\section{Laurence J. Zwiebel (USA)}

Vanderbilt University/Vanderbilt University Medical Center, Institutes for Chemical Biology \& Global Health, Center for Molecular Neuroscience, VU Station B 351634, Nashville, TN 37235-3582, USA. Email: I.zwiebel@vanderbilt.edu 


\section{Reviewers}

\section{Prof. J.R. Carlson (USA)}

Molecular, Cellular, and Developmental Biology, KBT 1132, Yale University, P.O. Box 208103, New Haven, CT 06520-8103, USA

\section{Dr. L. Cruz López (Mexico)}

Departamento de Entomología, El Colegio de la Frontera Sur Unidad Tapachula, Tapachula, Mexico

\section{Dr. H.M. Ferguson (United Kingdom)}

Division of Immunity and Infection \& Division of Environmental and Evolutionary Biology, Glasgow Biomedical Research Centre, University of Glasgow, 120 University Place, Glasgow G12 8TA, UK

Prof. B.S. Hansson (Germany)

Max Planck Institute for Chemical Ecology, Hans-Knöll-Straße 8, D-07745 Jena, Germany

\section{Dr. J.W. Hargrove (South Africa)}

SACEMA Director, DST/NRF Centre of Excellence in Epidemiological Modelling and Analysis, c/o STIAS, 19 Jonkershoekweg, Stellenbosch 7600, South Africa

Prof. J.G. Hildebrand (USA)

ARL Division of Neurobiology, The University of Arizona, PO Box 210077, Tucson, AZ 85721-0077, USA

\section{Dr. B.G.J. Knols (the Netherlands)}

Division of Infectious Diseases, Tropical Medicine \& AIDS, Academic Medical Centre, Amsterdam, the Netherlands

\section{Prof. M.J. Klowden (USA)}

University of Idaho Campus, Ag Science 233, Moscow, ID 83844-2339, USA

\section{Prof. B.A. Mullens (USA)}

Department of Entomology, University of California, Riverside, Ca 92506, USA

\section{Prof. D.E. Sonenshine (USA)}

Old Dominion University, Department of Biological Sciences, Norfolk, VA 23529 -0266, USA

\section{Prof. Dr. W. Takken (the Netherlands)}

Laboratory of Entomology, Wageningen University and Research Centre, PO Box 8031, 6700 EH Wageningen, the Netherlands

\section{Prof. S.J. Torr (United Kingdom)}

Natural Resources Institute, University of Greenwich at Medway, Central Avenue, Chatham Maritime, Chatham, Kent ME4, 4TB, UK

\section{Dr. J.J.A. van Loon (the Netherlands)}

Laboratory of Entomology, Wageningen University and Research Centre, PO Box 8031, $6700 \mathrm{EH}$ Wageningen, the Netherlands 



\section{Keyword index}

\section{A}

AAA pheromone - See: attractionaggregation-attachment pheromone

AaOR7 27

ABC PRO suction trap 233

acaricide 341

acaricide tags 341

acetic acid 48, 52, 151, 153, 158, 161, 163, $165,333,336$

- ethyl lactate 48

acetone $17,51,143,154,155,160,161,167$, $169,192,195,223,228,230,236,252$, $253,254,265,270,271,272,274,276$, $277,278,282,293,295,297,298,301,335$ acetophenone 26,28 acetothiophene 52 acetylphenone 52 acetylpyridine 52 acetylthiazole 52 across-fibre patterning 54 action potential 96,97 activation $115,118,119,121,250,258,268$, $271,294,295,301,309,315,328$ activator $146,194,205,320,402$ active

- orientation 250

- phase 122

- space 132

activity rhythm 115 adenosine triphosphate 66 adenotrophic viviparity 265 adenyl cyclase 19 adenylyl cyclase 66,68 Aedes 25, 32, 39, 44, 127, 130, 132, 193, 238 - aegypti 17, 18, 21, 22, 23, 24, 25, 27, 28, $29,30,31,39,41,44,47,49,52,53,54,63$, $65,67,69,70,72,73,74,75,91,92,93,94$, $97,99,100,101,102,105,118,120,121$, $122,124,126,127,128,130,133,136$, $143,144,145,146,147,148,150,151$, $152,153,154,155,156,157,165,167$, $168,169,228,231,351,352,354,356$, $358,365,366,367,368,369,370,371$, $372,373,374,375,376,377,378,379$, $380,382,384,385,386,388,390,391$, $399,400,401,402$
- aegyptigenome 68

- albopictus 156, 366, 370, 371, 372, 373, 376, 383

- caspius 189

- dalzieli 189

- hirsutus 189

- polynesiensis 355

- taeniorhynchus 94

- triseriatus 47, 371

Aethia cristatella 156, 337

Africa 248, 266, 279, 281, 283, 301, 365, 403

African horse sickness 220

age 53

agent-based model 404

AgGR 28, 29, 53

aggregation 69,224

aggregation pheromone $67,312,319$

AgOR 28, 29, 30, 32

AgOR7 26

AgOR8 28, 29, 53, 55

Agrotis segetum 70

AHSV 234, 238

aim-then-shoot 125

air entrainment 228

air pollutant 238

Akabane virus 220

alarm pheromone 316

alcohol 51,53, 163, 167, 191

aldehyde $46,163,167,168$

- aromatic 330

- C8 293

aliphatic acid 27

n-aliphatic aldehyde 318

aliphatic carboxylic acid 26, 47, 50, 143, 157, $158,163,168,169,195$

n-aliphatic carboxylic acid 317

allergic reaction 248

allo-aroma-dendr-9-ene 208

allomone $147,165,166,272,273,328,338$

alpha-isomethylionone 230, 232

Amazon basin 311

Amblyomma

- americanum 328, 330, 334, 335, 336, $337,340,342$

- cajennense 339

- cohaerens 333

- hebraeum 328, 332, 333, 334, 335, 336, $338,339,341$ 


$$
\begin{aligned}
& \text { - maculatum 330,339, } 341 \\
& \text { - variegatum 327, 328, 330, 331, 332, 333, }
\end{aligned}
$$
ammonium bicarbonate 237 amyl acetate 318 anaphylactic shock 248 anaplasmosis 327 anautogenous 104, 116 anemotactic response 280 anemotaxis $116,123,126,232$ Angola 183, 185, 186, 274 angular velocity 335 animal extract 223

Anopheles 25, 32, 39, 127, 130, 181, 197

- arabiensis 122, 128, 132, 133, 181, 182, 183, 184, 187, 188, 189, 191, 192, 193, 194,195

- aruni 182

- brucei 182

- bwambae 181, 182

- confusus 182

- coustani 184, 187, 189

- funestus 129, 181, 182, 185, 187, 188, $189,190,193,194,355$

- fuscivenosus 182

- gambiae 17, 18, 20, 21, 22, 23, 24, 25, 26, $27,28,29,30,31,39,41,44,45,47,48,49$, $51,52,53,54,63,67,69,70,74,94,106$, $107,118,120,121,122,126,128,129$, $145,146,147,148,150,157,161,165$, $167,168,181,182,183,184,188,189$, 190, 191, 192, 193, 194, 195, 260, 318, $352,355,358,399,400,401,402$

- gambiae genome 68

- gambiae sensu stricto 120, 122, 128, 129, $133,136,143,144,158,159,181,187$, 195,211

- leesoni 182

- maculipennis 41,44

- melas 181, 182, 183

- merus 181, 182, 184

- moucheti 185

- nili 185, 187, 190
- parensis 182

- pharoensis 186, 187, 188, 189, 193, 194

- punctulatus 352

- quadriannulatus 51, 128, 129, 130, 132, 162, 182, 184, 187, 189, 192, 194

- quadriannulatus species A 181

- quadriannulatus species B 181

- rivulorum 182

- rufipes 189

- sergenti 187, 189, 194

- squamosus 187, 189

- stephensi 41, 43, 50, 93, 94, 118, 356

- vaneedeni 182

- ziemanni 44, 187, 194

Antarctica 219

antenna $21,23,24,27,30,31,39,40,41,42$, $44,45,64,65,67,74,92,93,94,96,125$, $126,146,206,229,230,312$

antennal lobe $26,27,29,30,31,45,63,74$, 79,80

antennal pedicelus 314

Antheraea polyphemus 21, 23, 65

anthropophagic 133, 206

anthropophilic 43,122, 128, 132, 133, 144, $145,151,182,183,185,190,191,250$

- behaviour 402

anticoagulant 353

anti-coagulation factor 358

anti-platelet compound 353

anti-platelet factor 358

Apis mellifera $20,76,77,78$

apocrine sweat 255

apyrase $349,358,359$

Arachnidae 92

arbovirus 220

Argas

- persicus 333, 334, 341

- walkerae 333, 335

Argasidae 327

Argentina 311,368

armadillo 310

arrestant 146

arrestin 20

arrestment 115

- (assembly) pheromone 338

- pheromone 332, 333, 334, 339

asexual parasitaemia 351

Asia 365 
assembly pheromone 339,340

AtrAedes lure $374,375,378$

attractant $135,143,144,145,146,150,151$, $156,168,181,182,187,193,194,195$, $196,197,203,204,205,239,252,320,402$ attraction $132,133,135,136,144,166,189$, 205, 206, 207, 296, 309, 328, 355

attraction-aggregation-attachment (AAA) pheromone $328,333,334,338,339,340$, $341,342,343$

- component 331

attractiveness $187,188,208,210$

audio signals 209, 210

Australia 221, 222, 226, 235, 310, 327, 376, $378,383,386$

autogenous 104, 116, 248

autogeny 218

Autospike 95

axillary 331

axillary odour 47,53

axon 42, 45, 80, 92

Axonopus compressus 371

8-azaguanine 339

\section{B}

babesiosis 327,336

Bacillus thuringiensis var. israelensis 384

back pack aspirator 382

bait $131,144,147,167,182,187,189,190$, 191, 192, 193, 194, 195, 196, 197, 205

baited trap 320

baker yeast 321

bancroftian filariasis 144

$\beta$-barbatene 208

basic reproductive number 358

Bayrepel 235

Beauveria bassiana 341

behaviour 20, 30, 53, 65, 68, 73, 74, 80, 91, $94,99,106,115,117,118,120,122,123$, $125,129,136,182,184,203,204,205$, 207, 209

- defensive 304

- foraging 53

- manipulation 349

behavioural

- package 258

- resistance 403

- response $54,55,79$
- response, defensive 353

Belgium 220

Benin 183, 274

benzaldehyde $163,164,165,230,232,331$, 332,333

benzene 46,155

benzeneethanol 160,162

benzoic acid 154, 164, 333, 337

benzothiazole 160,161, 372

benzyl alcohol 70, 72, 163

beta trap 300

BG

- Lure trap 380, 381, 383

- Mesh lure trap 237

- Sentinel trap 367, 381, 382, 383, 391

biased random walk 270

bioassay $122,127,130,135,136,143,145$, $148,165,167,168,205,206,208,231$, $272,273,338,374$

biting

- inhibitor 255

- midge $91,94,95,96,97,98,101,102$, $124,144,402$

- periodicity 369

- site, preferential 223

- site selection 251

blend $48,55,128,135,143,150,155,157$, $165,167,169,182,187,191,193,195$, $206,207,231,270,276,316,337,400,402$

- natural and synthetic 13

- volatile 55

blood feeding $94,99,100,103,105,109$, $118,119,124,191,211,239,247,248$, 291, 293, 294, 327, 350

- parasite-induced changes in behaviour 351

- persistence 357

blood meal 53, 68, 91, 92, 99, 104, 115, 133, $145,146,150,205,206,236,259,273$, $276,296,311,319,358$

bluetongue virus (BTV) 13, 218, 220, 228, 234, 238, 239

body odour 30,188, 236

Bolivia 311

Bombyx mori 20, 80

Borrelia burgdorferi 353

Botswana 183, 185

brain $26,45,55,74,76,78,79,92,96$ 
Brazil 204, 205, 311, 365, 366, 368, 375, 376, $377,378,382,384,386,388,390,391$, 392,402

breath $46,47,122,135,151,155,156,161$, $187,188,189,205,235,253,333,379$

- exhaled 351

Breteau Index 366, 368

Brugia malayi 350

BTV - See: bluetongue virus

buffalo 268, 272, 273

Burkina Faso 183, 185, 186, 188, 189, 266, 275, 277, 280, 282

Burundi 183, 185, 186

bushbuck 275, 276

- urine 276

bushpig 272

1,3-butanediamine 155

2,3-butanediol 164, 165

2,3-butanedione 160, 162

butanoic acid 70, 72, 151, 153, 158, 160, $163,295,296,333,336$

1-butanol 160, 162

butanone 154, 156, 223, 265

2-butoxyethanol 48, 50,70,72

1-butylamine 52

2-butylamine 313

butylamine 26, 27, 51, 52, 313

butyric acid 313

8-butyrolactone 331

\section{C}

cabbage root fly 125

calf 188,189

Cameroon 183, 185, 186, 250, 260, 266

Canada 247, 248

canine skin 336

Cape Verde 183

capitate peg 27

- sensilla 29, 44, 48, 52, 53, 54

caproic acid 379, 381

6-caprolactone 331, 332

carbohydrate 46

carbon dioxide $\left(\mathrm{CO}_{2}\right) \quad 14,17,27,28,29,30$, $46,52,53,73,91,92,93,94,95,96,97,98$, $99,100,101,103,105,106,107,108,109$, $115,117,118,120,122,124,127,128$, $130,131,132,133,134,135,136,143$, $144,151,157,158,161,162,163,169$,
$181,187,188,189,190,191,192,193$, $194,195,204,205,206,207,223,228$, $229,230,231,233,234,236,237,238$, $239,247,250,251,252,253,254,255$, $256,257,258,259,260,265,270,271$, $272,273,276,277,281,282,291,293$, 294, 295, 296, 297, 298, 299, 301, 309, $315,316,317,318,319,320,321,327$, $328,330,335,336,339,340,341,343$, $367,370,379,380,381,382,383,399$, $401,402,403$

- plume 135

- receptors 54

carbon disulfide $154,156,164$

carboxylic acid $43,51,52,55,72,151,157$, $167,191,193,194,195,293,294,379,404$

- aromatic 154, 164

Caribbean 222

(E)- $\beta$-caryophyllene 208

cation channel 69

cattle 187, 191, 192, 195, 268, 273, 277

- faeces 293

- odour 277, 278, 293

- urine 301

CDC

- backpack aspirator 369,376

- gravid trap 370, 376

- light trap 14,382, 383

cembrene 203, 207

Central African Republic 183, 185, 275, 276

Central America 247

central nervous system (CNS) 71, 74, 97, 98, 106,229

Ceratopogonidae 101, 217

Cercopithecus aethiops 190

Chad 183, 185, 186

Chagas disease $14,309,310,311,321,350$

Chaitophorus stevensis 238

Chelydra serpentina 248

chemical

- cue 22,64, 143, 204

- ecology 55

- signal 18, 209, 211

- stimulus 92,99

chemokinetic responses 232

chemoreception 18, 30, 67

chemoreceptor 94

- neuron 92 
chemosensory

- information 74

- protein 21

- sensilla 23, 25, 27

- system 109

chemotactic response 232

chemotaxis 232

chicken 162, 166, 311, 314

Chikungunya 13, 17, 144, 402

Chile 311

China 221, 310

cholesterol 155, 163

cholinergic connection 77

cibarial pump 354

circadian

- clock 115, 309, 311, 319

- rhythm 117, 118, 148, 150, 223, 355

cis-vaccenyl acetate 23

citral 70,72

citronellol 48, 51, 293

climate change 218, 237

climatic condition 226

cluster analysis 388

Cnephia pecuarum 254

CNS - See: central nervous system

$\mathrm{CO}_{2}$ - See: carbon dioxide

cockroach 74, 76, 78, 319

coding pathway 78

Cola laurifolia 274

Colophospermum mopane 268

Columbia 369

common crane 259

community-based

- dengue control 392

- intervention 278

Congo 183, 185

- -Brazzaville 275, 277

- Democratic Republic of 183, 185, 186, 275,280

contact pheromone 204

Container Index 368

continuous feeding model 360

controlled-release device 373

convection 136

- current 117, 127, 134

- heat 42

convention

- current 126 copulation 209, 210, 225

co-receptor 24, 52

Côte d'Ivoire 183, 185, 186, 275, 280

counterflow geometry 234

coupled gas chromatographyelectroantennography 231

cow 21, 51, 190, 191

- odour 254

- urine 254, 255

crepuscular 223

m-cresol 293

p-cresol 47, 293, 295, 296, 372

crested auklet 337

Crimean Congo Haemorrhagic fever 327

crosstalk 76

Cuba 369

Culex 39, 44, 47, 122, 127, 132, 193, 194

- annulirostris 223

- antennatus 189

- duttoni 189

- molestus 104, 105

- nebulosus 189

- nigripalpus 165, 371

- pipiens 41, 54, 92, 93, 100, 103, 104, 105, 136, 371

- pipiens quinquefasciatus 17, 399

- quinquefasciatus 25, 27, 28, 30,31, 50, $55,63,69,70,72,73,74,93,94,107,118$, $131,143,144,146,147,150,162,163$, $165,166,168,169,192,211,352,355$, $369,371,372,373,400$

- tarsalis 131, 165, 372

- territans 41

Culicidae 366

Culicoides 102, 103, 217

- albicans 223

- barbosai 222

- bolitinos 225

- brevitarsis 218, 223, 224, 226

- chiopterus 220,225

- darlingtonae 230

- delta 223

- dewulfi 220, 225

- flavivenula 230

- floridensis 222

- furens 102, 222, 223, 231, 233, 234, 236

- glabellus 230

- heliconiae 230 
- henryi 221

- histrio 224

- hollensis 222, 223

- imicola 218, 219, 220, 226

- immaculatus 222

- impunctatus 218, 219, 220, 221, 223, $224,227,228,230,231,232,235,236$, 238, 239

- insinuates 230

- longior 221

- lupicaris 223

- marmoratus 221

- melleus 222, 223, 224

- mississippiensis 102, 103, 222, 234, 236

- molestus 221, 224

- nubeculosus 224, 225, 226, 230, 235, 238, 239

- obsoletus 218, 220, 225

- ornatus 221

- paraensis 230

- pseudodiabolicus 230

- pulicaris 220, 223, 225

- punctatus 225

- scoticus 220, 225

- sonorensis 239

- stellifer 102

- subimmaculatus 221,222, 224

- tissoti 222

- variipennis 226, 236

- variipennis sonorensis 227, 238

- vexans 223

Culiseta

- inornata 25, 26

- melanura 94

cuticular hydrocarbons 210

cyclic adenosine monophosphate 19,66 cyclic feeding model 360

cysteine 22

\section{D}

decanal 72, 152, 156, 230, 232, 372

(E)-2-decanal 165

decanoic acid 151, 153, 159, 161, 163

decanol 163

2-decanone 154

deer 223

DEET - See: N,N-diethyl-meta-toluamide dehydrolinalool 235 dendrite $18,24,31,32,39,42,43,44,64,69$

dengue $13,17,39,144,291,365,367,376$, $384,385,388,390,391,402,404$

- control 377,378

- fever 350, 368

- haemorrhagic fever 365

- risk 366

- transmission risk 368

- virus 366

Dermacentor variabilis $329,330,331,334$, $335,336,337$

desiccation 237

deterrent 15

diapause 54, 103

dichloromethane 155, 156, 167, 338

2,6-dichlorophenol 339, 341

Dictyoptera 74

differential attractiveness 227

dihydromyrcenol 230,232

dimethyl

- amine 313,318

- disulfide 143, 154, 156, 164, 167, 169

- sulfide 331

- trisulfide 155, 156, 164, 165, 294, 295, 296

diones 167

Dipetalogaster maxima 314

Diptera 74, 92

Dirofilaria

- immitis 350

- ursi 249

disability-adjusted life years 203

disease

- transmission 13, 14, 98, 143, 204

- vector $13,23,56,63,69,70,80,110,169$

dispersion 133

diurnal 120,150

Djibouti 183

2,3-docosanediol 164, 165

dodecanal 165

dodecanoic acid 151, 153, 159, 161, 163

n-dodecanoic acid 204

1-dodecanol 47, 158

doe urine 337

$\operatorname{dog} 351$

- ears 336

DOR67d 21

dose 
- -effect 252

- -response curve 188, 252, 295

- -response relationship 294

Drosophila 18, 20, 21, 22, 23, 24, 25, 26, 29, $30,31,45,53,106,117,121,130,146$, 210, 211

- melanogaster $17,44,45,67,68,71,76$, $77,78,79,80,106,211,238,400$

- melanogastor OR83b 69

dry ice $251,321,370,379$

dual-choice

- assay 187

- bioassay 372

- olfactometer 232

- test 189

dual-port olfactometer - See: olfactometer, dual-port

dung

- odour 294

- volatile 295, 296

\section{E}

E-2-heptenal 331

EAG - See: electroantennogram

East Coast fever 341

ectoparasite 327

ectopic expression 71

Egypt 186, 189

Ehrlichia (Cowdria) ruminantium 340

electric net $132,189,268,269,271,276$, 277,401

electroantennogram (EAG) 165, 167, 229

electroantennography $146,228,270$

electrophysiological 206

- response 23, 30

- study 26, 48, 54, 55, 95, 251, 293, 294

ELISA 259

emanation 162

encephalitis 17

endogenous

- activity 293

- physiological factor 318

- rhythm 269

endophagic 186

endophilic 182

epizootic haemorrhagic disease 220, 221

Equatorial Guinea 183, 185

equine enchephalosis 221 erhlichiosis 327,336

Eritrea 183, 185

ethanol 162

Ethiopia 183, 184, 185, 186, 191, 301

ethylamine 313,318

ethyl butyrate $48,70,72,156$

ethyl disulfide 154, 156, 164

ethylene glycol 163

2-ethylhexanoic acid 333, 337

2-ethylhexanol 230, 232

ethyl lactate 48

ethyl-L-lactate $48,70,72$

ethyl mercaptan 331

4-ethylphenol 50, 158, 224

ethyl propanoate 48

ethyl propionate 48

Eucalyptus citriodora 235

eugenol 48, 51, 70, 72

Europe 247

evolution 17, 24, 30

EVS trap 370, 382

excitation 39

exophagic 186, 224, 225

exophilic 184, 186, 224

extrinsic incubation period 227

$\mathbf{F}$

F3 trap 300

faeces $165,167,319$

fatty acid 17, 48, 130, 228, 379

- derivative 46

Fay-Prince trap $367,370,382$

feather 336

- odour 314

feeding

- aggregation 338

- persistence 349,356, 361

- site preference 257, 338

filament $124,128,151$

filiariasis 17

Flaviviridae 365

flea 359

flight

- path 119, 122, 132

- periodicity 294

- simulator 130

floating larval trap 369

fluoro-lactate 48 
fly 22

foot odour 190, 191, 193

fox 206

France 220, 225

French Polynesia 376

fruit fly 319

functional

- genomics 63

- group 45

- specificity 20

- type 49,55

furan 314,318

furfural 331

Fusca group 266, 267, 274, 279

\section{G}

GABA receptor antagonists 77

Gabon 183, 185, 186, 383

GAL4/UAS expression system 67

Gambia 183, 185, 186

game elimination 266

gametocytes 351,352

gas chromatography $145,228,270,312$

- -mass spectometry analysis 46,47

- -mass spectrometry 228

- -SSR 73

gene $17,20,21,23,24,29,31,39,44,45$, 55,67

- sequence 146

- silencing 358,400

genetically modified vector 400

genetics 14

genetic variation 280

genome $17,22,24,45,136$

- sequencing 63,80

genomic studies 64

geographic information system (GIS) 367, 384,390

- map 386, 391

- technology 239

geranyl acetone $47,50,143,154,156,160$,

$161,169,228,230,232,235$

Germany 220

Ghana 183, 185, 186

GIS: - See geographic information system

glandular secretion 337, 338

global warming 105 glomeruli $27,29,31,74,75,76,77,78,79$, 80

Glossina 303

- austeni 268, 273, 279, 280

- brevipalpis 274, 280

- fuscipes 265, 275, 276, 280

- fuscipes fuscipes 277, 280, 282

- longipalpis 273

- longipennis 274, 279, 280

- medicorum 274

- morsitans centralis 267,273

- morsitans morsitans 265, 267, 268, 273, 278, 280, 296

- morsitans submorsitans 267, 279, 282

- pallidipes 252, 253, 265, 267, 268, 273, $274,278,279,280,282,352,355$

- palpalis 265, 275, 281

- palpalis gambiensis 275, 277, 280

- palpalis palpalis 274, 277, 280

- swynnertoni 268, 273

- tachinoides 274, 275, 277, 280, 282

Glossinidae 292

glycerol 152,163

goat $187,191,272,273$

gonotrophic cycle $53,115,119,136,248$, 318

Gp - See: G-protein

GPCR - See: G-protein coupled receptor

G-protein (Gp) 19, 20, 25, 66

- coupled receptor (GPCR) 23, 45, 68

GR - See: gustatory receptor gene

grape berry moth 238

grass infusion $47,370,371,372,373,375$, 376

green leaf volatiles 204

GRN - See: gustatory receptor neuron

grooming 273

- behaviour 224

grooved peg 41, 44, 64, 309, 312, 313

- sensilla 42, 48, 49, 51, 52, 53, 54, 65

Grus grus 259

Guadeloupe 340, 341

guanine 333,339

Guinea 183, 185

Guinea Bissau 183

guinea pig 295, 311

gustatory

- neuron 44 
- organ 26,64

- receptor cell 332

- receptor gene (GR) 146

- receptor neuron (GRN) 27

- sensilla 251

\section{H}

Haemaphysalis leachi 338

haematin 333, 339

Haematobia irritans 293, 302

Haematobosca 301

haematophagy 291

haemostasis 353

haemostatic defence 358

Haller's organ 329, 330, 331, 332, 335

hamster 205, 206, 351

hard tick 327,328

Hawaii 219

hay infusion $370,371,376$

HBC - See: human biting catch

headspace extract 312

heartwater 327,336

heat $46,91,109,115,127,134,136,150$, $234,317,320,335,340$

heat/thermal stimuli 257

Heliothis virescens 20, 23

heptadecanal 165

heptadecane 333

n-heptadecane 224, 226

heptadecanoic acid 151, 154, 164

heptanal 72, 152, 163, 314, 331

heptane 155

heptanoic acid $70,72,151,153,159,160$, $163,332,333$

1-hepten-3-ol 50,152,156

heterodimer 24, 45, 66

heterogeneous odour plumes 381

heterologous

- expression 29,71

- platform 55

- system 21

heteromeric complex 20

(E, Z)-6,11-hexadecadienyl acetate 65

hexadecanal 165

hexadecanoic acid 151, 153, 159, 161, 164, 165

hexanal 152, 156, 163, 164, 165, 204, 331, $333,337,338$ hexane 165

hexanoic acid $68,153,155,156,158,163$, $169,190,332,333,336,337,379$

1-hexen-3-ol 50

4-hexen-1-ol 152, 156

high-throughput 32,165

Hippopotamus 276

homeostasis 45

homogeneous 129, 130,132, 151

homolog 24,68

honeybee 22

host

- cue 115, 127, 128, 132, 136, 143, 206

- defensive behaviour 257

- finding behaviour 133

- lethargy 353

- location 117, 129, 134, 135, 217

- location behaviour 239

- manipulation by parasites 359

- odour 14,117, 122, 127, 131, 134

- orientation 136

- -parasite relationships 299

- preference 43, 128, 146, 249, 259

- selection 272

- stimuli 119,133

host-seeking 20,26, 99, 100,101, 103, 125

- behaviour 69, 91, 92, 98, 100, 103, 105, $106,109,143,145,147,148,150,157$, $158,162,163,165,166,167,168,169$, $181,182,187,188,195,197,204,247$

- behaviour, indoor 401

- strategy 259

house-entering 132, 133, 135

house improvement 311

hovering 134

human

- biting catch (HBC) 403

- body odour 283

- breath 293, 294, 331, 335

- effluvia 314

- emanation 145

- feet 47

- landing catch 191, 192, 196

- landing collection 369, 382

- odour 255

- skin 46, 151, 156, 157, 162, 379

- skin emanation 26

- skin extract 379 
- skin odour 380,381

- sweat 250,313, 317

- sweat odour 316

- urine odour 314

humidity 134,136

hunter strategy 328

Hyalomma dromedarii 328,342

hydrogen sulfide 330,333

Hydrotaea irritans 228

3-hydroxy-2-butanone 160, 162

2-hydroxybenzaldehyde 331

3-hydroxybenzaldehyde 331

hydroxybenzaldehyde 332

2-hydroxybenzoic acid 154, 164

2-hydroxypentanoic acid 160

hygroreceptor 44

- cell 313

Hymenoptera 74,92

hypoxanthine 333,339

I

iguanas 311

Ilex vomitoria 236

immunomodulator 353

impala 272, 273

increased

- attractiveness of pregnant women 352

- biting behaviour 349

India 310

Indian Ocean 187

$1 \mathrm{H}$-indole 155

indole $30,47,49,50,52,53,55,70,72,160$, $161,336,372$

Indonesia 310

infectious diseases 17,39

infochemical 48

infrared radiation 314

inhibition 39

inosine 333, 339

insecticide-treated

- bed net 405

- cattle 278, 304

- target 265

in situ hybridisation 52,67

interception trap 252

interdigital gland substance 337

Intergovernmental Panel on Climate Change (IPCC) 237 interneuron 63

intrinsic attractiveness 352

intron 22

invitation effect 255

ionotropic 69

- response 20

IPCC - See: Intergovernmental Panel on

Climate Change

IR3535 404

isobutanoic acid 333

isobutylamine 313,318

isobutyric acid $313,316,317,318$

isopentanoic acid 336

isopentylamine 313

isoprene 335

isovaleric acid 313

Israel 186

Italy 376,383

Ivory Coast 278

Ixodes

- angustus 327

- canisuga 336

- neitzi 337

- persulcatus 336

- ricinus 328, 331, 332, 336, 337, 339, 340, 342

- rubicundus 340

- scapularis 333, 334, 336, 337, 339, 340, $341,342,343,353,400$

- texanus 329

- uriae 334, 337, 339

Ixodidae 327

\section{J}

Johnston's organ $75,125,130$

K

kairomone $28,69,70,71,115,134,136,143$, $147,151,157,161,165,166,167,169$, $181,189,194,195,196,203,205,207$, $235,270,271,272,273,280,281,293$, $297,299,302,304,328,330,337,340$, $399,401,402,403,404$

Kenya $183,184,185,186,190,270,273,275$, $278,280,301,352,355$

Kenyon cells 79

ketone $26,27,52,161,167,168,191,194$, 293 
kissing bug 403

klinokinesis 115, 116, 126, 127, 134, 232

- longitudinal 126

- transverse 126

klinokinetic response 280, 296

klipspringer antelope 337

knock-down spray 403

Kobus ellipsiprymnus 272

kudu 268, 272

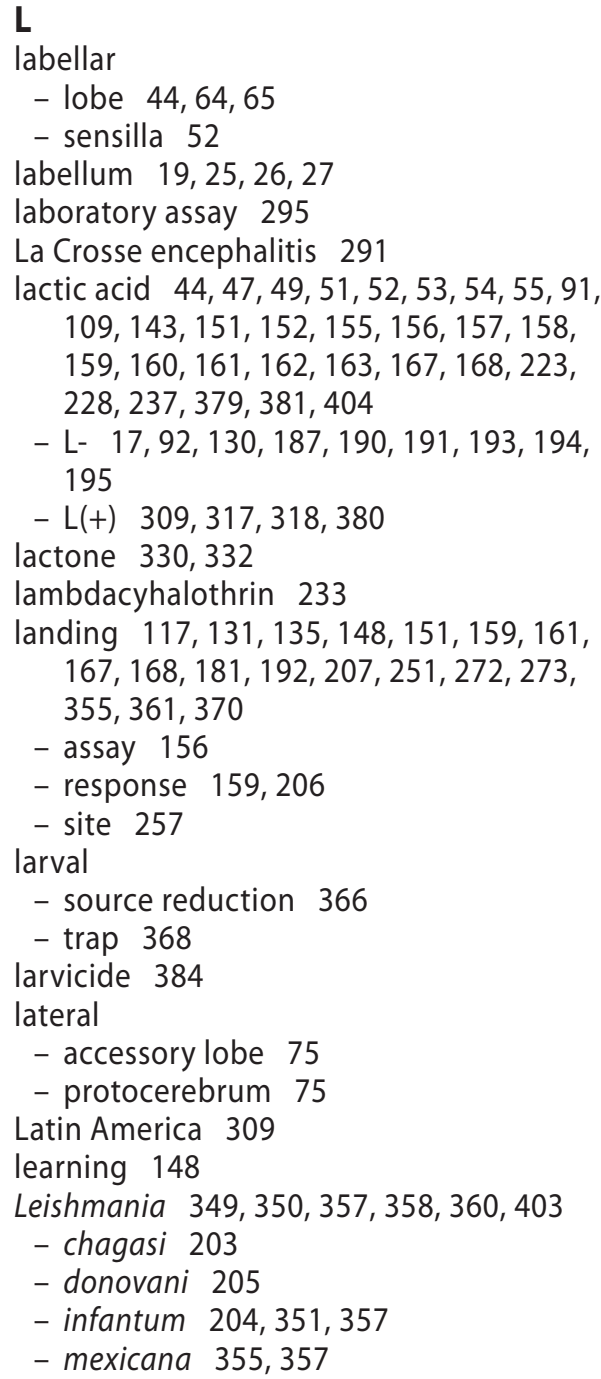

leishmaniasis 13, 17, 204, 206, 207, 209, 211 lekking site 206 lemon eucalyptus 235

lemongrass 191

Lepidoptera 65, 69, 74, 92

Leucocytozoon 249

- simondi 249

- smithi 249

Liberia 183, 185, 274, 277

light trap 370

Limburger cheese 47, 51, 190, 194

limonene $70,72,230,232,372$

linalool 70, 72, 73, 74, 230, 232, 235, 293

lipid extract 337

lipocalin 21

lizard 281

- monitor 276

- odour 277, 278

- urine 276

locomotion compensator 315

locomotor stimulant 335

long-range

- dispersal 374

- orientation 270

- response 268, 269

loon 249, 259, 260

lure 206, 207, 209

- and contaminate' strategy 404

- and kill 196

- and kill tactic 403

Lurex 237

Lurex3 237

LUSH 21, 22, 67

Lutzomyia

- carmelinoi 208

- evandroi 208

- evansi 205

- intermedia 205

- lenti 208

- lichyi 210

- longipalpis 203, 204, 205, 206, 207, 208, 209, 210, 211, 351, 355

- migonei 208

- neivai 208

- tejadai 208

- vexator 210

- whitmani 205, 206, 208

Luxembourg 220

Lyme disease 13, 327, 336, 338, 353

lymphatic filariasis 350 


\section{M}

macroglomerulus 76

Madagascar 183, 184, 185, 190

MAG protein 118

Malaise trap 299

malaria $13,17,39,63,133,144,181,182$, $183,185,187,195,196,291,350,351$, $352,355,357,358,360,361,384,402,404$

Malawi $183,184,185,186$

Malaysia 205, 310

Mali 183,185

mammalophilic 249, 256

Manduca 68

- sexta 76,77

mangrove 222,274

Mansonia 127, 188, 193, 194

- africana 41,189

- uniformis 41,129, 188, 189

mark-release-recapture 122,133

mass-spectrometry 145

mass trapping 196

mating 118, 209

- aggregation 206, 207

- behaviour 210, 211

- disruption 209

- swarm 236

Mauritania $183,185,186$

Mauritius 183, 184

maxillary palp $19,24,27,28,29,30,39,40$, $44,48,52,54,64,65,67,91,92,93,94$,

$102,103,104,127,146,229,230$

- sensilla 96, 97, 106

Mean Female Aedes Index (MFAl) 386, 387, 388

mechanical transmission 350

mechanism

- rate-sensitive 97

- tonic response 97

mechanoreception 116

mechanoreceptive anemotaxis 125

mechanoreceptor 125

mechanosensory cue 130

membrane potential 229

memory

- formation 79

- system 80

p-menthane-3 235

menthol 230, 232 menthone 70,72

meso-2,3-butanediol 164,165

metabotropic 69

metacyclic promastigote 357

Metarhizium anisopliae 341

methanoic acid 152

2-methoxyphenol 273

2-methyl-1-butanol 160, 162

3-methyl-1-butanol 50, 158, 160, 162

2-methyl-2-butanol 204

3-methyl-2-butylamine 313

3-methyl-2-hexanoic acid 236

3-methyl-2-hexenoic acid 50,189

(E/Z)-3-methyl-2-hexenoic acid 159, 161

4-methyl-2-methoxyphenol 273, 283

4-methyl-2-nitrophenol 331,336

6-methyl-5-hepten-2-one 47, 53, 143, 154, $156,160,161,169,228,230,232,235$

methyl-amine 313

2-methylbutanal 160,162

3-methylbutanal 160,162

2-methylbutanoic acid 160, 162

2-oxo-3-methylbutanoic acid 159, 161

3-methylbutanoic acid 151, 153, 158, 160, $162,163,187,191,194,332$

methylbutyrate 48

2-methylbutyric acid 313

4-methylcyclohexanol 48, 50,72

2-methyldocosane 224

8-methyldocosane 224

9-methylgermacrene 203, 204, 208

9-methylgermacrene-B 203, 208

S-9-methylgermacrene-B 204, 207

3-methylindole $47,53,70,72,334,336,372$, 373

4-methylnitrophenol 332

2-oxo-3-methylpentanoic acid 160, 161

2-oxo-4-methylpentanoic acid 160, 161

2-methylphenol 332, 336

3-methylphenol 70, 72, 223, 274, 276, 336

4-methylphenol 47, 50, 51, 53, 192, 195, $223,265,270,273,274,276,277,278$, 334,336

methylphenol 30

1S,2S'-2-methylpiperidinyl-3-cyclohexene-1carboxamide 342

2-methylpropanoic acid 158, 161, 332, 334

methyl propyl disulfide $155,156,164,165$ 
methyl salicylate $230,334,339,340,341$

methyl sesquiterpenes 208

methyl sulfide $154,156,164$

9-methyltricosane 224

3-methyl-a-himachalene 203, 207

Mexico 376

MFAI - See: Mean Female Aedes Index

microbiota 145,162

microfilariae 251

microfilarial periodicity 355

microsatellite DNA 281

microvasculature 355

midge 304

Midgeater trap 237

Midg-IT trap 237

migratory flight 133

Mimosa pigra 274

'miombo' woodland 267

miR-279 29

Mitragyna inermis 274

MM-X trap 149

moisture 46, 91, 109, 115, 150

molecular genetics 30

monkey 187,190

Morelia senegalensis 274

Morsitans group 265, 266, 267, 273, 274, $275,278,279$

mosquito $14,20,22,23,24,25,26,27,28$, $29,30,31,32,39,40,41,42,44,46,47,48$, $51,52,53,54,55,63,64,68,69,70,72,73$, $74,76,78,79,80,91,92,93,94,95,96,97$, $98,100,101,102,103,104,106,107,109$, $115,117,118,119,120,121,122,124$, $125,126,127,131,132,134,136,143$, $144,145,146,147,151,155,156,157$, $162,165,166,167,168,169,181,187$, $188,189,190,191,192,193,194,195$, $196,197,205,239,294,298,304,316$, $317,318,319,351,352,353,357,358$, $361,368,390,400,405$

- behaviour 17

- genes 18

- olfactory gene 21

Mosquito Magnet trap 233, 234, 237, 382 MosquitoTRAP 377

MosquiTRAP $365,367,371,372,375,376$, $378,384,385,388,391$ moth $74,76,78,117,122,123,124,126$, $128,135,298$

mouse 317,320

mouthpart 40,74

- sensilla 251

Mozambique 183, 184

Murray Valley encephalitis 291

Muscidae 101

mushroom body $75,79,80$

$\beta$-myrcene 164, 165

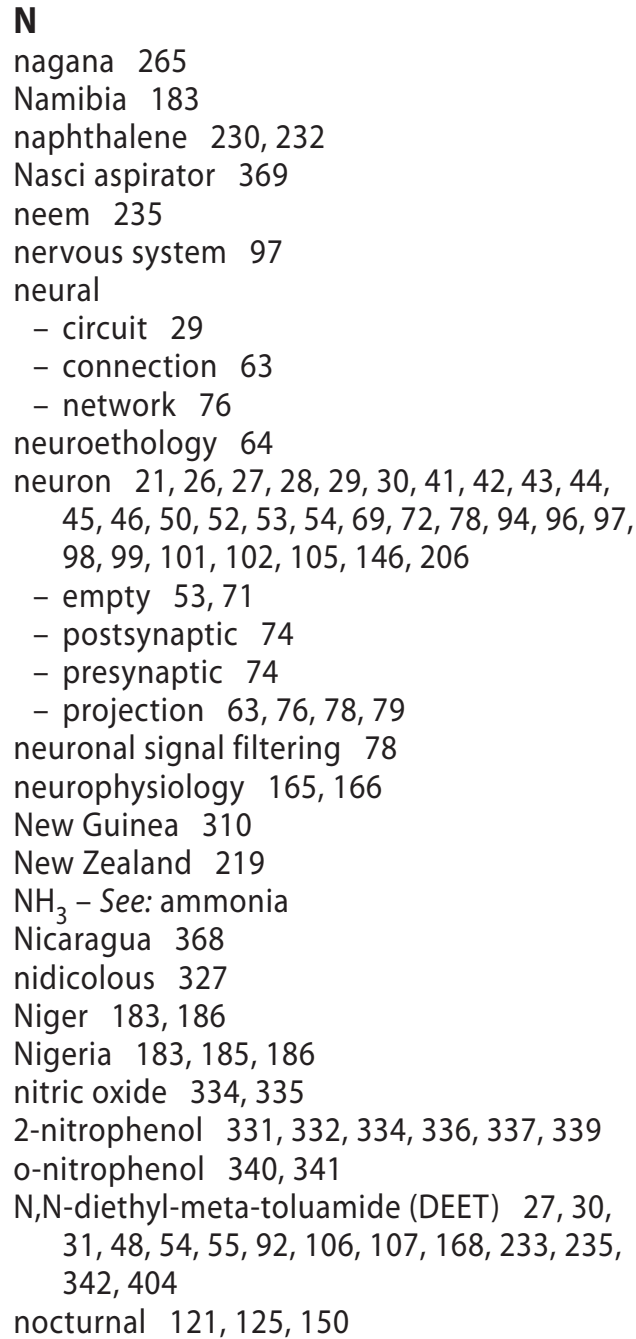


nonanal $70,72,143,147,152,156,162,163$, $164,165,230,232,314,316,317,372$, $373,374,375$

nonanoic acid 151, 153, 159, 161, 163, 332, $334,339,340$

2-nonanone 50

(E)-2-nonenal 230, 232

2-nonene 155

non-nidicolous 327,328

non-random feeding 352

non-vector biting fly 292

nuisance insect 13

nutritional state 118,150

Nzi trap 301

\section{0}

OBET - See: odour-baited entry trap

obligate haematophagy 319

OBP - See: odorant-binding protein

Obp genes 68

octadecanal 165

octadecanoic acid 151, 154, 164, 165

octan-1-ol 293

octanal $72,143,152,156,230,232,314$

octanoic acid 68, 153, 159, 160, 163, 253

1 - and 2-octanol 253

1-octen-3-ol 17, 27, 28, 29, 43, 48, 50, 52, 54, $55,70,72,91,92,106,109,132,156,162$, $187,189,190,192,194,195,207,223$, $228,230,232,233,234,236,237,253$, $265,270,293,294,295,297,298,300$, $301,316,317,318,334,336,337,339,404$

octenal 337

2-octene 155

7-octenoic acid 50,53,159, 161, 187, 189, 194,236

octenol $167,253,254,271,272,274,276$, 277, 278, 282

Odocoileus virginianus 336,337

odorant 14,30,39,44, 46, 76

- -binding protein (OBP) 17, 20, 21, 22, 24, $25,39,44,63,64,65,66,67,80,211,343$, 400

- degrading enzymes 20

- stimuli 26

odorant receptor (OR) 17, 19, 20, 23, 29, 30, $46,63,64,66,76,80,343$

- gene 45
- neuron (ORN) 19, 20, 23, 24, 26, 27, 28, $29,30,31,32,40,41,42,43,44,45,46,47$, $48,50,51,52,53,55,64,66,68,69,70,71$, $72,73,74,76,77,78,231$

- protein 17, 25

odour $48,51,55,71,77,79,115,116,118$, $123,125,129,133,162,165$

- -baited entry trap (OBET) 129, 132, 133, 136, 188, 189, 190, 191, 192, 194, 196

- -baited station 403

- -baited target 282

- -baited trap 14, 217, 239, 278, 283, 309, 401

- blend 17, 27, 46, 69, 71, 144, 145, 157, $166,169,236$

- blend, natural 315

- coding 31, 39, 45, 46, 63, 64, 78

- cue 121,122,134

- -degrading enzyme 343

- discrimination 79

- -mediated upwind anemotaxis 166

- -modulated anemotaxis 314

- -modulated behaviour 401

- plume $71,73,117,119,120,121,122$, $123,124,125,126,127,129,132,133$, $134,135,136,269,296$

- profile 147

- receptor cell (ORC) 312, 313, 314, 316

- source 124, 130

- space 24,30

- stimulus 45,73, 124

olfactometer $95,127,129,143,146,148$, $149,150,151,155,156,158,159,160$, $161,162,163,166,167,168,208,223$, $226,258,272,273,291,294,295,315$, $320,335,375,402$

- dual-port 152, 158, 163, 374

- Y-tube 152, 223, 230, 232, 379

olfactory

- behaviour 14, 15, 80

- coding 80

- cue 47, 119, 125, 146, 150, 165, 168, 182

- gene 20,399, 400

- lobe 55,74

- neuron 42, 106

- organ 52

- physiology 24,39

- processing 77,80 
- protein 18, 23

- receptor 31,39,45,68, 330

- receptor cell 309

- receptor gene 146

- receptor neuron 39, 92, 95, 230

- receptor neuron (ORN) 18

- receptor protein 44

- regulation 14

- response 31

- sensilla $27,41,43,44,48,49,312$

- sensilla type 229

- signal 78,91

- signalling 23,29,69

- signalling pathways 21

- signal transduction 29, 32, 66

- stimuli 144

- transduction 19, 20

ommatidia 256

Onchocerca

- cervicalis 220

- volvulus 248, 261, 350

opossum 310

opportunistic 128, 147, 156, 183, 205

optic lobe 26

optomotor

- anemotaxis 115, 125, 134, 269, 271

- response 123,126

OR - See: odorant receptor

OR7 24

OR83b 24, 44, 66, 68, 69

ORC - See: odour receptor cell

Oreotragus oreotragus 337

orientation $91,92,109,115,116,117,119$, $120,121,122,124,125,127,128,129$, $130,132,134,160$

- behaviour 78

ORN - See: odorant receptor neuron

Ornithodoros erraticus 333, 335

Ornithodoros turicata 340

ornithophilic 147, 249, 257

- species 254

orthokinesis $126,127,232$

orthokinetic response 280, 296

ortholog 29,69, 74

oviposition $47,48,49,50,51,53,54,55,68$, $69,80,99,118,133,136,203,204,205$, 208, 236, 238, 239, 319, 372, 373, 374, $375,376,384,400$
- attractant 365,367

- behaviour 26

- cue 375

- site 371

- site, artificial 370

- skip- 378

- trap 367

ovitrap $368,377,378$

ox

- blood 355

- odour 270, 271, 272, 274, 277, 301

- odour, natural 269

- urine 272

2-oxobutanoic acid 159, 161

oxocarboxylic acid 47, 50, 52

2-oxohexanoic acid 160, 161

2-oxooctanoic acid 160

2-oxopentanoic acid 159, 161

2-oxopropanoic acid 159

ozone 238

P

packed cell volume 354

Palpalis group 265, 266, 267, 274, 275, 278, $279,281,282,283$

Panama 205

Panicum maximum 371, 372, 373, 375

Panstrongylus megistus 310, 321

Papua New Guinea 352

Paraguay 311

Paralobesia viteana 238

parasite periodicity 355

parrafin oil 70,72

passive ambush tactic 328

Patagonia 219

pathway 21

PBP - See: pheromone-binding protein

Pediculus humanus 400

penguin 340

pentacosane 155

pentadecanal 165

pentadecanoic acid 151, 153, 164

pentanoic acid 151, 153, 158, 160, 334, 336, 337

2-pentanone 154, 156

3-pentanone 154, 332

peptide 22,64

peridomestic 311 
peripheral

- olfactory system $53,63,64,74$

- sensory neuron 103

- sensory system 101

Periplaneta americana 76

Peru 311, 368

phagostimulant 251

Phantom Midge 94

pharangeal pump 354

phasic response 73

phasic-tonic discharge pattern 102

phenol 46, 50, 53, 55, 163, 194, 223, 230,

$236,272,273,276,277,282,330,336$

phenylacetaldehyde 341

pheromone $20,21,23,27,32,46,65,69,70$, $71,80,122,123,124,126,135,204,205$, $206,210,211,224,226,236,238,328$

- -binding protein (PBP) 65

- -mediated behaviour 238

- plume 125, 128

- receptor 68

- receptor neuron 96

- tag 341

Phlebotomus 204

- argentipes 205, 207, 208, 210

- duboscai 208,210

- longipes 210

- martini 210

- orientalis 208, 210

- papatasi 208

physical cue 150

physiological state $53,117,118,135$

Phytoseiulus persimilis 74

Picaridin 404

pig 187, 191

pigeon 162,311

a-pinene $48,164,165$

$\mathrm{a}(+)$ pinene 314

plague 359

Plasmodium 181, 350, 353, 403

- chabaudi 351, 354, 356

- falciparum 181, 352, 353, 355

- gallinaceum 351, 356, 358

- knowlesi 181

- malariae 181

- ovale 181

- vivax 181

- yoelii nigeriensis 354, 356 plume $101,109,115,116,119,123,124$, $127,131,132,134,135,151,236,252$, 253, 270, 294, 380, 381 - See also: odour plume

- filamentous 73

- following $115,117,128,129$

- homogeneous 73

- intermittent 130,132

- structure 117, 135, 136

- temperature 234

Porapak 331

positive anemotaxis 314

Premise Index 366, 368

pre-orbital gland 272

presynaptic inhibition 78

Principe 266

probing time 354

proboscis $24,25,39,40,44,52,64,65,67$, 93, 104

promastigote secretory gel 357,358

propanal 163

propanoic acid $151,153,158,160,163,334$

propionic acid 48, 70, 72, 237

3-n-propylphenol 192, 195, 223, 233, 265, 270, 273, 274, 277, 278

Prosimulium decemarticulatum 260

Prostomoxys 301

proteophosphoglycan 357

prothoracic tarsi 251

protocerebrum 79,80

Pterocarpus santalinoides 274

pulsed stimulation 315

push-pull

- approach 341, 342

- effect 338

- strategy 235, 239, 404

- system 234

4-pyridinamine 155

pyridine $155,314,318$

pyruvate 334,337

pyruvic acid 48

Q

quality coding 77

questing 328

- behaviour 335,337 


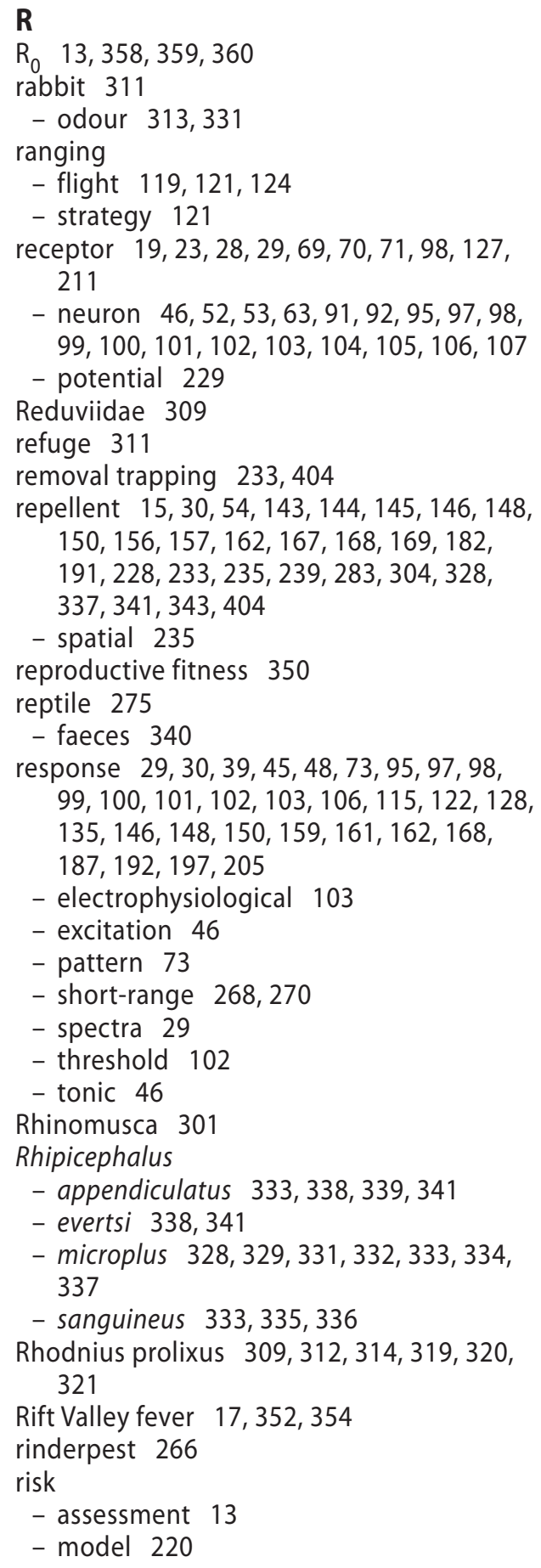

\author{
River Blindness 248 \\ RNAi 400 \\ - knock-down 211 \\ - strategy 68 \\ rolling fulcrum model 258 \\ Russia 221 \\ Rwanda 183, 185, 186
}

\section{$\mathbf{S}$}

Sabethes cayaneus 121

Saccharomyces cerevisiae 320

salicylaldehyde 334

saliva 251

salivary

- antigen 248

- gland protein 353

sandfly $69,124,203,204,205,206,207,208$, $209,211,357,358,400,403$

savanna 256

scanning electron micrograph 92,93

scent-marking behaviour 337

Sclerica bractaeda 371

Scotland 221, 226, 227, 231, 232, 402

scotophase 150, 319

screenhouse 190

screwworm fly 144,402

second messenger 66

secretion 331

semi-field 187, 188, 191, 193, 195, 197, 375, 378,401

- enclosure 401

- study 383

- test 374

semiochemical $21,47,107,115,117,143$, $144,145,146,148,150,165,166,168$, $209,211,231,235,239,341,402,404$

Senegal $183,185,186,190,274$

sensillum $18,21,24,26,30,40,42,44,46$, $47,52,55,63,64,66,67,68,69,71,74,92$, $93,94,95,99,146,206,211,229,329,330$

- ampullacea 41,42

- basiconica 41, 52, 64, 67, 92, 93, 94, 99, $102,104,229,230,309,312,314$

- chaetica 41

- coeloconica 41, 42, 43, 44, 64, 229

- coeloconica, large 42

- trichodea 23, 31, 41, 42, 43, 44, 48, 49, $53,54,64,65,67,69,70,72,73,107,312$ 
sensory

- cue 92, 259

- neuron 64,105, 106

- neuron membrane protein (SNMP) 19, $20,21,23$

- organ 64

- periphery 80

- physiology 399

- stimuli 30

- system 115, 146

Sergentomyia 204

- fallax 208

- minuta 208

servosphere $315,316,317,335$

sesquiterpene 208

sex pheromone 203, 207, 208, 209, 341

sexual dimorphism 74,80

sexually dimorphic macroglomeruli 78

Seychelles 184

sheep 187, 191

- odour 314

short-chain

- aliphatic amine 313

- carboxylic acid 313

- fatty acid 330

Sierra Leone 183, 185, 186

signal 91

- transduction 17, 18, 24, 63

signalling 204

- molecule 68

- pathway 20,66

Simuliidae 101

Simulium

- anatinum 250, 254, 260

- annulus 259

- arcticum 247, 248, 249, 252, 254, 256, 257

- aureum 260

- damnosum 250, 255, 256, 260

- euryadminiculum 249, 250, 254, 257, 259,260

- luggeri 248

- meridionale 254

- rugglesi 250, 254, 257, 259, 260

- venustum 248, 251, 252, 255

- vittatum 249, 251

Singapore 384

single sensillum
- electrophysiology 330, 332

- recording (SSR) 26, 27, 45, 69, 146, 206, 228,312

singleton 24

sit-and-wait strategy 121,268

skin 205, 206

- emanation 127, 129, 143, 145, 155, 157, $158,162,235$

- microbial flora 351

- odour 120, 128, 130,317, 335

- secretion 255

SkinLure 383

sleeping sickness 278

smell 39

snapping turtle 248

SNMP - See: sensory neuron membrane protein

sodium hydroxide 335

soft tick 327

solid phase micro extraction (SPME) 228, 372

solvent washings 228

Somalia 183, 184, 185, 186, 268, 274, 279, 280,282

sound 91

South Africa $183,184,185,186,266,268$, 280

South America 249

spider mite 74

SPME - See: solid phase micro extraction

Spodoptera littoralis 76

squalene $155,334,337$

Sri Lanka 207

SSR 71, 73 - See: single sensillum recording stable fly 291, 292, 294, 296, 297, 299, 302, 304

Staphylococcus epidermidis 145

steer hair extract 332

Stegomyia 368

sticky panel 266

sticky trap 252, 367, 372, 376, 377, 378, 391

Stomoxyinae 291, 299

Stomoxys 291, 293, 298, 301, 303

- calcitrans 236, 292, 300

- niger niger 300

- nigra 350

- sitiens 300

Stygeromyia 301 
styrene 155

suboesophageal ganglion 26, 27

suction trap 121

Sudan 183, 185, 186

sugar feeding 236

sulcatone 50

sulfide 164

surveillance $14,309,340$

- system 299

- tool 299

Swaziland 184

sweat 47, 51, 54, 130, 157, 161, 195

Sweden 259

Sweetscent dispenser 380

synergism 55, 152, 158, 159, 169, 194, 209

synergistic effect $47,167,379$

Syria 186

Syzygium guineense 274

\section{T}

Tabanidae 101, 121

Tanzania 183, 184, 185, 186, 188, 189, 191, $192,193,402$

targeted on-host control 341

temporal coding 73

tent trap 254

terpene $46,207,208,314$

terpinen-4-ol 314, 318

terpineol 51

- $a(+) \quad 314$

tetradecanal 165

tetradecanoic acid 151, 153, 159, 160, 164, 165

Thailand 368

The Gambia 191, 193

theileriosis 327,336

The Netherlands 220

thermal cue 117

thermoreceptor 126,340

thigmotaxis 311,312

a-thujone $48,51,70,72$

tick $14,69,74,327$

- -borne disease 13

- -borne encephalitis 327

- climbing bioassay 338

- repellent 342

tissue, olfactory 21

Togo 183, 185, 186 toluene 155

tonic spiking pattern 74

tourism 221

Toxorhynchites brevipalpis 121

transduction 20

- mechanism 68,80

- olfactory 18,44

- pathway 19

transgene 29,67

transmission 106

- risk 405

trap 14, 108, 117, 129, 131, 132, 144, 157, $162,181,187,188,189,190,191,192$, $193,194,195,196,205,207,208,232$, $233,236,237,261$

Triatoma

- brasiliensis 321

- dimidiata 309, 321

- infectans 350

- infestans 309, 310, 312, 313, 314, 315, $317,319,320,321$

- pallidipennis 321

- pseudomaculata 321

Triatominae 309,310

triatomine $69,315,317,319,320,321,356$

- bug 144, 309, 311

- trap 321

tridecanoic acid 153, 159, 161, 163

2,4,5-trimethylthiazole 48

Trypanosoma 265, 277

- brucei 282

- brucei gambiense 266, 282,350

- brucei rhodesiense 282, 350

- congolense 282,352, 355

- cruzi 309, 310, 318, 350, 356

- vivax 282, 355

trypanosomiasis $13,161,265,266,275,278$, $279,282,283,291$

tsetse $14,117,118,119,121,123,124,125$, $132,135,144,161,167,207,230,236$, 237, 253, 261, 265, 269, 270, 291, 292, $296,297,298,299,301,303,304,336$, $355,357,361,402,403,404,405$

- control 282

tuning $69,70,71$

- curve 71,77

- profile 71,79

Tunisia 208 
turbulence $116,122,125$

turbulent

- diffusion 120

- flow 124

- plume 157

- presentation 129

- stream 109

\section{U}

Uganda 183, 185, 186, 275, 282

Ulomyia fuliginosa 203

undecanal 165,338

undecanoic acid 151, 153, 163

2-undecanone 70,72

United Kingdom 220, 235

upwind

- anemotaxis $134,252,271,295,296,298$, 301

- flight 381

- flight behaviour 401

- movement 295

uric acid 334,340

urine $192,235,336,351$

- odour 313

uropygial

- gland 254, 260

- odour 250, 259

Ursus americanus 249

Uruguay 311

USA 221, 222, 223, 234, 235

\section{V}

11-cis vaccenyl acetate 67

vacuum distillation 228

Y-valerolactone 331, 332

Varanus niloticus niloticus 276

vasodilator 353,358

Vavoua trap 299, 301

vector

- behaviour, changes in 356

- -borne disease 106, 143, 144, 181

- control 14, 32, 405

- longevity 350

- manipulation 359

vectorial capacity $182,203,360$

ventilated pits 268

Vesicular Stomatitis Virus 249

visual
- contact 256

- cue 46, 117, 121, 125, 126, 132, 134, 136, 150,292

- resolution 121

- stimulus 117, 130, 247

voltinism 238

Vulpes vulpes 206

\section{W}

Wahlund effect 281

warthog 268, 269, 272, 276

water 48

waterbuck 272, 273

water buffalo 223, 230

waterfowl 259

weather

- effect of 226

West Africa 247

West Nile 13, 17, 39, 103, 144, 146, 165, 384

white-tailed deer 336,337

Wilhelmia equina 257

Wilton trap 367,370

wind

- speed 226

- tunnel 120,121, 122, 126, 128, 131, 135, $136,143,148,152,157,159,232,258$, 269, 294, 295, 296, 335, 381, 401, 402

Wuchereria bancrofti 350, 355

Wyomia smithii 41

\section{$\mathbf{X}$}

xanthine 334,339

Xenopus 27, 29, 31, 146

$\mathbf{Y}$

yeast-baited trap 321

yeast odour 320

yellow fever 17, 39, 354, 365, 368, 401, 402

Yersinia pestis 357,359

Y-tube - See: olfactometer, Y-tube

$\mathbf{Z}$

z-4-decanal 152,156

Zambia 183, 185, 186, 268, 278

Zanzibar 184

zebra 273

Zimbabwe 183, 184, 185, 186, 192, 268, 270, $273,278,279,280,300,301,303,341$ 
zoonosis 310

zoophagic 184

zoophilic 43, 129, 162, 183, 184, 186 










